\title{
Direct observation of knock-on in surface reactions at zero impact parameter
}

\author{
Matthew J. Timm§, Lydie Leung§, and John C. Polanyi* \\ Lash Miller Chemical Laboratories, Department of Chemistry, University of Toronto, 80 St. \\ George Street, Toronto, Ontario M5S 3H6, Canada
}

$\S$ Matthew J. Timm and Lydie Leung contributed equally to this work.

*e-mail: john.polanyi@utoronto.ca 
This file includes:

\section{Methods}

Supplementary Figures 1

Supplementary Tables 1-4 


\section{Contents}

1. Obtaining Minimum Energy Pathways for single and double $\mathrm{CF}_{2}$ knock-on ...........4

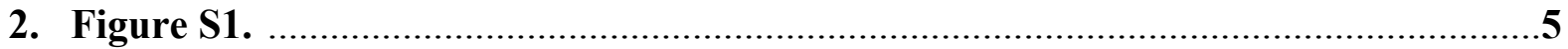

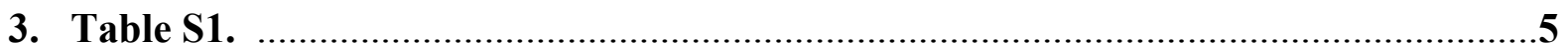

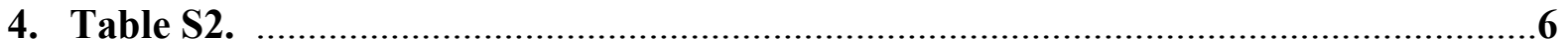

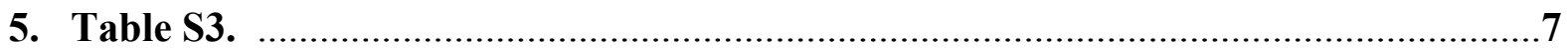

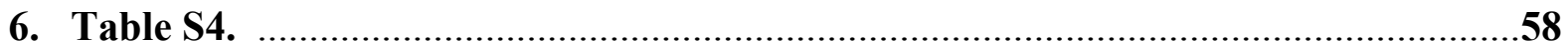




\section{Obtaining Minimum Energy Pathways for single and double $\mathrm{CF}_{2}$ knock-on.}

The minimum energy pathway (MEP) for single and double $\mathrm{CF}_{2}$ knock-on were obtained through climbing image nudged-elastic-band (CI-NEB) calculations.

For the CI-NEB calculations involving single $\mathrm{CF}_{2}$ knock-on, the initial state (IS) and final state (FS) corresponded to those in experiment (Figure 1a and Figure 1b respectively). Between the IS and the FS fourteen interstitial images were used. A local minimum was found along the reaction coordinate between the IS and FS (Figure S1, CI-NEB image 6) and was relaxed following the same procedure used for the IS and FS states. This minimum follows dissociation of the in-plane $\mathrm{C}-\mathrm{F}$ bond of the $\mathrm{CF}_{3}$ precursor. Four interstitial images were used to obtain the barrier between the IS and this local minimum. To obtain the barrier (TS) between the local minimum and the FS, 10 interstitial images were used. This is the barrier to $\mathrm{CF}_{2}$ knock-on.

For double $\mathrm{CF}_{2}$ knock-on, the initial state (IS) and final state (FS) corresponded to those observed in experiment, given in Figure $3 \mathrm{a}$ and Figure $3 \mathrm{~b}$ of the main text. Between the IS and FS, twenty-three interstitial images were used. Two local minima were found along the reaction coordinate between the IS and FS. The first minimum followed the dissociation of the in-plane $\mathrm{C}-\mathrm{F}$ bond of the $\mathrm{CF}_{3}$ precursor (Figure S2, CI-NEB image 7). The second local minimum followed the first knock-on (Figure S2, CI-NEB image 17). Both local minima were relaxed, following the same procedure used for the IS and FS states. Five interstitial images were used to obtain the first barrier, between the IS and the first minimum. The second barrier (TS1), between the first minimum and the second minimum, was obtained using nine interstitial images. The third barrier (TS2), between the second minimum and the FS, was obtained using nine interstitial images. This minimum energy pathway describes the mechanism for double knock-on reaction. 


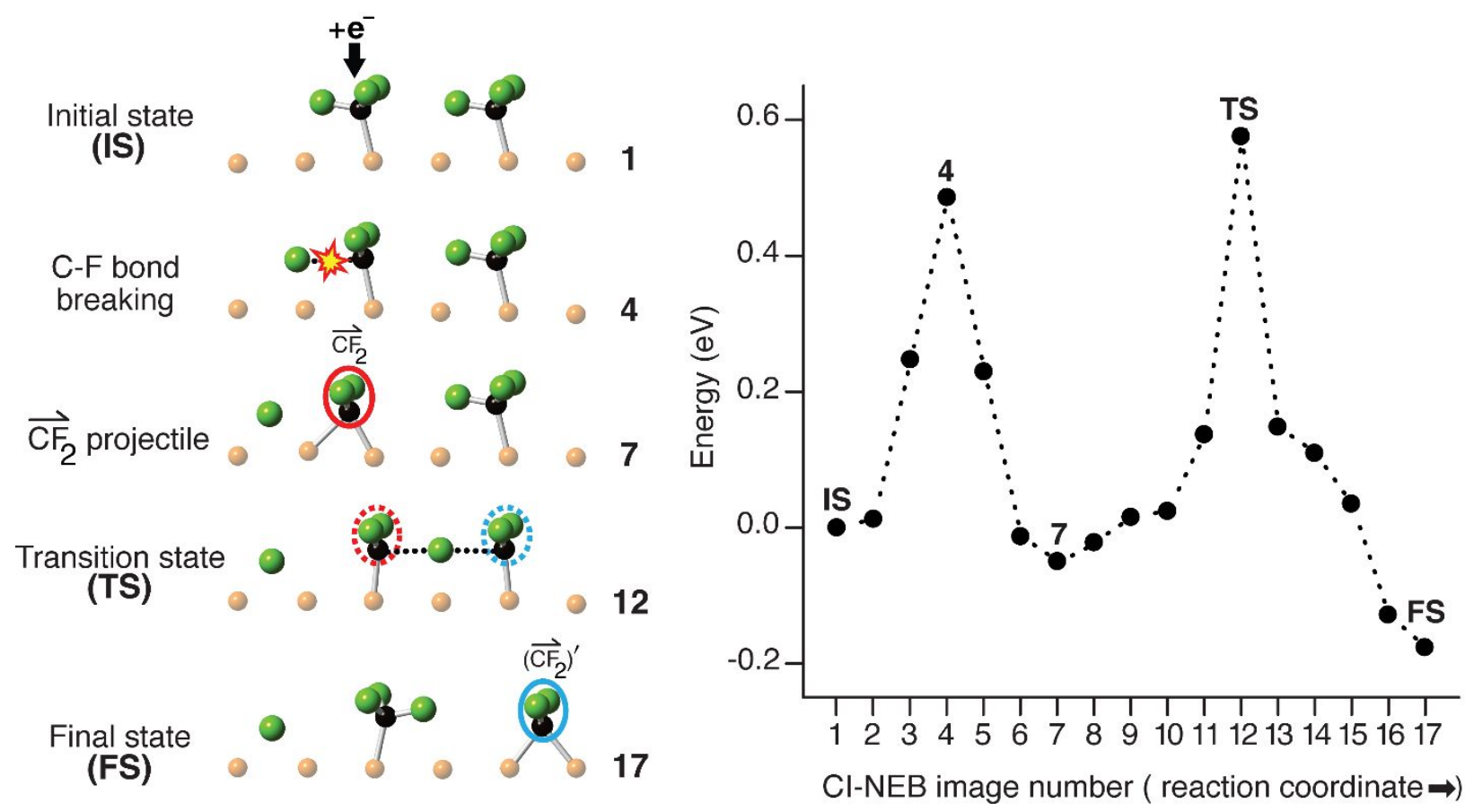

Figure S1. Minimum energy pathway (MEP) across the DFT potential energy surface obtained from CI-NEB calculations for $\mathrm{CF}_{2}$ knock-on. Two steps are required for knock-on. The first step involves creation of the $\mathrm{CF}_{2}$ projectile from dissociation of the $\mathrm{CF}_{3}$ precursor (image 3 ). The second step involves fluorine-exchange between the $\mathrm{CF}_{2}$ projectile (red) and $\mathrm{CF}_{3}$ target to form inverted $\left(\mathrm{CF}_{3}\right)^{\prime}$ and knocked-on $\left(\mathrm{CF}_{2}\right)^{\prime}$ (blue).

Table S1. $\mathrm{CF}_{2}$ single knock-on, $\mathrm{CI}-\mathrm{NEB}$ image number, calculated energy and calculated reaction barrier height.

\begin{tabular}{c|cc} 
CI-NEB image number & Energy Relative to IS (eV) & Barrier Height (eV) \\
\hline $\mathbf{1}$ & 0.0000 & \\
$\mathbf{2}$ & 0.0127 & \\
$\mathbf{3}$ & 0.2484 & 0.4878 \\
$\mathbf{4}$ & 0.4878 & \\
$\mathbf{5}$ & 0.2303 & \\
$\mathbf{6}$ & -0.0131 & \\
$\mathbf{7}$ & -0.0500 & \\
$\mathbf{8}$ & -0.0220 & \\
$\mathbf{9}$ & 0.0153 & \\
$\mathbf{1 0}$ & 0.0243 & \\
$\mathbf{1 1}$ & 0.1375 & \\
$\mathbf{1 2}$ & 0.5778 & \\
$\mathbf{1 3}$ & 0.1488 & \\
$\mathbf{1 4}$ & 0.1099 & \\
$\mathbf{1 5}$ & 0.0350 & \\
$\mathbf{1 7}$ & -0.1288 & \\
\hline
\end{tabular}


Table S2. $\mathrm{CF}_{2}$ Double Knock-On, CI-NEB image number, calculated energy and calculated reaction barrier height.

\begin{tabular}{|c|cc} 
CI-NEB image number & Energy Relative to IS (eV) & Barrier Height (eV) \\
\hline $\mathbf{1}$ & 0.0000 & \\
$\mathbf{3}$ & 0.0156 & \\
$\mathbf{4}$ & 0.2822 & 0.4888 \\
$\mathbf{5}$ & 0.4888 & \\
$\mathbf{6}$ & 0.2650 & \\
$\mathbf{7}$ & 0.0701 & \\
$\mathbf{8}$ & 0.0295 & \\
$\mathbf{9}$ & 0.0649 & \\
$\mathbf{1 0}$ & 0.0896 & \\
$\mathbf{1 1}$ & 0.0976 & \\
$\mathbf{1 2}$ & 0.1901 & \\
$\mathbf{1 3}$ & 0.5318 & \\
$\mathbf{1 4}$ & 0.4243 & \\
$\mathbf{1 5}$ & 0.1700 & \\
$\mathbf{1 6}$ & 0.1000 & \\
$\mathbf{1 8}$ & 0.0929 & \\
$\mathbf{1 9}$ & 0.0932 & \\
$\mathbf{2 0}$ & 0.0973 & \\
$\mathbf{2 1}$ & 0.1123 & \\
$\mathbf{2 2}$ & 0.2329 & \\
$\mathbf{2 3}$ & 0.4797 & \\
$\mathbf{2 4}$ & 0.5804 & \\
$\mathbf{2 5}$ & 0.1690 & \\
$\mathbf{2 6}$ & 0.1418 & \\
$\mathbf{2 7}$ & 0.1049 & \\
& -0.0452 & \\
& -0.1090 & \\
\hline
\end{tabular}


Table S3. $\mathrm{CF}_{2}$ Single Knock-On, element type, $\mathrm{x}$-coordinate, $\mathrm{y}$-coordinate, $\mathrm{z}$-coordinate for all 158 atoms in CI-NEB images 1 to 17.

CI-NEB Image 1

\begin{tabular}{|c|c|c|c|}
\hline Element & X-Coordinate $(\AA)$ & Y-Coordinate (§) & Z-Coordinate (§) \\
\hline $\mathbf{C}$ & 3.56654700865991 & 7.94626110906592 & 10.62703072854130 \\
\hline $\mathbf{C}$ & 3.56706259468629 & 13.08395592735320 & 10.62143697350860 \\
\hline $\mathbf{F}$ & 3.56819854896444 & 9.35828542997345 & 10.89034255142980 \\
\hline $\mathbf{F}$ & 2.46870116863259 & 7.49018575450469 & 11.32289650929990 \\
\hline $\mathbf{F}$ & 4.66268319967337 & 7.48725968725703 & 11.32372268825670 \\
\hline $\mathbf{F}$ & 3.56622290441861 & 14.51011018856590 & 10.87695173826370 \\
\hline $\mathbf{F}$ & 2.47103374672789 & 12.63964814012820 & 11.32078240454680 \\
\hline $\mathbf{F}$ & 4.66343875110618 & 12.64092222814700 & 11.32122416557160 \\
\hline $\mathbf{C u}$ & 0.00000000000000 & 0.00000000000000 & 3.56221131249991 \\
\hline $\mathrm{Cu}$ & 0.00000000000000 & 2.52252111840003 & 3.56221131249991 \\
\hline $\mathbf{C u}$ & 0.00000000000000 & 5.04504223680007 & 3.56221131249991 \\
\hline $\mathrm{Cu}$ & 0.00000000000000 & 7.56756335519993 & 3.56221131249991 \\
\hline $\mathbf{C u}$ & 0.00000000000000 & 10.09008447359990 & 3.56221131249991 \\
\hline $\mathrm{Cu}$ & 0.00000000000000 & 12.61260559199990 & 3.56221131249991 \\
\hline $\mathbf{C u}$ & 0.00000000000000 & 15.13512671040000 & 3.56221131249991 \\
\hline $\mathrm{Cu}$ & 0.00000000000000 & 17.65764782880000 & 3.56221131249991 \\
\hline $\mathrm{Cu}$ & 0.00000000000000 & 20.18016894719990 & 3.56221131249991 \\
\hline $\mathbf{C u}$ & 0.00000000000000 & 22.70269006559990 & 3.56221131249991 \\
\hline $\mathrm{Cu}$ & 3.56738357634323 & 0.00000000000000 & 3.56221131249991 \\
\hline $\mathrm{Cu}$ & 3.56738357634323 & 2.52252111840003 & 3.56221131249991 \\
\hline $\mathbf{C u}$ & 3.56738357634323 & 5.04504223680007 & 3.56221131249991 \\
\hline $\mathrm{Cu}$ & 3.56738357634323 & 7.56756335519993 & 3.56221131249991 \\
\hline $\mathrm{Cu}$ & 3.56738357634323 & 10.09008447359990 & 3.56221131249991 \\
\hline $\mathrm{Cu}$ & 3.56738357634323 & 12.61260559199990 & 3.56221131249991 \\
\hline $\mathrm{Cu}$ & 3.56738357634323 & 15.13512671040000 & 3.56221131249991 \\
\hline $\mathrm{Cu}$ & 3.56738357634323 & 17.65764782880000 & 3.56221131249991 \\
\hline $\mathbf{C u}$ & 3.56738357634323 & 20.18016894719990 & 3.56221131249991 \\
\hline $\mathrm{Cu}$ & 3.56738357634323 & 22.70269006559990 & 3.56221131249991 \\
\hline $\mathbf{C u}$ & 7.13476715482693 & 0.00000000000000 & 3.56221131249991 \\
\hline $\mathrm{Cu}$ & 7.13476715482693 & 2.52252111840003 & 3.56221131249991 \\
\hline $\mathrm{Cu}$ & 7.13476715482693 & 5.04504223680007 & 3.56221131249991 \\
\hline $\mathbf{C u}$ & 7.13476715482693 & 7.56756335519993 & 3.56221131249991 \\
\hline $\mathbf{C u}$ & 7.13476715482693 & 10.09008447359990 & 3.56221131249991 \\
\hline $\mathrm{Cu}$ & 7.13476715482693 & 12.61260559199990 & 3.56221131249991 \\
\hline $\mathrm{Cu}$ & 7.13476715482693 & 15.13512671040000 & 3.56221131249991 \\
\hline $\mathrm{Cu}$ & 7.13476715482693 & 17.65764782880000 & 3.56221131249991 \\
\hline $\mathrm{Cu}$ & 7.13476715482693 & 20.18016894719990 & 3.56221131249991 \\
\hline $\mathbf{C u}$ & 7.13476715482693 & 22.70269006559990 & 3.56221131249991 \\
\hline $\mathrm{Cu}$ & 1.78369178870677 & 1.26126055919993 & 4.82343353250005 \\
\hline $\mathbf{C u}$ & 1.78369178870677 & 3.78378167759996 & 4.82343353250005 \\
\hline $\mathbf{C u}$ & 1.78369178870677 & 6.30630279600000 & 4.82343353250005 \\
\hline $\mathrm{Cu}$ & 1.78369178870677 & 8.82882391440003 & 4.82343353250005 \\
\hline $\mathrm{Cu}$ & 1.78369178870677 & 11.35134503280000 & 4.82343353250005 \\
\hline $\mathrm{Cu}$ & 1.78369178870677 & 13.87386615119990 & 4.82343353250005 \\
\hline $\mathbf{C u}$ & 1.78369178870677 & 16.39638726959990 & 4.82343353250005 \\
\hline $\mathrm{Cu}$ & 1.78369178870677 & 18.91890838799990 & 4.82343353250005 \\
\hline $\mathrm{Cu}$ & 1.78369178870677 & 21.44142950640000 & 4.82343353250005 \\
\hline $\mathrm{Cu}$ & 1.78369178870677 & 23.96395062480000 & 4.82343353250005 \\
\hline $\mathbf{C u}$ & 5.35107536505000 & 1.26126055919993 & 4.82343353250005 \\
\hline
\end{tabular}




\begin{tabular}{|c|c|c|c|}
\hline $\mathbf{C u}$ & 5.35107536505000 & 3.78378167759996 & 4.82343353250005 \\
\hline $\mathbf{C u}$ & 5.35107536505000 & 6.30630279600000 & 4.82343353250005 \\
\hline $\mathbf{C u}$ & 5.35107536505000 & 8.82882391440003 & 4.82343353250005 \\
\hline $\mathbf{C u}$ & 5.35107536505000 & 11.35134503280000 & 4.82343353250005 \\
\hline $\mathrm{Cu}$ & 5.35107536505000 & 13.87386615119990 & 4.82343353250005 \\
\hline $\mathbf{C u}$ & 5.35107536505000 & 16.39638726959990 & 4.82343353250005 \\
\hline $\mathbf{C u}$ & 5.35107536505000 & 18.91890838799990 & 4.82343353250005 \\
\hline $\mathbf{C u}$ & 5.35107536505000 & 21.44142950640000 & 4.82343353250005 \\
\hline $\mathbf{C u}$ & 5.35107536505000 & 23.96395062480000 & 4.82343353250005 \\
\hline $\mathbf{C u}$ & 8.91845894246346 & 1.26126055919993 & 4.82343353250005 \\
\hline $\mathbf{C u}$ & 8.91845894246346 & 3.78378167759996 & 4.82343353250005 \\
\hline $\mathbf{C u}$ & 8.91845894246346 & 6.30630279600000 & 4.82343353250005 \\
\hline $\mathbf{C u}$ & 8.91845894246346 & 8.82882391440003 & 4.82343353250005 \\
\hline $\mathrm{Cu}$ & 8.91845894246346 & 11.35134503280000 & 4.82343353250005 \\
\hline $\mathrm{Cu}$ & 8.91845894246346 & 13.87386615119990 & 4.82343353250005 \\
\hline $\mathrm{Cu}$ & 8.91845894246346 & 16.39638726959990 & 4.82343353250005 \\
\hline $\mathrm{Cu}$ & 8.91845894246346 & 18.91890838799990 & 4.82343353250005 \\
\hline $\mathbf{C u}$ & 8.91845894246346 & 21.44142950640000 & 4.82343353250005 \\
\hline $\mathbf{C u}$ & 8.91845894246346 & 23.96395062480000 & 4.82343353250005 \\
\hline $\mathbf{C u}$ & 0.00233872401564 & 12720 & 632599 \\
\hline $\mathrm{Cu}$ & 10.70026568192440 & 58088 & 7588000 \\
\hline $\mathrm{Cu}$ & 10.69806915106760 & 5.03 & 6.09256 \\
\hline $\mathrm{Cu}$ & 10.69706303035580 & 895 & 25413 \\
\hline $\mathrm{Cu}$ & 10.69552477603530 & 74169461610 & 7772452 \\
\hline $\mathbf{C u}$ & 0.00155905615148 & 80210 & 621874 \\
\hline $\mathbf{C u}$ & 10.69900001011200 & 15.13794942618650 & 6.09466982151825 \\
\hline $\mathbf{C u}$ & 10.70083630048350 & 17.65362482618980 & 6.09798897995926 \\
\hline $\mathbf{C u}$ & 0.00308327975037 & 20.18096805690270 & 6.09437530098834 \\
\hline $\mathbf{C u}$ & 10.69964402751900 & 22.70312763613370 & 6.09406011908406 \\
\hline $\mathbf{C u}$ & 3.56736562067597 & 0.00200915417214 & 6.09348855329227 \\
\hline $\mathbf{C u}$ & 3.56739318716801 & 2.52302943320059 & 6.08923629508686 \\
\hline $\mathrm{Cu}$ & 3.56742344510544 & 5.04709899725720 & 6.10147311479868 \\
\hline $\mathbf{C u}$ & 3.56741674184976 & 7.57027395009167 & 6.13755759183053 \\
\hline $\mathbf{C u}$ & 3.56741466819020 & 10.08742040285930 & 6.13488227844701 \\
\hline $\mathbf{C u}$ & 3.56740265176300 & 12.61236604744940 & 6.14003280176090 \\
\hline $\mathrm{Cu}$ & 3.56741693736277 & 15.12359795320010 & 97311446 \\
\hline $\mathbf{C u}$ & 3.56740898441015 & 17.65501081339600 & 6.08897 \\
\hline $\mathrm{Cu}$ & 3.56740693809632 & 20.17999807718830 & 6.09448590695678 \\
\hline $\mathrm{Cu}$ & 3.56738854770298 & 22.70404643318960 & 6.09287977204592 \\
\hline $\mathbf{C u}$ & 7.13242904077274 & 25.22343235937390 & 6.09456912663985 \\
\hline $\mathrm{Cu}$ & 7.13664466135708 & 2.52278810082966 & 6.09676254315464 \\
\hline $\mathrm{Cu}$ & 7.13881411085872 & 5.03846025464647 & 6.09255392057190 \\
\hline $\mathbf{C u}$ & 7.13983016697939 & 7.56894287746128 & 6.10145456284498 \\
\hline $\mathbf{C u}$ & 7.14141890878447 & 10.09272143841560 & 6.09295246406980 \\
\hline $\mathbf{C u}$ & 7.13327625373073 & 12.60673650648210 & 6.10409682291832 \\
\hline $\mathbf{C u}$ & 7.13792485449400 & 15.13797521810890 & 6.09463357250274 \\
\hline $\mathbf{C u}$ & 7.13606117307552 & 17.65362255412790 & 6.09796904825854 \\
\hline $\mathrm{Cu}$ & 7.13164939433882 & 20.18099940732420 & 6.09437825187965 \\
\hline $\mathbf{C u}$ & 7.13723792445528 & 22.70312395557520 & 6.09404778742811 \\
\hline $\mathrm{Cu}$ & 1.78396833141506 & 1.25912704366430 & 7.38600455650923 \\
\hline $\mathbf{C u}$ & 1.78461550262739 & 3.78338532816827 & 7.38598100633007 \\
\hline $\mathrm{Cu}$ & 1.78101848526112 & 6.30041374156188 & 7.37773687901787 \\
\hline $\mathrm{Cu}$ & 1.77587721793481 & 8.82914099027826 & 7.37730614969199 \\
\hline $\mathrm{Cu}$ & 1.79510224909125 & 11.34163648346990 & 7.38734635655565 \\
\hline
\end{tabular}




\begin{tabular}{|c|c|c|c|}
\hline $\mathbf{C u}$ & 1.77326543311424 & 13.87641918560480 & 7.38174287011690 \\
\hline $\mathbf{C u}$ & 1.78922907088014 & 16.39319946176190 & 7.38870598916749 \\
\hline $\mathbf{C u}$ & 1.78144534874967 & 18.91905892790990 & 7.38566428085755 \\
\hline $\mathbf{C u}$ & 1.78561843951229 & 21.44506924061750 & 7.38414057375588 \\
\hline $\mathbf{C u}$ & 1.78543028272084 & 23.96266535484240 & 7.38670607370499 \\
\hline $\mathbf{C u}$ & 5.35081243468083 & 1.25907192000975 & 7.38595739291754 \\
\hline $\mathbf{C u}$ & 5.35025041682398 & 3.78332888675486 & 7.38594870392595 \\
\hline $\mathbf{C u}$ & 5.35384198230093 & 6.30039433145570 & 7.37767370433251 \\
\hline $\mathbf{C u}$ & 5.35903973729104 & 8.82909000435620 & 7.37718171796475 \\
\hline $\mathbf{C u}$ & 5.33986463057284 & 11.34162974076480 & 7.38710044752867 \\
\hline $\mathbf{C u}$ & 5.36164197551943 & 13.87645176431110 & 7.38167042400865 \\
\hline $\mathbf{C u}$ & 5.34561796155026 & 16.39319053191220 & 7.38869751514158 \\
\hline $\mathbf{C u}$ & 5.35338409076582 & 18.91906334173880 & 7.38563330009420 \\
\hline $\mathbf{C u}$ & 5.34920638095102 & 21.44508819098320 & 7.38415821363045 \\
\hline $\mathbf{C u}$ & 5.34934379697408 & 23.96262925588390 & 7.38669161603560 \\
\hline $\mathbf{C u}$ & 8.91846891605871 & 1.26390045437242 & 7.39370054035572 \\
\hline $\mathbf{C u}$ & 8.91845338029924 & 3.78049314335096 & 7.39366126052490 \\
\hline $\mathrm{Cu}$ & 8.91843741255743 & 10330 & 7.3974494 \\
\hline $\mathbf{C u}$ & 8.91845637591423 & 2767 & 7.3949705 \\
\hline $\mathbf{C u}$ & 8.91848657579658 & 6270630 & 7.402392 \\
\hline $\mathbf{C u}$ & 8.918 & 13 & 7.3 \\
\hline $\mathbf{C u}$ & 8.91 & 320 & 2648 \\
\hline $\mathbf{C u}$ & 8.9 & & 389 \\
\hline $\mathbf{C u}$ & 8.91844279794398 & 372006860 & 7.39292490289452 \\
\hline $\mathbf{C u}$ & 8.91846800685699 & 409270 & 7.39228955080519 \\
\hline $\mathbf{C u}$ & 10.70 & 25. & 8.609 \\
\hline $\mathbf{C u}$ & 0.005068 & 52 & 277877978 \\
\hline $\mathbf{C u}$ & 0.00 & 509 & 8.60 \\
\hline $\mathbf{C u}$ & 0.00 & 7.5 & 3122 \\
\hline $\mathbf{C u}$ & 0.00 & 10.0 & 6141 \\
\hline $\mathbf{C u}$ & 0.00 & 960 & 8472 \\
\hline $\mathbf{C u}$ & 10.70063 & 016250 & 77385912258 \\
\hline $\mathbf{C u}$ & 0.00463318403741 & 17.65263686049550 & 8.61395318204213 \\
\hline $\mathbf{C u}$ & 0.00016500865649 & 20.17920817007460 & 8.60693619599039 \\
\hline $\mathbf{C u}$ & 00149123366093 & 22.70417459458610 & 8.60861040090877 \\
\hline $\mathbf{C u}$ & 3.56739374927921 & 25.22133150439660 & 8.61527725664305 \\
\hline $\mathbf{C u}$ & 3.56740960698214 & 2.51216499531550 & 8.60905244484454 \\
\hline $\mathbf{C u}$ & 3.56747160740420 & 5.01818769196023 & 8.61127166838501 \\
\hline $\mathbf{C u}$ & 3.56739861016771 & 7.54205583643675 & 8.69388379271914 \\
\hline $\mathbf{C u}$ & 3.56760849442771 & 10.08808362261140 & 8.65308899203836 \\
\hline $\mathbf{C u}$ & 3.56763935467914 & 12.61163147771790 & 8.70183271341820 \\
\hline $\mathbf{C u}$ & 3.56747330456167 & 15.16144282347170 & 8.65699172331805 \\
\hline $\mathbf{C u}$ & 3.56742571863413 & 17.67477623483690 & 8.59768874422721 \\
\hline $\mathbf{C u}$ & 3.56744228094956 & 20.18802596006510 & 8.60852343197323 \\
\hline $\mathbf{C u}$ & 3.56736996383620 & 22.70483253358240 & 8.61251431237181 \\
\hline $\mathbf{C u}$ & 7.13527461965259 & 25.22492107186600 & 8.60982520181818 \\
\hline $\mathbf{C u}$ & 7.12970251127016 & 2.51952650297578 & 8.61401476092379 \\
\hline $\mathbf{C u}$ & 7.13265978158007 & 5.04410839893226 & 8.60634089995724 \\
\hline $\mathbf{C u}$ & 7.13004310614777 & 7.56995144525467 & 8.61503974618645 \\
\hline $\mathbf{C u}$ & 7.13223482135815 & 10.09364396042150 & 8.61344465555503 \\
\hline $\mathbf{C u}$ & 7.13022967339844 & 12.60963404123630 & 8.61943702717936 \\
\hline $\mathbf{C u}$ & 7.13629410552186 & 15.13010706768700 & 8.60779602488189 \\
\hline $\mathbf{C u}$ & 7.13011815154283 & 17.65265645836580 & 8.61399006258659 \\
\hline $\mathbf{C u}$ & 7.13455802358561 & 20.17923629313800 & $8.00 / 0112$ \\
\hline
\end{tabular}




\begin{tabular}{|c|c|c|c|}
\hline $\mathbf{C u}$ & 7.13326800212998 & 0770 & 47322 \\
\hline \multicolumn{4}{|c|}{ CI-NEB Image 2} \\
\hline Element & X-Coordinate (Å) & Y-Coordinate (§̊) & Z-Coordinate (Å) \\
\hline C & 3.56724058584757 & 7.98140381165116 & 10.62356565116320 \\
\hline C & 3.56726803109218 & 13.27686107664170 & 10.61056567408280 \\
\hline $\mathbf{F}$ & 3.56754405376285 & 9.39692444235784 & 10.88338955225370 \\
\hline $\mathbf{F}$ & 2.47037258405315 & 7.52847381576192 & 11.32189090373330 \\
\hline $\mathbf{F}$ & 4.66379505665652 & 7.52787561663861 & 11.32198823632740 \\
\hline $\mathbf{F}$ & 3.56721391311347 & 14.73240761445990 & 10.77846173795000 \\
\hline $\mathbf{F}$ & 2.47098876461676 & 12.89619637757770 & 11.34089595931490 \\
\hline $\mathbf{F}$ & 4.66342561227734 & 12.89628412536000 & 11.34113286790750 \\
\hline $\mathbf{C u}$ & 0.00000000000000 & 0.00000000000000 & 3.56221131249991 \\
\hline $\mathbf{C u}$ & 0.00000000000000 & & 3.56221131249991 \\
\hline $\mathbf{C u}$ & 0.00000000000000 & & 3.56221131249991 \\
\hline $\mathrm{Cu}$ & 0.00000000000000 & & 1249991 \\
\hline $\mathbf{C u}$ & 0.00000000000000 & 10.09008447359990 & 3.56221131249991 \\
\hline $\mathbf{C u}$ & 0.00000000000000 & 99990 & 19991 \\
\hline $\mathbf{C u}$ & 0.000 & 040000 & 49991 \\
\hline $\mathbf{C u}$ & 0.000 & 17.6 & 9991 \\
\hline $\mathrm{Cu}$ & 0.00 & 20.1 & 9991 \\
\hline $\mathrm{Cu}$ & 0.00 & 22.7 & 9991 \\
\hline $\mathbf{C u}$ & 3.56 & 0.0 & 9991 \\
\hline $\mathbf{C u}$ & 3.56 & 2.5 & 9991 \\
\hline $\mathbf{C u}$ & 3.56 & 5.0 & 9991 \\
\hline $\mathbf{C u}$ & 3.56 & 7.5 & 9991 \\
\hline $\mathbf{C u}$ & 3.56 & 10.0 & 9991 \\
\hline $\mathbf{C u}$ & 3.56 & 99990 & 49991 \\
\hline $\mathbf{C u}$ & 323 & 40000 & 49991 \\
\hline $\mathbf{C u}$ & 323 & 80000 & 49991 \\
\hline $\mathbf{C u}$ & $3.56^{\circ}$ & 20.1801689 & 3.56221131249991 \\
\hline $\mathbf{C u}$ & 634323 & 22.70269006559990 & 3.56221131249991 \\
\hline $\mathbf{C u}$ & 2693 & 00000 & 3.56221131249991 \\
\hline $\mathbf{C u}$ & 7.13476715482693 & 11840003 & 3.56221131249991 \\
\hline $\mathbf{C u}$ & 7.134 & 07 & 49991 \\
\hline $\mathbf{C u}$ & 7.13 & 7.5 & 9991 \\
\hline $\mathbf{C u}$ & 7.13 & 990 & 9991 \\
\hline $\mathbf{C u}$ & 7.13 & 99990 & 9991 \\
\hline $\mathbf{C u}$ & 693 & 0000 & 9991 \\
\hline $\mathbf{C u}$ & 482693 & 880000 & 49991 \\
\hline $\mathrm{Cu}$ & 15482693 & 20.18016894719990 & 3.56221131249991 \\
\hline $\mathbf{C u}$ & 5482693 & 22.70269006559990 & 31249991 \\
\hline $\mathbf{C u}$ & 1.78369178870677 & 1.26126055919993 & 4.82343353250005 \\
\hline $\mathbf{C u}$ & 1.78369178870677 & 3.78378167759996 & 4.82343353250005 \\
\hline $\mathbf{C u}$ & 1.78369178870677 & 6.30630279600000 & 4.82343353250005 \\
\hline $\mathbf{C u}$ & 1.78369178870677 & 8.82882391440003 & 4.82343353250005 \\
\hline $\mathbf{C u}$ & 1.78369178870677 & 11.35134503280000 & 4.82343353250005 \\
\hline $\mathbf{C u}$ & 1.78369178870677 & 13.87386615119990 & 4.82343353250005 \\
\hline $\mathbf{C u}$ & 1.78369178870677 & 16.39638726959990 & 4.82343353250005 \\
\hline $\mathbf{C u}$ & 1.78369178870677 & 18.91890838799990 & 4.82343353250005 \\
\hline $\mathbf{C u}$ & 1.78369178870677 & 21.44142950640000 & 4.82343353250005 \\
\hline $\mathbf{C u}$ & 1.78369178870677 & 23.96395062480000 & 4.82343353250005 \\
\hline $\mathbf{C u}$ & 5.35107536505000 & 1.26126055919993 & 4.82343353250005 \\
\hline $\mathbf{C u}$ & 5.35107536505000 & 3.78378167759996 & 4.82343353250005 \\
\hline $\mathbf{C u}$ & 5.35107536505000 & 6.30630279600000 & 4.82343353250005 \\
\hline
\end{tabular}




\begin{tabular}{|c|c|c|c|}
\hline $\mathbf{C u}$ & 5.35107536505000 & 8.82882391440003 & 4.82343353250005 \\
\hline $\mathbf{C u}$ & 5.35107536505000 & 11.35134503280000 & 4.82343353250005 \\
\hline $\mathbf{C u}$ & 5.35107536505000 & 13.87386615119990 & 4.82343353250005 \\
\hline $\mathbf{C u}$ & 5.35107536505000 & 16.39638726959990 & 4.82343353250005 \\
\hline $\mathbf{C u}$ & 5.35107536505000 & 18.91890838799990 & 4.82343353250005 \\
\hline $\mathbf{C u}$ & 5.35107536505000 & 21.44142950640000 & 4.82343353250005 \\
\hline $\mathbf{C u}$ & 5.35107536505000 & 23.96395062480000 & 4.82343353250005 \\
\hline $\mathbf{C u}$ & 8.91845894246346 & 1.26126055919993 & 4.82343353250005 \\
\hline $\mathbf{C u}$ & 8.91845894246346 & 3.78378167759996 & 4.82343353250005 \\
\hline $\mathbf{C u}$ & 8.91845894246346 & 6.30630279600000 & 4.82343353250005 \\
\hline $\mathbf{C u}$ & 8.91845894246346 & 8.82882391440003 & 4.82343353250005 \\
\hline $\mathbf{C u}$ & 8.91845894246346 & 11.35134503280000 & 4.82343353250005 \\
\hline $\mathbf{C u}$ & 8.91845894246346 & 13.87386615119990 & 4.82343353250005 \\
\hline $\mathrm{Cu}$ & 8.91845894246346 & 16.39638726959990 & 4.82343353250005 \\
\hline $\mathbf{C u}$ & 8.91845894246346 & 18.91890838799990 & 4.82343353250005 \\
\hline $\mathbf{C u}$ & 8.91845894246346 & 21.44142950640000 & 4.82343353250005 \\
\hline $\mathbf{C u}$ & 8.91845894246346 & 062480000 & 4.82343353250005 \\
\hline $\mathbf{C u}$ & 97259244 & 25.22443640071500 & 6.09578154122684 \\
\hline $\mathbf{C u}$ & 10.70072378535220 & 2.52406492429610 & 6.09787925755211 \\
\hline $\mathbf{C u}$ & 09712070 & $\$ 1607$ & 945136 \\
\hline $\mathbf{C u}$ & 10.69807415537950 & 005896 & 415274 \\
\hline $\mathbf{C u}$ & 10.69780120249040 & 7233020 & 06424632720 \\
\hline $\mathbf{C u}$ & 0.00 & 9740 & 72467 \\
\hline $\mathbf{C u}$ & 9500 & 1380 & 06212 \\
\hline $\mathbf{C u}$ & 10.6 & 0110 & 86308 \\
\hline $\mathbf{C u}$ & 1920 & 92410 & 7590862 \\
\hline $\mathbf{C u}$ & 10.69923318937980 & 22.70456830356630 & 6.09506868751607 \\
\hline $\mathbf{C u}$ & 3.56737584704129 & 0.00379704092632 & 6.09422052231241 \\
\hline $\mathbf{C u}$ & 7643021314 & 9124281 & 6.09020895349039 \\
\hline $\mathbf{C u}$ & 3.56737772900264 & 5.049034 & 6.10207183239843 \\
\hline $\mathbf{C u}$ & 3.56737859764059 & 7.57375761936376 & 6.13783327781630 \\
\hline $\mathbf{C u}$ & 96286 & 74800 & 6.13588909355513 \\
\hline $\mathbf{C u}$ & 3.56738142267593 & 12.61591357008620 & 6.14397755400507 \\
\hline $\mathbf{C u}$ & 3.56738531354186 & 15.12770818477670 & 6.12402544311353 \\
\hline $\mathbf{C u}$ & 3.56738250162321 & 17.65951002606180 & 6.08850141058809 \\
\hline $\mathbf{C u}$ & 3.56737741869955 & 20.18257011674820 & 6.09513502866506 \\
\hline $\mathbf{C u}$ & 3.56737578977667 & 22.70615296904100 & 6.09367063676530 \\
\hline $\mathbf{C u}$ & 7.13296536343662 & 25.22443712942940 & 6.09576414252153 \\
\hline $\mathbf{C u}$ & 7.13618557986334 & 2.52406666226625 & 6.09786221730092 \\
\hline $\mathbf{C u}$ & 7.13847373331303 & 5.04010863665404 & 6.09368932023440 \\
\hline $\mathbf{C u}$ & 7.13883376645301 & 7.57008271472174 & 6.10256150351097 \\
\hline $\mathbf{C u}$ & 7.13911076315851 & 10.09518955732800 & 6.09505207841968 \\
\hline $\mathbf{C u}$ & 7.13292400455328 & 12.60691259025860 & 6.10436693076262 \\
\hline $\mathbf{C u}$ & 7.13993837058973 & 15.13926952126630 & 6.09378339825582 \\
\hline $\mathbf{C u}$ & 7.13899453033318 & 17.65764469587940 & 6.09624708290218 \\
\hline $\mathbf{C u}$ & 7.13248752831935 & 20.18306416266200 & 6.09512191746654 \\
\hline $\mathbf{C u}$ & 7.13767755707951 & 22.70456830365640 & 6.09505778078468 \\
\hline $\mathbf{C u}$ & 1.78399239217312 & 1.26155348278948 & 7.38696977877819 \\
\hline $\mathbf{C u}$ & 1.78531971586690 & 3.78658866992608 & 7.38769879558367 \\
\hline $\mathbf{C u}$ & 1.78245480507923 & 6.30415435543577 & 7.37947805421636 \\
\hline $\mathbf{C u}$ & 1.77588965850196 & 8.83468450323563 & 7.37925410389243 \\
\hline $\mathbf{C u}$ & 1.80051427157879 & 11.35142987304710 & 7.39440662876776 \\
\hline $\mathbf{C u}$ & 1.77043948991344 & 13.88258020326100 & 7.37792853416945 \\
\hline $\mathbf{C u}$ & 1.78261260153778 & 16.40374096070110 & 7.37879806824422 \\
\hline
\end{tabular}




\begin{tabular}{|c|c|c|c|}
\hline $\mathbf{C u}$ & 1.77914662973883 & 18.92503532068510 & 7.38532498079100 \\
\hline $\mathbf{C u}$ & 1.78438280016263 & 21.44859988192830 & 7.38520661550538 \\
\hline $\mathbf{C u}$ & 1.78511544650343 & 23.96514876844420 & 7.38812775259664 \\
\hline $\mathbf{C u}$ & 5.35076780939822 & 1.26155272837430 & 7.38695138586653 \\
\hline $\mathbf{C u}$ & 5.34943638010075 & 3.78659108149317 & 7.38767711026563 \\
\hline $\mathbf{C u}$ & 5.35229147819546 & 6.30416084254129 & 7.37947737005850 \\
\hline $\mathbf{C u}$ & 5.35886661772608 & 8.83467321387568 & 7.37925609255420 \\
\hline $\mathbf{C u}$ & 5.33426053724232 & 11.35142648558800 & 7.39433964089736 \\
\hline $\mathbf{C u}$ & 5.36433505079097 & 13.88258453436180 & 7.37793833373731 \\
\hline $\mathbf{C u}$ & 5.35215601297424 & 16.40373924448770 & 7.37879084650105 \\
\hline $\mathbf{C u}$ & 5.35561852201606 & 18.92503759560370 & 7.38531485555720 \\
\hline $\mathbf{C u}$ & 5.35037871039315 & 21.44860113665120 & 7.38518835191264 \\
\hline $\mathbf{C u}$ & 5.34964555056096 & 23.96515080636990 & 7.38810924515219 \\
\hline $\mathbf{C u}$ & 8.91844068618293 & 1.26581947008621 & 7.39497599770495 \\
\hline $\mathbf{C u}$ & 8.91844164261813 & 3.78291880411846 & 7.39495610477405 \\
\hline $\mathbf{C u}$ & 8.91844334912293 & 6.30930116873800 & 7.39827677299747 \\
\hline $\mathbf{C u}$ & 8.91844395187333 & 8.83227734517829 & 7.39461881345891 \\
\hline $\mathbf{C u}$ & 8.91844213557062 & 11.35425038593810 & 7.40348459797340 \\
\hline $\mathbf{C u}$ & 8.91844117386000 & 13.87123677810390 & 7.39101535864286 \\
\hline $\mathbf{C u}$ & 8.91843987601261 & 54926277180 & 7.39624355532955 \\
\hline $\mathbf{C u}$ & 8.91843744545931 & 18.91743334 & 7.39460728384182 \\
\hline $\mathrm{Cu}$ & 8.91843932265864 & 21.44316891569100 & 7.39453114563131 \\
\hline $\mathbf{C u}$ & 8.91844027140892 & 23.96271114645550 & 7.39313991126043 \\
\hline $\mathbf{C u}$ & 10.70167484900400 & 0.00158207822190 & 8.61149445220646 \\
\hline $\mathbf{C u}$ & 0.00540772714871 & 2.52183163867241 & 8.61526766594558 \\
\hline $\mathbf{C u}$ & 0.00316328040436 & 5.04670786028746 & 8.60814071482716 \\
\hline $\mathbf{C u}$ & 0.00444747037293 & 7.57295605000986 & 8.61536682108623 \\
\hline $\mathbf{C u}$ & 0.00370442040233 & 10.09720374676960 & 8.61440235539664 \\
\hline $\mathbf{C u}$ & 0.00442672687348 & 12.61302187932410 & 8.61981302552135 \\
\hline $\mathrm{Cu}$ & 10.70071557390400 & 15.13464753254250 & 8.60642623806540 \\
\hline $\mathbf{C u}$ & 0.00330791943978 & 17.65761675701140 & 8.61473849454757 \\
\hline $\mathbf{C u}$ & -0.00033049054116 & 20.18299778722960 & 8.61036978946574 \\
\hline $\mathbf{C u}$ & 0.00086112539031 & 22.70668982786660 & 8.61111854324150 \\
\hline $\mathbf{C u}$ & 3.56739191112022 & 25.22474350036430 & 8.61604198180753 \\
\hline $\mathbf{C u}$ & 3.56739390358215 & 2.51628966455004 & 8.61012518769408 \\
\hline $\mathbf{C u}$ & 3.56738831766110 & 5.02573329408054 & 8.61311733160452 \\
\hline $\mathbf{C u}$ & 3.56736516306992 & 7.55721542920648 & 8.69392967674288 \\
\hline $\mathbf{C u}$ & 3.56741010052605 & 10.10658361444110 & 8.65580867870446 \\
\hline $\mathbf{C u}$ & 3.56743553348982 & 12.64466360730890 & 8.72480473227266 \\
\hline $\mathbf{C u}$ & 3.56740188869196 & 15.19371840262670 & 8.62441367710180 \\
\hline $\mathbf{C u}$ & 3.56739821299452 & 17.69250390219260 & 8.59140929198197 \\
\hline $\mathbf{C u}$ & 3.56739505581101 & 20.19558531362940 & 8.60953488143216 \\
\hline $\mathbf{C u}$ & 3.56739415234496 & 22.70835200459870 & 8.61405685406619 \\
\hline $\mathbf{C u}$ & 7.13520711991368 & 0.00158395070736 & 8.61150645113785 \\
\hline $\mathbf{C u}$ & 7.12931883500801 & 2.52183409465364 & 8.61526052345945 \\
\hline $\mathbf{C u}$ & 7.13156095105622 & 5.04670506834968 & 8.60814221358341 \\
\hline $\mathbf{C u}$ & 7.13028394110800 & 7.57294976163472 & 8.61535988793129 \\
\hline $\mathbf{C u}$ & 7.13102547256095 & 10.09720029751950 & 8.61439472058651 \\
\hline $\mathbf{C u}$ & 7.13030337339924 & 12.61301456670890 & 8.61980037807423 \\
\hline $\mathbf{C u}$ & 7.13617383804472 & 15.13464819442920 & 8.60645300566408 \\
\hline $\mathbf{C u}$ & 7.13141895637046 & 17.65762053443490 & 8.61474589409640 \\
\hline $\mathbf{C u}$ & 7.13505588179472 & 20.18300265658450 & 8.61038022782722 \\
\hline $\mathbf{C u}$ & 7.13386902660917 & 22.70669100621860 & 8.61112764774171 \\
\hline
\end{tabular}




\begin{tabular}{|c|c|c|c|}
\hline Element & X-Coordinate (Å) & Y-Coordinate (Å) & Z-Coordinate ( $($ ) \\
\hline C & 3.56731042868704 & 8.01009752818712 & 10.62807358922240 \\
\hline C & 3.56698997085292 & 13.30894487931510 & 10.60273962686690 \\
\hline $\mathbf{F}$ & 3.56736574556857 & 9.42756675010702 & 10.88928258229220 \\
\hline $\mathbf{F}$ & 2.47045852364782 & 7.55970264233295 & 11.32811358320100 \\
\hline $\mathbf{F}$ & 4.66402297345351 & 7.55957803034824 & 11.32826011384610 \\
\hline $\mathbf{F}$ & 3.56722954563392 & 15.06218272155480 & 10.60525456144630 \\
\hline $\mathbf{F}$ & 2.48758517822103 & 13.11335024662580 & 11.37693344980510 \\
\hline $\mathbf{F}$ & 4.64596384728331 & 13.11312134203230 & 11.37740164326430 \\
\hline $\mathbf{C u}$ & 0.00000000000000 & 0.00000000000000 & 3.56221131249991 \\
\hline $\mathbf{C u}$ & 0.00000000000000 & 2.52252111840003 & 3.56221131249991 \\
\hline $\mathbf{C u}$ & 0.00000000000000 & 5.04504223680007 & 3.56221131249991 \\
\hline $\mathbf{C u}$ & 0.00000000000000 & 7.56756335519993 & 3.56221131249991 \\
\hline $\mathbf{C u}$ & 0.00000000000000 & 10.09008447359990 & 3.56221131249991 \\
\hline $\mathbf{C u}$ & 0.00000000000000 & 12.61260559199990 & 3.56221131249991 \\
\hline $\mathbf{C u}$ & 0.00000000000000 & 15.13512671040000 & 3.56221131249991 \\
\hline $\mathbf{C u}$ & 0.00000000000000 & 17.65764782880000 & 3.56221131249991 \\
\hline $\mathbf{C u}$ & 0.00000000000000 & 20.18016894719990 & 3.56221131249991 \\
\hline $\mathbf{C u}$ & 0.00000000000000 & 22.70269006559990 & 3.56221131249991 \\
\hline $\mathbf{C u}$ & 3.56738357634323 & 0.00000000000000 & 21131249991 \\
\hline $\mathbf{C u}$ & 3.56738357634323 & 2.52252111840003 & 21131249991 \\
\hline $\mathbf{C u}$ & 3.56738357634323 & 23680007 & 3.56221131249991 \\
\hline $\mathbf{C u}$ & 3.56738357634323 & 7.56756335519993 & 3.56221131249991 \\
\hline $\mathbf{C u}$ & 3.56738357634323 & 10.09008447359990 & 3.56221131249991 \\
\hline $\mathbf{C u}$ & 3.56738357634323 & 12.61260559199990 & 3.56221131249991 \\
\hline $\mathbf{C u}$ & 3.56738357634323 & 15.13512671040000 & 3.56221131249991 \\
\hline $\mathrm{Cu}$ & 3.56738357634323 & 17.65764782880000 & 3.56221131249991 \\
\hline $\mathbf{C u}$ & 3.56738357634323 & 20.18016894719990 & 3.56221131249991 \\
\hline $\mathbf{C u}$ & 3.56738357634323 & 22.70269006559990 & 3.56221131249991 \\
\hline $\mathbf{C u}$ & 7.13476715482693 & 0.00000000000000 & 3.56221131249991 \\
\hline $\mathbf{C u}$ & 7.13476715482693 & 2.52252111840003 & 3.56221131249991 \\
\hline $\mathbf{C u}$ & 7.13476715482693 & 5.04504223680007 & 3.56221131249991 \\
\hline $\mathbf{C u}$ & 7.13476715482693 & 7.56756335519993 & 3.56221131249991 \\
\hline $\mathbf{C u}$ & 7.13476715482693 & 10.09008447359990 & 3.56221131249991 \\
\hline $\mathbf{C u}$ & 7.13476715482693 & 12.61260559199990 & 3.56221131249991 \\
\hline $\mathbf{C u}$ & 7.13476715482693 & 15.13512671040000 & 3.56221131249991 \\
\hline $\mathbf{C u}$ & 7.13476715482693 & 17.65764782880000 & 3.56221131249991 \\
\hline $\mathbf{C u}$ & 7.13476715482693 & 20.18016894719990 & 3.56221131249991 \\
\hline $\mathbf{C u}$ & 7.13476715482693 & 22.70269006559990 & 3.56221131249991 \\
\hline $\mathbf{C u}$ & 1.78369178870677 & 1.26126055919993 & 4.82343353250005 \\
\hline $\mathbf{C u}$ & 1.78369178870677 & 3.78378167759996 & 4.82343353250005 \\
\hline $\mathbf{C u}$ & 1.78369178870677 & 6.30630279600000 & 4.82343353250005 \\
\hline $\mathbf{C u}$ & 1.78369178870677 & 8.82882391440003 & 4.82343353250005 \\
\hline $\mathbf{C u}$ & 1.78369178870677 & 11.35134503280000 & 4.82343353250005 \\
\hline $\mathbf{C u}$ & 1.78369178870677 & 13.87386615119990 & 4.82343353250005 \\
\hline $\mathbf{C u}$ & 1.78369178870677 & 16.39638726959990 & 4.82343353250005 \\
\hline $\mathbf{C u}$ & 1.78369178870677 & 18.91890838799990 & 4.82343353250005 \\
\hline $\mathbf{C u}$ & 1.78369178870677 & 21.44142950640000 & 4.82343353250005 \\
\hline $\mathbf{C u}$ & 1.78369178870677 & 23.96395062480000 & 4.82343353250005 \\
\hline $\mathbf{C u}$ & 5.35107536505000 & 1.26126055919993 & 4.82343353250005 \\
\hline $\mathbf{C u}$ & 5.35107536505000 & 3.78378167759996 & 4.82343353250005 \\
\hline $\mathrm{Cu}$ & 5.35107536505000 & 6.30630279600000 & 4.82343353250005 \\
\hline $\mathbf{C u}$ & 5.35107536505000 & 8.82882391440003 & 4.82343353250005 \\
\hline $\mathbf{C u}$ & 5.35107536505000 & 11.35134503280000 & 4.82343353250005 \\
\hline
\end{tabular}




\begin{tabular}{|c|c|c|c|}
\hline $\mathbf{C u}$ & 5.35107536505000 & 13.87386615119990 & 4.82343353250005 \\
\hline $\mathbf{C u}$ & 5.35107536505000 & 16.39638726959990 & 4.82343353250005 \\
\hline $\mathbf{C u}$ & 5.35107536505000 & 18.91890838799990 & 4.82343353250005 \\
\hline $\mathbf{C u}$ & 5.35107536505000 & 21.44142950640000 & 4.82343353250005 \\
\hline $\mathbf{C u}$ & 5.35107536505000 & 23.96395062480000 & 4.82343353250005 \\
\hline $\mathbf{C u}$ & 8.91845894246346 & 1.26126055919993 & 4.82343353250005 \\
\hline $\mathbf{C u}$ & 8.91845894246346 & 3.78378167759996 & 4.82343353250005 \\
\hline $\mathbf{C u}$ & 8.91845894246346 & 6.30630279600000 & 4.82343353250005 \\
\hline $\mathbf{C u}$ & 8.91845894246346 & 8.82882391440003 & 4.82343353250005 \\
\hline $\mathbf{C u}$ & 8.91845894246346 & 11.35134503280000 & 4.82343353250005 \\
\hline $\mathbf{C u}$ & 8.91845894246346 & 13.87386615119990 & 4.82343353250005 \\
\hline $\mathbf{C u}$ & 8.91845894246346 & 16.39638726959990 & 4.82343353250005 \\
\hline $\mathbf{C u}$ & 8.91845894246346 & 18.91890838799990 & 4.82343353250005 \\
\hline $\mathbf{C u}$ & 8.91845894246346 & 21.44142950640000 & 4.82343353250005 \\
\hline $\mathrm{Cu}$ & 8.91845894246346 & 23.96395062480000 & 4.82343353250005 \\
\hline $\mathbf{C u}$ & 0.00031632548936 & 25.22403574485180 & 6.09637400752671 \\
\hline $\mathbf{C u}$ & 10.70151817593090 & 2.52403035000277 & 6.09863310894231 \\
\hline $\mathbf{C u}$ & 1253985960 & & 6.09486216471389 \\
\hline $\mathbf{C u}$ & 775523110590 & & 6.10226983581756 \\
\hline $\mathbf{C u}$ & 10.69824483170660 & $10.0965 \varsigma$ & 990071 \\
\hline $\mathbf{C u}$ & 211344 & 12.60744 & 449037 \\
\hline $\mathbf{C u}$ & 798990 & 15.1391469 & 3156532 \\
\hline $\mathbf{C u}$ & 99780 & 17.6 & 75131 \\
\hline $\mathbf{C u}$ & 4077484 & 9020 & 668530 \\
\hline $\mathbf{C u}$ & 10.69 & 29730 & 63558 \\
\hline $\mathbf{C u}$ & 7912221 & 8410 & 174450 \\
\hline $\mathbf{C u}$ & 522589013 & 2.524254 & 177467 \\
\hline $\mathbf{C u}$ & 680180303 & 8121 & 6.10077245317157 \\
\hline $\mathbf{C u}$ & 7971474 & 8586 & 6.14003198700952 \\
\hline $\mathbf{C u}$ & 3.56734270721085 & 10.08957438305020 & 6.13298467350798 \\
\hline $\mathbf{C u}$ & 3.56735095401863 & 12.61614698932550 & 6.15119533663731 \\
\hline $\mathbf{C u}$ & 33301 & 15.128363 & 6.13648252548582 \\
\hline $\mathbf{C u}$ & 3.56735233696855 & 17.65619920019960 & 6.10039120843920 \\
\hline $\mathbf{C u}$ & 3.56735946057546 & 20.18151788389540 & 6.09414365573851 \\
\hline $\mathbf{C u}$ & 3.56735616997131 & 22.70525725912080 & 6.09353368023604 \\
\hline $\mathbf{C u}$ & 7.13448222986944 & 25.22403583785330 & 6.09637616198890 \\
\hline $\mathbf{C u}$ & 7.13543159340868 & 2.52402146112927 & 6.09862740457784 \\
\hline $\mathbf{C u}$ & 7.13824480922220 & 4417877867 & 6.09486421745587 \\
\hline $\mathbf{C u}$ & 7.13920239924568 & 7.56902649864837 & 6.10228030487781 \\
\hline $\mathbf{C u}$ & 7.13871255771791 & 10.09659285059360 & 6.09531707368864 \\
\hline $\mathbf{C u}$ & 7.13222658884976 & 12.60744835077260 & 6.10152577867479 \\
\hline $\mathbf{C u}$ & 7.14098437139523 & 15.13914676877310 & 6.09408742161173 \\
\hline $\mathbf{C u}$ & 7.14028056322340 & 17.66017595749090 & 6.09619793796142 \\
\hline $\mathbf{C u}$ & 7.13052540834534 & 20.18181976276960 & 6.09762690205916 \\
\hline $\mathbf{C u}$ & 7.13769044330012 & 22.70424461631000 & 6.09595912231898 \\
\hline $\mathbf{C u}$ & 1.78397667331323 & 1.26072533780719 & 7.38776422371369 \\
\hline $\mathbf{C u}$ & 1.78632836438141 & 3.78630428207035 & 7.38780566261793 \\
\hline $\mathbf{C u}$ & 1.78458032418980 & 6.30631425262275 & 7.38053950003786 \\
\hline $\mathbf{C u}$ & 1.77305888365827 & 8.83536839468732 & 7.38010326671074 \\
\hline $\mathbf{C u}$ & 1.79950800617316 & 11.35336108171620 & 7.39617269711646 \\
\hline $\mathbf{C u}$ & 1.77197637909048 & 13.88326035832480 & 7.37509059662848 \\
\hline $\mathbf{C u}$ & 1.77832108377803 & 16.40659266702970 & 7.37304055724678 \\
\hline $\mathbf{C u}$ & 1.78262726199732 & 18.92114081521060 & 7.39106319038984 \\
\hline $\mathbf{C u}$ & 1.78461093916298 & 21.44703109695420 & 7.38586379365010 \\
\hline
\end{tabular}




\begin{tabular}{|c|c|c|c|}
\hline $\mathbf{C u}$ & 1.78530533412609 & 23.96511708919920 & 7.38859164121359 \\
\hline $\mathrm{Cu}$ & 5.35076654529743 & 1.26072219074907 & 7.38776365728654 \\
\hline $\mathbf{C u}$ & 5.34840880272170 & 3.78630059159556 & 7.38779966123021 \\
\hline $\mathbf{C u}$ & 5.35014934406418 & 6.30631273587240 & 7.38054696426550 \\
\hline $\mathbf{C u}$ & 5.36166847035614 & 8.83536568334667 & 7.38014802716054 \\
\hline $\mathbf{C u}$ & 5.33521828512192 & 11.35336348802950 & 7.39615902121779 \\
\hline $\mathbf{C u}$ & 5.36275458506969 & 13.88326262788050 & 7.37513368015698 \\
\hline $\mathbf{C u}$ & 5.35642089443626 & 16.40659403939800 & 7.37306605354758 \\
\hline $\mathbf{C u}$ & 5.35211498023692 & 18.92114254446570 & 7.39106302917775 \\
\hline $\mathbf{C u}$ & 5.35013598931439 & 21.44703912862980 & 7.38585976126538 \\
\hline $\mathbf{C u}$ & 5.34944235293955 & 23.96511819999490 & 7.38858906103488 \\
\hline $\mathbf{C u}$ & 8.91848545571035 & 1.26596002046707 & 7.39656477174833 \\
\hline $\mathbf{C u}$ & 8.91848958959528 & 3.78310310006784 & 7.39674374721103 \\
\hline $\mathbf{C u}$ & 8.91849840877745 & 6.30740252338190 & 7.39973037651974 \\
\hline $\mathbf{C u}$ & 8.91849490017575 & 8.83299715165774 & 7.39418317412932 \\
\hline $\mathbf{C u}$ & 8.9184923 & 32110 & 4710479 \\
\hline $\mathbf{C u}$ & 8.918489 & 6350 & 7497528 \\
\hline $\mathbf{C u}$ & 8.9184877 & 4800 & 2002207 \\
\hline $\mathbf{C u}$ & 401 & 270 & 15750 \\
\hline $\mathbf{C u}$ & 255 & 580 & 3379 \\
\hline $\mathbf{C u}$ & 8.9 & 740 & 54 \\
\hline $\mathbf{C u}$ & 10.7 & & 26 \\
\hline $\mathbf{C u}$ & 37 & & 8.6 \\
\hline $\mathbf{C u}$ & 0.00 & & 310764 \\
\hline $\mathbf{C u}$ & 0.00 & 157 & 8.6 \\
\hline $\mathbf{C u}$ & 19 & 10. & 8.6 \\
\hline $\mathbf{C u}$ & 0.0 & 580 & 8.6 \\
\hline $\mathbf{C u}$ & 10.7 & 480 & 4858 \\
\hline $\mathbf{C u}$ & 18 & 940 & 4657 \\
\hline $\mathbf{C u}$ & 42 & 9900 & 7995 \\
\hline $\mathbf{C u}$ & 12 & 630 & 2762 \\
\hline $\mathbf{C u}$ & 962 & 9190 & 633284 \\
\hline $\mathbf{C u}$ & 836 & 5404 & 779259456 \\
\hline $\mathbf{C u}$ & 2400 & 8703810742 & 8.61072208883362 \\
\hline $\mathbf{C u}$ & 25314 & 7888 & 8.70063171618119 \\
\hline $\mathbf{C u}$ & 5754980393 & 0891871100 & 8.65114589088314 \\
\hline $\mathbf{C u}$ & 56735894816504 & 12.64071759838740 & 8.73851055866562 \\
\hline $\mathbf{C u}$ & 56736970161500 & 15.20037619261420 & 8.62176754500142 \\
\hline $\mathbf{C u}$ & 3.56736325859878 & 17.69821357471750 & 8.61269211957259 \\
\hline $\mathbf{C u}$ & 3.56737163268680 & 20.19557234283260 & 8.61143051881782 \\
\hline $\mathbf{C u}$ & 3.56737044421241 & 22.70619230302290 & 8.61452406872346 \\
\hline $\mathbf{C u}$ & 13507564648518 & 0.0012164 & 8.61221144865847 \\
\hline $\mathbf{C u}$ & 7.12822275923636 & 2.52218843 & 8.61608193388563 \\
\hline $\mathbf{C u}$ & 776596 & 5.04670271080297 & 998128453 \\
\hline $\mathbf{C u}$ & 7.13196685248749 & 7.57360835618457 & 8.61495906017329 \\
\hline $\mathbf{C u}$ & 7.13341570862702 & 10.09882835986770 & 8.61599065374592 \\
\hline $\mathbf{C u}$ & 7.13072393182642 & 12.61400753697250 & 8.61621793210266 \\
\hline $\mathbf{C u}$ & 7.13605770534700 & 15.13446797258190 & 8.60714153916765 \\
\hline $\mathbf{C u}$ & 7.13243121797416 & 17.65758343643920 & 8.61633414296459 \\
\hline $\mathrm{Cu}$ & 7.13256799562674 & 20.18262495228710 & 8.61364300473670 \\
\hline $\mathbf{C u}$ & 7.13332477675107 & 22.70615042918490 & 8.61200546399590 \\
\hline \multicolumn{4}{|c|}{ I-NEB Image 4} \\
\hline 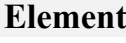 & $\mathrm{X}$-Coordinat & & \\
\hline $\mathrm{C}$ & 40 & & \\
\hline
\end{tabular}




\begin{tabular}{|c|c|c|c|}
\hline $\mathbf{C}$ & 3.56704242301441 & 13.07063034825350 & 10.58816468877320 \\
\hline $\mathbf{F}$ & 3.56740047668735 & 9.42855318072863 & 10.89505283757020 \\
\hline $\mathbf{F}$ & 2.47065416332146 & 7.55904429116039 & 11.32830167134310 \\
\hline $\mathbf{F}$ & 4.66399614974054 & 7.55895670529099 & 11.32832391097550 \\
\hline $\mathbf{F}$ & 3.56739358379568 & 15.47601001871280 & 10.51884769178210 \\
\hline $\mathbf{F}$ & 2.50179007581099 & 13.10150550335410 & 11.36694759794890 \\
\hline $\mathbf{F}$ & 4.63188115579675 & 13.10125093658210 & 11.36753724577890 \\
\hline $\mathbf{C u}$ & 0.00000000000000 & 0.00000000000000 & 3.56221131249991 \\
\hline $\mathbf{C u}$ & 0.00000000000000 & 2.52252111840003 & 3.56221131249991 \\
\hline $\mathbf{C u}$ & 0.00000000000000 & 5.04504223680007 & 3.56221131249991 \\
\hline $\mathbf{C u}$ & 0.00000000000000 & 7.56756335519993 & 3.56221131249991 \\
\hline $\mathbf{C u}$ & 0.00000000000000 & 10.09008447359990 & 3.56221131249991 \\
\hline $\mathbf{C u}$ & 0.00000000000000 & 12.61260559199990 & 3.56221131249991 \\
\hline $\mathbf{C u}$ & 0.00000000000000 & 15.13512671040000 & 3.56221131249991 \\
\hline $\mathbf{C u}$ & 0.00000000000000 & 17.65764782880000 & 3.56221131249991 \\
\hline $\mathrm{Cu}$ & 0.00000000000000 & 20.18016894719990 & 3.56221131249991 \\
\hline $\mathbf{C u}$ & 0.00000000000000 & 22.70269006559990 & 3.56221131249991 \\
\hline $\mathbf{C u}$ & 3.56738357634323 & 0.00000000000000 & 3.56221131249991 \\
\hline $\mathbf{C u}$ & 34323 & 2.52252111 & 3.56221131249991 \\
\hline $\mathbf{C u}$ & 3.56738 & 3680007 & 3.56221131249991 \\
\hline $\mathbf{C u}$ & 3.56738357634323 & 7.56756335 & 3.56221131249991 \\
\hline $\mathbf{C u}$ & 3.56738357634323 & 10.09008447359990 & 3.56221131249991 \\
\hline $\mathbf{C u}$ & 3.56738357634323 & 12.61260559199990 & 3.56221131249991 \\
\hline $\mathbf{C u}$ & 3.56738357634323 & 15.13512671040000 & 3.56221131249991 \\
\hline $\mathbf{C u}$ & 3.56738357634323 & 17.65764782880000 & 3.56221131249991 \\
\hline $\mathbf{C u}$ & 3.56738357634323 & 20.18016894719990 & 3.56221131249991 \\
\hline $\mathbf{C u}$ & 3.56738357634323 & 22.70269006559990 & 3.56221131249991 \\
\hline $\mathbf{C u}$ & 7.13476715482693 & 0.00000000000000 & 3.56221131249991 \\
\hline $\mathbf{C u}$ & 7.13476715482693 & 2.52252111840003 & 3.56221131249991 \\
\hline $\mathbf{C u}$ & 7.13476715482693 & 23680007 & 3.56221131249991 \\
\hline $\mathbf{C u}$ & 7.13476715482693 & 519993 & 3.56221131249991 \\
\hline $\mathbf{C u}$ & 7.13476715482693 & 10.09008447359990 & 3.56221131249991 \\
\hline $\mathbf{C u}$ & 7.13476715482693 & 12.61260559199990 & 3.56221131249991 \\
\hline $\mathbf{C u}$ & 7.13476715482693 & 15.13512671040000 & 3.56221131249991 \\
\hline $\mathbf{C u}$ & 7.13476715482693 & 17.65764782880000 & 3.56221131249991 \\
\hline $\mathrm{Cu}$ & 7.13476715482693 & 20.18016894719990 & 3.56221131249991 \\
\hline $\mathbf{C u}$ & 7.13476715482693 & 22.70269006559990 & 3.56221131249991 \\
\hline $\mathbf{C u}$ & 1.78369178870677 & 1.26126055919993 & 4.82343353250005 \\
\hline $\mathbf{C u}$ & 1.78369178870677 & 3.78378167759996 & 4.82343353250005 \\
\hline $\mathrm{Cu}$ & 1.78369178870677 & 6.30630279600000 & 4.82343353250005 \\
\hline $\mathbf{C u}$ & 1.78369178870677 & 8.82882391440003 & 4.82343353250005 \\
\hline $\mathbf{C u}$ & 1.78369178870677 & 11.35134503280000 & 4.82343353250005 \\
\hline $\mathbf{C u}$ & 1.78369178870677 & 13.87386615119990 & 4.82343353250005 \\
\hline $\mathbf{C u}$ & 1.78369178870677 & 16.39638726959990 & 53250005 \\
\hline $\mathbf{C u}$ & 1.783 & 18.918 & 250005 \\
\hline $\mathbf{C u}$ & 1.783 & 40000 & 4.82343353250005 \\
\hline $\mathbf{C u}$ & 1.783 & 23.96395 & 250005 \\
\hline $\mathbf{C u}$ & 5.35107536505000 & 1.26126055919993 & 4.82343353250005 \\
\hline $\mathbf{C u}$ & 5.35107536505000 & 3.78378167759996 & 4.82343353250005 \\
\hline $\mathbf{C u}$ & 5.35107536505000 & 6.30630279600000 & 4.82343353250005 \\
\hline $\mathbf{C u}$ & 5.35107536505000 & 8.82882391440003 & 4.82343353250005 \\
\hline $\mathbf{C u}$ & 5.35107536505000 & 11.35134503280000 & 4.82343353250005 \\
\hline $\mathbf{C u}$ & 5.35107536505000 & 13.87386615119990 & 4.82343353250005 \\
\hline $\mathbf{C u}$ & 5.35107536505000 & 16.39638726959990 & 4.82343353250005 \\
\hline
\end{tabular}




\begin{tabular}{|c|c|c|c|}
\hline $\mathbf{C u}$ & 5.35107536505000 & 18.91890838799990 & 4.82343353250005 \\
\hline $\mathbf{C u}$ & 5.35107536505000 & 21.44142950640000 & 4.82343353250005 \\
\hline $\mathbf{C u}$ & 5.35107536505000 & 23.96395062480000 & 4.82343353250005 \\
\hline $\mathbf{C u}$ & 8.91845894246346 & 1.26126055919993 & 4.82343353250005 \\
\hline $\mathbf{C u}$ & 8.91845894246346 & 3.78378167759996 & 4.82343353250005 \\
\hline $\mathbf{C u}$ & 8.91845894246346 & 6.30630279600000 & 4.82343353250005 \\
\hline $\mathbf{C u}$ & 8.91845894246346 & 8.82882391440003 & 4.82343353250005 \\
\hline $\mathbf{C u}$ & 8.91845894246346 & 11.35134503280000 & 4.82343353250005 \\
\hline $\mathbf{C u}$ & 8.91845894246346 & 13.87386615119990 & 4.82343353250005 \\
\hline $\mathbf{C u}$ & 8.91845894246346 & 16.39638726959990 & 4.82343353250005 \\
\hline $\mathbf{C u}$ & 8.91845894246346 & 18.91890838799990 & 4.82343353250005 \\
\hline $\mathbf{C u}$ & 8.91845894246346 & 21.44142950640000 & 4.82343353250005 \\
\hline $\mathbf{C u}$ & 8.91845894246346 & 23.96395062480000 & 4.82343353250005 \\
\hline $\mathrm{Cu}$ & 10.70077212073460 & 25.22391875611950 & 6.09640336586479 \\
\hline $\mathbf{C u}$ & 10.70201509980520 & 2.52375646660552 & 6.09872684993145 \\
\hline $\mathbf{C u}$ & 10.69963200439350 & 5.04029920853570 & 6.09522743457334 \\
\hline $\mathbf{C u}$ & 10.69629746090490 & & 6.10179647864740 \\
\hline $\mathbf{C u}$ & 687903674750 & 3172180600 & 6.09483139824909 \\
\hline $\mathbf{C u}$ & 6514998 & 12.60842295825380 & 6.10095279196659 \\
\hline $\mathbf{C u}$ & 5750 & 2610 & 474809 \\
\hline $\mathbf{C u}$ & 10.69689494744790 & 17.661 & 47106 \\
\hline $\mathbf{C u}$ & 4871716582 & 97060 & 6.0989263 \\
\hline $\mathbf{C u}$ & 33201550 & 9460 & 27759 \\
\hline $\mathbf{C u}$ & 1326154 & 550 & 322949 \\
\hline $\mathbf{C u}$ & 962 & 234 & 664203 \\
\hline $\mathbf{C u}$ & 010942 & 5692 & 066658 \\
\hline $\mathbf{C u}$ & 148134 & 9888 & 6.14054551559304 \\
\hline $\mathbf{C u}$ & 30816 & 10.08891478229880 & 6.13133592100385 \\
\hline $\mathbf{C u}$ & 0710396 & 12.6155 & 6.15390316075542 \\
\hline $\mathbf{C u}$ & 3.56738681539679 & 15.12822184832900 & 6.14875843399508 \\
\hline $\mathbf{C u}$ & 155407343 & 17.65252770332460 & 6.11054790707458 \\
\hline $\mathbf{C u}$ & 7075870 & 20.18 & 6.09310722707406 \\
\hline $\mathbf{C u}$ & 3.56737400918914 & 22.70357203045410 & 6.09369315193824 \\
\hline $\mathbf{C u}$ & 7.13615419587727 & 25.22392053822850 & 6.09640229534969 \\
\hline $\mathbf{C u}$ & 7.13490945169444 & 2.52375821333429 & 6.09871751738047 \\
\hline $\mathbf{C u}$ & 7.13729070245950 & 5.04030280533882 & 6.09522337342001 \\
\hline $\mathbf{C u}$ & 7.14061937200698 & 7.56852190122335 & 6.10180444462483 \\
\hline $\mathbf{C u}$ & 7.14003356751694 & 10.09683009312980 & 6.09483833079780 \\
\hline $\mathbf{C u}$ & 7.13067168040104 & 12.60842467206590 & 6.10093883984157 \\
\hline $\mathbf{C u}$ & 7.14186632751467 & 15.13784006833860 & 6.09476395082527 \\
\hline $\mathbf{C u}$ & 7.14002385726291 & 17.66107702492900 & 6.09730684430706 \\
\hline $\mathbf{C u}$ & 7.12832279780799 & 20.18147386324030 & 6.09890653205814 \\
\hline $\mathbf{C u}$ & 7.13766564819217 & 22.70380602507840 & 6.09630494413936 \\
\hline $\mathbf{C u}$ & 1.78365796263021 & 1.26000208499075 & 7.38823238819699 \\
\hline $\mathbf{C u}$ & 1.78703149345079 & 3.78519319615887 & 7.38763012248956 \\
\hline $\mathbf{C u}$ & 1.78519067958584 & 6.30764300015164 & 7.38062590944664 \\
\hline $\mathbf{C u}$ & 1.77107109479848 & 8.83353359331689 & 7.37994996303992 \\
\hline $\mathbf{C u}$ & 1.79507686501813 & 11.34903746286320 & 7.39361488221087 \\
\hline $\mathbf{C u}$ & 1.77805804339650 & 13.88525777509370 & 7.37550515078831 \\
\hline $\mathbf{C u}$ & 1.77723050014498 & 16.40362098056960 & 7.37349996709889 \\
\hline $\mathbf{C u}$ & 1.78774803874748 & 18.91555735914480 & 7.39507699129493 \\
\hline $\mathbf{C u}$ & 1.78527450315110 & 21.44602115625820 & 7.38634262848904 \\
\hline $\mathbf{C u}$ & 1.78505359911520 & 23.96600205947600 & 7.38845451595980 \\
\hline $\mathbf{C u}$ & 5.35109978129244 & 1.26000333560067 & 7.38822427922283 \\
\hline
\end{tabular}




\begin{tabular}{|c|c|c|c|}
\hline $\mathbf{C u}$ & 5.34772374568229 & 3.78519608911907 & 7.38761130516940 \\
\hline $\mathbf{C u}$ & 5.34956356287296 & 6.30764904428344 & 7.38062318847317 \\
\hline $\mathbf{C u}$ & 5.36368501696764 & 8.83353366925227 & 7.37998182665698 \\
\hline $\mathbf{C u}$ & 5.33967784159017 & 11.34904091548480 & 7.39359423228465 \\
\hline $\mathbf{C u}$ & 5.35670081192808 & 13.88525119633650 & 7.37552633011282 \\
\hline $\mathbf{C u}$ & 5.35753725016778 & 16.40361846446340 & 7.37351243940069 \\
\hline $\mathbf{C u}$ & 5.34701456323226 & 18.91555450620490 & 7.39506086142147 \\
\hline $\mathbf{C u}$ & 5.34948605998877 & 21.44602189118120 & 7.38632886476227 \\
\hline $\mathbf{C u}$ & 5.34970573864361 & 23.96600390808200 & 7.38844642539259 \\
\hline $\mathbf{C u}$ & 8.91846187815591 & 1.26580737076450 & 7.39702462430394 \\
\hline $\mathbf{C u}$ & 8.91846414208967 & 3.78286522274403 & 7.39804152499454 \\
\hline $\mathbf{C u}$ & 8.91846245072286 & 6.30638766534691 & 7.40023201885303 \\
\hline $\mathbf{C u}$ & 8.91845640573829 & 8.83281565493495 & 7.39426317533684 \\
\hline $\mathbf{C u}$ & 8.91845158385726 & 11.35554859211980 & 7.40021512157707 \\
\hline $\mathbf{C u}$ & 8.91844943992083 & & 7.39388568038536 \\
\hline $\mathbf{C u}$ & 8.91845204732587 & & 7.39737357695752 \\
\hline $\mathbf{C u}$ & 8.918 & & 7.40038626985550 \\
\hline $\mathbf{C u}$ & 8.918 & 517820 & 7.39624849283445 \\
\hline $\mathbf{C u}$ & 8.91 & 23.9 & 41704024 \\
\hline $\mathbf{C u}$ & 9960 & 633 & 4236 \\
\hline $\mathbf{C u}$ & 0.0 & & 5343 \\
\hline $\mathbf{C u}$ & 157 & & 8479 \\
\hline $\mathbf{C u}$ & 96 & & 266 \\
\hline $\mathrm{Cu}$ & 412 & 630 & 3328 \\
\hline $\mathbf{C u}$ & 107 & 12. & 5163 \\
\hline $\mathbf{C u}$ & 10.70 & 5510 & 8.60 \\
\hline $\mathbf{C u}$ & 0.0019 & 2990 & 8.616 \\
\hline $\mathbf{C u}$ & 0305 & 0580 & 8.61390 \\
\hline $\mathbf{C u}$ & 6097 & 22.7 & 8.612 \\
\hline $\mathbf{C u}$ & 52667 & 25.22305844543530 & 8.61701340303448 \\
\hline $\mathbf{C u}$ & 8859 & 496631 & 605638677 \\
\hline $\mathbf{C u}$ & 1060 & 7046 & 8.60833579891826 \\
\hline $\mathbf{C u}$ & 3349 & 7.56 & 8.70134161262274 \\
\hline $\mathbf{C u}$ & 455 & 710 & 8.649 \\
\hline $\mathbf{C u}$ & 98 & 000 & 8.73 \\
\hline $\mathbf{C u}$ & 31 & 330 & 1911 \\
\hline $\mathbf{C u}$ & 523369 & 408810 & 3987 \\
\hline $\mathbf{C u}$ & 399772359 & 20.19080193658910 & 8.60978741486255 \\
\hline $\mathbf{C u}$ & 3.56738459623504 & 22.70265271838280 & 8.61387410103745 \\
\hline $\mathbf{C u}$ & 7.13548409850787 & 0.00116032798294 & 8.61221592462246 \\
\hline $\mathbf{C u}$ & 7.12793921178924 & 2.52228679815053 & 8.61630378234652 \\
\hline $\mathbf{C u}$ & 7.12714324575613 & 5.04629151767720 & 8.60941879222557 \\
\hline $\mathbf{C u}$ & 7.13276935402429 & 7.57339718998927 & 8.61476369532713 \\
\hline $\mathbf{C u}$ & 7.13462057372118 & 10.09892841223880 & 8.61637104887330 \\
\hline $\mathbf{C u}$ & 7.13043027327682 & 12.61402333714810 & 8.61331576468975 \\
\hline $\mathbf{C u}$ & 7.13595795922186 & 15.13324822112060 & 8.60882045573288 \\
\hline $\mathbf{C u}$ & 7.13280677429899 & 17.65710136834750 & 8.61660102865664 \\
\hline $\mathbf{C u}$ & 7.13 & 20.1 & 707834355 \\
\hline $\mathbf{C u}$ & 7.13295853602364 & 22.70525598952360 & 8.61229422763413 \\
\hline \multicolumn{4}{|c|}{ CI-NEB image 5} \\
\hline ement & X-Coordinate (Å) & Å) & e (Å) \\
\hline C & 3.567 & 390168 & 68257730 \\
\hline C & 3.56696512623055 & 13.00624180280000 & 10.55163960417290 \\
\hline $\mathbf{F}$ & 3.56742274626228 & 9.42454757953031 & 10.89697171217240 \\
\hline
\end{tabular}




\begin{tabular}{|c|c|c|c|}
\hline $\mathbf{F}$ & 2.47029179197134 & 7.55597798969077 & 11.32812782525480 \\
\hline $\mathbf{F}$ & 4.66423447178680 & 7.55580016636164 & 11.32819972621630 \\
\hline $\mathbf{F}$ & 3.56738247861916 & 15.99006063992330 & 10.33723265696850 \\
\hline $\mathbf{F}$ & 2.50080742295424 & 13.13108549408700 & 11.33264518779830 \\
\hline $\mathbf{F}$ & 4.63269369887871 & 13.13085758597400 & 11.33327881585430 \\
\hline $\mathbf{C u}$ & 0.00000000000000 & 0.00000000000000 & 3.56221131249991 \\
\hline $\mathbf{C u}$ & 0.00000000000000 & 2.52252111840003 & 3.56221131249991 \\
\hline $\mathbf{C u}$ & 0.00000000000000 & 5.04504223680007 & 3.56221131249991 \\
\hline $\mathbf{C u}$ & 0.00000000000000 & 7.56756335519993 & 3.56221131249991 \\
\hline $\mathbf{C u}$ & 0.00000000000000 & 10.09008447359990 & 3.56221131249991 \\
\hline $\mathbf{C u}$ & 0.00000000000000 & 12.61260559199990 & 3.56221131249991 \\
\hline $\mathbf{C u}$ & 0.00000000000000 & 15.13512671040000 & 3.56221131249991 \\
\hline $\mathbf{C u}$ & 0.00000000000000 & 17.65764782880000 & 3.56221131249991 \\
\hline $\mathbf{C u}$ & 0.00000000000000 & 20.18016894719990 & 3.56221131249991 \\
\hline $\mathrm{Cu}$ & 0.00000000000000 & 22.70269006559990 & 3.56221131249991 \\
\hline $\mathbf{C u}$ & 3.56738357634323 & 0.00000000000000 & 3.56221131249991 \\
\hline $\mathbf{C u}$ & 3.56738 & 2.52252111840003 & 3.56221131249991 \\
\hline $\mathbf{C u}$ & 57634323 & 5.04504223680007 & 3.56221131249991 \\
\hline $\mathbf{C u}$ & 3.56738357634323 & & 1131249991 \\
\hline $\mathbf{C u}$ & 3.56738357634323 & 10.09008447359990 & 49991 \\
\hline $\mathbf{C u}$ & 3.56738357634323 & 12.61260559199990 & 49991 \\
\hline $\mathbf{C u}$ & 7634323 & 12671040000 & 3.56221131249991 \\
\hline $\mathbf{C u}$ & 323 & 80000 & 49991 \\
\hline $\mathrm{Cu}$ & 4323 & 1719990 & 249991 \\
\hline $\mathbf{C u}$ & 4323 & 22.70269006559990 & 249991 \\
\hline $\mathbf{C u}$ & 7.13476715482693 & 0.00000000000000 & 3.56221131249991 \\
\hline $\mathbf{C u}$ & 7.13476715482693 & 2.52252111840003 & 3.56221131249991 \\
\hline $\mathbf{C u}$ & 7.13476715482693 & 5.04504223680007 & 3.56221131249991 \\
\hline $\mathbf{C u}$ & 7.13476715482693 & 7.56756335519993 & 3.56221131249991 \\
\hline $\mathbf{C u}$ & 7.13476715482693 & 10.09008447359990 & 3.56221131249991 \\
\hline $\mathbf{C u}$ & 7.13476715482693 & 12.61260559199990 & 3.56221131249991 \\
\hline $\mathbf{C u}$ & 7.13476715482693 & 15.13512671040000 & 3.56221131249991 \\
\hline $\mathbf{C u}$ & 7.13476715482693 & 17.65764782880000 & 3.56221131249991 \\
\hline $\mathbf{C u}$ & 7.13476715482693 & 20.18016894719990 & 3.56221131249991 \\
\hline $\mathbf{C u}$ & 7.13476715482693 & 22.70269006559990 & 3.56221131249991 \\
\hline $\mathbf{C u}$ & 1.78369178 & 1.26126055919993 & 3250005 \\
\hline $\mathbf{C u}$ & 1.78369178870677 & 7759996 & 3250005 \\
\hline $\mathbf{C u}$ & 1.78369178870677 & 000 & 53250005 \\
\hline $\mathbf{C u}$ & 1.78369178870677 & 8.82882391440003 & 4.82343353250005 \\
\hline $\mathbf{C u}$ & 1.78369178870677 & 11.35134503280000 & 4.82343353250005 \\
\hline $\mathbf{C u}$ & 1.78369178870677 & 13.87386615119990 & 4.82343353250005 \\
\hline $\mathbf{C u}$ & 1.78369178870677 & 16.39638726959990 & 4.82343353250005 \\
\hline $\mathbf{C u}$ & 1.78369178870677 & 18.91890838799990 & 4.82343353250005 \\
\hline $\mathbf{C u}$ & 1.78369178870677 & 21.44142950640000 & 4.82343353250005 \\
\hline $\mathbf{C u}$ & 1.78369178870677 & 23.96395062480000 & 4.82343353250005 \\
\hline $\mathbf{C u}$ & 5.35107536505000 & 1.26126055919993 & 4.82343353250005 \\
\hline $\mathbf{C u}$ & 5.35107536505000 & 3.78378167759996 & 4.82343353250005 \\
\hline $\mathbf{C u}$ & 5.35107536505000 & 6.30630279600000 & 4.82343353250005 \\
\hline $\mathbf{C u}$ & 5.35107536505000 & 8.82882391440003 & 4.82343353250005 \\
\hline $\mathbf{C u}$ & 5.35107536505000 & 11.35134503280000 & 4.82343353250005 \\
\hline $\mathbf{C u}$ & 5.35107536505000 & 13.87386615119990 & 4.82343353250005 \\
\hline $\mathbf{C u}$ & 5.35107536505000 & 16.39638726959990 & 4.82343353250005 \\
\hline $\mathbf{C u}$ & 5.35107536505000 & 18.91890838799990 & 4.82343353250005 \\
\hline $\mathbf{C u}$ & 5.35107536505000 & 21.44142950640000 & 4.82343353250005 \\
\hline
\end{tabular}




\begin{tabular}{|c|c|c|c|}
\hline $\mathbf{C u}$ & 5.35107536505000 & 23.96395062480000 & 4.82343353250005 \\
\hline $\mathbf{C u}$ & 8.91845894246346 & 1.26126055919993 & 4.82343353250005 \\
\hline $\mathbf{C u}$ & 8.91845894246346 & 3.78378167759996 & 4.82343353250005 \\
\hline $\mathbf{C u}$ & 8.91845894246346 & 6.30630279600000 & 4.82343353250005 \\
\hline $\mathbf{C u}$ & 8.91845894246346 & 8.82882391440003 & 4.82343353250005 \\
\hline $\mathbf{C u}$ & 8.91845894246346 & 11.35134503280000 & 4.82343353250005 \\
\hline $\mathbf{C u}$ & 8.91845894246346 & 13.87386615119990 & 4.82343353250005 \\
\hline $\mathbf{C u}$ & 8.91845894246346 & 16.39638726959990 & 4.82343353250005 \\
\hline $\mathbf{C u}$ & 8.91845894246346 & 18.91890838799990 & 4.82343353250005 \\
\hline $\mathbf{C u}$ & 8.91845894246346 & 21.44142950640000 & 4.82343353250005 \\
\hline $\mathbf{C u}$ & 8.91845894246346 & 23.96395062480000 & 4.82343353250005 \\
\hline $\mathbf{C u}$ & 10.69926845526850 & 25.22368700792130 & 6.09582074465806 \\
\hline $\mathbf{C u}$ & 10.70166690435800 & 2.52300673508959 & 6.09820056540959 \\
\hline $\mathbf{C u}$ & 10.70077941854230 & 5.03987421310175 & 6.09504870861328 \\
\hline $\mathrm{Cu}$ & 10.69472797587760 & 7.56782120320029 & 6.10131802302023 \\
\hline $\mathbf{C u}$ & 10.69396648024020 & 10.09565127321590 & 6.09426688270700 \\
\hline $\mathbf{C u}$ & 0.00412638366153 & 12.60876331608240 & 6.10024456004431 \\
\hline $\mathbf{C u}$ & 10.69487378422480 & 15.13577161886930 & 6.09473071886219 \\
\hline $\mathbf{C u}$ & 10.69877596397980 & 17.66193777155280 & 6.09986482107209 \\
\hline $\mathbf{C u}$ & 0.00987831109157 & 20.178972 & 868256 \\
\hline $\mathbf{C u}$ & 10.70083049475970 & 22.70211097198140 & 021025 \\
\hline $\mathbf{C u}$ & 37871305557 & 0.00039608623879 & 119663 \\
\hline $\mathbf{C u}$ & 5130012 & 2.52 & 93698 \\
\hline $\mathrm{Cu}$ & 55882088 & 166241 & 096549 \\
\hline $\mathbf{C u}$ & 7897 & 4195 & 068142 \\
\hline $\mathbf{C u}$ & 3.56738046861278 & 10.08835488825340 & 6.13333561823138 \\
\hline $\mathbf{C u}$ & 3.56737927973673 & 12.61126422214380 & 6.15203848772094 \\
\hline $\mathbf{C u}$ & 3.56738425127893 & 15.12619060857440 & 6.14498673943765 \\
\hline $\mathbf{C u}$ & 3.56737699959071 & 17.64586993065550 & 6.13725170114892 \\
\hline $\mathbf{C u}$ & 3.56737430334503 & 20.17619140597240 & 6.09493734014483 \\
\hline $\mathbf{C u}$ & 3.56737798934433 & 22.70011829658060 & 6.09417868236550 \\
\hline $\mathbf{C u}$ & 7.13764979177293 & 25.22368959977000 & 6.09582137701974 \\
\hline $\mathbf{C u}$ & 7.13525079540516 & 2.52300783596542 & 6.09819132479607 \\
\hline $\mathbf{C u}$ & 7.13613635056763 & 5.03987643217383 & 6.09504340676640 \\
\hline $\mathbf{C u}$ & 7.14218249601193 & 7.56782217263599 & 6.10133012417892 \\
\hline $\mathbf{C u}$ & 7.14294138471121 & 717928190 & 6.09428101554470 \\
\hline $\mathbf{C u}$ & 7.13063411090359 & 12.60876331988630 & 6.10023318492348 \\
\hline $\mathbf{C u}$ & .14203384587876 & 15.13577014694820 & 6.0947 \\
\hline $\mathbf{C u}$ & 7.13814255795801 & 17.66193542560380 & 6.09986579165146 \\
\hline $\mathbf{C u}$ & 7.12489314602612 & 20.17897360851280 & 6.10151932709941 \\
\hline $\mathbf{C u}$ & 7.13608874721011 & 22.70211371268570 & 6.09648016342415 \\
\hline $\mathbf{C u}$ & 1.78395475058406 & 1.25827632458306 & 7.38822764979861 \\
\hline $\mathbf{C u}$ & 1.78711529754420 & 3.78317468196778 & 7.38686870950095 \\
\hline $\mathbf{C u}$ & 1.78524686234390 & 6.30756529882045 & 7.38071602030378 \\
\hline $\mathbf{C u}$ & 1.77027904304959 & 8.82971192159374 & 7.38009834215339 \\
\hline $\mathbf{C u}$ & 1.78970039264939 & 11.33957056783580 & 7.39069206848229 \\
\hline $\mathbf{C u}$ & 1.77923797802790 & 13.87897448983030 & 7.37106378714109 \\
\hline $\mathbf{C u}$ & 1.77723037382852 & 16.39579991971380 & 7.37848083882754 \\
\hline $\mathbf{C u}$ & 1.80204186164052 & 18.90130819914250 & 7.40898003353657 \\
\hline $\mathbf{C u}$ & 1.78792856147517 & 21.44018809317010 & 7.38806389133290 \\
\hline $\mathbf{C u}$ & 1.78555332589363 & 23.96473552495920 & 7.38747953331850 \\
\hline $\mathbf{C u}$ & 5.35080839060355 & 1.25827711757854 & 7.38821604298154 \\
\hline $\mathbf{C u}$ & 5.34764314454353 & 3.78317596510110 & 7.38684637242811 \\
\hline $\mathbf{C u}$ & 5.34950762368090 & 6.30757048552055 & 7.38071275165133 \\
\hline
\end{tabular}




\begin{tabular}{|c|c|c|c|}
\hline $\mathrm{Cu}$ & 5.36447521325160 & 8.82970974621358 & 7.38013262075971 \\
\hline $\mathrm{Cu}$ & 5.34505299749158 & 11.33957110533370 & 7.39067765194354 \\
\hline $\mathbf{C u}$ & 5.35551634386026 & 13.87896570892650 & 7.37108431302185 \\
\hline $\mathbf{C u}$ & 5.35753379872407 & 16.39579696487970 & 7.37849198324361 \\
\hline $\mathbf{C u}$ & 5.33272262877965 & 18.90130493177360 & 7.40893080166648 \\
\hline $\mathbf{C u}$ & 5.34683722855410 & 21.44019069352750 & 7.38803351676614 \\
\hline $\mathbf{C u}$ & 5.34921281966627 & 23.96473980338140 & 7.38746556401004 \\
\hline $\mathbf{C u}$ & 8.91845284014372 & 1.26465210113827 & 7.39721407835312 \\
\hline $\mathbf{C u}$ & 8.91845521218186 & 3.78232414769833 & 7.40031575314431 \\
\hline $\mathbf{C u}$ & 8.91845462782991 & 6.30552850526458 & 7.40083388467078 \\
\hline $\mathbf{C u}$ & 8.91845001373080 & 8.83157649313702 & 7.39437541878217 \\
\hline $\mathbf{C u}$ & 8.91844835425737 & 11.35548842263680 & 7.39883193076622 \\
\hline $\mathbf{C u}$ & 8.91844501228213 & 13.87073608059410 & 7.39535207113132 \\
\hline $\mathbf{C u}$ & 8.91844749779245 & 16.40098739037440 & 7.39385532181821 \\
\hline $\mathbf{C u}$ & 8.91845235722871 & 18.91940313816950 & 7.40248630094046 \\
\hline $\mathbf{C u}$ & 8.91845222 & 69040 & 12519 \\
\hline $\mathbf{C u}$ & 8.91845147390084 & 17544040 & 4601 \\
\hline $\mathbf{C u}$ & 10.70091013412550 & 68883550 & 3429 \\
\hline $\mathbf{C u}$ & 0.006456 & 92 & 8.6 \\
\hline $\mathbf{C u}$ & 324 & & 95 \\
\hline $\mathbf{C u}$ & 0.0 & & 157 \\
\hline $\mathbf{C u}$ & 10.7 & 70 & 335 \\
\hline $\mathbf{C u}$ & 48 & & 8.6 \\
\hline $\mathbf{C u}$ & 10.7 & 500 & 8.6 \\
\hline $\mathbf{C u}$ & 0.00 & 670 & 8.6 \\
\hline $\mathbf{C u}$ & 89 & 270 & 8.6 \\
\hline $\mathbf{C u}$ & 54 & 680 & 8.6 \\
\hline $\mathbf{C u}$ & 12 & 450 & 8.6 \\
\hline $\mathbf{C u}$ & 30 & & $8 .($ \\
\hline $\mathbf{C u}$ & 53 & 29 & 978 \\
\hline $\mathbf{C u}$ & 49 & 61 & 8.7 \\
\hline $\mathbf{C u}$ & 34 & 730 & 3946 \\
\hline $\mathbf{C u}$ & 67 & 53290 & 52705 \\
\hline $\mathbf{C u}$ & 912 & 29690 & 8.67348259004588 \\
\hline $\mathbf{C u}$ & 3599 & 17.6108644 & 8.75451085556613 \\
\hline $\mathbf{C u}$ & 56740174189999 & 20.16627778715530 & 8.61156481162405 \\
\hline $\mathbf{C u}$ & 56739377746399 & 22.69208025652590 & 8.61239731162351 \\
\hline $\mathbf{C u}$ & 7130085 & 0.00000970755435 & 8.61119114334738 \\
\hline $\mathbf{C u}$ & 7.12829349613206 & 2.52110828106544 & 8.61635242595246 \\
\hline $\mathbf{C u}$ & 12623118690244 & 5.04576877731504 & 8.60892076464211 \\
\hline $\mathbf{C u}$ & 13373580936901 & 7.57237004402686 & 8.61447239685676 \\
\hline $\mathbf{C u}$ & 2077 & 10.09761854490080 & 8.61660411399512 \\
\hline $\mathbf{C u}$ & 7.13070169343433 & 12.61280562197330 & 8.61200359366728 \\
\hline $\mathbf{C u}$ & 13679697497363 & 15.13028682917050 & 8.6078477 \\
\hline $\mathbf{C u}$ & 7.13387068316479 & 17.65362852164530 & 8.61628506504120 \\
\hline $\mathbf{C u}$ & 7.12871408838104 & 20.17775538447680 & 8.61280530796408 \\
\hline $\mathbf{C u}$ & 7.13173983020774 & 22.70245965488360 & 8.61187296844344 \\
\hline \multicolumn{4}{|c|}{ NEB Image 6} \\
\hline ement & X-Coordinate (§) & $\mathrm{Y}-\mathrm{Cc}$ & $\mathrm{Z}-\mathrm{C}$ \\
\hline C & 4405517 & 7.99945643180343 & 10.62762720583620 \\
\hline C & 3.56689553495619 & 13.20498309599550 & 10.42748318209320 \\
\hline $\mathbf{F}$ & 3.56741518908510 & 9.41124465621684 & 10.90134855656080 \\
\hline $\mathbf{F}$ & 2.46975439047691 & 7.54157040413085 & 11.32336957918460 \\
\hline $\mathbf{F}$ & 308 & 53 & \\
\hline
\end{tabular}




\begin{tabular}{|c|c|c|c|}
\hline $\mathbf{F}$ & 3.56734071689570 & 16.38793271507990 & 10.23115342340550 \\
\hline $\mathbf{F}$ & 2.49435152767016 & 13.30125244647660 & 11.22213463308720 \\
\hline $\mathbf{F}$ & 4.63907966815642 & 13.30110432875490 & 11.22267265197580 \\
\hline $\mathbf{C u}$ & 0.00000000000000 & 0.00000000000000 & 3.56221131249991 \\
\hline $\mathbf{C u}$ & 0.00000000000000 & 2.52252111840003 & 3.56221131249991 \\
\hline $\mathbf{C u}$ & 0.00000000000000 & 5.04504223680007 & 3.56221131249991 \\
\hline $\mathbf{C u}$ & 0.00000000000000 & 7.56756335519993 & 3.56221131249991 \\
\hline $\mathbf{C u}$ & 0.00000000000000 & 10.09008447359990 & 3.56221131249991 \\
\hline $\mathbf{C u}$ & 0.00000000000000 & 12.61260559199990 & 3.56221131249991 \\
\hline $\mathbf{C u}$ & 0.00000000000000 & 15.13512671040000 & 249991 \\
\hline $\mathbf{C u}$ & 0.00000000000000 & 17.65764782880000 & 21131249991 \\
\hline $\mathbf{C u}$ & 0.00000000000000 & 20.18016894719990 & 3.56221131249991 \\
\hline $\mathbf{C u}$ & 0.00000000000000 & 22.70269006559990 & 3.56221131249991 \\
\hline $\mathbf{C u}$ & 3.56738357634323 & 0.00000000000000 & 3.56221131249991 \\
\hline $\mathbf{C u}$ & 3.56738357634323 & 2.52252111840003 & 3.56221131249991 \\
\hline $\mathrm{Cu}$ & 3.56738357634323 & 5.04504223680007 & 3.56221131249991 \\
\hline $\mathbf{C u}$ & 7634323 & 5519993 & 249991 \\
\hline $\mathbf{C u}$ & 3.56738357634323 & 10.09008447359990 & 3.56221131249991 \\
\hline $\mathbf{C u}$ & 7634323 & 12.61260559199990 & 1249991 \\
\hline $\mathbf{C u}$ & 7634323 & 15.13512671040000 & 249991 \\
\hline $\mathbf{C u}$ & 3.56738357634323 & 17.65764782880000 & 3.56221131249991 \\
\hline $\mathbf{C u}$ & 3.56738357634323 & 20.18016894719990 & 3.56221131249991 \\
\hline $\mathbf{C u}$ & 3.56738357634323 & 22.70269006559990 & 3.56221131249991 \\
\hline $\mathbf{C u}$ & 7.13476715482693 & 0.00000000000000 & 3.56221131249991 \\
\hline $\mathbf{C u}$ & 7.13476715482693 & 2.52252111840003 & 3.56221131249991 \\
\hline $\mathbf{C u}$ & 7.13476715482693 & 5.04504223680007 & 3.56221131249991 \\
\hline $\mathbf{C u}$ & 7.13476715482693 & 7.56756335519993 & 3.56221131249991 \\
\hline $\mathbf{C u}$ & 7.13476715482693 & 10.09008447359990 & 3.56221131249991 \\
\hline $\mathbf{C u}$ & 7.13476715482693 & 12.61260559199990 & 3.56221131249991 \\
\hline $\mathbf{C u}$ & 7.13476715482693 & 15.13512671040000 & 3.56221131249991 \\
\hline $\mathbf{C u}$ & 7.13476715482693 & 17.65764782880000 & 3.56221131249991 \\
\hline $\mathbf{C u}$ & 7.13476715482693 & 20.18016894719990 & 3.56221131249991 \\
\hline $\mathbf{C u}$ & 7.13476715482693 & 22.70269006559990 & 3.56221131249991 \\
\hline $\mathbf{C u}$ & 1.78369178870677 & 1.26126055919993 & 4.82343353250005 \\
\hline $\mathbf{C u}$ & 1.78369178870677 & 3.78378167759996 & 4.82343353250005 \\
\hline $\mathbf{C u}$ & 1.78369178870677 & 6.30630279600000 & 4.82343353250005 \\
\hline $\mathbf{C u}$ & 1.78369178870677 & 91440003 & 50005 \\
\hline $\mathbf{C u}$ & 1.78369178870677 & 11.35134503280000 & 4.82343353250005 \\
\hline $\mathbf{C u}$ & 1.78369178870677 & 13.87386615119990 & 4.82343353250005 \\
\hline $\mathbf{C u}$ & 1.78369178870677 & 16.39638726959990 & 4.82343353250005 \\
\hline $\mathbf{C u}$ & 1.78369178870677 & 18.91890838799990 & 4.82343353250005 \\
\hline $\mathbf{C u}$ & 1.78369178870677 & 21.44142950640000 & 4.82343353250005 \\
\hline $\mathbf{C u}$ & 1.78369178870677 & 23.96395062480000 & 4.82343353250005 \\
\hline $\mathbf{C u}$ & 5.351 & 1.26 & 3250005 \\
\hline $\mathbf{C u}$ & 5.351 & 3.783781 & 50005 \\
\hline $\mathbf{C u}$ & 5.35 & 6.30 & 4.82343 \\
\hline $\mathbf{C u}$ & 05000 & 1440003 & 250005 \\
\hline $\mathbf{C u}$ & 5.35107536505000 & 11.35134503280000 & 4.82343353250005 \\
\hline $\mathbf{C u}$ & 5.35107536505000 & 13.87386615119990 & 4.82343353250005 \\
\hline $\mathbf{C u}$ & 5.35107536505000 & 16.39638726959990 & 4.82343353250005 \\
\hline $\mathbf{C u}$ & 5.35107536505000 & 18.91890838799990 & 4.82343353250005 \\
\hline $\mathbf{C u}$ & 5.35107536505000 & 21.44142950640000 & 4.82343353250005 \\
\hline $\mathbf{C u}$ & 5.35107536505000 & 23.96395062480000 & 4.82343353250005 \\
\hline $\mathbf{C u}$ & 8.91845894246346 & 1.26126055919993 & 4.82343353250005 \\
\hline
\end{tabular}




\begin{tabular}{|c|c|c|c|}
\hline $\mathbf{C u}$ & 8.91845894246346 & 3.78378167759996 & 4.82343353250005 \\
\hline $\mathbf{C u}$ & 8.91845894246346 & 6.30630279600000 & 4.82343353250005 \\
\hline $\mathbf{C u}$ & 8.91845894246346 & 8.82882391440003 & 4.82343353250005 \\
\hline $\mathbf{C u}$ & 8.91845894246346 & 11.35134503280000 & 4.82343353250005 \\
\hline $\mathrm{Cu}$ & 8.91845894246346 & 13.87386615119990 & 4.82343353250005 \\
\hline $\mathbf{C u}$ & 8.91845894246346 & 16.39638726959990 & 4.82343353250005 \\
\hline $\mathrm{Cu}$ & 8.91845894246346 & 18.91890838799990 & 4.82343353250005 \\
\hline $\mathbf{C u}$ & 8.91845894246346 & 21.44142950640000 & 4.82343353250005 \\
\hline $\mathbf{C u}$ & 8.91845894246346 & 23.96395062480000 & 4.82343353250005 \\
\hline $\mathbf{C u}$ & 10.69898915435910 & 25.22234393660630 & 6.09504461727304 \\
\hline $\mathbf{C u}$ & -0.00011659397148 & 2.52190547210736 & 6.09779093187481 \\
\hline $\mathbf{C u}$ & 10.70065336721510 & 5.03830437560382 & 6.09433448683963 \\
\hline $\mathbf{C u}$ & 10.69349613100750 & 7.56530682779588 & 6.10077701440571 \\
\hline $\mathrm{Cu}$ & 10.69143416392270 & 10.09275611509460 & 6.09347540370365 \\
\hline $\mathrm{Cu}$ & 0.00261023967703 & 12.60801274385370 & 6.09900543727790 \\
\hline $\mathrm{Cu}$ & 10.69615912562350 & 15.13518557541030 & 6.09466713569788 \\
\hline $\mathrm{Cu}$ & 10.70222739666140 & 17.65801274514430 & 6.10261472337219 \\
\hline $\mathbf{C u}$ & 0.00897201645492 & 20.17584456832550 & 6.10017843831247 \\
\hline $\mathbf{C u}$ & 8436556160 & 22.70030436151180 & 6.09568155873242 \\
\hline $\mathbf{C u}$ & 3.56737824183533 & 625955846 & 5078756 \\
\hline $\mathrm{Cu}$ & 3.56737778493832 & 2.52229865083043 & 2153704 \\
\hline $\mathrm{Cu}$ & 893775605 & 5.04765364811520 & 05768725 \\
\hline $\mathrm{Cu}$ & 3.56 & 246 & 917985 \\
\hline $\mathrm{Cu}$ & 9554 & 10.08 & 828133 \\
\hline $\mathbf{C u}$ & 96483 & 60350 & 6.130 \\
\hline $\mathbf{C u}$ & 3.56738081504846 & 15.12478872502380 & 6.16384395500707 \\
\hline $\mathbf{C u}$ & 3.56737184744821 & 17.63957129884050 & 6.14416892443910 \\
\hline $\mathbf{C u}$ & 3.56737118382046 & 20.17221651242410 & 6.09391311072410 \\
\hline $\mathbf{C u}$ & 3.56737617515610 & 22.69841286364440 & 6.09402568674953 \\
\hline $\mathbf{C u}$ & 7.13793286457943 & 25.22234705990330 & 6.09505740704038 \\
\hline $\mathbf{C u}$ & 7.13488715710468 & 2.52190799897819 & 6.09779156807573 \\
\hline $\mathrm{Cu}$ & 7.13626300089563 & 5.03831005983821 & 6.09434060754084 \\
\hline $\mathbf{C u}$ & 7.14341717824171 & 7.56530845365072 & 6.10080381671588 \\
\hline $\mathbf{C u}$ & 7.14547478672919 & 10.09275005816010 & 6.09350967732526 \\
\hline $\mathbf{C u}$ & 7.13215015079290 & 12.60801196941100 & 6.09901003809984 \\
\hline $\mathrm{Cu}$ & 7.14074980527828 & 15.13518241592730 & 6.09469161889728 \\
\hline $\mathbf{C u}$ & 7.13469631265153 & 17.65801203515600 & 6.10261652823071 \\
\hline $\mathrm{Cu}$ & 8618 & 20.17584892996140 & 870374417 \\
\hline $\mathrm{Cu}$ & 7.13693762557068 & 22.70030808222000 & 6.09568690222081 \\
\hline $\mathbf{C u}$ & 1.78409522495120 & 1.25570592421926 & 7.38747927184061 \\
\hline $\mathrm{Cu}$ & 1.78733133486712 & 3.78129713786719 & 7.38594821219152 \\
\hline $\mathrm{Cu}$ & 1.78465197051013 & 6.30408392713837 & 7.37988126791406 \\
\hline $\mathbf{C u}$ & 1.76963210098853 & 8.82390329292427 & 7.38007154459826 \\
\hline $\mathbf{C u}$ & 1.78268387135755 & 11.33147208723580 & 7.38322484033136 \\
\hline $\mathbf{C u}$ & 1.77788650630620 & 13.87973966504750 & 7.36874550156142 \\
\hline $\mathbf{C u}$ & 1.78724965106404 & 16.38204710310280 & 7.39193604950096 \\
\hline $\mathbf{C u}$ & 1.80076611592912 & 18.90189657283650 & 7.40099719605456 \\
\hline $\mathrm{Cu}$ & 1.78575658560595 & 21.43935815159190 & 7.38631280371841 \\
\hline $\mathbf{C u}$ & 1.78477544947470 & 23.96230657324740 & 7.38607892797262 \\
\hline $\mathrm{Cu}$ & 5.35065980361253 & 1.25570794553294 & 7.38749010996163 \\
\hline $\mathbf{C u}$ & 5.34741835759003 & 3.78130178778930 & 7.38595092426956 \\
\hline $\mathbf{C u}$ & 5.35008440981077 & 6.30409744234265 & 7.37992285892111 \\
\hline $\mathrm{Cu}$ & 5.36510776681093 & 8.82389421139392 & 7.38015128420298 \\
\hline $\mathrm{Cu}$ & 5.35206108696184 & 11.33147500217690 & 7.38326213949613 \\
\hline
\end{tabular}




\begin{tabular}{|c|c|c|c|}
\hline $\mathbf{C u}$ & 5.35684873581754 & 13.87973661053490 & 7.36880534852235 \\
\hline $\mathbf{C u}$ & 5.34749549465373 & 16.38204442527000 & 7.39195203004784 \\
\hline $\mathbf{C u}$ & 5.33398247278113 & 18.90189687721760 & 7.40096356612779 \\
\hline $\mathbf{C u}$ & 5.34899577194335 & 21.43936340547220 & 7.38630966022541 \\
\hline $\mathbf{C u}$ & 5.34997820045306 & 23.96231052732760 & 7.38608877588548 \\
\hline $\mathbf{C u}$ & 8.91846643060594 & 1.26266116732798 & 7.39656201761044 \\
\hline $\mathbf{C u}$ & 8.91846976645994 & 3.78058633654889 & 7.40175241992498 \\
\hline $\mathbf{C u}$ & 8.91847232173383 & 6.30211367121485 & 7.40057854890078 \\
\hline $\mathbf{C u}$ & 8.91846816414400 & 8.82877678810364 & 7.39405669806442 \\
\hline $\mathbf{C u}$ & 8.91845974467029 & 11.35384734829370 & 7.39859200299050 \\
\hline $\mathbf{C u}$ & 8.91845856307715 & 13.86707080740580 & 7.39473886524493 \\
\hline $\mathbf{C u}$ & 8.91846105720706 & 16.39817155002720 & 7.39211954619746 \\
\hline $\mathbf{C u}$ & 8.91846394006859 & 18.91404367074150 & 7.40224180255545 \\
\hline $\mathbf{C u}$ & 8.918467 & 21.43389605576610 & 7.3969 \\
\hline $\mathbf{C u}$ & 8.91846634138047 & 23.95490903876140 & 7.39497344446169 \\
\hline $\mathbf{C u}$ & 10.69992446556050 & -0.00271844269458 & 8.61007990021438 \\
\hline $\mathbf{C u}$ & 0.00 & 458 & 1033 \\
\hline $\mathrm{Cu}$ & 0.00 & 668 & 231 \\
\hline $\mathrm{Cu}$ & 0.00 & 272 & 7620 \\
\hline $\mathbf{C u}$ & 10.65 & 10. & 2085 \\
\hline $\mathbf{C u}$ & 602 & 7730 & 9991 \\
\hline $\mathbf{C u}$ & 10.6 & 6970 & 7871 \\
\hline $\mathbf{C u}$ & 0.00 & 8440 & 1648 \\
\hline $\mathbf{C u}$ & 385 & 9770 & 80808 \\
\hline $\mathbf{C u}$ & 568 & 22.6 & 3309 \\
\hline $\mathrm{Cu}$ & 6287 & -0.00 & 8.61 \\
\hline $\mathrm{Cu}$ & 1419219 & 331461 & 8.610937 \\
\hline $\mathbf{C u}$ & 990 & 5071 & 882920484 \\
\hline $\mathbf{C u}$ & 179225 & 7.56250086475274 & 8.69921431515552 \\
\hline $\mathbf{C u}$ & $3.56^{\circ}$ & 10.1084748 & 8.65713737755980 \\
\hline $\mathbf{C u}$ & 163 & 12.57117821902460 & 8.65010330625770 \\
\hline $\mathbf{C u}$ & 666 & 15.0 & 2894104 \\
\hline $\mathbf{C u}$ & 206 & 17.6 & 59781 \\
\hline $\mathbf{C u}$ & 517 & 20.17 & 3457 \\
\hline $\mathbf{C u}$ & 315 & 22.65 & 5564 \\
\hline $\mathbf{C u}$ & 80 & -0.0 & 8.61 \\
\hline $\mathbf{C u}$ & 418103303 & 2.5180763 & 8.61605776399628 \\
\hline $\mathbf{C u}$ & 382 & 327 & 8.60 \\
\hline $\mathbf{C u}$ & 7.13396858147506 & 7.56836647679182 & 8.61336834052158 \\
\hline $\mathbf{C u}$ & 7.13791692414780 & 10.09424888492780 & 8.61579983194885 \\
\hline $\mathbf{C u}$ & 5159059770 & 12.61000005991990 & 8.61061806416196 \\
\hline $\mathbf{C u}$ & 7.138 & 465397090 & 8.60697792696208 \\
\hline $\mathbf{C u}$ & 7.13205839717844 & 17.64727858690170 & 8.61412162721612 \\
\hline $\mathbf{C u}$ & 7.12738416697785 & 382510 & 8.61022515668631 \\
\hline $\mathbf{C u}$ & 7.13189211685176 & 22.69845067704400 & 8.61180131328299 \\
\hline \multicolumn{4}{|c|}{ CI-NEB Image 7} \\
\hline ement & $\mathrm{X}-\mathrm{Cc}$ & Y-Coordinate $(\AA)$ & Z-Coordinate (§) \\
\hline C & & & 9240 \\
\hline C & 3.56619562514859 & 13.52715705224350 & 10.32478773836150 \\
\hline $\mathbf{F}$ & & & 10.88710294028440 \\
\hline $\mathbf{F}$ & 2.47031777699580 & 7.59217161249964 & 11.32851320853510 \\
\hline $\mathbf{F}$ & 4.66439191580274 & 7.59214883323084 & 11.32860968230670 \\
\hline $\mathbf{F}$ & 5326 & 16.50418968067830 & 10.22987718439040 \\
\hline $\mathbf{F}$ & 2.48782379409238 & 13.59507357789270 & 11.12619790214690 \\
\hline
\end{tabular}




\begin{tabular}{|c|c|c|c|}
\hline $\mathbf{F}$ & 4.64275224890801 & 13.59380109561300 & 11.12860864364510 \\
\hline $\mathbf{C u}$ & 0.00000000000000 & 0.00000000000000 & 3.56221131249991 \\
\hline $\mathbf{C u}$ & 0.00000000000000 & 2.52252111840003 & 3.56221131249991 \\
\hline $\mathbf{C u}$ & 0.00000000000000 & 5.04504223680007 & 3.56221131249991 \\
\hline $\mathbf{C u}$ & 0.00000000000000 & 7.56756335519993 & 3.56221131249991 \\
\hline $\mathbf{C u}$ & 0.00000000000000 & 10.09008447359990 & 3.56221131249991 \\
\hline $\mathbf{C u}$ & 0.00000000000000 & 12.61260559199990 & 3.56221131249991 \\
\hline $\mathbf{C u}$ & 0.00000000000000 & 15.13512671040000 & 3.56221131249991 \\
\hline $\mathbf{C u}$ & 0.00000000000000 & 17.65764782880000 & 3.56221131249991 \\
\hline $\mathbf{C u}$ & 0.00000000000000 & 20.18016894719990 & 3.56221131249991 \\
\hline $\mathbf{C u}$ & 0.00000000000000 & 22.70269006559990 & 3.56221131249991 \\
\hline $\mathbf{C u}$ & 3.56738357634323 & 0.00000000000000 & 3.56221131249991 \\
\hline $\mathbf{C u}$ & 3.56738357634323 & 2.52252111840003 & 3.56221131249991 \\
\hline $\mathbf{C u}$ & 3.56738357634323 & 5.04504223680007 & 3.56221131249991 \\
\hline $\mathbf{C u}$ & 3.56738357634323 & 7.56756335519993 & 3.56221131249991 \\
\hline $\mathrm{Cu}$ & 3.56738357634323 & 10.09008447359990 & 3.56221131249991 \\
\hline $\mathbf{C u}$ & 3.56738357634323 & 12.61260559199990 & 3.56221131249991 \\
\hline $\mathbf{C u}$ & 3.56738357634323 & 15.13512671040000 & 3.56221131249991 \\
\hline $\mathbf{C u}$ & 3.56738357634323 & 17.65764782880000 & 3.56221131249991 \\
\hline $\mathbf{C u}$ & 7634323 & 20.18016894719990 & 3.56221 \\
\hline $\mathbf{C u}$ & 3.56738357634323 & 22.70269006 & 3.562211 \\
\hline $\mathbf{C u}$ & 5482693 & 0000000000 & 3.56221 \\
\hline $\mathbf{C u}$ & 82693 & 2.52 & 49991 \\
\hline $\mathrm{Cu}$ & 5482693 & 3680007 & 249991 \\
\hline $\mathbf{C u}$ & 482693 & 9993 & 3.56221 \\
\hline $\mathbf{C u}$ & 7.13476715482693 & 10.09008447359990 & 3.56221131249991 \\
\hline $\mathbf{C u}$ & 7.13476715482693 & 12.61260559199990 & 3.56221131249991 \\
\hline $\mathbf{C u}$ & 7.13476715482693 & 15.13512671040000 & 3.56221131249991 \\
\hline $\mathbf{C u}$ & 7.13476715482693 & 17.65764782880000 & 3.56221131249991 \\
\hline $\mathbf{C u}$ & 7.13476715482693 & 20.18016894719990 & 3.56221131249991 \\
\hline $\mathbf{C u}$ & 7.13476715482693 & 22.70269006559990 & 3.56221131249991 \\
\hline $\mathbf{C u}$ & 1.78369178870677 & 1.26126055919993 & 4.82343353250005 \\
\hline $\mathbf{C u}$ & 1.78369178870677 & 3.78378167759996 & 4.82343353250005 \\
\hline $\mathbf{C u}$ & 1.78369178870677 & 6.306302796 & 4.82343353250005 \\
\hline $\mathbf{C u}$ & 1.78369178870677 & 8.82882391440003 & 4.82343353250005 \\
\hline $\mathbf{C u}$ & 1.78369178870677 & 11.35134503280000 & 4.82343353250005 \\
\hline $\mathbf{C u}$ & 1.78369178870677 & 13.87386615119990 & 4.82343353250005 \\
\hline $\mathbf{C u}$ & 1.78369178870677 & 16.39638726959990 & 4.82343353250005 \\
\hline $\mathbf{C u}$ & 1.78369178870677 & 18.91890838799990 & 4.82343353250005 \\
\hline $\mathbf{C u}$ & 1.78369178870677 & 21.44142950640000 & 4.82343353250005 \\
\hline $\mathbf{C u}$ & 1.78369178870677 & 23.96395062480000 & 4.82343353250005 \\
\hline $\mathbf{C u}$ & 5.35107536505000 & 1.26126055919993 & 4.82343353250005 \\
\hline $\mathbf{C u}$ & 5.35107536505000 & 3.78378167759996 & 4.82343353250005 \\
\hline $\mathbf{C u}$ & 5.35107536505000 & 6.30630279600000 & 4.82343353250005 \\
\hline $\mathbf{C u}$ & 5.35107536505000 & 8.82882391440003 & 4.82343353250005 \\
\hline $\mathbf{C u}$ & 5.35107536505000 & 11.35134503280000 & 4.82343353250005 \\
\hline $\mathbf{C u}$ & 5.35107536505000 & 13.87386615119990 & 4.82343353250005 \\
\hline $\mathbf{C u}$ & 5.35107536505000 & 16.39638726959990 & 4.82343353250005 \\
\hline $\mathbf{C u}$ & 5.35107536505000 & 18.91890838799990 & 4.82343353250005 \\
\hline $\mathbf{C u}$ & 5.35107536505000 & 21.44142950640000 & 4.82343353250005 \\
\hline $\mathbf{C u}$ & 5.35107536505000 & 23.96395062480000 & 4.82343353250005 \\
\hline $\mathbf{C u}$ & 8.91845894246346 & 1.26126055919993 & 4.82343353250005 \\
\hline $\mathbf{C u}$ & 8.91845894246346 & 3.78378167759996 & 4.82343353250005 \\
\hline $\mathbf{C u}$ & 8.91845894246346 & 6.30630279600000 & 4.82343353250005 \\
\hline
\end{tabular}




\begin{tabular}{|c|c|c|c|}
\hline $\mathbf{C u}$ & 8.91845894246346 & 8.82882391440003 & 4.82343353250005 \\
\hline $\mathbf{C u}$ & 8.91845894246346 & 11.35134503280000 & 4.82343353250005 \\
\hline $\mathbf{C u}$ & 8.91845894246346 & 13.87386615119990 & 4.82343353250005 \\
\hline $\mathbf{C u}$ & 8.91845894246346 & 16.39638726959990 & 4.82343353250005 \\
\hline $\mathbf{C u}$ & 8.91845894246346 & 18.91890838799990 & 4.82343353250005 \\
\hline $\mathbf{C u}$ & 8.91845894246346 & 21.44142950640000 & 4.82343353250005 \\
\hline $\mathbf{C u}$ & 8.91845894246346 & 23.96395062480000 & 4.82343353250005 \\
\hline $\mathbf{C u}$ & 10.69915774066100 & 25.22453674688410 & 6.09481127373750 \\
\hline $\mathbf{C u}$ & 0.00046643425937 & 2.52449745231580 & 6.09775586830421 \\
\hline $\mathbf{C u}$ & 10.70065506921520 & 5.04070564895061 & 6.09430187869492 \\
\hline $\mathbf{C u}$ & 10.69466254122890 & 7.56775332583833 & 6.10106248375790 \\
\hline $\mathbf{C u}$ & 10.69343062513950 & 10.09564323649850 & 6.09509775830033 \\
\hline $\mathbf{C u}$ & 0.00012204308912 & 12.60964174705890 & 6.09809370855047 \\
\hline $\mathrm{Cu}$ & 10.69463948139920 & 15.14041325417990 & 6.09280477915318 \\
\hline $\mathbf{C u}$ & 10.70130808468650 & 17.66112612251940 & 6.10094386149322 \\
\hline $\mathbf{C u}$ & 0.00649447607901 & 20.17996338829450 & 6.09734760781028 \\
\hline $\mathbf{C u}$ & 10.69813449994790 & 22.70432117185380 & 6.09441282318696 \\
\hline $\mathbf{C u}$ & 40924216051 & 0.00593422998960 & 6.09292668406785 \\
\hline $\mathbf{C u}$ & 61538666 & 2.52517315257633 & 6.08977943579091 \\
\hline $\mathbf{C u}$ & 1252 & 205 & 6.0984 \\
\hline $\mathbf{C u}$ & 2175 & 593 & 140836 \\
\hline $\mathbf{C u}$ & 085 & 870 & 2884212272 \\
\hline $\mathbf{C u}$ & 303 & 6910 & 19888 \\
\hline $\mathbf{C u}$ & 659 & 29720 & 783200 \\
\hline $\mathbf{C u}$ & 991 & 2360 & 50403 \\
\hline $\mathbf{C u}$ & 1521 & 20.179694 & 6.09163256018525 \\
\hline $\mathbf{C u}$ & 3.56740655087857 & 22.70476565959890 & 6.09410001255321 \\
\hline $\mathbf{C u}$ & 7.13773757555920 & 25.22454292307490 & 6.09478751656139 \\
\hline $\mathbf{C u}$ & 7.13428349342837 & 21408938 & 6.09771897788778 \\
\hline $\mathbf{C u}$ & 7.13625130151568 & 5.04070337767038 & 6.09426913909184 \\
\hline $\mathbf{C u}$ & 7.14223430449505 & 7.56775 & 6.10105656512010 \\
\hline $\mathbf{C u}$ & 4806 & 8390 & 6.09509748305172 \\
\hline $\mathbf{C u}$ & 7.13462708702327 & 12.60964769592020 & 6.09808745163483 \\
\hline $\mathbf{C u}$ & 7.14225313833767 & 15.14041090255140 & 6.09280901611284 \\
\hline $\mathbf{C u}$ & 7.13560502040271 & 17.66112979268150 & 6.10092413459324 \\
\hline $\mathbf{C u}$ & 7.12826494801683 & 20.17997192604580 & 6.09730435856917 \\
\hline $\mathbf{C u}$ & 7.13876438453448 & 22.70433117138040 & 6.09438386099828 \\
\hline $\mathbf{C u}$ & 1.78351385223520 & 1.26184941292043 & 7.38707402368868 \\
\hline $\mathbf{C u}$ & 1.78811278867347 & 3.78803852061295 & 7.38623867949927 \\
\hline $\mathbf{C u}$ & 1.78626062216304 & 6.31011044197234 & 7.38083463668384 \\
\hline $\mathbf{C u}$ & 1.77202120585720 & 8.83251030760278 & 7.38115986069913 \\
\hline $\mathbf{C u}$ & 1.78570555000889 & 11.34692279094210 & 7.38669681542361 \\
\hline $\mathbf{C u}$ & 1.76873183560997 & 13.89349709133670 & 7.36059756394571 \\
\hline $\mathbf{C u}$ & 1.79059802594089 & 16.39299258497610 & 7.39191316009501 \\
\hline $\mathbf{C u}$ & 1.79691337562954 & 18.91783778363990 & 7.39027563100301 \\
\hline $\mathbf{C u}$ & 1.78226242303666 & 21.45165624608310 & 7.38339402008828 \\
\hline $\mathbf{C u}$ & 1.78337058606120 & 23.96992121474040 & 7.38597877563209 \\
\hline $\mathbf{C u}$ & 5.35129988018579 & 1.26184892982773 & 7.38704727244702 \\
\hline $\mathbf{C u}$ & 5.34669368700041 & 3.78803508435386 & 7.38619280355679 \\
\hline $\mathbf{C u}$ & 5.34854072376668 & 6.31010919055000 & 7.38080439616292 \\
\hline $\mathbf{C u}$ & 5.36275859449510 & 8.83251288164637 & 7.38118681603614 \\
\hline $\mathbf{C u}$ & 5.34908188782638 & 11.34691151976580 & 7.38668158863415 \\
\hline $\mathbf{C u}$ & 5.36603947702226 & 13.89349547710350 & 7.36068035708464 \\
\hline $\mathbf{C u}$ & 5.34418563087917 & 16.39300144931520 & 7.39189473189044 \\
\hline
\end{tabular}




\begin{tabular}{|c|c|c|c|}
\hline $\mathbf{C u}$ & 5.33788129256638 & 18.91785013372330 & 7.39021410482983 \\
\hline $\mathbf{C u}$ & 5.35254048015879 & 21.45166854613200 & 7.38336121908319 \\
\hline $\mathbf{C u}$ & 5.35143850846870 & 23.96993443772820 & 7.38595257383994 \\
\hline $\mathbf{C u}$ & 8.91841621110369 & 1.26747926888244 & 7.39539997878625 \\
\hline $\mathbf{C u}$ & 8.91842150909642 & 3.78524041285148 & 7.40090693780706 \\
\hline $\mathbf{C u}$ & 8.91842562347935 & 6.30597545935137 & 7.39998212137826 \\
\hline $\mathbf{C u}$ & 8.91842531430704 & 8.83354939728393 & 7.39324128859656 \\
\hline $\mathbf{C u}$ & 8.91842040210432 & 11.35827450037260 & 7.39953912101416 \\
\hline $\mathbf{C u}$ & 8.91841526843895 & 13.87283844098060 & 7.39324448282763 \\
\hline $\mathbf{C u}$ & 8.91841556040958 & 16.40545140419670 & 7.3935032 \\
\hline $\mathbf{C u}$ & 8.91842510984018 & 18.91837390995190 & 7.40148483212717 \\
\hline $\mathbf{C u}$ & 8.91842145680867 & 21.43901342451940 & 7.39629622051824 \\
\hline $\mathbf{C u}$ & 8.91841628481909 & 23.96021280453940 & 7.3936758 \\
\hline $\mathbf{C u}$ & 10.69856768995260 & 0.00223089792772 & 8.6107 \\
\hline $\mathbf{C u}$ & 0.00578135726465 & 54336 & 8.61511786239773 \\
\hline $\mathbf{C u}$ & 0.00904222115174 & 542 & 8.60872772 \\
\hline $\mathbf{C u}$ & 0.002 & 409 & 8.6 \\
\hline $\mathrm{Cu}$ & 10.70 & 10.0 & 8.6 \\
\hline $\mathrm{Cu}$ & 0.00 & 12. & 8.6 \\
\hline $\mathrm{Cu}$ & 10.6 & 900 & 8.6 \\
\hline $\mathbf{C u}$ & 146 & 6870 & 8.6 \\
\hline $\mathbf{C u}$ & 334 & 3360 & 7029 \\
\hline $\mathbf{C u}$ & 488 & 3430 & 9848 \\
\hline $\mathbf{C u}$ & 1381 & 852 & 03254 \\
\hline $\mathbf{C u}$ & 939 & 203 & 8.60 \\
\hline $\mathrm{Cu}$ & 843 & 115 & 8.60 \\
\hline $\mathrm{Cu}$ & 0171333 & 2610 & 8.70332650 \\
\hline $\mathrm{Cu}$ & 2147 & 8770 & 30497 \\
\hline $\mathbf{C u}$ & 508111 & 33040 & 8.63579985697113 \\
\hline $\mathbf{C u}$ & $3.56^{\circ}$ & 15.05 & 8.86061299066180 \\
\hline $\mathbf{C u}$ & 901 & 17.71 & 8.70193 \\
\hline $\mathbf{C u}$ & 3.56 & 20.20 & 58786 \\
\hline $\mathbf{C u}$ & 3.56 & 22.7 & 8.61 \\
\hline $\mathbf{C u}$ & 7.13 & 200 & 4290 \\
\hline $\mathbf{C u}$ & 7.12 & 96 & 7666 \\
\hline $\mathbf{C u}$ & 423 & 555 & 35200 \\
\hline $\mathbf{C u}$ & 7.132105 & 0992 & 8.61203803590020 \\
\hline $\mathbf{C u}$ & 423 & 630 & 8.61 \\
\hline $\mathbf{C u}$ & 7.13214194757104 & 12.61623928038960 & 8.61066680407659 \\
\hline $\mathbf{C u}$ & 7.13858443231075 & 15.13438914833250 & 8.60347064286249 \\
\hline $\mathbf{C u}$ & 7.12822175964287 & 17.65725522842750 & 8.61146550365349 \\
\hline $\mathbf{C u}$ & 7.12 & 20.1 & 4366712 \\
\hline $\mathbf{C u}$ & 7.13362642552036 & 22.70511779286600 & 8.61267021617971 \\
\hline \multicolumn{4}{|c|}{ CI-NEB Image 8} \\
\hline lement & X-Coordinate (§̊) & Y-Coordinate $(\AA)$ & Z-Coordinate $(\AA)$ \\
\hline C & 8194329675 & 7.91058263629455 & 10.62968037067820 \\
\hline C & 3.56 & 71144570 & 10.40956583131890 \\
\hline $\mathbf{F}$ & 3.5 & & 10.93 \\
\hline $\mathbf{F}$ & 2.47062239585832 & 7.42910299278103 & 11.31229169989460 \\
\hline $\mathbf{F}$ & & & 11.31230063855980 \\
\hline $\mathbf{F}$ & 3.56738022409872 & 16.43726268912590 & 10.22049205830070 \\
\hline $\mathbf{F}$ & 2.49359507043827 & 13.32111544955950 & 11.20761957009840 \\
\hline $\mathbf{F}$ & 4.64075916370764 & 13.32113642201930 & 11.20776829862590 \\
\hline $\mathbf{C u}$ & 0.00000000000000 & 0.00000000000000 & 3.56221130874985 \\
\hline
\end{tabular}




\begin{tabular}{|c|c|c|c|}
\hline $\mathbf{C u}$ & 0.00000000000000 & 2.52252112319280 & 3.56221130874985 \\
\hline $\mathbf{C u}$ & 0.00000000000000 & 5.04504224638560 & 3.56221130874985 \\
\hline $\mathbf{C u}$ & 0.00000000000000 & 7.56756339329007 & 3.56221130874985 \\
\hline $\mathbf{C u}$ & 0.00000000000000 & 10.09008449277100 & 3.56221130874985 \\
\hline $\mathrm{Cu}$ & 0.00000000000000 & 12.61260559199990 & 3.56221130874985 \\
\hline $\mathbf{C u}$ & 0.00000000000000 & 15.13512678658010 & 3.56221130874985 \\
\hline $\mathbf{C u}$ & 0.00000000000000 & 17.65764779070990 & 3.56221130874985 \\
\hline $\mathbf{C u}$ & 0.00000000000000 & 20.18016898529000 & 3.56221130874985 \\
\hline $\mathbf{C u}$ & 0.00000000000000 & 22.70268998941980 & 3.56221130874985 \\
\hline $\mathbf{C u}$ & 3.56738358436983 & 0.00000000000000 & 3.56221130874985 \\
\hline $\mathbf{C u}$ & 3.56738358436983 & 2.52252112319280 & 3.56221130874985 \\
\hline $\mathbf{C u}$ & 3.56738358436983 & 5.04504224638560 & 3.56221130874985 \\
\hline $\mathbf{C u}$ & 3.56738358436983 & 7.56756339329007 & 3.56221130874985 \\
\hline $\mathrm{Cu}$ & 3.56738358436983 & 10.09008449277100 & 3.56221130874985 \\
\hline $\mathrm{Cu}$ & 3.56738358436983 & 12.61260559199990 & 3.56221130874985 \\
\hline $\mathbf{C u}$ & 3.56738358436983 & 15.13512678658010 & 3.56221130874985 \\
\hline $\mathrm{Cu}$ & 3.56738358436983 & 4779070990 & 3.56221130874985 \\
\hline $\mathbf{C u}$ & 3.56738358436983 & 20.18016898529000 & 3.56221130874985 \\
\hline $\mathbf{C u}$ & 3.56738358436983 & 22.70268998941980 & 3.56221130874985 \\
\hline $\mathbf{C u}$ & 7.13476717066607 & 000000 & 21130874985 \\
\hline $\mathrm{Cu}$ & 7.13476717066607 & 2319280 & 1130874985 \\
\hline $\mathrm{Cu}$ & 7.13476717066607 & 3560 & 21130874985 \\
\hline $\mathrm{Cu}$ & 7.13476717066607 & 29007 & 74985 \\
\hline $\mathrm{Cu}$ & 7.13476717066607 & 10.09008449277100 & 130874985 \\
\hline $\mathbf{C u}$ & 7.13476717066607 & 199990 & 130874985 \\
\hline $\mathbf{C u}$ & 7.13476717066607 & 15.13512678658010 & 3.56221130874985 \\
\hline $\mathbf{C u}$ & 7.13476717066607 & 17.65764779070990 & 3.56221130874985 \\
\hline $\mathbf{C u}$ & 7.13476717066607 & 20.18016898529000 & 3.56221130874985 \\
\hline $\mathbf{C u}$ & 7.13476717066607 & 22.70268998941980 & 3.56221130874985 \\
\hline $\mathbf{C u}$ & 1.78369179266658 & 1.26126056147013 & 4.82343351925002 \\
\hline $\mathbf{C u}$ & 1.78369179266658 & 3.78378169677122 & 4.82343351925002 \\
\hline $\mathrm{Cu}$ & 1.78369179266658 & 6.30630279600000 & 4.82343351925002 \\
\hline $\mathbf{C u}$ & 1.78369179266658 & 8.82882389522878 & 4.82343351925002 \\
\hline $\mathbf{C u}$ & 1.78369179266658 & 11.35134499470990 & 4.82343351925002 \\
\hline $\mathbf{C u}$ & 1.78369179266658 & 13.87386618929000 & 4.82343351925002 \\
\hline $\mathrm{Cu}$ & 1.78369179266658 & 16.39638719341980 & 4.82343351925002 \\
\hline $\mathbf{C u}$ & 1.78369179266658 & 18.91890838799990 & 4.82343351925002 \\
\hline $\mathrm{Cu}$ & 1.78369179266658 & 21.44142958258010 & 4.82343351925002 \\
\hline $\mathrm{Cu}$ & 1.78369179266658 & 23.96395058670990 & 4.82343351925002 \\
\hline $\mathbf{C u}$ & 5.35107536505000 & 1.26126056147013 & 4.82343351925002 \\
\hline $\mathrm{Cu}$ & 5.35107536505000 & 3.78378169677122 & 4.82343351925002 \\
\hline $\mathrm{Cu}$ & 5.35107536505000 & 6.30630279600000 & 4.82343351925002 \\
\hline $\mathbf{C u}$ & 5.35107536505000 & 8.82882389522878 & 4.82343351925002 \\
\hline $\mathbf{C u}$ & 5.35107536505000 & 11.35134499470990 & 4.82343351925002 \\
\hline $\mathbf{C u}$ & 5.35107536505000 & 13.87386618929000 & 4.82343351925002 \\
\hline $\mathbf{C u}$ & 5.35107536505000 & 16.39638719341980 & 4.82343351925002 \\
\hline $\mathbf{C u}$ & 5.35107536505000 & 18.91890838799990 & 4.82343351925002 \\
\hline $\mathrm{Cu}$ & 5.35107536505000 & 21.44142958258010 & 4.82343351925002 \\
\hline $\mathbf{C u}$ & 5.35107536505000 & 23.96395058670990 & 4.82343351925002 \\
\hline $\mathbf{C u}$ & 8.91845887889266 & 1.26126056147013 & 4.82343351925002 \\
\hline $\mathbf{C u}$ & 8.91845887889266 & 3.78378169677122 & 4.82343351925002 \\
\hline $\mathbf{C u}$ & 8.91845887889266 & 6.30630279600000 & 4.82343351925002 \\
\hline $\mathrm{Cu}$ & 8.91845887889266 & 8.82882389522878 & 4.82343351925002 \\
\hline $\mathrm{Cu}$ & 8.91845887889266 & 11.35134499470990 & 4.82343351925002 \\
\hline
\end{tabular}




\begin{tabular}{|c|c|c|c|}
\hline $\mathbf{C u}$ & 8.91845887889266 & 13.87386618929000 & 4.82343351925002 \\
\hline $\mathbf{C u}$ & 8.91845887889266 & 16.39638719341980 & 4.82343351925002 \\
\hline $\mathbf{C u}$ & 8.91845887889266 & 18.91890838799990 & 4.82343351925002 \\
\hline $\mathbf{C u}$ & 8.91845887889266 & 21.44142958258010 & 4.82343351925002 \\
\hline $\mathbf{C u}$ & 8.91845887889266 & 23.96395058670990 & 4.82343351925002 \\
\hline $\mathbf{C u}$ & 10.69888210437340 & 25.22259811643330 & 6.09477988301840 \\
\hline $\mathbf{C u}$ & -0.00003491921165 & 2.52164346840649 & 6.09776595400766 \\
\hline $\mathbf{C u}$ & 10.69962033419340 & 5.03735869552884 & 6.09360103924593 \\
\hline $\mathbf{C u}$ & 10.69304415539990 & 7.56552631793644 & 6.10049851884442 \\
\hline $\mathbf{C u}$ & 10.69204434891390 & 10.09269070766580 & 6.09405540831003 \\
\hline $\mathbf{C u}$ & 0.00315847721279 & 12.60795051625570 & 6.09974156307903 \\
\hline $\mathbf{C u}$ & 10.69700823052050 & 15.13553609771500 & 6.09486142610032 \\
\hline $\mathbf{C u}$ & 0.00014247470364 & 17.65754619772000 & 6.10229942981308 \\
\hline $\mathrm{Cu}$ & 0.00729407810198 & 20.17690470856950 & 6.09858803722544 \\
\hline $\mathbf{C u}$ & 10.69887326741100 & 22.70133936282260 & 6.09507661171515 \\
\hline $\mathbf{C u}$ & 3.56738177285885 & & 6.09310323162705 \\
\hline $\mathbf{C u}$ & 040832558 & & 6.08952606089956 \\
\hline $\mathbf{C u}$ & 8113595328 & & 6.09846653900362 \\
\hline $\mathbf{C u}$ & 3.56738655937785 & & 6.13842419223571 \\
\hline $\mathbf{C u}$ & 8629189274 & 10.08428944022370 & 6.132 \\
\hline $\mathbf{C u}$ & 724675 & 71804630 & 6.12 \\
\hline $\mathbf{C u}$ & 434 & 15.1264 & 6.167 \\
\hline $\mathbf{C u}$ & 1743 & 17.6 & 5813 \\
\hline $\mathbf{C u}$ & 4026 & 4850 & 6.09 \\
\hline $\mathbf{C u}$ & 178 & 6170 & 3933 \\
\hline $\mathbf{C u}$ & 7.1380 & 25.22260009957230 & 6.09 \\
\hline $\mathbf{C u}$ & 7.13480343343052 & 2.52164462177038 & 6.09776030922789 \\
\hline $\mathbf{C u}$ & 25312286 & 5.03736 & 6.09359989940670 \\
\hline $\mathbf{C u}$ & 7.14386881227549 & 20849730 & 6.10051744745477 \\
\hline $\mathbf{C u}$ & 7.14486705076396 & 10.09268837373970 & 6.09407757944039 \\
\hline $\mathbf{C u}$ & 7.13160280742765 & 12.60794999674100 & 6.09973474883328 \\
\hline $\mathbf{C u}$ & 0601785210 & 15.13553400477640 & 6.09487159101129 \\
\hline $\mathbf{C u}$ & 7.13462976198732 & 17.65754405484730 & 6.10228964654871 \\
\hline $\mathbf{C u}$ & 7.12747774817408 & 20.17690804395080 & 6.09856427063426 \\
\hline $\mathbf{C u}$ & 7.13804510597750 & 22.70134217538490 & 6.09507890935872 \\
\hline $\mathbf{C u}$ & 1.78361808130732 & 1.25623458163916 & 7.38730983998846 \\
\hline $\mathbf{C u}$ & 1.78711591658909 & 3.78089684803449 & 7.38572952582429 \\
\hline $\mathbf{C u}$ & 1.78165089411742 & 6.30140268536687 & 7.37649951200612 \\
\hline $\mathbf{C u}$ & 1.77157457563864 & 8.82268324241123 & 7.38107621566494 \\
\hline $\mathbf{C u}$ & 1.78363596470742 & 11.33126147238290 & 7.38461907771956 \\
\hline $\mathbf{C u}$ & 1.77816139070068 & 13.88261350256960 & 7.36909283693003 \\
\hline $\mathbf{C u}$ & 1.79041196088760 & 16.38404010904550 & 7.39420611314394 \\
\hline $\mathbf{C u}$ & 1.79846977987625 & 18.90837328168640 & 7.39450521803946 \\
\hline $\mathbf{C u}$ & 1.78329272203876 & 21.44408016016330 & 7.38457475990045 \\
\hline $\mathbf{C u}$ & 1.78360995960142 & 23.96439049327170 & 7.38557516395558 \\
\hline $\mathbf{C u}$ & 5.35114583383971 & 1.25623420246151 & 7.38730773221947 \\
\hline $\mathbf{C u}$ & 5.34764481089889 & 3.78089751580443 & 7.38571494714701 \\
\hline $\mathbf{C u}$ & 5.35311155766698 & 6.30140494370878 & 7.37650864393276 \\
\hline $\mathbf{C u}$ & 5.36318747795948 & 8.82268386161738 & 7.38111825124029 \\
\hline $\mathbf{C u}$ & 5.35112381535350 & 11.33126106160770 & 7.38462957570357 \\
\hline $\mathbf{C u}$ & 5.35659311860402 & 13.88261482675580 & 7.36911354491970 \\
\hline $\mathbf{C u}$ & 5.34434721769428 & 16.38403777397070 & 7.39418994598762 \\
\hline $\mathbf{C u}$ & 5.33629064851316 & 18.90837379019590 & 7.39445364480198 \\
\hline $\mathbf{C u}$ & 5.35147017593616 & 21.44408436799930 & 7.38456153807464 \\
\hline
\end{tabular}




\begin{tabular}{|c|c|c|c|}
\hline $\mathbf{C u}$ & 5.35115461376035 & 6439299747270 & 7.38557520665774 \\
\hline $\mathbf{C u}$ & 8.91845902957675 & 1.26266087739815 & 7.39604539776997 \\
\hline $\mathbf{C u}$ & 8.91846267586900 & 3.77967110627126 & 7.40211697263036 \\
\hline $\mathbf{C u}$ & 8.91846255670016 & 6.30123462583243 & 7.39995424357214 \\
\hline $\mathbf{C u}$ & 8.91845967801646 & 8.82857425914792 & 7.39453180258138 \\
\hline $\mathbf{C u}$ & 8.91845651208119 & 11.35341136443510 & 7.39844941503145 \\
\hline $\mathbf{C u}$ & 8.91845484798974 & 13.86682396539480 & 7.39480846462868 \\
\hline $\mathbf{C u}$ & 8.91845552157520 & 16.39833084637550 & 7.39224358656398 \\
\hline $\mathbf{C u}$ & 8.91845857967490 & 18.91359278578510 & 7.4019 \\
\hline $\mathbf{C u}$ & 8.91845886279751 & 21.43448853500300 & 7.39702605641436 \\
\hline $\mathbf{C u}$ & 8.91845779610817 & 23.95524033592070 & 7.39479887448143 \\
\hline $\mathbf{C u}$ & 10.69918009397050 & 25.22288181071250 & 8.61050299267744 \\
\hline $\mathbf{C u}$ & 0.00584623057383 & 2.51728338616375 & 8.61656468411550 \\
\hline $\mathrm{Cu}$ & 3614 & 5.0 & 8.60 \\
\hline $\mathrm{Cu}$ & $0.0011 \varepsilon$ & 556 & 6304 \\
\hline $\mathrm{Cu}$ & 316366300 & 7310 & 8177 \\
\hline $\mathbf{C u}$ & 844 & 12.6 & 9915 \\
\hline $\mathbf{C u}$ & 10.6992 & 15.12620097659330 & 22295494 \\
\hline $\mathbf{C u}$ & 7885 & 17.6 & 3416 \\
\hline $\mathbf{C u}$ & 681 & 20.1 & 8.60 \\
\hline $\mathbf{C u}$ & 1726 & 6864330 & 26974778 \\
\hline $\mathbf{C u}$ & 442 & 25.21 & 8.61 \\
\hline $\mathbf{C u}$ & 56738410834760 & 2.51350022655532 & 8.61015618305485 \\
\hline $\mathbf{C u}$ & 0998 & 5.020161 & 8.60706573149247 \\
\hline $\mathbf{C u}$ & 57083 & 7156 & 8.68912037852568 \\
\hline $\mathbf{C u}$ & 56737181297728 & 10.10697806223180 & 8.66189623639329 \\
\hline $\mathbf{C u}$ & 3.567386 & 12.5733697 & 8.64220018011708 \\
\hline $\mathbf{C u}$ & 575 & 15.01 & 93767 \\
\hline $\mathbf{C u}$ & 608 & 17.69 & 43831 \\
\hline $\mathbf{C u}$ & 746 & 20.1 & 5826 \\
\hline $\mathbf{C u}$ & 026 & 22.69 & 1468 \\
\hline $\mathbf{C u}$ & 7.137 & 25.22 & 557284 \\
\hline $\mathbf{C u}$ & 7.128 & 2.51 & 7238852 \\
\hline $\mathbf{C u}$ & 7.126 & 5.04( & 8.60888 \\
\hline $\mathbf{C u}$ & 3280 & 7.56 & 8.61246 \\
\hline $\mathrm{Cu}$ & 7.13 & 796956440 & 10981165 \\
\hline $\mathbf{C u}$ & 7.1305 & 12.60998701749230 & 8.61036323880157 \\
\hline $\mathbf{C u}$ & 7.13 & 15.12620422711400 & 4854872717 \\
\hline $\mathbf{C u}$ & 7.130 & 2844157730 & 8.61231285906207 \\
\hline $\mathbf{C u}$ & 7.12813590349278 & 20.17211818801040 & 8.60930580054531 \\
\hline $\mathbf{C u}$ & 7.13305866489795 & 22.69953841106000 & 8.61250065422912 \\
\hline \multicolumn{4}{|c|}{ CI-NEB Image 9} \\
\hline ement & X-Coordinat & Y-Coordinate $(\AA)$ & te $(\AA)$ \\
\hline C & & & 10.6 \\
\hline C & 5362 & 12.9 & 10.5 \\
\hline $\mathbf{F}$ & 3.56737396148372 & & 10.93374377263040 \\
\hline $\mathbf{F}$ & & & 11.31841245857720 \\
\hline $\mathbf{F}$ & & & 11.31841696368670 \\
\hline $\mathbf{F}$ & 3.56738090781622 & 16.42020175783370 & 10.20289971166860 \\
\hline $\mathbf{F}$ & 2.49726945045450 & 12.99607521893790 & 11.30462447396860 \\
\hline $\mathbf{F}$ & 4.63625466485695 & 12.99621976391070 & 11.30501206006110 \\
\hline $\mathbf{C u}$ & 000000000 & 0.00000000000000 & 3.56221130499978 \\
\hline $\mathbf{C u}$ & 000000 & 2.52252112 & 3.56221130499978 \\
\hline $\mathbf{C u}$ & 0.00000000000000 & 5.04504225597115 & 3.56221130499978 \\
\hline
\end{tabular}




\begin{tabular}{|c|c|c|c|}
\hline $\mathbf{C u}$ & 0.00000000000000 & 7.56756343138021 & 3.56221130499978 \\
\hline $\mathbf{C u}$ & 0.00000000000000 & 10.09008451194210 & 3.56221130499978 \\
\hline $\mathbf{C u}$ & 0.00000000000000 & 12.61260559199990 & 3.56221130499978 \\
\hline $\mathbf{C u}$ & 0.00000000000000 & 15.13512686276020 & 3.56221130499978 \\
\hline $\mathbf{C u}$ & 0.00000000000000 & 17.65764775261970 & 3.56221130499978 \\
\hline $\mathbf{C u}$ & 0.00000000000000 & 20.18016902338020 & 3.56221130499978 \\
\hline $\mathbf{C u}$ & 0.00000000000000 & 22.70268991323970 & 3.56221130499978 \\
\hline $\mathbf{C u}$ & 3.56738359239644 & 0.00000000000000 & 3.56221130499978 \\
\hline $\mathbf{C u}$ & 3.56738359239644 & 2.52252112798557 & 3.56221130499978 \\
\hline $\mathbf{C u}$ & 3.56738359239644 & 5.04504225597115 & 3.56221130499978 \\
\hline $\mathbf{C u}$ & 3.56738359239644 & 7.56756343138021 & 3.56221130499978 \\
\hline $\mathbf{C u}$ & 3.56738359239644 & 10.09008451194210 & 3.56221130499978 \\
\hline $\mathbf{C u}$ & 3.56738359239644 & 12.61260559199990 & 3.56221130499978 \\
\hline $\mathbf{C u}$ & 3.56738359239644 & 15.13512686276020 & 3.56221130499978 \\
\hline $\mathbf{C u}$ & 3.56738359239644 & 17.65764775261970 & 3.56221130499978 \\
\hline $\mathbf{C u}$ & 3.56738359239644 & 20.18016902338020 & 3.56221130499978 \\
\hline $\mathbf{C u}$ & 3.56738359239644 & 22.70268991323970 & 3.56221130499978 \\
\hline $\mathbf{C u}$ & 7.13476718650522 & 0.00000000000000 & 130499978 \\
\hline $\mathbf{C u}$ & 7.13476718650522 & 2.5225 & 78 \\
\hline $\mathbf{C u}$ & .13476718650522 & 115 & 78 \\
\hline $\mathbf{C u}$ & 0522 & 21 & \\
\hline $\mathbf{C u}$ & 0522 & 10.0 & \\
\hline $\mathbf{C u}$ & & & 78 \\
\hline $\mathrm{Cu}$ & 7.13476718650522 & 15.1 & 21130499978 \\
\hline $\mathbf{C u}$ & 7.13476718650522 & 17.6 & 30499978 \\
\hline $\mathbf{C u}$ & 7.13 & 20.1 & 30499978 \\
\hline $\mathbf{C u}$ & 7.13476718650522 & 22.70268 & 99978 \\
\hline $\mathbf{C u}$ & 1.78 & 1.2 & 600000 \\
\hline $\mathbf{C u}$ & 1.78 & 3.7 & 0000 \\
\hline $\mathbf{C u}$ & 1.78 & 6.3 & 0000 \\
\hline $\mathbf{C u}$ & 1.7 & 8.8 & 00000 \\
\hline $\mathbf{C u}$ & 1.78 & 11.35134 & 50600000 \\
\hline $\mathbf{C u}$ & 1.78369179662638 & 13.87386622738020 & 4.82343350600000 \\
\hline $\mathbf{C u}$ & 1.78369179662638 & 16.39638711723970 & 4.82343350600000 \\
\hline $\mathbf{C u}$ & 1.78369179662638 & 18.91890838799990 & 4.82343350600000 \\
\hline $\mathbf{C u}$ & 1.78369179662638 & 21.44142965876020 & 4.82343350600000 \\
\hline $\mathbf{C u}$ & 1.78369179662638 & 23.96395054861970 & 4.82343350600000 \\
\hline $\mathbf{C u}$ & 5.35107536505000 & 1.26126056374033 & 4.82343350600000 \\
\hline $\mathbf{C u}$ & 5.35107536505000 & 3.78378171594229 & 4.82343350600000 \\
\hline $\mathbf{C u}$ & 5.35107536505000 & 6.30630279600000 & 4.82343350600000 \\
\hline $\mathbf{C u}$ & 5.35107536505000 & 8.82882387605753 & 4.82343350600000 \\
\hline $\mathbf{C u}$ & 5.35107536505000 & 11.35134495661970 & 4.82343350600000 \\
\hline $\mathbf{C u}$ & 5.35107536505000 & 13.87386622738020 & 4.82343350600000 \\
\hline $\mathbf{C u}$ & 5.35107536505000 & 16.39638711723970 & 4.82343350600000 \\
\hline $\mathbf{C u}$ & 5.35107536505000 & 18.91890838799990 & 4.82343350600000 \\
\hline $\mathbf{C u}$ & 5.35107536505000 & 21.44142965876020 & 4.82343350600000 \\
\hline $\mathbf{C u}$ & 5.35107536505000 & 23.96395054861970 & 4.82343350600000 \\
\hline $\mathbf{C u}$ & 8.91845881532186 & 1.26126056374033 & 4.82343350600000 \\
\hline $\mathbf{C u}$ & 8.91845881532186 & 3.78378171594229 & 4.82343350600000 \\
\hline $\mathbf{C u}$ & 8.91845881532186 & 6.30630279600000 & 4.82343350600000 \\
\hline $\mathbf{C u}$ & 8.91845881532186 & 8.82882387605753 & 4.82343350600000 \\
\hline $\mathbf{C u}$ & 8.91845881532186 & 11.35134495661970 & 4.82343350600000 \\
\hline $\mathbf{C u}$ & 8.91845881532186 & 13.87386622738020 & 343350600000 \\
\hline $\mathbf{C u}$ & 8.918458815 & 16.39638711723970 & 4.8234 \\
\hline
\end{tabular}




\begin{tabular}{|c|c|c|c|}
\hline $\mathbf{C u}$ & 8.91845881532186 & 18.91890838799990 & 4.82343350600000 \\
\hline $\mathbf{C u}$ & 8.91845881532186 & 21.44142965876020 & 4.82343350600000 \\
\hline $\mathbf{C u}$ & 8.91845881532186 & 23.96395054861970 & 4.82343350600000 \\
\hline $\mathbf{C u}$ & 10.69852495879110 & 25.22377020725970 & 6.09471493741500 \\
\hline $\mathrm{Cu}$ & 10.70148387080500 & 2.52220068333175 & 6.09755937111025 \\
\hline $\mathbf{C u}$ & 10.69978174718720 & 5.03848646770585 & 6.09382252448950 \\
\hline $\mathrm{Cu}$ & 10.69384568571600 & 7.56649800953470 & 6.10082937206173 \\
\hline $\mathbf{C u}$ & 10.69204709324340 & 10.09339919978170 & 6.09374093907438 \\
\hline $\mathbf{C u}$ & 0.00607607753479 & 12.61011198701500 & 6.10109596437887 \\
\hline $\mathbf{C u}$ & 10.69772666620570 & 15.13386807842210 & 6.09538348639437 \\
\hline $\mathbf{C u}$ & 10.70018660867960 & 17.65963589128360 & 6.10067281697995 \\
\hline $\mathbf{C u}$ & 0.00645826646256 & 20.17939292631810 & 6.09851369020564 \\
\hline $\mathbf{C u}$ & 10.69931151946920 & 22.70232520646810 & 6.09530175717524 \\
\hline $\mathrm{Cu}$ & 3.56738388715225 & 0.00157480408866 & 6.09312176954793 \\
\hline $\mathrm{Cu}$ & 3.56738279681622 & 2.52370986146433 & 6.08959475156647 \\
\hline $\mathrm{Cu}$ & 3.56738403872457 & 5.04835902432403 & 6.09864412174281 \\
\hline $\mathrm{Cu}$ & 8993071164 & & 6.13938977794651 \\
\hline $\mathbf{C u}$ & 38957812386 & 10.08626 & 6.13528599172179 \\
\hline $\mathbf{C u}$ & & 12.60920983413410 & 6.14047234457006 \\
\hline $\mathbf{C u}$ & 8171829974 & 630910 & 6.15481978485411 \\
\hline $\mathrm{Cu}$ & 7900467915 & 17.64517088907850 & 6.13887506616167 \\
\hline $\mathrm{Cu}$ & 915041459 & 20.17760468070720 & 6.09113636475147 \\
\hline $\mathrm{Cu}$ & 3.56 & 12470 & 45341 \\
\hline $\mathrm{Cu}$ & 9406605754 & 25.22377164789460 & 2280308376 \\
\hline $\mathbf{C u}$ & 634646620 & 2.522201 & 6.0975 \\
\hline $\mathbf{C u}$ & 7.13713775998380 & 5.03848926917893 & 6.09382128512302 \\
\hline $\mathbf{C u}$ & 7.14306722852610 & 7.56650125433100 & 6.10084612213867 \\
\hline $\mathbf{C u}$ & 7.14485836192548 & 10.09339964071540 & 6.09376374380100 \\
\hline $\mathbf{C u}$ & 7.12868230739741 & 12.61011176516540 & 6.10108221645547 \\
\hline $\mathbf{C u}$ & 7.13917974628375 & 15.13386648087810 & 6.09539567066559 \\
\hline $\mathbf{C u}$ & 7.13672970332654 & 17.65963188227880 & 6.10067143159779 \\
\hline $\mathrm{Cu}$ & 7.12831210357265 & 20.17939425092760 & 6.09849328085909 \\
\hline $\mathbf{C u}$ & 7.13760761005409 & 22.70232684684440 & 6.09530364199351 \\
\hline $\mathbf{C u}$ & 1.78361977645958 & 1.25808648822739 & 7.38739229254541 \\
\hline $\mathbf{C u}$ & 1.78653108975653 & 3.78141278560947 & 7.38581806580163 \\
\hline $\mathrm{Cu}$ & 05103 & 6.30 & 36861548 \\
\hline $\mathbf{C u}$ & 1.77226423339364 & 5123011 & 7.38208074546729 \\
\hline $\mathrm{Cu}$ & 1.78466614674736 & 11.33229465122370 & 7.38385756779816 \\
\hline $\mathrm{Cu}$ & 1.78690201259441 & 13.88360857522610 & 7.37875317583959 \\
\hline $\mathbf{C u}$ & 1.78258923897934 & 16.39194795310610 & 7.38485263815009 \\
\hline $\mathrm{Cu}$ & 1.79709824304461 & 18.91204628937210 & 7.39386504636153 \\
\hline $\mathrm{Cu}$ & 1.78341926024498 & 21.44639718824290 & 7.38441999915266 \\
\hline $\mathbf{C u}$ & 1.78373713004915 & 23.96706081678930 & 7.38543870835328 \\
\hline $\mathbf{C u}$ & 5.35114833857016 & 1.25808639323465 & 7.38739041380827 \\
\hline $\mathbf{C u}$ & 5.34823457256771 & 3.78141321393830 & 7.38580510534882 \\
\hline $\mathbf{C u}$ & 5.35311849824045 & 6.30325107380553 & 7.37768696186979 \\
\hline $\mathbf{C u}$ & 5.36250325401075 & 8.82494073038096 & 7.38212045103646 \\
\hline $\mathrm{Cu}$ & 5.35008430496303 & 11.33230469399180 & 7.38387666223281 \\
\hline $\mathbf{C u}$ & 5.34784208514120 & 13.88360750237330 & 7.37876757464008 \\
\hline $\mathbf{C u}$ & 5.35216500509243 & 16.39194179896540 & 7.38486059923048 \\
\hline $\mathbf{C u}$ & 5.33766520002191 & 18.91204688296090 & 7.39383081099081 \\
\hline $\mathrm{Cu}$ & 5.35134810251228 & 21.44640074777350 & 7.38440966072367 \\
\hline $\mathbf{C u}$ & 5.35103189496264 & 23.96706288914990 & 7.38543866856100 \\
\hline $\mathbf{C u}$ & 8.91846037593804 & 1.26387296580879 & 7.39671440186115 \\
\hline
\end{tabular}




\begin{tabular}{|c|c|c|c|}
\hline $\mathbf{C u}$ & 8.91846373651128 & 3.78086396444112 & 7.40192648427505 \\
\hline $\mathbf{C u}$ & 8.91846159419397 & 6.30381484310134 & 7.40046496290579 \\
\hline $\mathbf{C u}$ & 8.91845616390667 & 8.82933839050872 & 7.39498812332603 \\
\hline $\mathbf{C u}$ & 8.91845107001085 & 11.35481722458760 & 7.39671723589534 \\
\hline $\mathbf{C u}$ & 8.91844980144229 & 13.86743487588900 & 7.39562978382735 \\
\hline $\mathbf{C u}$ & 8.91845208461758 & 16.39779883209260 & 7.39053178474730 \\
\hline $\mathbf{C u}$ & 8.91845757313704 & 18.91713294879240 & 7.40217427825448 \\
\hline $\mathrm{Cu}$ & 8.91845958909747 & 21.43667650022150 & 7.39799503250827 \\
\hline $\mathrm{Cu}$ & 8.91845898786154 & 23.95699639835230 & 7.39579937578874 \\
\hline $\mathbf{C u}$ & 10.69975619204140 & 25.22450866169790 & 8.61037274310803 \\
\hline $\mathrm{Cu}$ & 0.00588484398677 & 2.51894497462344 & 8.61682925584479 \\
\hline $\mathrm{Cu}$ & 0.00763989168824 & 5.04300337690207 & 8.60863150524594 \\
\hline $\mathrm{Cu}$ & 0.00033561138683 & 7.56905462271831 & 8.61409266027397 \\
\hline $\mathrm{Cu}$ & 10.69985042436610 & 10.09486007940670 & 8.61559568888117 \\
\hline $\mathrm{Cu}$ & 6153221546 & 12.61117681084240 & 8.61010437036574 \\
\hline $\mathrm{Cu}$ & 10.69949093942650 & & 908535902 \\
\hline $\mathrm{Cu}$ & & & 784174706 \\
\hline $\mathrm{Cu}$ & 102320 & 20.1 & 45945 \\
\hline $\mathrm{Cu}$ & 384 & 22.7 & 41950 \\
\hline $\mathrm{Cu}$ & 768 & 25 & 0766 \\
\hline $\mathrm{Cu}$ & 995 & & 7213 \\
\hline $\mathrm{Cu}$ & 96 & & 769 \\
\hline $\mathrm{Cu}$ & 22 & & 708 \\
\hline $\mathrm{Cu}$ & 54 & 10. & 841 \\
\hline $\mathbf{C u}$ & & 12 & 223 \\
\hline $\mathbf{C u}$ & 794 & 15. & 722 \\
\hline $\mathrm{Cu}$ & 374 & 17. & 5085 \\
\hline $\mathbf{C u}$ & 253 & 20. & 3647 \\
\hline $\mathbf{C u}$ & 332 & 22.7 & 0225 \\
\hline $\mathbf{C u}$ & 689 & 25. & 1639 \\
\hline $\mathbf{C u}$ & 693 & 026 & 02779 \\
\hline $\mathbf{C u}$ & & 14 & 57248 \\
\hline $\mathbf{C u}$ & 30005415 & 92 & 7740 \\
\hline $\mathrm{Cu}$ & 7.13706632053711 & 10.094 & 2216513 \\
\hline $\mathrm{Cu}$ & 7.12937782857863 & 12.61117976649500 & 9452179339 \\
\hline $\mathrm{Cu}$ & 1233 & 800700 & 196324338 \\
\hline $\mathrm{Cu}$ & 7.133 & 979440 & 4822990314 \\
\hline $\mathrm{Cu}$ & 232 & 20.1 & 144257 \\
\hline $\mathbf{C u}$ & 7.13276623041084 & 22.70166913106090 & 8.61284532261915 \\
\hline \multicolumn{4}{|c|}{ CI-NEB Image 10} \\
\hline ement & inate $(\AA)$ & dinate $(\AA ̊)$ & Z-Coordinate (Å) \\
\hline C & 3.56747765891793 & 264594361 & 510652520 \\
\hline C & 3.56608087435570 & 12.63013683114540 & 10.54757346305140 \\
\hline $\mathbf{F}$ & 3.56736935023884 & 9.32518041434291 & 10.92581352451490 \\
\hline $\mathbf{F}$ & 2.47093319989241 & 7.44914331529322 & 11.32939678495260 \\
\hline $\mathbf{F}$ & 4.66419314232700 & 7.44935821090808 & 11.32927305107100 \\
\hline $\mathbf{F}$ & 3.56737955679020 & 16.42002217792080 & 10.19203543915240 \\
\hline $\mathbf{F}$ & 2.49698833931500 & 12.61896682755970 & 11.34165268727580 \\
\hline $\mathbf{F}$ & 4.63364358599047 & 12.61980449075960 & 11.34361994121750 \\
\hline $\mathbf{C u}$ & 0.00000000000000 & & 3.56221130124971 \\
\hline $\mathbf{C u}$ & 0.00000000000000 & & 3.56221130124971 \\
\hline $\mathbf{C u}$ & 0.00000000000000 & & 3.56221130124971 \\
\hline $\mathbf{C u}$ & 0.00000000000000 & 346947035 & 3.56221130124971 \\
\hline $\mathbf{C u}$ & 0.00000000000000 & 10.09008453111330 & 3.56221130124971 \\
\hline
\end{tabular}




\begin{tabular}{|c|c|c|c|}
\hline $\mathbf{C u}$ & 0.00000000000000 & 12.61260559199990 & 3.56221130124971 \\
\hline $\mathbf{C u}$ & 0.00000000000000 & 15.13512693894030 & 3.56221130124971 \\
\hline $\mathbf{C u}$ & 0.00000000000000 & 17.65764771452960 & 3.56221130124971 \\
\hline $\mathbf{C u}$ & 0.00000000000000 & 20.18016906147030 & 3.56221130124971 \\
\hline $\mathbf{C u}$ & 0.00000000000000 & 22.70268983705960 & 3.56221130124971 \\
\hline $\mathbf{C u}$ & 3.56738360042304 & 0.00000000000000 & 3.56221130124971 \\
\hline $\mathbf{C u}$ & 3.56738360042304 & 2.52252113277834 & 3.56221130124971 \\
\hline $\mathbf{C u}$ & 3.56738360042304 & 5.04504226555668 & 3.56221130124971 \\
\hline $\mathbf{C u}$ & 3.56738360042304 & 7.56756346947035 & 3.56221130124971 \\
\hline $\mathbf{C u}$ & 3.56738360042304 & 10.09008453111330 & 3.56221130124971 \\
\hline $\mathbf{C u}$ & 3.56738360042304 & 12.61260559199990 & 3.56221130124971 \\
\hline $\mathbf{C u}$ & 3.56738360042304 & 15.13512693894030 & 3.56221130124971 \\
\hline $\mathbf{C u}$ & 3.56738360042304 & 17.65764771452960 & 3.56221130124971 \\
\hline $\mathrm{Cu}$ & 3.56738360042304 & 20.18016906147030 & 3.56221130124971 \\
\hline $\mathbf{C u}$ & 3.56738360042304 & 22.70268983705960 & 3.56221130124971 \\
\hline $\mathbf{C u}$ & 7.13476720234444 & 0.00000000000000 & 3.56221130124971 \\
\hline $\mathbf{C u}$ & 7.13476720234444 & 2.52252113277834 & 3.56221130124971 \\
\hline $\mathbf{C u}$ & 7.13476720234444 & 5.04504226555668 & 3.56221130124971 \\
\hline $\mathbf{C u}$ & 7.13476720234444 & 7.56756346947035 & 3.56221130124971 \\
\hline $\mathbf{C u}$ & 7.13476720234444 & 3111330 & 124971 \\
\hline $\mathbf{C u}$ & 7.13476720234444 & 12.61260559199990 & 3.56221 \\
\hline $\mathbf{C u}$ & 7.13476720234444 & 15.13512693894030 & 3.562211 \\
\hline $\mathbf{C u}$ & 34444 & 2960 & 24971 \\
\hline $\mathbf{C u}$ & 34444 & 20.18016 & 3.56221130124971 \\
\hline $\mathbf{C u}$ & 34444 & 22.70268 & 3.5622113 \\
\hline $\mathbf{C u}$ & 1.78369180058619 & 1.26126056601053 & 4.8234334 \\
\hline $\mathbf{C u}$ & 1.78369180058619 & 73511336 & 4.82343349274998 \\
\hline $\mathbf{C u}$ & 1.78369180058619 & 9600000 & 4.82343349274998 \\
\hline $\mathbf{C u}$ & 1.78369180058619 & 8.82882385688628 & 4.82343349274998 \\
\hline $\mathbf{C u}$ & 1.78369180058619 & 11.35134491852960 & 4.82343349274998 \\
\hline $\mathbf{C u}$ & 1.78369180058619 & 13.87386626547030 & 4.82343349274998 \\
\hline $\mathbf{C u}$ & 1.78369180058619 & 16.39638704105960 & 4.82343349274998 \\
\hline $\mathbf{C u}$ & 1.78369180058619 & 18.91890838799990 & 4.82343349274998 \\
\hline $\mathbf{C u}$ & 1.78369180058619 & 21.44142973494030 & 4.82343349274998 \\
\hline $\mathbf{C u}$ & 1.78369180058619 & 23.96395051052960 & 4.82343349274998 \\
\hline $\mathbf{C u}$ & 5.35107536505000 & 1.26126056601053 & 4.82343349274998 \\
\hline $\mathbf{C u}$ & 5.35107536505000 & 3.78378173511336 & 4.82343349274998 \\
\hline $\mathbf{C u}$ & 5.35107536505000 & 6.30630279600000 & 4.82343349274998 \\
\hline $\mathbf{C u}$ & 5.35107536505000 & 8.82882385688628 & 4.82343349274998 \\
\hline $\mathbf{C u}$ & 5.35107536505000 & 11.35134491852960 & 4.82343349274998 \\
\hline $\mathbf{C u}$ & 5.35107536505000 & 13.87386626547030 & 4.82343349274998 \\
\hline $\mathbf{C u}$ & 5.35107536505000 & 16.39638704105960 & 4.82343349274998 \\
\hline $\mathbf{C u}$ & 5.35107536505000 & 18.91890838799990 & 4.82343349274998 \\
\hline $\mathbf{C u}$ & 5.35107536505000 & 21.44142973494030 & 4.82343349274998 \\
\hline $\mathbf{C u}$ & 5.35107536505000 & 23.96395051052960 & 4.82343349274998 \\
\hline $\mathbf{C u}$ & 8.91845875175106 & 1.26126056601053 & 4.82343349274998 \\
\hline $\mathbf{C u}$ & 8.91845875175106 & 3.78378173511336 & 4.82343349274998 \\
\hline $\mathbf{C u}$ & 8.91845875175106 & 6.30630279600000 & 4.82343349274998 \\
\hline $\mathbf{C u}$ & 8.91845875175106 & 8.82882385688628 & 4.82343349274998 \\
\hline $\mathbf{C u}$ & 8.91845875175106 & 11.35134491852960 & 4.82343349274998 \\
\hline $\mathbf{C u}$ & 8.91845875175106 & 13.87386626547030 & 4.82343349274998 \\
\hline $\mathbf{C u}$ & 8.91845875175106 & 16.39638704105960 & 4.82343349274998 \\
\hline $\mathbf{C u}$ & 8.91845875175106 & 18.91890838799990 & 4.82343349274998 \\
\hline $\mathbf{C u}$ & 8.91845875175106 & 21.44142973494030 & 4.82343349274998 \\
\hline
\end{tabular}




\begin{tabular}{|c|c|c|c|}
\hline $\mathbf{C u}$ & 8.91845875175106 & 23.96395051052960 & 4.82343349274998 \\
\hline $\mathbf{C u}$ & 10.69852457680890 & 25.22434908841010 & 6.09480657556208 \\
\hline $\mathbf{C u}$ & 10.70120208207260 & 2.52250978927688 & 6.09750607829472 \\
\hline $\mathbf{C u}$ & 10.69987957654450 & 5.03917116549661 & 6.09404225004446 \\
\hline $\mathrm{Cu}$ & 10.69469491202760 & 7.56703753293509 & 6.10117115829057 \\
\hline $\mathbf{C u}$ & 10.69210274244240 & 10.09340001967130 & 6.09371839234392 \\
\hline $\mathrm{Cu}$ & 0.00677865982270 & 12.61149070237910 & 6.10157944773944 \\
\hline $\mathbf{C u}$ & 10.69785345755250 & 15.13343088097730 & 6.09521972996996 \\
\hline $\mathbf{C u}$ & 10.69890259238390 & 17.66078136414660 & 6.09963365756594 \\
\hline $\mathbf{C u}$ & 0.00599654755163 & 20.18100810110060 & 6.09835318876093 \\
\hline $\mathbf{C u}$ & 10.69932473471750 & 22.70298480801790 & 6.09547305822427 \\
\hline $\mathbf{C u}$ & 3.56738895464060 & 0.00219469235546 & 6.09337270242026 \\
\hline $\mathbf{C u}$ & 3.56738829386016 & 2.52510461830979 & 6.08979412629648 \\
\hline $\mathrm{Cu}$ & 3.56738926278735 & 5.04944523882758 & 6.09909557801903 \\
\hline $\mathrm{Cu}$ & 3.56739347167540 & 7.56982450623123 & 6.14115144720094 \\
\hline $\mathrm{Cu}$ & 3.56739587417782 & 10.08810277095280 & 6.13756375120037 \\
\hline $\mathrm{Cu}$ & 8938858757 & 12.61016409067130 & 6.14432607875475 \\
\hline $\mathbf{C u}$ & 8727407527 & 31962400300 & 6.14881926423466 \\
\hline $\mathbf{C u}$ & & 17.64825097958760 & 6.13732782251947 \\
\hline $\mathbf{C u}$ & 3.56738478505648 & 20.17980183611900 & 6.09061 \\
\hline $\mathrm{Cu}$ & 3.56738874305690 & 22.70232186837140 & 6.0938 \\
\hline $\mathrm{Cu}$ & 868432517 & 25.22435049604250 & 6.09481265178854 \\
\hline $\mathrm{Cu}$ & 7.13 & 355 & 6.09 \\
\hline $\mathrm{Cu}$ & 00845 & 5.039173 & 6.0940 \\
\hline $\mathbf{C u}$ & 7.14222444395599 & 2996909 & 20416882 \\
\hline $\mathbf{C u}$ & 7.14480111102898 & 10.09340070270520 & 6.09374021035528 \\
\hline $\mathbf{C u}$ & 7.12798843047629 & 12.61148810151580 & 6.10155798701390 \\
\hline $\mathbf{C u}$ & 7.13905527276165 & 15.13343223191370 & 6.09522911302905 \\
\hline $\mathbf{C u}$ & 7.13801648842385 & 17.66077659732620 & 6.09963489443660 \\
\hline $\mathbf{C u}$ & 7.12877863724974 & 20.18100819331330 & 6.09833346899928 \\
\hline $\mathbf{C u}$ & 7.13759911330216 & 22.70298591741730 & 6.09547366323501 \\
\hline $\mathrm{Cu}$ & 1.78348933654930 & 1.25947788744076 & 7.38752298288488 \\
\hline $\mathbf{C u}$ & 1.78613818049658 & 3.78214187742680 & 7.38613657565748 \\
\hline $\mathbf{C u}$ & 1.78196189696374 & 6.30437974409650 & 7.37896658257396 \\
\hline $\mathbf{C u}$ & 1.77389399444262 & 8.82658040443832 & 7.38364998117738 \\
\hline $\mathrm{Cu}$ & 83838015 & 11.33411768607220 & 1218519091 \\
\hline $\mathbf{C u}$ & 1.79076458638590 & 15303587090 & 7.38393412701164 \\
\hline $\mathrm{Cu}$ & 1.77873374461067 & 16.39642641691680 & 441861124 \\
\hline $\mathrm{Cu}$ & 1.79603221418989 & 18.91511239437070 & 7.39279537460244 \\
\hline $\mathbf{C u}$ & 1.78334600041061 & 21.44842682072370 & 7.38429693804335 \\
\hline $\mathrm{Cu}$ & 1.78370021613072 & 23.96874094218000 & 7.38561436918060 \\
\hline $\mathrm{Cu}$ & 5.35129484060696 & 1.25947872485631 & 7.38751935347784 \\
\hline $\mathbf{C u}$ & 5.34864232056718 & 3.78214121591586 & 7.38612252735229 \\
\hline $\mathbf{C u}$ & 5.35282256228969 & 6.30438066580801 & 7.37897243084479 \\
\hline $\mathbf{C u}$ & 5.36088772237381 & 8.82658382017699 & 7.38368038253509 \\
\hline $\mathbf{C u}$ & 5.35100548052619 & 11.33413061697190 & 7.38154321911486 \\
\hline $\mathbf{C u}$ & 5.34400068791612 & 13.88515234952370 & 7.38393100862503 \\
\hline $\mathrm{Cu}$ & 5.35603500814425 & 16.39642232794070 & 7.37935942360512 \\
\hline $\mathbf{C u}$ & 5.33874534337922 & 18.91510840656320 & 7.39275684263556 \\
\hline $\mathrm{Cu}$ & 5.35143534247955 & 21.44842926259220 & 7.38428645606826 \\
\hline $\mathbf{C u}$ & 5.35108396214286 & 23.96874242557430 & 7.38561013944822 \\
\hline $\mathbf{C u}$ & 8.91846331380199 & 1.26480148767878 & 7.39702463463980 \\
\hline $\mathrm{Cu}$ & 8.91846838390306 & 3.78180044137552 & 7.40141192195698 \\
\hline $\mathrm{Cu}$ & 8.91846494199007 & 6.30545770544503 & 7.40041155354231 \\
\hline
\end{tabular}




\begin{tabular}{|c|c|c|c|}
\hline $\mathbf{C u}$ & 8.91845467545405 & 8.82973565450581 & 7.39538293219062 \\
\hline $\mathbf{C u}$ & 8.91844637720741 & 11.35583210645320 & 7.39601271984845 \\
\hline $\mathrm{Cu}$ & 8.91844713105571 & 13.86844684383810 & 7.39607008203849 \\
\hline $\mathbf{C u}$ & 8.91845293962059 & 16.39818773162050 & 7.38991375365295 \\
\hline $\mathbf{C u}$ & 8.91846079567287 & 18.91935978051990 & 7.40224724142231 \\
\hline $\mathrm{Cu}$ & 8.91846293114791 & 21.43823068689340 & 7.39860815705002 \\
\hline $\mathbf{C u}$ & 8.91846174819611 & 23.95837973457960 & 7.39624264922869 \\
\hline $\mathrm{Cu}$ & 10.69993549859200 & 25.22571060192040 & 8.61064889577809 \\
\hline $\mathrm{Cu}$ & 0.00567259802952 & 2.52018850139394 & 8.61698985918699 \\
\hline $\mathbf{C u}$ & 0.00716449823353 & 5.04434539455656 & 8.60875873580748 \\
\hline $\mathrm{Cu}$ & 0.00042927854094 & 7.57042390601838 & 8.61495340513335 \\
\hline $\mathrm{Cu}$ & 10.70002192282860 & 10.09568747567780 & 8.61510681148257 \\
\hline $\mathrm{Cu}$ & 0.00571052816976 & 12.61226111216000 & 8.61012765451494 \\
\hline $\mathrm{Cu}$ & 10.69977524121120 & & 8.60733943036430 \\
\hline $\mathrm{Cu}$ & 0.00086400408618 & & 989286536 \\
\hline $\mathrm{Cu}$ & 3626563270 & & 8.61034539507724 \\
\hline $\mathrm{Cu}$ & 0.00 & & 712068 \\
\hline $\mathrm{Cu}$ & 115 & & 882569536 \\
\hline $\mathrm{Cu}$ & 1948 & & 082448 \\
\hline $\mathrm{Cu}$ & 595 & & 3323 \\
\hline $\mathrm{Cu}$ & 257 & & 115 \\
\hline $\mathrm{Cu}$ & 445 & 10. & 194 \\
\hline $\mathrm{Cu}$ & & & 015 \\
\hline $\mathrm{Cu}$ & 74 & 15 & 225 \\
\hline $\mathbf{C u}$ & & & 716 \\
\hline $\mathbf{C u}$ & 39 & 20. & 8520 \\
\hline $\mathrm{Cu}$ & 555 & 22.7 & 8663 \\
\hline $\mathbf{C u}$ & 547 & 25.2 & 6912 \\
\hline $\mathbf{C u}$ & 11 & 50 & 0530 \\
\hline $\mathbf{C u}$ & 400 & 175 & 9704 \\
\hline $\mathbf{C u}$ & 781 & 71 & 26415 \\
\hline $\mathbf{C u}$ & & 690 & 77832 \\
\hline $\mathbf{C u}$ & 6892 & 12.6 & 91744 \\
\hline $\mathrm{Cu}$ & 7.13713307098997 & 15.1286435360 & 6332877 \\
\hline $\mathrm{Cu}$ & 7.13390258644121 & 17.65312940093070 & 8.60974459815825 \\
\hline $\mathrm{Cu}$ & 43902037 & 20.1 & 8.61031625443736 \\
\hline $\mathbf{C u}$ & 7.13281783647902 & & 0186400 \\
\hline \multicolumn{4}{|c|}{ CI-NEB Image 11} \\
\hline lement & X-Coordinate (Å) & Y-Coordinate (Å) & Z-Coordinate (§̊) \\
\hline $\mathbf{C}$ & 3.56899024732378 & 7.97928658356795 & 10.64511501688860 \\
\hline $\mathrm{C}$ & 3.56725096728228 & 12.43950199943760 & 10.59098223244950 \\
\hline $\mathbf{F}$ & 3.56784226468765 & 9.57184036918646 & 10.71778117994780 \\
\hline $\mathbf{F}$ & 2.48746035738295 & 7.67190538637025 & 11.40832525494210 \\
\hline $\mathbf{F}$ & 4.65287839842971 & 7.67325921114688 & 11.40561489791690 \\
\hline $\mathbf{F}$ & 3.56729260417861 & 16.41752790567130 & 10.18951635933600 \\
\hline $\mathbf{F}$ & 2.50107375964150 & 12.39405811548440 & 11.38387565305950 \\
\hline $\mathbf{F}$ & 4.63336073985296 & 12.39415709967900 & 11.38395579826390 \\
\hline $\mathbf{C u}$ & 0.00000000000000 & 0.00000000000000 & 3.56221129749964 \\
\hline $\mathrm{Cu}$ & 0.00000000000000 & 2.52252113757111 & 3.56221129749964 \\
\hline $\mathbf{C u}$ & 0.00000000000000 & & 3.56221129749964 \\
\hline $\mathbf{C u}$ & 0.00000000000000 & & 3.56221129749964 \\
\hline $\mathbf{C u}$ & 0.00000000000000 & 55028440 & 3.56221129749964 \\
\hline $\mathbf{C u}$ & 0.00000000000000 & 12.61260559199990 & 3.56221129749964 \\
\hline $\mathbf{C u}$ & 0.00000000000000 & 15.13512701512040 & 3.56221129749964 \\
\hline
\end{tabular}




\begin{tabular}{|c|c|c|c|}
\hline $\mathbf{C u}$ & 0.00000000000000 & 17.65764767643950 & 3.56221129749964 \\
\hline $\mathrm{Cu}$ & 0.00000000000000 & 20.18016909956040 & 3.56221129749964 \\
\hline $\mathbf{C u}$ & 0.00000000000000 & 22.70268976087950 & 3.56221129749964 \\
\hline $\mathbf{C u}$ & 3.56738360844965 & 0.00000000000000 & 3.56221129749964 \\
\hline $\mathbf{C u}$ & 3.56738360844965 & 2.52252113757111 & 3.56221129749964 \\
\hline $\mathbf{C u}$ & 3.56738360844965 & 5.04504227514222 & 3.56221129749964 \\
\hline $\mathbf{C u}$ & 3.56738360844965 & 7.56756350756048 & 3.56221129749964 \\
\hline $\mathbf{C u}$ & 3.56738360844965 & 10.09008455028440 & 3.56221129749964 \\
\hline $\mathbf{C u}$ & 3.56738360844965 & 12.61260559199990 & 3.56221129749964 \\
\hline $\mathbf{C u}$ & 3.56738360844965 & 15.13512701512040 & 3.56221129749964 \\
\hline $\mathbf{C u}$ & 3.56738360844965 & 17.65764767643950 & 3.56221129749964 \\
\hline $\mathbf{C u}$ & 3.56738360844965 & 20.18016909956040 & 3.56221129749964 \\
\hline $\mathbf{C u}$ & 3.56738360844965 & 22.70268976087950 & 3.56221129749964 \\
\hline $\mathrm{Cu}$ & 7.13476721818367 & 0.00000000000000 & 3.56221129749964 \\
\hline $\mathbf{C u}$ & 7.13476721818367 & 2.52252113757111 & 3.56221129749964 \\
\hline $\mathrm{Cu}$ & 7.13476721818367 & 5.04504227514222 & 3.56221129749964 \\
\hline $\mathbf{C u}$ & 7.13476721818367 & 7.56756350756048 & 3.56221129749964 \\
\hline $\mathbf{C u}$ & 7.13476721818367 & 10.0900 & 3.56221129749964 \\
\hline $\mathbf{C u}$ & 7.13476721818367 & 12.61260559199990 & 3.56221129749964 \\
\hline $\mathbf{C u}$ & 7.13476721818367 & 701512040 & 3.56221129749964 \\
\hline $\mathrm{Cu}$ & 7.13476721818367 & 17.6576476 & 3.56221129749964 \\
\hline $\mathbf{C u}$ & 7.13476721818367 & 20.1801690 & 3.56221129749964 \\
\hline $\mathrm{Cu}$ & 6721818367 & 76087950 & 29749964 \\
\hline $\mathbf{C u}$ & 1.78369180454599 & 1.26126056828074 & 47949996 \\
\hline $\mathbf{C u}$ & 1.78369180454599 & 3.78 & 347949996 \\
\hline $\mathbf{C u}$ & 1.78369180454599 & 9600000 & 4.82343347949996 \\
\hline $\mathbf{C u}$ & 1.78369180454599 & 8.82882383771503 & 4.82343347949996 \\
\hline $\mathbf{C u}$ & 1.78369180454599 & 11.35134488043950 & 4.82343347949996 \\
\hline $\mathbf{C u}$ & 1.78369180454599 & 13.87386630356040 & 4.82343347949996 \\
\hline $\mathbf{C u}$ & 1.78369180454599 & 16.39638696487950 & 4.82343347949996 \\
\hline $\mathbf{C u}$ & 1.78369180454599 & 18.91890838799990 & 4.82343347949996 \\
\hline $\mathbf{C u}$ & 1.78369180454599 & 21.44142981112040 & 4.82343347949996 \\
\hline $\mathbf{C u}$ & 1.78369180454599 & 23.96395047243950 & 4.82343347949996 \\
\hline $\mathbf{C u}$ & 5.35107536505000 & 1.26126056828074 & 4.82343347949996 \\
\hline $\mathrm{Cu}$ & 5.35107536505000 & 3.78378175428443 & 4.82343347949996 \\
\hline $\mathbf{C u}$ & 5.35107536505000 & 6.30630279600000 & 4.82343347949996 \\
\hline $\mathbf{C u}$ & 5.35107536505000 & 8.82882383771503 & 4.82343347949996 \\
\hline $\mathbf{C u}$ & 5.35107536505000 & 11.35134488043950 & 4.82343347949996 \\
\hline $\mathbf{C u}$ & 5.35107536505000 & 13.87386630356040 & 4.82343347949996 \\
\hline $\mathbf{C u}$ & 5.35107536505000 & 16.39638696487950 & 4.82343347949996 \\
\hline $\mathbf{C u}$ & 5.35107536505000 & 18.91890838799990 & 4.82343347949996 \\
\hline $\mathbf{C u}$ & 5.35107536505000 & 21.44142981112040 & 4.82343347949996 \\
\hline $\mathbf{C u}$ & 5.35107536505000 & 23.96395047243950 & 4.82343347949996 \\
\hline $\mathbf{C u}$ & 8.91845868818027 & 1.26126056828074 & 4.82343347949996 \\
\hline $\mathbf{C u}$ & 8.91845868818027 & 3.78378175428443 & 4.82343347949996 \\
\hline $\mathbf{C u}$ & 8.91845868818027 & 6.30630279600000 & 4.82343347949996 \\
\hline $\mathbf{C u}$ & 8.91845868818027 & 8.82882383771503 & 4.82343347949996 \\
\hline $\mathbf{C u}$ & 8.91845868818027 & 11.35134488043950 & 4.82343347949996 \\
\hline $\mathbf{C u}$ & 8.91845868818027 & 13.87386630356040 & 4.82343347949996 \\
\hline $\mathbf{C u}$ & 8.91845868818027 & 16.39638696487950 & 4.82343347949996 \\
\hline $\mathbf{C u}$ & 8.91845868818027 & 18.91890838799990 & 4.82343347949996 \\
\hline $\mathbf{C u}$ & 8.91845868818027 & 21.44142981112040 & 4.82343347949996 \\
\hline $\mathbf{C u}$ & 8.91845868818027 & 23.96395047243950 & 4.82343347949996 \\
\hline $\mathbf{C u}$ & 10.69860692679490 & 25.22384160211990 & 6.09518196198266 \\
\hline
\end{tabular}




\begin{tabular}{|c|c|c|c|}
\hline $\mathbf{C u}$ & 10.70004261737970 & 2.52158099370018 & 6.09643427834026 \\
\hline $\mathbf{C u}$ & 10.70128023649000 & 5.03990416242962 & 6.09396460299120 \\
\hline $\mathbf{C u}$ & 10.69744894872220 & 7.56639345802580 & 6.09832360697926 \\
\hline $\mathbf{C u}$ & 10.69378068774670 & 10.09222125161250 & 6.09364319233507 \\
\hline $\mathrm{Cu}$ & 0.00782878695447 & 12.61239599216130 & 6.10275015929003 \\
\hline $\mathbf{C u}$ & 10.69940443309160 & 15.13112616943930 & 6.09654294707896 \\
\hline $\mathrm{Cu}$ & 10.69836675101950 & 17.65986894623030 & 6.09883166374876 \\
\hline $\mathbf{C u}$ & 0.00496022066003 & 20.18092855184570 & 6.09821638585494 \\
\hline $\mathbf{C u}$ & 10.69947713819440 & 22.70248754217930 & 6.09574768985216 \\
\hline $\mathbf{C u}$ & 3.56739013881001 & 0.00117094164102 & 6.09369640055943 \\
\hline $\mathbf{C u}$ & 3.56739014956530 & 2.52504348241685 & 6.09059131138882 \\
\hline $\mathbf{C u}$ & 3.56738761636769 & 5.04662087795146 & 6.09677351737467 \\
\hline $\mathbf{C u}$ & 3.56737611966561 & 7.56757490523711 & 6.15311624314711 \\
\hline $\mathrm{Cu}$ & 3.56737869950638 & 10.08899057205340 & 6.15042072779660 \\
\hline $\mathrm{Cu}$ & 3.56738018918394 & 12.60883060053970 & 6.15410697208075 \\
\hline $\mathrm{Cu}$ & 3.56738059263644 & 15.12961240883290 & 6.14512657867084 \\
\hline $\mathrm{Cu}$ & 3.56738146967386 & 17.64959092269980 & 6.13772126913625 \\
\hline $\mathbf{C u}$ & 07290 & 20.18019700831590 & 6.08972270500108 \\
\hline $\mathbf{C u}$ & & 22.70101607702440 & 6.09392574718547 \\
\hline $\mathbf{C u}$ & 403439 & 352811270 & 165636815 \\
\hline $\mathrm{Cu}$ & 544765 & 87131 & 6.09 \\
\hline $\mathrm{Cu}$ & 946252 & 72961 & 6.0939733 \\
\hline $\mathrm{Cu}$ & 91859 & 6003 & 6780 \\
\hline $\mathrm{Cu}$ & 591658696 & 10.09221094416700 & 24551 \\
\hline $\mathrm{Cu}$ & 3644 & 57350 & 24681 \\
\hline $\mathbf{C u}$ & 7.13751276133000 & 15.13113240856030 & 6.09655425774538 \\
\hline $\mathbf{C u}$ & 7.13855044273873 & 17.65987318385980 & 6.09884074113177 \\
\hline $\mathbf{C u}$ & 7.12980686908679 & 20.18093435998050 & 6.09819905687406 \\
\hline $\mathbf{C u}$ & 7.13744040228652 & 22.70249092972760 & 6.09574521277564 \\
\hline $\mathbf{C u}$ & 1.78258202016810 & 1.25862319845136 & 7.38754940717104 \\
\hline $\mathbf{C u}$ & 1.78350228917814 & 3.77921390850092 & 7.38608784242798 \\
\hline $\mathrm{Cu}$ & 1.78419814237308 & 6.30257144174831 & 7.38278229070835 \\
\hline $\mathbf{C u}$ & 1.77673167605690 & 8.82297172135589 & 7.38246478603503 \\
\hline $\mathbf{C u}$ & 1.78461254342940 & 11.33549693086650 & 7.38271043281658 \\
\hline $\mathbf{C u}$ & 1.79734369444585 & 13.88111890044780 & 7.39421438229001 \\
\hline $\mathrm{Cu}$ & 1.77617148288445 & 51361466010 & 405016115 \\
\hline $\mathbf{C u}$ & 55332187 & 18.91457358012780 & 7.39255488567707 \\
\hline $\mathrm{Cu}$ & 1.78348566173006 & 21.44782364225950 & 7.38425220923968 \\
\hline $\mathrm{Cu}$ & 1.78351397026889 & 23.96787842690980 & 7.38620905935124 \\
\hline $\mathbf{C u}$ & 5.35219464270308 & 1.25862723859828 & 7.38755632159136 \\
\hline $\mathrm{Cu}$ & 5.35126597481139 & 3.77922279207708 & 7.38609998974541 \\
\hline $\mathrm{Cu}$ & 5.35054812122055 & 6.30257978285585 & 7.38282328482366 \\
\hline $\mathbf{C u}$ & 5.35800423779472 & 8.82296989549122 & 7.38250992925475 \\
\hline $\mathbf{C u}$ & 5.35013060309015 & 11.33549188424800 & 7.38273706922170 \\
\hline $\mathbf{C u}$ & 5.33740797080854 & 13.88111429939390 & 7.39419949285076 \\
\hline $\mathbf{C u}$ & 5.35858663631280 & 16.39661518377180 & 7.37598076997472 \\
\hline $\mathbf{C u}$ & 5.33938353740780 & 18.91457339876990 & 7.39253258975246 \\
\hline $\mathrm{Cu}$ & 5.35128811212495 & 21.44782702104330 & 7.38424575908365 \\
\hline $\mathbf{C u}$ & 5.35126307971144 & 23.96788212399180 & 7.38620850631065 \\
\hline $\mathrm{Cu}$ & 8.91846323382799 & 1.26225006376309 & 7.39698361798321 \\
\hline $\mathbf{C u}$ & 8.91847372236237 & 3.78200483290681 & 7.39828458013101 \\
\hline $\mathrm{Cu}$ & 8.91848712745153 & 6.30494813945352 & 7.39727653450707 \\
\hline $\mathrm{Cu}$ & 8.91848138960749 & 8.82770918648828 & 7.39396458776589 \\
\hline $\mathrm{Cu}$ & 8.91847585290241 & 11.35416885220260 & 7.39517431900887 \\
\hline
\end{tabular}




\begin{tabular}{|c|c|c|c|}
\hline $\mathbf{C u}$ & 8.91846611487800 & 13.86822353217570 & 7.39789491946670 \\
\hline $\mathbf{C u}$ & 8.91846200021283 & 16.39585291733320 & 7.39066357228482 \\
\hline $\mathbf{C u}$ & 8.91846223159520 & 18.91924800353640 & 7.40116852345773 \\
\hline $\mathbf{C u}$ & 8.91846078371147 & 21.43788450571330 & 7.39835432058645 \\
\hline $\mathbf{C u}$ & 8.91845778848677 & 23.95786747492280 & 7.39736384194735 \\
\hline $\mathbf{C u}$ & 10.70098238983280 & -0.00064335808342 & 8.61161198863419 \\
\hline $\mathbf{C u}$ & 0.00291089116452 & 2.51984385751886 & 8.61607223840490 \\
\hline $\mathbf{C u}$ & 0.00487149307081 & 5.04358133850467 & 8.60933952628271 \\
\hline $\mathbf{C u}$ & 0.00079082811358 & 7.56839181536305 & 8.61094265621541 \\
\hline $\mathbf{C u}$ & 10.70053366771990 & 10.09265642023530 & 8.61271107652215 \\
\hline $\mathbf{C u}$ & 0.00497799181918 & 12.61049169972360 & 8.61176359781682 \\
\hline $\mathbf{C u}$ & 10.70119231007250 & 15.12754253661550 & 8.60955683936848 \\
\hline $\mathbf{C u}$ & 10.70221868135870 & 17.65220204849740 & 8.60953452836987 \\
\hline $\mathbf{C u}$ & 0.00626821511535 & 20.17787793047660 & 8.60996047532871 \\
\hline $\mathbf{C u}$ & 0.002559 & 22.70242440046790 & 8.61333636206719 \\
\hline $\mathbf{C u}$ & 3.56739179316222 & 25.22142393656030 & 8.61470520181238 \\
\hline $\mathbf{C u}$ & & & 8.61171093046330 \\
\hline $\mathbf{C u}$ & 2761 & & 8.60283334226984 \\
\hline $\mathbf{C u}$ & 049 & 3468 & 962769 \\
\hline $\mathbf{C u}$ & 46 & 630 & 155 \\
\hline $\mathbf{C u}$ & 294 & 12. & 5237 \\
\hline $\mathbf{C u}$ & 361 & 860 & 3072 \\
\hline $\mathbf{C u}$ & 89 & 390 & 344 \\
\hline $\mathrm{Cu}$ & 08 & 350 & 5063 \\
\hline $\mathbf{C u}$ & 96 & 840 & 1477 \\
\hline $\mathbf{C u}$ & 763 & -0.06 & 3105 \\
\hline $\mathbf{C u}$ & 7.13 & 202 & 8.61 \\
\hline $\mathbf{C u}$ & 589 & 552 & 8.60 \\
\hline $\mathbf{C u}$ & 159 & 7.56 & 78554 \\
\hline $\mathbf{C u}$ & 891 & 9180 & 018082 \\
\hline $\mathbf{C u}$ & 6957 & 84930 & 99300 \\
\hline $\mathbf{C u}$ & 082 & 4420 & 532260 \\
\hline $\mathbf{C u}$ & 757 & 17.652207 & 40219 \\
\hline $\mathbf{C u}$ & 471 & 2750 & 32791 \\
\hline $\mathbf{C u}$ & 7.13220523894741 & 657480 & 8.61332679563881 \\
\hline \multicolumn{4}{|c|}{ CI-NEB Image 12} \\
\hline lement & X-Coordinate $(\AA ̊)$ & late $(\AA)$ & dinate $(\AA ̊)$ \\
\hline C & 3.56930861115036 & 7.68622777685401 & 10.64245950554920 \\
\hline $\mathrm{C}$ & 3.56729463461661 & 12.33872845281630 & 10.62513952837850 \\
\hline $\mathbf{F}$ & 3.56763488874783 & 10.07436832663450 & 10.61260352771550 \\
\hline $\mathbf{F}$ & 2.50707367743307 & 7.62173089185237 & 11.42856678650520 \\
\hline $\mathbf{F}$ & 4.63395649940532 & 7.62230915881623 & 11.42526800507430 \\
\hline $\mathbf{F}$ & 3.56721215459557 & 16.41233037084320 & 10.19107668369230 \\
\hline $\mathbf{F}$ & 2.50213345071405 & 12.40633080318340 & 11.40651953069780 \\
\hline $\mathbf{F}$ & 4.63235362115828 & 12.40651535781330 & 11.40662309836070 \\
\hline $\mathbf{C u}$ & 0.00000000000000 & 0.00000000000000 & 3.56221129374958 \\
\hline $\mathbf{C u}$ & 0.00000000000000 & 2.52252114236388 & 3.56221129374958 \\
\hline $\mathbf{C u}$ & 0.00 & 2775 & 3.56221129374958 \\
\hline $\mathbf{C u}$ & 0.00000000000000 & 7.56756354565062 & 3.56221129374958 \\
\hline $\mathbf{C u}$ & 0.000 & 10.09 & 74958 \\
\hline $\mathbf{C u}$ & 0000000000 & 12.61260559199990 & 3.56221129374958 \\
\hline $\mathbf{C u}$ & 0.00000000000000 & 15.13512709130050 & 3.56221129374958 \\
\hline $\mathbf{C u}$ & 0.00000000000000 & 17.65764763834930 & 3.56221129374958 \\
\hline $\mathbf{C u}$ & 0.00000000000000 & 20.18016913765060 & 3.56221129374958 \\
\hline
\end{tabular}




\begin{tabular}{|c|c|c|c|}
\hline $\mathbf{C u}$ & 0.00000000000000 & 22.70268968469940 & 3.56221129374958 \\
\hline $\mathbf{C u}$ & 3.56738361647625 & 0.00000000000000 & 3.56221129374958 \\
\hline $\mathbf{C u}$ & 3.56738361647625 & 2.52252114236388 & 3.56221129374958 \\
\hline $\mathbf{C u}$ & 3.56738361647625 & 5.04504228472775 & 3.56221129374958 \\
\hline $\mathrm{Cu}$ & 3.56738361647625 & 7.56756354565062 & 3.56221129374958 \\
\hline $\mathbf{C u}$ & 3.56738361647625 & 10.09008456945550 & 3.56221129374958 \\
\hline $\mathbf{C u}$ & 3.56738361647625 & 12.61260559199990 & 3.56221129374958 \\
\hline $\mathbf{C u}$ & 3.56738361647625 & 15.13512709130050 & 3.56221129374958 \\
\hline $\mathbf{C u}$ & 3.56738361647625 & 17.65764763834930 & 3.56221129374958 \\
\hline $\mathbf{C u}$ & 3.56738361647625 & 20.18016913765060 & 3.56221129374958 \\
\hline $\mathbf{C u}$ & 3.56738361647625 & 22.70268968469940 & 3.56221129374958 \\
\hline $\mathbf{C u}$ & 7.13476723402289 & 0.00000000000000 & 3.56221129374958 \\
\hline $\mathbf{C u}$ & 7.13476723402289 & 2.52252114236388 & 3.56221129374958 \\
\hline $\mathrm{Cu}$ & 7.13476723402289 & 5.04504228472775 & 3.56221129374958 \\
\hline $\mathrm{Cu}$ & 7.13476723402289 & 7.56756354565062 & 3.56221129374958 \\
\hline $\mathbf{C u}$ & 7.13476723402289 & 10.09008456945550 & 3.56221129374958 \\
\hline $\mathbf{C u}$ & 7.13476723402289 & 12.61260559199990 & 3.56221129374958 \\
\hline $\mathbf{C u}$ & 7.13476723402289 & 15.13512709130050 & 3.56221129374958 \\
\hline $\mathbf{C u}$ & 7.13476723402289 & 17.65764763834930 & 3.56221129374958 \\
\hline $\mathbf{C u}$ & 7.13476723402289 & 20.18016913765060 & 3.56221 \\
\hline $\mathrm{Cu}$ & 7.13476723402289 & 22.70268968469940 & 3.56221129374958 \\
\hline $\mathrm{Cu}$ & 1.78369180 & 1.26126057055094 & 4.82343346624993 \\
\hline $\mathrm{Cu}$ & 50580 & 5550 & 4993 \\
\hline $\mathrm{Cu}$ & 1.7836918 & 9600000 & 4.823 \\
\hline $\mathbf{C u}$ & 1.7836918 & 4377 & 24993 \\
\hline $\mathbf{C u}$ & 1.78369180850580 & 11.35134484234930 & 4.82343346624993 \\
\hline $\mathbf{C u}$ & 1.78369180850580 & 13.87386634165060 & 4.82343346624993 \\
\hline $\mathbf{C u}$ & 1.78369180850580 & 16.39638688869940 & 4.82343346624993 \\
\hline $\mathbf{C u}$ & 1.78369180850580 & 18.91890838799990 & 4.82343346624993 \\
\hline $\mathbf{C u}$ & 1.78369180850580 & 21.44142988730050 & 4.82343346624993 \\
\hline $\mathbf{C u}$ & 1.78369180850580 & 23.96395043434930 & 4.82343346624993 \\
\hline $\mathrm{Cu}$ & 5.35107536505000 & 1.26126057055094 & 4.82343346624993 \\
\hline $\mathbf{C u}$ & 5.35107536505000 & 3.78378177345550 & 4.82343346624993 \\
\hline $\mathrm{Cu}$ & 5.35107536505000 & 6.30630279 & 4.82343346624993 \\
\hline $\mathrm{Cu}$ & 5.35107536505000 & 8.82882381854377 & 4.82343346624993 \\
\hline $\mathbf{C u}$ & 5.35107536505000 & 11.35134484234930 & 4.82343346624993 \\
\hline $\mathbf{C u}$ & 5.35107536505000 & 13.87386634165060 & 4.82343346624993 \\
\hline $\mathrm{Cu}$ & 5.35107536505000 & 16.39638688869940 & 4.82343346624993 \\
\hline $\mathrm{Cu}$ & 5.35107536505000 & 18.91890838799990 & 4.82343346624993 \\
\hline $\mathbf{C u}$ & 5.35107536505000 & 21.44142988730050 & 4.82343346624993 \\
\hline $\mathrm{Cu}$ & 5.35107536505000 & 23.96395043434930 & 4.82343346624993 \\
\hline $\mathrm{Cu}$ & 8.91845862460946 & 1.26126057055094 & 4.82343346624993 \\
\hline $\mathbf{C u}$ & 8.91845862460946 & 3.78378177345550 & 4.82343346624993 \\
\hline $\mathbf{C u}$ & 8.91845862460946 & 6.30630279600000 & 4.82343346624993 \\
\hline $\mathbf{C u}$ & 8.91845862460946 & 8.82882381854377 & 4.82343346624993 \\
\hline $\mathbf{C u}$ & 8.91845862460946 & 11.35134484234930 & 4.82343346624993 \\
\hline $\mathbf{C u}$ & 8.91845862460946 & 13.87386634165060 & 4.82343346624993 \\
\hline $\mathrm{Cu}$ & 8.91845862460946 & 16.39638688869940 & 4.82343346624993 \\
\hline $\mathbf{C u}$ & 8.91845862460946 & 18.91890838799990 & 4.82343346624993 \\
\hline $\mathbf{C u}$ & 8.91845862460946 & 21.44142988730050 & 4.82343346624993 \\
\hline $\mathbf{C u}$ & 8.91845862460946 & 23.96395043434930 & 4.82343346624993 \\
\hline $\mathrm{Cu}$ & 10.69998170050440 & 25.22422374264810 & 6.09591341906000 \\
\hline $\mathrm{Cu}$ & 10.69828611081620 & 2.52126353538223 & 6.09569000772245 \\
\hline $\mathbf{C u}$ & 10.70199280067450 & 5.04141056402606 & 6.09384882178654 \\
\hline
\end{tabular}




\begin{tabular}{|c|c|c|c|}
\hline $\mathbf{C u}$ & 10.69965919872770 & 7.56739923796283 & 6.09625980175492 \\
\hline $\mathrm{Cu}$ & 10.69402426353770 & 10.08991751652230 & 6.09291453884765 \\
\hline $\mathbf{C u}$ & 0.00658652708537 & 12.61456332025230 & 6.10195541262282 \\
\hline $\mathbf{C u}$ & 10.70096531872820 & 15.13095618115840 & 6.09663603406757 \\
\hline $\mathrm{Cu}$ & 10.69944207850820 & 17.65966021666510 & 6.09842246277715 \\
\hline $\mathbf{C u}$ & 0.00330613440984 & 20.18102784550420 & 6.09801872343761 \\
\hline $\mathbf{C u}$ & 10.69995898783050 & 22.70192585016730 & 6.09617246756960 \\
\hline $\mathbf{C u}$ & 3.56739756233391 & 0.00087376299022 & 6.09384540066784 \\
\hline $\mathbf{C u}$ & 3.56739673349303 & 2.52505280581961 & 6.09145487937536 \\
\hline $\mathbf{C u}$ & 3.56739173381244 & 5.04413806417763 & 6.09394967069422 \\
\hline $\mathbf{C u}$ & 3.56737438419881 & 7.56594252509651 & 6.15870867047454 \\
\hline $\mathbf{C u}$ & 3.56737623844722 & 10.09035637268210 & 6.15840086428178 \\
\hline $\mathbf{C u}$ & 3.56737822243174 & 12.60637513933000 & 6.15591132852505 \\
\hline $\mathrm{Cu}$ & 3.56738033019438 & 15.12956433482270 & 6.14332030796038 \\
\hline $\mathrm{Cu}$ & 3.56738468347404 & 17.64989388250740 & 6.13832381676272 \\
\hline $\mathrm{Cu}$ & 3.56739094653407 & 20.18122260153750 & 6.09031127356594 \\
\hline $\mathrm{Cu}$ & 3.56739749182417 & 22.70090997968020 & 6.09416246302925 \\
\hline $\mathbf{C u}$ & 7.13692124823100 & 25.22422472708100 & 6.09590515925889 \\
\hline $\mathbf{C u}$ & 7.13862182137574 & & 6.09569197924398 \\
\hline $\mathbf{C u}$ & 122858944 & 2527131 & 5148819 \\
\hline $\mathrm{Cu}$ & 7.13724768928931 & 7.56739315287000 & 6.096 \\
\hline $\mathrm{Cu}$ & 06363 & 0587819030 & 6.0929436 \\
\hline $\mathrm{Cu}$ & 7.12 & 12.6 & 6.10 \\
\hline $\mathrm{Cu}$ & 7.13594795993321 & 15.130965 & 523197 \\
\hline $\mathbf{C u}$ & 7.13746678650694 & 201970 & 6.09 \\
\hline $\mathbf{C u}$ & 7.13145108607460 & 20.18103576381020 & 6.09800004745593 \\
\hline $\mathbf{C u}$ & 7.13694574614986 & 22.70193081132840 & 6.09616336605352 \\
\hline $\mathbf{C u}$ & 1.78239682254305 & 1.26055164090400 & 7.38787428675615 \\
\hline $\mathbf{C u}$ & 1.78189627002681 & 3.77808109867782 & 7.38589600499659 \\
\hline $\mathbf{C u}$ & 1.78415769910332 & 6.30261548341740 & 7.38272489008945 \\
\hline $\mathbf{C u}$ & 1.78116825721333 & 8.82444328786903 & 7.37887632344156 \\
\hline $\mathrm{Cu}$ & 1.78037174249938 & 11.33704938952140 & 7.38101927925156 \\
\hline $\mathbf{C u}$ & 1.80055251937240 & 13.87719523706840 & 7.39597660219112 \\
\hline $\mathbf{C u}$ & 1.77513405233277 & 16.39617518147470 & 7.37414270739827 \\
\hline $\mathbf{C u}$ & 1.79506757110649 & 18.91486521093720 & 7.39290127386930 \\
\hline $\mathrm{Cu}$ & 1.78343856825386 & 21.44686868362750 & 7.38462989933942 \\
\hline $\mathbf{C u}$ & 1.78338224693835 & 23.96757940410000 & 7.38673628721498 \\
\hline $\mathrm{Cu}$ & 5.35238116181953 & 1.26055 & 7.38788228440220 \\
\hline $\mathrm{Cu}$ & 5.35287153972180 & 3.77808921020062 & 7.38591113097850 \\
\hline $\mathbf{C u}$ & 5.35057866012356 & 6.30262826479385 & 7.38277405885398 \\
\hline $\mathrm{Cu}$ & 5.35356672048152 & 8.82443755912279 & 7.37891330554583 \\
\hline $\mathrm{Cu}$ & 5.35436805210678 & 11.33703958859390 & 7.38104851372901 \\
\hline $\mathbf{C u}$ & 5.33419716035054 & 13.87719654950800 & 7.39595367706238 \\
\hline $\mathbf{C u}$ & 5.35962174211141 & 16.39617864122430 & 7.37418010638039 \\
\hline $\mathbf{C u}$ & 5.33969838458549 & 18.91486667113130 & 7.39288038323894 \\
\hline $\mathbf{C u}$ & 5.35133739084923 & 21.44687245617040 & 7.38462222565529 \\
\hline $\mathbf{C u}$ & 5.35139856530284 & 23.96758412298260 & 7.38673576834639 \\
\hline $\mathrm{Cu}$ & 8.91844921076098 & 1.26045457629676 & 7.39677146709766 \\
\hline $\mathbf{C u}$ & 8.91846573183946 & 3.78222959197605 & 7.39656487994020 \\
\hline $\mathrm{Cu}$ & 8.91848858640536 & 6.30504696094446 & 7.39559385556885 \\
\hline $\mathbf{C u}$ & 8.91849203661430 & 8.82669931569968 & 7.39580226923734 \\
\hline $\mathrm{Cu}$ & 8.91847388148295 & 11.35371959258310 & 7.39422636857444 \\
\hline $\mathrm{Cu}$ & 8.91846078700260 & 13.87022263103580 & 7.39964345428025 \\
\hline $\mathrm{Cu}$ & 8.91845405535203 & 16.39503823203710 & 7.39186969697113 \\
\hline
\end{tabular}




\begin{tabular}{|c|c|c|c|}
\hline $\mathbf{C u}$ & 8.91845017586037 & 18.91932791424810 & 8740623704 \\
\hline $\mathbf{C u}$ & 8.91844652406251 & 21.43811089938480 & 7.39674487472611 \\
\hline $\mathbf{C u}$ & 8.91844374646466 & 23.95825065129640 & 7.39831899376643 \\
\hline $\mathbf{C u}$ & 0.00012105279079 & -0.00105921752331 & 8.61206264902226 \\
\hline $\mathbf{C u}$ & 0.00182974267541 & 2.52036535339636 & 8.61456785739298 \\
\hline $\mathbf{C u}$ & 0.00430273898954 & 5.04401109913367 & 8.60983603392860 \\
\hline $\mathbf{C u}$ & 0.00178340532470 & 7.56803453800666 & 8.60744294060069 \\
\hline $\mathbf{C u}$ & 10.70080621379210 & 10.09115456809090 & 8.61218740396874 \\
\hline $\mathbf{C u}$ & 0.00431540245534 & 12.61065375871250 & 8.61206215099396 \\
\hline $\mathbf{C u}$ & 10.70221199806530 & 15.12803514449710 & 8.6105188 \\
\hline $\mathbf{C u}$ & 2643563770 & 17.65219432033860 & 8.610608 \\
\hline $\mathbf{C u}$ & 0.00570284554054 & 20.17806919314490 & 8.60964859349545 \\
\hline $\mathbf{C u}$ & 0.00312863190666 & 22.70232853055260 & 8.61306765613809 \\
\hline $\mathrm{Cu}$ & 86078 & 25.22040616782070 & 8.61418716 \\
\hline $\mathrm{Cu}$ & 62962 & 2.5115 & 91555 \\
\hline $\mathbf{C u}$ & 252 & 199 & 8148 \\
\hline $\mathbf{C u}$ & 934 & 641 & 4687 \\
\hline $\mathbf{C u}$ & $3.56^{\circ}$ & 33840 & 8.677 \\
\hline $\mathbf{C u}$ & 3.56 & 7390 & 8.727 \\
\hline $\mathbf{C u}$ & 3.56 & 5940 & 8.71 \\
\hline $\mathbf{C u}$ & 3.56 & 17.7 & $8.71 \mathrm{~s}$ \\
\hline $\mathbf{C u}$ & 6505 & 1860 & 8.59 \\
\hline $\mathbf{C u}$ & 3.56740226505876 & 22.70577772958940 & 47836569 \\
\hline $\mathbf{C u}$ & 7.13463323177612 & -0.00105942009377 & 8.61205933571908 \\
\hline $\mathbf{C u}$ & 10923244 & 2.52036766174331 & 8.61456030473859 \\
\hline $\mathbf{C u}$ & 7.130 & 5.04401202937865 & 8.60979127554897 \\
\hline $\mathbf{C u}$ & 7.1330 & 74733 & 8.60739783823051 \\
\hline $\mathbf{C u}$ & 7.136 & 10.09 & 866257 \\
\hline $\mathbf{C u}$ & 7.130 & 12.61 & 80622 \\
\hline $\mathbf{C u}$ & 7.134 & 15.12 & 65471 \\
\hline $\mathbf{C u}$ & 7.13 & 17.6 & 3277 \\
\hline $\mathbf{C u}$ & 2218 & 20.1 & 83371 \\
\hline $\mathbf{C u}$ & 7.13161678868876 & 580 & 7578910 \\
\hline \multicolumn{4}{|c|}{ CI-NEB Image 13} \\
\hline ement & X-Coordinate $(\AA)$ & $\mathbf{Y}-\mathbf{C}$ & Z-C \\
\hline C & 3.56946982713915 & 7.5766 & 30254160 \\
\hline C & 3.56733740294696 & 12.04652526631250 & 10.62410903687260 \\
\hline $\mathbf{F}$ & 206496 & 21674320 & 10.77306636985270 \\
\hline $\mathbf{F}$ & 2.50 & 09540 & 11.39671413487010 \\
\hline $\mathbf{F}$ & 4.63775004089897 & 7.61070404230684 & 11.39398037279350 \\
\hline $\mathbf{F}$ & 3.56730 & 16.41385638778900 & 10.18791048971580 \\
\hline $\mathbf{F}$ & 2.47479948545006 & 12.41230954840780 & 11.36203602676610 \\
\hline $\mathbf{F}$ & 4.65973644731465 & 12.41238226965300 & 11.36220323788430 \\
\hline $\mathbf{C u}$ & 0.00 & & 951 \\
\hline $\mathbf{C u}$ & & & 951 \\
\hline $\mathbf{C u}$ & & & 951 \\
\hline $\mathbf{C u}$ & & & 9951 \\
\hline $\mathbf{C u}$ & & 58862650 & 3.56221128999951 \\
\hline $\mathbf{C u}$ & 0.00000000000000 & 12.61260559199990 & 3.56221128999951 \\
\hline $\mathbf{C u}$ & & 15.13512716748060 & 3.56221128999951 \\
\hline $\mathbf{C u}$ & 0.00000000000000 & 17.65764760025920 & 3.56221128999951 \\
\hline $\mathbf{C u}$ & 0.00000000000000 & 20.18016917574070 & 3.56221128999951 \\
\hline $\mathbf{C u}$ & 0.00000000000000 & 22.70268960851930 & 3.56221128999951 \\
\hline $\mathbf{C u}$ & 3.56738362450286 & 0.00000000000000 & 3.56221128999951 \\
\hline
\end{tabular}




\begin{tabular}{|c|c|c|c|}
\hline $\mathbf{C u}$ & 3.56738362450286 & 2.52252114715664 & 3.56221128999951 \\
\hline $\mathbf{C u}$ & 3.56738362450286 & 5.04504229431329 & 3.56221128999951 \\
\hline $\mathbf{C u}$ & 3.56738362450286 & 7.56756358374076 & 3.56221128999951 \\
\hline $\mathbf{C u}$ & 3.56738362450286 & 10.09008458862650 & 3.56221128999951 \\
\hline $\mathbf{C u}$ & 3.56738362450286 & 12.61260559199990 & 3.56221128999951 \\
\hline $\mathbf{C u}$ & 3.56738362450286 & 15.13512716748060 & 3.56221128999951 \\
\hline $\mathbf{C u}$ & 3.56738362450286 & 17.65764760025920 & 3.56221128999951 \\
\hline $\mathbf{C u}$ & 3.56738362450286 & 20.18016917574070 & 3.56221128999951 \\
\hline $\mathbf{C u}$ & 3.56738362450286 & 22.70268960851930 & 3.56221128999951 \\
\hline $\mathbf{C u}$ & 7.13476724986203 & 0.00000000000000 & 3.56221128999951 \\
\hline $\mathbf{C u}$ & 7.13476724986203 & 2.52252114715664 & 3.56221128999951 \\
\hline $\mathbf{C u}$ & 7.13476724986203 & 5.04504229431329 & 3.56221128999951 \\
\hline $\mathbf{C u}$ & 7.13476724986203 & 7.56756358374076 & 3.56221128999951 \\
\hline $\mathbf{C u}$ & 7.13476724986203 & 10.09008458862650 & 3.56221128999951 \\
\hline $\mathbf{C u}$ & 7.13476724986203 & 12.61260559199990 & 3.56221128999951 \\
\hline $\mathbf{C u}$ & 7.13476724986203 & 15.13512716748060 & 3.56221128999951 \\
\hline $\mathbf{C u}$ & 7.13476724986203 & 4760025920 & 3.56221128999951 \\
\hline $\mathbf{C u}$ & 7.13476724986203 & 20.18016917574070 & 3.56221128999951 \\
\hline $\mathbf{C u}$ & 724986203 & 22.70268 & 3.56221128999951 \\
\hline $\mathbf{C u}$ & 46560 & 1.261260 & 345299991 \\
\hline $\mathbf{C u}$ & 181246560 & 658 & 5299991 \\
\hline $\mathbf{C u}$ & 1.783 & 6.30 & 4.82343 \\
\hline $\mathbf{C u}$ & 1.78 & 8.82 & 99991 \\
\hline $\mathbf{C u}$ & 46560 & 30425920 & 45299991 \\
\hline $\mathbf{C u}$ & 5560 & 74070 & 299991 \\
\hline $\mathbf{C u}$ & 1.78369 & 16.39638681251930 & 4.82343345299991 \\
\hline $\mathbf{C u}$ & 1.78369181246560 & 18.9189083 & 4.82343345299991 \\
\hline $\mathbf{C u}$ & 1.78369181246560 & 21.44142996348060 & 4.82343345299991 \\
\hline $\mathbf{C u}$ & 1.78369181246560 & 23.96395039625920 & 4.82343345299991 \\
\hline $\mathbf{C u}$ & 5.35107536505000 & 1.26126057282114 & 4.82343345299991 \\
\hline $\mathbf{C u}$ & 5.35107536505000 & 3.78378179262658 & 4.82343345299991 \\
\hline $\mathbf{C u}$ & 6505000 & 6.306 & 4.82343345299991 \\
\hline $\mathbf{C u}$ & 5.35107536505000 & 8.82882379937252 & 4.82343345299991 \\
\hline $\mathbf{C u}$ & 5.35107536505000 & 11.35134480425920 & 4.82343345299991 \\
\hline $\mathbf{C u}$ & 5.35107536505000 & 13.87386637974070 & 4.82343345299991 \\
\hline $\mathbf{C u}$ & 5.35107536505000 & 16.39638681251930 & 4.82343345299991 \\
\hline $\mathbf{C u}$ & 5.35107536505000 & 18.91890838799990 & 4.82343345299991 \\
\hline $\mathbf{C u}$ & 5.35107536505000 & 21.44142996348060 & 4.82343345299991 \\
\hline $\mathbf{C u}$ & 5.35107536505000 & 23.96395039625920 & 4.82343345299991 \\
\hline $\mathbf{C u}$ & 8.91845856103867 & 1.26126057282114 & 4.82343345299991 \\
\hline $\mathbf{C u}$ & 8.91845856103867 & 3.78378179262658 & 4.82343345299991 \\
\hline $\mathbf{C u}$ & 8.91845856103867 & 6.30630279600000 & 4.82343345299991 \\
\hline $\mathbf{C u}$ & 8.91845856103867 & 8.82882379937252 & 4.82343345299991 \\
\hline $\mathbf{C u}$ & 8.91845856103867 & 11.35134480425920 & 4.82343345299991 \\
\hline $\mathbf{C u}$ & 8.91845856103867 & 13.87386637974070 & 4.82343345299991 \\
\hline $\mathbf{C u}$ & 8.91845856103867 & 16.39638681251930 & 4.82343345299991 \\
\hline $\mathbf{C u}$ & 8.91845856103867 & 18.91890838799990 & 4.82343345299991 \\
\hline $\mathbf{C u}$ & 8.91845856103867 & 21.44142996348060 & 4.82343345299991 \\
\hline $\mathbf{C u}$ & 8.91845856103867 & 23.96395039625920 & 4.82343345299991 \\
\hline $\mathbf{C u}$ & 10.70269326776030 & -0.00059976654715 & 6.09634750515209 \\
\hline $\mathbf{C u}$ & 10.69734035911370 & 2.52183878996686 & 6.09614373516786 \\
\hline $\mathbf{C u}$ & 10.70050391793970 & 5.04100879276219 & 6.09390823890077 \\
\hline $\mathbf{C u}$ & 10.70157197252150 & 7.56901050166882 & 6.09705811771767 \\
\hline $\mathbf{C u}$ & 10.69282270774520 & 10.08734835348770 & 6.09221611701274 \\
\hline
\end{tabular}




\begin{tabular}{|c|c|c|c|}
\hline $\mathbf{C u}$ & 0.00408231934820 & 12.61740232463920 & 6.10519934535322 \\
\hline $\mathbf{C u}$ & 10.70161182975280 & 15.13351147895000 & 6.09726685808796 \\
\hline $\mathbf{C u}$ & 10.70058347362280 & 17.65852375000320 & 6.09910719068715 \\
\hline $\mathbf{C u}$ & 0.00240770890676 & 20.18122287946920 & 6.09733692414193 \\
\hline $\mathrm{Cu}$ & 10.70006457366550 & 22.70149524328510 & 6.09576714769400 \\
\hline $\mathbf{C u}$ & 3.56736053668520 & -0.00058126591852 & 6.09375284160006 \\
\hline $\mathbf{C u}$ & 3.56736212604835 & 2.52548402490056 & 6.09232121034735 \\
\hline $\mathbf{C u}$ & 3.56735858004336 & 5.04480934971295 & 6.10132024298541 \\
\hline $\mathbf{C u}$ & 3.56735084038870 & 7.56095018591959 & 6.15437762657327 \\
\hline $\mathbf{C u}$ & 3.56735106136745 & 10.09199840127820 & 6.13894187568815 \\
\hline $\mathbf{C u}$ & 3.56735229918171 & 12.60464210744580 & 6.14577492647275 \\
\hline $\mathbf{C u}$ & 3.56735282639637 & 15.12774651190170 & 6.14699796710762 \\
\hline $\mathbf{C u}$ & 3.56735299180162 & 17.64934843979830 & 6.13641258974145 \\
\hline $\mathrm{Cu}$ & 3.56735329959176 & 20.18080852460110 & 6.08896663371127 \\
\hline $\mathrm{Cu}$ & 3.56735826830292 & 22.70112550260020 & 6.09311129338581 \\
\hline $\mathrm{Cu}$ & 7.13424440265942 & -0.00059572893163 & 6.09636275652275 \\
\hline $\mathrm{Cu}$ & 7.13959905168565 & & 6.09617492755319 \\
\hline $\mathbf{C u}$ & 7.13645081683094 & & 6.09393184976011 \\
\hline $\mathbf{C u}$ & 7.13537015079841 & & 6.09709408923342 \\
\hline $\mathbf{C u}$ & 7.14413321496232 & 10.08734669 & 6.09225196057840 \\
\hline $\mathrm{Cu}$ & 7.13070782158315 & 12.61739220050700 & 6.10520326028449 \\
\hline $\mathrm{Cu}$ & 7.1353299 & 15.13351591329480 & 6.09727116571912 \\
\hline $\mathrm{Cu}$ & 7.136 & 7910 & 6.09 \\
\hline $\mathrm{Cu}$ & 7.13238086670462 & 20.18122623 & 6.09733 \\
\hline $\mathbf{C u}$ & 10222546 & 22.70149870235910 & 6.09578178328191 \\
\hline $\mathbf{C u}$ & 1.78298060694987 & 1.26244153285941 & 7.38873611217211 \\
\hline $\mathbf{C u}$ & 1.78416362300471 & 3.77754815448961 & 7.38945946836325 \\
\hline $\mathbf{C u}$ & 1.78099129337873 & 6.29716338280077 & 7.37938116468363 \\
\hline $\mathbf{C u}$ & 1.78472293314448 & 8.82703612459326 & 7.37882305136929 \\
\hline $\mathbf{C u}$ & 1.77243422867654 & 11.33886589107670 & 7.38344878947483 \\
\hline $\mathbf{C u}$ & 1.80441410634989 & 13.87563265349790 & 7.39675199387346 \\
\hline $\mathrm{Cu}$ & 1.77954879506594 & 16.39595967504480 & 7.37407823514482 \\
\hline $\mathbf{C u}$ & 1.79495089996259 & 18.91611055361430 & 7.39278179931385 \\
\hline $\mathbf{C u}$ & 1.78330374898176 & 21.44495583556160 & 7.38432560337764 \\
\hline $\mathbf{C u}$ & 1.78474658715748 & 23.96578309432800 & 7.38625633368981 \\
\hline $\mathrm{Cu}$ & 5.35175837037927 & 1.262444 & 202452 \\
\hline $\mathbf{C u}$ & 5.35057864079985 & 3.77755093912747 & 7.38946740558453 \\
\hline $\mathrm{Cu}$ & 5.35376381964843 & 6.29715343033208 & 7.37937853008946 \\
\hline $\mathrm{Cu}$ & 5.35003473485790 & 8.82705334264988 & 7.37879690951501 \\
\hline $\mathbf{C u}$ & 5.36229690846294 & 11.33885760198160 & 7.38346720248496 \\
\hline $\mathrm{Cu}$ & 5.33032154470559 & 13.87563260512320 & 7.39669441222437 \\
\hline $\mathrm{Cu}$ & 5.35518354469683 & 16.39595910149730 & 7.37407699019226 \\
\hline $\mathbf{C u}$ & 5.33978475424031 & 18.91611453841230 & 7.39274881158939 \\
\hline $\mathbf{C u}$ & 5.35143688453160 & 21.44496169505510 & 7.38431500186117 \\
\hline $\mathbf{C u}$ & 5.34999442562900 & 23.96579111060830 & 7.38625310858709 \\
\hline $\mathbf{C u}$ & 8.91848353110661 & 1.26081529827855 & 7.39854903827771 \\
\hline $\mathbf{C u}$ & 8.91849614874902 & 3.78054061814435 & 7.39587456098571 \\
\hline $\mathrm{Cu}$ & 8.91851262511064 & 6.30613493132443 & 7.39268444706450 \\
\hline $\mathbf{C u}$ & 8.91850769769716 & 8.82503654568262 & 7.39679172821586 \\
\hline $\mathbf{C u}$ & 8.91849018561436 & 11.35345953041330 & 7.39270698286403 \\
\hline $\mathbf{C u}$ & 8.91847450510342 & 13.87116254405260 & 7.40402600537735 \\
\hline $\mathrm{Cu}$ & 8.91846817058937 & 16.39518963231370 & 7.39415492471283 \\
\hline $\mathrm{Cu}$ & 8.91846993767432 & 18.91678774777680 & 7.39969238589478 \\
\hline $\mathbf{C u}$ & 8.91847450685421 & 21.43808664487260 & 7.39631258916851 \\
\hline
\end{tabular}




\begin{tabular}{|c|c|c|c|}
\hline $\mathbf{C u}$ & 8.91847687448165 & 23.95881879263220 & 473552433 \\
\hline $\mathbf{C u}$ & 0.00119868033309 & -0.00129354464550 & 8.61207960268090 \\
\hline $\mathbf{C u}$ & 0.00127463934243 & 2.52056330799646 & 8.61391065993576 \\
\hline $\mathbf{C u}$ & 0.00331702344906 & 5.04415491464431 & 8.60842344140387 \\
\hline $\mathbf{C u}$ & 0.00384915848862 & 7.56803428040729 & 8.60604256887770 \\
\hline $\mathbf{C u}$ & 10.69948221416710 & 10.08906238440140 & 8.61171528090101 \\
\hline $\mathbf{C u}$ & 0.00401899658137 & 12.61061576354980 & 8.61748934713411 \\
\hline $\mathbf{C u}$ & 0.00408433893096 & 15.12861533488690 & 8.60918157133659 \\
\hline $\mathbf{C u}$ & 0.00156646820750 & 17.65277921937900 & 8.61229312 \\
\hline $\mathbf{C u}$ & 0.00290180314876 & 20.17738400472720 & 8.60900454 \\
\hline $\mathbf{C u}$ & 0.00299691033983 & 22.70226437989260 & 8.61243058126689 \\
\hline $\mathbf{C u}$ & 3.56735655253947 & -0.00445031084976 & 8.61383 \\
\hline $\mathbf{C u}$ & 5201989153 & 2.51172869056084 & 8.61323739676890 \\
\hline $\mathrm{Cu}$ & 30636955 & 195931581 & 8.6180 \\
\hline $\mathrm{Cu}$ & 7447462 & 7.528 & 8.724 \\
\hline $\mathbf{C u}$ & 5744793 & 10.0 & 8.644 \\
\hline $\mathbf{C u}$ & 7640 & 1310 & 8.7 \\
\hline $\mathbf{C u}$ & 049317 & $15.10 s$ & 8.718 \\
\hline $\mathbf{C u}$ & 3.56 & 17.7 & 8.71 \\
\hline $\mathbf{C u}$ & 3.56 & 36390 & 8.59 \\
\hline $\mathbf{C u}$ & 98605 & 22.7066 & 8.611 \\
\hline $\mathbf{C u}$ & 6873 & -0.001 & 8.612 \\
\hline $\mathbf{C u}$ & 7.1335 & 961 & 8.613 \\
\hline $\mathbf{C u}$ & 7.131508450 & 5.0441 & 8.60839859633223 \\
\hline $\mathbf{C u}$ & 7.1310419 & 665606 & 8.60600283685505 \\
\hline $\mathbf{C u}$ & 7.137 & 10.08906536191960 & 8.61171622118676 \\
\hline $\mathbf{C u}$ & 7.130756315 & 12.6106 & 8.61748411137511 \\
\hline $\mathbf{C u}$ & 7.13 & 15.1 & 8.609 \\
\hline $\mathbf{C u}$ & 7.13 & 17.6 & 6111 \\
\hline $\mathbf{C u}$ & 7.13 & 20.1 & 8.60 \\
\hline $\mathbf{C u}$ & 7.13177805043673 & 22.7 & 8.61243294615605 \\
\hline \multicolumn{4}{|c|}{ CI-NEB Image 14} \\
\hline ement & X-Coordinate $(\AA)$ & Å) & $\mathrm{Z}-\mathrm{C}$ \\
\hline $\mathbf{C}$ & 3.56956029378049 & 7.28956979992706 & 2809080 \\
\hline C & 3.56733205043602 & 12.05038470627660 & 10.61605109529030 \\
\hline $\mathbf{F}$ & 317942 & 10.62066799194300 & 10.85291398393210 \\
\hline $\mathbf{F}$ & 2.501191 & 2654154969 & 11.33703808815810 \\
\hline $\mathbf{F}$ & 4.63 & 11692920 & 11.33471629778010 \\
\hline $\mathbf{F}$ & 3.56 & 16.41380237917020 & 10.18648978583330 \\
\hline $\mathbf{F}$ & 2.47044591871937 & 12.47805117797520 & 11.32317146148690 \\
\hline $\mathbf{F}$ & 4.66415455897308 & 12.47806588351840 & 11.32324770996350 \\
\hline $\mathbf{C u}$ & 0.000000 & & 21128624945 \\
\hline $\mathbf{C u}$ & 0.00 & & 3.56221128624945 \\
\hline $\mathbf{C u}$ & 0.00 & & 24945 \\
\hline $\mathbf{C u}$ & & & 1945 \\
\hline $\mathbf{C u}$ & & & 945 \\
\hline $\mathbf{C u}$ & & & 945 \\
\hline $\mathbf{C u}$ & & 66070 & 624945 \\
\hline $\mathrm{Cu}$ & 0.00000000000000 & 17.65764756216900 & 3.56221128624945 \\
\hline $\mathbf{C u}$ & & 20.18016921383090 & 3.56221128624945 \\
\hline $\mathbf{C u}$ & 0.00000000000000 & 22.70268953233920 & 3.56221128624945 \\
\hline $\mathbf{C u}$ & 3.56738363252946 & 0.00000000000000 & 3.56221128624945 \\
\hline $\mathbf{C u}$ & 8363252946 & 2.52252115194941 & 3.56221128624945 \\
\hline $\mathbf{C u}$ & 3.56738363252946 & 5.04504230389882 & 3.56221128624945 \\
\hline
\end{tabular}




\begin{tabular}{|c|c|c|c|}
\hline $\mathbf{C u}$ & 3.56738363252946 & 7.56756362183090 & 3.56221128624945 \\
\hline $\mathbf{C u}$ & 3.56738363252946 & 10.09008460779760 & 3.56221128624945 \\
\hline $\mathbf{C u}$ & 3.56738363252946 & 12.61260559199990 & 3.56221128624945 \\
\hline $\mathbf{C u}$ & 3.56738363252946 & 15.13512724366070 & 3.56221128624945 \\
\hline $\mathbf{C u}$ & 3.56738363252946 & 17.65764756216900 & 3.56221128624945 \\
\hline $\mathbf{C u}$ & 3.56738363252946 & 20.18016921383090 & 3.56221128624945 \\
\hline $\mathbf{C u}$ & 3.56738363252946 & 22.70268953233920 & 3.56221128624945 \\
\hline $\mathbf{C u}$ & 7.13476726570118 & 0.00000000000000 & 3.56221128624945 \\
\hline $\mathbf{C u}$ & 7.13476726570118 & 2.52252115194941 & 3.56221128624945 \\
\hline $\mathbf{C u}$ & 7.13476726570118 & 5.04504230389882 & 3.56221128624945 \\
\hline $\mathbf{C u}$ & 7.13476726570118 & 7.56756362183090 & 3.56221128624945 \\
\hline $\mathbf{C u}$ & 7.13476726570118 & 10.09008460779760 & 3.56221128624945 \\
\hline $\mathbf{C u}$ & 7.13476726570118 & 12.61260559199990 & 3.56221128624945 \\
\hline $\mathbf{C u}$ & 7.13476726570118 & 15.13512724366070 & 3.56221128624945 \\
\hline $\mathbf{C u}$ & 7.13476726570118 & 17.65764756216900 & 3.56221128624945 \\
\hline $\mathrm{Cu}$ & 7.13476726570118 & 20.18016921383090 & 3.56221128624945 \\
\hline $\mathbf{C u}$ & 7.13476726570118 & 22.70268953233920 & 3.56221128624945 \\
\hline $\mathbf{C u}$ & 1.78369181642541 & 1.26126057509134 & 4.82343343974989 \\
\hline $\mathbf{C u}$ & 1.78369181642541 & 3.78378181179765 & 4.82343343974989 \\
\hline $\mathbf{C u}$ & 81642541 & 000 & 343974989 \\
\hline $\mathbf{C u}$ & 1.78369181642541 & 0127 & 4.82343343974989 \\
\hline $\mathbf{C u}$ & 181642541 & 11.3513 & 4.82343343974989 \\
\hline $\mathbf{C u}$ & 1.78 & 090 & 74989 \\
\hline $\mathbf{C u}$ & 1.78 & 3920 & 74989 \\
\hline $\mathbf{C u}$ & 541 & 9990 & 974989 \\
\hline $\mathbf{C u}$ & 1.78365 & 66070 & 4.82343343974989 \\
\hline $\mathbf{C u}$ & 1.78369181642541 & 23.96395 & 4.82343343974989 \\
\hline $\mathbf{C u}$ & 5.35107536505000 & 1.26126057509134 & 4.82343343974989 \\
\hline $\mathbf{C u}$ & 36505000 & 3.7837 & 4.82343343974989 \\
\hline $\mathbf{C u}$ & 36505000 & 6.306302 & 4.82343343974989 \\
\hline $\mathbf{C u}$ & 5.35107536505000 & 8.82882378020127 & 4.82343343974989 \\
\hline $\mathbf{C u}$ & 5.3510 & 76616910 & 4.82343343974989 \\
\hline $\mathbf{C u}$ & 5.35107536505000 & 13.87386641783090 & 4.82343343974989 \\
\hline $\mathbf{C u}$ & 5.35107536505000 & 16.39638673633920 & 4.82343343974989 \\
\hline $\mathbf{C u}$ & 5.35107536505000 & 18.91890838799990 & 4.82343343974989 \\
\hline $\mathbf{C u}$ & 5.35107536505000 & 21.44143003966070 & 4.82343343974989 \\
\hline $\mathbf{C u}$ & 5.35107536505000 & 23.96395035816900 & 4.82343343974989 \\
\hline $\mathbf{C u}$ & 8.91845849746787 & 1.26126057509134 & 4.82343343974989 \\
\hline $\mathbf{C u}$ & 8.91845849746787 & 3.78378181179765 & 4.82343343974989 \\
\hline $\mathbf{C u}$ & 8.91845849746787 & 6.30630279600000 & 4.82343343974989 \\
\hline $\mathbf{C u}$ & 8.91845849746787 & 8.82882378020127 & 4.82343343974989 \\
\hline $\mathbf{C u}$ & 8.91845849746787 & 11.35134476616910 & 4.82343343974989 \\
\hline $\mathbf{C u}$ & 8.91845849746787 & 13.87386641783090 & 4.82343343974989 \\
\hline $\mathbf{C u}$ & 8.91845849746787 & 16.39638673633920 & 4.82343343974989 \\
\hline $\mathbf{C u}$ & 8.91845849746787 & 18.91890838799990 & 4.82343343974989 \\
\hline $\mathbf{C u}$ & 8.91845849746787 & 21.44143003966070 & 4.82343343974989 \\
\hline $\mathbf{C u}$ & 8.91845849746787 & 23.96395035816900 & 4.82343343974989 \\
\hline $\mathbf{C u}$ & 0.00148693423656 & 0.00070410596916 & 6.09662343817551 \\
\hline $\mathbf{C u}$ & 10.69968900465870 & 2.52245887308576 & 6.09799157006796 \\
\hline $\mathbf{C u}$ & 10.69874178228850 & 5.03923320055001 & 6.09281805699568 \\
\hline $\mathbf{C u}$ & 10.69981608431290 & 7.57179639830897 & 6.09553909641590 \\
\hline $\mathbf{C u}$ & 10.69148597604760 & 10.08788827638080 & 6.09149836141767 \\
\hline $\mathbf{C u}$ & 0.00214185293009 & 12.61880397022470 & 6.10532233200215 \\
\hline $\mathbf{C u}$ & 10.70168696213040 & 15.13470513798780 & 6.09705170426445 \\
\hline
\end{tabular}




\begin{tabular}{|c|c|c|c|}
\hline $\mathbf{C u}$ & 10.70131929310290 & 17.65880760215870 & 6.09957961850045 \\
\hline $\mathrm{Cu}$ & 0.00279758631849 & 20.18196647581870 & 6.09705551719588 \\
\hline $\mathbf{C u}$ & 10.69986185686730 & 22.70249771474690 & 6.09531696205708 \\
\hline $\mathbf{C u}$ & 3.56737580091317 & 0.00246641647635 & 6.09427862207859 \\
\hline $\mathrm{Cu}$ & 3.56737646980811 & 2.52934363387131 & 6.09237147673666 \\
\hline $\mathbf{C u}$ & 3.56737300785869 & 5.04791576527480 & 6.11106247781739 \\
\hline $\mathbf{C u}$ & 3.56737558828025 & 7.56213717543177 & 6.14191756772739 \\
\hline $\mathbf{C u}$ & 3.56737978758273 & 10.09592526829110 & 6.13120306204828 \\
\hline $\mathbf{C u}$ & 3.56737601569304 & 12.60588068057370 & 6.14057813681762 \\
\hline $\mathbf{C u}$ & 3.56737264360835 & 15.12949958210200 & 6.14704556369310 \\
\hline $\mathbf{C u}$ & 3.56737515807824 & 17.65188980923430 & 6.13584897231342 \\
\hline $\mathbf{C u}$ & 3.56737664256498 & 20.18113015766400 & 6.08910129956993 \\
\hline $\mathbf{C u}$ & 3.56737592674108 & 22.70307105498270 & 6.09376036628808 \\
\hline $\mathrm{Cu}$ & 7.13327578800436 & 0.00070074900510 & 6.09661863160148 \\
\hline $\mathrm{Cu}$ & 7.13723304375271 & 2.52245583601469 & 6.09799263896150 \\
\hline $\mathrm{Cu}$ & 7.13819609256973 & 5.03923633652196 & 6.09282126155034 \\
\hline $\mathrm{Cu}$ & 7.13710123069805 & & 6.09555492813645 \\
\hline $\mathbf{C u}$ & 7.14543821796230 & 10.08789190306980 & 6.09152058327032 \\
\hline $\mathbf{C u}$ & 7.13261486940473 & 12.61879857655230 & 6.10532182941273 \\
\hline $\mathbf{C u}$ & 7.13522100062512 & 15.13470740837440 & 6.0970 \\
\hline $\mathrm{Cu}$ & 7.13558811334279 & 17.65880783714650 & 6.09957 \\
\hline $\mathrm{Cu}$ & 7.13195792390884 & 20.18196964800620 & 6.0970456 \\
\hline $\mathrm{Cu}$ & 7.137 & 22.702 & 371731 \\
\hline $\mathrm{Cu}$ & 1.78485583117539 & 1.26678219411210 & 7.38954 \\
\hline $\mathbf{C u}$ & 1.789626 & 3.78 & 7.39701927996946 \\
\hline $\mathbf{C u}$ & 1.77280395500857 & 6.29840752533793 & 7.36848263212943 \\
\hline $\mathbf{C u}$ & 1.78564993514333 & 8.83115710966392 & 7.37775659433425 \\
\hline $\mathbf{C u}$ & 1.76859462564014 & 11.34083342279340 & 7.38086078902926 \\
\hline $\mathbf{C u}$ & 1.80504466633523 & 13.87650326345880 & 7.39500586301626 \\
\hline $\mathbf{C u}$ & 1.78123705307826 & 16.39683197469430 & 7.37390496332003 \\
\hline $\mathbf{C u}$ & 1.79575971763249 & 18.91783470891860 & 7.39308431464361 \\
\hline $\mathrm{Cu}$ & 1.78310675374183 & 21.44679976834450 & 7.38410249578055 \\
\hline $\mathbf{C u}$ & 1.78533625022551 & 23.96749067472920 & 7.38619640834716 \\
\hline $\mathbf{C u}$ & 5.34989479589845 & 1.26677662965767 & 7.38953586876939 \\
\hline $\mathbf{C u}$ & 5.34514142844075 & 3.78579479345146 & 7.39698899160281 \\
\hline $\mathrm{Cu}$ & 5.36198543285139 & 6.29840237652876 & 7.36846379905310 \\
\hline $\mathbf{C u}$ & 5.34912737786346 & 8.83117968318051 & 7.37774740539887 \\
\hline $\mathrm{Cu}$ & 5.36615483951879 & 11.34083511527550 & 7.38089652058840 \\
\hline $\mathrm{Cu}$ & 5.32970310143339 & 13.87650362104110 & 7.39495815414566 \\
\hline $\mathbf{C u}$ & 5.35351013141344 & 16.39683399159480 & 7.37389580870353 \\
\hline $\mathrm{Cu}$ & 5.33898639679260 & 18.91783974195390 & 7.39304948329077 \\
\hline $\mathrm{Cu}$ & 5.35164201449517 & 21.44680218438720 & 7.38410080025413 \\
\hline $\mathbf{C u}$ & 5.34941041178557 & 23.96748981600140 & 7.38619137426701 \\
\hline $\mathbf{C u}$ & 8.91846209554013 & 1.26387969166669 & 7.39897851990625 \\
\hline $\mathbf{C u}$ & 8.91847619859221 & 3.78048395138260 & 7.39575665166750 \\
\hline $\mathbf{C u}$ & 8.91848687292074 & 6.30832671999633 & 7.38967612303986 \\
\hline $\mathbf{C u}$ & 8.91848467530266 & 8.82704888246787 & 7.39696738760183 \\
\hline $\mathrm{Cu}$ & 8.91846733904981 & 11.35462789540650 & 7.39156902187148 \\
\hline $\mathbf{C u}$ & 8.91845437446051 & 13.87442071800920 & 7.40463839101857 \\
\hline $\mathbf{C u}$ & 8.91844739759116 & 16.39689923791480 & 7.39560650212229 \\
\hline $\mathbf{C u}$ & 8.91844968781995 & 18.91755047440720 & 7.39935348632273 \\
\hline $\mathrm{Cu}$ & 8.91845396070221 & 21.44015741624060 & 7.39633236770425 \\
\hline $\mathrm{Cu}$ & 8.91845625243502 & 23.96110400850120 & 7.39843327551719 \\
\hline $\mathbf{C u}$ & 0.00204690657885 & 0.00128589173506 & 8.61128741261058 \\
\hline
\end{tabular}




\begin{tabular}{|c|c|c|c|}
\hline $\mathbf{C u}$ & 0.00258123728848 & 353650354650 & 8.61417208773077 \\
\hline $\mathbf{C u}$ & 0.00104053824962 & 5.04704637165376 & 8.60693397731846 \\
\hline $\mathbf{C u}$ & 0.00368448729740 & 7.57033362875463 & 8.60410045135990 \\
\hline $\mathbf{C u}$ & 10.69886644794510 & 10.09009972185260 & 8.61202034783567 \\
\hline $\mathbf{C u}$ & 0.00384738738259 & 12.61232114119400 & 8.61752665231821 \\
\hline $\mathbf{C u}$ & 0.00596042212270 & 15.13039131867230 & 8.60817443659426 \\
\hline $\mathbf{C u}$ & 0.00282897720366 & 17.65510424837110 & 8.61227901450779 \\
\hline $\mathbf{C u}$ & 0.00183400200299 & 20.17884405740550 & 8.60827271389477 \\
\hline $\mathbf{C u}$ & 0.00275289801347 & 22.70448438385590 & 8.61207381305521 \\
\hline $\mathbf{C u}$ & 3.56737118499617 & 0.00176290079387 & 8.61443354549699 \\
\hline $\mathbf{C u}$ & 3.56738127696368 & 2.52277052935381 & 8.61512656700022 \\
\hline $\mathbf{C u}$ & 3.56742564634101 & 5.05181635451379 & 8.64735909025710 \\
\hline $\mathbf{C u}$ & 3.56724069597961 & 7.55632167314759 & 8.68664980760576 \\
\hline $\mathbf{C u}$ & 3.56737098162223 & 10.04945164634410 & 8.64422115544060 \\
\hline $\mathbf{C u}$ & 3.56737608150030 & 12.58560202408730 & 8.70550040554020 \\
\hline $\mathbf{C u}$ & 3.56738186234286 & 15.10693296141230 & 8.72065621156330 \\
\hline $\mathbf{C u}$ & 3.56 & 17.71546603160150 & 061786144 \\
\hline $\mathbf{C u}$ & 575 & 73730 & 776040 \\
\hline $\mathbf{C u}$ & 5201 & 95540 & 47977 \\
\hline $\mathbf{C u}$ & 7.13 & 12765 & 43758 \\
\hline $\mathbf{C u}$ & 7.13 & 334 & 8806 \\
\hline $\mathbf{C u}$ & 7.13 & 988 & 4763 \\
\hline $\mathbf{C u}$ & 7 & 384 & 788 \\
\hline $\mathrm{Cu}$ & 80 & 5090 & 7607 \\
\hline $\mathbf{C u}$ & 67 & 5200 & 4560 \\
\hline $\mathbf{C u}$ & 7.1 & 2880 & 38805 \\
\hline $\mathbf{C u}$ & 7.13 & 17.6 & 63962 \\
\hline $\mathbf{C u}$ & 7.13 & 4420 & 701064 \\
\hline $\mathbf{C u}$ & 7.13199725386984 & 8592800 & 8.61206099814684 \\
\hline \multicolumn{4}{|c|}{ CI-NEB Image 15} \\
\hline lement & X-Coordinate (Å) & $\mathrm{Y}-\mathrm{Cc}$ & $\mathrm{Z}-\mathrm{C}$ \\
\hline $\mathbf{C}$ & 01454 & 6.919094 & 10.40774194106420 \\
\hline C & 3.567 & 12.06762770846380 & 10.61302843272870 \\
\hline $\mathbf{F}$ & 3.56 & 33110 & 7547932360 \\
\hline $\mathbf{F}$ & 2.49 & 323 & 11.21 \\
\hline $\mathbf{F}$ & 4.6 & 590 & 11.2 \\
\hline $\mathbf{F}$ & 775 & 77970 & 3909950 \\
\hline $\mathbf{F}$ & 269 & 0110 & 11.3 \\
\hline $\mathbf{F}$ & 3123 & 12.50674389310810 & 11.31436097450910 \\
\hline $\mathbf{C u}$ & 0.00000000000000 & 0.00000000000000 & 3.56221128249938 \\
\hline $\mathbf{C u}$ & 0.00000000000000 & 2.52 & 3.56221128249938 \\
\hline $\mathbf{C u}$ & 0.00000000000000 & 5.04504231348436 & 3.56221128249938 \\
\hline $\mathbf{C u}$ & 0.00000000000000 & 7.56756365992104 & 3.56221128249938 \\
\hline $\mathbf{C u}$ & 0.00000000000000 & 10.09008462696870 & 3.56221128249938 \\
\hline $\mathbf{C u}$ & 0.00000000000000 & 12.61260559199990 & 3.56221128249938 \\
\hline $\mathbf{C u}$ & 0.00000000000000 & 15.13512731984080 & 3.56221128249938 \\
\hline $\mathbf{C u}$ & 0.00000000000000 & 17.65764752407890 & 3.56221128249938 \\
\hline $\mathbf{C u}$ & 0.00 & 20.18 & 3.56221128249938 \\
\hline $\mathbf{C u}$ & 0.00000000000000 & 22.70268945615910 & 3.56221128249938 \\
\hline $\mathbf{C u}$ & 606 & 0000 & 249938 \\
\hline $\mathbf{C u}$ & 3.56738364055606 & 2.52252115674218 & 3.56221128249938 \\
\hline $\mathbf{C u}$ & 3.56738364055606 & 5.04504231348436 & 3.56221128249938 \\
\hline $\mathbf{C u}$ & 3.56738364055606 & 7.56756365992104 & 3.56221128249938 \\
\hline $\mathbf{C u}$ & 3.56738364055606 & 10.09008462696870 & 3.56221128249938 \\
\hline
\end{tabular}




\begin{tabular}{|c|c|c|c|}
\hline $\mathrm{Cu}$ & 3.56738364055606 & 12.61260559199990 & 3.56221128249938 \\
\hline $\mathrm{Cu}$ & 3.56738364055606 & 15.13512731984080 & 3.56221128249938 \\
\hline $\mathbf{C u}$ & 3.56738364055606 & 17.65764752407890 & 3.56221128249938 \\
\hline $\mathbf{C u}$ & 3.56738364055606 & 20.18016925192100 & 3.56221128249938 \\
\hline $\mathbf{C u}$ & 3.56738364055606 & 22.70268945615910 & 3.56221128249938 \\
\hline $\mathbf{C u}$ & 7.13476728154033 & 0.00000000000000 & 3.56221128249938 \\
\hline $\mathbf{C u}$ & 7.13476728154033 & 2.52252115674218 & 3.56221128249938 \\
\hline $\mathbf{C u}$ & 7.13476728154033 & 5.04504231348436 & 3.56221128249938 \\
\hline $\mathbf{C u}$ & 7.13476728154033 & 7.56756365992104 & 3.56221128249938 \\
\hline $\mathbf{C u}$ & 7.13476728154033 & 10.09008462696870 & 3.56221128249938 \\
\hline $\mathbf{C u}$ & 7.13476728154033 & 12.61260559199990 & 3.56221128249938 \\
\hline $\mathbf{C u}$ & 7.13476728154033 & 15.13512731984080 & 3.56221128249938 \\
\hline $\mathbf{C u}$ & 7.13476728154033 & 17.65764752407890 & 3.56221128249938 \\
\hline $\mathbf{C u}$ & 7.13476728154033 & 20.18016925192100 & 3.56221128249938 \\
\hline $\mathbf{C u}$ & 7.13476728154033 & 22.70268945615910 & 3.56221128249938 \\
\hline $\mathbf{C u}$ & 1.78369182038521 & 1.26126057736154 & 4.82343342649986 \\
\hline $\mathbf{C u}$ & 1.78369182038521 & 3.78378183096872 & 4.82343342649986 \\
\hline $\mathbf{C u}$ & 1.78369182038521 & 6.30630279600000 & 4.82343342649986 \\
\hline $\mathbf{C u}$ & 1.78369182038521 & 3002 & 342649986 \\
\hline $\mathbf{C u}$ & 1.78369182038521 & 07890 & 342649986 \\
\hline $\mathbf{C u}$ & 3521 & 2100 & 986 \\
\hline $\mathbf{C u}$ & 8521 & 5910 & 9986 \\
\hline $\mathbf{C u}$ & 1.78 & & 19986 \\
\hline $\mathbf{C u}$ & 1.78369182038521 & 3011584080 & 4.82343342649986 \\
\hline $\mathbf{C u}$ & 8521 & 23.9639503 & 4.82343342649986 \\
\hline $\mathbf{C u}$ & 5.35 & 154 & 4.82343342649986 \\
\hline $\mathbf{C u}$ & 5.351 & 3.78 & 4.82343342649986 \\
\hline $\mathbf{C u}$ & 5.35 & 6.3( & 342649986 \\
\hline $\mathbf{C u}$ & 5.35 & 002 & 9986 \\
\hline $\mathbf{C u}$ & 5.35 & 7890 & 342649986 \\
\hline $\mathbf{C u}$ & 5.35 & 2100 & 42649986 \\
\hline $\mathbf{C u}$ & 5000 & 5910 & 4.82343342649986 \\
\hline $\mathbf{C u}$ & 5.35107536505000 & 18.91890838799990 & 4.82343342649986 \\
\hline $\mathbf{C u}$ & 5.35107536505000 & 21.44143011584080 & 4.82343342649986 \\
\hline $\mathbf{C u}$ & 5.35107536505000 & 23.96395032007890 & 4.82343342649986 \\
\hline $\mathbf{C u}$ & 8.91845843389707 & 1.26126057736154 & 4.82343342649986 \\
\hline $\mathbf{C u}$ & 8.91845843389707 & 3.78378183096872 & 4.82343342649986 \\
\hline $\mathbf{C u}$ & 8.91845843389707 & 6.30630279600000 & 4.82343342649986 \\
\hline $\mathbf{C u}$ & 8.91845843389707 & 8.82882376103002 & 4.82343342649986 \\
\hline $\mathbf{C u}$ & 8.91845843389707 & 11.35134472807890 & 4.82343342649986 \\
\hline $\mathbf{C u}$ & 8.91845843389707 & 13.87386645592100 & 4.82343342649986 \\
\hline $\mathbf{C u}$ & 8.91845843389707 & 16.39638666015910 & 4.82343342649986 \\
\hline $\mathbf{C u}$ & 8.91845843389707 & 18.91890838799990 & 4.82343342649986 \\
\hline $\mathbf{C u}$ & 8.91845843389707 & 21.44143011584080 & 4.82343342649986 \\
\hline $\mathbf{C u}$ & 8.91845843389707 & 23.96395032007890 & 4.82343342649986 \\
\hline $\mathbf{C u}$ & 0.00235127350742 & 0.00389297889913 & 6.09665381507715 \\
\hline $\mathbf{C u}$ & 0.00308091473070 & 2.52482910683784 & 6.10191966643955 \\
\hline $\mathbf{C u}$ & 10.69798995561410 & 5.03623901576429 & 6.09215911004167 \\
\hline $\mathbf{C u}$ & 10.69451168776260 & 7.57532347529945 & 6.09290794245997 \\
\hline $\mathbf{C u}$ & 10.69114659231090 & 10.08976246182380 & 6.09179736649278 \\
\hline $\mathbf{C u}$ & 0.00073800807262 & 12.62073063399910 & 6.10334934262505 \\
\hline $\mathbf{C u}$ & 10.70147956271880 & 15.13560434099440 & 6.09571182335878 \\
\hline $\mathbf{C u}$ & 10.70205015146070 & 17.65967346969100 & 6.09932116648515 \\
\hline $\mathbf{C u}$ & 0.00393549118756 & 20.18339687607740 & 5521025 \\
\hline
\end{tabular}




\begin{tabular}{|c|c|c|c|}
\hline $\mathbf{C u}$ & 10.69958318029920 & 22.70423572727920 & 6.09441540984336 \\
\hline $\mathbf{C u}$ & 3.56737776532666 & 0.00895763330099 & 6.09623252505890 \\
\hline $\mathbf{C u}$ & 3.56738074633488 & 2.53641653872582 & 6.09387861858204 \\
\hline $\mathbf{C u}$ & 3.56738519765512 & 5.05356013267964 & 6.13640251071859 \\
\hline $\mathrm{Cu}$ & 3.56738898281954 & 7.56359822910124 & 6.11837985568702 \\
\hline $\mathbf{C u}$ & 3.56739192002047 & 10.09963087555620 & 6.12629878564369 \\
\hline $\mathbf{C u}$ & 3.56738182097480 & 12.60761074128550 & 6.13913931021566 \\
\hline $\mathbf{C u}$ & 3.56737506591024 & 15.13248229680700 & 6.14637441004205 \\
\hline $\mathbf{C u}$ & 3.56737504510100 & 17.65605562509180 & 6.13533541255015 \\
\hline $\mathbf{C u}$ & 3.56737511270834 & 20.17912738016440 & 6.08965531727543 \\
\hline $\mathbf{C u}$ & 3.56737660609393 & 22.70544324285860 & 6.09604983553412 \\
\hline $\mathbf{C u}$ & 7.13242557633874 & 0.00388906758434 & 6.09664363529350 \\
\hline $\mathbf{C u}$ & 7.13170404916168 & 2.52482441051412 & 6.10189947351693 \\
\hline $\mathrm{Cu}$ & 7.13895480041085 & 5.03624471747749 & 6.09215293933511 \\
\hline $\mathrm{Cu}$ & 7.14241572613372 & 7.57533273107612 & 6.09292129754974 \\
\hline $\mathrm{Cu}$ & 7.14577468486862 & 10.08976408144600 & 6.09181779248747 \\
\hline $\mathrm{Cu}$ & 7.13402820727028 & 12.62072300836490 & 6.10334710399811 \\
\hline $\mathbf{C u}$ & 7.13543950191149 & 15.13560181734110 & 6.09570460276805 \\
\hline $\mathbf{C u}$ & 7.13487077404810 & 17.65966828137680 & 6.09931640799067 \\
\hline $\mathbf{C u}$ & $7.130833 \varepsilon$ & 20.1834008 & 00205085 \\
\hline $\mathrm{Cu}$ & 7.13733709976009 & 22.70423398989060 & 5949086 \\
\hline $\mathrm{Cu}$ & 1177484 & 1.27492849120578 & 7.3913 \\
\hline $\mathrm{Cu}$ & 1.803 & 3.80 & 7.4 \\
\hline $\mathrm{Cu}$ & 2853983 & 6.30 & 7.354 \\
\hline $\mathbf{C u}$ & 1.78457 & 21614 & 17295655 \\
\hline $\mathbf{C u}$ & 1.76752790802056 & 11.34646129165610 & 7.37778261663361 \\
\hline $\mathbf{C u}$ & 1.80463088539381 & 13.87956327462900 & 7.39194643962572 \\
\hline $\mathbf{C u}$ & 1.78109985273164 & 16.39826336635090 & 7.37296677345842 \\
\hline $\mathbf{C u}$ & 1.79622237630306 & 18.91991450337540 & 7.39300396779872 \\
\hline $\mathbf{C u}$ & 1.78230924765027 & 21.45076800191880 & 7.38331718329464 \\
\hline $\mathbf{C u}$ & 1.78575107614618 & 23.97216641428500 & 7.38617827625694 \\
\hline $\mathrm{Cu}$ & 5.34512635481086 & 1.27491975588058 & 7.39136269446638 \\
\hline $\mathbf{C u}$ & 5.33109258538507 & 3.80605505480469 & 7.41499859785681 \\
\hline $\mathbf{C u}$ & 5.37521532254440 & 6.30482428118276 & 7.35489848064046 \\
\hline $\mathbf{C u}$ & 5.35020935895987 & 8.83460276623602 & 7.37757893960430 \\
\hline $\mathrm{Cu}$ & 5.36724222224745 & 11.34645974438480 & 7.37781773798517 \\
\hline $\mathbf{C u}$ & 5.33013013426550 & 13.87956154830900 & 7.39190340142589 \\
\hline $\mathrm{Cu}$ & 5.35365830085375 & 16.39826220324350 & 7.37295989789235 \\
\hline $\mathrm{Cu}$ & 5.33853677765123 & 18.91991684077780 & 7.39296642610316 \\
\hline $\mathbf{C u}$ & 5.35244969456949 & 21.45077015103660 & 7.38331717216280 \\
\hline $\mathrm{Cu}$ & 5.34900934170250 & 23.97216467938440 & 7.38616858126717 \\
\hline $\mathrm{Cu}$ & 8.91846747746659 & 1.26928417885312 & 7.39778873815261 \\
\hline $\mathbf{C u}$ & 8.91847501260250 & 3.78041930036923 & 7.39616803520577 \\
\hline $\mathbf{C u}$ & 8.91847513863218 & 6.31026938471477 & 7.38616994088834 \\
\hline $\mathbf{C u}$ & 8.91847886310864 & 8.83094781342895 & 7.39633187179265 \\
\hline $\mathbf{C u}$ & 8.91846575734859 & 11.35556774253070 & 7.39042337017264 \\
\hline $\mathbf{C u}$ & 8.91846135142853 & 13.87854213615830 & 7.40206656319232 \\
\hline $\mathrm{Cu}$ & 8.91845918811225 & 16.39850664947900 & 7.39629106672207 \\
\hline $\mathbf{C u}$ & 8.91846092097185 & 18.91952418528230 & 7.39854082378776 \\
\hline $\mathbf{C u}$ & 8.91846398196053 & 21.44334688080480 & 7.39687907720448 \\
\hline $\mathbf{C u}$ & 8.91846428700993 & 23.96458086542550 & 7.39659654058469 \\
\hline $\mathrm{Cu}$ & 0.00285559084958 & 0.00631110133938 & 8.60892537427635 \\
\hline $\mathrm{Cu}$ & 0.00700555673423 & 2.52873792815902 & 8.61332290129054 \\
\hline $\mathbf{C u}$ & 10.70055278474570 & 5.05198682242129 & 8.60555211771086 \\
\hline
\end{tabular}




\begin{tabular}{|c|c|c|c|}
\hline $\mathbf{C u}$ & 10.70273428236010 & 35125 & 8.60333856019343 \\
\hline $\mathbf{C u}$ & 10.69972333530480 & 10.09294387397950 & 8.61105722728423 \\
\hline $\mathbf{C u}$ & 0.00474986789988 & 12.61495257723540 & 8.61407153649529 \\
\hline $\mathbf{C u}$ & 0.00631072408102 & 15.13241080226250 & 8.60722751696420 \\
\hline $\mathbf{C u}$ & 0.00204796528862 & 17.65852260171780 & 8.61108605361726 \\
\hline $\mathbf{C u}$ & 0.00063630234336 & 20.18097935267710 & 8.60720461110139 \\
\hline $\mathbf{C u}$ & 0.00217977425020 & 22.70817611312730 & 8.61111511522532 \\
\hline $\mathbf{C u}$ & 3.56738644084609 & 0.01542385419271 & 8.61586224535660 \\
\hline $\mathbf{C u}$ & 3.56741230359025 & 2.55481501634770 & 8.62163826137881 \\
\hline $\mathbf{C u}$ & 3.56754782292609 & 5.12638412228148 & 8.76188295597031 \\
\hline $\mathbf{C u}$ & 3.56730666891607 & 7.59672897952038 & 8.63763586981623 \\
\hline $\mathbf{C u}$ & 3.56738452375859 & 10.06264753410990 & 8.64403896713945 \\
\hline $\mathbf{C u}$ & 3.56738063480493 & 12.58871170059450 & 324935655 \\
\hline $\mathbf{C u}$ & 3.56738904574081 & 15.10947197846140 & 8.71947570730396 \\
\hline $\mathbf{C u}$ & 3.56738992620213 & 17.71931700007370 & 8.72001931545936 \\
\hline $\mathbf{C u}$ & 3.56738606507763 & & 8.59447466436466 \\
\hline $\mathbf{C u}$ & & 74052076560 & 8.61146627494542 \\
\hline $\mathbf{C u}$ & 7.1 & & 8.60890469886378 \\
\hline $\mathbf{C u}$ & 7.12 & & 944606804 \\
\hline $\mathbf{C u}$ & 7.13 & & 9452 \\
\hline $\mathrm{Cu}$ & 7.1 & & 76791 \\
\hline $\mathbf{C u}$ & 46 & 900 & 55782 \\
\hline $\mathbf{C u}$ & 97 & 12 & 759 \\
\hline $\mathrm{Cu}$ & 27 & 15. & 3554 \\
\hline $\mathbf{C u}$ & 91 & 17. & 3428 \\
\hline $\mathbf{C u}$ & 75 & 820 & 3208008 \\
\hline $\mathbf{C u}$ & 7.13259003375370 & 17840115060 & 8.61110714863352 \\
\hline \multicolumn{4}{|c|}{ CI-NEB Image 16} \\
\hline lement & X-Coordinat & $\mathrm{Y}-\mathrm{Cc}$ & Z-C \\
\hline C & 402993 & 6.54709 & 10.294002 \\
\hline C & $3.56^{\circ}$ & 12.09411 & 10.60978867306730 \\
\hline $\mathbf{F}$ & 5761 & 10.671276 & 10.86205388994290 \\
\hline $\mathbf{F}$ & 37603 & 2039667 & 11.10932868083380 \\
\hline $\mathbf{F}$ & 4.64 & 6.64 & 86630 \\
\hline $\mathbf{F}$ & 3.56 & 49550 & 5796720 \\
\hline $\mathbf{F}$ & 16 & 300 & 65250 \\
\hline $\mathbf{F}$ & 4.6 & 12.5 & 71000 \\
\hline $\mathbf{C u}$ & 00 & 00 & 4931 \\
\hline $\mathbf{C u}$ & 000000 & 153495 & 1127874931 \\
\hline $\mathbf{C u}$ & 0.00000000000000 & 5.04504232306990 & 3.56221127874931 \\
\hline $\mathbf{C u}$ & 0.00000000000000 & 7.56 & 1127874931 \\
\hline $\mathbf{C u}$ & 0.00000000000000 & 10.09008464613970 & 3.56221127874931 \\
\hline $\mathbf{C u}$ & 0.00000000000000 & 12.61260559199990 & 3.56221127874931 \\
\hline $\mathbf{C u}$ & 0.00000000000000 & 15.13512739602090 & 3.56221127874931 \\
\hline $\mathbf{C u}$ & 0.00000000000000 & 17.65764748598880 & 3.56221127874931 \\
\hline $\mathbf{C u}$ & 0.00000000000000 & 20.18016929001110 & 3.56221127874931 \\
\hline $\mathbf{C u}$ & 0.00000000000000 & 22.70268937997900 & 3.56221127874931 \\
\hline $\mathbf{C u}$ & 858267 & 0.00 & 3.56221127874931 \\
\hline $\mathbf{C u}$ & 3.56738364858267 & 2.52252116153495 & 3.56221127874931 \\
\hline $\mathbf{C u}$ & 267 & 2306990 & 1127874931 \\
\hline $\mathbf{C u}$ & 3.56738364858267 & 7.56756369801118 & 3.56221127874931 \\
\hline $\mathbf{C u}$ & 3.56738364858267 & 10.09008464613970 & 3.56221127874931 \\
\hline $\mathbf{C u}$ & 3.56738364858267 & 12.61260559199990 & 3.56221127874931 \\
\hline $\mathbf{C u}$ & 3.56738364858267 & 15.13512739602090 & 3.56221127874931 \\
\hline
\end{tabular}




\begin{tabular}{|c|c|c|c|}
\hline $\mathbf{C u}$ & 3.56738364858267 & 17.65764748598880 & 3.56221127874931 \\
\hline $\mathbf{C u}$ & 3.56738364858267 & 20.18016929001110 & 3.56221127874931 \\
\hline $\mathbf{C u}$ & 3.56738364858267 & 22.70268937997900 & 3.56221127874931 \\
\hline $\mathbf{C u}$ & 7.13476729737947 & 0.00000000000000 & 3.56221127874931 \\
\hline $\mathbf{C u}$ & 7.13476729737947 & 2.52252116153495 & 3.56221127874931 \\
\hline $\mathbf{C u}$ & 7.13476729737947 & 5.04504232306990 & 3.56221127874931 \\
\hline $\mathbf{C u}$ & 7.13476729737947 & 7.56756369801118 & 3.56221127874931 \\
\hline $\mathbf{C u}$ & 7.13476729737947 & 10.09008464613970 & 3.56221127874931 \\
\hline $\mathbf{C u}$ & 7.13476729737947 & 12.61260559199990 & 3.56221127874931 \\
\hline $\mathbf{C u}$ & 7.13476729737947 & 15.13512739602090 & 3.56221127874931 \\
\hline $\mathbf{C u}$ & 7.13476729737947 & 17.65764748598880 & 3.56221127874931 \\
\hline $\mathbf{C u}$ & 7.13476729737947 & 20.18016929001110 & 3.56221127874931 \\
\hline $\mathbf{C u}$ & 7.13476729737947 & 22.70268937997900 & 3.56221127874931 \\
\hline $\mathbf{C u}$ & 1.78369182434502 & 1.26126057963175 & 4.82343341324984 \\
\hline $\mathbf{C u}$ & 1.78369182434502 & 3.78378185013979 & 4.82343341324984 \\
\hline $\mathrm{Cu}$ & 1.78369182434502 & 6.30630279600000 & 4.82343341324984 \\
\hline $\mathbf{C u}$ & 1.78369182434502 & 8.82882374185877 & 4.82343341324984 \\
\hline $\mathbf{C u}$ & 82434502 & 11.35134468998880 & 4.82343341324984 \\
\hline $\mathbf{C u}$ & 1.78369182434502 & 13.87386649401110 & \\
\hline $\mathbf{C u}$ & 1.78369182434502 & 7900 & 24984 \\
\hline $\mathbf{C u}$ & 1.78369182434502 & 9990 & 4.8234 \\
\hline $\mathbf{C u}$ & 1.783 & 21.4414301 & 4.82343341324984 \\
\hline $\mathbf{C u}$ & 1502 & 23.9 & 4984 \\
\hline $\mathbf{C u}$ & 5.351 & 175 & 4.823 \\
\hline $\mathbf{C u}$ & 5000 & 979 & 24984 \\
\hline $\mathbf{C u}$ & 36505000 & 9600000 & 341324984 \\
\hline $\mathbf{C u}$ & 5.35107536505000 & 8.82882374185877 & 4.82343341324984 \\
\hline $\mathbf{C u}$ & 5.35107536505000 & 11.35134468998880 & 4.82343341324984 \\
\hline $\mathbf{C u}$ & 36505000 & 13.87386649401110 & 4.82343341324984 \\
\hline $\mathbf{C u}$ & 6505000 & 16.39638658 & 4.82343341324984 \\
\hline $\mathbf{C u}$ & 5.35107536505000 & 18.91890838799990 & 4.82343341324984 \\
\hline $\mathbf{C u}$ & 6505000 & 2090 & 4.82343341324984 \\
\hline $\mathbf{C u}$ & 5.35107536505000 & 23.96395028198880 & 4.82343341324984 \\
\hline $\mathbf{C u}$ & 8.91845837032627 & 1.26126057963175 & 4.82343341324984 \\
\hline $\mathbf{C u}$ & 8.91845837032627 & 3.78378185013979 & 4.82343341324984 \\
\hline $\mathbf{C u}$ & 8.91845837032627 & 0000 & 4.82343341324984 \\
\hline $\mathbf{C u}$ & 8.91845837032627 & 8.82882374185877 & 4.82343341324984 \\
\hline $\mathbf{C u}$ & 8.91845837032627 & 11.35134468998880 & 4.82343341324984 \\
\hline $\mathbf{C u}$ & 8.91845837032627 & 13.87386649401110 & 4.82343341324984 \\
\hline $\mathbf{C u}$ & 8.91845837032627 & 16.39638658397900 & 4.82343341324984 \\
\hline $\mathbf{C u}$ & 8.91845837032627 & 18.91890838799990 & 4.82343341324984 \\
\hline $\mathbf{C u}$ & 8.91845837032627 & 21.44143019202090 & 4.82343341324984 \\
\hline $\mathbf{C u}$ & 8.91845837032627 & 23.96395028198880 & 4.82343341324984 \\
\hline $\mathbf{C u}$ & 0.00081314271155 & 0.00602028867842 & 6.09625107394723 \\
\hline $\mathbf{C u}$ & 0.00322632025735 & 2.52606644090384 & 6.10239752892631 \\
\hline $\mathbf{C u}$ & 10.69846071158600 & 5.03601664125819 & 6.09214609709307 \\
\hline $\mathbf{C u}$ & 10.69044717908180 & 7.57769186739328 & 6.09110178699401 \\
\hline $\mathbf{C u}$ & 10.69145862611770 & 10.09156813014560 & 6.09312853234246 \\
\hline $\mathbf{C u}$ & 0.00141289637522 & 12.62278147220200 & 6.10285864930422 \\
\hline $\mathbf{C u}$ & 10.70088524469440 & 15.13674290280890 & 6.09482957780363 \\
\hline $\mathbf{C u}$ & 10.70086459180380 & 17.66034678301640 & 6.09859386269615 \\
\hline $\mathbf{C u}$ & 0.00473632514661 & 20.18416887810560 & 6.09675238526751 \\
\hline $\mathbf{C u}$ & 10.70062471894080 & 22.70501794419590 & 6.09416950988412 \\
\hline $\mathbf{C u}$ & 3.56737976112824 & 0.01197698829258 & 6.09766560757146 \\
\hline
\end{tabular}




\begin{tabular}{|c|c|c|c|}
\hline $\mathbf{C u}$ & 3.56737983335224 & 2.53891453731927 & 6.09453225816917 \\
\hline $\mathbf{C u}$ & 3.56738523088833 & 5.05665043388882 & 6.14326874470420 \\
\hline $\mathbf{C u}$ & 3.56738993873287 & 7.56641430233595 & 6.11032771132988 \\
\hline $\mathbf{C u}$ & 3.56739169978133 & 10.09984898454290 & 6.12608310849365 \\
\hline $\mathrm{Cu}$ & 3.56738342262329 & 12.60839865428550 & 6.13700287775097 \\
\hline $\mathbf{C u}$ & 3.56737874112825 & 15.13527388150150 & 6.14653458920916 \\
\hline $\mathbf{C u}$ & 3.56737927456829 & 17.66049400301690 & 6.13499514070614 \\
\hline $\mathbf{C u}$ & 3.56738035902888 & 20.18046756193620 & 6.08862429874759 \\
\hline $\mathbf{C u}$ & 3.56738217034657 & 22.70702755778650 & 6.09651274449490 \\
\hline $\mathbf{C u}$ & 7.13396370918020 & 0.00601670320960 & 6.09624196645363 \\
\hline $\mathbf{C u}$ & 7.13155623744311 & 2.52606505363050 & 6.10237467970250 \\
\hline $\mathbf{C u}$ & 7.13847287877982 & 5.03602356736249 & 6.09214700948992 \\
\hline $\mathbf{C u}$ & 7.14647783261553 & 7.57769565695514 & 6.09112404556609 \\
\hline $\mathrm{Cu}$ & 7.14545929370846 & 10.09156887793300 & 6.09315071514836 \\
\hline $\mathrm{Cu}$ & 7.13335836861577 & 12.62277610860960 & 6.10285145136623 \\
\hline $\mathrm{Cu}$ & 7.13603705652125 & 15.13674294354350 & 6.09482492371991 \\
\hline $\mathrm{Cu}$ & 7.13605690779317 & 17.66034284807490 & 6.09859214967662 \\
\hline $\mathbf{C u}$ & 4273927 & 20.18417175142680 & 6.09673891331860 \\
\hline $\mathbf{C u}$ & 7.13629693589652 & 22.70501677643260 & 6.09416923152498 \\
\hline $\mathbf{C u}$ & 1.79091700670378 & 1.277 & 76355 \\
\hline $\mathrm{Cu}$ & 1.80556893058664 & 3.810912 & 838048066 \\
\hline $\mathrm{Cu}$ & 1.752034 & 1906611330 & 7.34689647769486 \\
\hline $\mathrm{Cu}$ & 1.786 & 8.83 & 5441 \\
\hline $\mathrm{Cu}$ & 1370912429 & 11.34 & 7.37661124292222 \\
\hline $\mathrm{Cu}$ & 11305 & 4290 & 788984 \\
\hline $\mathbf{C u}$ & 1.78041931138888 & 16.40140112388860 & 7.37228130950463 \\
\hline $\mathbf{C u}$ & 1.79615008731705 & 18.92164743500140 & 7.39288208085331 \\
\hline $\mathbf{C u}$ & 1.78261885247881 & 21.45348077372120 & 7.38287611465240 \\
\hline $\mathbf{C u}$ & 1.78583808368088 & 23.97649204993710 & 7.38610006746473 \\
\hline $\mathbf{C u}$ & 5.34385932481832 & 1.27763837706971 & 7.39235513507659 \\
\hline $\mathbf{C u}$ & 5.32922539795359 & 3.81090954096928 & 7.41533897973509 \\
\hline $\mathrm{Cu}$ & 5.38276083040684 & 6.31355539470857 & 7.34690804179730 \\
\hline $\mathbf{C u}$ & 5.34877100217226 & 8.83431424107515 & 7.38370741856683 \\
\hline $\mathbf{C u}$ & 5.36635876274390 & 11.34874690239840 & 7.37664389554310 \\
\hline $\mathbf{C u}$ & 5.33087034934838 & 13.88357701589600 & 7.38931714641980 \\
\hline $\mathrm{Cu}$ & 5.35434619645437 & 16.40140143954010 & 7.37227841486433 \\
\hline $\mathbf{C u}$ & 5.33861964228018 & 18.92164770053880 & 7.39284474248312 \\
\hline $\mathrm{Cu}$ & 5.35215084807574 & 21.45348136372090 & 7.38287067620915 \\
\hline $\mathrm{Cu}$ & 5.34893169545553 & 23.97648908548600 & 7.38608622864434 \\
\hline $\mathbf{C u}$ & 8.91846560487189 & 1.27273075284426 & 7.39651850589985 \\
\hline $\mathrm{Cu}$ & 8.91847003325463 & 3.78257725776121 & 7.39668040073252 \\
\hline $\mathrm{Cu}$ & 8.91847048952646 & 6.31306599179463 & 7.38492726616767 \\
\hline $\mathbf{C u}$ & 8.91847078811845 & 8.83600877294951 & 7.39546710189874 \\
\hline $\mathbf{C u}$ & 8.91846627619931 & 11.35736022713010 & 7.39234116283636 \\
\hline $\mathbf{C u}$ & 8.91846468294158 & 13.88096542660500 & 7.40203092255915 \\
\hline $\mathbf{C u}$ & 8.91846149445833 & 16.40014661166460 & 7.39657904322892 \\
\hline $\mathbf{C u}$ & 8.91846017931815 & 18.92203973763820 & 7.39855819566420 \\
\hline $\mathrm{Cu}$ & 8.91846199759622 & 21.44544383380620 & 7.39784470361064 \\
\hline $\mathbf{C u}$ & 8.91846312078181 & 23.96770925794240 & 7.39578017900045 \\
\hline $\mathrm{Cu}$ & 0.00248677451960 & 0.00972957361626 & 8.60865727779728 \\
\hline $\mathbf{C u}$ & 0.00879521335334 & 2.53250519608185 & 8.61227276174709 \\
\hline $\mathbf{C u}$ & 10.69978825057760 & 5.05620899182458 & 8.60241191212865 \\
\hline $\mathrm{Cu}$ & 10.69952461497870 & 7.57832189540979 & 8.60454632353514 \\
\hline $\mathrm{Cu}$ & 10.70071849877480 & 10.09591171494290 & 8.61172780885522 \\
\hline
\end{tabular}




\begin{tabular}{|c|c|c|c|}
\hline $\mathbf{C u}$ & 0.00594692766768 & 12.61735955850460 & 0062633 \\
\hline $\mathbf{C u}$ & 0.00676439257855 & 15.13473068258120 & 8.60708835681314 \\
\hline $\mathbf{C u}$ & 10.70238480144960 & 17.66203649217110 & 8.61068450718031 \\
\hline $\mathbf{C u}$ & 0.00068514556066 & 20.18405984710570 & 8.60758013006985 \\
\hline $\mathbf{C u}$ & 0.00281399095759 & 22.71097747821880 & 8.60988985613200 \\
\hline $\mathbf{C u}$ & 3.56739029691587 & 0.02336492251745 & 8.61711246919517 \\
\hline $\mathbf{C u}$ & 3.56741197145546 & 2.57196763251075 & 8.62458593824484 \\
\hline $\mathbf{C u}$ & 3.56743589944332 & 5.12572352310285 & 8.79778641568601 \\
\hline $\mathbf{C u}$ & 3.56736496122749 & 7.59357667010217 & 8.63795693843815 \\
\hline $\mathbf{C u}$ & 3.56738246197635 & 10.07018271387040 & 8.64353551536274 \\
\hline $\mathbf{C u}$ & 3.56738670542574 & 12.59629979413090 & 8.69419325931876 \\
\hline $\mathbf{C u}$ & 3.56739188589436 & 15.11173516899210 & 8.71821597730341 \\
\hline $\mathbf{C u}$ & 3.56739292346323 & 17.72245081092120 & 8.72113772637067 \\
\hline $\mathbf{C u}$ & 3.56739273897036 & 20.21428990980130 & 8.59325906855516 \\
\hline $\mathbf{C u}$ & 3.56738802151922 & 22.71728326283530 & 8.61030316433343 \\
\hline $\mathbf{C u}$ & 7.13228069772706 & & 8.60863789536303 \\
\hline $\mathbf{C u}$ & 7.12 & & 145723115 \\
\hline $\mathbf{C u}$ & 7.13 & & 24338051 \\
\hline $\mathbf{C u}$ & 7.13 & & 64386039 \\
\hline $\mathbf{C u}$ & 7.13 & 200 & 970 \\
\hline $\mathbf{C u}$ & 7.12 & 7010 & 851 \\
\hline $\mathrm{Cu}$ & 7.12 & 2090 & 415 \\
\hline $\mathrm{Cu}$ & 78 & 240 & 443 \\
\hline $\mathrm{Cu}$ & 7.1 & 500 & 9150 \\
\hline $\mathbf{C u}$ & 7.13195710781189 & 8990 & 2119 \\
\hline \multicolumn{4}{|c|}{ CI-NEB Image 17} \\
\hline lement & X-Coordinate (Å) & $\mathrm{Y}-\mathrm{Co}$ & $\mathrm{Z}-\mathrm{C}$ \\
\hline C & 3.56 & 6.294 & 10.26 \\
\hline C & 3.56 & 12.15 & 19580 \\
\hline $\mathbf{F}$ & 3.566 & 10.736 & 3785040 \\
\hline $\mathbf{F}$ & 2.48 & 78869 & 56119370 \\
\hline $\mathbf{F}$ & 7462 & $6.28656 \mathrm{C}$ & 11.08830561925380 \\
\hline $\mathbf{F}$ & 3.567 & 16.41116749654900 & 10.18130724527610 \\
\hline $\mathbf{F}$ & 2.47 & 4409840 & 2015245920 \\
\hline $\mathbf{F}$ & 4.66 & 12.6 & 65600090 \\
\hline $\mathbf{C u}$ & 0.0 & 00 & 9996 \\
\hline $\mathbf{C u}$ & 0.00 & 790 & 7499996 \\
\hline $\mathbf{C u}$ & 000 & 79 & 9996 \\
\hline $\mathbf{C u}$ & 000000000 & 0060 & 27499996 \\
\hline $\mathbf{C u}$ & 0.00000000000000 & 10.09008466 & 127499996 \\
\hline $\mathbf{C u}$ & 0.00000000000000 & 12.61260559199990 & 3.56221127499996 \\
\hline $\mathbf{C u}$ & 0.00000000000000 & 15.13512747220130 & 3.56221127499996 \\
\hline $\mathbf{C u}$ & 0.00000000000000 & 17.65764744789940 & 3.56221127499996 \\
\hline $\mathbf{C u}$ & 0.00000000000000 & 20.18016932810050 & 3.56221127499996 \\
\hline $\mathbf{C u}$ & 0.00000000000000 & 22.70268930379860 & 3.56221127499996 \\
\hline $\mathbf{C u}$ & 3.56738365660942 & 0.00000000000000 & 3.56221127499996 \\
\hline $\mathbf{C u}$ & 3.56738365660942 & 2.52252116632790 & 3.56221127499996 \\
\hline $\mathbf{C u}$ & 3.56 & 579 & 3.56221127499996 \\
\hline $\mathbf{C u}$ & 3.56738365660942 & 6373610060 & 3.56221127499996 \\
\hline $\mathbf{C u}$ & 60942 & 10.0900 & 7499996 \\
\hline $\mathbf{C u}$ & 3.56738365660942 & 12.61260559199990 & 3.56221127499996 \\
\hline $\mathbf{C u}$ & 3.56738365660942 & 15.13512747220130 & 3.56221127499996 \\
\hline $\mathbf{C u}$ & 3.56738365660942 & 17.65764744789940 & 3.56221127499996 \\
\hline $\mathbf{C u}$ & 3.56738365660942 & 20.18016932810050 & 3.56221127499996 \\
\hline
\end{tabular}




\begin{tabular}{|c|c|c|c|}
\hline $\mathbf{C u}$ & 3.56738365660942 & 22.70268930379860 & 3.56221127499996 \\
\hline $\mathbf{C u}$ & 7.13476731321877 & 0.00000000000000 & 3.56221127499996 \\
\hline $\mathbf{C u}$ & 7.13476731321877 & 2.52252116632790 & 3.56221127499996 \\
\hline $\mathbf{C u}$ & 7.13476731321877 & 5.04504233265579 & 3.56221127499996 \\
\hline $\mathrm{Cu}$ & 7.13476731321877 & 7.56756373610060 & 3.56221127499996 \\
\hline $\mathbf{C u}$ & 7.13476731321877 & 10.09008466531150 & 3.56221127499996 \\
\hline $\mathbf{C u}$ & 7.13476731321877 & 12.61260559199990 & 3.56221127499996 \\
\hline $\mathbf{C u}$ & 7.13476731321877 & 15.13512747220130 & 3.56221127499996 \\
\hline $\mathbf{C u}$ & 7.13476731321877 & 17.65764744789940 & 3.56221127499996 \\
\hline $\mathbf{C u}$ & 7.13476731321877 & 20.18016932810050 & 3.56221127499996 \\
\hline $\mathbf{C u}$ & 7.13476731321877 & 22.70268930379860 & 3.56221127499996 \\
\hline $\mathbf{C u}$ & 1.78369182830467 & 1.26126058190266 & 4.82343339999999 \\
\hline $\mathbf{C u}$ & 1.78369182830467 & 3.78378186931158 & 4.82343339999999 \\
\hline $\mathrm{Cu}$ & 1.78369182830467 & 6.30630279600000 & 4.82343339999999 \\
\hline $\mathrm{Cu}$ & 1.78369182830467 & 8.82882372268841 & 4.82343339999999 \\
\hline $\mathrm{Cu}$ & 1.78369182830467 & 11.35134465189930 & 4.82343339999999 \\
\hline $\mathrm{Cu}$ & 182830467 & 13.87386653210060 & 4.82343339999999 \\
\hline $\mathbf{C u}$ & 182830467 & 16.39638650779860 & 4.82343339999999 \\
\hline $\mathbf{C u}$ & 1.78369182830467 & 18.91890838799990 & 4.82343339999999 \\
\hline $\mathbf{C u}$ & 1.78369182830467 & 20130 & 339999999 \\
\hline $\mathrm{Cu}$ & 1.78369182830467 & 23.9639502 & 4.82343339999999 \\
\hline $\mathrm{Cu}$ & 5.351 & 1.261260581 & 4.82343339999999 \\
\hline $\mathrm{Cu}$ & 5.351 & 1158 & 99999 \\
\hline $\mathrm{Cu}$ & 36505000 & 0000 & 39999999 \\
\hline $\mathrm{Cu}$ & 505000 & 8841 & 9999999 \\
\hline $\mathbf{C u}$ & 5.35107536505000 & 11.35134465189930 & 4.82343339999999 \\
\hline $\mathbf{C u}$ & 5.35107536505000 & 13.87386653210060 & 4.82343339999999 \\
\hline $\mathbf{C u}$ & 5.35107536505000 & 16.39638650779860 & 4.82343339999999 \\
\hline $\mathbf{C u}$ & 5.35107536505000 & 18.91890838799990 & 4.82343339999999 \\
\hline $\mathbf{C u}$ & 5.35107536505000 & 21.44143026820130 & 4.82343339999999 \\
\hline $\mathbf{C u}$ & 5.35107536505000 & 23.96395024389930 & 4.82343339999999 \\
\hline $\mathrm{Cu}$ & 8.91845830675569 & 1.26126058190266 & 4.82343339999999 \\
\hline $\mathbf{C u}$ & 8.91845830675569 & 3.78378186931158 & 4.82343339999999 \\
\hline $\mathbf{C u}$ & 8.91845830675569 & 6.30630279600000 & 4.82343339999999 \\
\hline $\mathrm{Cu}$ & 8.91845830675569 & 8.82882372268841 & 4.82343339999999 \\
\hline $\mathrm{Cu}$ & 5830675569 & 65189930 & 4.82343339999999 \\
\hline $\mathbf{C u}$ & 30675569 & 3210060 & 39999999 \\
\hline $\mathrm{Cu}$ & 5830675569 & 16.39638650779860 & 4.82343339999999 \\
\hline $\mathrm{Cu}$ & 8.91845830675569 & 18.91890838799990 & 4.82343339999999 \\
\hline $\mathbf{C u}$ & 8.91845830675569 & 21.44143026820130 & 4.82343339999999 \\
\hline $\mathrm{Cu}$ & 8.91845830675569 & 23.96395024389930 & 4.82343339999999 \\
\hline $\mathrm{Cu}$ & 10.70149591386660 & 0.00275459067410 & 6.09530803028495 \\
\hline $\mathbf{C u}$ & 10.70128345511950 & 2.52192150353755 & 6.09899245766479 \\
\hline $\mathbf{C u}$ & 10.69824943582790 & 5.03546202149694 & 6.08989142596710 \\
\hline $\mathbf{C u}$ & 10.69510449351590 & 7.57873893834631 & 6.09161839022174 \\
\hline $\mathbf{C u}$ & 10.69765364984220 & 10.08812319560880 & 6.09603232320308 \\
\hline $\mathbf{C u}$ & 0.00272779933643 & 12.61964126409530 & 6.10324249959916 \\
\hline $\mathrm{Cu}$ & 10.70075507183410 & 15.13543401296640 & 6.09414375479076 \\
\hline $\mathbf{C u}$ & 10.70117074264780 & 17.65876460812880 & 6.09822441175996 \\
\hline $\mathrm{Cu}$ & 0.00483576793050 & 20.18224831205960 & 6.09649623435063 \\
\hline $\mathbf{C u}$ & 10.70107435889820 & 22.70269019006700 & 6.09349732901685 \\
\hline $\mathrm{Cu}$ & 3.56740434808189 & 0.00745655712281 & 6.09706976591771 \\
\hline $\mathrm{Cu}$ & 3.56741371761966 & 2.53084758087071 & 6.09013086937375 \\
\hline $\mathrm{Cu}$ & 3.56750506838352 & 5.04990765128220 & 6.12519913679691 \\
\hline
\end{tabular}




\begin{tabular}{|c|c|c|c|}
\hline $\mathbf{C u}$ & 3.56757716113852 & 7.56149593661911 & 6.12628644030778 \\
\hline $\mathbf{C u}$ & 3.56749585706554 & 10.09405675459270 & 6.12925415918536 \\
\hline $\mathbf{C u}$ & 3.56739232231325 & 12.60522750697670 & 6.13417686922427 \\
\hline $\mathbf{C u}$ & 3.56740832578338 & 15.13385333143650 & 6.14616058551153 \\
\hline $\mathbf{C u}$ & 3.56740506151935 & 17.65899800580630 & 6.13469366939211 \\
\hline $\mathbf{C u}$ & 3.56738073581702 & 20.17828931645090 & 6.08806826331632 \\
\hline $\mathbf{C u}$ & 3.56740295177885 & 22.70456498204410 & 6.09609371709895 \\
\hline $\mathbf{C u}$ & 7.13590233432626 & 0.00274414157701 & 6.09530356643491 \\
\hline $\mathbf{C u}$ & 7.13598736036441 & 2.52195574805156 & 6.09898475382382 \\
\hline $\mathbf{C u}$ & 7.14060826131491 & 5.03558160525798 & 6.08996960055358 \\
\hline $\mathbf{C u}$ & 7.14542679147486 & 7.57856395712442 & 6.09176893551248 \\
\hline $\mathbf{C u}$ & 7.14163993469284 & 10.08813468491610 & 6.09606751915738 \\
\hline $\mathbf{C u}$ & 7.13193357545223 & 12.61960993531760 & 6.10324126938538 \\
\hline $\mathbf{C u}$ & 7.13704506407969 & 15.13545158116760 & 6.09415884420200 \\
\hline $\mathbf{C u}$ & 7.13636830857451 & 17.65876922419730 & 6.09822380838656 \\
\hline $\mathbf{C u}$ & 7.12984483758914 & 20.18226187988380 & 6.09648836056882 \\
\hline $\mathbf{C u}$ & 7.13647725966722 & 22.70267491103540 & 6.09350570007944 \\
\hline $\mathbf{C u}$ & 1.78685206853322 & 1.26677976872255 & 7.38932152356371 \\
\hline $\mathbf{C u}$ & 1.79555305590317 & 3.79344599976233 & 7.39686029939869 \\
\hline $\mathbf{C u}$ & 1.75163773610301 & 6.308 & 052400893 \\
\hline $\mathrm{Cu}$ & 1.79951934173823 & 759836 & 7.40128 \\
\hline $\mathbf{C u}$ & 1.773360 & 001890 & 7.37867423829185 \\
\hline $\mathrm{Cu}$ & 1.802 & 44410 & 7.38 \\
\hline $\mathbf{C u}$ & 1.7804006 & 898570 & 30057907 \\
\hline $\mathbf{C u}$ & 1.79614134079975 & 760000 & 7.392 \\
\hline $\mathbf{C u}$ & 1.78250911013238 & 21.44988877736450 & 7.38229199840725 \\
\hline $\mathbf{C u}$ & 1.78444883441304 & 23.97020004301240 & 7.38561704458292 \\
\hline $\mathbf{C u}$ & 5.34805147994468 & 1.26681043960653 & 7.38918864094461 \\
\hline $\mathbf{C u}$ & 5.33991685868821 & 3.79386722661941 & 7.39665743262052 \\
\hline $\mathbf{C u}$ & 5.38477622651989 & 6.30867565487316 & 7.34362108005566 \\
\hline $\mathbf{C u}$ & 5.33643259872871 & 8.82099470806962 & 7.40052883304758 \\
\hline $\mathbf{C u}$ & 5.36190626869261 & 11.33993334735190 & 7.37828380654614 \\
\hline $\mathbf{C u}$ & 5.33232562423958 & 13.88139776747170 & 7.38595148071859 \\
\hline $\mathbf{C u}$ & 5.35461543326280 & 16.39941309620810 & 7.37173901742575 \\
\hline $\mathbf{C u}$ & 5.33877295282403 & 18.91912621901360 & 7.39256901899126 \\
\hline $\mathbf{C u}$ & 5.35238644333146 & 21.44997208122190 & 7.38218802714114 \\
\hline $\mathbf{C u}$ & 5.35047049167807 & 23.97018712388770 & 7.38547086879995 \\
\hline $\mathbf{C u}$ & 8.91860336399944 & 1.26795061790119 & 7.39620079604042 \\
\hline $\mathbf{C u}$ & 8.91916380509810 & 3.77981641334469 & 7.39533437330306 \\
\hline $\mathbf{C u}$ & 8.91996596272823 & 6.31183015831811 & 7.38326960541942 \\
\hline $\mathbf{C u}$ & 8.91977739596266 & 8.83414483760254 & 7.39508177020033 \\
\hline $\mathbf{C u}$ & 8.91891349382084 & 11.35241490097870 & 7.39287969109138 \\
\hline $\mathbf{C u}$ & 8.91860586471849 & 13.87739435586040 & 7.40223998821076 \\
\hline $\mathbf{C u}$ & 8.91873874891545 & 16.39788132606660 & 7.39638663351521 \\
\hline $\mathbf{C u}$ & 8.91859443647673 & 18.91974941335570 & 7.39827924698970 \\
\hline $\mathbf{C u}$ & 8.91857069759656 & 21.44233136534380 & 7.39765794685127 \\
\hline $\mathbf{C u}$ & 8.91863607718383 & 23.96355736787380 & 7.39505881981624 \\
\hline $\mathbf{C u}$ & 0.00100993231010 & 0.00501547002000 & 8.61000787953688 \\
\hline $\mathbf{C u}$ & 0.00675456339811 & 2.52645366302596 & 8.61096727473960 \\
\hline $\mathbf{C u}$ & 10.70094074122250 & 5.04979860840420 & 8.59767928860737 \\
\hline $\mathbf{C u}$ & 10.70130989182970 & 7.57338485567371 & 8.60519052088596 \\
\hline $\mathbf{C u}$ & 0.00147671310365 & 10.09153777755070 & 8.61257972827232 \\
\hline $\mathbf{C u}$ & 0.00786893324062 & 12.61375251905110 & 8.61026252078972 \\
\hline $\mathbf{C u}$ & 0.00678501339607 & 15.13230740075260 & 8.60608247773474 \\
\hline
\end{tabular}




$\begin{array}{lllll}\mathbf{C u} & 10.70199848427620 & 17.65951242472700 & 8.61082684676493 \\ \mathbf{C u} & 0.00070439156235 & 20.18095183634760 & 8.60736850213586 \\ \mathbf{C u} & 0.00228130098824 & 22.70747889122760 & 8.60943138004360 \\ \mathbf{C u} & 3.56740196279645 & 0.01123455138118 & 8.61574885065378 \\ \mathbf{C u} & 3.56744055153125 & 2.54011809876846 & 8.61340114169068 \\ \mathbf{C u} & 3.56744042961407 & 5.05274377202725 & 8.70654144510294 \\ \mathbf{C u} & 3.56747597363290 & 7.53709152579059 & 8.70652653060906 \\ \mathbf{C u} & 3.56745241555288 & 10.04810806511340 & 8.65806022474685 \\ \mathbf{C u} & 3.56744680274859 & 12.59353216670870 & 8.68219372851392 \\ \mathbf{C u} & 3.56740022833634 & 15.10900061557310 & 8.71578897833919 \\ \mathbf{C u} & 3.56739515106243 & 17.72004447803500 & 8.72111475306594 \\ \mathbf{C u} & 3.56739991436900 & 20.21045519191380 & 8.59227701557865 \\ \mathbf{C u} & 3.56739788363561 & 22.71086789659790 & 8.60905656455836 \\ \mathbf{C u} & 7.13375642437534 & 0.00502907890190 & 8.60999491045244 \\ \mathbf{C u} & 7.12801802463722 & 2.52649519031833 & 8.61089708155007 \\ \mathbf{C u} & 7.13793129732605 & 5.04982530862237 & 8.59761097770213 \\ \mathbf{C u} & 7.13695134592673 & 7.57336311132920 & 8.60509853348194 \\ \mathbf{C u} & 7.13331671413358 & 10.09151484295550 & 8.61250247591017 \\ \mathbf{C u} & 7.12689763249682 & 12.61373972097630 & 8.61020911147606 \\ \mathbf{C u} & 7.12798432420314 & 15.13229994504460 & 8.60604970182701 \\ \mathbf{C u} & 7.13516512241931 & 17.65951629885320 & 8.61081020382703 \\ \mathbf{C u} & 7.13405271333903 & 20.18094721550640 & 8.60733893282260 \\ \mathbf{C u} & 7.13248391489325 & 22.70747162339710 & 8.60941326304238\end{array}$


Table S4. $\mathrm{CF}_{2}$ Double Knock-On, element type, $\mathrm{x}$-coordinate, y-coordinate, z-coordinate for all 222 atoms in CI-NEB images 1 to 27.

\section{CI-NEB Image 1}

Element X-Coordinate $(\AA)$

F

F

F

F

F

C

C

C

$\mathrm{Cu}$

$\mathrm{Cu}$

$\mathrm{Cu}$

$\mathrm{Cu}$

Cu

$\mathrm{Cu}$

$\mathrm{Cu}$

$\mathrm{Cu}$

Cu

$\mathrm{Cu}$

Cu

Cu

$\mathrm{Cu}$

$\mathrm{Cu}$

$\mathrm{Cu}$

$\mathrm{Cu}$

$\mathrm{Cu}$

$\mathrm{Cu}$

$\mathrm{Cu}$

Cu $\quad 3.56727419238642$

Cu $\quad 3.56727419238642$

Cu $\quad 3.56727419238642$

Cu $\quad 3.56727419238642$

$\mathrm{Cu} \quad 3.56727419238642$

Cu $\quad 3.56727419238642$

$\mathrm{Cu} \quad 3.56727419238642$

Cu $\quad 3.56727419238642$

Cu $\quad 3.56727419238642$

Cu 7.13454838477291

Cu 7.13454838477291

Cu 7.13454838477291

Cu 7.13454838477291

Cu 7.13454838477291

Cu 7.13454838477291

Cu 7.13454838477291

Cu 7.13454838477291

Cu 7.13454838477291
Y-Coordinate (§)

12.61836921059020

12.61837936329530

10.74927781770270

17.65518311559750

17.65515697673720

15.79308971152360

22.77672150746770

22.77665395729910

20.90838591963430

12.17802107997690

17.22611544615250

22.32588856280290

0.00000000000000

2.52244512012115

5.04488953395804

7.56733465407887

10.08977871477400

12.61222383489520

15.13466895501540

17.65711336885320

20.17955848897460

22.70200254966930

25.22444766979040

27.74689208362550

30.26933720374670

32.79178232386990

0.00000000000000

2.52244512012115

5.04488953395804

7.56733465407887

10.08977871477400

12.61222383489520

15.13466895501540

17.65711336885320

20.17955848897460

22.70200254966930

25.22444766979040

27.74689208362550

30.26933720374670

32.79178232386990

0.00000000000000

2.52244512012115

5.04488953395804

7.56733465407887

10.08977871477400

12.61222383489520

15.13466895501540

17.65711336885320

20.17955848897460
Z-Coordinate (Å)

7.75299288463032

7.75299486465544

7.30499559444517

7.75583818821348

7.75586352358093

7.27349911942982

7.75642508555011

7.75640539697827

7.31326729354012

7.05512331975690

7.05103048024868

7.05907144639313

0.00000000000000

0.00000000000000

0.00000000000000

0.00000000000000

0.00000000000000

0.00000000000000

0.00000000000000

0.00000000000000

0.00000000000000

0.00000000000000

0.00000000000000

0.00000000000000

0.00000000000000

0.00000000000000

0.00000000000000

0.00000000000000

0.00000000000000

0.00000000000000

0.00000000000000

0.00000000000000

0.00000000000000

0.00000000000000

0.00000000000000

0.00000000000000

0.00000000000000

0.00000000000000

0.00000000000000

0.00000000000000

0.00000000000000

0.00000000000000

0.00000000000000

0.00000000000000

0.00000000000000

0.00000000000000

0.00000000000000

0.00000000000000

0.00000000000000 


\begin{tabular}{|c|c|c|c|}
\hline $\mathrm{Cu}$ & 7.13454838477291 & 22.70200254966930 & 0.00000000000000 \\
\hline $\mathbf{C u}$ & 7.13454838477291 & 25.22444766979040 & 0.00000000000000 \\
\hline $\mathbf{C u}$ & 7.13454838477291 & 27.74689208362550 & 0.00000000000000 \\
\hline $\mathbf{C u}$ & 7.13454838477291 & 30.26933720374670 & 0.00000000000000 \\
\hline $\mathbf{C u}$ & 7.13454838477291 & 32.79178232386990 & 0.00000000000000 \\
\hline $\mathbf{C u}$ & 1.78363955761358 & 1.26122414920059 & 1.26122399999993 \\
\hline $\mathbf{C u}$ & 1.78363955761358 & 3.78366926932196 & 1.26122399999993 \\
\hline $\mathbf{C u}$ & 1.78363955761358 & 6.30611333001662 & 1.26122399999993 \\
\hline $\mathbf{C u}$ & 1.78363955761358 & 8.82855845013778 & 1.26122399999993 \\
\hline $\mathbf{C u}$ & 1.78363955761358 & 11.35100286397460 & 1.26122399999993 \\
\hline $\mathbf{C u}$ & 1.78363955761358 & 13.87344798409460 & 1.26122399999993 \\
\hline $\mathbf{C u}$ & 1.78363955761358 & 16.39589310421720 & 1.26122399999993 \\
\hline $\mathbf{C u}$ & 1.78363955761358 & 18.91833716491180 & 1.26122399999993 \\
\hline $\mathrm{Cu}$ & 1.78363955761358 & 21.44078263817520 & 1.26122399999993 \\
\hline $\mathbf{C u}$ & 1.78363955761358 & 23.96322669886980 & 1.26122399999993 \\
\hline $\mathbf{C u}$ & 1.78363955761358 & 26.48567181899090 & 1.26122399999993 \\
\hline $\mathbf{C u}$ & 1.78363955761358 & 29.00811693911060 & 1.26122399999993 \\
\hline $\mathbf{C u}$ & 1.78363955761358 & 31.53056135294700 & 1.26122399999993 \\
\hline $\mathbf{C u}$ & 1.78363955761358 & 34.05300647307050 & 1.26122399999993 \\
\hline $\mathbf{C u}$ & 5.35091375000000 & 1.26122414920059 & 1.26122399999993 \\
\hline $\mathbf{C u}$ & 5.35091375000000 & 3.78366926932196 & 1.26122399999993 \\
\hline $\mathbf{C u}$ & 5.35091375000000 & 6.30611333001662 & 1.26122399999993 \\
\hline $\mathbf{C u}$ & 5.35091375000000 & 013778 & 22399999993 \\
\hline $\mathrm{Cu}$ & 5.35091375000000 & 286397460 & 22399999993 \\
\hline $\mathrm{Cu}$ & 5.3509 & 409460 & 2399999993 \\
\hline $\mathrm{Cu}$ & 5000000 & 21720 & 1.26122399999993 \\
\hline $\mathbf{C u}$ & 5.35091375000000 & 18.91833716491180 & 1.26122399999993 \\
\hline $\mathbf{C u}$ & 5.35091375000000 & 21.44078263817520 & 1.26122399999993 \\
\hline $\mathbf{C u}$ & 5.35091375000000 & 23.96322669886980 & 1.26122399999993 \\
\hline $\mathrm{Cu}$ & 5.35091375000000 & 26.48567181899090 & 1.26122399999993 \\
\hline $\mathbf{C u}$ & 5.35091375000000 & 29.00811693911060 & 1.26122399999993 \\
\hline $\mathrm{Cu}$ & 5.35091375000000 & 31.53056135294700 & 1.26122399999993 \\
\hline $\mathrm{Cu}$ & 5.35091375000000 & 34.05300647307050 & 1.26122399999993 \\
\hline $\mathbf{C u}$ & 8.91818794238642 & 1.26122414920059 & 1.26122399999993 \\
\hline $\mathbf{C u}$ & 8.91818794238642 & 3.78366926932196 & 1.26122399999993 \\
\hline $\mathbf{C u}$ & 8.91818794238642 & 6.30611333001662 & 1.26122399999993 \\
\hline $\mathbf{C u}$ & 8.91818794238642 & 8.82855845013778 & 1.26122399999993 \\
\hline $\mathbf{C u}$ & 8.91818794238642 & 11.35100286397460 & 1.26122399999993 \\
\hline $\mathbf{C u}$ & 8.91818794238642 & 13.87344798409460 & 1.26122399999993 \\
\hline $\mathbf{C u}$ & 8.91818794238642 & 16.39589310421720 & 1.26122399999993 \\
\hline $\mathbf{C u}$ & 8.91818794238642 & 18.91833716491180 & 1.26122399999993 \\
\hline $\mathbf{C u}$ & 8.91818794238642 & 21.44078263817520 & 1.26122399999993 \\
\hline $\mathbf{C u}$ & 8.91818794238642 & 23.96322669886980 & 1.26122399999993 \\
\hline $\mathbf{C u}$ & 8.91818794238642 & 26.48567181899090 & 1.26122399999993 \\
\hline $\mathbf{C u}$ & 8.91818794238642 & 29.00811693911060 & 1.26122399999993 \\
\hline $\mathbf{C u}$ & 8.91818794238642 & 31.53056135294700 & 1.26122399999993 \\
\hline $\mathbf{C u}$ & 8.91818794238642 & 34.05300647307050 & 1.26122399999993 \\
\hline $\mathbf{C u}$ & 0.00151991824166 & 0.00032027696697 & 2.53847073723977 \\
\hline $\mathbf{C u}$ & 10.70070165887970 & 2.52324511658211 & 2.54121105991417 \\
\hline $\mathbf{C u}$ & 10.70180546935590 & 5.04570529690417 & 2.54065683172154 \\
\hline $\mathbf{C u}$ & 10.70145707276700 & 7.56774031850397 & 2.54418877489142 \\
\hline $\mathbf{C u}$ & 10.69957105246630 & 10.08933015289570 & 2.53934428813320 \\
\hline $\mathbf{C u}$ & 10.69972971720530 & 12.61653244009690 & 2.54900838654345 \\
\hline $\mathbf{C u}$ & 10.69730973942950 & 15.13699590324340 & 2.53841124598915 \\
\hline
\end{tabular}




\begin{tabular}{|c|c|c|c|}
\hline $\mathbf{C u}$ & 10.69825381099910 & 17.65938667324150 & 2.54724663725419 \\
\hline $\mathbf{C u}$ & 10.69834343251370 & 20.17927343405540 & 2.53845019280132 \\
\hline $\mathbf{C u}$ & 10.69708269179280 & 22.70268212452820 & 2.54668869473098 \\
\hline $\mathbf{C u}$ & 10.69997863453130 & 25.22791773239980 & 2.53862098792453 \\
\hline $\mathbf{C u}$ & 10.70020331236850 & 27.74921548564240 & 2.54331928157358 \\
\hline $\mathbf{C u}$ & 0.00085816918551 & 30.26988127616670 & 2.54112053461771 \\
\hline $\mathbf{C u}$ & 10.70084161928590 & 32.79279735552080 & 2.54145782850086 \\
\hline $\mathbf{C u}$ & 3.56729354480622 & 0.00172753144960 & 2.54116840945342 \\
\hline $\mathbf{C u}$ & 3.56732029062812 & 2.52379516205691 & 2.54212517199734 \\
\hline $\mathbf{C u}$ & 3.56725052585768 & 5.04625183264936 & 2.54456057203359 \\
\hline $\mathbf{C u}$ & 3.56727110045613 & 7.56730536184162 & 2.53966532278900 \\
\hline $\mathbf{C u}$ & 3.56728540208279 & 10.09401788245790 & 2.57536423222779 \\
\hline $\mathbf{C u}$ & 3.56729191098249 & 12.61756750079530 & 2.57767677879708 \\
\hline $\mathbf{C u}$ & 3.56729333417779 & 15.14493051708160 & 2.58192223903203 \\
\hline $\mathbf{C u}$ & 3.56732188513716 & 17.65932343178760 & 2.57845488553257 \\
\hline $\mathbf{C u}$ & 3.56729993475549 & 20.18153312682110 & 2.58354863545302 \\
\hline $\mathbf{C u}$ & 3.56727243608311 & 22.70003570839780 & 2.58023582295132 \\
\hline $\mathbf{C u}$ & 3.56727226069411 & 25.22193941209360 & 2.55284513679520 \\
\hline $\mathbf{C u}$ & 3.56726629830354 & 27.74809235448420 & 2.54163817280904 \\
\hline $\mathbf{C u}$ & 3.56726299758029 & 30.27042775520920 & 2.5433010 \\
\hline $\mathbf{C u}$ & 607034710 & 32.79110771045510 & 2.541187856 \\
\hline $\mathbf{C u}$ & 872187 & 0.00026 & 2.53848473884290 \\
\hline $\mathbf{C u}$ & 570 & 443 & 2.54117412697373 \\
\hline $\mathbf{C u}$ & 7.13461116946708 & 5.04565254031839 & 2.54072060091675 \\
\hline $\mathbf{C u}$ & 7.13490062028923 & 7.56773152044853 & 2.54412748908005 \\
\hline $\mathbf{C u}$ & 7.13683018186651 & 10.08932873147440 & 2.53928286351066 \\
\hline $\mathbf{C u}$ & 7.13660787967267 & 12.61652938863030 & 2.54900206284239 \\
\hline $\mathbf{C u}$ & 7.13906669481251 & 15.13698943333020 & 2.53843024084960 \\
\hline $\mathbf{C u}$ & 501205 & 17.65940007882040 & 2.54724085599232 \\
\hline $\mathbf{C u}$ & 7.13796220450911 & 20.17938150011690 & 2.53842521746027 \\
\hline $\mathbf{C u}$ & 52418892 & 22.70269437631550 & 2.54666411299290 \\
\hline $\mathbf{C u}$ & 7.13638722143378 & 25.22785284678100 & 2.53864602389771 \\
\hline $\mathbf{C u}$ & 7.13611012630570 & 27.74930593213990 & 2.54342263381602 \\
\hline $\mathbf{C u}$ & 7.13370620038474 & 30.26977908925300 & 2.54101706844636 \\
\hline $\mathbf{C u}$ & 7.13560573369242 & 32.79278152674170 & 2.54148083425885 \\
\hline $\mathbf{C u}$ & 1.78484711901105 & 1.26366339241930 & 3.85368597477389 \\
\hline $\mathbf{C u}$ & 1.78187912890182 & 3.78527155229993 & 3.85526867723735 \\
\hline $\mathbf{C u}$ & 1.77940746068826 & 6.30382960058219 & 3.85705675710054 \\
\hline $\mathbf{C u}$ & 1.78777299344669 & 8.82967090562964 & 3.85486746084098 \\
\hline $\mathbf{C u}$ & 1.77905603325042 & 11.35164365683940 & 3.84086271602254 \\
\hline $\mathbf{C u}$ & 1.78913030585630 & 13.88093283743320 & 3.84673869825516 \\
\hline $\mathbf{C u}$ & 1.77570522535649 & 16.40069649673960 & 3.84208784398597 \\
\hline $\mathbf{C u}$ & 1.78793241825803 & 18.92626529560820 & 3.84986571400104 \\
\hline $\mathbf{C u}$ & 1.77523827447108 & 21.44361665324370 & 3.84144223968919 \\
\hline $\mathbf{C u}$ & 1.78457380786373 & 23.97017233598020 & 3.84360762970442 \\
\hline $\mathbf{C u}$ & 1.78140588122562 & 26.49271968834770 & 3.85595212373062 \\
\hline $\mathbf{C u}$ & 1.78232370913053 & 29.01207264707460 & 3.85699340459340 \\
\hline $\mathbf{C u}$ & 1.78197364948993 & 31.53301528743700 & 3.85623537169758 \\
\hline $\mathbf{C u}$ & 1.78466745618015 & 34.05438021217620 & 3.85303608808205 \\
\hline $\mathbf{C u}$ & 5.34972433809009 & 1.26378919465950 & 3.85374890548963 \\
\hline $\mathbf{C u}$ & 5.35270588087529 & 3.78528117863355 & 3.85531845474092 \\
\hline $\mathbf{C u}$ & 5.35517919691645 & 6.30381082021128 & 3.85704788872313 \\
\hline $\mathbf{C u}$ & 5.34678580999216 & 8.82961896692425 & 3.85476009819527 \\
\hline $\mathbf{C u}$ & 5.35549142475191 & 11.35167488753460 & 3.84079192822886 \\
\hline
\end{tabular}




\begin{tabular}{|c|c|c|c|}
\hline $\mathrm{Cu}$ & 5.34547025357341 & 13.88091456717270 & 3.84668543269796 \\
\hline $\mathbf{C u}$ & 5.35886056346169 & 16.40070619217860 & 3.84198492664784 \\
\hline $\mathrm{Cu}$ & 5.34659339173966 & 18.92630552819790 & 3.84991280360998 \\
\hline $\mathbf{C u}$ & 5.35929033507941 & 21.44359417278390 & 3.84162616136243 \\
\hline $\mathbf{C u}$ & 5.34995006604138 & 23.97013220582520 & 3.84364265297193 \\
\hline $\mathbf{C u}$ & 5.35314830312124 & 26.49273827341630 & 3.85592743124075 \\
\hline $\mathrm{Cu}$ & 5.35214552241147 & 29.01203102724910 & 3.85704398857618 \\
\hline $\mathrm{Cu}$ & 5.35256615671577 & 31.53293795515920 & 3.85627109960151 \\
\hline $\mathbf{C u}$ & 5.34986683780583 & 34.05435899740020 & 3.85303956058634 \\
\hline $\mathbf{C u}$ & 8.91820563505813 & 1.26213468870814 & 3.85006565776046 \\
\hline $\mathbf{C u}$ & 8.91821888308601 & 3.78587189224455 & 3.85223029816187 \\
\hline $\mathrm{Cu}$ & 8.91823353182946 & 6.30697415967895 & 3.84956637378000 \\
\hline $\mathrm{Cu}$ & 8.91821301405838 & 8.82787860207567 & 3.85445665974861 \\
\hline $\mathrm{Cu}$ & 8.91814030868024 & 11.35440766079660 & 3.85217241283215 \\
\hline $\mathrm{Cu}$ & 8.91819111498140 & 13.87628811836880 & 3.85892622088055 \\
\hline $\mathrm{Cu}$ & 8.91817974222060 & 16.40091178543840 & 3.85650740868507 \\
\hline $\mathrm{Cu}$ & 8.91813932746144 & 18.91944215790540 & 3.85746698340423 \\
\hline $\mathbf{C u}$ & 8.91818844116206 & 21.44339011303180 & 3.85545676531809 \\
\hline $\mathbf{C u}$ & 8.91817084569752 & 23.96654956198560 & 3.85191303824478 \\
\hline $\mathrm{Cu}$ & 8.91818819208561 & 26.49184032468780 & 3.85247451367407 \\
\hline $\mathrm{Cu}$ & 8.91818065851280 & 29.01048240479090 & 48535 \\
\hline $\mathbf{C u}$ & 8.91818758593923 & 31.53499536731210 & 811484 \\
\hline $\mathrm{Cu}$ & 8.91817722775895 & 34.05605539994850 & 3.849898 \\
\hline $\mathrm{Cu}$ & 0.00251168671215 & 0.00237754698872 & 5.05560646971683 \\
\hline $\mathrm{Cu}$ & 0.00220854257098 & 2.52539769012148 & 5.05804438864671 \\
\hline $\mathrm{Cu}$ & 10.69769244295690 & 5.04777317130534 & 5.05972721851747 \\
\hline $\mathbf{C u}$ & 0.00216341772719 & 7.56928485328755 & 5.06119720876123 \\
\hline $\mathrm{Cu}$ & 0.00422526142944 & 10.09202273413250 & 5.04997508903443 \\
\hline $\mathbf{C u}$ & 0.01039518882953 & 12.61419350116180 & 5.05966367798179 \\
\hline $\mathrm{Cu}$ & 0.00714064728931 & 15.13710951202690 & 5.05316982565525 \\
\hline $\mathrm{Cu}$ & 0.00376262683395 & 17.66336990652880 & 5.06322213029448 \\
\hline $\mathrm{Cu}$ & 0.00552822952083 & 20.18243513078170 & 5.05447589104240 \\
\hline $\mathrm{Cu}$ & 0.00622487276119 & 22.70902897750230 & 5.05946964052859 \\
\hline $\mathbf{C u}$ & 0.00269631496933 & 25.22678104061010 & 5.05280740821290 \\
\hline $\mathbf{C u}$ & 10.70129638598540 & 27.75245514922630 & 5.06426274955099 \\
\hline $\mathbf{C u}$ & 10.69823217668210 & 30.27096508149870 & 5.05786218048478 \\
\hline $\mathrm{Cu}$ & 0.00325857132754 & 32.79546359337090 & 5.05858287841501 \\
\hline $\mathbf{C u}$ & 3.56730759293946 & 0.00169782993817 & 5.06248091067831 \\
\hline $\mathrm{Cu}$ & 3.56725506295556 & 2.52574039074842 & 5.05904011820465 \\
\hline $\mathbf{C u}$ & 3.56730864982142 & 5.04279476334865 & 5.06139863468628 \\
\hline $\mathrm{Cu}$ & 3.56727746836322 & 7.55444130840760 & 5.04506004546209 \\
\hline $\mathbf{C u}$ & 3.56729928839810 & 10.06987697799810 & 5.09955533526256 \\
\hline $\mathbf{C u}$ & 3.56728588909962 & 12.60950973433510 & 5.12973966451659 \\
\hline $\mathbf{C u}$ & 3.56729147933806 & 15.13438943661750 & 5.09668397396073 \\
\hline $\mathbf{C u}$ & 3.56736062063472 & 17.67154841866620 & 5.12913373150330 \\
\hline $\mathrm{Cu}$ & 3.56726931314396 & 20.19186849374150 & 5.10066410547411 \\
\hline $\mathrm{Cu}$ & 3.56718457822833 & 22.72850249865530 & 5.12854787705878 \\
\hline $\mathrm{Cu}$ & 3.56726106876280 & 25.24854981069200 & 5.05989724504616 \\
\hline $\mathbf{C u}$ & 3.56718286613491 & 27.75826704908160 & 5.05883312209948 \\
\hline $\mathrm{Cu}$ & 3.56734550270639 & 30.27618678923570 & 5.06029459210942 \\
\hline $\mathbf{C u}$ & 3.56727275127564 & 32.79617844996270 & 5.05825425063885 \\
\hline $\mathbf{C u}$ & 7.13207286549087 & 0.00249758408807 & 5.05555076808093 \\
\hline $\mathbf{C u}$ & 7.13236181850998 & 2.52538208187041 & 5.05800656308691 \\
\hline $\mathbf{C u}$ & 7.13862168168244 & 5.04780250268574 & 5.05974679564058 \\
\hline
\end{tabular}




\begin{tabular}{|c|c|c|c|}
\hline $\mathbf{C u}$ & 7.13243474980457 & 7.56931693332420 & 5.06116269254100 \\
\hline $\mathrm{Cu}$ & 7.13031702519729 & 10.09204176766500 & 5.04996352332175 \\
\hline $\mathrm{Cu}$ & 7.12403351933761 & 12.61426691323650 & 5.05962613639194 \\
\hline $\mathrm{Cu}$ & 7.12741346492330 & 15.13711654670130 & 5.05320614679345 \\
\hline $\mathbf{C u}$ & 7.13074195105108 & 17.66335093410010 & 5.06318298223275 \\
\hline $\mathbf{C u}$ & 7.12909037546599 & 20.18243468691800 & 5.05457858507790 \\
\hline $\mathbf{C u}$ & 7.12835720053355 & 22.70902566959710 & 5.05945295430151 \\
\hline $\mathbf{C u}$ & 7.13184118592357 & 25.22675304939150 & 5.05279461285689 \\
\hline $\mathrm{Cu}$ & 7.13507813537986 & 27.75246349082160 & 5.06428244475511 \\
\hline $\mathrm{Cu}$ & 7.13810004508859 & 30.27094317644120 & 5.05785242223753 \\
\hline $\mathrm{Cu}$ & 7.13131003216376 & 32.79547554705280 & 5.05857098490519 \\
\hline \multicolumn{4}{|c|}{ CI-NEB Image 2} \\
\hline Element & X-Coordinate $(\AA)$ & Y-Coordinate $(\AA)$ & Z-Coordinate (§̊) \\
\hline $\mathbf{F}$ & 4.66232073626575 & 12.36294656892700 & 7.79407249513580 \\
\hline $\mathbf{F}$ & 2.47220221306465 & 12.36294934403530 & 7.79404069682092 \\
\hline $\mathbf{F}$ & 3.56727073555239 & 10.52786205802130 & 7.21625596505343 \\
\hline $\mathbf{F}$ & 4.66305409311777 & 17.64821653322860 & 7.75736061826553 \\
\hline $\mathbf{F}$ & 2.47147773777677 & 17.64822159569200 & 7.75733911330849 \\
\hline $\mathbf{F}$ & 3.56726741525010 & 15.78314743037950 & 7.28511234397130 \\
\hline $\mathbf{F}$ & 4.66370264362885 & 22.75894400226290 & 7.75380839603056 \\
\hline $\mathbf{F}$ & 2.47083079776547 & 22.75894678356300 & 7.75378483741204 \\
\hline $\mathbf{F}$ & 3.56726846599969 & 20.89167934863420 & 7.30557084951549 \\
\hline C & 3.56727188027595 & 11.98798955056180 & 7.06191800087694 \\
\hline $\mathbf{C}$ & 3.56727201174230 & 17.21551125010070 & 7.05451246404666 \\
\hline $\mathbf{C}$ & 3.56727363545395 & 22.30967977024670 & 7.05552621796349 \\
\hline $\mathrm{Cu}$ & 0.00000000000000 & 0.00000000000000 & 0.00000000000000 \\
\hline $\mathrm{Cu}$ & 0.00000000000000 & 2.52244512012115 & 0.00000000000000 \\
\hline $\mathrm{Cu}$ & 0.00000000000000 & 5.04488953395804 & 0.00000000000000 \\
\hline $\mathrm{Cu}$ & 0.00000000000000 & 7.56733465407884 & 0.00000000000000 \\
\hline $\mathrm{Cu}$ & 0.00000000000000 & 10.08977871477400 & 0.00000000000000 \\
\hline $\mathrm{Cu}$ & 0.00000000000000 & 12.61222383489520 & 0.00000000000000 \\
\hline $\mathrm{Cu}$ & 0.00000000000000 & 15.13466895501510 & 0.00000000000000 \\
\hline $\mathrm{Cu}$ & 0.00000000000000 & 17.65711336885320 & 0.00000000000000 \\
\hline $\mathrm{Cu}$ & 0.00000000000000 & 20.17955848897460 & 0.00000000000000 \\
\hline $\mathrm{Cu}$ & 0.00000000000000 & 22.70200254966930 & 0.00000000000000 \\
\hline $\mathbf{C u}$ & 0.00000000000000 & 25.22444766979040 & 0.00000000000000 \\
\hline $\mathbf{C u}$ & 0.00000000000000 & 27.74689208362510 & 0.00000000000000 \\
\hline $\mathbf{C u}$ & 0.00000000000000 & 30.26933720374630 & 0.00000000000000 \\
\hline $\mathrm{Cu}$ & 0.00000000000000 & 32.79178232386990 & 0.00000000000000 \\
\hline $\mathbf{C u}$ & 3.56727419238642 & 0.00000000000000 & 0.00000000000000 \\
\hline $\mathbf{C u}$ & 3.56727419238642 & 2.52244512012115 & 0.00000000000000 \\
\hline $\mathrm{Cu}$ & 3.56727419238642 & 5.04488953395804 & 0.00000000000000 \\
\hline $\mathrm{Cu}$ & 3.56727419238642 & 7.56733465407884 & 0.00000000000000 \\
\hline $\mathrm{Cu}$ & 3.56727419238642 & 10.08977871477400 & 0.00000000000000 \\
\hline $\mathbf{C u}$ & 3.56727419238642 & 12.61222383489520 & 0.00000000000000 \\
\hline $\mathrm{Cu}$ & 3.56727419238642 & 15.13466895501510 & 0.00000000000000 \\
\hline $\mathbf{C u}$ & 3.56727419238642 & 17.65711336885320 & 0.00000000000000 \\
\hline $\mathrm{Cu}$ & 3.56727419238642 & 20.17955848897460 & 0.00000000000000 \\
\hline $\mathrm{Cu}$ & 3.56727419238642 & 22.70200254966930 & 0.00000000000000 \\
\hline $\mathrm{Cu}$ & 3.56727419238642 & 25.22444766979040 & 0.00000000000000 \\
\hline $\mathbf{C u}$ & 3.56727419238642 & 27.74689208362510 & 0.00000000000000 \\
\hline $\mathrm{Cu}$ & 3.56727419238642 & 30.26933720374630 & 0.00000000000000 \\
\hline $\mathrm{Cu}$ & 3.56727419238642 & 32.79178232386990 & 0.00000000000000 \\
\hline $\mathrm{Cu}$ & 7.13454838477291 & 0.00000000000000 & 0.00000000000000 \\
\hline
\end{tabular}




\begin{tabular}{|c|c|c|c|}
\hline $\mathrm{Cu}$ & 7.13454838477291 & 2.52244512012115 & 0.00000000000000 \\
\hline $\mathbf{C u}$ & 7.13454838477291 & 5.04488953395804 & 0.00000000000000 \\
\hline $\mathbf{C u}$ & 7.13454838477291 & 7.56733465407884 & 0.00000000000000 \\
\hline $\mathbf{C u}$ & 7.13454838477291 & 10.08977871477400 & 0.00000000000000 \\
\hline $\mathbf{C u}$ & 7.13454838477291 & 12.61222383489520 & 0.00000000000000 \\
\hline $\mathbf{C u}$ & 7.13454838477291 & 15.13466895501510 & 0.00000000000000 \\
\hline $\mathbf{C u}$ & 7.13454838477291 & 17.65711336885320 & 0.00000000000000 \\
\hline $\mathbf{C u}$ & 7.13454838477291 & 20.17955848897460 & 0.00000000000000 \\
\hline $\mathbf{C u}$ & 7.13454838477291 & 22.70200254966930 & 0.00000000000000 \\
\hline $\mathbf{C u}$ & 7.13454838477291 & 25.22444766979040 & 0.00000000000000 \\
\hline $\mathbf{C u}$ & 7.13454838477291 & 27.74689208362510 & 0.00000000000000 \\
\hline $\mathrm{Cu}$ & 7.13454838477291 & 30.26933720374630 & 0.00000000000000 \\
\hline $\mathbf{C u}$ & 7.13454838477291 & 32.79178232386990 & 0.00000000000000 \\
\hline $\mathrm{Cu}$ & 1.78363955761358 & 1.26122414920059 & 1.26122399999993 \\
\hline $\mathbf{C u}$ & 1.78363955761358 & 3.78366926932194 & 1.26122399999993 \\
\hline $\mathbf{C u}$ & 1.78363955761358 & 6.30611333001662 & 1.26122399999993 \\
\hline $\mathbf{C u}$ & 1.78363955761358 & 8.82855845013778 & 1.26122399999993 \\
\hline $\mathbf{C u}$ & 1.78363955761358 & 11.35100286397460 & 1.26122399999993 \\
\hline $\mathbf{C u}$ & 1.78363955761358 & 13.87344798409430 & 1.26122399999993 \\
\hline $\mathbf{C u}$ & 1.78363955761358 & 16.39589310421720 & 1.26122399999993 \\
\hline $\mathbf{C u}$ & 1.78363955761358 & 18.91833716491180 & 1.26122399999993 \\
\hline $\mathbf{C u}$ & 1.78363955761358 & 21.44078263817520 & 1.26122399999993 \\
\hline $\mathbf{C u}$ & 1.7836395 & 23.96322669886980 & 1.26122399999993 \\
\hline $\mathrm{Cu}$ & 1.783639 & 26.48567181899090 & 1.26122399999993 \\
\hline $\mathrm{Cu}$ & 1.783 & 11020 & 1.26122399999993 \\
\hline $\mathrm{Cu}$ & 1.78363955761358 & 31.53056135294660 & 1.26122399999993 \\
\hline $\mathbf{C u}$ & 1.78363955761358 & 34.05300647307050 & 1.26122399999993 \\
\hline $\mathbf{C u}$ & 5.35091375000000 & 1.26122414920059 & 1.26122399999993 \\
\hline $\mathbf{C u}$ & 5.35091375000000 & 3.78366926932194 & 1.26122399999993 \\
\hline $\mathbf{C u}$ & 5.35091375000000 & 6.30611333001662 & 1.26122399999993 \\
\hline $\mathrm{Cu}$ & 5.35091375000000 & 8.82855845013778 & 1.26122399999993 \\
\hline $\mathrm{Cu}$ & 5.35091375000000 & 11.35100286397460 & 1.26122399999993 \\
\hline $\mathrm{Cu}$ & 5.35091375000000 & 13.87344798409430 & 1.26122399999993 \\
\hline $\mathbf{C u}$ & 5.35091375000000 & 16.39589310421720 & 1.26122399999993 \\
\hline $\mathbf{C u}$ & 5.35091375000000 & 18.91833716491180 & 1.26122399999993 \\
\hline $\mathbf{C u}$ & 5.35091375000000 & 21.44078263817520 & 1.26122399999993 \\
\hline $\mathbf{C u}$ & 5.35091375000000 & 23.96322669886980 & 1.26122399999993 \\
\hline $\mathbf{C u}$ & 5.35091375000000 & 26.48567181899090 & 1.26122399999993 \\
\hline $\mathbf{C u}$ & 5.35091375000000 & 29.00811693911020 & 1.26122399999993 \\
\hline $\mathbf{C u}$ & 5.35091375000000 & 31.53056135294660 & 1.26122399999993 \\
\hline $\mathbf{C u}$ & 5.35091375000000 & 34.05300647307050 & 1.26122399999993 \\
\hline $\mathbf{C u}$ & 8.91818794238642 & 1.26122414920059 & 1.26122399999993 \\
\hline $\mathbf{C u}$ & 8.91818794238642 & 3.78366926932194 & 1.26122399999993 \\
\hline $\mathbf{C u}$ & 8.91818794238642 & 6.30611333001662 & 1.26122399999993 \\
\hline $\mathbf{C u}$ & 8.91818794238642 & 8.82855845013778 & 1.26122399999993 \\
\hline $\mathbf{C u}$ & 8.91818794238642 & 11.35100286397460 & 1.26122399999993 \\
\hline $\mathbf{C u}$ & 8.91818794238642 & 13.87344798409430 & 1.26122399999993 \\
\hline $\mathbf{C u}$ & 8.91818794238642 & 16.39589310421720 & 1.26122399999993 \\
\hline $\mathbf{C u}$ & 8.91818794238642 & 18.91833716491180 & 1.26122399999993 \\
\hline $\mathbf{C u}$ & 8.91818794238642 & 21.44078263817520 & 1.26122399999993 \\
\hline $\mathbf{C u}$ & 8.91818794238642 & 23.96322669886980 & 1.26122399999993 \\
\hline $\mathbf{C u}$ & 8.91818794238642 & 26.48567181899090 & 1.26122399999993 \\
\hline $\mathbf{C u}$ & 8.91818794238642 & 29.00811693911020 & 1.26122399999993 \\
\hline $\mathbf{C u}$ & 8.91818794238642 & 31.53056135294660 & 1.26122399999993 \\
\hline
\end{tabular}




\begin{tabular}{|c|c|c|c|}
\hline $\mathbf{C u}$ & 8.91818794238642 & 34.05300647307050 & 1.26122399999993 \\
\hline $\mathbf{C u}$ & 0.00118329499970 & -0.00148071599509 & 2.53797575461536 \\
\hline $\mathbf{C u}$ & 10.70081158758220 & 2.52155296693684 & 2.54142013752473 \\
\hline $\mathbf{C u}$ & -0.00095660212463 & 5.04310684969316 & 2.54119752167073 \\
\hline $\mathbf{C u}$ & -0.00221991615071 & 7.56485461372668 & 2.54217572717417 \\
\hline $\mathbf{C u}$ & 10.69838010955130 & 10.08826732781860 & 2.53847981169245 \\
\hline $\mathbf{C u}$ & 10.70078176180910 & 12.61536130510440 & 2.54885886396641 \\
\hline $\mathbf{C u}$ & 10.70035522886550 & 15.13358990567330 & 2.53981868357463 \\
\hline $\mathbf{C u}$ & 10.69868448334700 & 17.65601367927350 & 2.54740370693885 \\
\hline $\mathbf{C u}$ & 10.69773191916120 & 20.17709891982780 & 2.53838282735317 \\
\hline $\mathbf{C u}$ & 10.69735509054750 & 22.70116550508770 & 2.54682674364570 \\
\hline $\mathbf{C u}$ & 10.70010356765990 & 25.22642813837220 & 2.53860692844292 \\
\hline $\mathbf{C u}$ & 10.70032072276190 & 27.74841724393890 & 2.54341816133950 \\
\hline $\mathrm{Cu}$ & 0.00067602371731 & 30.26856121869920 & 2.54075969421261 \\
\hline $\mathbf{C u}$ & 10.70091355376700 & 32.79133033071090 & 2.54079887829035 \\
\hline $\mathbf{C u}$ & 3.56726020388026 & -0.00000446795441 & 2.54106015059453 \\
\hline $\mathbf{C u}$ & 3.56728444191898 & 2.52142762799553 & 2.54208560376109 \\
\hline $\mathbf{C u}$ & 3.56727828186056 & 5.04315070666125 & 2.54513832509151 \\
\hline $\mathbf{C u}$ & 3.56728208365740 & 7.56426069178873 & 2.53961318572030 \\
\hline $\mathbf{C u}$ & 3.56726878895051 & 10.08989268034220 & 2.57045264779324 \\
\hline $\mathbf{C u}$ & 3.56726499726690 & 12.61458504991070 & 2.58538750723379 \\
\hline $\mathbf{C u}$ & 3.56727131045485 & 15.14153887616450 & 2.58459385627409 \\
\hline $\mathbf{C u}$ & 3.56728325961319 & 598187020 & 838148298 \\
\hline $\mathrm{Cu}$ & 3.56727863951134 & 2444426090 & 34441 \\
\hline $\mathrm{Cu}$ & 3.56728169864752 & 22.69824009388380 & 88603 \\
\hline $\mathrm{Cu}$ & 3.56727249393309 & 25.22054540905340 & 2.55297512820594 \\
\hline $\mathbf{C u}$ & 3.56727202058507 & 27.74656483986020 & 2.54175704064880 \\
\hline $\mathbf{C u}$ & 3.56727583012215 & 30.26977640262740 & 2.54265689822659 \\
\hline $\mathrm{Cu}$ & 3.56726139183409 & 32.78967531042400 & 2.54124310787263 \\
\hline $\mathrm{Cu}$ & 7.13337884956448 & -0.00148537045944 & 2.53797500783965 \\
\hline $\mathrm{Cu}$ & 7.13557491885954 & 2.52154646449792 & 2.54143617596697 \\
\hline $\mathrm{Cu}$ & 7.13550689761290 & 5.04309896259770 & 2.54121214359013 \\
\hline $\mathrm{Cu}$ & 7.13675691014570 & 7.56484406027172 & 2.54219581848483 \\
\hline $\mathbf{C u}$ & 7.13805651440140 & 10.08827167623720 & 2.53848756838124 \\
\hline $\mathbf{C u}$ & 7.13559492634452 & 12.61537491684010 & 2.54884748890271 \\
\hline $\mathbf{C u}$ & 7.13602427457080 & 15.13360112328010 & 2.53978689872822 \\
\hline $\mathbf{C u}$ & 7.13768355723089 & 17.65600840190000 & 2.54740670177776 \\
\hline $\mathbf{C u}$ & 7.13865426034821 & 20.17710878927770 & 2.53839824962810 \\
\hline $\mathbf{C u}$ & 7.13904126034916 & 22.70117340832020 & 2.54683184540735 \\
\hline $\mathbf{C u}$ & 7.13626233228524 & 25.22643795266730 & 2.53861649946609 \\
\hline $\mathbf{C u}$ & 7.13606463512399 & 27.74842234640560 & 2.54345915744143 \\
\hline $\mathbf{C u}$ & 7.13386522552616 & 30.26855749872710 & 2.54073414478265 \\
\hline $\mathbf{C u}$ & 7.13547931882962 & 32.79133554544640 & 2.54080759423550 \\
\hline $\mathbf{C u}$ & 1.78394864960653 & 1.25799546472461 & 3.85338874388427 \\
\hline $\mathrm{Cu}$ & 1.78078634416849 & 3.77941429448956 & 3.85613733326191 \\
\hline $\mathbf{C u}$ & 1.77728185648862 & 6.29724140326161 & 3.85551346510185 \\
\hline $\mathbf{C u}$ & 1.78105832188209 & 8.81904957433993 & 3.84659556586440 \\
\hline $\mathbf{C u}$ & 1.77771764313504 & 11.34511593283290 & 3.83980230646640 \\
\hline $\mathbf{C u}$ & 1.79621109172245 & 13.86991302480650 & 3.85524684908414 \\
\hline $\mathbf{C u}$ & 1.77700160811515 & 16.39220187545740 & 3.84365469769070 \\
\hline $\mathbf{C u}$ & 1.78765313309453 & 18.91922123099280 & 3.84947004438805 \\
\hline $\mathbf{C u}$ & 1.77555048896876 & 21.43842936403210 & 3.84015908859468 \\
\hline $\mathbf{C u}$ & 1.78529135203579 & 23.96680242621890 & 3.84317289975505 \\
\hline $\mathbf{C u}$ & 1.78103782250650 & 26.48980551556890 & 3.85635494251338 \\
\hline
\end{tabular}




\begin{tabular}{|c|c|c|c|}
\hline $\mathbf{C u}$ & 1.78193478309958 & 29.00998146822260 & 3.85686498021491 \\
\hline $\mathbf{C u}$ & 1.78218666182194 & 31.52930761377160 & 3.85625023324282 \\
\hline $\mathbf{C u}$ & 1.78478880276873 & 34.04971929174540 & 3.85262194137090 \\
\hline $\mathbf{C u}$ & 5.35057549634240 & 1.25797011832682 & 3.85342306831892 \\
\hline $\mathbf{C u}$ & 5.35375834295793 & 3.77941345299389 & 3.85614317965654 \\
\hline $\mathbf{C u}$ & 5.35726661423796 & 6.29722116843326 & 3.85557618037747 \\
\hline $\mathbf{C u}$ & 5.35348937691839 & 8.81905511199790 & 3.84661160802751 \\
\hline $\mathbf{C u}$ & 5.35684293075119 & 11.34512050700980 & 3.83978107379074 \\
\hline $\mathbf{C u}$ & 5.33832833954254 & 13.86991603649820 & 3.85521742682254 \\
\hline $\mathbf{C u}$ & 5.35755022373264 & 16.39220854890630 & 3.84365933418703 \\
\hline $\mathbf{C u}$ & 5.34692083919606 & 18.91926491555300 & 3.84950904863885 \\
\hline $\mathbf{C u}$ & 5.35902390135657 & 21.43845891904350 & 3.84017833162937 \\
\hline $\mathbf{C u}$ & 5.34927382240503 & 23.96683085952880 & 3.84318668550931 \\
\hline $\mathbf{C u}$ & 5.35349332452513 & 26.48982406868200 & 3.85638005704093 \\
\hline $\mathbf{C u}$ & 5.35260735367211 & 29.00999231126270 & 3.85689292694691 \\
\hline $\mathbf{C u}$ & 5.35235602099201 & 31.52930756883170 & 3.85624429876724 \\
\hline $\mathbf{C u}$ & 5.34975526902711 & 34.04969229531580 & 3.85261575422377 \\
\hline $\mathbf{C u}$ & 8.91819331128087 & 1.25807671403479 & 3.84973695325889 \\
\hline $\mathbf{C u}$ & 8.91819801408147 & 3.78207072942741 & 3.85313123200126 \\
\hline $\mathbf{C u}$ & 8.91819214033446 & 6.30291750018964 & 3.85025770837407 \\
\hline $\mathbf{C u}$ & 8.91820073196382 & 1617 & 69535 \\
\hline $\mathbf{C u}$ & 8.91819237636312 & 11. & 3.8 \\
\hline $\mathbf{C u}$ & 78312 & 13.87270650950840 & 3.85800580247044 \\
\hline $\mathbf{C u}$ & 8.91818 & 16.39428787255350 & 3.85530528838475 \\
\hline $\mathbf{C u}$ & 8.91818259325385 & 18.91330745497540 & 3.85905510559584 \\
\hline $\mathbf{C u}$ & 8.91820871215314 & 21.43997168373290 & 3.85559104908038 \\
\hline $\mathbf{C u}$ & 8.91820588080039 & 23.96207889826670 & 3.85245644139378 \\
\hline $\mathbf{C u}$ & 8.91820031678519 & 26.48845746474510 & 3.85199332800260 \\
\hline $\mathbf{C u}$ & 8.91820202751666 & 29.007963 & 29102853 \\
\hline $\mathbf{C u}$ & 8.91819223222404 & 31.53059201273960 & 3.85008221088251 \\
\hline $\mathbf{C u}$ & 8.91821168570502 & 34.05254292976100 & 3.85006864867847 \\
\hline $\mathbf{C u}$ & 0.00234617671465 & -0.00341853056349 & 5.05578286695780 \\
\hline $\mathbf{C u}$ & 0.00108930874884 & 2.51946553695160 & 5.05761378208160 \\
\hline $\mathbf{C u}$ & 10.69693628722790 & 5.04284430285572 & 5.06156872336058 \\
\hline $\mathbf{C u}$ & 0.00036983481507 & 7.56346466876680 & 5.06080544762184 \\
\hline $\mathbf{C u}$ & 0.00268274246348 & 10.08652963950760 & 5.04919998406569 \\
\hline $\mathbf{C u}$ & 0.00919500397142 & 12.60990672076010 & 5.05965018122623 \\
\hline $\mathbf{C u}$ & 0.00830034140292 & 15.13069428290930 & 5.05347946225574 \\
\hline $\mathbf{C u}$ & 0.00393091794286 & 17.65533919037550 & 5.06271049710915 \\
\hline $\mathbf{C u}$ & 0.00618144462248 & 20.17689840205550 & 5.05368737362959 \\
\hline $\mathbf{C u}$ & 0.00822239837416 & 22.70440580014930 & 5.05932313645774 \\
\hline $\mathbf{C u}$ & 0.00298239433479 & 25.22298624941320 & 5.05230701329926 \\
\hline $\mathbf{C u}$ & 10.70082414419880 & 27.74890314740730 & 5.06420726131126 \\
\hline $\mathbf{C u}$ & 10.69804718570820 & 30.26714360596520 & 5.05849295163061 \\
\hline $\mathbf{C u}$ & 0.00274615036955 & 32.79012793730910 & 5.05794573115564 \\
\hline $\mathbf{C u}$ & 3.56725917535397 & -0.00541041773962 & 5.06207483449952 \\
\hline $\mathbf{C u}$ & 3.56727369443900 & 2.51701372701608 & 5.05765094472261 \\
\hline $\mathbf{C u}$ & 3.56726027584479 & 5.03250052566650 & 5.06056968292846 \\
\hline $\mathbf{C u}$ & 3.56727141694016 & 7.53679136491423 & 5.04070110321293 \\
\hline $\mathbf{C u}$ & 3.56725602284935 & 10.03813760490580 & 5.06985706156656 \\
\hline $\mathbf{C u}$ & 3.56727585779897 & 12.57644978639170 & 5.16579068133958 \\
\hline $\mathbf{C u}$ & 3.56728189752687 & 15.11626075596760 & 5.10565274789947 \\
\hline $\mathbf{C u}$ & 3.56728178217911 & 17.65715576816300 & 5.13158660314918 \\
\hline $\mathbf{C u}$ & 3.56727739061184 & 20.18138195841290 & $5.094 / 00$ \\
\hline
\end{tabular}




\begin{tabular}{|c|c|c|c|}
\hline $\mathbf{C u}$ & 3.56728760889666 & 22.72134362790450 & 5.12751322244964 \\
\hline $\mathbf{C u}$ & 3.56725944814340 & 25.24420644773330 & 5.05952857211151 \\
\hline $\mathbf{C u}$ & 3.56732445138678 & 27.75430737155610 & 5.05849998678869 \\
\hline $\mathbf{C u}$ & 3.56726301454470 & 30.27091073564790 & 5.06081840116661 \\
\hline $\mathbf{C u}$ & 3.56727853263190 & 32.79001174024330 & 5.05911993346432 \\
\hline $\mathbf{C u}$ & 7.13221286981535 & -0.00340842237043 & 5.05578361052273 \\
\hline $\mathbf{C u}$ & 7.13347070377940 & 2.51947169903501 & 5.05761633826008 \\
\hline $\mathbf{C u}$ & 7.13945883790906 & 5.04283323088544 & 5.06158099524263 \\
\hline $\mathbf{C u}$ & 7.13418077065235 & 7.56346280940941 & 5.06080427552145 \\
\hline $\mathbf{C u}$ & 7.13186452888003 & 10.08651400701140 & 5.04918596941514 \\
\hline $\mathbf{C u}$ & 7.12533760475576 & 12.60989416097260 & 5.05962995660060 \\
\hline $\mathbf{C u}$ & 7.12622454699753 & 15.13071339240810 & 5.05346240688560 \\
\hline $\mathbf{C u}$ & 7.13062270181724 & 17.65532935176750 & 5.06272227795554 \\
\hline $\mathbf{C u}$ & 7.12837532778526 & 20.17691149599090 & 5.05368627331006 \\
\hline $\mathbf{C u}$ & 7.12638530935776 & 22.70443679598310 & 93024 \\
\hline $\mathbf{C u}$ & 7.13158929777243 & 25.22302794325730 & 5.05229897889085 \\
\hline $\mathbf{C u}$ & 2772853 & 27.74892465488860 & 74227229 \\
\hline $\mathrm{Cu}$ & 273905 & 30.26715222098270 & 2434455 \\
\hline $\mathbf{C u}$ & 7.13179475024663 & 32.79014948151330 & 5.05794542954245 \\
\hline \multicolumn{4}{|c|}{ CI-NEB Image 3} \\
\hline Element & X-Coordinate (Å) & Y-Coordinate (§̊) & Z-Coordinate (Å) \\
\hline $\mathbf{F}$ & 4.64380714610906 & 12.16896732267680 & 7.82970814002198 \\
\hline $\mathbf{F}$ & 2.49086650232661 & 12.16897102711380 & 7.82979776305057 \\
\hline $\mathbf{F}$ & 3.56728822779905 & 10.17902079638020 & 7.03701090427658 \\
\hline $\mathbf{F}$ & 4.66294428737091 & 17.64958600882040 & 7.76148061956838 \\
\hline $\mathbf{F}$ & 2.47159605202442 & 17.64951623703540 & 7.76145315391121 \\
\hline $\mathbf{F}$ & 3.56733617354057 & 15.78231722582110 & 7.29751866315903 \\
\hline $\mathbf{F}$ & 4.66369906695260 & 22.75527783704180 & 7.74986477199115 \\
\hline $\mathbf{F}$ & 2.47087177352789 & 22.75527785308090 & 7.74985635351042 \\
\hline $\mathbf{F}$ & 3.56728634589584 & 20.88701991959690 & 7.30692149038700 \\
\hline $\mathrm{C}$ & 3.56730075280990 & 11.99734915463580 & 7.05503135072210 \\
\hline C & 3.56729184810400 & 17.21571183163660 & 7.05895857674411 \\
\hline C & 3.56728828274177 & 22.30355378360050 & 7.05293496491712 \\
\hline $\mathbf{C u}$ & 0.00000000000000 & 0.00000000000000 & 0.00000000000000 \\
\hline $\mathbf{C u}$ & 0.00000000000000 & 2.52244512012115 & 0.00000000000000 \\
\hline $\mathbf{C u}$ & 0.00000000000000 & 5.04488953395804 & 0.00000000000000 \\
\hline $\mathbf{C u}$ & 0.00000000000000 & 7.56733465407884 & 0.00000000000000 \\
\hline $\mathbf{C u}$ & 0.00000000000000 & 10.08977871477400 & 0.00000000000000 \\
\hline $\mathbf{C u}$ & 0.00000000000000 & 12.61222383489520 & 0.00000000000000 \\
\hline $\mathbf{C u}$ & 0.00000000000000 & 15.13466895501510 & 0.00000000000000 \\
\hline $\mathbf{C u}$ & 0.00000000000000 & 17.65711336885320 & 0.00000000000000 \\
\hline $\mathbf{C u}$ & 0.00000000000000 & 20.17955848897460 & 0.00000000000000 \\
\hline $\mathbf{C u}$ & 0.00000000000000 & 22.70200254966930 & 0.00000000000000 \\
\hline $\mathbf{C u}$ & 0.00000000000000 & 25.22444766979040 & 0.00000000000000 \\
\hline $\mathbf{C u}$ & 0.00000000000000 & 27.74689208362510 & 0.00000000000000 \\
\hline $\mathbf{C u}$ & 0.00000000000000 & 30.26933720374630 & 0.00000000000000 \\
\hline $\mathbf{C u}$ & 0.00000000000000 & 32.79178232386990 & 0.00000000000000 \\
\hline $\mathbf{C u}$ & 3.56727419238642 & 0.00000000000000 & 0.00000000000000 \\
\hline $\mathbf{C u}$ & 3.56727419238642 & 2.52244512012115 & 0.00000000000000 \\
\hline $\mathbf{C u}$ & 3.56727419238642 & 5.04488953395804 & 0.00000000000000 \\
\hline $\mathbf{C u}$ & 3.56727419238642 & 7.56733465407884 & 0.00000000000000 \\
\hline $\mathbf{C u}$ & 3.56727419238642 & 10.08977871477400 & 0.00000000000000 \\
\hline $\mathbf{C u}$ & 3.56727419238642 & 12.61222383489520 & 0.00000000000000 \\
\hline $\mathbf{C u}$ & 3.56727419238642 & 15.13466895501510 & 0.00000000000000 \\
\hline
\end{tabular}




\begin{tabular}{|c|c|c|c|}
\hline $\mathbf{C u}$ & 3.56727419238642 & 17.65711336885320 & 0.00000000000000 \\
\hline $\mathbf{C u}$ & 3.56727419238642 & 20.17955848897460 & 0.00000000000000 \\
\hline $\mathbf{C u}$ & 3.56727419238642 & 22.70200254966930 & 0.00000000000000 \\
\hline $\mathbf{C u}$ & 3.56727419238642 & 25.22444766979040 & 0.00000000000000 \\
\hline $\mathbf{C u}$ & 3.56727419238642 & 27.74689208362510 & 0.00000000000000 \\
\hline $\mathbf{C u}$ & 3.56727419238642 & 30.26933720374630 & 0.00000000000000 \\
\hline $\mathbf{C u}$ & 3.56727419238642 & 32.79178232386990 & 0.00000000000000 \\
\hline $\mathbf{C u}$ & 7.13454838477291 & 0.00000000000000 & 0.00000000000000 \\
\hline $\mathbf{C u}$ & 7.13454838477291 & 2.52244512012115 & 0.00000000000000 \\
\hline $\mathbf{C u}$ & 7.13454838477291 & 5.04488953395804 & 0.00000000000000 \\
\hline $\mathbf{C u}$ & 7.13454838477291 & 7.56733465407884 & 0.00000000000000 \\
\hline $\mathrm{Cu}$ & 7.13454838477291 & 10.08977871477400 & 0.00000000000000 \\
\hline $\mathrm{Cu}$ & 7.13454838477291 & 12.61222383489520 & 0.00000000000000 \\
\hline $\mathrm{Cu}$ & 7.13454838477291 & 15.13466895501510 & 0.00000000000000 \\
\hline $\mathbf{C u}$ & 7.13454838477291 & 17.65711336885320 & 0.00000000000000 \\
\hline $\mathbf{C u}$ & 7.13454838477291 & 20.17955848897460 & 0.00000000000000 \\
\hline $\mathbf{C u}$ & 7.13454838477291 & 22.70200254966930 & 0.00000000000000 \\
\hline $\mathbf{C u}$ & 7.13454838477291 & 25.22444766979040 & 0.00000000000000 \\
\hline $\mathbf{C u}$ & 7.13454838477291 & 27.74689208362510 & 0.00000000000000 \\
\hline $\mathbf{C u}$ & 7.13454838477291 & 30.26933720374630 & 0.00000000000000 \\
\hline $\mathbf{C u}$ & 7.13454838477291 & 32.79178232386990 & 0.00000000000000 \\
\hline $\mathbf{C u}$ & 1.78363955761358 & 1.26122414920059 & 1.26122399999993 \\
\hline $\mathbf{C u}$ & 1.78363955761358 & 3.78366926932194 & 1.26122399999993 \\
\hline $\mathrm{Cu}$ & 1.78363955761358 & 333001662 & 1.26122399999993 \\
\hline $\mathrm{Cu}$ & 1.78363955761358 & 3778 & 2399999993 \\
\hline $\mathrm{Cu}$ & 1.78363955761358 & 11.35100286397460 & 1.26122399999993 \\
\hline $\mathbf{C u}$ & 1.78363955761358 & 13.87344798409430 & 1.26122399999993 \\
\hline $\mathbf{C u}$ & 1.78363955761358 & 16.39589310421720 & 1.26122399999993 \\
\hline $\mathbf{C u}$ & 1.78363955761358 & 18.91833716491180 & 1.26122399999993 \\
\hline $\mathrm{Cu}$ & 1.78363955761358 & 21.44078263817520 & 1.26122399999993 \\
\hline $\mathbf{C u}$ & 1.78363955761358 & 23.96322669886980 & 1.26122399999993 \\
\hline $\mathrm{Cu}$ & 1.78363955761358 & 26.48567181899090 & 1.26122399999993 \\
\hline $\mathrm{Cu}$ & 1.78363955761358 & 29.00811693911020 & 1.26122399999993 \\
\hline $\mathbf{C u}$ & 1.78363955761358 & 31.53056135294660 & 1.26122399999993 \\
\hline $\mathbf{C u}$ & 1.78363955761358 & 34.05300647307050 & 1.26122399999993 \\
\hline $\mathbf{C u}$ & 5.35091375000000 & 1.26122414920059 & 1.26122399999993 \\
\hline $\mathbf{C u}$ & 5.35091375000000 & 3.78366926932194 & 1.26122399999993 \\
\hline $\mathbf{C u}$ & 5.35091375000000 & 6.30611333001662 & 1.26122399999993 \\
\hline $\mathbf{C u}$ & 5.35091375000000 & 8.82855845013778 & 1.26122399999993 \\
\hline $\mathbf{C u}$ & 5.35091375000000 & 11.35100286397460 & 1.26122399999993 \\
\hline $\mathbf{C u}$ & 5.35091375000000 & 13.87344798409430 & 1.26122399999993 \\
\hline $\mathbf{C u}$ & 5.35091375000000 & 16.39589310421720 & 1.26122399999993 \\
\hline $\mathbf{C u}$ & 5.35091375000000 & 18.91833716491180 & 1.26122399999993 \\
\hline $\mathbf{C u}$ & 5.35091375000000 & 21.44078263817520 & 1.26122399999993 \\
\hline $\mathbf{C u}$ & 5.35091375000000 & 23.96322669886980 & 1.26122399999993 \\
\hline $\mathbf{C u}$ & 5.35091375000000 & 26.48567181899090 & 1.26122399999993 \\
\hline $\mathbf{C u}$ & 5.35091375000000 & 29.00811693911020 & 1.26122399999993 \\
\hline $\mathbf{C u}$ & 5.35091375000000 & 31.53056135294660 & 1.26122399999993 \\
\hline $\mathbf{C u}$ & 5.35091375000000 & 34.05300647307050 & 1.26122399999993 \\
\hline $\mathrm{Cu}$ & 8.91818794238642 & 1.26122414920059 & 1.26122399999993 \\
\hline $\mathbf{C u}$ & 8.91818794238642 & 3.78366926932194 & 1.26122399999993 \\
\hline $\mathbf{C u}$ & 8.91818794238642 & 6.30611333001662 & 1.26122399999993 \\
\hline $\mathbf{C u}$ & 8.91818794238642 & 8.82855845013778 & 1.26122399999993 \\
\hline $\mathbf{C u}$ & 8.91818794238642 & 11.35100286397460 & 1.26122399999993 \\
\hline
\end{tabular}




\begin{tabular}{|c|c|c|c|}
\hline $\mathbf{C u}$ & 8.91818794238642 & 13.87344798409430 & 1.26122399999993 \\
\hline $\mathbf{C u}$ & 8.91818794238642 & 16.39589310421720 & 1.26122399999993 \\
\hline $\mathbf{C u}$ & 8.91818794238642 & 18.91833716491180 & 1.26122399999993 \\
\hline $\mathbf{C u}$ & 8.91818794238642 & 21.44078263817520 & 1.26122399999993 \\
\hline $\mathbf{C u}$ & 8.91818794238642 & 23.96322669886980 & 1.26122399999993 \\
\hline $\mathbf{C u}$ & 8.91818794238642 & 26.48567181899090 & 1.26122399999993 \\
\hline $\mathbf{C u}$ & 8.91818794238642 & 29.00811693911020 & 1.26122399999993 \\
\hline $\mathbf{C u}$ & 8.91818794238642 & 31.53056135294660 & 1.26122399999993 \\
\hline $\mathbf{C u}$ & 8.91818794238642 & 34.05300647307050 & 1.26122399999993 \\
\hline $\mathbf{C u}$ & 0.00107746482096 & -0.00051389889215 & 2.53827662033251 \\
\hline $\mathbf{C u}$ & 10.70075101158860 & 2.52321628567225 & 2.54101984352054 \\
\hline $\mathbf{C u}$ & 0.00007626835966 & 5.04433434445921 & 2.54181387428326 \\
\hline $\mathbf{C u}$ & -0.00312381712884 & 7.56400631920520 & 2.54090584380569 \\
\hline $\mathbf{C u}$ & 10.69715353832350 & 10.08867582604860 & 2.53835738215669 \\
\hline $\mathbf{C u}$ & 10.70096530498610 & 12.61505532353370 & 2.54586675849540 \\
\hline $\mathbf{C u}$ & 10.70071741014710 & 15.13345585397350 & 2.53958562213724 \\
\hline $\mathbf{C u}$ & 10.69864101715880 & 17.65585863172110 & 2.54615452250285 \\
\hline $\mathbf{C u}$ & 10.69721445572630 & 20.17749549999910 & 2.53849598120186 \\
\hline $\mathbf{C u}$ & 10.69727293605400 & 22.70181298467540 & 2.54647250111683 \\
\hline $\mathbf{C u}$ & 10.70013410330040 & 25.22650848579960 & 2.53878289734131 \\
\hline $\mathbf{C u}$ & 67719880 & 27.74857550803070 & 2.54350486303425 \\
\hline $\mathbf{C u}$ & 0.00035562060499 & 30.26889122206560 & 2.54108880813583 \\
\hline $\mathbf{C u}$ & 10.70123537567160 & 32.79247720003580 & 78121843 \\
\hline $\mathbf{C u}$ & 565956138 & 0.00088636319534 & 2019411 \\
\hline $\mathrm{Cu}$ & 3.56728092271879 & 2.52245213116965 & 774220 \\
\hline $\mathrm{Cu}$ & 3.56727901144589 & 5.04509370417673 & 17718651 \\
\hline $\mathbf{C u}$ & 3.56726965867605 & 7.56694835053522 & 2.54928474330880 \\
\hline $\mathbf{C u}$ & 3.56727724008837 & 10.08879278279940 & 2.58131487040065 \\
\hline $\mathrm{Cu}$ & 3.56727625423496 & 12.61464638070310 & 2.59424121022558 \\
\hline $\mathrm{Cu}$ & 3.56726965656185 & 15.14201228575540 & 2.58170369308741 \\
\hline $\mathrm{Cu}$ & 3.56727120842966 & 17.65282031513580 & 2.58068683067471 \\
\hline $\mathbf{C u}$ & 3.56727881115179 & 20.17583025293930 & 2.57998937382116 \\
\hline $\mathrm{Cu}$ & 3.56728019196869 & 22.69895274876760 & 2.57887031352526 \\
\hline $\mathbf{C u}$ & 3.56728001080924 & 25.22098308595420 & 2.55307526195540 \\
\hline $\mathbf{C u}$ & 3.56729515398314 & 27.74657920236770 & 2.54220042876673 \\
\hline $\mathbf{C u}$ & 3.56728675040177 & 30.27042869984160 & 2.54334769235903 \\
\hline $\mathbf{C u}$ & 3.56727445678057 & 32.78952845364310 & 2.54202861368082 \\
\hline $\mathbf{C u}$ & 7.13348094170894 & -0.00051414090111 & 2.53828182287060 \\
\hline $\mathbf{C u}$ & 7.13562455111824 & 2.52319916387523 & 2.54104459123754 \\
\hline $\mathbf{C u}$ & 7.13448779890626 & 5.04434757444228 & 2.54182740057459 \\
\hline $\mathbf{C u}$ & 7.13766455212848 & 7.56400897718862 & 2.54091892234364 \\
\hline $\mathbf{C u}$ & 7.13921701909466 & 10.08867195274590 & 2.53837322783410 \\
\hline $\mathbf{C u}$ & 7.13540698597810 & 12.61504867107410 & 2.54587064162243 \\
\hline $\mathbf{C u}$ & 7.13567605762790 & 15.13343815341060 & 2.53958247299525 \\
\hline $\mathbf{C u}$ & 7.13773595357495 & 17.65585848682010 & 2.54615563576970 \\
\hline $\mathbf{C u}$ & 7.13916609185194 & 20.17749789095070 & 2.53850172671418 \\
\hline $\mathbf{C u}$ & 7.13909678392886 & 22.70181283311770 & 2.54647510350973 \\
\hline $\mathbf{C u}$ & 7.13624819915115 & 25.22650823038530 & 2.53878296980462 \\
\hline $\mathbf{C u}$ & 7.13598397750813 & 27.74858631044850 & 2.54351526894303 \\
\hline $\mathbf{C u}$ & 7.13419249074294 & 30.26890203590610 & 2.54109178038789 \\
\hline $\mathbf{C u}$ & 7.13513639117095 & 32.79249498472240 & 2.54122982366022 \\
\hline $\mathbf{C u}$ & 1.78320235193666 & 1.26119646471099 & 3.85357384990734 \\
\hline $\mathbf{C u}$ & 1.78168478953787 & 3.78352437654761 & 3.85488495742315 \\
\hline $\mathbf{C u}$ & 1.78069410868253 & 6.30119276674059 & 3.85785056955420 \\
\hline
\end{tabular}




\begin{tabular}{|c|c|c|c|}
\hline $\mathbf{C u}$ & 1.77802725850307 & 8.81877668483108 & 3.83826947610966 \\
\hline $\mathbf{C u}$ & 1.77881364889624 & 11.34614768287820 & 3.83701115342858 \\
\hline $\mathbf{C u}$ & 1.79575029167135 & 13.87017315295060 & 3.85611861565504 \\
\hline $\mathbf{C u}$ & 1.77628100260174 & 16.39207834432760 & 3.84315784314063 \\
\hline $\mathbf{C u}$ & 1.78711010656695 & 18.91829703878120 & 3.84930531089741 \\
\hline $\mathbf{C u}$ & 1.77491852367403 & 21.43824784798820 & 3.83920597219409 \\
\hline $\mathbf{C u}$ & 1.78492326695925 & 23.96713003515030 & 3.84333511038068 \\
\hline $\mathbf{C u}$ & 1.78002594666374 & 26.48990747517420 & 3.85702485669870 \\
\hline $\mathbf{C u}$ & 1.78193411528025 & 29.01049562573250 & 3.85704567723669 \\
\hline $\mathbf{C u}$ & 1.78176525454223 & 31.53167727761270 & 3.85675505935399 \\
\hline $\mathbf{C u}$ & 1.78502967328441 & 34.05280124721770 & 3.85283827808023 \\
\hline $\mathbf{C u}$ & 5.35134892933976 & 1.26119982536764 & 3.85357706049260 \\
\hline $\mathbf{C u}$ & 5.35286620787861 & 3.78352193487657 & 3.85488611994002 \\
\hline $\mathbf{C u}$ & 5.35385346849368 & 6.30120265730470 & 3.85785743472224 \\
\hline $\mathbf{C u}$ & 5.35652014556656 & 8.81878193057709 & 3.83828694043762 \\
\hline $\mathbf{C u}$ & 5.35573062640051 & 11.34613080230850 & 3.83702132948640 \\
\hline $\mathbf{C u}$ & 5.33881581389067 & 13.87017990653310 & 3.85611980473807 \\
\hline $\mathbf{C u}$ & 5.35827972375419 & 16.39209022140000 & 3.84316419328308 \\
\hline $\mathbf{C u}$ & 5.34741589396366 & 18.91830106486370 & 3.84927999550678 \\
\hline $\mathbf{C u}$ & 5.35963435960518 & 21.43825220097130 & 3.83921937085386 \\
\hline $\mathbf{C u}$ & 5.34963440926439 & 23.96714011458540 & 3.84331657204125 \\
\hline $\mathbf{C u}$ & 5.35453873159907 & 26.48991920927010 & 3.85701864618895 \\
\hline $\mathbf{C u}$ & 5.35261433596081 & 29.01050722828780 & 797659927 \\
\hline $\mathbf{C u}$ & 5.35280468218138 & 58980001440 & 734000481 \\
\hline $\mathbf{C u}$ & 5.3495286 & 34.05280146385080 & 3.85282561139937 \\
\hline $\mathbf{C u}$ & 8.91818905148669 & 1.26044570920993 & 3.85009097194414 \\
\hline $\mathbf{C u}$ & 8.91819092933779 & 3.78549455637038 & 3.85328881646413 \\
\hline $\mathbf{C u}$ & 8.91817447235099 & 6.30220890487942 & 3.85194398226464 \\
\hline $\mathbf{C u}$ & 8.91817712760463 & 8.82395898357878 & 3.85575572248054 \\
\hline $\mathbf{C u}$ & 8.91818110537744 & 11.35271534070770 & 3.84931727668586 \\
\hline $\mathbf{C u}$ & 8.91818483052983 & 13.87375611217330 & 3.85573750972677 \\
\hline $\mathbf{C u}$ & 8.91818661054379 & 16.39373993559160 & 3.85524249771333 \\
\hline $\mathbf{C u}$ & 8.91819062916269 & 18.91328466485360 & 3.85907130906583 \\
\hline $\mathbf{C u}$ & 8.91818774361579 & 21.44038571173210 & 3.85565006520962 \\
\hline $\mathbf{C u}$ & 8.91818986187981 & 23.96220034130520 & 3.85247954010064 \\
\hline $\mathbf{C u}$ & 8.91818167612987 & 26.49030511696860 & 3.85286773560568 \\
\hline $\mathbf{C u}$ & 8.91818328270000 & 29.00923124516670 & 3.85127030678727 \\
\hline $\mathbf{C u}$ & 8.91818528315600 & 31.53389681154420 & 3.85114152986605 \\
\hline $\mathbf{C u}$ & 8.91819241774967 & 34.05464589599250 & 3.85009762162974 \\
\hline $\mathbf{C u}$ & 0.00171781844990 & -0.00111502115583 & 5.05590446779648 \\
\hline $\mathbf{C u}$ & 0.00175166913898 & 2.52438611728848 & 5.05760082826332 \\
\hline $\mathbf{C u}$ & 10.69957271586840 & 5.04583980335915 & 5.06298107473001 \\
\hline $\mathbf{C u}$ & 0.00178713092866 & 7.56585298026899 & 5.05849470072971 \\
\hline $\mathbf{C u}$ & 0.00278312955091 & 10.08882234997470 & 5.04774983051133 \\
\hline $\mathbf{C u}$ & 0.00866766349892 & 12.60980260565920 & 5.05432775290327 \\
\hline $\mathbf{C u}$ & 0.00712816214315 & 15.13051567645450 & 5.05404412506594 \\
\hline $\mathbf{C u}$ & 0.00443756508380 & 17.65442740039560 & 5.06143284086985 \\
\hline $\mathbf{C u}$ & 0.00721851018001 & 20.17654961073540 & 5.05383653701481 \\
\hline $\mathbf{C u}$ & 0.00714328848343 & 22.70490476972520 & 5.05955396786267 \\
\hline $\mathbf{C u}$ & 0.00140897467138 & 25.22327965826730 & 5.05336845952165 \\
\hline $\mathbf{C u}$ & 10.70032261846930 & 27.75031407512140 & 5.06526335460194 \\
\hline $\mathbf{C u}$ & 10.69840995500790 & 30.26891898217900 & 5.05789514079945 \\
\hline $\mathbf{C u}$ & 0.00259985321805 & 32.79405736131990 & 5.05886325501320 \\
\hline $\mathbf{C u}$ & 3.56727755916331 & -0.00096471438802 & 5.06111855916586 \\
\hline
\end{tabular}




\begin{tabular}{|c|c|c|c|}
\hline $\mathbf{C u}$ & 3.56726797636629 & 2.52111116942971 & 5.05718407093419 \\
\hline $\mathbf{C u}$ & 3.56727313470508 & 5.03377142442856 & 5.06177502735337 \\
\hline $\mathbf{C u}$ & 3.56726945275074 & 7.53134444574011 & 5.05639172779037 \\
\hline $\mathbf{C u}$ & 3.56726194426874 & 10.03104123486190 & 5.07147760506507 \\
\hline $\mathbf{C u}$ & 3.56727516477791 & 12.58419515351350 & 5.17639559963450 \\
\hline $\mathbf{C u}$ & 3.56726440015828 & 15.11959224243660 & 5.10175769757096 \\
\hline $\mathbf{C u}$ & 3.56728308409649 & 17.65368593664840 & 5.13349516352925 \\
\hline $\mathbf{C u}$ & 3.56727001363500 & 20.17943130603110 & 5.09183976405392 \\
\hline $\mathbf{C u}$ & 3.56728532703635 & 22.71747187771560 & 5.12510664099279 \\
\hline $\mathbf{C u}$ & 3.56728875889193 & 25.24299315903740 & 5.05834110409927 \\
\hline $\mathbf{C u}$ & 3.56726820139931 & 27.75482012973460 & 5.05870506069191 \\
\hline $\mathbf{C u}$ & 3.56726836549933 & 30.27467277411020 & 5.05993972268007 \\
\hline $\mathbf{C u}$ & 3.56727668404554 & 32.79483730583970 & 5.05940693008615 \\
\hline $\mathbf{C u}$ & 7.13283912249902 & -0.00110674172744 & 5.05589442807748 \\
\hline $\mathbf{C u}$ & 7.13280398682422 & 2.52439287456535 & 05317800 \\
\hline $\mathbf{C u}$ & 7.13679685490780 & 5.04584894002516 & 5.06298637349343 \\
\hline $\mathbf{C u}$ & 7.13273630273292 & 7.56585502417772 & 565174 \\
\hline $\mathrm{Cu}$ & 7.13175228824822 & 10.08884246272110 & 67609 \\
\hline $\mathbf{C u}$ & 7.12588177152039 & 12.60979774650430 & 5.0543 \\
\hline $\mathbf{C u}$ & 7.12739363471159 & 15.13052996588650 & 5.05404995016705 \\
\hline $\mathbf{C u}$ & 7.13010459845606 & 17.65442953742420 & 5.06142468023505 \\
\hline $\mathbf{C u}$ & 7.12734444350059 & 20.17655098472880 & 5.05383096542533 \\
\hline $\mathbf{C u}$ & 7.12740862778612 & 22.70488264843360 & 5.05955038093142 \\
\hline $\mathbf{C u}$ & 7.13313791665689 & 25.22329605697790 & 5.05337536622732 \\
\hline $\mathbf{C u}$ & 7.13606208950999 & 27.75034538615540 & 5.06528425587014 \\
\hline $\mathbf{C u}$ & 7.13797635741895 & 30.26894068430090 & 5.05791243976854 \\
\hline $\mathbf{C u}$ & 7.13194991892096 & 32.79407777062610 & 5.05886473330232 \\
\hline \multicolumn{4}{|c|}{ CI-NEB Image 4} \\
\hline Element & X-Coordinate $(\AA ̊)$ & Y-Coordinate ( $(\AA)$ & Z-Coordinate (§̊) \\
\hline $\mathbf{F}$ & 4.63110823876603 & 12.18398828412050 & 7.81546840670322 \\
\hline $\mathbf{F}$ & 2.50359433178281 & 12.18400182914350 & 7.81558523890778 \\
\hline $\mathbf{F}$ & 3.56728099969380 & 9.76342641951035 & 6.95944430554881 \\
\hline $\mathbf{F}$ & 4.66286292815147 & 17.65091020236360 & 7.76277205174700 \\
\hline $\mathbf{F}$ & 2.47165255305462 & 17.65080127116970 & 7.76273865082737 \\
\hline $\mathbf{F}$ & 3.56736128085109 & 15.78263022415310 & 7.30211410705985 \\
\hline $\mathbf{F}$ & 4.66363891484516 & 22.75509657007840 & 7.74892091254208 \\
\hline $\mathbf{F}$ & 2.47093206230535 & 22.75509751391070 & 7.74891523226816 \\
\hline $\mathbf{F}$ & 3.56728695768183 & 20.88633263441890 & 7.30851243312968 \\
\hline $\mathbf{C}$ & 3.56731795232690 & 12.22415402417270 & 7.03803369159821 \\
\hline C & 3.56729097381812 & 17.21771007724580 & 7.05980735728836 \\
\hline C & 3.56728763513305 & 22.30197549836900 & 7.05249600496006 \\
\hline $\mathbf{C u}$ & 0.00000000000000 & 0.00000000000000 & 0.00000000000000 \\
\hline $\mathbf{C u}$ & 0.00000000000000 & 2.52244512012115 & 0.00000000000000 \\
\hline $\mathbf{C u}$ & 0.00000000000000 & 5.04488953395804 & 0.00000000000000 \\
\hline $\mathbf{C u}$ & 0.00000000000000 & 7.56733465407884 & 0.00000000000000 \\
\hline $\mathbf{C u}$ & 0.00000000000000 & 10.08977871477400 & 0.00000000000000 \\
\hline $\mathbf{C u}$ & 0.00000000000000 & 12.61222383489520 & 0.00000000000000 \\
\hline $\mathbf{C u}$ & 0.00000000000000 & 15.13466895501510 & 0.00000000000000 \\
\hline $\mathbf{C u}$ & 0.00000000000000 & 17.65711336885320 & 0.00000000000000 \\
\hline $\mathbf{C u}$ & 0.00000000000000 & 20.17955848897460 & 0.00000000000000 \\
\hline $\mathbf{C u}$ & 0.00000000000000 & 22.70200254966930 & 0.00000000000000 \\
\hline $\mathbf{C u}$ & 0.00000000000000 & 25.22444766979040 & 0.00000000000000 \\
\hline $\mathbf{C u}$ & 0.00000000000000 & 27.74689208362510 & 0.00000000000000 \\
\hline $\mathbf{C u}$ & 0.00000000000000 & 30.26933720374630 & 0.00000000000000 \\
\hline
\end{tabular}




\begin{tabular}{|c|c|c|c|}
\hline $\mathbf{C u}$ & 0.00000000000000 & 32.79178232386990 & 0.00000000000000 \\
\hline $\mathbf{C u}$ & 3.56727419238642 & 0.00000000000000 & 0.00000000000000 \\
\hline $\mathrm{Cu}$ & 3.56727419238642 & 2.52244512012115 & 0.00000000000000 \\
\hline $\mathbf{C u}$ & 3.56727419238642 & 5.04488953395804 & 0.00000000000000 \\
\hline $\mathbf{C u}$ & 3.56727419238642 & 7.56733465407884 & 0.00000000000000 \\
\hline $\mathbf{C u}$ & 3.56727419238642 & 10.08977871477400 & 0.00000000000000 \\
\hline $\mathbf{C u}$ & 3.56727419238642 & 12.61222383489520 & 0.00000000000000 \\
\hline $\mathbf{C u}$ & 3.56727419238642 & 15.13466895501510 & 0.00000000000000 \\
\hline $\mathbf{C u}$ & 3.56727419238642 & 17.65711336885320 & 0.00000000000000 \\
\hline $\mathbf{C u}$ & 3.56727419238642 & 20.17955848897460 & 0.00000000000000 \\
\hline $\mathbf{C u}$ & 3.56727419238642 & 22.70200254966930 & 0.00000000000000 \\
\hline $\mathbf{C u}$ & 3.56727419238642 & 25.22444766979040 & 0.00000000000000 \\
\hline $\mathbf{C u}$ & 3.56727419238642 & 27.74689208362510 & 0.00000000000000 \\
\hline $\mathbf{C u}$ & 3.56727419238642 & 30.26933720374630 & 0.00000000000000 \\
\hline $\mathbf{C u}$ & 3.56727419238642 & 32.79178232386990 & 0.00000000000000 \\
\hline $\mathbf{C u}$ & 7.13454838477291 & 0.00000000000000 & 0.00000000000000 \\
\hline $\mathbf{C u}$ & 7.13454838477291 & 2.52244512012115 & 0.00000000000000 \\
\hline $\mathbf{C u}$ & 7.13454838477291 & 5.04488953395804 & 0.00000000000000 \\
\hline $\mathbf{C u}$ & 7.13454838477291 & 7.56733465407884 & 0.00000000000000 \\
\hline $\mathbf{C u}$ & 7.13454838477291 & 10.08977871477400 & 0.00000000000000 \\
\hline $\mathbf{C u}$ & 7.13454838477291 & 12.61222383489520 & 0000000000 \\
\hline $\mathbf{C u}$ & 7.13454838477291 & 15.13466895501510 & 0.00000000000000 \\
\hline $\mathbf{C u}$ & 7.13454838477291 & 17.65711336885320 & 0000000 \\
\hline $\mathbf{C u}$ & 7.13454838477291 & 20.17955848897460 & 000000 \\
\hline $\mathbf{C u}$ & 7.13454838477291 & 22.70200254966930 & 0000000 \\
\hline $\mathrm{Cu}$ & 7.13454838477291 & 766979040 & 0000000000 \\
\hline $\mathrm{Cu}$ & 7.13454838477291 & 27.74689208362510 & 0.00000000000000 \\
\hline $\mathbf{C u}$ & 7.13454838477291 & 30.26933720374630 & 0.00000000000000 \\
\hline $\mathbf{C u}$ & 7.13454838477291 & 32.79178232386990 & 0.00000000000000 \\
\hline $\mathbf{C u}$ & 1.78363955761358 & 1.26122414920059 & 1.26122399999993 \\
\hline $\mathbf{C u}$ & 1.78363955761358 & 3.78366926932194 & 1.26122399999993 \\
\hline $\mathbf{C u}$ & 1.78363955761358 & 6.30611333001662 & 1.26122399999993 \\
\hline $\mathrm{Cu}$ & 1.78363955761358 & 8.82855845013778 & 1.26122399999993 \\
\hline $\mathbf{C u}$ & 1.78363955761358 & 11.35100286397460 & 1.26122399999993 \\
\hline $\mathbf{C u}$ & 1.78363955761358 & 13.87344798409430 & 1.26122399999993 \\
\hline $\mathbf{C u}$ & 1.78363955761358 & 16.39589310421720 & 1.26122399999993 \\
\hline $\mathbf{C u}$ & 1.78363955761358 & 18.91833716491180 & 1.26122399999993 \\
\hline $\mathbf{C u}$ & 1.78363955761358 & 21.44078263817520 & 1.26122399999993 \\
\hline $\mathbf{C u}$ & 1.78363955761358 & 23.96322669886980 & 1.26122399999993 \\
\hline $\mathbf{C u}$ & 1.78363955761358 & 26.48567181899090 & 1.26122399999993 \\
\hline $\mathbf{C u}$ & 1.78363955761358 & 29.00811693911020 & 1.26122399999993 \\
\hline $\mathbf{C u}$ & 1.78363955761358 & 31.53056135294660 & 1.26122399999993 \\
\hline $\mathbf{C u}$ & 1.78363955761358 & 34.05300647307050 & 1.26122399999993 \\
\hline $\mathbf{C u}$ & 5.35091375000000 & 1.26122414920059 & 1.26122399999993 \\
\hline $\mathbf{C u}$ & 5.35091375000000 & 3.78366926932194 & 1.26122399999993 \\
\hline $\mathbf{C u}$ & 5.35091375000000 & 6.30611333001662 & 1.26122399999993 \\
\hline $\mathbf{C u}$ & 5.35091375000000 & 8.82855845013778 & 1.26122399999993 \\
\hline $\mathbf{C u}$ & 5.35091375000000 & 11.35100286397460 & 1.26122399999993 \\
\hline $\mathrm{Cu}$ & 5.35091375000000 & 13.87344798409430 & 1.26122399999993 \\
\hline $\mathbf{C u}$ & 5.35091375000000 & 16.39589310421720 & 1.26122399999993 \\
\hline $\mathbf{C u}$ & 5.35091375000000 & 18.91833716491180 & 1.26122399999993 \\
\hline $\mathbf{C u}$ & 5.35091375000000 & 21.44078263817520 & 1.26122399999993 \\
\hline $\mathbf{C u}$ & 5.35091375000000 & 23.96322669886980 & 1.26122399999993 \\
\hline $\mathbf{C u}$ & 5.35091375000000 & 26.48567181899090 & 1.26122399999993 \\
\hline
\end{tabular}




\begin{tabular}{|c|c|c|c|}
\hline $\mathrm{Cu}$ & 5.35091375000000 & 29.00811693911020 & 1.26122399999993 \\
\hline $\mathbf{C u}$ & 5.35091375000000 & 31.53056135294660 & 1.26122399999993 \\
\hline $\mathbf{C u}$ & 5.35091375000000 & 34.05300647307050 & 1.26122399999993 \\
\hline $\mathbf{C u}$ & 8.91818794238642 & 1.26122414920059 & 1.26122399999993 \\
\hline $\mathbf{C u}$ & 8.91818794238642 & 3.78366926932194 & 1.26122399999993 \\
\hline $\mathbf{C u}$ & 8.91818794238642 & 6.30611333001662 & 1.26122399999993 \\
\hline $\mathbf{C u}$ & 8.91818794238642 & 8.82855845013778 & 1.26122399999993 \\
\hline $\mathbf{C u}$ & 8.91818794238642 & 11.35100286397460 & 1.26122399999993 \\
\hline $\mathbf{C u}$ & 8.91818794238642 & 13.87344798409430 & 1.26122399999993 \\
\hline $\mathbf{C u}$ & 8.91818794238642 & 16.39589310421720 & 1.26122399999993 \\
\hline $\mathbf{C u}$ & 8.91818794238642 & 18.91833716491180 & 1.26122399999993 \\
\hline $\mathbf{C u}$ & 8.91818794238642 & 21.44078263817520 & 1.26122399999993 \\
\hline $\mathbf{C u}$ & 8.91818794238642 & 23.96322669886980 & 1.26122399999993 \\
\hline $\mathrm{Cu}$ & 8.91818794238642 & 26.48567181899090 & 1.26122399999993 \\
\hline $\mathbf{C u}$ & 8.91818794238642 & 29.00811693911020 & 1.26122399999993 \\
\hline $\mathbf{C u}$ & 8.91818794238642 & 31.53056135294660 & 1.26122399999993 \\
\hline $\mathbf{C u}$ & 8.91818794238642 & 34.05300647307050 & 1.26122399999993 \\
\hline $\mathbf{C u}$ & 0.00153057443230 & -0.00013765164649 & 2.53845610867687 \\
\hline $\mathbf{C u}$ & 10.70074429800070 & 2.52390734552997 & 2.54109989211388 \\
\hline $\mathbf{C u}$ & 0.00126112020729 & 5.04452336703330 & 2.54278719805473 \\
\hline $\mathbf{C u}$ & -0.00227781162578 & 7.56400769718898 & 2.54158533332817 \\
\hline $\mathbf{C u}$ & 10.69747868900890 & 10.08906253092220 & 2.53881856323406 \\
\hline $\mathbf{C u}$ & 10.70108833786990 & 51753260760 & 2421597 \\
\hline $\mathbf{C u}$ & 10.699784735 & 4519777020 & 2080329 \\
\hline $\mathrm{Cu}$ & 10.6987631 & 94440 & 554860 \\
\hline $\mathrm{Cu}$ & 10.69704802802960 & 027740 & 5729144 \\
\hline $\mathbf{C u}$ & 10.69704046054420 & 22.70224427004040 & 2.54626722783298 \\
\hline $\mathbf{C u}$ & 10.70040480492500 & 25.22661925514670 & 2.53890703241973 \\
\hline $\mathbf{C u}$ & 10.70069561225250 & 27.74821276066390 & 2.54374073548028 \\
\hline $\mathrm{Cu}$ & 0.00025963998475 & 30.26912143364580 & 2.54146357287517 \\
\hline $\mathrm{Cu}$ & 10.70134689151670 & 32.79277491729230 & 2.54125294731950 \\
\hline $\mathbf{C u}$ & 3.56727493688337 & 0.00123088511697 & 2.54129388923310 \\
\hline $\mathrm{Cu}$ & 3.56727613269197 & 2.52261715683674 & 2.54178566122899 \\
\hline $\mathbf{C u}$ & 3.56728078320703 & 5.04571647292425 & 2.54082715414784 \\
\hline $\mathbf{C u}$ & 3.56727710577766 & 7.57021414922874 & 2.55978269020084 \\
\hline $\mathbf{C u}$ & 3.56726954235290 & 10.09024470536610 & 2.59280078705167 \\
\hline $\mathbf{C u}$ & 3.56727050086438 & 12.61603930868960 & 2.59610206054616 \\
\hline $\mathbf{C u}$ & 3.56727656124062 & 15.14243677771910 & 2.58085479734124 \\
\hline $\mathbf{C u}$ & 3.56727825602309 & 17.65230836344060 & 2.58157378032204 \\
\hline $\mathbf{C u}$ & 3.56728116695618 & 20.17601336040940 & 2.57914785573500 \\
\hline $\mathbf{C u}$ & 3.56728563890063 & 22.69931200420130 & 2.57798280274690 \\
\hline $\mathbf{C u}$ & 3.56728079525846 & 25.22086649035100 & 2.55308484337611 \\
\hline $\mathbf{C u}$ & 3.56728139722216 & 27.74729277981990 & 2.54255466308302 \\
\hline $\mathbf{C u}$ & 3.56728144584723 & 30.27077285785480 & 2.54336108878395 \\
\hline $\mathrm{Cu}$ & 3.56727586839011 & 32.78955765204020 & 2.54251543514341 \\
\hline $\mathbf{C u}$ & 7.13301307913530 & -0.00014166607205 & 2.53845854486176 \\
\hline $\mathbf{C u}$ & 7.13562813028268 & 2.52389723396091 & 2.54109814685048 \\
\hline $\mathbf{C u}$ & 7.13328519376771 & 5.04452040934956 & 2.54278562939219 \\
\hline $\mathbf{C u}$ & 7.13681067167771 & 7.56398982513479 & 2.54157918176950 \\
\hline $\mathbf{C u}$ & 7.13889069487109 & 10.08905507771200 & 2.53882388779178 \\
\hline $\mathbf{C u}$ & 7.13528118967577 & 12.61460303627900 & 2.54507773955484 \\
\hline $\mathbf{C u}$ & 7.13658824307102 & 15.13393841363870 & 2.53881971457652 \\
\hline $\mathbf{C u}$ & 7.13761245831076 & 17.65625581724320 & 2.54546284068228 \\
\hline $\mathbf{C u}$ & 7.13933071389059 & 20.17800638326020 & 2.53843570876370 \\
\hline
\end{tabular}




\begin{tabular}{|c|c|c|c|}
\hline $\mathrm{Cu}$ & 7.13934462517165 & 22.70223647951290 & 2.54627276907319 \\
\hline $\mathbf{C u}$ & 7.13597860131028 & 25.22662052496110 & 2.53890420039272 \\
\hline $\mathbf{C u}$ & 7.13567949861377 & 27.74822528860870 & 2.54374894043254 \\
\hline $\mathbf{C u}$ & 7.13428654098620 & 30.26912534700570 & 2.54146492987897 \\
\hline $\mathbf{C u}$ & 7.13502406930245 & 32.79277535082320 & 2.54125726255409 \\
\hline $\mathbf{C u}$ & 1.78333680469927 & 1.26218359972638 & 3.85361914447466 \\
\hline $\mathbf{C u}$ & 1.78239936753376 & 3.78494404531779 & 3.85480262934952 \\
\hline $\mathbf{C u}$ & 1.78613152377749 & 6.30468836430753 & 3.86049307391900 \\
\hline $\mathbf{C u}$ & 1.77854587541422 & 8.82206572744408 & 3.83723202823326 \\
\hline $\mathbf{C u}$ & 1.78344806564707 & 11.34629659674980 & 3.83823890247212 \\
\hline $\mathbf{C u}$ & 1.79175015591826 & 13.87306134948010 & 3.85370768430159 \\
\hline $\mathbf{C u}$ & 1.77581895049682 & 16.39356194324730 & 3.84245730543437 \\
\hline $\mathbf{C u}$ & 1.78682021251936 & 18.91933990789720 & 3.84931167021192 \\
\hline $\mathrm{Cu}$ & 1.77476187435058 & 21.43866209202840 & 3.83872591546229 \\
\hline $\mathbf{C u}$ & 1.78514589983620 & 23.96730268429510 & 3.84370735369080 \\
\hline $\mathbf{C u}$ & 1.77964465292329 & 26.48968079543540 & 3.85689340462329 \\
\hline $\mathbf{C u}$ & 1.78230757290585 & 29.01038463351670 & 3.85691719996140 \\
\hline $\mathbf{C u}$ & 1.78189835478890 & 31.53225276548560 & 3.85659468119130 \\
\hline $\mathbf{C u}$ & 1.78474027846905 & 34.05312127484020 & 3.85310508621212 \\
\hline $\mathbf{C u}$ & 5.35121424535164 & 1.26218809456833 & 3.85360913379962 \\
\hline $\mathbf{C u}$ & 5.35214084757940 & 3.78495485885827 & 3.85479081896848 \\
\hline $\mathbf{C u}$ & 5.34842049810209 & 6.30470359433394 & 3.86048108966099 \\
\hline $\mathbf{C u}$ & 5.35599708308638 & 8.8220602 & 5387873 \\
\hline $\mathbf{C u}$ & 5.35110526043365 & 11.34626682805980 & 104289326 \\
\hline $\mathbf{C u}$ & 5.34279030996955 & 13.8730478 & 3.8537 \\
\hline $\mathbf{C u}$ & 5.35873652474122 & 16.39356683230430 & 3.842445 \\
\hline $\mathbf{C u}$ & 5.34772819365441 & 18.91935123466670 & 3.84931932828142 \\
\hline $\mathbf{C u}$ & 5.35979189633009 & 21.43866713985180 & 3.83876005058024 \\
\hline $\mathbf{C u}$ & 5.34942508065578 & 23.96728585940210 & 3.84369257488934 \\
\hline $\mathbf{C u}$ & 5.35491660993779 & 26.48969312287170 & 3.85689253061465 \\
\hline $\mathbf{C u}$ & 5.35224368702212 & 29.01039734244790 & 3.85693425051629 \\
\hline $\mathbf{C u}$ & 5.35263088096890 & 31.53224679294150 & 3.85659468969500 \\
\hline $\mathbf{C u}$ & 5.34980919600867 & 34.05312992103500 & 3.85310677367902 \\
\hline $\mathbf{C u}$ & 8.91818123466820 & 1.26128323061824 & 3.85016160020538 \\
\hline $\mathbf{C u}$ & 8.91818684080977 & 3.78586257750092 & 3.85211258315008 \\
\hline $\mathbf{C u}$ & 8.91817834540163 & 6.30246512504062 & 3.85251915507448 \\
\hline $\mathbf{C u}$ & 8.91818071302192 & 8.82505410424480 & 3.85694147075379 \\
\hline $\mathbf{C u}$ & 8.91817943020542 & 11.35300411795360 & 3.84906562459037 \\
\hline $\mathbf{C u}$ & 8.91818312319542 & 13.87410027265140 & 3.85487248583788 \\
\hline $\mathbf{C u}$ & 8.91818833109429 & 16.39378199739550 & 3.85535949738489 \\
\hline $\mathbf{C u}$ & 8.91820965049921 & 18.91356579349910 & 3.85888372983123 \\
\hline $\mathbf{C u}$ & 8.91819457865124 & 21.44072970301720 & 3.85585151926993 \\
\hline $\mathbf{C u}$ & 8.91819322518790 & 23.96230894678060 & 3.85202165410251 \\
\hline $\mathbf{C u}$ & 8.91819633241757 & 26.49110873465340 & 3.85358466944986 \\
\hline $\mathbf{C u}$ & 8.91818885047318 & 29.00928875004700 & 3.85102881617274 \\
\hline $\mathbf{C u}$ & 8.91818594080468 & 31.53447882077780 & 3.85130486352804 \\
\hline $\mathbf{C u}$ & 8.91818755914514 & 34.05411098229740 & 3.85026691226023 \\
\hline $\mathbf{C u}$ & 0.00137453703498 & -0.00091993956196 & 5.05572114578599 \\
\hline $\mathbf{C u}$ & 0.00211680052407 & 2.52510261797342 & 5.05776942521763 \\
\hline $\mathbf{C u}$ & 10.70110926644600 & 5.04731688714019 & 5.06254899303317 \\
\hline $\mathbf{C u}$ & 0.00311003472844 & 7.56708057549577 & 5.05734013133773 \\
\hline $\mathbf{C u}$ & 0.00364182153072 & 10.09040613050890 & 5.04824343326382 \\
\hline $\mathbf{C u}$ & 0.00821176319324 & 12.60977496613690 & 5.05120895681633 \\
\hline $\mathbf{C u}$ & 0.00616740538958 & 15.13097501811650 & 5.05445712167713 \\
\hline
\end{tabular}




\begin{tabular}{|c|c|c|c|}
\hline $\mathbf{C u}$ & 0.00482469437448 & 17.65400539014290 & 5.06099721211797 \\
\hline $\mathbf{C u}$ & 0.00802223819896 & 20.17644260182300 & 5.05413398340542 \\
\hline $\mathbf{C u}$ & 0.00669172685846 & 22.70495078758320 & 5.05965819495750 \\
\hline $\mathbf{C u}$ & 0.00109820133695 & 25.22320179257250 & 5.05376648639212 \\
\hline $\mathbf{C u}$ & 10.70043486127360 & 27.75037185686380 & 5.06535062525965 \\
\hline $\mathbf{C u}$ & 10.69896915526070 & 30.26929876600190 & 5.05748668902273 \\
\hline $\mathbf{C u}$ & 0.00257932787492 & 32.79469927857660 & 5.05918217075575 \\
\hline $\mathbf{C u}$ & 3.56727708193159 & 35.31376519468440 & 5.06035542625198 \\
\hline $\mathbf{C u}$ & 3.56727282623978 & 2.52393353562742 & 5.05753698768662 \\
\hline $\mathbf{C u}$ & 3.56728431841728 & 5.03984338074086 & 5.061 \\
\hline $\mathbf{C u}$ & 3.56727122287364 & 7.54882998738608 & 5.08656085149020 \\
\hline $\mathbf{C u}$ & 3.56725731862353 & 10.06075868974810 & 5.10818912881328 \\
\hline $\mathbf{C u}$ & 3.56726409474685 & 12.60911127007300 & 5.17154794584428 \\
\hline $\mathrm{Cu}$ & 3.56728867400360 & 15.12472363638880 & 5.09821417529530 \\
\hline $\mathbf{C u}$ & 3.56725910779947 & 17.65359896067870 & 5.13345063077017 \\
\hline $\mathrm{Cu}$ & 3.56726398736831 & 20.18019027768540 & 5.09125208281675 \\
\hline $\mathrm{Cu}$ & 3.56729431813687 & 22.71779625550080 & 5.12493870412246 \\
\hline $\mathbf{C u}$ & 3.56728562819131 & 25.24351489144240 & 5.05864244216885 \\
\hline $\mathbf{C u}$ & 3.56727200102929 & 27.75476508269470 & 5.05873755901609 \\
\hline $\mathbf{C u}$ & 3.56727374262393 & 30.27556485946210 & 5.05944113668193 \\
\hline $\mathbf{C u}$ & 3.56728041877866 & 32.79536544706450 & 5.05971282536720 \\
\hline $\mathbf{C u}$ & 7.13317628384477 & -0.00091324639768 & 5.05572556367794 \\
\hline $\mathbf{C u}$ & 7.13243148803956 & 2.52511252236841 & 5.05776877892522 \\
\hline $\mathbf{C u}$ & 7.13525410674131 & 5.04732396291390 & 5.06255282779246 \\
\hline $\mathbf{C u}$ & 7.13141112946203 & 7.56707099485344 & 5.05736369323214 \\
\hline $\mathbf{C u}$ & 7.13094259624404 & 10.09041474015850 & 5.04827868822773 \\
\hline $\mathbf{C u}$ & 7.12630777344673 & 12.60978066326000 & 5.05120412082920 \\
\hline $\mathbf{C u}$ & 7.12836818819672 & 15.13097583232700 & 5.05447880508115 \\
\hline $\mathbf{C u}$ & 7.12971652828158 & 17.65401123404060 & 5.06098911039073 \\
\hline $\mathbf{C u}$ & 7.12654723011777 & 20.17645211803720 & 5.05415196895314 \\
\hline $\mathbf{C u}$ & 7.12787370462056 & 22.70496858353650 & 5.05965537578602 \\
\hline $\mathbf{C u}$ & 7.13346484254681 & 25.22322205506200 & 5.05376762597688 \\
\hline $\mathbf{C u}$ & 7.13594399312400 & 27.75039409535920 & 5.06536463948860 \\
\hline $\mathbf{C u}$ & 7.13740351209457 & 30.26932506950410 & 5.05749660636162 \\
\hline $\mathbf{C u}$ & 7.13197002748285 & 32.79471941956640 & 5.05917210921660 \\
\hline \multicolumn{4}{|c|}{ CI-NEB Image 5} \\
\hline Element & X-Coordinate (Å) & Y-Coordinate $(\AA)$ & Z-Coordinate (§̊) \\
\hline $\mathbf{F}$ & 4.63201330507736 & 12.16159337887510 & 7.78476570820007 \\
\hline $\mathbf{F}$ & 2.50270463982393 & 12.16159498186920 & 7.78487447294860 \\
\hline $\mathbf{F}$ & 3.56727732403417 & 9.22985865907685 & 6.77930836957561 \\
\hline $\mathbf{F}$ & 4.66322141177495 & 17.65436892884680 & 7.76314990975864 \\
\hline $\mathbf{F}$ & 2.47132148068195 & 17.65427390572970 & 7.76314601439007 \\
\hline $\mathbf{F}$ & 3.56735587993276 & 15.78701844902420 & 7.30571635057313 \\
\hline $\mathbf{F}$ & 4.66369148307675 & 22.75525959046790 & 7.74774001563972 \\
\hline $\mathbf{F}$ & 2.47087064651556 & 22.75526359962070 & 7.74773107812896 \\
\hline $\mathbf{F}$ & 3.56728031032824 & 20.88621688861160 & 7.31120300393248 \\
\hline C & 3.56731911970146 & 12.28135833959640 & 7.00474963830028 \\
\hline C & 3.56729313559876 & 17.21973389550480 & 7.06094575743797 \\
\hline C & 3.56728342899704 & 22.30016374290140 & 7.05223082194107 \\
\hline $\mathbf{C u}$ & 0.00000000000000 & 0.00000000000000 & 0.00000000000000 \\
\hline $\mathrm{Cu}$ & 0.00000000000000 & 2.52244512012115 & 0.00000000000000 \\
\hline $\mathbf{C u}$ & 0.00000000000000 & 5.04488953395804 & 0.00000000000000 \\
\hline $\mathbf{C u}$ & 0.00000000000000 & 7.56733465407884 & 0.00000000000000 \\
\hline $\mathbf{C u}$ & 0.00000000000000 & 10.08977871477400 & 0.00000000000000 \\
\hline
\end{tabular}




\begin{tabular}{|c|c|c|c|}
\hline $\mathbf{C u}$ & 0.00000000000000 & 12.61222383489520 & 0.00000000000000 \\
\hline $\mathbf{C u}$ & 0.00000000000000 & 15.13466895501510 & 0.00000000000000 \\
\hline $\mathbf{C u}$ & 0.00000000000000 & 17.65711336885320 & 0.00000000000000 \\
\hline $\mathbf{C u}$ & 0.00000000000000 & 20.17955848897460 & 0.00000000000000 \\
\hline $\mathbf{C u}$ & 0.00000000000000 & 22.70200254966930 & 0.00000000000000 \\
\hline $\mathbf{C u}$ & 0.00000000000000 & 25.22444766979040 & 0.00000000000000 \\
\hline $\mathbf{C u}$ & 0.00000000000000 & 27.74689208362510 & 0.00000000000000 \\
\hline $\mathbf{C u}$ & 0.00000000000000 & 30.26933720374630 & 0.00000000000000 \\
\hline $\mathbf{C u}$ & 0.00000000000000 & 32.79178232386990 & 0.00000000000000 \\
\hline $\mathbf{C u}$ & 3.56727419238642 & 0.00000000000000 & 0.00000000000000 \\
\hline $\mathbf{C u}$ & 3.56727419238642 & 2.52244512012115 & 0.00000000000000 \\
\hline $\mathbf{C u}$ & 3.56727419238642 & 5.04488953395804 & 0.00000000000000 \\
\hline $\mathbf{C u}$ & 3.56727419238642 & 7.56733465407884 & 0.00000000000000 \\
\hline $\mathbf{C u}$ & 3.56727419238642 & 10.08977871477400 & 0.00000000000000 \\
\hline $\mathbf{C u}$ & 3.56727419238642 & 12.61222383489520 & 0.00000000000000 \\
\hline $\mathbf{C u}$ & 3.56727419238642 & 15.13466895501510 & 0.00000000000000 \\
\hline $\mathbf{C u}$ & 3.56727419238642 & 17.65711336885320 & 0.00000000000000 \\
\hline $\mathbf{C u}$ & 3.56727419238642 & 20.17955848897460 & 0.00000000000000 \\
\hline $\mathbf{C u}$ & 3.56727419238642 & 22.70200254966930 & 0.00000000000000 \\
\hline $\mathbf{C u}$ & 3.56727419238642 & 25.22444766979040 & 0.00000000000000 \\
\hline $\mathbf{C u}$ & 3.56727419238642 & 27.74689208362510 & 0.00000000000000 \\
\hline $\mathbf{C u}$ & 3.56727419238642 & 30.26933720374630 & 0.00000000000000 \\
\hline $\mathbf{C u}$ & 3.56727419238642 & 32.79178232386990 & 0.00000000000000 \\
\hline $\mathbf{C u}$ & 7.13454838477291 & 0.00000000000000 & 0.00000000000000 \\
\hline $\mathbf{C u}$ & 7.13454838477291 & 2.52244512012115 & 0.00000000000000 \\
\hline $\mathbf{C u}$ & 7.13454838477291 & 95804 & 0.00000000000000 \\
\hline $\mathbf{C u}$ & 7.13454838477291 & 7.56733465407884 & 0.00000000000000 \\
\hline $\mathbf{C u}$ & 7.13454838477291 & 10.08977871477400 & 0.00000000000000 \\
\hline $\mathbf{C u}$ & 7.13454838477291 & 12.61222383489520 & 0.00000000000000 \\
\hline $\mathbf{C u}$ & 7.13454838477291 & 15.13466895501510 & 0.00000000000000 \\
\hline $\mathbf{C u}$ & 7.13454838477291 & 17.65711336885320 & 0.00000000000000 \\
\hline $\mathbf{C u}$ & 7.13454838477291 & 20.17955848897460 & 0.00000000000000 \\
\hline $\mathbf{C u}$ & 7.13454838477291 & 22.70200254966930 & 0.00000000000000 \\
\hline $\mathbf{C u}$ & 7.13454838477291 & 25.22444766979040 & 0.00000000000000 \\
\hline $\mathbf{C u}$ & 7.13454838477291 & 27.74689208362510 & 0.00000000000000 \\
\hline $\mathbf{C u}$ & 7.13454838477291 & 30.26933720374630 & 0.00000000000000 \\
\hline $\mathbf{C u}$ & 7.13454838477291 & 32.79178232386990 & 0.00000000000000 \\
\hline $\mathbf{C u}$ & 1.78363955761358 & 1.26122414920059 & 1.26122399999993 \\
\hline $\mathbf{C u}$ & 1.78363955761358 & 3.78366926932194 & 1.26122399999993 \\
\hline $\mathbf{C u}$ & 1.78363955761358 & 6.30611333001662 & 1.26122399999993 \\
\hline $\mathbf{C u}$ & 1.78363955761358 & 8.82855845013778 & 1.26122399999993 \\
\hline $\mathbf{C u}$ & 1.78363955761358 & 11.35100286397460 & 1.26122399999993 \\
\hline $\mathbf{C u}$ & 1.78363955761358 & 13.87344798409430 & 1.26122399999993 \\
\hline $\mathbf{C u}$ & 1.78363955761358 & 16.39589310421720 & 1.26122399999993 \\
\hline $\mathbf{C u}$ & 1.78363955761358 & 18.91833716491180 & 1.26122399999993 \\
\hline $\mathbf{C u}$ & 1.78363955761358 & 21.44078263817520 & 1.26122399999993 \\
\hline $\mathbf{C u}$ & 1.78363955761358 & 23.96322669886980 & 1.26122399999993 \\
\hline $\mathrm{Cu}$ & 1.78363955761358 & 26.48567181899090 & 1.26122399999993 \\
\hline $\mathbf{C u}$ & 1.78363955761358 & 29.00811693911020 & 1.26122399999993 \\
\hline $\mathbf{C u}$ & 1.78363955761358 & 31.53056135294660 & 1.26122399999993 \\
\hline $\mathbf{C u}$ & 1.78363955761358 & 34.05300647307050 & 1.26122399999993 \\
\hline $\mathbf{C u}$ & 5.35091375000000 & 1.26122414920059 & 1.26122399999993 \\
\hline $\mathbf{C u}$ & 5.35091375000000 & 3.78366926932194 & 1.26122399999993 \\
\hline $\mathbf{C u}$ & 5.35091375000000 & 6.30611333001662 & 1.26122399999993 \\
\hline
\end{tabular}




\begin{tabular}{|c|c|c|c|}
\hline $\mathbf{C u}$ & 5.35091375000000 & 8.82855845013778 & 1.26122399999993 \\
\hline $\mathbf{C u}$ & 5.35091375000000 & 11.35100286397460 & 1.26122399999993 \\
\hline $\mathbf{C u}$ & 5.35091375000000 & 13.87344798409430 & 1.26122399999993 \\
\hline $\mathbf{C u}$ & 5.35091375000000 & 16.39589310421720 & 1.26122399999993 \\
\hline $\mathbf{C u}$ & 5.35091375000000 & 18.91833716491180 & 1.26122399999993 \\
\hline $\mathbf{C u}$ & 5.35091375000000 & 21.44078263817520 & 1.26122399999993 \\
\hline $\mathbf{C u}$ & 5.35091375000000 & 23.96322669886980 & 1.26122399999993 \\
\hline $\mathbf{C u}$ & 5.35091375000000 & 26.48567181899090 & 1.26122399999993 \\
\hline $\mathbf{C u}$ & 5.35091375000000 & 29.00811693911020 & 1.26122399999993 \\
\hline $\mathbf{C u}$ & 5.35091375000000 & 31.53056135294660 & 1.26122399999993 \\
\hline $\mathbf{C u}$ & 5.35091375000000 & 34.05300647307050 & 1.26122399999993 \\
\hline $\mathbf{C u}$ & 8.91818794238642 & 1.26122414920059 & 1.26122399999993 \\
\hline $\mathbf{C u}$ & 8.91818794238642 & 3.78366926932194 & 1.26122399999993 \\
\hline $\mathbf{C u}$ & 8.91818794238642 & 6.30611333001662 & 1.26122399999993 \\
\hline $\mathbf{C u}$ & 8.91818794238642 & 8.82855845013778 & 1.26122399999993 \\
\hline $\mathrm{Cu}$ & 8.91818794238642 & 11.35100286397460 & 1.26122399999993 \\
\hline $\mathbf{C u}$ & 8.91818794238642 & 13.87344798409430 & 1.26122399999993 \\
\hline $\mathbf{C u}$ & 8.91818794238642 & 16.39589310421720 & 1.26122399999993 \\
\hline $\mathbf{C u}$ & 8.91818794238642 & 18.91833716491180 & 1.26122399999993 \\
\hline $\mathbf{C u}$ & 8.91818794238642 & 21.44078263817520 & 1.26122399999993 \\
\hline $\mathbf{C u}$ & 8.91818794238642 & 23.96322669886980 & 9999993 \\
\hline $\mathbf{C u}$ & 8.91818794238642 & 26.48567181899090 & 1.26122399999993 \\
\hline $\mathbf{C u}$ & 8.91818794238642 & 29.00811693911020 & 1.26122399999993 \\
\hline $\mathbf{C u}$ & 8.91818794238642 & 31.53056135294660 & 1.26122399999993 \\
\hline $\mathbf{C u}$ & 8.91818794238642 & 34.05300647307050 & 1.26122399999993 \\
\hline $\mathbf{C u}$ & 0.00191588082711 & 0.00080895500717 & 2.53840737775931 \\
\hline $\mathbf{C u}$ & 10.70087342585040 & 2.52505000332411 & 2.54126503560073 \\
\hline $\mathbf{C u}$ & 0.00488697851069 & 5.04622218978454 & 2.54463369889166 \\
\hline $\mathbf{C u}$ & -0.00013086131513 & 7.56462215565302 & 2.54302082838820 \\
\hline $\mathbf{C u}$ & 10.69855959134130 & 10.08936329740420 & 2.53875785247986 \\
\hline $\mathbf{C u}$ & 10.69978869337150 & 12.61576133314350 & 2.54455249120918 \\
\hline $\mathbf{C u}$ & 10.69804372314240 & 15.13513026941380 & 2.53780656221241 \\
\hline $\mathbf{C u}$ & 10.69895024889260 & 17.65699533273840 & 2.54519753052998 \\
\hline $\mathbf{C u}$ & 10.69674674625650 & 20.17835862255010 & 2.53808538745251 \\
\hline $\mathbf{C u}$ & 10.69686982026830 & 22.70260666005890 & 2.54627559012773 \\
\hline $\mathbf{C u}$ & 10.70018993342190 & 25.22662892342840 & 2.53882726757326 \\
\hline $\mathbf{C u}$ & 10.70048688380390 & 27.74731776119110 & 2.54373203115860 \\
\hline $\mathbf{C u}$ & 10.70239891623180 & 30.26932276812360 & 2.54168166578862 \\
\hline $\mathbf{C u}$ & 10.70122109375990 & 32.79297486192090 & 2.54145418001030 \\
\hline $\mathbf{C u}$ & 3.56727338870605 & 0.00323595191007 & 2.54230008371966 \\
\hline $\mathbf{C u}$ & 3.56727464326706 & 2.52471496021942 & 2.54249475482593 \\
\hline $\mathbf{C u}$ & 3.56727785276069 & 5.05041901496172 & 2.54221166809545 \\
\hline $\mathbf{C u}$ & 3.56726976298249 & 7.57632412577575 & 2.58626475945352 \\
\hline $\mathbf{C u}$ & 3.56726694652642 & 10.09376312474380 & 2.58699372776523 \\
\hline $\mathbf{C u}$ & 3.56727193859929 & 12.62011714438770 & 2.59097148322114 \\
\hline $\mathbf{C u}$ & 3.56727630894069 & 15.14310848593070 & 2.58352326771860 \\
\hline $\mathbf{C u}$ & 3.56727824832022 & 17.65250386895920 & 2.58346462601197 \\
\hline $\mathbf{C u}$ & 3.56728132650332 & 20.17689205601480 & 2.57906707888954 \\
\hline $\mathbf{C u}$ & 3.56728087962677 & 22.69836714810120 & 2.57721487637464 \\
\hline $\mathbf{C u}$ & 3.56728039786809 & 25.22072573297570 & 2.55290426082539 \\
\hline $\mathbf{C u}$ & 3.56728248157498 & 27.74879734984780 & 2.54222246907550 \\
\hline $\mathbf{C u}$ & 3.56727795564545 & 30.27084299790910 & 2.54262308064081 \\
\hline $\mathbf{C u}$ & 3.56728168819943 & 32.79097606594430 & 2.54285779146227 \\
\hline $\mathbf{C u}$ & 7.13263514041927 & 0.00081820723061 & 2.53842330008242 \\
\hline
\end{tabular}




\begin{tabular}{|c|c|c|c|}
\hline $\mathbf{C u}$ & 7.13549981230974 & 2.52507179723914 & 2.54125566226905 \\
\hline $\mathbf{C u}$ & 7.12965393617331 & 5.04623184945550 & 2.54463435436386 \\
\hline $\mathrm{Cu}$ & 7.13467347219719 & 7.56462819416639 & 2.54302514722548 \\
\hline $\mathbf{C u}$ & 7.13780087879736 & 10.08936797064690 & 2.53875624654172 \\
\hline $\mathbf{C u}$ & 7.13658624418637 & 12.61575732558630 & 2.54455718826521 \\
\hline $\mathbf{C u}$ & 7.13833646811961 & 15.13512839831270 & 2.53781484278182 \\
\hline $\mathbf{C u}$ & 7.13743178304277 & 17.65700144631870 & 2.54520257934919 \\
\hline $\mathbf{C u}$ & 7.13963391161733 & 20.17836280162740 & 2.53810260311265 \\
\hline $\mathbf{C u}$ & 7.13951252485539 & 22.70260487681750 & 2.54628539575104 \\
\hline $\mathbf{C u}$ & 7.13619619931016 & 25.22662937358880 & 2.53883098648039 \\
\hline $\mathbf{C u}$ & 7.13589531534080 & 27.74730988817170 & 2.54373580136040 \\
\hline $\mathbf{C u}$ & 7.13397848520652 & 30.26932297151500 & 2.54169155875227 \\
\hline $\mathbf{C u}$ & 7.13515049201890 & 32.79297971778620 & 2.54145976626821 \\
\hline $\mathrm{Cu}$ & 1.78496313601661 & 1.26518102723186 & 3.85318908112084 \\
\hline $\mathbf{C u}$ & 1.78468503040225 & 3.79137843709807 & 3.85612017441862 \\
\hline $\mathbf{C u}$ & 1.80194527150832 & 6.32000566631885 & 3.87262536510184 \\
\hline $\mathbf{C u}$ & 1.78097358284376 & 8.82882093317834 & 3.83934330201155 \\
\hline $\mathbf{C u}$ & 1.78301178356064 & 11.35375926843040 & 3.83497701387744 \\
\hline $\mathbf{C u}$ & 1.78668984551079 & 13.88019794200350 & 3.85023668232665 \\
\hline $\mathbf{C u}$ & 1.77450975686824 & 16.39736658542000 & 3.84292767709691 \\
\hline $\mathbf{C u}$ & 1.78657065425552 & 18.92167785781700 & 3.85031292037424 \\
\hline $\mathbf{C u}$ & 1.77528614754897 & 21.43873880927160 & 3.83815169264032 \\
\hline $\mathbf{C u}$ & 1.78555982321972 & 23.96781669554350 & 56064825 \\
\hline $\mathbf{C u}$ & 1.77973392730917 & 26.4896 & 6132003 \\
\hline $\mathbf{C u}$ & 5288785 & 29.010520 & 70703 \\
\hline $\mathbf{C u}$ & 1.78226472978971 & 31.53330472641240 & 3.85649915441791 \\
\hline $\mathbf{C u}$ & 1.78476631527821 & 34.05454411211090 & 3.85368690866029 \\
\hline $\mathbf{C u}$ & 5.34958913279282 & 1.26517815394392 & 3.85318617883176 \\
\hline $\mathbf{C u}$ & 5.34985607989143 & 3.79137615829199 & 3.85612426856407 \\
\hline $\mathbf{C u}$ & 5.33258558376713 & 6.32001097347625 & 3.87262247850815 \\
\hline $\mathbf{C u}$ & 5.35355773323670 & 8.82882783479888 & 3.83934820986410 \\
\hline $\mathbf{C u}$ & 5.35153129853263 & 11.35376189386760 & 3.83497305310970 \\
\hline $\mathbf{C u}$ & 5.34785862214560 & 13.88020022812190 & 3.85024746773042 \\
\hline $\mathbf{C u}$ & 5.36003430224063 & 16.39738917839460 & 3.84292202441449 \\
\hline $\mathbf{C u}$ & 5.34798462334065 & 18.92168467118270 & 3.85031998017331 \\
\hline $\mathbf{C u}$ & 5.35927111477643 & 21.43874127322360 & 3.83817611913628 \\
\hline $\mathbf{C u}$ & 5.34899189605165 & 23.96781725279300 & 3.84382479900548 \\
\hline $\mathbf{C u}$ & 5.35483325578131 & 26.48961321345380 & 3.85630807996424 \\
\hline $\mathbf{C u}$ & 5.35125530641067 & 29.01051624218970 & 3.85654771224680 \\
\hline $\mathbf{C u}$ & 5.35228069588910 & 31.53330757512520 & 3.85650001081846 \\
\hline $\mathbf{C u}$ & 5.34977953959132 & 34.05455260041620 & 3.85368606544338 \\
\hline $\mathbf{C u}$ & 8.91818092002029 & 1.26373515410385 & 3.85032995558326 \\
\hline $\mathbf{C u}$ & 8.91818292257135 & 3.78647112980060 & 3.85138110343055 \\
\hline $\mathbf{C u}$ & 8.91817974047386 & 6.30423457313869 & 3.85276143199420 \\
\hline $\mathbf{C u}$ & 8.91819098765070 & 8.82639975818703 & 3.85736926094228 \\
\hline $\mathbf{C u}$ & 8.91818651201346 & 11.35410457182560 & 3.84928768445515 \\
\hline $\mathbf{C u}$ & 8.91818702348669 & 13.87368036658140 & 3.85402204753193 \\
\hline $\mathbf{C u}$ & 8.91818856279864 & 16.39461889965250 & 3.85546698779817 \\
\hline $\mathbf{C u}$ & 8.91819811941751 & 18.91413181731720 & 3.85894817086552 \\
\hline $\mathbf{C u}$ & 8.91818508333020 & 21.44102687319200 & 3.85579137060012 \\
\hline $\mathbf{C u}$ & 8.91819472761166 & 23.96260524289920 & 3.85157737837149 \\
\hline $\mathbf{C u}$ & 8.91819945084656 & 26.49181522872990 & 3.85442623480663 \\
\hline $\mathbf{C u}$ & 8.91818629557956 & 29.00984339931840 & 3.85075760872687 \\
\hline $\mathbf{C u}$ & 8.91818697152143 & 31.53492308909540 & 3.85109312686056 \\
\hline
\end{tabular}




\begin{tabular}{|c|c|c|c|}
\hline $\mathbf{C u}$ & 8.91818963249948 & 34.05410507712440 & 3.85025389351291 \\
\hline $\mathbf{C u}$ & 0.00160947290193 & 0.00096978791744 & 5.05508570275802 \\
\hline $\mathrm{Cu}$ & 0.00370841874638 & 2.52607453253654 & 5.05713183072114 \\
\hline $\mathrm{Cu}$ & 10.70475322750990 & 5.05155951166764 & 5.05977949921876 \\
\hline $\mathbf{C u}$ & 0.00395791904679 & 7.56961251396644 & 5.05765532675323 \\
\hline $\mathbf{C u}$ & 0.00421165997533 & 10.09281931545550 & 5.04756511248216 \\
\hline $\mathbf{C u}$ & 0.00730148555582 & 12.61122306554680 & 5.05107855822163 \\
\hline $\mathrm{Cu}$ & 0.00464582018790 & 15.13235173742600 & 5.05534104289795 \\
\hline $\mathbf{C u}$ & 0.00318216280180 & 17.65551469048190 & 5.06184487470891 \\
\hline $\mathrm{Cu}$ & 0.00784843879618 & 20.17685414141030 & 5.05404789081419 \\
\hline $\mathbf{C u}$ & 0.00758060069126 & 22.70492588704830 & 5.05933113302886 \\
\hline $\mathbf{C u}$ & 0.00150171517442 & 25.22383460242930 & 5.05366166648212 \\
\hline $\mathbf{C u}$ & 10.70129092153210 & 27.75107721720000 & 5.06418568934033 \\
\hline $\mathrm{Cu}$ & 10.69965143347270 & 30.26999233394820 & 5.05680139414763 \\
\hline $\mathrm{Cu}$ & 0.00280591590787 & 32.79577738706700 & 5.05906672693927 \\
\hline $\mathrm{Cu}$ & 3.56726726353348 & 35.31851307084530 & 5.06051515758407 \\
\hline $\mathrm{Cu}$ & 3.56727555690625 & 20836120 & 5.05804111664172 \\
\hline $\mathrm{Cu}$ & 3.56726452922751 & 50590856 & 5.06425206516276 \\
\hline $\mathrm{Cu}$ & 3.56726068044633 & 7.62097880904379 & 5.20299886474875 \\
\hline $\mathrm{Cu}$ & 6275762913 & 10.14173000040580 & 5.11229202928307 \\
\hline $\mathbf{C u}$ & 3.56725603407754 & 12.63670864101660 & 5.15756839845264 \\
\hline $\mathbf{C u}$ & 3.56727344780659 & 15.12945209484230 & 5.10017052153442 \\
\hline $\mathrm{Cu}$ & 3.56726658639015 & 17.65507446445750 & 5.13390769945860 \\
\hline $\mathrm{Cu}$ & 3.56728938751936 & 20.18024057872180 & 5.09269516713325 \\
\hline $\mathrm{Cu}$ & 3.56727538169276 & 22.71918844301000 & 5.12516262157804 \\
\hline $\mathrm{Cu}$ & 3.56728092170708 & 25.24445268688940 & 5.05853703128594 \\
\hline $\mathrm{Cu}$ & 3.56728273962720 & 27.75504963863110 & 5.05821443368596 \\
\hline $\mathrm{Cu}$ & 3.56727175107678 & 30.27719855046790 & 5.05884916691814 \\
\hline $\mathrm{Cu}$ & 3.56726761424572 & 32.79692561674600 & 5.05937518968854 \\
\hline $\mathrm{Cu}$ & 7.13293712595928 & 0.00096453038886 & 5.05513547174170 \\
\hline $\mathrm{Cu}$ & 7.13084406843173 & 2.52607983378236 & 5.05711461428742 \\
\hline $\mathrm{Cu}$ & 7.13160273757135 & 5.05157082153637 & 5.05977487126957 \\
\hline $\mathrm{Cu}$ & 7.13057066397109 & 7.56962017601127 & 5.05765257322705 \\
\hline $\mathrm{Cu}$ & 7.13033280193109 & 10.09282347237930 & 5.04754767723205 \\
\hline $\mathrm{Cu}$ & 7.12723436299951 & 12.61122462905420 & 5.05108234010761 \\
\hline $\mathbf{C u}$ & 7.12990201562685 & 15.13233931212520 & 5.05533666666653 \\
\hline $\mathbf{C u}$ & 7.13138337231939 & 17.65552311379100 & 5.06184054503676 \\
\hline $\mathbf{C u}$ & 7.12672368630146 & 20.17685702891190 & 5.05404921197273 \\
\hline $\mathrm{Cu}$ & 7.12698278479956 & 22.70495202334300 & 5.05932558991400 \\
\hline $\mathrm{Cu}$ & 7.13307702890205 & 25.22384473954200 & 5.05365615345990 \\
\hline $\mathbf{C u}$ & 7.13510881250426 & 27.75108877681200 & 5.06419399895231 \\
\hline $\mathrm{Cu}$ & 9157753 & 30.27000438172050 & 5.05681102515048 \\
\hline $\mathrm{Cu}$ & 7.13174901802789 & 32.79577551081460 & 5.05906148166018 \\
\hline \multicolumn{4}{|c|}{ CI-NEB Image 6} \\
\hline Element & X-Coordinate $(\AA)$ & Y-Coordinate $(\AA)$ & Z-Coordinate (§̊) \\
\hline $\mathbf{F}$ & 4.63711309274412 & 11.94705934749190 & 7.69326522259379 \\
\hline $\mathbf{F}$ & 2.49760092375465 & 11.94709096806080 & 7.69337649714914 \\
\hline $\mathbf{F}$ & 3.56727851612406 & 8.83978539353762 & 6.68789649575735 \\
\hline $\mathbf{F}$ & 4.66353617545645 & 17.66595130440430 & 7.75890388545991 \\
\hline $\mathbf{F}$ & 2.47100421972444 & 17.66594975062290 & 7.75889061012886 \\
\hline $\mathbf{F}$ & 3.56727533925388 & 15.79716803077200 & 7.31300990884275 \\
\hline $\mathbf{F}$ & 4.66375492464511 & 22.75714006755040 & 7.74509255259777 \\
\hline $\mathbf{F}$ & 2.47078696676143 & 22.75712757355480 & 7.74508359048969 \\
\hline $\mathbf{F}$ & 3.56728403826846 & 20.88776126681160 & 7.31191904573972 \\
\hline
\end{tabular}




\begin{tabular}{|c|c|c|c|}
\hline C & 3.56731748847443 & 12.06712024360390 & 6.90466805947595 \\
\hline C & 3.56727471103778 & 17.22441159410400 & 7.06052570207824 \\
\hline C & 3.56727646927564 & 22.30039161805650 & 7.05065469077649 \\
\hline $\mathbf{C u}$ & 0.00000000000000 & 0.00000000000000 & 0.00000000000000 \\
\hline $\mathrm{Cu}$ & 0.00000000000000 & 2.52244512012115 & 0.00000000000000 \\
\hline $\mathbf{C u}$ & 0.00000000000000 & 5.04488953395804 & 0.00000000000000 \\
\hline $\mathrm{Cu}$ & 0.00000000000000 & 7.56733465407884 & 0.00000000000000 \\
\hline $\mathbf{C u}$ & 0.00000000000000 & 10.08977871477400 & 0.00000000000000 \\
\hline $\mathbf{C u}$ & 0.00000000000000 & 12.61222383489520 & 0.00000000000000 \\
\hline $\mathbf{C u}$ & 0.00000000000000 & 15.13466895501510 & 0.00000000000000 \\
\hline $\mathbf{C u}$ & 0.00000000000000 & 17.65711336885320 & 0.00000000000000 \\
\hline $\mathbf{C u}$ & 0.00000000000000 & 20.17955848897460 & 0.00000000000000 \\
\hline $\mathbf{C u}$ & 0.00000000000000 & 22.70200254966930 & 0.00000000000000 \\
\hline $\mathbf{C u}$ & 0.00000000000000 & 25.22444766979040 & 0.00000000000000 \\
\hline $\mathbf{C u}$ & 0.00000000000000 & 27.74689208362510 & 0.00000000000000 \\
\hline $\mathbf{C u}$ & 0.00000000000000 & 30.26933720374630 & 0.00000000000000 \\
\hline $\mathbf{C u}$ & 0.00000000000000 & 32.79178232386990 & 0.00000000000000 \\
\hline $\mathbf{C u}$ & 3.56727419238642 & 0.00000000000000 & 0.00000000000000 \\
\hline $\mathbf{C u}$ & 3.56727419238642 & 2.52244512012115 & 0.00000000000000 \\
\hline $\mathbf{C u}$ & 3.56727419238642 & 5.04488953395804 & 0.00000000000000 \\
\hline $\mathbf{C u}$ & 3.56727419238642 & 7.56733465407884 & 0.00000000000000 \\
\hline $\mathrm{Cu}$ & 3.56727419238642 & 10.08977871477400 & 0.00000000000000 \\
\hline $\mathbf{C u}$ & 3.56727419238642 & 12.61222383489520 & 0.00000000000000 \\
\hline $\mathbf{C u}$ & 3.56727419238642 & 15.13466895501510 & 0.00000000000000 \\
\hline $\mathbf{C u}$ & 3.56727419238642 & 17.65711336885320 & 0.00000000000000 \\
\hline $\mathbf{C u}$ & 3.56727419238642 & 20.17955848897460 & 0.00000000000000 \\
\hline $\mathbf{C u}$ & 3.56727419238642 & 22.70200254966930 & 0.00000000000000 \\
\hline $\mathbf{C u}$ & 3.56727419238642 & 25.22444766979040 & 0.00000000000000 \\
\hline $\mathbf{C u}$ & 3.56727419238642 & 27.74689208362510 & 0.00000000000000 \\
\hline $\mathrm{Cu}$ & 3.56727419238642 & 30.26933720374630 & 0.00000000000000 \\
\hline $\mathbf{C u}$ & 3.56727419238642 & 86990 & 0.00000000000000 \\
\hline $\mathbf{C u}$ & 7.13454838477291 & 0.00000000000000 & 0.00000000000000 \\
\hline $\mathbf{C u}$ & 7.13454838477291 & 2.52244512012115 & 0.00000000000000 \\
\hline $\mathbf{C u}$ & 7.13454838477291 & 5.04488953395804 & 0.00000000000000 \\
\hline $\mathbf{C u}$ & 7.13454838477291 & 7.56733465407884 & 0.00000000000000 \\
\hline $\mathbf{C u}$ & 7.13454838477291 & 10.08977871477400 & 0.00000000000000 \\
\hline $\mathrm{Cu}$ & 7.13454838477291 & 12.61222383489520 & 0.00000000000000 \\
\hline $\mathbf{C u}$ & 7.13454838477291 & 15.13466895501510 & 0.00000000000000 \\
\hline $\mathbf{C u}$ & 7.13454838477291 & 17.65711336885320 & 0.00000000000000 \\
\hline $\mathbf{C u}$ & 7.13454838477291 & 20.17955848897460 & 0.00000000000000 \\
\hline $\mathbf{C u}$ & 7.13454838477291 & 22.70200254966930 & 0.00000000000000 \\
\hline $\mathbf{C u}$ & 7.13454838477291 & 25.22444766979040 & 0.00000000000000 \\
\hline $\mathbf{C u}$ & 7.13454838477291 & 27.74689208362510 & 0.00000000000000 \\
\hline $\mathbf{C u}$ & 7.13454838477291 & 30.26933720374630 & 0.00000000000000 \\
\hline $\mathbf{C u}$ & 7.13454838477291 & 32.79178232386990 & 0.00000000000000 \\
\hline $\mathbf{C u}$ & 1.78363955761358 & 1.26122414920059 & 1.26122399999993 \\
\hline $\mathbf{C u}$ & 1.78363955761358 & 3.78366926932194 & 1.26122399999993 \\
\hline $\mathbf{C u}$ & 1.78363955761358 & 6.30611333001662 & 1.26122399999993 \\
\hline $\mathbf{C u}$ & 1.78363955761358 & 8.82855845013778 & 1.26122399999993 \\
\hline $\mathbf{C u}$ & 1.78363955761358 & 11.35100286397460 & 1.26122399999993 \\
\hline $\mathbf{C u}$ & 1.78363955761358 & 13.87344798409430 & 1.26122399999993 \\
\hline $\mathrm{Cu}$ & 1.78363955761358 & 16.39589310421720 & 1.26122399999993 \\
\hline $\mathrm{Cu}$ & 1.78363955761358 & 18.91833716491180 & 1.26122399999993 \\
\hline $\mathbf{C u}$ & 1.78363955761358 & 21.44078263817520 & 1.26122399999993 \\
\hline
\end{tabular}




\begin{tabular}{|c|c|c|c|}
\hline $\mathrm{Cu}$ & 1.78363955761358 & 23.96322669886980 & 1.26122399999993 \\
\hline $\mathrm{Cu}$ & 1.78363955761358 & 26.48567181899090 & 1.26122399999993 \\
\hline $\mathbf{C u}$ & 1.78363955761358 & 29.00811693911020 & 1.26122399999993 \\
\hline $\mathrm{Cu}$ & 1.78363955761358 & 31.53056135294660 & 1.26122399999993 \\
\hline $\mathbf{C u}$ & 1.78363955761358 & 34.05300647307050 & 1.26122399999993 \\
\hline $\mathbf{C u}$ & 5.35091375000000 & 1.26122414920059 & 1.26122399999993 \\
\hline $\mathbf{C u}$ & 5.35091375000000 & 3.78366926932194 & 1.26122399999993 \\
\hline $\mathrm{Cu}$ & 5.35091375000000 & 6.30611333001662 & 1.26122399999993 \\
\hline $\mathbf{C u}$ & 5.35091375000000 & 8.82855845013778 & 1.26122399999993 \\
\hline $\mathbf{C u}$ & 5.35091375000000 & 11.35100286397460 & 1.26122399999993 \\
\hline $\mathbf{C u}$ & 5.35091375000000 & 13.87344798409430 & 1.26122399999993 \\
\hline $\mathbf{C u}$ & 5.35091375000000 & 16.39589310421720 & 1.26122399999993 \\
\hline $\mathbf{C u}$ & 5.35091375000000 & 18.91833716491180 & 1.26122399999993 \\
\hline $\mathbf{C u}$ & 5.35091375000000 & 21.44078263817520 & 1.26122399999993 \\
\hline $\mathrm{Cu}$ & 5.35091375000000 & 23.96322669886980 & 1.26122399999993 \\
\hline $\mathrm{Cu}$ & 5.35091375000000 & 26.48567181899090 & 1.26122399999993 \\
\hline $\mathrm{Cu}$ & 5.35091375000000 & 29.00811693911020 & 1.26122399999993 \\
\hline $\mathbf{C u}$ & 5.35091375000000 & 31.53056135294660 & 1.26122399999993 \\
\hline $\mathrm{Cu}$ & 5.35091375000000 & 34.05300647307050 & 1.26122399999993 \\
\hline $\mathbf{C u}$ & 8.91818794238642 & 2414920059 & 1.2612239 \\
\hline $\mathbf{C u}$ & 8.91818794238642 & 32194 & 93 \\
\hline $\mathbf{C u}$ & 8.91818794238642 & 662 & 1.2 \\
\hline $\mathbf{C u}$ & 4238642 & 8.82855845013778 & 1.26122399999993 \\
\hline $\mathrm{Cu}$ & 8.91818794238642 & 11.35100286397460 & 1.26122399999993 \\
\hline $\mathrm{Cu}$ & 8.91818794238642 & 13.87344798409430 & 1.26122399999993 \\
\hline $\mathbf{C u}$ & 8.91818794238642 & 16.39589310421720 & 1.26122399999993 \\
\hline $\mathrm{Cu}$ & 8.91818794238642 & 18.91833716491180 & 1.26122399999993 \\
\hline $\mathrm{Cu}$ & 8.91818794238642 & 21.44078263817520 & 1.26122399999993 \\
\hline $\mathrm{Cu}$ & 8.91818794238642 & 23.96322669886980 & 1.26122399999993 \\
\hline $\mathrm{Cu}$ & 8.91818794238642 & 26.48567181899090 & 1.26122399999993 \\
\hline $\mathrm{Cu}$ & 8.91818794238642 & 29.00811693911020 & 1.26122399999993 \\
\hline $\mathrm{Cu}$ & 8.91818794238642 & 31.53056135294660 & 1.26122399999993 \\
\hline $\mathbf{C u}$ & 8.91818794238642 & 34.05300647307050 & 1.26122399999993 \\
\hline $\mathbf{C u}$ & 0.00184939439068 & 0.00241268027978 & 2.53816569764389 \\
\hline $\mathbf{C u}$ & 10.69975077034450 & 2.52691423208635 & 2.54130435932221 \\
\hline $\mathbf{C u}$ & 0.00319499841401 & 5.04877192587052 & 2.54262910971603 \\
\hline $\mathrm{Cu}$ & 0.00182942798144 & 7.56842772874166 & 2.54437556008771 \\
\hline $\mathbf{C u}$ & 10.70121243363270 & 10.09078854091990 & 2.53892993705501 \\
\hline $\mathrm{Cu}$ & 10.70073358115850 & 12.61768432025470 & 2.54459295182112 \\
\hline $\mathbf{C u}$ & 10.69660769912910 & 15.13742710202730 & 2.53711611224832 \\
\hline $\mathrm{Cu}$ & 10.69904520386450 & 17.65926713269300 & 2.54462351512324 \\
\hline $\mathbf{C u}$ & 10.69670027538830 & 20.18021302569030 & 2.53731941515520 \\
\hline $\mathbf{C u}$ & 10.69675495728880 & 22.70441849465110 & 2.54613527068108 \\
\hline $\mathbf{C u}$ & 10.69985066465250 & 25.22805280375440 & 2.53820161336249 \\
\hline $\mathbf{C u}$ & 10.70018437677850 & 27.74817867992880 & 2.54322407220889 \\
\hline $\mathbf{C u}$ & 10.70215020627350 & 30.26993186085510 & 2.54161768133998 \\
\hline $\mathbf{C u}$ & 10.70102003743120 & 32.79359586846690 & 2.54117478119609 \\
\hline $\mathbf{C u}$ & 3.56727400953788 & 0.00566628688274 & 2.54221541120164 \\
\hline $\mathrm{Cu}$ & 3.56727673303957 & 2.52665391767622 & 2.54256041886994 \\
\hline $\mathbf{C u}$ & 3.56727632828431 & 5.05146901879096 & 2.54112412687194 \\
\hline $\mathrm{Cu}$ & 3.56726939492288 & 7.57930498373029 & 2.58994851170870 \\
\hline $\mathbf{C u}$ & 3.56727632876326 & 10.09546058849870 & 2.60626392613129 \\
\hline $\mathbf{C u}$ & 3.56727509794585 & 12.61997225204100 & 2.57484845759094 \\
\hline $\mathrm{Cu}$ & 3.56728348803982 & 15.14590868223620 & 2.58315254 \\
\hline
\end{tabular}




\begin{tabular}{|c|c|c|c|}
\hline $\mathbf{C u}$ & 3.56727960585684 & 17.65504456558390 & 2.58488476970964 \\
\hline $\mathbf{C u}$ & 3.56728205129566 & 20.17922059115150 & 2.57918308899858 \\
\hline $\mathbf{C u}$ & 3.56727961115458 & 22.69903884051440 & 2.57656656891643 \\
\hline $\mathbf{C u}$ & 3.56728184893908 & 25.22080285552850 & 2.55182407267416 \\
\hline $\mathbf{C u}$ & 3.56727755075357 & 27.74926675500470 & 2.54162212561969 \\
\hline $\mathbf{C u}$ & 3.56727517843072 & 30.27170072495750 & 2.54149563906396 \\
\hline $\mathbf{C u}$ & 3.56727745197015 & 32.79341755837620 & 2.54265245234426 \\
\hline $\mathbf{C u}$ & 7.13270291383800 & 0.00240928083280 & 2.53816291536480 \\
\hline $\mathbf{C u}$ & 7.13663054814922 & 2.52690515606940 & 2.54130950740091 \\
\hline $\mathbf{C u}$ & 7.13136123812150 & 5.04876525103315 & 2.54261826778771 \\
\hline $\mathbf{C u}$ & 7.13272039745819 & 7.56841805018454 & 2.54437382539314 \\
\hline $\mathbf{C u}$ & 7.13517569575707 & 10.09077970958720 & 2.53892703525882 \\
\hline $\mathbf{C u}$ & 7.13564336273085 & 12.61769031721370 & 2.54459439607501 \\
\hline $\mathrm{Cu}$ & 7.13978481964558 & 15.13743161467660 & 2.53712168475510 \\
\hline $\mathbf{C u}$ & 7.13733577073518 & 17.65925971068800 & 2.54463255245750 \\
\hline $\mathbf{C u}$ & 7.13967638402018 & 20.18022094086750 & 2.53732982013840 \\
\hline $\mathbf{C u}$ & 7.13962839678082 & 22.70442539912830 & 2.54614198064578 \\
\hline $\mathbf{C u}$ & 7.13652735841986 & 25.22805431335480 & 2.53820548987212 \\
\hline $\mathbf{C u}$ & 7.13619508948009 & 27.74818027784470 & 2.54322454616669 \\
\hline $\mathbf{C u}$ & 7.13422770075185 & 30.26992852751640 & 2.54162848109264 \\
\hline $\mathbf{C u}$ & 7.13535933667979 & 32.79359454854050 & 2.54117508646415 \\
\hline $\mathbf{C u}$ & 1.78495713470674 & 1.26794005953774 & 3.85221715546012 \\
\hline $\mathbf{C u}$ & 1.78145563164011 & 3.79244203851278 & 6428466540 \\
\hline $\mathbf{C u}$ & 179551899 & 07085 & 5068546535 \\
\hline $\mathrm{Cu}$ & 1.79087906098354 & 8.8398 & 330441 \\
\hline $\mathrm{Cu}$ & 1.78426846082271 & 11.35145150118360 & 3933839253 \\
\hline $\mathbf{C u}$ & 1.78437612580952 & 13.88671951773220 & 3.84552439695990 \\
\hline $\mathbf{C u}$ & 1.77382615040580 & 16.40210776535320 & 3.84392544170941 \\
\hline $\mathrm{Cu}$ & 1.78682677504131 & 18.92495074596310 & 3.85052633255841 \\
\hline $\mathrm{Cu}$ & 1.77537152124298 & 21.44133868937620 & 3.83707789119851 \\
\hline $\mathrm{Cu}$ & 1.78613330094274 & 23.97083825094120 & 3.84303240444020 \\
\hline $\mathbf{C u}$ & 1.77983777824404 & 26.49194425408580 & 3.85509988574190 \\
\hline $\mathrm{Cu}$ & 1.78336695812820 & 29.01234065562410 & 3.85549405694443 \\
\hline $\mathbf{C u}$ & 1.78255944111160 & 31.53545873738980 & 3.85585535584924 \\
\hline $\mathbf{C u}$ & 1.78408935071246 & 34.05684277290920 & 3.85334247461696 \\
\hline $\mathbf{C u}$ & 5.34958633374229 & 1.26792833958927 & 3.85221827490387 \\
\hline $\mathbf{C u}$ & 5.35310414447981 & 3.79243431302058 & 3.85405537029688 \\
\hline $\mathbf{C u}$ & 5.33587185040079 & 6.31920782086945 & 3.86472256497543 \\
\hline $\mathbf{C u}$ & 5.34366850072538 & 8.83977846616503 & 3.84888194680833 \\
\hline $\mathbf{C u}$ & 5.35029018969862 & 11.35144949716930 & 3.83647476857453 \\
\hline $\mathbf{C u}$ & 5.35016798383743 & 13.88671425691540 & 3.84554053133233 \\
\hline $\mathbf{C u}$ & 5.36072632713968 & 16.40211368669170 & 3.84395394268039 \\
\hline $\mathbf{C u}$ & 5.34773584966301 & 18.92494300290570 & 3.85051320601741 \\
\hline $\mathbf{C u}$ & 5.35918370479168 & 21.44133551260260 & 3.83709238538871 \\
\hline $\mathrm{Cu}$ & 5.34841930796076 & 23.97083128713540 & 3.84303287409978 \\
\hline $\mathbf{C u}$ & 5.35471314888415 & 26.49194618854670 & 3.85511031335414 \\
\hline $\mathbf{C u}$ & 5.35118924176016 & 29.01233389022570 & 3.85550102536209 \\
\hline $\mathbf{C u}$ & 5.35199729104210 & 31.53545996345580 & 3.85586087218411 \\
\hline $\mathbf{C u}$ & 5.35045204648570 & 34.05684395380180 & 3.85334759421675 \\
\hline $\mathbf{C u}$ & 8.91818952023353 & 1.26833964964196 & 3.84961009674894 \\
\hline $\mathbf{C u}$ & 8.91818524627472 & 3.79172415465018 & 3.85192937230271 \\
\hline $\mathbf{C u}$ & 8.91818575879069 & 6.31171441950883 & 3.85208835524860 \\
\hline $\mathbf{C u}$ & 8.91818365777069 & 8.83310856485849 & 3.85580203996485 \\
\hline $\mathbf{C u}$ & 8.91817873469042 & 11.35885417635330 & 3.84738059892274 \\
\hline
\end{tabular}




\begin{tabular}{|c|c|c|c|}
\hline $\mathrm{Cu}$ & 8.91819521866242 & 13.87657966434390 & 3.85193017689340 \\
\hline $\mathbf{C u}$ & 8.91819650213879 & 16.39921814716960 & 3.85478257305954 \\
\hline $\mathbf{C u}$ & 8.91819130997035 & 18.91845634571470 & 3.85903225414757 \\
\hline $\mathbf{C u}$ & 8.91819076386329 & 21.44324793879980 & 3.85552145346651 \\
\hline $\mathbf{C u}$ & 8.91819611363442 & 23.96412834603250 & 3.85104786842493 \\
\hline $\mathbf{C u}$ & 8.91819194835455 & 26.49287208147120 & 3.85425048175886 \\
\hline $\mathbf{C u}$ & 8.91819119067196 & 29.01053309596390 & 3.85102746905702 \\
\hline $\mathbf{C u}$ & 8.91819135964409 & 31.53593269237960 & 3.85097265644585 \\
\hline $\mathbf{C u}$ & 8.91819503192503 & 34.05544945561120 & 3.84996968944786 \\
\hline $\mathbf{C u}$ & 0.00173076178238 & 0.00520837662679 & 5.05351087953302 \\
\hline $\mathbf{C u}$ & 0.00334889728715 & 2.53007376834140 & 5.05787134880958 \\
\hline $\mathbf{C u}$ & 0.00316519359135 & 5.05959673277275 & 5.05705762918647 \\
\hline $\mathbf{C u}$ & 0.00474392359103 & 7.57753315007250 & 5.05456563866416 \\
\hline $\mathbf{C u}$ & 0.00440964122641 & 10.09803293005820 & 5.04670294818816 \\
\hline $\mathbf{C u}$ & 0.00742912700143 & 12.61653436511220 & 5.05010562078155 \\
\hline $\mathbf{C u}$ & 0.00246541935290 & 15.13708151814420 & 5.05523572009651 \\
\hline $\mathbf{C u}$ & 0.00149853173144 & 17.66074555123020 & 5.06242614696971 \\
\hline $\mathbf{C u}$ & 0.00787706403340 & 20.18031508420360 & 5.05317466039856 \\
\hline $\mathbf{C u}$ & 0.00903901263075 & 22.70699224770850 & 5.05867786580656 \\
\hline $\mathbf{C u}$ & 0.00234731339833 & 25.22546368768250 & 5.05254276646359 \\
\hline $\mathbf{C u}$ & 10.70159848380990 & 27.75249217668340 & 1101531685 \\
\hline $\mathbf{C u}$ & 10.70030169220880 & 30.27170896534430 & 9830139010 \\
\hline $\mathbf{C u}$ & 37385610 & 240515800 & 790995 \\
\hline $\mathbf{C u}$ & 1601980 & 35.32209450208390 & 018535 \\
\hline $\mathbf{C u}$ & 481625691 & 5163 & 61992 \\
\hline $\mathbf{C u}$ & 48802589 & 372 & 3558 \\
\hline $\mathbf{C u}$ & 8489695530 & 792 & 5901 \\
\hline $\mathbf{C u}$ & 54790117 & 916700 & 15287 \\
\hline $\mathbf{C u}$ & 447443134 & 12.64753491580840 & 551875 \\
\hline $\mathbf{C u}$ & 3.56726794697347 & 15.12321528997260 & 5.10574471648343 \\
\hline $\mathbf{C u}$ & 3.56727020253546 & 17.65421350417210 & 5.13276080089597 \\
\hline $\mathbf{C u}$ & 3.56727546563413 & 20.18155986670110 & 5.09268011132182 \\
\hline $\mathbf{C u}$ & 3.56727427611261 & 22.72174055124360 & 5.12394803727552 \\
\hline $\mathbf{C u}$ & 3.56728477755510 & 25.24774885333850 & 5.05760338503628 \\
\hline $\mathbf{C u}$ & 3.56727982673528 & 27.75824073695710 & 5.05690210020977 \\
\hline $\mathbf{C u}$ & 3.56727505313732 & 30.28045863646510 & 5.05712441605876 \\
\hline $\mathbf{C u}$ & 3.56727351014234 & 32.79954850074160 & 5.05823236006708 \\
\hline $\mathbf{C u}$ & 7.13282410232544 & 0.00520663225837 & 5.05349789406608 \\
\hline $\mathbf{C u}$ & 7.13119287633800 & 2.53005991677829 & 5.05787619756155 \\
\hline $\mathbf{C u}$ & 7.13138666188638 & 5.05959933336055 & 5.05705464988550 \\
\hline $\mathbf{C u}$ & 7.12979892562413 & 7.57752751296825 & 5.05456589859089 \\
\hline $\mathbf{C u}$ & 7.13012510707593 & 10.09802760732630 & 5.04669900845336 \\
\hline $\mathbf{C u}$ & 7.12712731151488 & 12.61654774491340 & 5.05010584227551 \\
\hline $\mathbf{C u}$ & 7.13209271911380 & 15.13708918142610 & 5.05523567209349 \\
\hline $\mathbf{C u}$ & 7.13306332256231 & 17.66075529684710 & 5.06243589914939 \\
\hline $\mathbf{C u}$ & 7.12666696934289 & 20.18031689987050 & 5.05317550762912 \\
\hline $\mathbf{C u}$ & 7.12551704855801 & 22.70699793031220 & 5.05867906426569 \\
\hline $\mathrm{Cu}$ & 7.13220756000996 & 25.22547534024070 & 5.05252099552726 \\
\hline $\mathbf{C u}$ & 7.13479035306070 & 27.75249209210070 & 5.06371307830416 \\
\hline $\mathbf{C u}$ & 7.13608253606690 & 30.27171145397200 & 5.05660360671706 \\
\hline $\mathbf{C u}$ & 7.13123175712462 & 32.79754973605590 & 5.05847122125896 \\
\hline \multicolumn{4}{|c|}{ CI-NEB Image 7} \\
\hline Element & X-Coordinate (Å) & Y-Coordinate ( $\AA$ ) & Z-Coordinate ( $(\AA)$ \\
\hline $\mathbf{F}$ & 4.64323994852686 & 11.65383471970930 & 7.58775524678153 \\
\hline
\end{tabular}




\begin{tabular}{|c|c|c|c|}
\hline $\mathbf{F}$ & 2.49133803702215 & 11.65383769484240 & 7.58779975003149 \\
\hline $\mathbf{F}$ & 3.56727507478444 & 8.73791050084457 & 6.69186181328470 \\
\hline $\mathbf{F}$ & 4.66359262732319 & 17.66071936483860 & 7.75375283250083 \\
\hline $\mathbf{F}$ & 2.47094911521080 & 17.66071899754970 & 7.75373217285789 \\
\hline $\mathbf{F}$ & 3.56727598409607 & 15.79016355859120 & 7.31867560857747 \\
\hline $\mathbf{F}$ & 4.66377435349050 & 22.75696038244510 & 7.74642675486386 \\
\hline $\mathbf{F}$ & 2.47075721998508 & 22.75695737397610 & 7.74639818257377 \\
\hline $\mathbf{F}$ & 3.56727610276367 & 20.88638410769670 & 7.31909091134122 \\
\hline C & 3.56727141564128 & 11.72090570359170 & 6.78602487802046 \\
\hline C & 3.56727731699713 & 17.21334034849970 & 7.05889526341718 \\
\hline C & 3.56727853294904 & 22.29733286070510 & 7.05375542314186 \\
\hline $\mathbf{C u}$ & 0.00000000000000 & 0.00000000000000 & 0.00000000000000 \\
\hline $\mathbf{C u}$ & 0.00000000000000 & 2.52244512012115 & 0.00000000000000 \\
\hline $\mathbf{C u}$ & 0.00000000000000 & 5.04488953395804 & 0.00000000000000 \\
\hline $\mathbf{C u}$ & 0.00000000000000 & 7.56733465407891 & 0.00000000000000 \\
\hline $\mathbf{C u}$ & 0.00000000000000 & 10.08977871477400 & 0.00000000000000 \\
\hline $\mathbf{C u}$ & 0.00000000000000 & 12.61222383489520 & 0.00000000000000 \\
\hline $\mathbf{C u}$ & 0.00000000000000 & 15.13466895501500 & 0.00000000000000 \\
\hline $\mathbf{C u}$ & 0.00000000000000 & 17.65711336885320 & 0.00000000000000 \\
\hline $\mathbf{C u}$ & 0.00000000000000 & 20.17955848897460 & 0.00000000000000 \\
\hline $\mathbf{C u}$ & 0.00000000000000 & 22.70200254966930 & 0.00000000000000 \\
\hline $\mathbf{C u}$ & 0.00000000000000 & 25.22444766979040 & 0.00000000000000 \\
\hline $\mathbf{C u}$ & 0.00000000000000 & 27.74689208362520 & 0.00000000000000 \\
\hline $\mathbf{C u}$ & 0.00000000000000 & 30.26933720374630 & 0.00000000000000 \\
\hline $\mathbf{C u}$ & 0.00000000000000 & 32.79178232386990 & 0.00000000000000 \\
\hline $\mathbf{C u}$ & 3.56727419238642 & 0.00000000000000 & 0.00000000000000 \\
\hline $\mathbf{C u}$ & 3.56727419238642 & 2.52244512012115 & 0.00000000000000 \\
\hline $\mathbf{C u}$ & 3.56727419238642 & 5.04488953395804 & 0.00000000000000 \\
\hline $\mathbf{C u}$ & 3.56727419238642 & 7.56733465407891 & 0.00000000000000 \\
\hline $\mathbf{C u}$ & 3.56727419238642 & 10.08977871477400 & 0.00000000000000 \\
\hline $\mathbf{C u}$ & 3.56727419238642 & 12.61222383489520 & 0.0000 \\
\hline $\mathbf{C u}$ & 8642 & 500 & 0000 \\
\hline $\mathbf{C u}$ & 3.56727419238642 & 17.65711336885320 & 0.00000000000000 \\
\hline $\mathbf{C u}$ & 3.56727419238642 & 20.17955848897460 & 0.00000000000000 \\
\hline $\mathbf{C u}$ & 3.56727419238642 & 22.70200254966930 & 0.00000000000000 \\
\hline $\mathbf{C u}$ & 3.56727419238642 & 25.22444766979040 & 0.00000000000000 \\
\hline $\mathrm{Cu}$ & 3.56727419238642 & 27.74689208362520 & 0.00000000000000 \\
\hline $\mathbf{C u}$ & 3.56727419238642 & 30.26933720374630 & 0.00000000000000 \\
\hline $\mathbf{C u}$ & 3.56727419238642 & 32.79178232386990 & 0.00000000000000 \\
\hline $\mathbf{C u}$ & 7.13454838477291 & 0.00000000000000 & 0.00000000000000 \\
\hline $\mathbf{C u}$ & 7.13454838477291 & 2.52244512012115 & 0.00000000000000 \\
\hline $\mathbf{C u}$ & 7.13454838477291 & 5.04488953395804 & 0.00000000000000 \\
\hline $\mathbf{C u}$ & 7.13454838477291 & 7.56733465407891 & 0.00000000000000 \\
\hline $\mathbf{C u}$ & 7.13454838477291 & 10.08977871477400 & 0.00000000000000 \\
\hline $\mathbf{C u}$ & 7.13454838477291 & 12.61222383489520 & 0.00000000000000 \\
\hline $\mathbf{C u}$ & 7.13454838477291 & 15.13466895501500 & 0.00000000000000 \\
\hline $\mathbf{C u}$ & 7.13454838477291 & 17.65711336885320 & 0.00000000000000 \\
\hline $\mathbf{C u}$ & 7.13454838477291 & 20.17955848897460 & 0.00000000000000 \\
\hline $\mathbf{C u}$ & 7.13454838477291 & 22.70200254966930 & 0.00000000000000 \\
\hline $\mathbf{C u}$ & 7.13454838477291 & 25.22444766979040 & 0.00000000000000 \\
\hline $\mathbf{C u}$ & 7.13454838477291 & 27.74689208362520 & 0.00000000000000 \\
\hline $\mathbf{C u}$ & 7.13454838477291 & 30.26933720374630 & 0.00000000000000 \\
\hline $\mathbf{C u}$ & 7.13454838477291 & 32.79178232386990 & 0.00000000000000 \\
\hline $\mathrm{Cu}$ & 1.78363955761358 & 1.26122414920059 & 1.26122399999993 \\
\hline
\end{tabular}




\begin{tabular}{|c|c|c|c|}
\hline $\mathbf{C u}$ & 1.78363955761358 & 3.78366926932198 & 1.26122395 \\
\hline $\mathbf{C u}$ & 1.78363955761358 & 6.30611333001662 & 1.26122399999993 \\
\hline $\mathbf{C u}$ & 1.78363955761358 & 8.82855845013778 & 1.26122399999993 \\
\hline $\mathbf{C u}$ & 1.78363955761358 & 11.35100286397460 & 1.26122399999993 \\
\hline $\mathrm{Cu}$ & 1.78363955761358 & 13.87344798409420 & 1.26122399999993 \\
\hline $\mathbf{C u}$ & 1.78363955761358 & 16.39589310421720 & 1.26122399999993 \\
\hline $\mathrm{Cu}$ & 1.78363955761358 & 18.91833716491180 & 1.26122399999993 \\
\hline $\mathbf{C u}$ & 1.78363955761358 & 21.44078263817520 & 1.26122399999993 \\
\hline $\mathbf{C u}$ & 1.78363955761358 & 23.96322669886980 & 1.26122399999993 \\
\hline $\mathbf{C u}$ & 1.78363955761358 & 26.48567181899090 & 1.26122399999993 \\
\hline $\mathbf{C u}$ & 1.78363955761358 & 29.00811693911030 & 1.26122399999993 \\
\hline $\mathbf{C u}$ & 1.78363955761358 & 31.53056135294660 & 1.26122399999993 \\
\hline $\mathbf{C u}$ & 1.78363955761358 & 34.05300647307050 & 1.26122399999993 \\
\hline $\mathbf{C u}$ & 5.35091375000000 & 1.26122414920059 & 1.26122399999993 \\
\hline $\mathbf{C u}$ & 5.35091375000000 & 3.78366926932198 & 1.26122399999993 \\
\hline $\mathbf{C u}$ & 5.35091375000000 & 6.30611333001662 & 1.26122399999993 \\
\hline $\mathbf{C u}$ & 5.35091375000000 & 8.82855845013778 & 1.26122399999993 \\
\hline $\mathbf{C u}$ & 5.35091375000000 & 11.35100286397460 & 1.26122399999993 \\
\hline $\mathbf{C u}$ & 5.35091375000000 & 13.87344798409420 & 1.26122399999993 \\
\hline $\mathbf{C u}$ & 5.35091375000000 & 16.39589310421720 & 1.26122399999993 \\
\hline $\mathbf{C u}$ & 5.35091375000000 & 18.91833716491180 & 1.26122399999993 \\
\hline $\mathbf{C u}$ & 5.35091375000000 & 21.44078263817520 & 1.26122399999993 \\
\hline $\mathbf{C u}$ & 5.35091375000000 & 23.96322669886980 & 1.26122399999993 \\
\hline $\mathbf{C u}$ & 5.35091375000000 & 26.48567181899090 & 1.26122399999993 \\
\hline $\mathbf{C u}$ & 5.35091375000000 & 29.00811693911030 & 1.26122399999993 \\
\hline $\mathbf{C u}$ & 5.35091375000000 & 31.53056135294660 & 1.26122399999993 \\
\hline $\mathbf{C u}$ & 5.35091375000000 & 34.05300647307050 & 1.26122399999993 \\
\hline $\mathbf{C u}$ & 8.91818794238642 & 1.26122414920059 & 1.26122399999993 \\
\hline $\mathbf{C u}$ & 8.91818794238642 & 3.78366926932198 & 1.26122399999993 \\
\hline $\mathbf{C u}$ & 8.91818794238642 & 6.30611333001662 & 1.26122399999993 \\
\hline $\mathbf{C u}$ & 8.91818794238642 & 8.82855845013778 & 1.26122399999993 \\
\hline $\mathbf{C u}$ & 8.91818794238642 & 11.35100286397460 & 1.26122399999993 \\
\hline $\mathbf{C u}$ & 8.91818794238642 & 13.87344798409420 & 1.26122399999993 \\
\hline $\mathbf{C u}$ & 8.91818794238642 & 16.39589310421720 & 1.26122399999993 \\
\hline $\mathbf{C u}$ & 8.91818794238642 & 18.91833716491180 & 1.26122399999993 \\
\hline $\mathbf{C u}$ & 8.91818794238642 & 21.44078263817520 & 1.26122399999993 \\
\hline $\mathbf{C u}$ & 8.91818794238642 & 23.96322669886980 & 1.26122399999993 \\
\hline $\mathbf{C u}$ & 8.91818794238642 & 26.48567181899090 & 1.26122399999993 \\
\hline $\mathbf{C u}$ & 8.91818794238642 & 29.00811693911030 & 1.26122399999993 \\
\hline $\mathbf{C u}$ & 8.91818794238642 & 31.53056135294660 & 1.26122399999993 \\
\hline $\mathbf{C u}$ & 8.91818794238642 & 34.05300647307050 & 1.26122399999993 \\
\hline $\mathbf{C u}$ & 0.00189777661729 & 0.00023651471671 & 2.53756819942914 \\
\hline $\mathbf{C u}$ & 10.70002382671170 & 2.52374924986340 & 2.54028316556369 \\
\hline $\mathbf{C u}$ & 0.00182192413932 & 5.04603590849299 & 2.54168028005159 \\
\hline $\mathbf{C u}$ & 0.00297784889417 & 7.56580376310957 & 2.54450632473709 \\
\hline $\mathbf{C u}$ & 10.69986943299120 & 10.08662432621960 & 2.53802620266104 \\
\hline $\mathbf{C u}$ & 10.69862257244410 & 12.61534081755970 & 2.54331562999161 \\
\hline $\mathbf{C u}$ & 10.69771116551270 & 15.13484217827400 & 2.53766696415091 \\
\hline $\mathbf{C u}$ & 10.69949861234580 & 17.65698186244510 & 2.54371210123718 \\
\hline $\mathbf{C u}$ & 10.69679141094420 & 20.17857031694280 & 2.53749575730059 \\
\hline $\mathbf{C u}$ & 10.69719804199490 & 22.70325355073080 & 2.54618919791753 \\
\hline $\mathbf{C u}$ & 10.70004312685660 & 25.22719872125810 & 2.53801511336469 \\
\hline $\mathbf{C u}$ & 10.70031012698830 & 27.74806956365110 & 2.54301779557500 \\
\hline $\mathbf{C u}$ & 10.70122869518490 & 30.26892971758060 & 2.54127568001670 \\
\hline
\end{tabular}




\begin{tabular}{|c|c|c|c|}
\hline $\mathrm{Cu}$ & 10.70119505211400 & 32.79158209151930 & 2.54058764301362 \\
\hline $\mathrm{Cu}$ & 3.56727214697599 & 0.00239945986408 & 2.54107116038678 \\
\hline $\mathrm{Cu}$ & 3.56726153621253 & 2.52365795180843 & 2.54134719523535 \\
\hline $\mathbf{C u}$ & 3.56726082017724 & 5.04649161766959 & 2.54148400647373 \\
\hline $\mathbf{C u}$ & 3.56726113351523 & 7.57568758874644 & 2.58778431254338 \\
\hline $\mathbf{C u}$ & 3.56726903468702 & 10.09100116539200 & 2.61786663588752 \\
\hline $\mathbf{C u}$ & 3.56723946829901 & 12.61198305583330 & 2.56140785901405 \\
\hline $\mathrm{Cu}$ & 3.56728352733658 & 15.14439898228500 & 2.58095386003789 \\
\hline $\mathbf{C u}$ & 3.56727902321993 & 17.65264195622520 & 2.58414206091571 \\
\hline $\mathrm{Cu}$ & 3.56727185171820 & 20.17594556590600 & 2.58041128874793 \\
\hline $\mathbf{C u}$ & 3.56727610100913 & 22.69746570684530 & 2.57665406971904 \\
\hline $\mathbf{C u}$ & 3.56728315916320 & 25.21839109451820 & 2.55050024948144 \\
\hline $\mathbf{C u}$ & 3.56730459666801 & 27.74705191904210 & 2.54014352175983 \\
\hline $\mathbf{C u}$ & 3.56727392601087 & 30.27020471443600 & 2.54115082132830 \\
\hline $\mathrm{Cu}$ & 3.56727678636456 & 32.79048304033540 & 2.54156289236270 \\
\hline $\mathbf{C u}$ & 7.13265439681751 & 0.00024394321018 & 2.53756630862621 \\
\hline $\mathbf{C u}$ & 4300372858 & 2.52374151053195 & 30744074 \\
\hline $\mathbf{C u}$ & 7.13273242508471 & 5.04602580805154 & 2.54167 \\
\hline $\mathbf{C u}$ & 6203817894 & 7.56579269950537 & 2.54450305592133 \\
\hline $\mathbf{C u}$ & 7.13649828196479 & 10.08661004510350 & 2.53802568849968 \\
\hline $\mathbf{C u}$ & 7.13775150588781 & 12.61535540033040 & 2.54329064782957 \\
\hline $\mathbf{C u}$ & 7.13863014949311 & 15.13484216618140 & 2.53767476976633 \\
\hline $\mathbf{C u}$ & 7.13688351321246 & 17.65700121009660 & 2.54373293855106 \\
\hline $\mathrm{Cu}$ & 7.13956240693236 & 20.17855960926560 & 2.53742139469733 \\
\hline $\mathbf{C u}$ & 7.13917877120117 & 22.70324524577350 & 2.54617389889190 \\
\hline $\mathrm{Cu}$ & 7.13635748031307 & 25.22717335037200 & 2.53802678928275 \\
\hline $\mathbf{C u}$ & 7.13607366502448 & 27.74808154170450 & 2.54303553921561 \\
\hline $\mathrm{Cu}$ & 7.13513932690202 & 30.26893772126040 & 2.54123014130238 \\
\hline $\mathbf{C u}$ & 7.13518517229178 & 32.79159474252930 & 2.54058548418094 \\
\hline $\mathbf{C u}$ & 1.78525944323059 & 1.26153505141384 & 3.85148408120279 \\
\hline $\mathbf{C u}$ & 1.77996094978959 & 3.78490195284303 & 3.85291584163240 \\
\hline $\mathrm{Cu}$ & 1.79435236394381 & 6.30865894292417 & 3.85972946486322 \\
\hline $\mathrm{Cu}$ & 1.79661988248453 & 8.83577665854417 & 3.85545964654930 \\
\hline $\mathbf{C u}$ & 1.77533682645173 & 11.34140216245280 & 3.82793166053048 \\
\hline $\mathbf{C u}$ & 1.78757655444078 & 13.87752546077780 & 3.84559809327525 \\
\hline $\mathbf{C u}$ & 1.77535934681245 & 16.39460996580730 & 3.84289113812355 \\
\hline $\mathrm{Cu}$ & 1.78748036460945 & 18.92011381857680 & 3.85037920389261 \\
\hline $\mathbf{C u}$ & 1.77589111700690 & 21.43718636012070 & 3.83799828652531 \\
\hline $\mathbf{C u}$ & 1.78672816906390 & 23.96755600581740 & 3.84263254815026 \\
\hline $\mathbf{C u}$ & 1.77936718155512 & 26.48969837893080 & 3.85516475850810 \\
\hline $\mathrm{Cu}$ & 1.78177035649868 & 29.01003255407260 & 3.85490462214419 \\
\hline $\mathrm{Cu}$ & 1.78268154062318 & 31.53038761645320 & 3.85598025116957 \\
\hline $\mathrm{Cu}$ & 1.78463229546183 & 34.05156403902870 & 3.85202677245029 \\
\hline $\mathbf{C u}$ & 5.34926808825477 & 1.26154911758861 & 3.85152614333737 \\
\hline $\mathbf{C u}$ & 5.35460549564771 & 3.78491186715684 & 3.85298338517333 \\
\hline $\mathbf{C u}$ & 5.34015197431773 & 6.30863634779191 & 3.85970390460125 \\
\hline $\mathbf{C u}$ & 5.33788927131594 & 8.83576333830749 & 3.85545571839235 \\
\hline $\mathbf{C u}$ & 5.35922509107461 & 11.34143783142570 & 3.82796743034043 \\
\hline $\mathbf{C u}$ & 5.34697219970290 & 13.87752254474880 & 3.84560620179481 \\
\hline $\mathrm{Cu}$ & 5.35924570419570 & 16.39458226759260 & 3.84292761413856 \\
\hline $\mathbf{C u}$ & 5.34705321146205 & 18.92009780199030 & 3.85037594704888 \\
\hline $\mathbf{C u}$ & 5.35862852602293 & 21.43716065268500 & 3.83799342144123 \\
\hline $\mathbf{C u}$ & 5.34784454869821 & 23.96756036780710 & 3.84262023860382 \\
\hline $\mathbf{C u}$ & 5.35519417196963 & 26.48971807185700 & 3.85519679524117 \\
\hline
\end{tabular}




\begin{tabular}{|c|c|c|c|}
\hline $\mathrm{Cu}$ & 5.35275640316507 & 29.01002156090800 & 3.85491460807882 \\
\hline $\mathrm{Cu}$ & 5.35187640216614 & 31.53038874382690 & 3.85598833483176 \\
\hline $\mathrm{Cu}$ & 5.34990504542845 & 34.05156840182690 & 3.85202207582500 \\
\hline $\mathbf{C u}$ & 8.91819604437574 & 1.26280696931304 & 3.85019869215164 \\
\hline $\mathbf{C u}$ & 8.91819346979287 & 3.78566936828035 & 3.85259079818950 \\
\hline $\mathbf{C u}$ & 8.91813003419290 & 6.30505219994452 & 3.85155999729022 \\
\hline $\mathrm{Cu}$ & 8.91819075297781 & 8.82598069237652 & 3.85655326419164 \\
\hline $\mathrm{Cu}$ & 8.91821672109995 & 11.35470449970150 & 3.84784198051104 \\
\hline $\mathbf{C u}$ & 8.91816750964304 & 13.87159539233910 & 3.85274526273650 \\
\hline $\mathbf{C u}$ & 8.91820194082056 & 16.39349464078360 & 3.85394457674238 \\
\hline $\mathbf{C u}$ & 8.91820414636900 & 18.91498852798850 & 3.85919844480273 \\
\hline $\mathrm{Cu}$ & 8.91819893397700 & 21.44188450565490 & 3.85614861238808 \\
\hline $\mathrm{Cu}$ & 8.91819259805895 & 23.96133069150070 & 3.85124230804167 \\
\hline $\mathrm{Cu}$ & 8.91820195449598 & 26.49045912099970 & 3.85478374093182 \\
\hline $\mathbf{C u}$ & 8.91819665243468 & 29.00887147963560 & 3.85159216463605 \\
\hline $\mathrm{Cu}$ & 8.91818120049725 & 31.53488431570910 & 3.85146996375328 \\
\hline $\mathrm{Cu}$ & 8.91820535052112 & 34.05378320856250 & 3.85132228379705 \\
\hline $\mathbf{C u}$ & 0.00423948768540 & 0.00034608680867 & 5.05361373442553 \\
\hline $\mathbf{C u}$ & 0.00478360554058 & 2.52403984185460 & 5.05584534119252 \\
\hline $\mathrm{Cu}$ & 0.00112487015289 & 5.05217737349671 & 5.05653098076878 \\
\hline $\mathrm{Cu}$ & 0.00469543941456 & 7.57070036472476 & 37310 \\
\hline $\mathbf{C u}$ & 0.00285092873132 & 10.09127401767140 & 550150 \\
\hline $\mathrm{Cu}$ & 0.00761605801670 & 12.61143239779990 & 5.05056659966335 \\
\hline $\mathrm{Cu}$ & 0.00376464915909 & 15.13123049930580 & 5.05521947701376 \\
\hline $\mathrm{Cu}$ & 0.00277396837118 & 17.65599251066200 & 5.05828048255501 \\
\hline $\mathrm{Cu}$ & 0.00795648953301 & 20.17739062354920 & 5.05311653677610 \\
\hline $\mathbf{C u}$ & 0.01037510866749 & 22.70412374786820 & 5.05866099247067 \\
\hline $\mathrm{Cu}$ & 0.00340778998573 & 25.22235340185180 & 5.05087786676195 \\
\hline $\mathbf{C u}$ & 10.70017995907570 & 27.75003240072280 & 5.06435698097685 \\
\hline $\mathrm{Cu}$ & 10.69859936970990 & 30.26846948284700 & 5.05719344920934 \\
\hline $\mathrm{Cu}$ & 0.00454559159546 & 32.79411529077570 & 5.05721682124359 \\
\hline $\mathrm{Cu}$ & 3.56726077676760 & 35.31251316254510 & 5.05783673063456 \\
\hline $\mathrm{Cu}$ & 3.56726482748578 & 2.52184036345324 & 5.05452129612343 \\
\hline $\mathbf{C u}$ & 3.56725184060158 & 5.02948735470463 & 5.05152648495907 \\
\hline $\mathbf{C u}$ & 3.56726601032536 & 7.53169666841591 & 5.15004477020320 \\
\hline $\mathbf{C u}$ & 3.56723965409056 & 10.17867623945550 & 5.31822435351525 \\
\hline $\mathrm{Cu}$ & 3.56725909125535 & 12.62814694243360 & 5.09301423190773 \\
\hline $\mathbf{C u}$ & 3.56727926996454 & 15.10400719163980 & 5.10895996064662 \\
\hline $\mathrm{Cu}$ & 3.56727203778509 & 17.63966792809290 & 5.13050684742400 \\
\hline $\mathbf{C u}$ & 3.56727013953136 & 20.17142918009370 & 5.09513943817127 \\
\hline $\mathrm{Cu}$ & 3.56729315470983 & 22.71264221001220 & 5.12574695507130 \\
\hline $\mathbf{C u}$ & 3.56729024161352 & 25.24205568274390 & 5.05632385372374 \\
\hline $\mathbf{C u}$ & 3.56726742987129 & 27.75366306859680 & 5.05346573438882 \\
\hline $\mathbf{C u}$ & 3.56727408974468 & 30.27229745594650 & 5.05654495600504 \\
\hline $\mathbf{C u}$ & 3.56727234087953 & 32.79395148606140 & 5.05605618556763 \\
\hline $\mathbf{C u}$ & 7.13031090651942 & 0.00033376913231 & 5.05356641572346 \\
\hline $\mathrm{Cu}$ & 7.12980444600284 & 2.52403683558234 & 5.05585923340604 \\
\hline $\mathrm{Cu}$ & 7.13339860477852 & 5.05218462399039 & 5.05652753963487 \\
\hline $\mathbf{C u}$ & 7.12983816123145 & 7.57070266944563 & 5.05357548405108 \\
\hline $\mathrm{Cu}$ & 7.13174468009047 & 10.09125627079510 & 5.04580164746262 \\
\hline $\mathbf{C u}$ & 7.12692896736432 & 12.61138736147340 & 5.05054180114519 \\
\hline $\mathbf{C u}$ & 7.13084620352642 & 15.13125922815270 & 5.05523683425423 \\
\hline $\mathbf{C u}$ & 7.13180018485100 & 17.65598111987380 & 5.05827479480097 \\
\hline $\mathbf{C u}$ & 7.12656653502139 & 20.17734575882510 & 5.05306404793855 \\
\hline
\end{tabular}




\begin{tabular}{|c|c|c|c|}
\hline $\mathbf{C u}$ & 7.12418986822133 & 22.70407948649930 & 5.05860999915457 \\
\hline $\mathbf{C u}$ & 7.13118157644511 & 25.22232901170420 & 5.05088483958682 \\
\hline $\mathbf{C u}$ & 7.13623480589684 & 27.75003596903620 & 5.06438449534872 \\
\hline $\mathbf{C u}$ & 7.13780311387889 & 30.26844873134610 & 5.05720127096484 \\
\hline $\mathbf{C u}$ & 7.13001366760758 & 32.79410704603870 & 5.05721020336721 \\
\hline \multicolumn{4}{|c|}{ CI-NEB Image 8} \\
\hline Element & X-Coordinate $(\AA))$ & Y-Coordinate (§̊) & Z-Coordinate (Å) \\
\hline $\mathbf{F}$ & 4.63803622670561 & 11.93308553404070 & 7.67426050034597 \\
\hline $\mathbf{F}$ & 2.49679612184893 & 11.93307497037670 & 7.67442584057338 \\
\hline $\mathbf{F}$ & 3.56727633301966 & 8.79561126403257 & 6.67943398575730 \\
\hline $\mathbf{F}$ & 4.66350596964910 & 17.66454534847000 & 7.75740174 \\
\hline $\mathbf{F}$ & 2.47103990353009 & 17.66454571010870 & 7.75738673623954 \\
\hline $\mathbf{F}$ & 3.56727649295996 & 15.79501742258330 & 7.31391635124071 \\
\hline $\mathbf{F}$ & 4.66377903618250 & 22.75657340952730 & 7.74457847744652 \\
\hline $\mathbf{F}$ & 2.47076508992896 & 22.75657399512230 & 7.74456334184378 \\
\hline $\mathbf{F}$ & 3.56727699455711 & 20.88646637082040 & 7.31449366649300 \\
\hline C & 3.56735257275035 & 12.03683791089880 & 6.88239086575141 \\
\hline $\mathrm{C}$ & 3.56727757702278 & 17.22156643583550 & 7.05978438606726 \\
\hline C & 3.56727676031836 & 22.29825992344640 & 7.05107533590491 \\
\hline $\mathbf{C u}$ & 0.00000000000000 & 0.00000000000000 & 0.00000000000000 \\
\hline $\mathbf{C u}$ & 0.00000000000000 & 2.52244512012115 & 0.00000000000000 \\
\hline $\mathbf{C u}$ & 0.00000000000000 & 5.04488953395804 & 0.00000000000000 \\
\hline $\mathbf{C u}$ & 0.00000000000000 & 7.56733465407878 & 0.00000000000000 \\
\hline $\mathbf{C u}$ & 0.00000000000000 & 10.08977871477400 & 0.00000000000000 \\
\hline $\mathbf{C u}$ & 0.00000000000000 & 12.61222383489520 & 0.00000000000000 \\
\hline $\mathbf{C u}$ & 0.00000000000000 & 15.13466895501450 & 0.00000000000000 \\
\hline $\mathbf{C u}$ & 0.00000000000000 & 17.65711336885320 & 0.00000000000000 \\
\hline $\mathbf{C u}$ & 0.00000000000000 & 20.17955848897460 & 0.00000000000000 \\
\hline $\mathbf{C u}$ & 0.00000000000000 & 22.70200254966930 & 0.00000000000000 \\
\hline $\mathbf{C u}$ & 0.00000000000000 & 25.22444766979040 & 0.00000000000000 \\
\hline $\mathbf{C u}$ & 0.00000000000000 & 27.74689208362390 & 0.00000000000000 \\
\hline $\mathbf{C u}$ & 0.00000000000000 & 30.26933720374510 & 0.00000000000000 \\
\hline $\mathbf{C u}$ & 0.00000000000000 & 32.79178232386990 & 0.00000000000000 \\
\hline $\mathbf{C u}$ & 3.56727419238642 & 0.00000000000000 & 0.00000000000000 \\
\hline $\mathbf{C u}$ & 3.56727419238642 & 2.52244512012115 & 0.00000000000000 \\
\hline $\mathbf{C u}$ & 3.56727419238642 & 5.04488953395804 & 0.00000000000000 \\
\hline $\mathbf{C u}$ & 3.56727419238642 & 7.56733465407878 & 0.00000000000000 \\
\hline $\mathbf{C u}$ & 3.56727419238642 & 10.08977871477400 & 0.00000000000000 \\
\hline $\mathbf{C u}$ & 3.56727419238642 & 12.61222383489520 & 0.00000000000000 \\
\hline $\mathbf{C u}$ & 3.56727419238642 & 15.13466895501450 & 0.00000000000000 \\
\hline $\mathbf{C u}$ & 3.56727419238642 & 17.65711336885320 & 0.00000000000000 \\
\hline $\mathbf{C u}$ & 3.56727419238642 & 20.17955848897460 & 0.00000000000000 \\
\hline $\mathbf{C u}$ & 3.56727419238642 & 22.70200254966930 & 0.00000000000000 \\
\hline $\mathbf{C u}$ & 3.56727419238642 & 25.22444766979040 & 0.00000000000000 \\
\hline $\mathbf{C u}$ & 3.56727419238642 & 27.74689208362390 & 0.00000000000000 \\
\hline $\mathbf{C u}$ & 3.56727419238642 & 30.26933720374510 & 0.00000000000000 \\
\hline $\mathbf{C u}$ & 3.56727419238642 & 32.79178232386990 & 0.00000000000000 \\
\hline $\mathbf{C u}$ & 7.13454838477291 & 0.00000000000000 & 0.00000000000000 \\
\hline $\mathbf{C u}$ & 7.13454838477291 & 2.52244512012115 & 0.00000000000000 \\
\hline $\mathbf{C u}$ & 7.13454838477291 & 5.04488953395804 & 0.00000000000000 \\
\hline $\mathbf{C u}$ & 7.13454838477291 & 7.56733465407878 & 0.00000000000000 \\
\hline $\mathbf{C u}$ & 7.13454838477291 & 10.08977871477400 & 0.00000000000000 \\
\hline $\mathbf{C u}$ & 7.13454838477291 & 12.61222383489520 & 0.00000000000000 \\
\hline $\mathbf{C u}$ & 7.13454838477291 & 15.13466895501450 & 0.00000000000000 \\
\hline
\end{tabular}




\begin{tabular}{|c|c|c|c|}
\hline $\mathrm{Cu}$ & 7.13454838477291 & 17.65711336885320 & 0.00000000000000 \\
\hline $\mathrm{Cu}$ & 7.13454838477291 & 20.17955848897460 & 0.00000000000000 \\
\hline $\mathrm{Cu}$ & 7.13454838477291 & 22.70200254966930 & 0.00000000000000 \\
\hline $\mathbf{C u}$ & 7.13454838477291 & 25.22444766979040 & 0.00000000000000 \\
\hline $\mathbf{C u}$ & 7.13454838477291 & 27.74689208362390 & 0.00000000000000 \\
\hline $\mathbf{C u}$ & 7.13454838477291 & 30.26933720374510 & 0.00000000000000 \\
\hline $\mathrm{Cu}$ & 7.13454838477291 & 32.79178232386990 & 0.00000000000000 \\
\hline $\mathrm{Cu}$ & 1.78363955761358 & 1.26122414920059 & 1.26122399999993 \\
\hline $\mathrm{Cu}$ & 1.78363955761358 & 3.78366926932192 & 1.26122399999993 \\
\hline $\mathrm{Cu}$ & 1.78363955761358 & 6.30611333001662 & 1.26122399999993 \\
\hline $\mathbf{C u}$ & 1.78363955761358 & 8.82855845013778 & 1.26122399999993 \\
\hline $\mathrm{Cu}$ & 1.78363955761358 & 11.35100286397460 & 1.26122399999993 \\
\hline $\mathrm{Cu}$ & 1.78363955761358 & 13.87344798409370 & 1.26122399999993 \\
\hline $\mathrm{Cu}$ & 1.78363955761358 & 16.39589310421720 & 1.26122399999993 \\
\hline $\mathrm{Cu}$ & 1.78363955761358 & 18.91833716491180 & 1.26122399999993 \\
\hline $\mathrm{Cu}$ & 1.78363955761358 & 21.44078263817520 & 1.26122399999993 \\
\hline $\mathrm{Cu}$ & 1.78363955761358 & 23.96322669886980 & 1.26122399999993 \\
\hline $\mathbf{C u}$ & 1.78363955761358 & 26.48567181899090 & 1.26122399999993 \\
\hline $\mathbf{C u}$ & 1.78363955761358 & 29.00811693910900 & 1.26122399999993 \\
\hline $\mathrm{Cu}$ & 1.78363955761358 & 31.53056135294540 & 1.26122399999993 \\
\hline $\mathrm{Cu}$ & 1.783639 & 34.05300647307050 & 1.26122399999993 \\
\hline $\mathbf{C u}$ & 5.35091375000000 & 1.26122414920059 & 1.26122399999993 \\
\hline $\mathrm{Cu}$ & 5.35091375000000 & 3.78366926932192 & 1.26122399999993 \\
\hline $\mathrm{Cu}$ & 5.35091375000000 & 6.30611333001662 & 1.26122399999993 \\
\hline $\mathrm{Cu}$ & 5.35091375000000 & 8.82855845013778 & 1.26122399999993 \\
\hline $\mathrm{Cu}$ & 5.35091375000000 & 11.35100286397460 & 1.26122399999993 \\
\hline $\mathbf{C u}$ & 5.35091375000000 & 13.87344798409370 & 1.26122399999993 \\
\hline $\mathrm{Cu}$ & 5.35091375000000 & 16.39589310421720 & 1.26122399999993 \\
\hline $\mathbf{C u}$ & 5.35091375000000 & 18.91833716491180 & 1.26122399999993 \\
\hline $\mathrm{Cu}$ & 5.35091375000000 & 21.44078263817520 & 1.26122399999993 \\
\hline $\mathrm{Cu}$ & 5.35091375000000 & 23.96322669886980 & 1.26122399999993 \\
\hline $\mathrm{Cu}$ & 5.35091375000000 & 26.48567181899090 & 1.26122399999993 \\
\hline $\mathrm{Cu}$ & 5.35091375000000 & 29.00811693910900 & 1.26122399999993 \\
\hline $\mathbf{C u}$ & 5.35091375000000 & 31.53056135294540 & 1.26122399999993 \\
\hline $\mathbf{C u}$ & 5.35091375000000 & 34.05300647307050 & 1.26122399999993 \\
\hline $\mathbf{C u}$ & 8.91818794238642 & 1.26122414920059 & 1.26122399999993 \\
\hline $\mathrm{Cu}$ & 8.91818794238642 & 3.78366926932192 & 1.26122399999993 \\
\hline $\mathbf{C u}$ & 8.91818794238642 & 6.30611333001662 & 1.26122399999993 \\
\hline $\mathrm{Cu}$ & 8.91818794238642 & 8.82855845013778 & 1.26122399999993 \\
\hline $\mathbf{C u}$ & 8.91818794238642 & 11.35100286397460 & 1.26122399999993 \\
\hline $\mathrm{Cu}$ & 8.91818794238642 & 13.87344798409370 & 1.26122399999993 \\
\hline $\mathbf{C u}$ & 8.91818794238642 & 16.39589310421720 & 1.26122399999993 \\
\hline $\mathbf{C u}$ & 8.91818794238642 & 18.91833716491180 & 1.26122399999993 \\
\hline $\mathbf{C u}$ & 8.91818794238642 & 21.44078263817520 & 1.26122399999993 \\
\hline $\mathbf{C u}$ & 8.91818794238642 & 23.96322669886980 & 1.26122399999993 \\
\hline $\mathrm{Cu}$ & 8.91818794238642 & 26.48567181899090 & 1.26122399999993 \\
\hline $\mathrm{Cu}$ & 8.91818794238642 & 29.00811693910900 & 1.26122399999993 \\
\hline $\mathrm{Cu}$ & 8.91818794238642 & 31.53056135294540 & 1.26122399999993 \\
\hline $\mathbf{C u}$ & 8.91818794238642 & 34.05300647307050 & 1.26122399999993 \\
\hline $\mathrm{Cu}$ & 0.00184137899452 & 0.00086699252272 & 2.53806754523277 \\
\hline $\mathbf{C u}$ & 10.69973410598240 & 2.52443299338496 & 2.54056636365488 \\
\hline $\mathbf{C u}$ & 0.00278753424660 & 5.04621251231778 & 2.54177930190723 \\
\hline $\mathbf{C u}$ & 0.00168442950218 & 7.56651168218059 & 2.54392198991237 \\
\hline $\mathbf{C u}$ & 10.70111944889360 & 10.08844226988620 & 2.53843152897469 \\
\hline
\end{tabular}




\begin{tabular}{|c|c|c|c|}
\hline $\mathbf{C u}$ & 10.70027366808010 & 12.61501172068270 & 2.54435820519208 \\
\hline $\mathbf{C u}$ & 10.69623383601630 & 15.13597455750470 & 2.53698633358233 \\
\hline $\mathbf{C u}$ & 10.69886083333260 & 17.65771136566820 & 2.54472213206047 \\
\hline $\mathbf{C u}$ & 10.69648489744190 & 20.17900791689570 & 2.53743031204854 \\
\hline $\mathbf{C u}$ & 10.69688141719740 & 22.70335153995970 & 2.54623667718956 \\
\hline $\mathbf{C u}$ & 10.69993203739280 & 25.22747567084820 & 2.53810450270902 \\
\hline $\mathbf{C u}$ & 10.70008571018480 & 27.74761086797330 & 2.54307327077056 \\
\hline $\mathbf{C u}$ & 10.70197237187510 & 30.26930881735270 & 2.54154148425456 \\
\hline $\mathbf{C u}$ & 10.70097586655830 & 32.79236650271200 & 2.54097990871282 \\
\hline $\mathbf{C u}$ & 3.56727525206043 & 0.00335714697433 & 2.54183058166939 \\
\hline $\mathbf{C u}$ & 3.56727879209919 & 2.52389503116304 & 2.54209003362464 \\
\hline $\mathbf{C u}$ & 3.56729678945519 & 5.04815999570991 & 2.54083748255177 \\
\hline $\mathbf{C u}$ & 3.56727445187103 & 7.57694219504644 & 2.58928836919326 \\
\hline $\mathrm{Cu}$ & 3.56729287465383 & 10.09345844906260 & 2.61052364582656 \\
\hline $\mathbf{C u}$ & 3.56727827155733 & 12.61843977827240 & 2.57125494732008 \\
\hline $\mathbf{C u}$ & 3.56728596284405 & 15.14506876222790 & 2.58319612219664 \\
\hline $\mathbf{C u}$ & 3.56728151842414 & 17.65336268622230 & 2.58524564817143 \\
\hline $\mathbf{C u}$ & 3.56728644595233 & 20.17713174950680 & 2.57922861469135 \\
\hline $\mathbf{C u}$ & 3.56724543302121 & 22.69771754282660 & 2.57659091642660 \\
\hline $\mathbf{C u}$ & 3.56727998983649 & 25.21926092268110 & 2.55179488853218 \\
\hline $\mathbf{C u}$ & 3.56727875172828 & 27.74809345265080 & 2.54126109211067 \\
\hline $\mathbf{C u}$ & 3.56726827022934 & 30.27012811737740 & 2.54141490294735 \\
\hline $\mathbf{C u}$ & 3.56727730777271 & 610300310 & 140213749 \\
\hline $\mathrm{Cu}$ & 7.13270550268877 & 720203333 & 1120266 \\
\hline $\mathrm{Cu}$ & 3073172 & 2.52 & 48656214 \\
\hline $\mathrm{Cu}$ & 7.13175 & 267 & 2.54177817203105 \\
\hline $\mathbf{C u}$ & 7.13286536261589 & 7.56649943078787 & 2.54391745657127 \\
\hline $\mathbf{C u}$ & 7.13525965546758 & 10.08845160954040 & 2.53842415180878 \\
\hline $\mathbf{C u}$ & 7.13610222760543 & 12.61500518411050 & 2.54436364559817 \\
\hline $\mathrm{Cu}$ & 7.14014169071976 & 15.13592835371640 & 2.53701414634680 \\
\hline $\mathrm{Cu}$ & 7.13752615642863 & 17.65770468024220 & 2.54474756543304 \\
\hline $\mathrm{Cu}$ & 7.13988842779772 & 20.17900663905220 & 2.53741794409500 \\
\hline $\mathrm{Cu}$ & 7.13950005182368 & 22.70335058989200 & 2.54624876944012 \\
\hline $\mathbf{C u}$ & 7.13642509061942 & 25.22745126529320 & 2.53807495395974 \\
\hline $\mathbf{C u}$ & 7.13627187322860 & 27.74757431166660 & 2.54306453044601 \\
\hline $\mathbf{C u}$ & 7.13440589797768 & 30.26929841092860 & 2.54157863604974 \\
\hline $\mathbf{C u}$ & 7.13540776373862 & 32.79236824603230 & 2.54097477256485 \\
\hline $\mathbf{C u}$ & 1.78499810269193 & 1.26324168382548 & 3.85163530685248 \\
\hline $\mathbf{C u}$ & 1.78058984887849 & 3.78593241251989 & 3.85308128729576 \\
\hline $\mathbf{C u}$ & 1.79623380856395 & 6.31150231256777 & 3.86185857263913 \\
\hline $\mathbf{C u}$ & 1.79206190063354 & 8.83598488344728 & 3.85029035612994 \\
\hline $\mathbf{C u}$ & 1.78303327422757 & 11.34586018830970 & 3.83579439802375 \\
\hline $\mathbf{C u}$ & 1.78266945914522 & 13.88365642138200 & 3.84321954543464 \\
\hline $\mathbf{C u}$ & 1.77318940688798 & 16.39890088148260 & 3.84398607845993 \\
\hline $\mathbf{C u}$ & 1.78614560300788 & 18.92222357069280 & 3.85056847597745 \\
\hline $\mathbf{C u}$ & 1.77550489350090 & 21.43825564064810 & 3.83699011886204 \\
\hline $\mathbf{C u}$ & 1.78620272816123 & 23.96840478081410 & 3.84302836690397 \\
\hline $\mathbf{C u}$ & 1.77987561214812 & 26.49003248239160 & 3.85498595745326 \\
\hline $\mathbf{C u}$ & 1.78315200825086 & 29.01048659941610 & 3.85512672640980 \\
\hline $\mathbf{C u}$ & 1.78280017885129 & 31.53191915562780 & 3.85570109270468 \\
\hline $\mathbf{C u}$ & 1.78446780259452 & 34.05307473286520 & 3.85290881175739 \\
\hline $\mathbf{C u}$ & 5.34955474268862 & 1.26323235216177 & 3.85161876514012 \\
\hline $\mathbf{C u}$ & 5.35396672062634 & 3.78593187009543 & 3.85309100083360 \\
\hline $\mathbf{C u}$ & 5.33829270181556 & 6.31148947482896 & 3.86186485316687 \\
\hline
\end{tabular}




\begin{tabular}{|c|c|c|c|}
\hline $\mathbf{C u}$ & 5.34248816095738 & 8.83598884981888 & 3.85027393238517 \\
\hline $\mathbf{C u}$ & 5.35149050327087 & 11.34587609547280 & 3.83580122444078 \\
\hline $\mathbf{C u}$ & 5.35187941250591 & 13.88364924702520 & 3.84323575965239 \\
\hline $\mathbf{C u}$ & 5.36137567509787 & 16.39890756929450 & 3.84400751734052 \\
\hline $\mathbf{C u}$ & 5.34837492523668 & 18.92219960091850 & 3.85060487154381 \\
\hline $\mathbf{C u}$ & 5.35904333256090 & 21.43825737292680 & 3.83698804292174 \\
\hline $\mathbf{C u}$ & 5.34839670231409 & 23.96840050104990 & 3.84301090030952 \\
\hline $\mathbf{C u}$ & 5.35467722395612 & 26.49002092506600 & 3.85499605437078 \\
\hline $\mathbf{C u}$ & 5.35138405211415 & 29.01047428462700 & 3.85516158356041 \\
\hline $\mathbf{C u}$ & 5.35172971859110 & 31.53190148881610 & 3.85567975880486 \\
\hline $\mathbf{C u}$ & 5.35008442286633 & 34.05306411913410 & 3.85290590479971 \\
\hline $\mathbf{C u}$ & 8.91817978052362 & 1.26465803506951 & 3.84992933309602 \\
\hline $\mathbf{C u}$ & 8.91818997873366 & 3.78589352256709 & 3.85166505949400 \\
\hline $\mathrm{Cu}$ & 8.91819089097819 & 6.30583334644451 & 3.85105482780592 \\
\hline $\mathbf{C u}$ & 8.91818823088432 & 8.82843867178798 & 3.85605766131477 \\
\hline $\mathbf{C u}$ & 8.91819131560668 & 11.35479894831540 & 3.84788407903427 \\
\hline $\mathbf{C u}$ & 8.91820688002206 & 13.87141042236110 & 3.85195437004516 \\
\hline $\mathbf{C u}$ & 8.91820267714439 & 16.39532467076610 & 3.85494761460040 \\
\hline $\mathbf{C u}$ & 8.91821135227431 & 18.91537746637770 & 3.85935153305760 \\
\hline $\mathbf{C u}$ & 8.91819096561280 & 21.44181259729660 & 3.85532706798404 \\
\hline $\mathbf{C u}$ & 8.91817935419166 & 23.96231760337890 & 3.85101521886042 \\
\hline $\mathbf{C u}$ & 8.91822404662504 & 26.49154637646330 & 3.85419489994107 \\
\hline $\mathbf{C u}$ & 8.91817744086624 & 017582540 & 8154236670 \\
\hline $\mathbf{C u}$ & 8.91816838405117 & 234661210 & 219740 \\
\hline $\mathbf{C u}$ & 8.91819024433181 & 2463100 & 18462 \\
\hline $\mathrm{Cu}$ & 0.00239439273125 & 35.31577692309170 & 5.05377707726987 \\
\hline $\mathbf{C u}$ & 0.00399887841666 & 2.52526178144267 & 5.05764587926969 \\
\hline $\mathbf{C u}$ & 0.00236122174775 & 5.05278778685765 & 5.05614186130173 \\
\hline $\mathbf{C u}$ & 0.00449036513471 & 7.57164675921067 & 5.05321537711201 \\
\hline $\mathrm{Cu}$ & 0.00431586921599 & 10.09313881072750 & 5.04705142626711 \\
\hline $\mathrm{Cu}$ & 0.00754866134742 & 12.61142056079730 & 5.05034210142675 \\
\hline $\mathbf{C u}$ & 0.00169269330857 & 15.13232113526520 & 5.05604431602617 \\
\hline $\mathrm{Cu}$ & 0.00115885607500 & 17.65722980040510 & 5.06265882556135 \\
\hline $\mathbf{C u}$ & 0.00720369778719 & 20.17809355087070 & 5.05332369730562 \\
\hline $\mathbf{C u}$ & 0.00960362777965 & 22.70465960365670 & 5.05872935283710 \\
\hline $\mathbf{C u}$ & 0.00263691903697 & 25.22362229960600 & 5.05158437638064 \\
\hline $\mathbf{C u}$ & 10.70114716561170 & 27.75069335404000 & 5.06323365563075 \\
\hline $\mathbf{C u}$ & 10.69996806712980 & 30.26914709237910 & 5.05684899925513 \\
\hline $\mathbf{C u}$ & 0.00427705081671 & 32.79486065750070 & 5.05780528358884 \\
\hline $\mathbf{C u}$ & 3.56727838742360 & 35.31509642939080 & 5.05859230829908 \\
\hline $\mathbf{C u}$ & 3.56729496379098 & 2.52556659960075 & 5.05674341163579 \\
\hline $\mathbf{C u}$ & 3.56727014602259 & 5.03378528823914 & 5.05241648571417 \\
\hline $\mathbf{C u}$ & 3.56728476361139 & 7.54406795206722 & 5.16209322056100 \\
\hline $\mathbf{C u}$ & 3.56727170864050 & 10.17686052126910 & 5.25866672963665 \\
\hline $\mathbf{C u}$ & 3.56725459877544 & 12.64327683907720 & 5.09853862515673 \\
\hline $\mathbf{C u}$ & 3.56727181566965 & 15.11749892285440 & 5.10604435117274 \\
\hline $\mathbf{C u}$ & 3.56727711624165 & 17.64927898144270 & 5.13171693965963 \\
\hline $\mathbf{C u}$ & 3.56727474906316 & 20.17683863209240 & 5.09254713748596 \\
\hline $\mathbf{C u}$ & 3.56728962469413 & 22.71714680691010 & 5.12397018202460 \\
\hline $\mathbf{C u}$ & 3.56727561432702 & 25.24418454416130 & 5.05755336665379 \\
\hline $\mathbf{C u}$ & 3.56726390536240 & 27.75513074137630 & 5.05608135947132 \\
\hline $\mathbf{C u}$ & 3.56723369414890 & 30.27620235175200 & 5.05854667931684 \\
\hline $\mathbf{C u}$ & 3.56727023024805 & 32.79535230853480 & 5.05803138258206 \\
\hline $\mathbf{C u}$ & 7.13214659064935 & 35.31577669449570 & 5.05376327010312 \\
\hline
\end{tabular}




\begin{tabular}{|c|c|c|c|}
\hline $\mathrm{Cu}$ & 7.13050498602030 & 2.52532379605948 & 5.05769204094968 \\
\hline $\mathrm{Cu}$ & 7.13222219132088 & 5.05284249946179 & 5.05612016606024 \\
\hline $\mathbf{C u}$ & 7.13004777085834 & 7.57163789057677 & 5.05317590463641 \\
\hline $\mathbf{C u}$ & 7.13024264895897 & 10.09314394775550 & 5.04704927305284 \\
\hline $\mathbf{C u}$ & 7.12702995973491 & 12.61138701829620 & 5.05030673342399 \\
\hline $\mathbf{C u}$ & 7.13289486517559 & 15.13230559275640 & 5.05604199096564 \\
\hline $\mathbf{C u}$ & 7.13343319878305 & 17.65721024918220 & 5.06268076097667 \\
\hline $\mathbf{C u}$ & 7.12730553595683 & 20.17806336336390 & 5.05334385356006 \\
\hline $\mathbf{C u}$ & 7.12496811881066 & 22.70464627626660 & 5.05872542955901 \\
\hline $\mathbf{C u}$ & 7.13194413930175 & 25.22360308191240 & 5.05157810661704 \\
\hline $\mathbf{C u}$ & 7.13524188835899 & 27.75066919482230 & 5.06325763007853 \\
\hline $\mathbf{C u}$ & 7.13640907446555 & 30.26912973344340 & 5.05685440238745 \\
\hline $\mathbf{C u}$ & 7.13028122104017 & 32.79480361534300 & 5.05781514504122 \\
\hline \multicolumn{4}{|c|}{ CI-NEB Image 9} \\
\hline Element & X-Coordinate (Å) & Y-Coordinate (§̊) & ate $(\AA)$ \\
\hline $\mathbf{F}$ & 4.63508284563763 & 12.22781912709100 & 7.75732983112446 \\
\hline $\mathbf{F}$ & 2.50000733310482 & 12.22792295282820 & 7.75757543651923 \\
\hline $\mathbf{F}$ & 3.56728170141910 & 8.80933587421063 & 6.66262383059670 \\
\hline $\mathbf{F}$ & 4.66324754891413 & 17.66140207118660 & 7.76415384868878 \\
\hline $\mathbf{F}$ & 2.47130753702793 & 17.66140333266570 & 7.76413433855537 \\
\hline $\mathbf{F}$ & 3.56728055424930 & 15.79316302416990 & 7.31148147466318 \\
\hline $\mathbf{F}$ & 4.66380105905620 & 22.75548604919490 & 7.74660044004568 \\
\hline $\mathbf{F}$ & 2.47075220993658 & 22.75548678401040 & 7.74659213278741 \\
\hline $\mathbf{F}$ & 3.56727815101617 & 20.88560674669280 & 7.31504125216585 \\
\hline C & 3.56745676256324 & 12.32553828782900 & 6.96985742859789 \\
\hline $\mathbf{C}$ & 3.56728355603906 & 17.22422149230120 & 7.06325809527184 \\
\hline C & 3.56727889243740 & 22.29802491610970 & 7.05243068789768 \\
\hline $\mathbf{C u}$ & 0.00000000000000 & 0.00000000000000 & 0.00000000000000 \\
\hline $\mathbf{C u}$ & 0.00000000000000 & 2.52244512012115 & 0.00000000000000 \\
\hline $\mathbf{C u}$ & 0.00000000000000 & 5.04488953395804 & 0.00000000000000 \\
\hline $\mathbf{C u}$ & 0.00000000000000 & 7.56733465407878 & 0.00000000000000 \\
\hline $\mathbf{C u}$ & 0.00000000000000 & 10.08977871477400 & 0.00000000000000 \\
\hline $\mathbf{C u}$ & 0.00000000000000 & 12.61222383489520 & 0.00000000000000 \\
\hline $\mathbf{C u}$ & 0.00000000000000 & 15.13466895501450 & 0.00000000000000 \\
\hline $\mathbf{C u}$ & 0.00000000000000 & 17.65711336885320 & 0.00000000000000 \\
\hline $\mathbf{C u}$ & 0.00000000000000 & 20.17955848897460 & 0.00000000000000 \\
\hline $\mathbf{C u}$ & 0.00000000000000 & 22.70200254966930 & 0.00000000000000 \\
\hline $\mathbf{C u}$ & 0.00000000000000 & 25.22444766979040 & 0.00000000000000 \\
\hline $\mathbf{C u}$ & 0.00000000000000 & 27.74689208362340 & 0.00000000000000 \\
\hline $\mathbf{C u}$ & 0.00000000000000 & 30.26933720374460 & 0.00000000000000 \\
\hline $\mathbf{C u}$ & 0.00000000000000 & 32.79178232386990 & 0.00000000000000 \\
\hline $\mathrm{Cu}$ & 3.56727419238642 & 0.00000000000000 & 0.00000000000000 \\
\hline $\mathbf{C u}$ & 3.56727419238642 & 2.52244512012115 & 0.00000000000000 \\
\hline $\mathrm{Cu}$ & 3.56727419238642 & 5.04488953395804 & 0.00000000000000 \\
\hline $\mathbf{C u}$ & 3.56727419238642 & 7.56733465407878 & 0.00000000000000 \\
\hline $\mathbf{C u}$ & 3.56727419238642 & 10.08977871477400 & 0.00000000000000 \\
\hline $\mathbf{C u}$ & 3.56727419238642 & 12.61222383489520 & 0.00000000000000 \\
\hline $\mathbf{C u}$ & 3.56727419238642 & 15.13466895501450 & 0.00000000000000 \\
\hline $\mathbf{C u}$ & 3.56727419238642 & 17.65711336885320 & 0.00000000000000 \\
\hline $\mathrm{Cu}$ & 3.56727419238642 & 20.17955848897460 & 0.00000000000000 \\
\hline $\mathbf{C u}$ & 3.56727419238642 & 22.70200254966930 & 0.00000000000000 \\
\hline $\mathbf{C u}$ & 3.56727419238642 & 25.22444766979040 & 0.00000000000000 \\
\hline $\mathbf{C u}$ & 3.56727419238642 & 27.74689208362340 & 0.00000000000000 \\
\hline $\mathbf{C u}$ & 3.56727419238642 & 30.26933720374460 & 0.00000000000000 \\
\hline
\end{tabular}




\begin{tabular}{|c|c|c|c|}
\hline $\mathbf{C u}$ & 3.56727419238642 & 32.79178232386990 & 0.00000000000000 \\
\hline $\mathbf{C u}$ & 7.13454838477291 & 0.00000000000000 & 0.00000000000000 \\
\hline $\mathbf{C u}$ & 7.13454838477291 & 2.52244512012115 & 0.00000000000000 \\
\hline $\mathbf{C u}$ & 7.13454838477291 & 5.04488953395804 & 0.00000000000000 \\
\hline $\mathbf{C u}$ & 7.13454838477291 & 7.56733465407878 & 0.00000000000000 \\
\hline $\mathbf{C u}$ & 7.13454838477291 & 10.08977871477400 & 0.00000000000000 \\
\hline $\mathbf{C u}$ & 7.13454838477291 & 12.61222383489520 & 0.00000000000000 \\
\hline $\mathbf{C u}$ & 7.13454838477291 & 15.13466895501450 & 0.00000000000000 \\
\hline $\mathbf{C u}$ & 7.13454838477291 & 17.65711336885320 & 0.00000000000000 \\
\hline $\mathbf{C u}$ & 7.13454838477291 & 20.17955848897460 & 0.00000000000000 \\
\hline $\mathbf{C u}$ & 7.13454838477291 & 22.70200254966930 & 0.00000000000000 \\
\hline $\mathbf{C u}$ & 7.13454838477291 & 25.22444766979040 & 0.00000000000000 \\
\hline $\mathrm{Cu}$ & 7.13454838477291 & 27.74689208362340 & 0.00000000000000 \\
\hline $\mathrm{Cu}$ & 7.13454838477291 & 30.26933720374460 & 0.00000000000000 \\
\hline $\mathbf{C u}$ & 7.13454838477291 & 32.79178232386990 & 0.00000000000000 \\
\hline $\mathbf{C u}$ & 1.78363955761358 & 1.26122414920059 & 1.26122399999993 \\
\hline $\mathbf{C u}$ & 1.78363955761358 & 3.78366926932192 & 1.26122399999993 \\
\hline $\mathbf{C u}$ & 1.78363955761358 & 6.30611333001662 & 1.26122399999993 \\
\hline $\mathbf{C u}$ & 1.78363955761358 & 8.82855845013778 & 1.26122399999993 \\
\hline $\mathbf{C u}$ & 1.78363955761358 & 11.35100286397460 & 1.26122399999993 \\
\hline $\mathbf{C u}$ & 1.78363955761358 & 13.87344798409370 & 1.26122399999993 \\
\hline $\mathbf{C u}$ & 1.78363955761358 & 16.39589310421720 & 1.26122399999993 \\
\hline $\mathbf{C u}$ & 1.783639 & 716491180 & 1.26122399999993 \\
\hline $\mathrm{Cu}$ & 1.783639 & 21.44078263817520 & 1.26122399999993 \\
\hline $\mathrm{Cu}$ & 1.783 & 23.96322669886980 & 1.26122399999993 \\
\hline $\mathrm{Cu}$ & 1.78363955761358 & 26.48567181899090 & 1.26122399999993 \\
\hline $\mathbf{C u}$ & 1.78363955761358 & 29.00811693910850 & 1.26122399999993 \\
\hline $\mathbf{C u}$ & 1.78363955761358 & 31.53056135294490 & 1.26122399999993 \\
\hline $\mathbf{C u}$ & 1.78363955761358 & 34.05300647307050 & 1.26122399999993 \\
\hline $\mathbf{C u}$ & 5.35091375000000 & 1.26122414920059 & 1.26122399999993 \\
\hline $\mathbf{C u}$ & 5.35091375000000 & 3.78366926932192 & 1.26122399999993 \\
\hline $\mathbf{C u}$ & 5.35091375000000 & 6.30611333001662 & 1.26122399999993 \\
\hline $\mathrm{Cu}$ & 5.35091375000000 & 8.82855845013778 & 1.26122399999993 \\
\hline $\mathbf{C u}$ & 5.35091375000000 & 11.35100286397460 & 1.26122399999993 \\
\hline $\mathbf{C u}$ & 5.35091375000000 & 13.87344798409370 & 1.26122399999993 \\
\hline $\mathbf{C u}$ & 5.35091375000000 & 16.39589310421720 & 1.26122399999993 \\
\hline $\mathbf{C u}$ & 5.35091375000000 & 18.91833716491180 & 1.26122399999993 \\
\hline $\mathbf{C u}$ & 5.35091375000000 & 21.44078263817520 & 1.26122399999993 \\
\hline $\mathbf{C u}$ & 5.35091375000000 & 23.96322669886980 & 1.26122399999993 \\
\hline $\mathbf{C u}$ & 5.35091375000000 & 26.48567181899090 & 1.26122399999993 \\
\hline $\mathbf{C u}$ & 5.35091375000000 & 29.00811693910850 & 1.26122399999993 \\
\hline $\mathbf{C u}$ & 5.35091375000000 & 31.53056135294490 & 1.26122399999993 \\
\hline $\mathbf{C u}$ & 5.35091375000000 & 34.05300647307050 & 1.26122399999993 \\
\hline $\mathbf{C u}$ & 8.91818794238642 & 1.26122414920059 & 1.26122399999993 \\
\hline $\mathbf{C u}$ & 8.91818794238642 & 3.78366926932192 & 1.26122399999993 \\
\hline $\mathbf{C u}$ & 8.91818794238642 & 6.30611333001662 & 1.26122399999993 \\
\hline $\mathbf{C u}$ & 8.91818794238642 & 8.82855845013778 & 1.26122399999993 \\
\hline $\mathbf{C u}$ & 8.91818794238642 & 11.35100286397460 & 1.26122399999993 \\
\hline $\mathbf{C u}$ & 8.91818794238642 & 13.87344798409370 & 1.26122399999993 \\
\hline $\mathbf{C u}$ & 8.91818794238642 & 16.39589310421720 & 1.26122399999993 \\
\hline $\mathbf{C u}$ & 8.91818794238642 & 18.91833716491180 & 1.26122399999993 \\
\hline $\mathbf{C u}$ & 8.91818794238642 & 21.44078263817520 & 1.26122399999993 \\
\hline $\mathbf{C u}$ & 8.91818794238642 & 23.96322669886980 & 1.26122399999993 \\
\hline $\mathbf{C u}$ & 8.91818794238642 & 26.48567181899090 & 1.26122399999993 \\
\hline
\end{tabular}




\begin{tabular}{|c|c|c|c|}
\hline $\mathrm{Cu}$ & 8.91818794238642 & 29.00811693910850 & 1.26122399999993 \\
\hline $\mathbf{C u}$ & 8.91818794238642 & 31.53056135294490 & 1.26122399999993 \\
\hline $\mathbf{C u}$ & 8.91818794238642 & 34.05300647307050 & 1.26122399999993 \\
\hline $\mathbf{C u}$ & 0.00157149890101 & 35.31398360596210 & 2.53843801474688 \\
\hline $\mathbf{C u}$ & 10.69961126685870 & 2.52329143367196 & 2.54069914888899 \\
\hline $\mathbf{C u}$ & 0.00286129834476 & 5.04471841478807 & 2.54201003377214 \\
\hline $\mathbf{C u}$ & -0.00017790862788 & 7.56530683358002 & 2.54230400129964 \\
\hline $\mathbf{C u}$ & 10.70072510119690 & 10.08950863286660 & 2.53893235802086 \\
\hline $\mathbf{C u}$ & 10.70237394798730 & 12.61410254471010 & 2.54507708004664 \\
\hline $\mathbf{C u}$ & 10.69659715976640 & 15.13519997247230 & 2.53703012922411 \\
\hline $\mathbf{C u}$ & 10.69933738221530 & 17.65716818415760 & 2.54533445878708 \\
\hline $\mathbf{C u}$ & 10.69649282027120 & 20.17838317103890 & 2.53775939525395 \\
\hline $\mathbf{C u}$ & 10.69709346093630 & 22.70222462022210 & 2.54623671335866 \\
\hline $\mathrm{Cu}$ & 10.69982520362510 & 25.22665432275620 & 2.53830748080949 \\
\hline $\mathbf{C u}$ & 10.69975454413350 & 27.74650312150370 & 2.54325261997122 \\
\hline $\mathbf{C u}$ & 10.70245489688230 & 30.26879224626630 & 2.54152479170906 \\
\hline $\mathbf{C u}$ & 10.70085759650940 & 32.79163481644530 & 2.54096338203075 \\
\hline $\mathbf{C u}$ & 3.56728230590529 & 0.00215710947992 & 2.54195785957195 \\
\hline $\mathbf{C u}$ & 3.56727719171419 & 2.52208601661879 & 2.54223815355600 \\
\hline $\mathbf{C u}$ & 3.56727995220810 & 5.04660795072511 & 2.54051877194734 \\
\hline $\mathbf{C u}$ & 3.56728357085098 & 7.57294031532297 & 2.58630410890582 \\
\hline $\mathbf{C u}$ & 3.56727535135370 & 10.09055239243900 & 2.59706963745878 \\
\hline $\mathbf{C u}$ & 8235172233 & 12.61966485743210 & 2.58076548153943 \\
\hline $\mathrm{Cu}$ & 62638789 & 15.1430974 & 3077550 \\
\hline $\mathrm{Cu}$ & 019833161 & 17.6522 & 07556667 \\
\hline $\mathrm{Cu}$ & 3.56728611388116 & 20.176493 & 2.57925431345656 \\
\hline $\mathbf{C u}$ & 3.56726143566815 & 22.69669991224000 & 2.57661164202509 \\
\hline $\mathbf{C u}$ & 3.56727732983948 & 25.21925682290700 & 2.55210996705941 \\
\hline $\mathbf{C u}$ & 3.56728538666902 & 27.74786706321960 & 2.54097725166808 \\
\hline $\mathrm{Cu}$ & 3.56727919310235 & 30.26872132511130 & 2.54094479235224 \\
\hline $\mathrm{Cu}$ & 3.56727336697173 & 32.79077464995440 & 2.54252130574650 \\
\hline $\mathrm{Cu}$ & 7.13297450537859 & 35.31398118479520 & 2.53843848264999 \\
\hline $\mathrm{Cu}$ & 7.13676664275832 & 2.52328250735441 & 2.54069693191585 \\
\hline $\mathbf{C u}$ & 7.13168831682150 & 5.04471245870671 & 2.54201125775596 \\
\hline $\mathbf{C u}$ & 7.13473046074425 & 7.56530463113174 & 2.54231751894359 \\
\hline $\mathbf{C u}$ & 7.13565004128445 & 10.08950893547310 & 2.53893073771768 \\
\hline $\mathbf{C u}$ & 7.13400432473777 & 12.61411041128120 & 2.54507305653598 \\
\hline $\mathbf{C u}$ & 7.13978015069996 & 15.13520519033760 & 2.53702706395036 \\
\hline $\mathbf{C u}$ & 7.13704539326292 & 17.65717465530920 & 2.54533723983448 \\
\hline $\mathbf{C u}$ & 7.13988637563820 & 20.17839240458100 & 2.53776525298413 \\
\hline $\mathbf{C u}$ & 7.13928159185546 & 22.70222306898570 & 2.54624016176832 \\
\hline $\mathbf{C u}$ & 7.13654754796316 & 25.22665177284400 & 2.53831222254655 \\
\hline $\mathbf{C u}$ & 7.13661220682961 & 27.74649815146350 & 2.54325901406553 \\
\hline $\mathbf{C u}$ & 7.13391091126346 & 30.26879219388100 & 2.54152064925500 \\
\hline $\mathbf{C u}$ & 7.13551473681590 & 32.79164194390020 & 2.54096132395125 \\
\hline $\mathrm{Cu}$ & 1.78404844151495 & 1.26029404658367 & 3.85259391821194 \\
\hline $\mathbf{C u}$ & 1.78016909651660 & 3.78197399879541 & 3.85354498368422 \\
\hline $\mathbf{C u}$ & 1.79582042514594 & 6.30706229475315 & 3.86132119559137 \\
\hline $\mathbf{C u}$ & 1.78402805700558 & 8.82849505908970 & 3.84020885053308 \\
\hline $\mathbf{C u}$ & 1.78960829379000 & 11.34423936692400 & 3.84198228161035 \\
\hline $\mathbf{C u}$ & 1.78385632327172 & 13.88132644011640 & 3.84487370627871 \\
\hline $\mathbf{C u}$ & 1.77411402506985 & 16.39721271930720 & 3.84455869632550 \\
\hline $\mathbf{C u}$ & 1.78601936958422 & 18.92150309282090 & 3.85086376420726 \\
\hline $\mathbf{C u}$ & 1.77644285989938 & 21.43745192115070 & 3.83746946238145 \\
\hline
\end{tabular}




\begin{tabular}{|c|c|c|c|}
\hline $\mathbf{C u}$ & 1.78583776583774 & 23.96719541992180 & 3.84368382179695 \\
\hline $\mathbf{C u}$ & 1.77983389953019 & 26.48814328523600 & 3.85507911867059 \\
\hline $\mathbf{C u}$ & 1.78383665089588 & 29.00902542973470 & 3.85492360234715 \\
\hline $\mathbf{C u}$ & 1.78305250290586 & 31.53007759068580 & 3.85570143973812 \\
\hline $\mathbf{C u}$ & 1.78433473170307 & 34.05025603486950 & 3.85342463714221 \\
\hline $\mathbf{C u}$ & 5.35050686125134 & 1.26028981777623 & 3.85259186201728 \\
\hline $\mathbf{C u}$ & 5.35438446234379 & 3.78196960659909 & 3.85355716912120 \\
\hline $\mathbf{C u}$ & 5.33873899633618 & 6.30705465292708 & 3.86130279591966 \\
\hline $\mathbf{C u}$ & 5.35051858576399 & 8.82850242225901 & 3.84019642799210 \\
\hline $\mathbf{C u}$ & 5.34495433322268 & 11.34423609437900 & 3.84195482604485 \\
\hline $\mathbf{C u}$ & 5.35070681531733 & 13.88132499860260 & 3.84487313065315 \\
\hline $\mathbf{C u}$ & 5.36046257066294 & 16.39721234604870 & 3.84456600787859 \\
\hline $\mathbf{C u}$ & 5.34855819766599 & 18.92150741778930 & 3.85086863177454 \\
\hline $\mathbf{C u}$ & 5.35810741608269 & 21.43744355323240 & 3.83748377313110 \\
\hline $\mathbf{C u}$ & 5.34871900775358 & 23.96718876601130 & 3.84368539304929 \\
\hline $\mathbf{C u}$ & 5.35471822890577 & 26.48813037850370 & 3.85508251606249 \\
\hline $\mathbf{C u}$ & 5.35071537812311 & 29.00902729009520 & 3.85493555284992 \\
\hline $\mathbf{C u}$ & 5.35149015909864 & 31.53008015858550 & 3.85573138960768 \\
\hline $\mathbf{C u}$ & 5.35022067013643 & 34.05025399223630 & 3.85341558764255 \\
\hline $\mathbf{C u}$ & 8.91818586979301 & 1.26277809341145 & 3.84969683230656 \\
\hline $\mathbf{C u}$ & 8.91818752087794 & 3.78386643328518 & 3.85084824045077 \\
\hline $\mathbf{C u}$ & 8.91818713178714 & 6.30421588729688 & 3.85101367320195 \\
\hline $\mathbf{C u}$ & 8.91818181456904 & 1810882 & 3.855 \\
\hline $\mathrm{Cu}$ & 8.91818228286661 & 11.35439207406330 & 3.848 \\
\hline $\mathrm{Cu}$ & 8.91818842664534 & 006752860 & 3.8520 \\
\hline $\mathrm{Cu}$ & 8.91818841509354 & 16.39458633087730 & 3.8550058 \\
\hline $\mathbf{C u}$ & 8.91819399221984 & 18.91414328722320 & 3.85851132686403 \\
\hline $\mathbf{C u}$ & 8.91819285314679 & 21.44073623482550 & 3.85553395690768 \\
\hline $\mathbf{C u}$ & 8.91819614112064 & 23.96137879891780 & 3.85099363677143 \\
\hline $\mathbf{C u}$ & 8.91818614324007 & 26.49027180280270 & 3.85438922363866 \\
\hline $\mathbf{C u}$ & 8.91818767789007 & 29.00840662817390 & 3.85102855006831 \\
\hline $\mathbf{C u}$ & 8.91818881381780 & 31.53175449476410 & 3.85144432512729 \\
\hline $\mathrm{Cu}$ & 8.91819233267576 & 34.05020099180940 & 3.85037339683236 \\
\hline $\mathrm{Cu}$ & 0.00076091408988 & 35.31225279667210 & 5.05439557772215 \\
\hline $\mathrm{Cu}$ & 0.00220699195602 & 2.52286570834420 & 5.05858704092046 \\
\hline $\mathbf{C u}$ & 0.00222318700447 & 5.04990679055011 & 5.05644913334950 \\
\hline $\mathbf{C u}$ & 0.00346029247734 & 7.56856143688034 & 5.05197202191438 \\
\hline $\mathbf{C u}$ & 0.00477128740099 & 10.09180860818180 & 5.04799237288804 \\
\hline $\mathbf{C u}$ & 0.00792661689169 & 12.60955935169950 & 5.04973121612135 \\
\hline $\mathbf{C u}$ & 0.00260981060547 & 15.13141674670960 & 5.05536174834208 \\
\hline $\mathbf{C u}$ & 0.00118767114524 & 17.65560583624240 & 5.06303788142632 \\
\hline $\mathbf{C u}$ & 0.00718923622230 & 20.17683392658460 & 5.05352822745015 \\
\hline $\mathbf{C u}$ & 0.00913854209421 & 22.70348561516140 & 5.05860806631656 \\
\hline $\mathbf{C u}$ & 0.00215182491054 & 25.22217363850990 & 5.05285680965298 \\
\hline $\mathbf{C u}$ & 10.70198772410270 & 27.74932072293220 & 5.06274145848769 \\
\hline $\mathbf{C u}$ & 10.70064166663430 & 30.26705866024700 & 5.05686843153742 \\
\hline $\mathbf{C u}$ & 0.00398640880793 & 32.79119310160480 & 5.05880075348119 \\
\hline $\mathbf{C u}$ & 3.56728156577346 & 35.31249433525290 & 5.05819446650132 \\
\hline $\mathrm{Cu}$ & 3.56727461457863 & 2.52244189336074 & 5.05654630941406 \\
\hline $\mathrm{Cu}$ & 3.56726830827486 & 5.02905407713432 & 5.05172327908830 \\
\hline $\mathbf{C u}$ & 3.56727209447192 & 7.53546213640528 & 5.16027342674441 \\
\hline $\mathbf{C u}$ & 3.56728692360963 & 10.12753580342220 & 5.18807093469298 \\
\hline $\mathbf{C u}$ & 3.56728400165784 & 12.62375207277440 & 5.12476910774194 \\
\hline $\mathbf{C u}$ & 3.56727935223963 & 15.11875624146160 & 5.10444758604198 \\
\hline
\end{tabular}




\begin{tabular}{|c|c|c|c|}
\hline $\mathbf{C u}$ & 3.56729705849856 & 17.65091196180890 & 5.13438844158492 \\
\hline $\mathbf{C u}$ & 3.56727967686781 & 20.17652363026230 & 5.09337069277475 \\
\hline $\mathbf{C u}$ & 3.56727604615900 & 22.71712749658840 & 5.12522603682767 \\
\hline $\mathbf{C u}$ & 3.56727785408277 & 25.24250711750670 & 5.05797044606141 \\
\hline $\mathbf{C u}$ & 3.56727163532005 & 27.75300839190570 & 5.05570701669230 \\
\hline $\mathbf{C u}$ & 3.56725896272960 & 30.27442457741450 & 5.05851872163602 \\
\hline $\mathbf{C u}$ & 3.56725950390184 & 32.79113608985190 & 5.05901411066261 \\
\hline $\mathbf{C u}$ & 7.13378955937062 & 35.31224932109550 & 5.05438662127681 \\
\hline $\mathbf{C u}$ & 7.13234378442178 & 2.52285582071929 & 5.05857810376958 \\
\hline $\mathbf{C u}$ & 7.13232491637612 & 5.04989313478380 & 5.05643957584505 \\
\hline $\mathbf{C u}$ & 7.13108766863531 & 7.56854366809601 & 5.05197159691515 \\
\hline $\mathbf{C u}$ & 7.12977237233099 & 10.09181741669130 & 5.04798674689143 \\
\hline $\mathbf{C u}$ & 7.12664155609892 & 12.60955665881480 & 5.04972186353525 \\
\hline $\mathbf{C u}$ & 7.13195154124143 & 15.13141657706350 & 5.05535662727237 \\
\hline $\mathbf{C u}$ & 7.13339140117500 & 17.65560660642850 & 57104124 \\
\hline $\mathbf{C u}$ & 7.12738234460803 & 20.17684352146700 & 5.05353461528953 \\
\hline $\mathbf{C u}$ & 40805467054 & 22.70348802115210 & 996343 \\
\hline $\mathrm{Cu}$ & 7.13240470288773 & 25.22216376522880 & 980091 \\
\hline $\mathbf{C u}$ & 7.13438787418974 & 27.74931092805550 & 5.06273746795637 \\
\hline $\mathbf{C u}$ & 7.13574635903318 & 30.26706339311000 & 5.05686101282630 \\
\hline $\mathbf{C u}$ & 7.13058322534704 & 32.79120219376400 & 5.05880212419541 \\
\hline \multicolumn{4}{|c|}{ CI-NEB Image 10} \\
\hline Element & X-Coordinate (Å) & Y-Coordinate (Å) & Z-Coordinate (Å) \\
\hline $\mathbf{F}$ & 4.63454077114076 & 12.56292263296310 & 7.79095097525766 \\
\hline $\mathbf{F}$ & 2.50114494357499 & 12.56294540784930 & 7.79158682836409 \\
\hline $\mathbf{F}$ & 3.56727800534446 & 8.80906247662347 & 6.65517824880949 \\
\hline $\mathbf{F}$ & 4.66301149091720 & 17.65521539769210 & 7.77216539862424 \\
\hline $\mathbf{F}$ & 2.47154512286755 & 17.65521949521830 & 7.77214059652720 \\
\hline $\mathbf{F}$ & 3.56727990124384 & 15.78853038324660 & 7.31205535066336 \\
\hline $\mathbf{F}$ & 4.66381125856656 & 22.75438978428790 & 7.74847329819570 \\
\hline $\mathbf{F}$ & 2.47073128925543 & 22.75439650333220 & 7.74845254495942 \\
\hline $\mathbf{F}$ & 3.56726978391269 & 20.88477109859270 & 7.31504903587283 \\
\hline C & 3.56760484329843 & 12.57349198565910 & 6.99870908149521 \\
\hline C & 3.56728427405446 & 17.22304902817190 & 7.06855109973869 \\
\hline C & 3.56727591187193 & 22.29802488183510 & 7.05362323578558 \\
\hline $\mathbf{C u}$ & 0.00000000000000 & 0.00000000000000 & 0.00000000000000 \\
\hline $\mathbf{C u}$ & 0.00000000000000 & 2.52244512012115 & 0.00000000000000 \\
\hline $\mathbf{C u}$ & 0.00000000000000 & 5.04488953395804 & 0.00000000000000 \\
\hline $\mathbf{C u}$ & 0.00000000000000 & 7.56733465407878 & 0.00000000000000 \\
\hline $\mathbf{C u}$ & 0.00000000000000 & 10.08977871477400 & 0.00000000000000 \\
\hline $\mathbf{C u}$ & 0.00000000000000 & 12.61222383489520 & 0.00000000000000 \\
\hline $\mathbf{C u}$ & 0.00000000000000 & 15.13466895501450 & 0.00000000000000 \\
\hline $\mathbf{C u}$ & 0.00000000000000 & 17.65711336885320 & 0.00000000000000 \\
\hline $\mathbf{C u}$ & 0.00000000000000 & 20.17955848897460 & 0.00000000000000 \\
\hline $\mathbf{C u}$ & 0.00000000000000 & 22.70200254966930 & 0.00000000000000 \\
\hline $\mathbf{C u}$ & 0.00000000000000 & 25.22444766979040 & 0.00000000000000 \\
\hline $\mathbf{C u}$ & 0.00000000000000 & 27.74689208362290 & 0.00000000000000 \\
\hline $\mathbf{C u}$ & 0.00000000000000 & 30.26933720374410 & 0.00000000000000 \\
\hline $\mathbf{C u}$ & 0.00000000000000 & 32.79178232386990 & 0.00000000000000 \\
\hline $\mathbf{C u}$ & 3.56727419238642 & 0.00000000000000 & 0.00000000000000 \\
\hline $\mathbf{C u}$ & 3.56727419238642 & 2.52244512012115 & 0.00000000000000 \\
\hline $\mathbf{C u}$ & 3.56727419238642 & 5.04488953395804 & 0.00000000000000 \\
\hline $\mathbf{C u}$ & 3.56727419238642 & 7.56733465407878 & 0.00000000000000 \\
\hline $\mathbf{C u}$ & 3.56727419238642 & 10.08977871477400 & 0.00000000000000 \\
\hline
\end{tabular}




\begin{tabular}{|c|c|c|c|}
\hline $\mathbf{C u}$ & 3.56727419238642 & 12.61222383489520 & 0.00000000000000 \\
\hline $\mathbf{C u}$ & 3.56727419238642 & 15.13466895501450 & 0.00000000000000 \\
\hline $\mathbf{C u}$ & 3.56727419238642 & 17.65711336885320 & 0.00000000000000 \\
\hline $\mathbf{C u}$ & 3.56727419238642 & 20.17955848897460 & 0.00000000000000 \\
\hline $\mathbf{C u}$ & 3.56727419238642 & 22.70200254966930 & 0.00000000000000 \\
\hline $\mathbf{C u}$ & 3.56727419238642 & 25.22444766979040 & 0.00000000000000 \\
\hline $\mathbf{C u}$ & 3.56727419238642 & 27.74689208362290 & 0.00000000000000 \\
\hline $\mathbf{C u}$ & 3.56727419238642 & 30.26933720374410 & 0.00000000000000 \\
\hline $\mathbf{C u}$ & 3.56727419238642 & 32.79178232386990 & 0.00000000000000 \\
\hline $\mathbf{C u}$ & 7.13454838477291 & 0.00000000000000 & 0.00000000000000 \\
\hline $\mathbf{C u}$ & 7.13454838477291 & 2.52244512012115 & 0.00000000000000 \\
\hline $\mathrm{Cu}$ & 7.13454838477291 & 5.04488953395804 & 0.00000000000000 \\
\hline $\mathrm{Cu}$ & 7.13454838477291 & 7.56733465407878 & 0.00000000000000 \\
\hline $\mathrm{Cu}$ & 7.13454838477291 & 10.08977871477400 & 0.00000000000000 \\
\hline $\mathbf{C u}$ & 7.13454838477291 & 12.61222383489520 & 0.00000000000000 \\
\hline $\mathbf{C u}$ & 7.13454838477291 & 15.13466895501450 & 0.00000000000000 \\
\hline $\mathbf{C u}$ & 7.13454838477291 & 17.65711336885320 & 0.00000000000000 \\
\hline $\mathbf{C u}$ & 7.13454838477291 & 20.17955848897460 & 0.00000000000000 \\
\hline $\mathbf{C u}$ & 7.13454838477291 & 22.70200254966930 & 0.00000000000000 \\
\hline $\mathbf{C u}$ & 7.13454838477291 & 25.22444766979040 & 0.00000000000000 \\
\hline $\mathbf{C u}$ & 7.13454838477291 & 27.74689208362290 & 0.00000000000000 \\
\hline $\mathbf{C u}$ & 7.13454838477291 & 30.26933720374410 & 0.00000000000000 \\
\hline $\mathbf{C u}$ & 7.13454838477291 & 32.791 & 0.00000000000000 \\
\hline $\mathrm{Cu}$ & 1.78363955761358 & 1.26122 & 1.26122399999993 \\
\hline $\mathrm{Cu}$ & 5761358 & 3.7836692 & 22399999993 \\
\hline $\mathrm{Cu}$ & 1.78363955761358 & 6.30611333001662 & 1.26122399999993 \\
\hline $\mathbf{C u}$ & 1.78363955761358 & 8.82855845013778 & 1.26122399999993 \\
\hline $\mathbf{C u}$ & 1.78363955761358 & 11.35100286397460 & 1.26122399999993 \\
\hline $\mathbf{C u}$ & 1.78363955761358 & 13.87344798409370 & 1.26122399999993 \\
\hline $\mathrm{Cu}$ & 1.78363955761358 & 16.39589310421720 & 1.26122399999993 \\
\hline $\mathrm{Cu}$ & 1.78363955761358 & 18.91833716491180 & 1.26122399999993 \\
\hline $\mathrm{Cu}$ & 1.78363955761358 & 21.44078263817520 & 1.26122399999993 \\
\hline $\mathrm{Cu}$ & 1.78363955761358 & 23.96322669886980 & 1.26122399999993 \\
\hline $\mathbf{C u}$ & 1.78363955761358 & 26.48567181899090 & 1.26122399999993 \\
\hline $\mathbf{C u}$ & 1.78363955761358 & 29.00811693910800 & 1.26122399999993 \\
\hline $\mathbf{C u}$ & 1.78363955761358 & 31.53056135294440 & 1.26122399999993 \\
\hline $\mathbf{C u}$ & 1.78363955761358 & 34.05300647307050 & 1.26122399999993 \\
\hline $\mathbf{C u}$ & 5.35091375000000 & 1.26122414920059 & 1.26122399999993 \\
\hline $\mathbf{C u}$ & 5.35091375000000 & 3.78366926932192 & 1.26122399999993 \\
\hline $\mathbf{C u}$ & 5.35091375000000 & 6.30611333001662 & 1.26122399999993 \\
\hline $\mathbf{C u}$ & 5.35091375000000 & 8.82855845013778 & 1.26122399999993 \\
\hline $\mathbf{C u}$ & 5.35091375000000 & 11.35100286397460 & 1.26122399999993 \\
\hline $\mathbf{C u}$ & 5.35091375000000 & 13.87344798409370 & 1.26122399999993 \\
\hline $\mathbf{C u}$ & 5.35091375000000 & 16.39589310421720 & 1.26122399999993 \\
\hline $\mathbf{C u}$ & 5.35091375000000 & 18.91833716491180 & 1.26122399999993 \\
\hline $\mathbf{C u}$ & 5.35091375000000 & 21.44078263817520 & 1.26122399999993 \\
\hline $\mathbf{C u}$ & 5.35091375000000 & 23.96322669886980 & 1.26122399999993 \\
\hline $\mathbf{C u}$ & 5.35091375000000 & 26.48567181899090 & 1.26122399999993 \\
\hline $\mathbf{C u}$ & 5.35091375000000 & 29.00811693910800 & 1.26122399999993 \\
\hline $\mathrm{Cu}$ & 5.35091375000000 & 31.53056135294440 & 1.26122399999993 \\
\hline $\mathbf{C u}$ & 5.35091375000000 & 34.05300647307050 & 1.26122399999993 \\
\hline $\mathbf{C u}$ & 8.91818794238642 & 1.26122414920059 & 1.26122399999993 \\
\hline $\mathbf{C u}$ & 8.91818794238642 & 3.78366926932192 & 1.26122399999993 \\
\hline $\mathbf{C u}$ & 8.91818794238642 & 6.30611333001662 & 1.26122399999993 \\
\hline
\end{tabular}




\begin{tabular}{|c|c|c|c|}
\hline $\mathbf{C u}$ & 8.91818794238642 & 8.82855845013778 & 1.2612239 \\
\hline $\mathbf{C u}$ & 8.91818794238642 & 11.35100286397460 & 1.26122399999993 \\
\hline $\mathbf{C u}$ & 8.91818794238642 & 13.87344798409370 & 1.26122399999993 \\
\hline $\mathbf{C u}$ & 8.91818794238642 & 16.39589310421720 & 1.26122399999993 \\
\hline $\mathbf{C u}$ & 8.91818794238642 & 18.91833716491180 & 1.26122399999993 \\
\hline $\mathbf{C u}$ & 8.91818794238642 & 21.44078263817520 & 1.26122399999993 \\
\hline $\mathbf{C u}$ & 8.91818794238642 & 23.96322669886980 & 1.26122399999993 \\
\hline $\mathbf{C u}$ & 8.91818794238642 & 26.48567181899090 & 1.26122399999993 \\
\hline $\mathbf{C u}$ & 8.91818794238642 & 29.00811693910800 & 1.26122399999993 \\
\hline $\mathbf{C u}$ & 8.91818794238642 & 31.53056135294440 & 1.26122399999993 \\
\hline $\mathbf{C u}$ & 8.91818794238642 & 34.05300647307050 & 1.261223999 \\
\hline $\mathbf{C u}$ & 0.00147184607531 & 35.31339190464360 & 2.53812008515763 \\
\hline $\mathbf{C u}$ & 10.69940666956650 & 2.52312568545808 & 2.54079221935663 \\
\hline $\mathbf{C u}$ & 0.00294694897648 & 5.04392265533951 & 2.54241430772550 \\
\hline $\mathrm{Cu}$ & -0.00080993487724 & 7.56490657286146 & 2.54206680779442 \\
\hline $\mathrm{Cu}$ & 10.70126557882460 & 10.08952301161680 & 2.53925189976290 \\
\hline $\mathrm{Cu}$ & 10.70318333758230 & 12.61279975224340 & 2.54468638874482 \\
\hline $\mathbf{C u}$ & 10.69648922941630 & 15.13502333252880 & 2.53664147680221 \\
\hline $\mathbf{C u}$ & 10.69969702173400 & 17.65725502094980 & 2.54571999888825 \\
\hline $\mathbf{C u}$ & 10.69685245801690 & 20.17827287616840 & 2.53798381739597 \\
\hline $\mathbf{C u}$ & 10.69723440183300 & 22.70193174745090 & 2.54646078104477 \\
\hline $\mathbf{C u}$ & 10.69984755647440 & 25.22641151599920 & 2.53847879437952 \\
\hline $\mathbf{C u}$ & 10.69960001523070 & 27.74612092070980 & 2.54326338492459 \\
\hline $\mathbf{C u}$ & 0.00100742396254 & 30.26837180619970 & 2.54147884199894 \\
\hline $\mathbf{C u}$ & 10.70084517965230 & 32.79108759891900 & 2.54081498799676 \\
\hline $\mathbf{C u}$ & 3.56727330074441 & 0.00141893165521 & 2.54195318994686 \\
\hline $\mathbf{C u}$ & 3.56727936625166 & 2.52103031121832 & 2.54251683401659 \\
\hline $\mathbf{C u}$ & 3.56727673898712 & 5.04569231822600 & 2.54074432017689 \\
\hline $\mathbf{C u}$ & 3.56727242005576 & 7.57119799530357 & 2.58536626956272 \\
\hline $\mathbf{C u}$ & 3.56728405344507 & 10.08906952401850 & 2.59390443163033 \\
\hline $\mathbf{C u}$ & 3.56727064696908 & 12.61948632920460 & 2.58380952127559 \\
\hline $\mathbf{C u}$ & 3.56726595613003 & 15.14125132759680 & 2.58646564930334 \\
\hline $\mathbf{C u}$ & 3.56727536174217 & 17.65099029663940 & 2.58776618569191 \\
\hline $\mathbf{C u}$ & 3.56727798352887 & 20.17563564854770 & 2.57979896896793 \\
\hline $\mathbf{C u}$ & 3.56728191725593 & 22.69597753140790 & 2.57716508616715 \\
\hline $\mathbf{C u}$ & 3.56727640411686 & 25.21929577583220 & 2.55214536670560 \\
\hline $\mathbf{C u}$ & 3.56728214296229 & 27.74788351175830 & 2.54108015399718 \\
\hline $\mathbf{C u}$ & 3.56727916675934 & 30.26852505148270 & 2.54087944224414 \\
\hline $\mathbf{C u}$ & 3.56727057159826 & 32.79075235209270 & 2.54259007891596 \\
\hline $\mathbf{C u}$ & 7.13307679305607 & 35.31338678825110 & 2.53812475303861 \\
\hline $\mathbf{C u}$ & 7.13697701785055 & 2.52312690700271 & 2.54079269948462 \\
\hline $\mathbf{C u}$ & 7.13161403631457 & 5.04391920993250 & 2.54241557982031 \\
\hline $\mathbf{C u}$ & 7.13537007726902 & 7.56490540160623 & 2.54205383906438 \\
\hline $\mathbf{C u}$ & 7.13511273144557 & 10.08951186509390 & 2.53926029978976 \\
\hline $\mathbf{C u}$ & 7.13319769617369 & 12.61280272760170 & 2.54468474441962 \\
\hline $\mathbf{C u}$ & 7.13988623623792 & 15.13500462627350 & 2.53664107810698 \\
\hline $\mathbf{C u}$ & 7.13667501131857 & 17.65723430482120 & 2.54572530445400 \\
\hline $\mathbf{C u}$ & 7.13952884101662 & 20.17825843461520 & 2.53800949100632 \\
\hline $\mathbf{C u}$ & 7.13914013144639 & 22.70191729007160 & 2.54646047582177 \\
\hline $\mathbf{C u}$ & 7.13653373786277 & 25.22642517112030 & 2.53848338336619 \\
\hline $\mathbf{C u}$ & 7.13679590324581 & 27.74614447856600 & 2.54329088885188 \\
\hline $\mathbf{C u}$ & 7.13354685327232 & 30.26839522695990 & 2.54145550666054 \\
\hline $\mathbf{C u}$ & 7.13554365856120 & 32.79108860648910 & 2.54081023208885 \\
\hline $\mathbf{C u}$ & 1.78384441918622 & 1.25853363410240 & 3.85279098025561 \\
\hline
\end{tabular}




\begin{tabular}{|c|c|c|c|}
\hline $\mathbf{C u}$ & 1.78013328959328 & 3.77900979669388 & 3.85395339292934 \\
\hline $\mathbf{C u}$ & 1.79578966385928 & 6.30449042612391 & 3.86084852039403 \\
\hline $\mathbf{C u}$ & 1.78246042250278 & 8.82569578431568 & 3.83756662343955 \\
\hline $\mathbf{C u}$ & 1.79292337539059 & 11.34274544962680 & 3.84696451401163 \\
\hline $\mathbf{C u}$ & 1.78245989174699 & 13.87990953796900 & 3.84308851265108 \\
\hline $\mathbf{C u}$ & 1.77502330976637 & 16.39572724385460 & 3.84618410197870 \\
\hline $\mathbf{C u}$ & 1.78670189436345 & 18.92044400007290 & 3.85196546643926 \\
\hline $\mathbf{C u}$ & 1.77717307705379 & 21.43717866864680 & 3.83789262864551 \\
\hline $\mathbf{C u}$ & 1.78577439625723 & 23.96689915256080 & 3.84404084488556 \\
\hline $\mathbf{C u}$ & 1.77986102730325 & 26.48758647109980 & 3.85521882868673 \\
\hline $\mathbf{C u}$ & 1.78397537017455 & 29.00855984765400 & 3.85544987488536 \\
\hline $\mathbf{C u}$ & 1.78287050662152 & 31.52957091412280 & 3.85585411859850 \\
\hline $\mathbf{C u}$ & 1.78382710899398 & 34.04935590800620 & 3.85358745195742 \\
\hline $\mathrm{Cu}$ & 5.35072696860079 & 1.25852529238615 & 3.85275604975404 \\
\hline $\mathbf{C u}$ & 5.35441346110838 & 3.77902542808565 & 3.85396668221710 \\
\hline $\mathbf{C u}$ & 5.33876879642680 & 6.30447800329413 & 3.86080645619801 \\
\hline $\mathbf{C u}$ & 5.35210107283048 & 8.82568936591146 & 3.83755789219745 \\
\hline $\mathbf{C u}$ & 5.34162860946487 & 11.34275392539940 & 3.84695642473755 \\
\hline $\mathbf{C u}$ & 5.35206976458012 & 13.87988851761700 & 3.84309526128023 \\
\hline $\mathbf{C u}$ & 5.35951758875710 & 16.39570851332220 & 3.84615369604564 \\
\hline $\mathbf{C u}$ & 5.34785983279898 & 18.92043918784290 & 3.85197034999930 \\
\hline $\mathbf{C u}$ & 5.35738917559971 & 21.43715931852390 & 3.83790936580998 \\
\hline $\mathbf{C u}$ & 5.34877972087421 & 23.96690536827260 & 3.84403456259448 \\
\hline $\mathbf{C u}$ & 5.354688 & 26.48758964579280 & 7705971 \\
\hline $\mathrm{Cu}$ & 189018954 & 29.0085478 & 3.85 \\
\hline $\mathrm{Cu}$ & 5.35167538427484 & 31.52957748665620 & 852260237 \\
\hline $\mathbf{C u}$ & 5.35071400745906 & 34.04936000809820 & 3.85357656517576 \\
\hline $\mathbf{C u}$ & 8.91818972168176 & 1.26151359462897 & 3.84917355381224 \\
\hline $\mathrm{Cu}$ & 8.91819267599210 & 3.78182583158455 & 3.85176875606143 \\
\hline $\mathrm{Cu}$ & 8.91819435584300 & 6.30296497923787 & 3.85110079180203 \\
\hline $\mathrm{Cu}$ & 8.91818432803347 & 8.82771345258081 & 3.85529097307796 \\
\hline $\mathrm{Cu}$ & 8.91818777215494 & 11.35315960774430 & 3.84806543666366 \\
\hline $\mathrm{Cu}$ & 8.91819818947997 & 13.87016787365820 & 3.85053447209626 \\
\hline $\mathbf{C u}$ & 8.91817208117490 & 16.39458837518350 & 3.85494768545209 \\
\hline $\mathbf{C u}$ & 8.91817582976600 & 18.91417975461710 & 3.85843543169086 \\
\hline $\mathbf{C u}$ & 8.91820218480324 & 21.44007643397260 & 3.85594190010431 \\
\hline $\mathbf{C u}$ & 8.91817941527672 & 23.96116653455380 & 3.85136362781391 \\
\hline $\mathbf{C u}$ & 8.91818136163615 & 26.48937241473970 & 3.85437515781632 \\
\hline $\mathbf{C u}$ & 8.91818063400680 & 29.00779858238190 & 3.85040528655001 \\
\hline $\mathbf{C u}$ & 8.91818384387469 & 31.53035723310340 & 3.85075814972538 \\
\hline $\mathbf{C u}$ & 8.91818838661469 & 34.04875458350720 & 3.84971276696603 \\
\hline $\mathbf{C u}$ & -0.00008344846338 & 35.31073590777060 & 5.05405974412079 \\
\hline $\mathbf{C u}$ & 0.00182053262014 & 2.52032448569594 & 5.05937423699698 \\
\hline $\mathbf{C u}$ & 0.00229632953001 & 5.04768609844016 & 5.05691862996773 \\
\hline $\mathrm{Cu}$ & 0.00383993808711 & 7.56675333034502 & 5.05138319129173 \\
\hline $\mathbf{C u}$ & 0.00490029358557 & 10.09084963845250 & 5.04874083240749 \\
\hline $\mathbf{C u}$ & 0.00760498394892 & 12.60839728820320 & 5.04885504710446 \\
\hline $\mathbf{C u}$ & 0.00199465041169 & 15.13102011015410 & 5.05378564766802 \\
\hline $\mathbf{C u}$ & 0.00114921009333 & 17.65505390226310 & 5.06308173379745 \\
\hline $\mathbf{C u}$ & 0.00769451549279 & 20.17639680140410 & 5.05380337096880 \\
\hline $\mathbf{C u}$ & 0.00928855626061 & 22.70315617223680 & 5.05862087996314 \\
\hline $\mathbf{C u}$ & 0.00224985118440 & 25.22144870059970 & 5.05345602647692 \\
\hline $\mathbf{C u}$ & 10.70181476601700 & 27.74835395966600 & 5.06254620080478 \\
\hline $\mathbf{C u}$ & 10.70032527469130 & 30.26618656327180 & 5.05679945190680 \\
\hline
\end{tabular}




\begin{tabular}{|c|c|c|c|}
\hline $\mathbf{C u}$ & 0.00297502189254 & 32.78979455641010 & 5.05918634857847 \\
\hline $\mathbf{C u}$ & 3.56726838254270 & 35.30926945374650 & 5.05807879818293 \\
\hline $\mathbf{C u}$ & 3.56726967122537 & 2.51807826294942 & 5.05767209109283 \\
\hline $\mathbf{C u}$ & 3.56727972143268 & 5.02396955404878 & 5.05085028885071 \\
\hline $\mathbf{C u}$ & 3.56730179406182 & 7.52803565043093 & 5.16061266723373 \\
\hline $\mathbf{C u}$ & 3.56729448561970 & 10.11641571177290 & 5.17432473843593 \\
\hline $\mathbf{C u}$ & 3.56719111002637 & 12.61350731800420 & 5.13254579798212 \\
\hline $\mathbf{C u}$ & 3.56726641301003 & 15.11621630978200 & 5.10567079467544 \\
\hline $\mathbf{C u}$ & 3.56730090663297 & 17.64936673985050 & 5.13868393254968 \\
\hline $\mathbf{C u}$ & 3.56725310676018 & 20.17554107963240 & 5.09468578868809 \\
\hline $\mathbf{C u}$ & 3.56728301482696 & 22.71717374914670 & 5.12642324744804 \\
\hline $\mathbf{C u}$ & 3.56726680670667 & 25.24168576389350 & 5.05831696909086 \\
\hline $\mathbf{C u}$ & 3.56727347363523 & 27.75195426796160 & 5.05601770690631 \\
\hline $\mathbf{C u}$ & 3.56727678637211 & 30.27366022321460 & 5.05834974489352 \\
\hline $\mathbf{C u}$ & 3.56726928131490 & 32.78887994202410 & 5.0595 \\
\hline $\mathbf{C u}$ & 7.13462751619935 & 35.31073106235560 & 5.05406751473869 \\
\hline $\mathbf{C u}$ & 7.13272314888629 & 2.52031828490040 & 5.05938269 \\
\hline $\mathrm{Cu}$ & 7.13226149855264 & 5.04768966601413 & 5.05692763590123 \\
\hline $\mathbf{C u}$ & 7.13068775710420 & 7.56676713556445 & 5.05135939734767 \\
\hline $\mathbf{C u}$ & 7.12964839148539 & 10.09086486054740 & 67439 \\
\hline $\mathbf{C u}$ & 7.12696340043385 & 12.60839576545650 & 5.04884660955087 \\
\hline $\mathbf{C u}$ & 7.13254450001993 & 15.13099347565120 & 5.05376679662440 \\
\hline $\mathbf{C u}$ & 7.13333063718306 & 17.65499919487480 & 5.06308512347321 \\
\hline $\mathbf{C u}$ & 7.12685769439646 & 20.17636454111600 & 5.05386560672050 \\
\hline $\mathbf{C u}$ & 7.12526668392185 & 22.70311357732370 & 5.05861331725999 \\
\hline $\mathbf{C u}$ & 7.13227895533104 & 25.22143485707380 & 5.05346038212206 \\
\hline $\mathbf{C u}$ & 7.13457917615914 & 27.74835885737150 & 5.06257537353394 \\
\hline $\mathbf{C u}$ & 7.13604801693182 & 30.26620014129820 & 5.05680094711927 \\
\hline $\mathbf{C u}$ & 7.13155563934630 & 32.78978861674140 & 5.05919992231355 \\
\hline \multicolumn{4}{|c|}{ CI-NEB Image 11} \\
\hline Element & X-Coordinate (§̊) & Y-Coordinate (§̊) & Z-Coordinate (Å) \\
\hline $\mathbf{F}$ & 4.63232199562518 & 12.76052104631270 & 7.82364348326476 \\
\hline $\mathbf{F}$ & 2.50280593805596 & 12.76031299665800 & 7.82382107049968 \\
\hline $\mathbf{F}$ & 3.56727547442863 & 8.81250336145380 & 6.65106568099976 \\
\hline $\mathbf{F}$ & 4.65128321087575 & 17.44825724468270 & 7.84301041231325 \\
\hline $\mathbf{F}$ & 2.48333072228798 & 17.44826500179180 & 7.84306062451627 \\
\hline $\mathbf{F}$ & 3.56728284463724 & 15.56861968470410 & 7.14237287842722 \\
\hline $\mathbf{F}$ & 4.66371163965845 & 22.76239489604680 & 7.75948401750293 \\
\hline $\mathbf{F}$ & 2.47081837386522 & 22.76239226898170 & 7.75946239872231 \\
\hline $\mathbf{F}$ & 3.56727274326482 & 20.89092165666270 & 7.33217893189600 \\
\hline C & 3.56749786572616 & 12.73568608083100 & 7.03286353373424 \\
\hline C & 3.56728874889903 & 17.15787774810700 & 7.08194919705419 \\
\hline C & 3.56727296623356 & 22.30432216405930 & 7.06506787932237 \\
\hline $\mathbf{C u}$ & 0.00000000000000 & 0.00000000000000 & 0.00000000000000 \\
\hline $\mathbf{C u}$ & 0.00000000000000 & 2.52244512012115 & 0.00000000000000 \\
\hline $\mathbf{C u}$ & 0.00000000000000 & 5.04488953395804 & 0.00000000000000 \\
\hline $\mathbf{C u}$ & 0.00000000000000 & 7.56733465407878 & 0.00000000000000 \\
\hline $\mathbf{C u}$ & 0.00000000000000 & 10.08977871477400 & 0.00000000000000 \\
\hline $\mathbf{C u}$ & 0.00000000000000 & 12.61222383489520 & 0.00000000000000 \\
\hline $\mathbf{C u}$ & 0.00000000000000 & 15.13466895501450 & 0.00000000000000 \\
\hline $\mathbf{C u}$ & 0.00000000000000 & 17.65711336885320 & 0.00000000000000 \\
\hline $\mathbf{C u}$ & 0.00000000000000 & 20.17955848897460 & 0.00000000000000 \\
\hline $\mathbf{C u}$ & 0.00000000000000 & 22.70200254966930 & 0.00000000000000 \\
\hline $\mathbf{C u}$ & 0.00000000000000 & 25.22444766979040 & 0.00000000000000 \\
\hline
\end{tabular}




\begin{tabular}{|c|c|c|c|}
\hline $\mathbf{C u}$ & 0.00000000000000 & 27.74689208362240 & 0.00000000000000 \\
\hline $\mathbf{C u}$ & 0.00000000000000 & 30.26933720374360 & 0.00000000000000 \\
\hline $\mathbf{C u}$ & 0.00000000000000 & 32.79178232386990 & 0.00000000000000 \\
\hline $\mathbf{C u}$ & 3.56727419238642 & 0.00000000000000 & 0.00000000000000 \\
\hline $\mathbf{C u}$ & 3.56727419238642 & 2.52244512012115 & 0.00000000000000 \\
\hline $\mathbf{C u}$ & 3.56727419238642 & 5.04488953395804 & 0.00000000000000 \\
\hline $\mathbf{C u}$ & 3.56727419238642 & 7.56733465407878 & 0.00000000000000 \\
\hline $\mathbf{C u}$ & 3.56727419238642 & 10.08977871477400 & 0.00000000000000 \\
\hline $\mathbf{C u}$ & 3.56727419238642 & 12.61222383489520 & 0.00000000000000 \\
\hline $\mathbf{C u}$ & 3.56727419238642 & 15.13466895501450 & 0.00000000000000 \\
\hline $\mathbf{C u}$ & 3.56727419238642 & 17.65711336885320 & 0.00000000000000 \\
\hline $\mathbf{C u}$ & 3.56727419238642 & 20.17955848897460 & 0.00000000000000 \\
\hline $\mathbf{C u}$ & 3.56727419238642 & 22.70200254966930 & 0.00000000000000 \\
\hline $\mathbf{C u}$ & 3.56727419238642 & 25.22444766979040 & 0.00000000000000 \\
\hline $\mathbf{C u}$ & 3.56727419238642 & 27.74689208362240 & 0.00000000000000 \\
\hline $\mathbf{C u}$ & 3.56727419238642 & 30.26933720374360 & 0.00000000000000 \\
\hline $\mathbf{C u}$ & 3.56727419238642 & 32.79178232386990 & 0.00000000000000 \\
\hline $\mathbf{C u}$ & 7.13454838477291 & 0.00000000000000 & 0.00000000000000 \\
\hline $\mathbf{C u}$ & 7.13454838477291 & 2.52244512012115 & 0.00000000000000 \\
\hline $\mathbf{C u}$ & 7.13454838477291 & 5.04488953395804 & 0.00000000000000 \\
\hline $\mathbf{C u}$ & 7.13454838477291 & 7.56733465407878 & 0000000000 \\
\hline $\mathbf{C u}$ & 7.13454838477291 & 10.08977871477400 & 0.00000000000000 \\
\hline $\mathbf{C u}$ & 7.13454838477291 & 12.61222383489520 & 0000000 \\
\hline $\mathrm{Cu}$ & 7.13454838477291 & 15.13466895501450 & 000000 \\
\hline $\mathbf{C u}$ & 7.13454838477291 & 17.65711336885320 & 0000000 \\
\hline $\mathrm{Cu}$ & 7.13454838477291 & 20.17955848897460 & 0000000000 \\
\hline $\mathrm{Cu}$ & 7.13454838477291 & 22.70200254966930 & 0.00000000000000 \\
\hline $\mathbf{C u}$ & 7.13454838477291 & 25.22444766979040 & 0.00000000000000 \\
\hline $\mathbf{C u}$ & 7.13454838477291 & 27.74689208362240 & 0.00000000000000 \\
\hline $\mathbf{C u}$ & 7.13454838477291 & 30.26933720374360 & 0.00000000000000 \\
\hline $\mathbf{C u}$ & 7.13454838477291 & 32.79178232386990 & 0.00000000000000 \\
\hline $\mathbf{C u}$ & 1.78363955761358 & 1.26122414920059 & 1.26122399999993 \\
\hline $\mathrm{Cu}$ & 1.78363955761358 & 3.78366926932192 & 1.26122399999993 \\
\hline $\mathrm{Cu}$ & 1.78363955761358 & 6.30611333001662 & 1.26122399999993 \\
\hline $\mathbf{C u}$ & 1.78363955761358 & 8.82855845013778 & 1.26122399999993 \\
\hline $\mathbf{C u}$ & 1.78363955761358 & 11.35100286397460 & 1.26122399999993 \\
\hline $\mathbf{C u}$ & 1.78363955761358 & 13.87344798409370 & 1.26122399999993 \\
\hline $\mathbf{C u}$ & 1.78363955761358 & 16.39589310421720 & 1.26122399999993 \\
\hline $\mathbf{C u}$ & 1.78363955761358 & 18.91833716491180 & 1.26122399999993 \\
\hline $\mathbf{C u}$ & 1.78363955761358 & 21.44078263817520 & 1.26122399999993 \\
\hline $\mathbf{C u}$ & 1.78363955761358 & 23.96322669886980 & 1.26122399999993 \\
\hline $\mathbf{C u}$ & 1.78363955761358 & 26.48567181899090 & 1.26122399999993 \\
\hline $\mathbf{C u}$ & 1.78363955761358 & 29.00811693910750 & 1.26122399999993 \\
\hline $\mathbf{C u}$ & 1.78363955761358 & 31.53056135294390 & 1.26122399999993 \\
\hline $\mathbf{C u}$ & 1.78363955761358 & 34.05300647307050 & 1.26122399999993 \\
\hline $\mathbf{C u}$ & 5.35091375000000 & 1.26122414920059 & 1.26122399999993 \\
\hline $\mathbf{C u}$ & 5.35091375000000 & 3.78366926932192 & 1.26122399999993 \\
\hline $\mathrm{Cu}$ & 5.35091375000000 & 6.30611333001662 & 1.26122399999993 \\
\hline $\mathrm{Cu}$ & 5.35091375000000 & 8.82855845013778 & 1.26122399999993 \\
\hline $\mathrm{Cu}$ & 5.35091375000000 & 11.35100286397460 & 1.26122399999993 \\
\hline $\mathbf{C u}$ & 5.35091375000000 & 13.87344798409370 & 1.26122399999993 \\
\hline $\mathbf{C u}$ & 5.35091375000000 & 16.39589310421720 & 1.26122399999993 \\
\hline $\mathbf{C u}$ & 5.35091375000000 & 18.91833716491180 & 1.26122399999993 \\
\hline $\mathbf{C u}$ & 5.35091375000000 & 21.44078263817520 & 1.26122399999993 \\
\hline
\end{tabular}




\begin{tabular}{|c|c|c|c|}
\hline $\mathbf{C u}$ & 5.35091375000000 & 23.96322669886980 & 1.26122399999993 \\
\hline $\mathbf{C u}$ & 5.35091375000000 & 26.48567181899090 & 1.26122399999993 \\
\hline $\mathbf{C u}$ & 5.35091375000000 & 29.00811693910750 & 1.26122399999993 \\
\hline $\mathbf{C u}$ & 5.35091375000000 & 31.53056135294390 & 1.26122399999993 \\
\hline $\mathbf{C u}$ & 5.35091375000000 & 34.05300647307050 & 1.26122399999993 \\
\hline $\mathbf{C u}$ & 8.91818794238642 & 1.26122414920059 & 1.26122399999993 \\
\hline $\mathbf{C u}$ & 8.91818794238642 & 3.78366926932192 & 1.26122399999993 \\
\hline $\mathbf{C u}$ & 8.91818794238642 & 6.30611333001662 & 1.26122399999993 \\
\hline $\mathbf{C u}$ & 8.91818794238642 & 8.82855845013778 & 1.26122399999993 \\
\hline $\mathbf{C u}$ & 8.91818794238642 & 11.35100286397460 & 1.26122399999993 \\
\hline $\mathbf{C u}$ & 8.91818794238642 & 13.87344798409370 & 1.26122399999993 \\
\hline $\mathbf{C u}$ & 8.91818794238642 & 16.39589310421720 & 1.26122399999993 \\
\hline $\mathbf{C u}$ & 8.91818794238642 & 18.91833716491180 & 1.26122399999993 \\
\hline $\mathbf{C u}$ & 8.91818794238642 & 21.44078263817520 & 1.26122399999993 \\
\hline $\mathbf{C u}$ & 8.91818794238642 & 23.96322669886980 & 1.26122399999993 \\
\hline $\mathbf{C u}$ & 8.91818794238642 & 26.48567181899090 & 1.26122399999993 \\
\hline $\mathbf{C u}$ & 8.91818794238642 & 29.00811693910750 & 1.26122399999993 \\
\hline $\mathbf{C u}$ & 8.91818794238642 & 31.53056135294390 & 1.26122399999993 \\
\hline $\mathbf{C u}$ & 8.91818794238642 & 34.05300647307050 & 1.26122399999993 \\
\hline $\mathbf{C u}$ & 0.00143894400083 & 35.31364514616620 & 2.53836885847685 \\
\hline $\mathbf{C u}$ & 10.69930954941740 & 2.52339877868270 & 2.54071821693275 \\
\hline $\mathbf{C u}$ & 0.00271404948209 & 5.04407335578874 & 2.54241372236932 \\
\hline $\mathbf{C u}$ & 10.70060983764270 & 785417155 & 94917901 \\
\hline $\mathbf{C u}$ & 10.70133237891020 & 10.09052700811780 & 764316 \\
\hline $\mathbf{C u}$ & 10.70305264013820 & 5728490 & 615409 \\
\hline $\mathrm{Cu}$ & 10.69664924970130 & 486587540 & 557330905 \\
\hline $\mathrm{Cu}$ & 10.70099599932730 & 17.65863318699130 & 2.54335738053048 \\
\hline $\mathbf{C u}$ & 10.69849484585960 & 20.17917573431230 & 2.53850383596270 \\
\hline $\mathbf{C u}$ & 10.69716464661520 & 22.70265793140250 & 2.54566824269628 \\
\hline $\mathbf{C u}$ & 10.70019208565640 & 25.22737935483800 & 2.53938290827659 \\
\hline $\mathbf{C u}$ & 10.70024304156910 & 27.74680265737900 & 2.54365662144493 \\
\hline $\mathbf{C u}$ & 0.00094291266553 & 30.26884632966800 & 2.54148435608390 \\
\hline $\mathrm{Cu}$ & 10.70073771622100 & 32.79143866615990 & 2.54085717865675 \\
\hline $\mathrm{Cu}$ & 3.56727681210911 & 0.00119321566079 & 2.54254073568654 \\
\hline $\mathrm{Cu}$ & 3.56727645151158 & 2.52057750186529 & 2.54305809416574 \\
\hline $\mathbf{C u}$ & 3.56727263885516 & 5.04509713406323 & 2.54059493481280 \\
\hline $\mathbf{C u}$ & 3.56727284717578 & 7.57030622431227 & 2.58378754200016 \\
\hline $\mathbf{C u}$ & 3.56728735358193 & 10.08912636564500 & 2.58914078815324 \\
\hline $\mathbf{C u}$ & 3.56728422309386 & 12.62089735486200 & 2.59092932850545 \\
\hline $\mathbf{C u}$ & 3.56727921368820 & 15.13960680066040 & 2.59471570554874 \\
\hline $\mathbf{C u}$ & 3.56727857408144 & 17.65287528160070 & 2.60157740094940 \\
\hline $\mathbf{C u}$ & 3.56727935637916 & 20.17824963422660 & 2.57840604163400 \\
\hline $\mathbf{C u}$ & 3.56728249347152 & 22.69696370980860 & 2.58076378098618 \\
\hline $\mathbf{C u}$ & 3.56727689482813 & 25.21956806057140 & 2.55299542088957 \\
\hline $\mathbf{C u}$ & 3.56728299061172 & 27.74859090627710 & 2.54219464325897 \\
\hline $\mathbf{C u}$ & 3.56727752505261 & 30.26832264544350 & 2.54167274222030 \\
\hline $\mathbf{C u}$ & 3.56727739491117 & 32.79046560818740 & 2.54310111239324 \\
\hline $\mathbf{C u}$ & 7.13311158221441 & 35.31363944789690 & 2.53837458898112 \\
\hline $\mathrm{Cu}$ & 7.13706782020666 & 2.52339846108158 & 2.54072192173531 \\
\hline $\mathrm{Cu}$ & 7.13183801917850 & 5.04406320058500 & 2.54240358599038 \\
\hline $\mathbf{C u}$ & 7.13576572659167 & 7.56503258589193 & 2.54205795098221 \\
\hline $\mathbf{C u}$ & 7.13505462328183 & 10.09051914581980 & 2.53986967978812 \\
\hline $\mathbf{C u}$ & 7.13333061600897 & 12.61166983365910 & 2.54482969372801 \\
\hline $\mathbf{C u}$ & 7.13973326917045 & 15.13599189020090 & 2.53707358257681 \\
\hline
\end{tabular}




\begin{tabular}{|c|c|c|c|}
\hline $\mathrm{Cu}$ & 7.13538328378530 & 17.65863113933910 & 2.54336248351221 \\
\hline $\mathbf{C u}$ & 7.13788455836323 & 20.17917620804340 & 2.53850625917095 \\
\hline $\mathrm{Cu}$ & 7.13921406309082 & 22.70265585132500 & 2.54566969310088 \\
\hline $\mathbf{C u}$ & 7.13617924371764 & 25.22738624632120 & 2.53938626789433 \\
\hline $\mathbf{C u}$ & 7.13612879370622 & 27.74679856552780 & 2.54365624832969 \\
\hline $\mathbf{C u}$ & 7.13360761918989 & 30.26884764739150 & 2.54148498049728 \\
\hline $\mathbf{C u}$ & 7.13563957702129 & 32.79143770456860 & 2.54086048988936 \\
\hline $\mathbf{C u}$ & 1.78306633825432 & 1.25920294548049 & 3.85325098873946 \\
\hline $\mathbf{C u}$ & 1.77910724451419 & 3.77926097432140 & 3.85502904437296 \\
\hline $\mathbf{C u}$ & 1.79553434160372 & 6.30399087236461 & 3.86080767064657 \\
\hline $\mathbf{C u}$ & 1.78079340872030 & 8.82453824222320 & 3.83381175392708 \\
\hline $\mathbf{C u}$ & 1.79728102731833 & 11.34483532601160 & 3.85280370596505 \\
\hline $\mathbf{C u}$ & 1.78051744192404 & 13.87769274349290 & 3.84245753736844 \\
\hline $\mathrm{Cu}$ & 1.77674132283313 & 16.39955520509140 & 3.84697219512414 \\
\hline $\mathbf{C u}$ & 1.78898076247672 & 18.92251170079090 & 3.85536442078696 \\
\hline $\mathbf{C u}$ & 1.77631984487262 & 21.44100073074060 & 3.83979846624935 \\
\hline $\mathbf{C u}$ & 1.78547587960566 & 23.96784242137040 & 3.84684291897746 \\
\hline $\mathbf{C u}$ & 1.78036008791817 & 26.48874263846230 & 3.85626377976932 \\
\hline $\mathbf{C u}$ & 1.78384729468426 & 29.00933259409820 & 3.85678784523377 \\
\hline $\mathbf{C u}$ & 1.78238381980594 & 31.53019793287670 & 3.85656755431045 \\
\hline $\mathbf{C u}$ & 1.78362465494730 & 34.04958686529740 & 3.85367600856997 \\
\hline $\mathbf{C u}$ & 5.35148709750917 & 1.25920620706997 & 3.85324734997593 \\
\hline $\mathbf{C u}$ & 5.35544160004268 & 3.77926709258422 & 36160012 \\
\hline $\mathbf{C u}$ & 5.33899937500359 & 6.30399 & 287272 \\
\hline $\mathbf{C u}$ & 5.35375529223052 & 8.82453679925238 & 826457 \\
\hline $\mathbf{C u}$ & 5.33728615586494 & 11.34482379985570 & 3.85277 \\
\hline $\mathbf{C u}$ & 5.35404927137391 & 13.87770626108060 & 3.84244787485669 \\
\hline $\mathbf{C u}$ & 5.35781778321805 & 16.39956141503770 & 3.84698520815331 \\
\hline $\mathbf{C u}$ & 5.34557012224292 & 18.92250975790490 & 3.85535498034440 \\
\hline $\mathbf{C u}$ & 5.35823409281778 & 21.44100437626100 & 3.83980715897829 \\
\hline $\mathbf{C u}$ & 5.34906794408910 & 23.96784420421060 & 3.84685191948781 \\
\hline $\mathbf{C u}$ & 5.35418858041655 & 26.48873979786420 & 3.85625759009176 \\
\hline $\mathbf{C u}$ & 5.35070336158243 & 29.00933208597260 & 3.85678942385626 \\
\hline $\mathbf{C u}$ & 5.35216784673454 & 31.53019616557160 & 3.85657179299465 \\
\hline $\mathbf{C u}$ & 5.35092589610436 & 34.04957556048310 & 3.85368468661239 \\
\hline $\mathbf{C u}$ & 8.91818899855449 & 1.26184266161653 & 3.84927526943259 \\
\hline $\mathbf{C u}$ & 8.91818860291905 & 3.78377142746116 & 3.85079911487213 \\
\hline $\mathbf{C u}$ & 8.91819884002082 & 6.30361037173404 & 3.85206446076116 \\
\hline $\mathbf{C u}$ & 8.91819350368986 & 8.82891893016927 & 3.85612614606759 \\
\hline $\mathbf{C u}$ & 8.91819568273791 & 11.35373693243870 & 3.84977360125660 \\
\hline $\mathbf{C u}$ & 8.91819380475418 & 13.87097278829590 & 3.85112718322391 \\
\hline $\mathbf{C u}$ & 8.91819200920967 & 16.39798344706030 & 3.85330816040474 \\
\hline $\mathbf{C u}$ & 8.91818922567511 & 18.91856847695380 & 3.85519976257360 \\
\hline $\mathbf{C u}$ & 8.91819465677709 & 21.44089796959110 & 3.85516960752334 \\
\hline $\mathbf{C u}$ & 8.91818739572893 & 23.96296824297780 & 3.85104385514909 \\
\hline $\mathbf{C u}$ & 8.91819223844500 & 26.49149102599160 & 3.85458602798889 \\
\hline $\mathbf{C u}$ & 8.91818994120753 & 29.00820237327320 & 3.85072605298917 \\
\hline $\mathbf{C u}$ & 8.91818869970651 & 31.53211266922440 & 3.85040858025599 \\
\hline $\mathbf{C u}$ & 8.91818911655105 & 34.04965382382050 & 3.85032968537964 \\
\hline $\mathbf{C u}$ & -0.00048020034721 & 35.31164296383990 & 5.05501315833069 \\
\hline $\mathbf{C u}$ & 10.70131261243320 & 2.52136673403872 & 5.05960813565276 \\
\hline $\mathbf{C u}$ & 0.00172297542652 & 5.04830253944375 & 5.05815395280345 \\
\hline $\mathbf{C u}$ & 0.00360707973521 & 7.56769707731871 & 5.05148391256484 \\
\hline $\mathbf{C u}$ & 0.00634650457008 & 10.09189799122350 & 5.04837553729297 \\
\hline
\end{tabular}




\begin{tabular}{|c|c|c|c|}
\hline $\mathbf{C u}$ & 0.00765720003256 & 12.60996629897980 & 5.05009859390669 \\
\hline $\mathbf{C u}$ & 10.70192778128830 & 15.13421644715320 & 5.05153322661762 \\
\hline $\mathbf{C u}$ & 0.00076962403701 & 17.65802664633810 & 5.05837352386602 \\
\hline $\mathbf{C u}$ & 0.00711869336251 & 20.17856491357590 & 5.05473645765473 \\
\hline $\mathbf{C u}$ & 0.00559837969607 & 22.70457870299890 & 5.05860575017460 \\
\hline $\mathbf{C u}$ & 0.00139492927122 & 25.22391888354960 & 5.05468448247183 \\
\hline $\mathbf{C u}$ & 10.70137430519000 & 27.74962850728310 & 5.06449896601674 \\
\hline $\mathbf{C u}$ & 10.69906082037280 & 30.26731650393350 & 5.05744228701047 \\
\hline $\mathbf{C u}$ & 0.00227290808243 & 32.79045318989610 & 5.05963546540175 \\
\hline $\mathbf{C u}$ & 3.56727547444555 & 35.30990083546490 & 145892 \\
\hline $\mathbf{C u}$ & 3.56727590700863 & 2.51780355973873 & 5.05765557342531 \\
\hline $\mathbf{C u}$ & 3.56727122229875 & 5.02229957672042 & 5.05086195859407 \\
\hline $\mathbf{C u}$ & 3.56726787944996 & 7.52371065045584 & 5.16101358099673 \\
\hline $\mathrm{Cu}$ & 3.56727889894913 & 10.10198730628450 & 5.15899325330801 \\
\hline $\mathbf{C u}$ & 3.56731227229566 & 12.60075786889580 & 5.15982526899661 \\
\hline $\mathrm{Cu}$ & 3.56727503210994 & 15.11600940331690 & 5.09322019671501 \\
\hline $\mathrm{Cu}$ & 3.56726500345249 & 17.66591105699980 & 5.17266884137654 \\
\hline $\mathbf{C u}$ & 3.56727413360883 & 20.18472074465250 & 5.09387612307938 \\
\hline $\mathbf{C u}$ & 3.56727813439996 & 22.71864211646850 & 5.13513414489496 \\
\hline $\mathbf{C u}$ & 3.56727726903145 & 25.24406722429940 & 5.06006502890884 \\
\hline $\mathbf{C u}$ & 3.56727445493143 & 27.75345005982140 & 5.05909874517183 \\
\hline $\mathbf{C u}$ & 3.56727274449980 & 30.27462428516080 & 5.0596570 \\
\hline $\mathbf{C u}$ & 3.56727553076826 & 32.79024320453200 & 5.06012596269363 \\
\hline $\mathbf{C u}$ & 7.13502991825306 & 35.31164692149340 & 5.05501054445346 \\
\hline $\mathbf{C u}$ & 7.13506470821348 & 2.52137273492796 & 5.05960569563913 \\
\hline $\mathbf{C u}$ & 7.13282135338239 & 5.04830967596178 & 5.05814479139692 \\
\hline $\mathbf{C u}$ & 7.13093865379090 & 7.56769686217953 & 5.05147105919250 \\
\hline $\mathbf{C u}$ & 7.12821507985146 & 10.09188812883320 & 5.04836339878598 \\
\hline $\mathbf{C u}$ & 7.12690351510539 & 12.60996010119600 & 5.05008064508389 \\
\hline $\mathbf{C u}$ & 7.13446441090451 & 15.13420949590810 & 5.05153728697492 \\
\hline $\mathbf{C u}$ & 7.13379067157646 & 17.65803033767190 & 5.05837782133526 \\
\hline $\mathbf{C u}$ & 7.12743970936255 & 20.17857612372410 & 5.05472474501990 \\
\hline $\mathbf{C u}$ & 7.12896295646644 & 22.70458843074300 & 5.05859938530780 \\
\hline $\mathbf{C u}$ & 7.13315577827575 & 25.22393265719050 & 5.05467950476145 \\
\hline $\mathbf{C u}$ & 7.13501013110920 & 27.74963194420790 & 5.06449894200755 \\
\hline $\mathbf{C u}$ & 7.13732727444779 & 30.26731901656890 & 5.05744465883245 \\
\hline $\mathbf{C u}$ & 7.13227352200613 & 32.79045305977430 & 5.05965140425253 \\
\hline \multicolumn{4}{|c|}{ CI-NEB Image 12} \\
\hline Element & X-Coordinate (Å) & Y-Coordinate (§̊) & Z-Coordinate $(\AA ̊)$ \\
\hline $\mathbf{F}$ & 4.63152238900224 & 12.75438220984300 & 7.84086282644152 \\
\hline $\mathbf{F}$ & 2.50377916340217 & 12.75413124954250 & 7.84103864531402 \\
\hline $\mathbf{F}$ & 3.56727843060136 & 8.82033917744098 & 6.65001892071552 \\
\hline $\mathbf{F}$ & 4.63122379274665 & 17.47655588479170 & 7.85734804804881 \\
\hline $\mathbf{F}$ & 2.50339635463063 & 17.47657527305990 & 7.85740275265344 \\
\hline $\mathbf{F}$ & 3.56728722237326 & 15.12539090813330 & 7.04727421844308 \\
\hline $\mathbf{F}$ & 4.66357342748199 & 22.76249128738100 & 7.76384719626181 \\
\hline $\mathbf{F}$ & 2.47095652674584 & 22.76248935623650 & 7.76382685978936 \\
\hline $\mathbf{F}$ & 3.56727131422366 & 20.88980858612660 & 7.33809336006880 \\
\hline C & 3.56757438080010 & 12.81570299386170 & 7.06109854493163 \\
\hline $\mathbf{C}$ & 3.56728981557940 & 17.42378585069590 & 7.07726675245427 \\
\hline C & 3.56727215791030 & 22.30789851800380 & 7.06707920413928 \\
\hline $\mathbf{C u}$ & 0.00000000000000 & 0.00000000000000 & 0.00000000000000 \\
\hline $\mathbf{C u}$ & 0.00000000000000 & 2.52244512012115 & 0.00000000000000 \\
\hline $\mathbf{C u}$ & 0.00000000000000 & 5.04488953395804 & 0.00000000000000 \\
\hline
\end{tabular}




\begin{tabular}{|c|c|c|c|}
\hline $\mathbf{C u}$ & 0.00000000000000 & 7.56733465407854 & 0.00000000000000 \\
\hline $\mathbf{C u}$ & 0.00000000000000 & 10.08977871477400 & 0.00000000000000 \\
\hline $\mathbf{C u}$ & 0.00000000000000 & 12.61222383489520 & 0.00000000000000 \\
\hline $\mathbf{C u}$ & 0.00000000000000 & 15.13466895501400 & 0.00000000000000 \\
\hline $\mathbf{C u}$ & 0.00000000000000 & 17.65711336885320 & 0.00000000000000 \\
\hline $\mathbf{C u}$ & 0.00000000000000 & 20.17955848897460 & 0.00000000000000 \\
\hline $\mathbf{C u}$ & 0.00000000000000 & 22.70200254966930 & 0.00000000000000 \\
\hline $\mathbf{C u}$ & 0.00000000000000 & 25.22444766979040 & 0.00000000000000 \\
\hline $\mathbf{C u}$ & 0.00000000000000 & 27.74689208362170 & 0.00000000000000 \\
\hline $\mathbf{C u}$ & 0.00000000000000 & 30.26933720374280 & 0.00000000000000 \\
\hline $\mathbf{C u}$ & 0.00000000000000 & 32.79178232386990 & 0.00000000000000 \\
\hline $\mathbf{C u}$ & 3.56727419238642 & 0.00000000000000 & 0.00000000000000 \\
\hline $\mathbf{C u}$ & 3.56727419238642 & 2.52244512012115 & 0.00000000000000 \\
\hline $\mathrm{Cu}$ & 3.56727419238642 & 5.04488953395804 & 0.00000000000000 \\
\hline $\mathbf{C u}$ & 3.56727419238642 & 7.56733465407854 & 0.00000000000000 \\
\hline $\mathbf{C u}$ & 3.56727419238642 & 10.08977871477400 & 0.00000000000000 \\
\hline $\mathbf{C u}$ & 3.56727419238642 & 12.61222383489520 & 0.00000000000000 \\
\hline $\mathbf{C u}$ & 3.56727419238642 & 15.13466895501400 & 0.00000000000000 \\
\hline $\mathbf{C u}$ & 3.56727419238642 & 17.65711336885320 & 0.00000000000000 \\
\hline $\mathbf{C u}$ & 3.56727419238642 & 20.17955848897460 & 0.00000000000000 \\
\hline $\mathbf{C u}$ & 3.56727419238642 & 22.70200254966930 & 0.00000000000000 \\
\hline $\mathbf{C u}$ & 3.56727419238642 & 25.22444766979040 & 0.00000000000000 \\
\hline $\mathbf{C u}$ & 3.56727419238642 & 27.74689208362170 & 0.00000000000000 \\
\hline $\mathbf{C u}$ & 3.56727419238642 & 30.26933720374280 & 0.00000000000000 \\
\hline $\mathrm{Cu}$ & 3.56727419238642 & 32.791782 & 0.00000000000000 \\
\hline $\mathrm{Cu}$ & 7.13454838477291 & 0.00000000000000 & 0000000000 \\
\hline $\mathbf{C u}$ & 7.13454838477291 & 2.52244512012115 & 0.00000000000000 \\
\hline $\mathbf{C u}$ & 7.13454838477291 & 5.04488953395804 & 0.00000000000000 \\
\hline $\mathbf{C u}$ & 7.13454838477291 & 7.56733465407854 & 0.00000000000000 \\
\hline $\mathrm{Cu}$ & 7.13454838477291 & 10.08977871477400 & 0.00000000000000 \\
\hline $\mathrm{Cu}$ & 7.13454838477291 & 12.61222383489520 & 0.00000000000000 \\
\hline $\mathrm{Cu}$ & 7.13454838477291 & 15.13466895501400 & 0.00000000000000 \\
\hline $\mathrm{Cu}$ & 7.13454838477291 & 17.65711336885320 & 0.00000000000000 \\
\hline $\mathbf{C u}$ & 7.13454838477291 & 20.17955848897460 & 0.00000000000000 \\
\hline $\mathbf{C u}$ & 7.13454838477291 & 22.70200254966930 & 0.00000000000000 \\
\hline $\mathbf{C u}$ & 7.13454838477291 & 25.22444766979040 & 0.00000000000000 \\
\hline $\mathbf{C u}$ & 7.13454838477291 & 27.74689208362170 & 0.00000000000000 \\
\hline $\mathbf{C u}$ & 7.13454838477291 & 30.26933720374280 & 0.00000000000000 \\
\hline $\mathbf{C u}$ & 7.13454838477291 & 32.79178232386990 & 0.00000000000000 \\
\hline $\mathbf{C u}$ & 1.78363955761358 & 1.26122414920059 & 1.26122399999993 \\
\hline $\mathbf{C u}$ & 1.78363955761358 & 3.78366926932167 & 1.26122399999993 \\
\hline $\mathbf{C u}$ & 1.78363955761358 & 6.30611333001662 & 1.26122399999993 \\
\hline $\mathbf{C u}$ & 1.78363955761358 & 8.82855845013778 & 1.26122399999993 \\
\hline $\mathbf{C u}$ & 1.78363955761358 & 11.35100286397460 & 1.26122399999993 \\
\hline $\mathbf{C u}$ & 1.78363955761358 & 13.87344798409320 & 1.26122399999993 \\
\hline $\mathrm{Cu}$ & 1.78363955761358 & 16.39589310421720 & 1.26122399999993 \\
\hline $\mathbf{C u}$ & 1.78363955761358 & 18.91833716491180 & 1.26122399999993 \\
\hline $\mathbf{C u}$ & 1.78363955761358 & 21.44078263817520 & 1.26122399999993 \\
\hline $\mathbf{C u}$ & 1.78363955761358 & 23.96322669886980 & 1.26122399999993 \\
\hline $\mathbf{C u}$ & 1.78363955761358 & 26.48567181899090 & 1.26122399999993 \\
\hline $\mathbf{C u}$ & 1.78363955761358 & 29.00811693910680 & 1.26122399999993 \\
\hline $\mathbf{C u}$ & 1.78363955761358 & 31.53056135294310 & 1.26122399999993 \\
\hline $\mathbf{C u}$ & 1.78363955761358 & 34.05300647307050 & 1.26122399999993 \\
\hline $\mathbf{C u}$ & 5.35091375000000 & 1.26122414920059 & 1.26122399999993 \\
\hline
\end{tabular}




\begin{tabular}{|c|c|c|c|}
\hline $\mathbf{C u}$ & 5.35091375000000 & 3.78366926932167 & 1.26122399999993 \\
\hline $\mathbf{C u}$ & 5.35091375000000 & 6.30611333001662 & 1.26122399999993 \\
\hline $\mathbf{C u}$ & 5.35091375000000 & 8.82855845013778 & 1.26122399999993 \\
\hline $\mathbf{C u}$ & 5.35091375000000 & 11.35100286397460 & 1.26122399999993 \\
\hline $\mathbf{C u}$ & 5.35091375000000 & 13.87344798409320 & 1.26122399999993 \\
\hline $\mathbf{C u}$ & 5.35091375000000 & 16.39589310421720 & 1.26122399999993 \\
\hline $\mathbf{C u}$ & 5.35091375000000 & 18.91833716491180 & 1.26122399999993 \\
\hline $\mathbf{C u}$ & 5.35091375000000 & 21.44078263817520 & 1.26122399999993 \\
\hline $\mathbf{C u}$ & 5.35091375000000 & 23.96322669886980 & 1.26122399999993 \\
\hline $\mathbf{C u}$ & 5.35091375000000 & 26.48567181899090 & 1.26122399999993 \\
\hline $\mathbf{C u}$ & 5.35091375000000 & 29.00811693910680 & 1.26122399999993 \\
\hline $\mathbf{C u}$ & 5.35091375000000 & 31.53056135294310 & 1.26122399999993 \\
\hline $\mathbf{C u}$ & 5.35091375000000 & 34.05300647307050 & 1.26122399999993 \\
\hline $\mathbf{C u}$ & 8.91818794238642 & 1.26122414920059 & 1.26122399999993 \\
\hline $\mathbf{C u}$ & 8.91818794238642 & 3.78366926932167 & 1.26122399999993 \\
\hline $\mathbf{C u}$ & 8.91818794238642 & 6.30611333001662 & 1.26122399999993 \\
\hline $\mathbf{C u}$ & 8.91818794238642 & 8.82855845013778 & 1.26122399999993 \\
\hline $\mathbf{C u}$ & 8.91818794238642 & 11.35100286397460 & 1.26122399999993 \\
\hline $\mathbf{C u}$ & 8.91818794238642 & 13.87344798409320 & 1.26122399999993 \\
\hline $\mathbf{C u}$ & 8.91818794238642 & 16.3958931 & 39999 \\
\hline $\mathbf{C u}$ & 8.91818794238642 & 716491180 & 1.26122399999993 \\
\hline $\mathbf{C u}$ & 94238642 & 21.44078263817520 & 1.26122399999993 \\
\hline $\mathbf{C u}$ & 8.91818794238642 & 23.96322669886980 & 1.26122399999993 \\
\hline $\mathbf{C u}$ & 8.91818794238642 & 26.48567181899090 & 1.26122399999993 \\
\hline $\mathbf{C u}$ & 8.91818794238642 & 29.00811693910680 & 1.26122399999993 \\
\hline $\mathbf{C u}$ & 8.91818794238642 & 31.53056135294310 & 1.26122399999993 \\
\hline $\mathbf{C u}$ & 8.91818794238642 & 34.05300647307050 & 1.26122399999993 \\
\hline $\mathbf{C u}$ & 0.00153289893152 & 35.31373245441130 & 2.53846954007226 \\
\hline $\mathbf{C u}$ & 10.69928326924730 & 2.52410902634329 & 2.54072498800560 \\
\hline $\mathbf{C u}$ & 0.00256489249724 & 5.04457380073532 & 2.54250113281072 \\
\hline $\mathbf{C u}$ & 10.70068398238780 & 7.56506459674233 & 2.54236419181497 \\
\hline $\mathbf{C u}$ & 10.70108850112110 & 10.09066565132250 & 2.53998109918973 \\
\hline $\mathbf{C u}$ & 0.00041803466272 & 12.61075920987800 & 2.54421981209719 \\
\hline $\mathbf{C u}$ & 10.69597907761580 & 15.13618333689630 & 2.53696369868425 \\
\hline $\mathbf{C u}$ & 10.70124979307240 & 17.65869914971030 & 2.54268264884769 \\
\hline $\mathbf{C u}$ & 10.69852517424230 & 20.17880940625300 & 2.53834358935125 \\
\hline $\mathbf{C u}$ & 10.69667844474690 & 22.70257033317570 & 2.54477722459807 \\
\hline $\mathbf{C u}$ & 10.69986555699160 & 25.22797015711680 & 2.53958404454750 \\
\hline $\mathbf{C u}$ & 10.70060096349710 & 27.74720758877330 & 2.54370084211502 \\
\hline $\mathbf{C u}$ & 0.00084563558241 & 30.26908194797850 & 2.54133488898562 \\
\hline $\mathbf{C u}$ & 10.70075381871850 & 32.79106390905190 & 2.54074815445622 \\
\hline $\mathbf{C u}$ & 3.56727492778306 & 0.00049946684082 & 2.54266008607380 \\
\hline $\mathbf{C u}$ & 3.56727423658949 & 2.52073340555474 & 2.54363213782030 \\
\hline $\mathbf{C u}$ & 3.56727368810143 & 5.04478212297747 & 2.54052080319592 \\
\hline $\mathbf{C u}$ & 3.56727293132724 & 7.57028269492267 & 2.58222732342469 \\
\hline $\mathbf{C u}$ & 3.56727403350834 & 10.08925767702750 & 2.58663918817635 \\
\hline $\mathbf{C u}$ & 3.56727713461888 & 12.62159161838980 & 2.59434217455909 \\
\hline $\mathbf{C u}$ & 3.56727466790458 & 15.13819577730790 & 2.60556280748684 \\
\hline $\mathbf{C u}$ & 3.56727601156964 & 17.65321129697030 & 2.60475567402610 \\
\hline $\mathbf{C u}$ & 3.56727745260845 & 20.17904508319040 & 2.57603180758916 \\
\hline $\mathbf{C u}$ & 3.56727660804831 & 22.69707606310610 & 2.58244854336841 \\
\hline $\mathbf{C u}$ & 3.56727482594601 & 25.21942507707500 & 2.55257877465349 \\
\hline $\mathbf{C u}$ & 3.56727682277521 & 27.74883739414620 & 2.54221595092449 \\
\hline $\mathbf{C u}$ & 3.56727640939491 & 30.26860096970490 & 2.54265147735182 \\
\hline
\end{tabular}




\begin{tabular}{|c|c|c|c|}
\hline $\mathrm{Cu}$ & 3.56727716694486 & 32.79033356495110 & 2.54319750727896 \\
\hline $\mathbf{C u}$ & 7.13301789336824 & 35.31371966392050 & 2.53848275450039 \\
\hline $\mathbf{C u}$ & 7.13709758085492 & 2.52410809844079 & 2.54072151141160 \\
\hline $\mathbf{C u}$ & 7.13198645249611 & 5.04457452236763 & 2.54249792459134 \\
\hline $\mathbf{C u}$ & 7.13569474731826 & 7.56506447878242 & 2.54236019226538 \\
\hline $\mathbf{C u}$ & 7.13529367795922 & 10.09065896056090 & 2.53997788075994 \\
\hline $\mathbf{C u}$ & 7.13413396740588 & 12.61075306774420 & 2.54421936565369 \\
\hline $\mathbf{C u}$ & 7.14040154214971 & 15.13617849581390 & 2.53697065937048 \\
\hline $\mathbf{C u}$ & 7.13512791693120 & 17.65869307628820 & 2.54269127374506 \\
\hline $\mathbf{C u}$ & 7.13785331302733 & 20.17880734466090 & 2.53833892349158 \\
\hline $\mathbf{C u}$ & 7.13969684401486 & 22.70257652982270 & 2.54477378743099 \\
\hline $\mathrm{Cu}$ & 7.13651648913418 & 25.22797287981180 & 2.53958332726130 \\
\hline $\mathrm{Cu}$ & 7.13577619724994 & 27.74720842708050 & 2.54370079476301 \\
\hline $\mathrm{Cu}$ & 7.13370652979186 & 30.26909116783790 & 2.54133181324186 \\
\hline $\mathrm{Cu}$ & 7.13562359254375 & 32.79106544438530 & 2.54075170735832 \\
\hline $\mathrm{Cu}$ & 1.78283056691665 & 1.25925917165138 & 3.85356368915393 \\
\hline $\mathrm{Cu}$ & 1.77867278106929 & 3.77941505727507 & 3.85550820700772 \\
\hline $\mathbf{C u}$ & 1.79574785228806 & 6.30451291173362 & 3.86072583037919 \\
\hline $\mathbf{C u}$ & 1.78032397300542 & 8.82507017303704 & 3.83298661196620 \\
\hline $\mathbf{C u}$ & 1.79846446096036 & 11.34697294227870 & 3.85438591949789 \\
\hline $\mathbf{C u}$ & 1.77821573296388 & 13.87744762489260 & 3.84186301953654 \\
\hline $\mathbf{C u}$ & 1.77940591612161 & 16.39761608588260 & 3.84613931431809 \\
\hline $\mathbf{C u}$ & 1.78771235835155 & 18.92254009217210 & 3.85477353952856 \\
\hline $\mathbf{C u}$ & 1.77511217504675 & 21.44228594049050 & 3.84002624274200 \\
\hline $\mathbf{C u}$ & 1.78450833049491 & 23.96782678925620 & 3.84726676578502 \\
\hline $\mathbf{C u}$ & 1.78054973090957 & 26.48891062260050 & 3.85618121005573 \\
\hline $\mathbf{C u}$ & 1.78358119616195 & 29.00922720475800 & 3.85728091528364 \\
\hline $\mathbf{C u}$ & 1.78236724742154 & 31.53001578840360 & 3.85657941653604 \\
\hline $\mathbf{C u}$ & 1.78352018934366 & 34.04947830879740 & 3.85371207246151 \\
\hline $\mathbf{C u}$ & 5.35171664357607 & 1.25926303701717 & 3.85356373736699 \\
\hline $\mathbf{C u}$ & 5.35587632349600 & 3.77942275195224 & 3.85550562180079 \\
\hline $\mathbf{C u}$ & 5.33879246634236 & 6.30451478147673 & 3.86070452189153 \\
\hline $\mathbf{C u}$ & 5.35422195998445 & 8.82507181010737 & 3.83299478008971 \\
\hline $\mathbf{C u}$ & 5.33611142594028 & 11.34695523451250 & 3.85436744432551 \\
\hline $\mathbf{C u}$ & 5.35634350753821 & 13.87745540660720 & 3.84186305014023 \\
\hline $\mathbf{C u}$ & 5.35514530250216 & 16.39761145243310 & 3.84615565809712 \\
\hline $\mathbf{C u}$ & 5.34683733458037 & 18.92253643179210 & 3.85477660661777 \\
\hline $\mathbf{C u}$ & 5.35944164463783 & 21.44229102570920 & 3.84002957165331 \\
\hline $\mathbf{C u}$ & 5.35004538435251 & 23.96783326488260 & 3.84726440691734 \\
\hline $\mathbf{C u}$ & 5.35399859987386 & 26.48891669767620 & 3.85618003826904 \\
\hline $\mathbf{C u}$ & 5.35096841436855 & 29.00922562798090 & 3.85728179709202 \\
\hline $\mathbf{C u}$ & 5.35219070697524 & 31.53000978890120 & 3.85658015667593 \\
\hline $\mathbf{C u}$ & 5.35102770032677 & 34.04947899267860 & 3.85371288318036 \\
\hline $\mathbf{C u}$ & 8.91818949827741 & 1.26136418241790 & 3.85016701504664 \\
\hline $\mathbf{C u}$ & 8.91818908782662 & 3.78442133515761 & 3.85029525564790 \\
\hline $\mathbf{C u}$ & 8.91819002199152 & 6.30339129634394 & 3.85248016479128 \\
\hline $\mathbf{C u}$ & 8.91819239488832 & 8.82828933265684 & 3.85651166045576 \\
\hline $\mathbf{C u}$ & 8.91819266932999 & 11.35281367962850 & 3.85067737539335 \\
\hline $\mathrm{Cu}$ & 8.91819001334924 & 13.87133676381910 & 3.85203396249390 \\
\hline $\mathbf{C u}$ & 8.91819163525514 & 16.39904643780840 & 3.85324639525692 \\
\hline $\mathrm{Cu}$ & 8.91818821697592 & 18.92020514350340 & 3.85244720563726 \\
\hline $\mathbf{C u}$ & 8.91819166687212 & 21.43998713410440 & 3.85541103144963 \\
\hline $\mathbf{C u}$ & 8.91819404831311 & 23.96333883388560 & 3.85104705270304 \\
\hline $\mathbf{C u}$ & 8.91819192792428 & 26.49126963757600 & 3.85506909595400 \\
\hline
\end{tabular}




\begin{tabular}{|c|c|c|c|}
\hline $\mathrm{Cu}$ & 8.91818947681567 & 29.00745804916140 & 3.85028950437457 \\
\hline $\mathbf{C u}$ & 8.91818764452541 & 31.53322950801980 & 3.85003538575303 \\
\hline $\mathbf{C u}$ & 8.91818776015857 & 34.05057691545190 & 3.85034241018604 \\
\hline $\mathbf{C u}$ & 10.70115358759820 & 35.31213056733810 & 5.05563436184481 \\
\hline $\mathbf{C u}$ & 10.70057247297950 & 2.52151343617001 & 5.05949951758672 \\
\hline $\mathbf{C u}$ & 0.00175464927508 & 5.04785400712941 & 5.05862871991973 \\
\hline $\mathbf{C u}$ & 0.00366466957598 & 7.56763059677045 & 5.05184237044514 \\
\hline $\mathbf{C u}$ & 0.00725864998410 & 10.09207427224900 & 5.04748449796062 \\
\hline $\mathbf{C u}$ & 0.00798497104787 & 12.61065774183700 & 5.0505622 \\
\hline $\mathbf{C u}$ & 10.70069908249080 & 15.13601286471930 & 5.05157 \\
\hline $\mathbf{C u}$ & 0.00034370578721 & 17.65837431617620 & 12002128 \\
\hline $\mathrm{Cu}$ & 0.00643328678321 & 20.17892282147640 & 5.05579630984421 \\
\hline $\mathbf{C u}$ & 0.00381867882237 & 22.70400101485030 & 5.05827570475067 \\
\hline $\mathrm{Cu}$ & 0.00154178409530 & 25.22401004809620 & 5.05513920638427 \\
\hline $\mathbf{C u}$ & 10.70091151579590 & 27.74963665420870 & 5.06553392037967 \\
\hline $\mathbf{C u}$ & 10.69854899324380 & 30.26746006054150 & 5.05746520404651 \\
\hline $\mathrm{Cu}$ & 0.00180862305821 & 32.79024261831590 & 5.0597 \\
\hline $\mathbf{C u}$ & 3.56727406014865 & 35.31005163859030 & 5.05834426622424 \\
\hline $\mathbf{C u}$ & 3.56727612517242 & 2.51709792641731 & 5.05766767292586 \\
\hline $\mathbf{C u}$ & 3.56727141412598 & 5.02201675158478 & 5.05001373496670 \\
\hline $\mathbf{C u}$ & 3.56726725167369 & 7.52202193632666 & 5.16298529206847 \\
\hline $\mathbf{C u}$ & 3.56726808649897 & 10.09380936732150 & 5.14833964360665 \\
\hline $\mathbf{C u}$ & 3.56731888652140 & 12.60092634666880 & 5.16305437565928 \\
\hline $\mathbf{C u}$ & 3.56727613598662 & 15.13569257478190 & 5.11309606495182 \\
\hline $\mathbf{C u}$ & 3.56727630353198 & 17.67538017233140 & 5.18005820706558 \\
\hline $\mathbf{C u}$ & 3.56727540818996 & 20.18759171861050 & 5.08777251076832 \\
\hline $\mathbf{C u}$ & 3.56728130721985 & 22.71719291041220 & 5.13604578754907 \\
\hline $\mathbf{C u}$ & 3.56727558352474 & 25.24459715822950 & 5.05977969360176 \\
\hline $\mathbf{C u}$ & 3.56727594058174 & 27.75292126388860 & 5.06093502497202 \\
\hline $\mathbf{C u}$ & 3.56727266277641 & 30.27375513415360 & 5.06063328843807 \\
\hline $\mathbf{C u}$ & 3.56727647621920 & 32.79133233748750 & 5.06046931910636 \\
\hline $\mathbf{C u}$ & 7.13521922106394 & 35.31213198605930 & 5.05562709767480 \\
\hline $\mathbf{C u}$ & 7.13580325654792 & 2.52151811769069 & 5.05949909439571 \\
\hline $\mathbf{C u}$ & 7.13278843364382 & 5.04785905371606 & 5.05862063438680 \\
\hline $\mathbf{C u}$ & 7.13087838549241 & 7.56762600635702 & 5.05182995418859 \\
\hline $\mathbf{C u}$ & 7.12730366704714 & 10.09207011649580 & 5.04748420771155 \\
\hline $\mathbf{C u}$ & 7.12657865807108 & 12.61065284267920 & 5.05054404511694 \\
\hline $\mathbf{C u}$ & 7.13568939941330 & 15.13600656931040 & 5.05157966746706 \\
\hline $\mathbf{C u}$ & 7.13421223665324 & 17.65837524051230 & 5.05485762871511 \\
\hline $\mathbf{C u}$ & 7.12812304501576 & 20.17893527960330 & 5.05578417277703 \\
\hline $\mathbf{C u}$ & 7.13073868458045 & 22.70401603035560 & 5.05827156062533 \\
\hline $\mathbf{C u}$ & 7.13301521674784 & 25.22402021259800 & 5.05513340456424 \\
\hline $\mathbf{C u}$ & 7.13546861520619 & 27.74963951485700 & 5.06553739845797 \\
\hline $\mathbf{C u}$ & 7.13783442235945 & 30.26745871496700 & 5.05747498682157 \\
\hline $\mathbf{C u}$ & 7.13274042677855 & 32.79024467488610 & 5.05975320347330 \\
\hline \multicolumn{4}{|c|}{ CI-NEB Image 13} \\
\hline Element & X-Coordinate $(\AA)$ & Y-Coordinate $(\AA)$ & Z-Coordinate (§̊) \\
\hline $\mathbf{F}$ & 4.63777556446617 & 12.77237530170830 & 7.84416422480409 \\
\hline $\mathbf{F}$ & 2.49739000279014 & 12.77219831274710 & 7.84444452603518 \\
\hline $\mathbf{F}$ & 3.56727911043577 & 8.82068518733388 & 6.64569849454186 \\
\hline $\mathbf{F}$ & 4.63032685696418 & 17.47231449910620 & 7.84586966715444 \\
\hline $\mathbf{F}$ & 2.50429826388462 & 17.47233186473950 & 7.84592515536975 \\
\hline $\mathbf{F}$ & 3.56730007075086 & 14.88251185943250 & 7.06970309615033 \\
\hline $\mathbf{F}$ & 4.66364802384033 & 22.76326764564060 & 7.76427098314149 \\
\hline
\end{tabular}




\begin{tabular}{|c|c|c|c|}
\hline $\mathbf{F}$ & 2.47088540198673 & 22.76326626774890 & 7.76425187048757 \\
\hline $\mathbf{F}$ & 3.56727283542848 & 20.89054808305800 & 7.33951069968378 \\
\hline C & 3.56747058179691 & 12.93955602151760 & 7.07125962485804 \\
\hline $\mathbf{C}$ & 3.56729224661055 & 17.48516363832470 & 7.06067355579098 \\
\hline C & 3.56727339372585 & 22.30861021218360 & 7.06773380498225 \\
\hline $\mathbf{C u}$ & 0.00000000000000 & 0.00000000000000 & 0.00000000000000 \\
\hline $\mathbf{C u}$ & 0.00000000000000 & 2.52244512012115 & 0.00000000000000 \\
\hline $\mathbf{C u}$ & 0.00000000000000 & 5.04488953395804 & 0.00000000000000 \\
\hline $\mathbf{C u}$ & 0.00000000000000 & 7.56733465407854 & 0.00000000000000 \\
\hline $\mathbf{C u}$ & 0.00000000000000 & 10.08977871477400 & 0.00000000000000 \\
\hline $\mathbf{C u}$ & 0.00000000000000 & 12.61222383489520 & 0.00000000000000 \\
\hline $\mathrm{Cu}$ & 0.00000000000000 & 15.13466895501400 & 0.00000000000000 \\
\hline $\mathbf{C u}$ & 0.00000000000000 & 17.65711336885320 & 0.00000000000000 \\
\hline $\mathbf{C u}$ & 0.00000000000000 & 20.17955848897460 & 0.00000000000000 \\
\hline $\mathbf{C u}$ & 0.00000000000000 & 22.70200254966930 & 0.00000000000000 \\
\hline $\mathbf{C u}$ & 0.00000000000000 & 25.22444766979040 & 0.00000000000000 \\
\hline $\mathrm{Cu}$ & 0.00000000000000 & 27.74689208362190 & 0.00000000000000 \\
\hline $\mathbf{C u}$ & 0.00000000000000 & 30.26933720374310 & 0.00000000000000 \\
\hline $\mathbf{C u}$ & 0.00000000000000 & 32.79178232386990 & 0.00000000000000 \\
\hline $\mathbf{C u}$ & 3.56727419238642 & 0.00000000000000 & 0.00000000000000 \\
\hline $\mathbf{C u}$ & 3.56727419238642 & 2.52244512012115 & 0.00000000000000 \\
\hline $\mathbf{C u}$ & 3.56727419238642 & 5.04488953395804 & 0.00000000000000 \\
\hline $\mathbf{C u}$ & 3.56727419238642 & 7.56733465407854 & 0.00000000000000 \\
\hline $\mathbf{C u}$ & 3.56727419238642 & 10.08977871477400 & 0.00000000000000 \\
\hline $\mathbf{C u}$ & 3.56727419238642 & 12.61222383489520 & 0.00000000000000 \\
\hline $\mathbf{C u}$ & 3.56727419238642 & 15.13466895501400 & 0.00000000000000 \\
\hline $\mathbf{C u}$ & 3.56727419238642 & 17.65711336885320 & 0.00000000000000 \\
\hline $\mathbf{C u}$ & 3.56727419238642 & 20.17955848897460 & 0.00000000000000 \\
\hline $\mathbf{C u}$ & 3.56727419238642 & 22.70200254966930 & 0.00000000000000 \\
\hline $\mathbf{C u}$ & 3.56727419238642 & 25.22444766979040 & 0.00000000000000 \\
\hline $\mathbf{C u}$ & 3.56727419238642 & 27.74689208362190 & 0.00000000000000 \\
\hline $\mathbf{C u}$ & 3.56727419238642 & 30.26933720374310 & 0.00000000000000 \\
\hline $\mathbf{C u}$ & 3.56727419238642 & 32.79178232386990 & 0.00000000000000 \\
\hline $\mathbf{C u}$ & 7.13454838477291 & 0.00000000000000 & 0.00000000000000 \\
\hline $\mathbf{C u}$ & 7.13454838477291 & 2.52244512012115 & 0.00000000000000 \\
\hline $\mathbf{C u}$ & 7.13454838477291 & 5.04488953395804 & 0.00000000000000 \\
\hline $\mathbf{C u}$ & 7.13454838477291 & 7.56733465407854 & 0.00000000000000 \\
\hline $\mathbf{C u}$ & 7.13454838477291 & 10.08977871477400 & 0.00000000000000 \\
\hline $\mathbf{C u}$ & 7.13454838477291 & 12.61222383489520 & 0.00000000000000 \\
\hline $\mathbf{C u}$ & 7.13454838477291 & 15.13466895501400 & 0.00000000000000 \\
\hline $\mathbf{C u}$ & 7.13454838477291 & 17.65711336885320 & 0.00000000000000 \\
\hline $\mathbf{C u}$ & 7.13454838477291 & 20.17955848897460 & 0.00000000000000 \\
\hline $\mathbf{C u}$ & 7.13454838477291 & 22.70200254966930 & 0.00000000000000 \\
\hline $\mathbf{C u}$ & 7.13454838477291 & 25.22444766979040 & 0.00000000000000 \\
\hline $\mathbf{C u}$ & 7.13454838477291 & 27.74689208362190 & 0.00000000000000 \\
\hline $\mathbf{C u}$ & 7.13454838477291 & 30.26933720374310 & 0.00000000000000 \\
\hline $\mathbf{C u}$ & 7.13454838477291 & 32.79178232386990 & 0.00000000000000 \\
\hline $\mathbf{C u}$ & 1.78363955761358 & 1.26122414920059 & 1.26122399999993 \\
\hline $\mathbf{C u}$ & 1.78363955761358 & 3.78366926932167 & 1.26122399999993 \\
\hline $\mathbf{C u}$ & 1.78363955761358 & 6.30611333001662 & 1.26122399999993 \\
\hline $\mathbf{C u}$ & 1.78363955761358 & 8.82855845013778 & 1.26122399999993 \\
\hline $\mathbf{C u}$ & 1.78363955761358 & 11.35100286397460 & 1.26122399999993 \\
\hline $\mathbf{C u}$ & 1.78363955761358 & 13.87344798409320 & 1.26122399999993 \\
\hline $\mathbf{C u}$ & 1.78363955761358 & 16.39589310421720 & 1.26122399999993 \\
\hline
\end{tabular}




\begin{tabular}{|c|c|c|c|}
\hline $\mathrm{Cu}$ & 1.78363955761358 & 18.91833716491180 & 1.26122399999993 \\
\hline $\mathrm{Cu}$ & 1.78363955761358 & 21.44078263817520 & 1.26122399999993 \\
\hline $\mathrm{Cu}$ & 1.78363955761358 & 23.96322669886980 & 1.26122399999993 \\
\hline $\mathbf{C u}$ & 1.78363955761358 & 26.48567181899090 & 1.26122399999993 \\
\hline $\mathbf{C u}$ & 1.78363955761358 & 29.00811693910700 & 1.26122399999993 \\
\hline $\mathbf{C u}$ & 1.78363955761358 & 31.53056135294340 & 1.26122399999993 \\
\hline $\mathrm{Cu}$ & 1.78363955761358 & 34.05300647307050 & 1.26122399999993 \\
\hline $\mathrm{Cu}$ & 5.35091375000000 & 1.26122414920059 & 1.26122399999993 \\
\hline $\mathbf{C u}$ & 5.35091375000000 & 3.78366926932167 & 1.26122399999993 \\
\hline $\mathbf{C u}$ & 5.35091375000000 & 6.30611333001662 & 1.26122399999993 \\
\hline $\mathbf{C u}$ & 5.35091375000000 & 8.82855845013778 & 1.26122399999993 \\
\hline $\mathrm{Cu}$ & 5.35091375000000 & 11.35100286397460 & 1.26122399999993 \\
\hline $\mathrm{Cu}$ & 5.35091375000000 & 13.87344798409320 & 1.26122399999993 \\
\hline $\mathrm{Cu}$ & 5.35091375000000 & 16.39589310421720 & 1.26122399999993 \\
\hline $\mathbf{C u}$ & 5.35091375000000 & 18.91833716491180 & 1.26122399999993 \\
\hline $\mathbf{C u}$ & 5.35091375000000 & 21.44078263817520 & 1.26122399999993 \\
\hline $\mathrm{Cu}$ & 5.35091375000000 & 23.96322669886980 & 1.26122399999993 \\
\hline $\mathbf{C u}$ & 5.35091375000000 & 26.48567181899090 & 1.26122399999993 \\
\hline $\mathbf{C u}$ & 5.35091375000000 & 29.00811693910700 & 1.26122399999993 \\
\hline $\mathrm{Cu}$ & 5.35091375000000 & 135294340 & 1.26122399999993 \\
\hline $\mathbf{C u}$ & 5.35091375000000 & 300647307050 & 1.26122399999993 \\
\hline $\mathbf{C u}$ & 8.91818794238642 & 1.26122414920059 & 1.26122399999993 \\
\hline $\mathrm{Cu}$ & 8.91818794238642 & 6926932167 & 2399999993 \\
\hline $\mathrm{Cu}$ & 8.91818794238642 & 01662 & 2399999993 \\
\hline $\mathrm{Cu}$ & 8.91818794238642 & 3778 & 99993 \\
\hline $\mathrm{Cu}$ & 8.91818794238642 & 11.35 & 1.26122399999993 \\
\hline $\mathrm{Cu}$ & 8.91818794238642 & 13.87344798409320 & 1.26122399999993 \\
\hline $\mathrm{Cu}$ & 8.91818794238642 & 16.39589310421720 & 1.26122399999993 \\
\hline $\mathbf{C u}$ & 8.91818794238642 & 18.91833716491180 & 1.26122399999993 \\
\hline $\mathrm{Cu}$ & 8.91818794238642 & 21.44078263817520 & 1.26122399999993 \\
\hline $\mathrm{Cu}$ & 8.91818794238642 & 23.96322669886980 & 1.26122399999993 \\
\hline $\mathrm{Cu}$ & 8.91818794238642 & 26.48567181899090 & 1.26122399999993 \\
\hline $\mathrm{Cu}$ & 8.91818794238642 & 29.00811693910700 & 1.26122399999993 \\
\hline $\mathrm{Cu}$ & 8.91818794238642 & 31.53056135294340 & 1.26122399999993 \\
\hline $\mathrm{Cu}$ & 8.91818794238642 & 34.05300647307050 & 1.26122399999993 \\
\hline $\mathrm{Cu}$ & 0.00166303484283 & 35.31381770077740 & 2.53890515112895 \\
\hline $\mathrm{Cu}$ & 10.69923227181210 & 2.52425784217990 & 2.54075424543228 \\
\hline $\mathbf{C u}$ & 0.00262546002951 & 5.04494571612633 & 2.54224104192033 \\
\hline $\mathrm{Cu}$ & 10.70072977432940 & 7.56518025611028 & 2.54270728456753 \\
\hline $\mathbf{C u}$ & 10.70098677431440 & 10.09042251189150 & 2.54003419908532 \\
\hline $\mathbf{C u}$ & 0.00021127341342 & 12.61070779857480 & 2.54421026575056 \\
\hline $\mathbf{C u}$ & 10.69618217629980 & 15.13620402253240 & 2.53716970498086 \\
\hline $\mathbf{C u}$ & 10.70156626256140 & 17.65825963387080 & 2.54288702834770 \\
\hline $\mathbf{C u}$ & 10.69830883961120 & 20.17859729431370 & 2.53820044566278 \\
\hline $\mathbf{C u}$ & 10.69653488428310 & 22.70279102473520 & 2.54444387944633 \\
\hline $\mathbf{C u}$ & 10.69971141841550 & 25.22821793021880 & 2.53959295083227 \\
\hline $\mathbf{C u}$ & 10.70078452428980 & 27.74745118682130 & 2.54367097846633 \\
\hline $\mathbf{C u}$ & 0.00080412322286 & 30.26889995783830 & 2.54121728626705 \\
\hline $\mathrm{Cu}$ & 10.70091939228390 & 32.79060558457180 & 2.54072688253251 \\
\hline $\mathrm{Cu}$ & 3.56727639756777 & 0.00049820360199 & 2.54237937043099 \\
\hline $\mathbf{C u}$ & 3.56727571625346 & 2.52121608240024 & 2.54410077413460 \\
\hline $\mathbf{C u}$ & 3.56727927390292 & 5.04464179592296 & 2.54091566285839 \\
\hline $\mathbf{C u}$ & 3.56727523674871 & 7.57022824702024 & 2.58137485347717 \\
\hline $\mathbf{C u}$ & 3.56727487704477 & 10.09005865278180 & 2.58638270542733 \\
\hline
\end{tabular}




\begin{tabular}{|c|c|c|c|}
\hline $\mathbf{C u}$ & 3.56727723522144 & 12.62204740010910 & 2.59313563763849 \\
\hline $\mathrm{Cu}$ & 3.56727920225825 & 15.13864154824020 & 2.60422205984156 \\
\hline $\mathbf{C u}$ & 3.56728059051836 & 17.65376300888550 & 2.60417535374581 \\
\hline $\mathbf{C u}$ & 3.56727971579984 & 20.17909153042410 & 2.57708011732282 \\
\hline $\mathbf{C u}$ & 3.56727864104973 & 22.69670590123130 & 2.58324686565365 \\
\hline $\mathbf{C u}$ & 3.56727712073960 & 25.21919749436920 & 2.55206109116089 \\
\hline $\mathbf{C u}$ & 3.56727703368887 & 27.74873046419630 & 2.54208659015149 \\
\hline $\mathbf{C u}$ & 3.56727833908120 & 30.26841138994840 & 2.54269217377996 \\
\hline $\mathbf{C u}$ & 3.56727675733206 & 32.79031930414370 & 2.54323238394033 \\
\hline $\mathbf{C u}$ & 7.13288736835751 & 35.31381406122780 & 2.53891094065568 \\
\hline $\mathbf{C u}$ & 7.13714470451096 & 2.52425803952473 & 2.54076035499177 \\
\hline $\mathbf{C u}$ & 7.13193233006061 & 5.04494201699774 & 2.54223270874412 \\
\hline $\mathbf{C u}$ & 7.13564525889075 & 7.56518190616467 & 2.54270675789438 \\
\hline $\mathrm{Cu}$ & 7.13539357099919 & 10.09041736324860 & 2.54003454843458 \\
\hline $\mathbf{C u}$ & 7.13433760328039 & 12.61070608754670 & 2.54420767121421 \\
\hline $\mathbf{C u}$ & 7.14019305501406 & 15.13619842562780 & 2.53718094083989 \\
\hline $\mathbf{C u}$ & 7.13481031907708 & 17.65825365315760 & 2.54289543824893 \\
\hline $\mathbf{C u}$ & 7.13808897543357 & 20.17861936427180 & 2.53820367401309 \\
\hline $\mathbf{C u}$ & 7.13984167549448 & 22.70278758983660 & 2.54444316638775 \\
\hline $\mathbf{C u}$ & 7.13665705368495 & 25.22822393203700 & 2.53959319722119 \\
\hline $\mathbf{C u}$ & 7.13559696612519 & 27.74746120653210 & 2.54368042717873 \\
\hline $\mathbf{C u}$ & 7.13374605247413 & 30.26890214188970 & 2.54121934570019 \\
\hline $\mathbf{C u}$ & 7.13545299880775 & 32.79059896024210 & 2.54074069072026 \\
\hline $\mathbf{C u}$ & 1.78301970772692 & 1.25936561401382 & 35915741 \\
\hline $\mathbf{C u}$ & 783864378 & 7288 & 450107 \\
\hline $\mathrm{Cu}$ & 1.79569594805215 & 13107 & 2356334424 \\
\hline $\mathbf{C u}$ & 1.78064933149555 & 8.82563274215371 & 3.83270371432194 \\
\hline $\mathbf{C u}$ & 1.79870490271472 & 11.34745863815840 & 3.85456131737470 \\
\hline $\mathrm{Cu}$ & 1.77628807171418 & 13.87711463746200 & 3.84201549864055 \\
\hline $\mathrm{Cu}$ & 1.78125844607713 & 16.39747827039730 & 3.84744279338666 \\
\hline $\mathrm{Cu}$ & 1.78681671861408 & 18.92384100896480 & 3.85372471833062 \\
\hline $\mathbf{C u}$ & 1.77478539257687 & 21.44306209570080 & 3.83973485312928 \\
\hline $\mathrm{Cu}$ & 1.78430646207599 & 23.96848663958670 & 3.84734448571033 \\
\hline $\mathbf{C u}$ & 1.78059364671691 & 26.48896177664950 & 3.85612938989077 \\
\hline $\mathbf{C u}$ & 1.78354840965195 & 29.00913027156100 & 3.85733589884034 \\
\hline $\mathbf{C u}$ & 1.78255288666442 & 31.52963893986290 & 3.85629293728782 \\
\hline $\mathbf{C u}$ & 1.78368551782577 & 34.04932422806700 & 3.85368309352873 \\
\hline $\mathbf{C u}$ & 5.35152456015782 & 1.25936008846688 & 3.85344908548601 \\
\hline $\mathbf{C u}$ & 5.35614163404602 & 3.77959894787911 & 3.85562906206260 \\
\hline $\mathbf{C u}$ & 5.33884944494343 & 6.30464216973238 & 3.86052356988570 \\
\hline $\mathbf{C u}$ & 5.35389761113359 & 8.82563009386049 & 3.83270901504212 \\
\hline $\mathbf{C u}$ & 5.33585604598882 & 11.34744650758900 & 3.85454182658977 \\
\hline $\mathbf{C u}$ & 5.35825753713987 & 13.87711307774860 & 3.84202262267130 \\
\hline $\mathbf{C u}$ & 5.35329459001188 & 16.39747055951770 & 3.84746123988218 \\
\hline $\mathrm{Cu}$ & 5.34773817279396 & 18.92383668840410 & 3.85372384802504 \\
\hline $\mathbf{C u}$ & 5.35977158958715 & 21.44306419641120 & 3.83974137032444 \\
\hline $\mathbf{C u}$ & 5.35024681520157 & 23.96849024546410 & 3.84734248227912 \\
\hline $\mathbf{C u}$ & 5.35396910789227 & 26.48897129190680 & 3.85612954317348 \\
\hline $\mathbf{C u}$ & 5.35100381139146 & 29.00912921684020 & 3.85733560555099 \\
\hline $\mathbf{C u}$ & 5.35200180273115 & 31.52963766993360 & 3.85629652099207 \\
\hline $\mathbf{C u}$ & 5.35087147513434 & 34.04932733768380 & 3.85370052503943 \\
\hline $\mathbf{C u}$ & 8.91818835869504 & 1.26071340148903 & 3.85034161945745 \\
\hline $\mathbf{C u}$ & 8.91818982778891 & 3.78419945066949 & 3.84984035970735 \\
\hline $\mathbf{C u}$ & 8.91819219157752 & 6.30359257095038 & 3.85210874754204 \\
\hline
\end{tabular}




\begin{tabular}{|c|c|c|c|}
\hline $\mathbf{C u}$ & 8.91819314099809 & 8.82780122897600 & 3.85616679879070 \\
\hline $\mathbf{C u}$ & 8.91817935959058 & 11.35290834551020 & 3.85103095850703 \\
\hline $\mathbf{C u}$ & 8.91818786766456 & 13.87149722365680 & 3.85202828782473 \\
\hline $\mathbf{C u}$ & 8.91819145992658 & 16.39938415827370 & 3.85299417723055 \\
\hline $\mathbf{C u}$ & 8.91818999177761 & 18.91957203401440 & 3.85202765389953 \\
\hline $\mathbf{C u}$ & 8.91819282301699 & 21.43936552823880 & 3.85540363754940 \\
\hline $\mathbf{C u}$ & 8.91818670709765 & 23.96333770288520 & 3.85104323274843 \\
\hline $\mathbf{C u}$ & 8.91819075129071 & 26.49094836959740 & 3.85545849798839 \\
\hline $\mathbf{C u}$ & 8.91819002559195 & 29.00701436153400 & 3.84950976140798 \\
\hline $\mathbf{C u}$ & 8.91818833318338 & 31.53392856101960 & 3.84980246825885 \\
\hline $\mathbf{C u}$ & 8.91819025768967 & 34.05116848168430 & 3.85030262000527 \\
\hline $\mathbf{C u}$ & -0.00026039704114 & 35.31218893629420 & 5.05562839805995 \\
\hline $\mathbf{C u}$ & 10.70059581626930 & 2.52158870846953 & 5.05916791950493 \\
\hline $\mathbf{C u}$ & 0.00139424188718 & 5.04767027860521 & 5.05841078655567 \\
\hline $\mathbf{C u}$ & 0.00423816074667 & 7.56715324893849 & 5.05199247032142 \\
\hline $\mathbf{C u}$ & 0.00781485334514 & 10.09257895730590 & 5.04698315378883 \\
\hline $\mathbf{C u}$ & 0.00825358719831 & 12.61072847247810 & 5.05206916901559 \\
\hline $\mathbf{C u}$ & 10.70091600992340 & 15.13671033921820 & 5.051 \\
\hline $\mathbf{C u}$ & 0.00039941880587 & 17.65869174804470 & 5.0545 \\
\hline $\mathbf{C u}$ & 0.00624031106790 & 20.17911030900740 & 5.05620035153162 \\
\hline $\mathbf{C u}$ & 0.00395745443554 & 22.70365287179600 & 5.05813319053017 \\
\hline $\mathbf{C u}$ & 0.00153285721788 & 25.22359055083990 & 5.05546864368176 \\
\hline $\mathbf{C u}$ & 10.70094551310710 & 27.74965513782990 & 5.06577934495572 \\
\hline $\mathbf{C u}$ & 10.69864322946100 & 30.26751795869920 & 5.05725400968614 \\
\hline $\mathbf{C u}$ & 0.00239002529977 & 32.78992490955330 & 5.05949980668713 \\
\hline $\mathrm{Cu}$ & 3.56727529487503 & 35.30999797677300 & 5.05812365795884 \\
\hline $\mathbf{C u}$ & 3.56728097042375 & 2.51680063149200 & 5.05758553342175 \\
\hline $\mathbf{C u}$ & 3.56727277991455 & 5.02218913219791 & 5.04978210465652 \\
\hline $\mathbf{C u}$ & 3.56727336310966 & 7.52098884015048 & 5.16262812282036 \\
\hline $\mathbf{C u}$ & 3.56727016300565 & 10.09543267729750 & 5.14792749962824 \\
\hline $\mathbf{C u}$ & 3.56729029018579 & 12.60677307787740 & 5.15570679468442 \\
\hline $\mathbf{C u}$ & 3.56726133493853 & 15.14846304764710 & 5.11290545759288 \\
\hline $\mathbf{C u}$ & 3.56727220129996 & 17.68040185251150 & 5.17945018616114 \\
\hline $\mathbf{C u}$ & 3.56727751325184 & 20.18721567731440 & 5.08962634553292 \\
\hline $\mathbf{C u}$ & 3.56728025153817 & 22.71691066209340 & 5.13618843565412 \\
\hline $\mathbf{C u}$ & 3.56727681671522 & 25.24472551668410 & 5.05996244981322 \\
\hline $\mathbf{C u}$ & 3.56727574603171 & 27.75264135381880 & 5.06119538085886 \\
\hline $\mathbf{C u}$ & 3.56727579890552 & 30.27374514744900 & 5.06090658036605 \\
\hline $\mathbf{C u}$ & 3.56727677221163 & 32.79178346642380 & 5.06039681276903 \\
\hline $\mathrm{Cu}$ & 7.13480807393779 & 35.31219119206310 & 5.05562565305929 \\
\hline $\mathbf{C u}$ & 7.13578262298402 & 2.52159170355723 & 5.05916839664747 \\
\hline $\mathrm{Cu}$ & 7.13315298388724 & 5.04767009350976 & 5.05840512475929 \\
\hline $\mathbf{C u}$ & 7.13030649428982 & 7.56714792445326 & 5.05198011802820 \\
\hline $\mathbf{C u}$ & 7.12674531900577 & 10.09257178198630 & 5.04697850423708 \\
\hline $\mathbf{C u}$ & 7.12630961252734 & 12.61072194212700 & 5.05205487577104 \\
\hline $\mathbf{C u}$ & 7.13547419018225 & 15.13670313548280 & 5.05183305678893 \\
\hline $\mathbf{C u}$ & 7.13415801167938 & 17.65869289316640 & 5.05457259553060 \\
\hline $\mathbf{C u}$ & 7.12831650392821 & 20.17911745250280 & 5.05618499223196 \\
\hline $\mathrm{Cu}$ & 7.13059982533193 & 22.70366338228810 & 5.05812901730971 \\
\hline $\mathrm{Cu}$ & 7.13302316667201 & 25.22360157302310 & 5.05546301090638 \\
\hline $\mathbf{C u}$ & 7.13543558288380 & 27.74965767040400 & 5.06578069405272 \\
\hline $\mathbf{C u}$ & 7.13772977577120 & 30.26752405743550 & 5.05726738279970 \\
\hline $\mathbf{C u}$ & 7.13215137866939 & 32.78992224391920 & 5.05951028356431 \\
\hline
\end{tabular}




\begin{tabular}{|c|c|c|c|}
\hline Element & X-Coordinate (§̊) & Y-Coordinate (§̊) & Z-Coordinate (§) \\
\hline $\mathbf{F}$ & 4.65149100465174 & 12.78560176006700 & 7.83133900103838 \\
\hline $\mathbf{F}$ & 2.48351470676886 & 12.78545965955320 & 7.83156755348131 \\
\hline $\mathbf{F}$ & 3.56728223120419 & 8.81827080380533 & 6.64054103809456 \\
\hline $\mathbf{F}$ & 4.63172912275293 & 17.47068016062560 & 7.83715515975527 \\
\hline $\mathbf{F}$ & 2.50297105071053 & 17.47059813431390 & 7.83727560961081 \\
\hline $\mathbf{F}$ & 3.56730740114294 & 14.65754761336210 & 7.13917134588967 \\
\hline $\mathbf{F}$ & 4.66382501940287 & 22.76498209155980 & 7.76458804216237 \\
\hline $\mathbf{F}$ & 2.47071353151992 & 22.76497147144390 & 7.76456045877975 \\
\hline $\mathbf{F}$ & 3.56727449788638 & 20.89289766999130 & 7.34068536557550 \\
\hline C & 3.56740322474757 & 13.08579556662900 & 7.07182450120393 \\
\hline C & 3.56730372632361 & 17.51631687663460 & 7.04759942328688 \\
\hline $\mathrm{C}$ & 3.56727589685399 & 22.30995927542970 & 7.06850870015923 \\
\hline $\mathrm{Cu}$ & 0.00000000000000 & 0.00000000000000 & 0.00000000000000 \\
\hline $\mathbf{C u}$ & 0.00000000000000 & 2.52244512012115 & 0.00000000000000 \\
\hline $\mathbf{C u}$ & 0.00000000000000 & 5.04488953395804 & 0.00000000000000 \\
\hline $\mathrm{Cu}$ & 0.00000000000000 & 7.56733465407854 & 0.00000000000000 \\
\hline $\mathrm{Cu}$ & 0.00000000000000 & 10.08977871477400 & 0.00000000000000 \\
\hline $\mathbf{C u}$ & 0.00000000000000 & 12.61222383489520 & 0.00000000000000 \\
\hline $\mathrm{Cu}$ & 0.00000000000000 & 15.13466895501400 & 0.00000000000000 \\
\hline $\mathrm{Cu}$ & 0.00000000000000 & 17.65711336885320 & 0.00000000000000 \\
\hline $\mathrm{Cu}$ & 0.00000000000000 & 20.17955848897460 & 0.00000000000000 \\
\hline $\mathrm{Cu}$ & 0.00000000000000 & 22.70200254966930 & 0.00000000000000 \\
\hline $\mathrm{Cu}$ & 0.00000000000000 & 25.22444766979040 & 0.00000000000000 \\
\hline $\mathrm{Cu}$ & 0.00000000000000 & 27.74689208362220 & 0.00000000000000 \\
\hline $\mathrm{Cu}$ & 0.00000000000000 & 30.26933720374330 & 0.00000000000000 \\
\hline $\mathrm{Cu}$ & 0.00000000000000 & 32.79178232386990 & 0.00000000000000 \\
\hline $\mathrm{Cu}$ & 3.56727419238642 & 0.00000000000000 & 0.00000000000000 \\
\hline $\mathrm{Cu}$ & 3.56727419238642 & 2.52244512012115 & 0.00000000000000 \\
\hline $\mathrm{Cu}$ & 3.56727419238642 & 5.04488953395804 & 0.00000000000000 \\
\hline $\mathrm{Cu}$ & 3.56727419238642 & 7.56733465407854 & 0.00000000000000 \\
\hline $\mathrm{Cu}$ & 3.56727419238642 & 10.08977871477400 & 0.00000000000000 \\
\hline $\mathbf{C u}$ & 3.56727419238642 & 12.61222383489520 & 0.00000000000000 \\
\hline $\mathrm{Cu}$ & 3.56727419238642 & 15.13466895501400 & 0.00000000000000 \\
\hline $\mathbf{C u}$ & 3.56727419238642 & 17.65711336885320 & 0.00000000000000 \\
\hline $\mathrm{Cu}$ & 3.56727419238642 & 20.17955848897460 & 0.00000000000000 \\
\hline $\mathbf{C u}$ & 3.56727419238642 & 22.70200254966930 & 0.00000000000000 \\
\hline $\mathrm{Cu}$ & 3.56727419238642 & 25.22444766979040 & 0.00000000000000 \\
\hline $\mathrm{Cu}$ & 3.56727419238642 & 27.74689208362220 & 0.00000000000000 \\
\hline $\mathrm{Cu}$ & 3.56727419238642 & 30.26933720374330 & 0.00000000000000 \\
\hline $\mathrm{Cu}$ & 3.56727419238642 & 32.79178232386990 & 0.00000000000000 \\
\hline $\mathrm{Cu}$ & 7.13454838477291 & 0.00000000000000 & 0.00000000000000 \\
\hline $\mathbf{C u}$ & 7.13454838477291 & 2.52244512012115 & 0.00000000000000 \\
\hline $\mathrm{Cu}$ & 7.13454838477291 & 5.04488953395804 & 0.00000000000000 \\
\hline $\mathbf{C u}$ & 7.13454838477291 & 7.56733465407854 & 0.00000000000000 \\
\hline $\mathrm{Cu}$ & 7.13454838477291 & 10.08977871477400 & 0.00000000000000 \\
\hline $\mathrm{Cu}$ & 7.13454838477291 & 12.61222383489520 & 0.00000000000000 \\
\hline $\mathrm{Cu}$ & 7.13454838477291 & 15.13466895501400 & 0.00000000000000 \\
\hline $\mathbf{C u}$ & 7.13454838477291 & 17.65711336885320 & 0.00000000000000 \\
\hline $\mathrm{Cu}$ & 7.13454838477291 & 20.17955848897460 & 0.00000000000000 \\
\hline $\mathrm{Cu}$ & 7.13454838477291 & 22.70200254966930 & 0.00000000000000 \\
\hline $\mathrm{Cu}$ & 7.13454838477291 & 25.22444766979040 & 0.00000000000000 \\
\hline $\mathrm{Cu}$ & 7.13454838477291 & 27.74689208362220 & 0.00000000000000 \\
\hline $\mathbf{C u}$ & 7.13454838477291 & 30.26933720374330 & 0.00000000000000 \\
\hline
\end{tabular}




\begin{tabular}{|c|c|c|c|}
\hline $\mathrm{Cu}$ & 7.13454838477291 & 32.79178232386990 & 0.00000000000000 \\
\hline $\mathrm{Cu}$ & 1.78363955761358 & 1.26122414920059 & 1.26122399999993 \\
\hline $\mathrm{Cu}$ & 1.78363955761358 & 3.78366926932167 & 1.26122399999993 \\
\hline $\mathbf{C u}$ & 1.78363955761358 & 6.30611333001662 & 1.26122399999993 \\
\hline $\mathbf{C u}$ & 1.78363955761358 & 8.82855845013778 & 1.26122399999993 \\
\hline $\mathbf{C u}$ & 1.78363955761358 & 11.35100286397460 & 1.26122399999993 \\
\hline $\mathbf{C u}$ & 1.78363955761358 & 13.87344798409320 & 1.26122399999993 \\
\hline $\mathrm{Cu}$ & 1.78363955761358 & 16.39589310421720 & 1.26122399999993 \\
\hline $\mathbf{C u}$ & 1.78363955761358 & 18.91833716491180 & 1.26122399999993 \\
\hline $\mathbf{C u}$ & 1.78363955761358 & 21.44078263817520 & 1.26122399999993 \\
\hline $\mathbf{C u}$ & 1.78363955761358 & 23.96322669886980 & 1.26122399999993 \\
\hline $\mathbf{C u}$ & 1.78363955761358 & 26.48567181899090 & 1.26122399999993 \\
\hline $\mathbf{C u}$ & 1.78363955761358 & 29.00811693910730 & 1.26122399999993 \\
\hline $\mathbf{C u}$ & 1.78363955761358 & 31.53056135294360 & 1.26122399999993 \\
\hline $\mathrm{Cu}$ & 1.78363955761358 & 34.05300647307050 & 1.26122399999993 \\
\hline $\mathbf{C u}$ & 5.35091375000000 & 1.26122414920059 & 1.26122399999993 \\
\hline $\mathbf{C u}$ & 5.35091375000000 & 69926932167 & 1.26122399999993 \\
\hline $\mathbf{C u}$ & 5.35091375000000 & 6.3061 & 1.26122399999993 \\
\hline $\mathbf{C u}$ & 5.35091375000000 & 8.82855845013778 & 1.26122399999993 \\
\hline $\mathbf{C u}$ & 5.35091375000000 & 11.35100286397460 & 1.26122399999993 \\
\hline $\mathbf{C u}$ & 5.35091375000000 & 13.87344798409320 & 1.26122399999993 \\
\hline $\mathbf{C u}$ & 5.35091375000000 & 16.39589310421720 & 1.26122399999993 \\
\hline $\mathbf{C u}$ & 5.35091375000000 & 18.91833716491180 & 1.26122399999993 \\
\hline $\mathrm{Cu}$ & 5.35091375000000 & 21.44078263817520 & 1.26122399999993 \\
\hline $\mathbf{C u}$ & 5.35091375000000 & 23.96322669886980 & 1.26122399999993 \\
\hline $\mathrm{Cu}$ & 5.35091375000000 & 26.48567181899090 & 1.26122399999993 \\
\hline $\mathbf{C u}$ & 5.35091375000000 & 29.00811693910730 & 1.26122399999993 \\
\hline $\mathrm{Cu}$ & 5.35091375000000 & 31.53056135294360 & 1.26122399999993 \\
\hline $\mathbf{C u}$ & 5.35091375000000 & 34.05300647307050 & 1.26122399999993 \\
\hline $\mathbf{C u}$ & 8.91818794238642 & 1.26122414920059 & 1.26122399999993 \\
\hline $\mathbf{C u}$ & 8.91818794238642 & 3.78366926932167 & 1.26122399999993 \\
\hline $\mathrm{Cu}$ & 8.91818794238642 & 6.30611333001662 & 1.26122399999993 \\
\hline $\mathrm{Cu}$ & 8.91818794238642 & 8.82855845013778 & 1.26122399999993 \\
\hline $\mathbf{C u}$ & 8.91818794238642 & 11.35100286397460 & 1.26122399999993 \\
\hline $\mathbf{C u}$ & 8.91818794238642 & 13.87344798409320 & 1.26122399999993 \\
\hline $\mathbf{C u}$ & 8.91818794238642 & 16.39589310421720 & 1.26122399999993 \\
\hline $\mathrm{Cu}$ & 8.91818794238642 & 18.91833716491180 & 1.26122399999993 \\
\hline $\mathbf{C u}$ & 8.91818794238642 & 21.44078263817520 & 1.26122399999993 \\
\hline $\mathbf{C u}$ & 8.91818794238642 & 23.96322669886980 & 1.26122399999993 \\
\hline $\mathrm{Cu}$ & 8.91818794238642 & 26.48567181899090 & 1.26122399999993 \\
\hline $\mathrm{Cu}$ & 8.91818794238642 & 29.00811693910730 & 1.26122399999993 \\
\hline $\mathbf{C u}$ & 8.91818794238642 & 31.53056135294360 & 1.26122399999993 \\
\hline $\mathrm{Cu}$ & 8.91818794238642 & 34.05300647307050 & 1.26122399999993 \\
\hline $\mathbf{C u}$ & 0.00174435476525 & 35.31395804130010 & 2.53925847579168 \\
\hline $\mathbf{C u}$ & 10.69913477771030 & 2.52423263733075 & 2.54079497879308 \\
\hline $\mathbf{C u}$ & 0.00293722805315 & 5.04519362069888 & 2.54202996033917 \\
\hline $\mathbf{C u}$ & 10.70071256893420 & 7.56514289324491 & 2.54291081387342 \\
\hline $\mathbf{C u}$ & 10.70084241154070 & 10.09018776495640 & 2.54014516808004 \\
\hline $\mathbf{C u}$ & -0.00003738256668 & 12.61096972653750 & 2.54520662911561 \\
\hline $\mathrm{Cu}$ & 10.69662348130030 & 15.13617816504050 & 2.53720144735816 \\
\hline $\mathbf{C u}$ & 10.70163160872910 & 17.65792875470390 & 2.54308625534822 \\
\hline $\mathbf{C u}$ & 10.69824711829240 & 20.17852303266740 & 2.53799446824352 \\
\hline $\mathbf{C u}$ & 10.69654178351820 & 22.70302711515910 & 2.54417717430027 \\
\hline $\mathbf{C u}$ & 10.69971381160030 & 25.22831134904820 & 2.53954015600802 \\
\hline
\end{tabular}




\begin{tabular}{|c|c|c|c|}
\hline $\mathbf{C u}$ & 10.70100842747110 & 27.74772732263170 & 2.54370865072 \\
\hline $\mathbf{C u}$ & 0.00089636209249 & 30.26886651319210 & 2.541058987943 \\
\hline $\mathbf{C u}$ & 10.70114761411990 & 32.79024898020760 & 2.540712667377 \\
\hline $\mathbf{C u}$ & 3.56727572156260 & 0.00066784019650 & 2.541751247 \\
\hline $\mathbf{C u}$ & 3.56727753469562 & 2.52188335155476 & 2.54417214398 \\
\hline $\mathbf{C u}$ & 3.56728320829141 & 5.04462645673487 & 2.54104531 \\
\hline $\mathbf{C u}$ & 3.56727728587167 & 7.57016966475109 & 2.58065230 \\
\hline $\mathbf{C u}$ & 3.56727906076126 & 10.09090545437940 & 2.586918 \\
\hline $\mathbf{C u}$ & 3.56728212664363 & 12.62235108289210 & \\
\hline $\mathbf{C u}$ & 3.56727971575472 & 15.13815850554390 & \\
\hline $\mathbf{C u}$ & 3.56727606868422 & 17.65489769437510 & \\
\hline $\mathbf{C u}$ & 3.56727671687895 & 20.17966118735270 & \\
\hline $\mathbf{C u}$ & 3.56727545665720 & 22.69631101903660 & \\
\hline $\mathbf{C u}$ & 3.56727349084953 & 25.21906526774980 & 2.551934 \\
\hline $\mathbf{C u}$ & 3.56727327096592 & 27.74878642454520 & 2.5416728 \\
\hline $\mathbf{C u}$ & 3.56727422071287 & 30.26813707568110 & 2.54251 \\
\hline $\mathbf{C u}$ & 65049202 & 9788240 & 2.5 \\
\hline $\mathbf{C u}$ & 7.13281030009733 & 2811410 & 2.5 \\
\hline $\mathbf{C u}$ & 7.13724 & 3636 & 2.5 \\
\hline $\mathbf{C u}$ & 506 & 5.0 & 2. \\
\hline $\mathbf{C u}$ & 7.135663 & 7.56 & 2.5 \\
\hline $\mathbf{C u}$ & 7.135536 & 10.0901 & 2.5 \\
\hline $\mathbf{C u}$ & 7.134585 & 12.6109 & 2.54 \\
\hline $\mathbf{C u}$ & 7.13975007269730 & 15.13617339424230 & 2.537 \\
\hline $\mathbf{C u}$ & 7.13474569401678 & 80960 & 2.543 \\
\hline $\mathbf{C u}$ & 7.138154( & 31137930 & 2.53 \\
\hline $\mathrm{Cu}$ & 7.13984102891641 & 22.70302788287020 & 2.544180881584 \\
\hline $\mathbf{C u}$ & 7.13662788592978 & 25.22829426189970 & 2.53 \\
\hline $\mathbf{C u}$ & 7.13537383954473 & 27.74773173946010 & 2.543712953507 \\
\hline $\mathbf{C u}$ & 7.13365826867506 & 30.26886972001510 & 2.5410638577 \\
\hline $\mathbf{C u}$ & 7.13523857755957 & 32.79025239873830 & 2.5407251503 \\
\hline $\mathbf{C u}$ & 1.78336053654040 & 818931 & 3.853 \\
\hline $\mathbf{C u}$ & 1.778297 & 5489 & 3.8 \\
\hline $\mathbf{C u}$ & 1.79596923417844 & 9739 & 3. \\
\hline $\mathbf{C u}$ & 1.78120169200537 & 68019916 & 3.832 \\
\hline $\mathbf{C u}$ & 1.79958981714356 & 65966870 & 3.8 \\
\hline $\mathbf{C u}$ & 1.77527145100628 & 13.87595615146780 & 3.843109210 \\
\hline $\mathbf{C u}$ & 1.78119474603848 & 16.39795879782270 & 3.848104102 \\
\hline $\mathbf{C u}$ & 1.78603438012587 & 18.92505785351560 & 3.852887565 \\
\hline $\mathbf{C u}$ & 1.77489938390075 & 21.44357329052370 & $3.839850754 \mathrm{C}$ \\
\hline $\mathbf{C u}$ & 6897063 & 23.9690 & 3.847 \\
\hline $\mathbf{C u}$ & 1.780 & 26.4 & 3 \\
\hline $\mathbf{C u}$ & 782221771 & 29.00918 & 3.857 \\
\hline $\mathbf{C u}$ & 1.78282485587059 & 31.52947225890030 & 3.8559023612 \\
\hline $\mathbf{C u}$ & 1.78387069891161 & 34.04936443400660 & 366146825 \\
\hline $\mathbf{C u}$ & 5.35118600057998 & 1.25955552942721 & 3.853237988464 \\
\hline $\mathbf{C u}$ & 5.35625024957534 & 3.77966415803754 & 3.855677008072 \\
\hline $\mathbf{C u}$ & 5.33858321573247 & 6.30445786406768 & \\
\hline $\mathbf{C u}$ & 5.35334761789085 & 8.82605186739073 & 3.832223743641 \\
\hline $\mathbf{C u}$ & 5.33496806932186 & 11.34 & \\
\hline $\mathbf{C u}$ & 5.35927932343753 & 13.87595772486970 & \\
\hline $\mathbf{C u}$ & 5.35335201056593 & 16.39795919581270 & \\
\hline $\mathbf{C u}$ & 5.34850365339146 & 18.92507109470640 & 3.8528935897 \\
\hline $\mathbf{C u}$ & 5.35965355215776 & 21.44357185048770 & 3.839865350718 \\
\hline
\end{tabular}




\begin{tabular}{|c|c|c|c|}
\hline $\mathrm{Cu}$ & 5.35028552683397 & 23.96908517371870 & 3.84739413284722 \\
\hline $\mathbf{C u}$ & 5.35386620664591 & 26.48900943883080 & 3.85584533312386 \\
\hline $\mathrm{Cu}$ & 5.35076345152994 & 29.00919285514920 & 3.85714816265754 \\
\hline $\mathbf{C u}$ & 5.35172260136422 & 31.52948269930310 & 3.85590676102283 \\
\hline $\mathbf{C u}$ & 5.35068445845439 & 34.04936022058730 & 3.85364134748199 \\
\hline $\mathbf{C u}$ & 8.91819267332449 & 1.26036241781336 & 3.85027812040650 \\
\hline $\mathbf{C u}$ & 8.91818884416141 & 3.78406707962662 & 3.84944783483989 \\
\hline $\mathbf{C u}$ & 8.91818949205432 & 6.30386301368148 & 3.85154181629738 \\
\hline $\mathbf{C u}$ & 8.91819162751113 & 8.82723170332008 & 3.85598527534747 \\
\hline $\mathbf{C u}$ & 8.91818752358191 & 11.35365569340350 & 3.85232979493607 \\
\hline $\mathbf{C u}$ & 8.91818974693759 & 13.87171681418580 & 3.85209466249423 \\
\hline $\mathbf{C u}$ & 8.91819084225927 & 16.39955181302360 & 3.85266337241477 \\
\hline $\mathbf{C u}$ & 8.91819049731133 & 18.91874063957350 & 3.85159223012007 \\
\hline $\mathrm{Cu}$ & 8.91819853425065 & 21.43880118473410 & 3.85528310487799 \\
\hline $\mathbf{C u}$ & 8.91819466588325 & 23.96323948998850 & 3.85099452274753 \\
\hline $\mathbf{C u}$ & 8.91818700356189 & 26.49074885196470 & 3.85576151247609 \\
\hline $\mathbf{C u}$ & 8.91819272418520 & 29.00674399038070 & 3.84867810273003 \\
\hline $\mathbf{C u}$ & 8.91819390773128 & 31.53448340737100 & 3.84993253346120 \\
\hline $\mathbf{C u}$ & 8.91819432071960 & 34.05166707412870 & 3.85024459950353 \\
\hline $\mathbf{C u}$ & 0.00049454972256 & 35.31242728820600 & 5.05556434472602 \\
\hline $\mathbf{C u}$ & 10.70087990035040 & 2.52170065999878 & 5.05865070209776 \\
\hline $\mathbf{C u}$ & 0.00112674943090 & 5.04783200183371 & 5.05807824319161 \\
\hline $\mathbf{C u}$ & 0.00507467802177 & 691759136 & 31830 \\
\hline $\mathbf{C u}$ & 0.00831922542536 & 7309360 & 78405 \\
\hline $\mathbf{C u}$ & 0.00868805 & 260990 & 81163 \\
\hline $\mathbf{C u}$ & -0.00051955632964 & 070990 & 7867060 \\
\hline $\mathbf{C u}$ & 10.70197724734190 & 17.65928553018390 & 5.05460828247676 \\
\hline $\mathbf{C u}$ & 0.00574990442900 & 20.17914634829260 & 5.05662514923801 \\
\hline $\mathbf{C u}$ & 0.00422049953907 & 22.70361453174560 & 5.05779746369461 \\
\hline $\mathbf{C u}$ & 0.00179357078566 & 25.22307645941110 & 5.05544742497095 \\
\hline $\mathbf{C u}$ & -0.00067913916462 & 27.74962894156350 & 5.06570416984620 \\
\hline $\mathbf{C u}$ & 10.69889096292780 & 30.26772758897250 & 5.05657706975976 \\
\hline $\mathbf{C u}$ & 0.00315267860782 & 32.78992919649110 & 5.05927156362621 \\
\hline $\mathbf{C u}$ & 3.56727933367142 & 35.30998480550390 & 5.05775751108040 \\
\hline $\mathbf{C u}$ & 3.56726774103267 & 2.51681081693285 & 5.05750192700547 \\
\hline $\mathbf{C u}$ & 3.56727834924039 & 5.02215129444996 & 5.04969420515528 \\
\hline $\mathbf{C u}$ & 3.56727915473808 & 7.51998997881025 & 5.16180851173343 \\
\hline $\mathbf{C u}$ & 3.56727329066601 & 10.09913500152040 & 5.15178508165739 \\
\hline $\mathbf{C u}$ & 3.56728684049931 & 12.60963268625440 & 5.15561586138209 \\
\hline $\mathbf{C u}$ & 3.56726182183799 & 15.15684339274300 & 5.09458988888257 \\
\hline $\mathbf{C u}$ & 3.56725943006922 & 17.67872622307800 & 5.17577102724702 \\
\hline $\mathbf{C u}$ & 3.56727357301779 & 20.18448110459430 & 5.09318735803090 \\
\hline $\mathbf{C u}$ & 3.56727748310211 & 22.71652164086430 & 5.13641984809264 \\
\hline $\mathbf{C u}$ & 3.56727383043134 & 25.24440873209960 & 5.06056515784855 \\
\hline $\mathbf{C u}$ & 3.56729232856451 & 27.75243560216550 & 5.06113920313793 \\
\hline $\mathbf{C u}$ & 3.56727348197432 & 30.27390554130390 & 5.06083352347181 \\
\hline $\mathbf{C u}$ & 3.56726582667384 & 32.79202672690700 & 5.06031144078112 \\
\hline $\mathbf{C u}$ & 7.13404962341744 & 35.31242028028450 & 5.05555120328433 \\
\hline $\mathbf{C u}$ & 7.13550053816967 & 2.52170038148126 & 5.05865788626746 \\
\hline $\mathbf{C u}$ & 7.13342139966809 & 5.04783328820757 & 5.05807189682209 \\
\hline $\mathbf{C u}$ & 7.12947642936693 & 7.56654742216526 & 5.05199345438349 \\
\hline $\mathbf{C u}$ & 7.12623670274843 & 10.09290598756880 & 5.04666447368363 \\
\hline $\mathbf{C u}$ & 7.12586839429378 & 12.61073670116820 & 5.05512937141864 \\
\hline $\mathbf{C u}$ & 7.13507821674255 & 15.13730611800610 & 5.05212489267438 \\
\hline
\end{tabular}




\begin{tabular}{|c|c|c|c|}
\hline $\mathbf{C u}$ & 7.13440517104861 & 17.65928302845850 & 5.05460605117756 \\
\hline $\mathbf{C u}$ & 7.12880714073464 & 20.17914551849810 & 5.05661888663651 \\
\hline $\mathbf{C u}$ & 7.13032220245200 & 22.70359313339690 & 5.05780346456054 \\
\hline $\mathbf{C u}$ & 7.13276114524164 & 25.22307750506500 & 5.05544875186854 \\
\hline $\mathbf{C u}$ & 7.13523480255327 & 27.74963560784030 & 5.06571383263753 \\
\hline $\mathbf{C u}$ & 7.13749263155372 & 30.26771536679130 & 5.05658435748669 \\
\hline $\mathbf{C u}$ & 7.13139923392359 & 32.78992539493890 & 5.05927700635320 \\
\hline \multicolumn{4}{|c|}{ CI-NEB Image 15} \\
\hline Element & X-Coordinate (Å) & Y-Coordinate (§̊) & Z-Coordinate (Å) \\
\hline $\mathbf{F}$ & 4.66232975666667 & 12.68178223133270 & 7.78705860734801 \\
\hline $\mathbf{F}$ & 2.47250697796866 & 12.68166008730300 & 7.78721857800312 \\
\hline $\mathbf{F}$ & 3.56728021302168 & 8.81631554297030 & 6.63197245403157 \\
\hline $\mathbf{F}$ & 4.63285311342115 & 17.54437157651000 & 7.80648662427962 \\
\hline $\mathbf{F}$ & 2.50178298419944 & 17.54441844517040 & 7.80655104055691 \\
\hline $\mathbf{F}$ & 3.56727614576939 & 14.53595007480600 & 7.27577114946527 \\
\hline $\mathbf{F}$ & 4.66388669069751 & 22.77307122435280 & 7.76860376025602 \\
\hline $\mathbf{F}$ & 2.47065492030152 & 22.77307186420280 & 7.76857097656068 \\
\hline $\mathbf{F}$ & 3.56727821669746 & 20.90049067709370 & 7.34729683756034 \\
\hline C & 3.56734157538910 & 13.08953364387680 & 79537138 \\
\hline C & 3.56729528431454 & 17.58145656079000 & 7.01560683749209 \\
\hline C & 3.56728131091117 & 22.31735378557500 & 7.07305679246510 \\
\hline $\mathbf{C u}$ & 0.00000000000000 & 0.00000000000000 & 0.00000000000000 \\
\hline $\mathbf{C u}$ & 0.00000000000000 & 2.52244512012115 & 0.00000000000000 \\
\hline $\mathbf{C u}$ & 0.00000000000000 & 5.04488953395804 & 0.00000000000000 \\
\hline $\mathbf{C u}$ & 0.00000000000000 & 7.56733465407854 & 0.00000000000000 \\
\hline $\mathbf{C u}$ & 0.00000000000000 & 10.08977871477400 & 0.00000000000000 \\
\hline $\mathbf{C u}$ & 0.00000000000000 & 12.61222383489520 & 0.00000000000000 \\
\hline $\mathbf{C u}$ & 0.00000000000000 & 15.13466895501400 & 0.00000000000000 \\
\hline $\mathbf{C u}$ & 0.00000000000000 & 17.65711336885320 & 0.00000000000000 \\
\hline $\mathbf{C u}$ & 0.00000000000000 & 20.17955848897460 & 0.00000000000000 \\
\hline $\mathbf{C u}$ & 0.00000000000000 & 22.70200254966930 & 0.00000000000000 \\
\hline $\mathbf{C u}$ & 0.00000000000000 & 25.22444766979040 & 0.00000000000000 \\
\hline $\mathbf{C u}$ & 0.00000000000000 & 27.74689208362240 & 0.00000000000000 \\
\hline $\mathbf{C u}$ & 0.00000000000000 & 30.26933720374360 & 0.00000000000000 \\
\hline $\mathbf{C u}$ & 0.00000000000000 & 32.79178232386990 & 0.00000000000000 \\
\hline $\mathbf{C u}$ & 3.56727419238642 & 0.00000000000000 & 0.00000000000000 \\
\hline $\mathbf{C u}$ & 3.56727419238642 & 2.52244512012115 & 0.00000000000000 \\
\hline $\mathbf{C u}$ & 3.56727419238642 & 5.04488953395804 & 0.00000000000000 \\
\hline $\mathbf{C u}$ & 3.56727419238642 & 7.56733465407854 & 0.00000000000000 \\
\hline $\mathbf{C u}$ & 3.56727419238642 & 10.08977871477400 & 0.00000000000000 \\
\hline $\mathbf{C u}$ & 3.56727419238642 & 12.61222383489520 & 0.00000000000000 \\
\hline $\mathbf{C u}$ & 3.56727419238642 & 15.13466895501400 & 0.00000000000000 \\
\hline $\mathbf{C u}$ & 3.56727419238642 & 17.65711336885320 & 0.00000000000000 \\
\hline $\mathbf{C u}$ & 3.56727419238642 & 20.17955848897460 & 0.00000000000000 \\
\hline $\mathbf{C u}$ & 3.56727419238642 & 22.70200254966930 & 0.00000000000000 \\
\hline $\mathbf{C u}$ & 3.56727419238642 & 25.22444766979040 & 0.00000000000000 \\
\hline $\mathbf{C u}$ & 3.56727419238642 & 27.74689208362240 & 0.00000000000000 \\
\hline $\mathbf{C u}$ & 3.56727419238642 & 30.26933720374360 & 0.00000000000000 \\
\hline $\mathbf{C u}$ & 3.56727419238642 & 32.79178232386990 & 0.00000000000000 \\
\hline $\mathbf{C u}$ & 7.13454838477291 & 0.00000000000000 & 0.00000000000000 \\
\hline $\mathbf{C u}$ & 7.13454838477291 & 2.52244512012115 & 0.00000000000000 \\
\hline $\mathrm{Cu}$ & 7.13454838477291 & 5.04488953395804 & 0.00000000000000 \\
\hline $\mathbf{C u}$ & 7.13454838477291 & 7.56733465407854 & 0.00000000000000 \\
\hline $\mathbf{C u}$ & 7.13454838477291 & 10.08977871477400 & 0.00000000000000 \\
\hline
\end{tabular}




\begin{tabular}{|c|c|c|c|}
\hline $\mathrm{Cu}$ & 7.13454838477291 & 12.61222383489520 & 0.00000000000000 \\
\hline $\mathrm{Cu}$ & 7.13454838477291 & 15.13466895501400 & 0.00000000000000 \\
\hline $\mathrm{Cu}$ & 7.13454838477291 & 17.65711336885320 & 0.00000000000000 \\
\hline $\mathbf{C u}$ & 7.13454838477291 & 20.17955848897460 & 0.00000000000000 \\
\hline $\mathbf{C u}$ & 7.13454838477291 & 22.70200254966930 & 0.00000000000000 \\
\hline $\mathbf{C u}$ & 7.13454838477291 & 25.22444766979040 & 0.00000000000000 \\
\hline $\mathrm{Cu}$ & 7.13454838477291 & 27.74689208362240 & 0.00000000000000 \\
\hline $\mathrm{Cu}$ & 7.13454838477291 & 30.26933720374360 & 0.00000000000000 \\
\hline $\mathbf{C u}$ & 7.13454838477291 & 32.79178232386990 & 0.00000000000000 \\
\hline $\mathrm{Cu}$ & 1.78363955761358 & 1.26122414920059 & 1.26122399999993 \\
\hline $\mathbf{C u}$ & 1.78363955761358 & 3.78366926932167 & 1.26122399999993 \\
\hline $\mathrm{Cu}$ & 1.78363955761358 & 6.30611333001662 & 1.26122399999993 \\
\hline $\mathrm{Cu}$ & 1.78363955761358 & 8.82855845013778 & 1.26122399999993 \\
\hline $\mathrm{Cu}$ & 1.78363955761358 & 11.35100286397460 & 1.26122399999993 \\
\hline $\mathrm{Cu}$ & 1.78363955761358 & 13.87344798409320 & 1.26122399999993 \\
\hline $\mathrm{Cu}$ & 1.78363955761358 & 16.39589310421720 & 1.26122399999993 \\
\hline $\mathrm{Cu}$ & 1.78363955761358 & 18.91833716491180 & 1.26122399999993 \\
\hline $\mathbf{C u}$ & 1.78363955761358 & 21.44078263817520 & 1.26122399999993 \\
\hline $\mathbf{C u}$ & 1.78363955761358 & 23.96322669886980 & 1.26122399999993 \\
\hline $\mathrm{Cu}$ & 1.78363955761358 & 26.48567181899090 & 1.26122399999993 \\
\hline $\mathrm{Cu}$ & 1.783639 & 29.00811693910750 & 1.26122399999993 \\
\hline $\mathbf{C u}$ & 1.78363955761358 & 31.53056135294390 & 1.26122399999993 \\
\hline $\mathrm{Cu}$ & 1.78363955761358 & 34.05300647307050 & 1.26122399999993 \\
\hline $\mathrm{Cu}$ & 5.35091375000000 & 1.26122414920059 & 1.26122399999993 \\
\hline $\mathrm{Cu}$ & 5.35091375000000 & 3.78366926932167 & 1.26122399999993 \\
\hline $\mathbf{C u}$ & 5.35091375000000 & 6.30611333001662 & 1.26122399999993 \\
\hline $\mathbf{C u}$ & 5.35091375000000 & 8.82855845013778 & 1.26122399999993 \\
\hline $\mathrm{Cu}$ & 5.35091375000000 & 11.35100286397460 & 1.26122399999993 \\
\hline $\mathbf{C u}$ & 5.35091375000000 & 13.87344798409320 & 1.26122399999993 \\
\hline $\mathrm{Cu}$ & 5.35091375000000 & 16.39589310421720 & 1.26122399999993 \\
\hline $\mathrm{Cu}$ & 5.35091375000000 & 18.91833716491180 & 1.26122399999993 \\
\hline $\mathrm{Cu}$ & 5.35091375000000 & 21.44078263817520 & 1.26122399999993 \\
\hline $\mathrm{Cu}$ & 5.35091375000000 & 23.96322669886980 & 1.26122399999993 \\
\hline $\mathbf{C u}$ & 5.35091375000000 & 26.48567181899090 & 1.26122399999993 \\
\hline $\mathbf{C u}$ & 5.35091375000000 & 29.00811693910750 & 1.26122399999993 \\
\hline $\mathbf{C u}$ & 5.35091375000000 & 31.53056135294390 & 1.26122399999993 \\
\hline $\mathrm{Cu}$ & 5.35091375000000 & 34.05300647307050 & 1.26122399999993 \\
\hline $\mathbf{C u}$ & 8.91818794238642 & 1.26122414920059 & 1.26122399999993 \\
\hline $\mathrm{Cu}$ & 8.91818794238642 & 3.78366926932167 & 1.26122399999993 \\
\hline $\mathbf{C u}$ & 8.91818794238642 & 6.30611333001662 & 1.26122399999993 \\
\hline $\mathrm{Cu}$ & 8.91818794238642 & 8.82855845013778 & 1.26122399999993 \\
\hline $\mathbf{C u}$ & 8.91818794238642 & 11.35100286397460 & 1.26122399999993 \\
\hline $\mathbf{C u}$ & 8.91818794238642 & 13.87344798409320 & 1.26122399999993 \\
\hline $\mathbf{C u}$ & 8.91818794238642 & 16.39589310421720 & 1.26122399999993 \\
\hline $\mathbf{C u}$ & 8.91818794238642 & 18.91833716491180 & 1.26122399999993 \\
\hline $\mathbf{C u}$ & 8.91818794238642 & 21.44078263817520 & 1.26122399999993 \\
\hline $\mathbf{C u}$ & 8.91818794238642 & 23.96322669886980 & 1.26122399999993 \\
\hline $\mathrm{Cu}$ & 8.91818794238642 & 26.48567181899090 & 1.26122399999993 \\
\hline $\mathbf{C u}$ & 8.91818794238642 & 29.00811693910750 & 1.26122399999993 \\
\hline $\mathrm{Cu}$ & 8.91818794238642 & 31.53056135294390 & 1.26122399999993 \\
\hline $\mathbf{C u}$ & 8.91818794238642 & 34.05300647307050 & 1.26122399999993 \\
\hline $\mathbf{C u}$ & 0.00181798558986 & 35.31386988298970 & 2.53944943619749 \\
\hline $\mathbf{C u}$ & 10.69905210443250 & 2.52394599717052 & 2.54083535836111 \\
\hline $\mathbf{C u}$ & 0.00315128440760 & 5.04529771144049 & 2.54199475679784 \\
\hline
\end{tabular}




\begin{tabular}{|c|c|c|c|}
\hline $\mathbf{C u}$ & 10.70070585345920 & 7.56524791642033 & 2.54313371962154 \\
\hline $\mathbf{C u}$ & 10.70059380039300 & 10.09072441220590 & 2.54064930857196 \\
\hline $\mathrm{Cu}$ & 10.70129388109470 & 12.61183196769220 & 2.54834970575974 \\
\hline $\mathbf{C u}$ & 10.69595652932230 & 15.13607150065300 & 2.53676963162518 \\
\hline $\mathbf{C u}$ & 10.70079838082850 & 17.65808746575140 & 2.54290756595053 \\
\hline $\mathbf{C u}$ & 10.69793374184900 & 20.17948025396400 & 2.53737259797327 \\
\hline $\mathbf{C u}$ & 10.69721960347760 & 22.70349996559380 & 2.54415292499292 \\
\hline $\mathbf{C u}$ & 10.69987396595390 & 25.22840744135670 & 2.53934179451049 \\
\hline $\mathbf{C u}$ & 10.70124691094600 & 27.74786063954730 & 2.54389827676865 \\
\hline $\mathbf{C u}$ & 0.00087295698894 & 30.26877388520150 & 2.54085087446982 \\
\hline $\mathbf{C u}$ & 10.70135660436200 & 32.79003261585730 & 2.54074427520777 \\
\hline $\mathbf{C u}$ & 3.56727634951644 & 0.00095344735686 & 2.54029400698798 \\
\hline $\mathbf{C u}$ & 3.56727424012257 & 2.52266859196322 & 2.54366597023589 \\
\hline $\mathbf{C u}$ & 3.56727991999286 & 5.04483693141173 & 2.54098446863122 \\
\hline $\mathbf{C u}$ & 3.56728185349842 & 7.57053655388153 & 2.57971364237368 \\
\hline $\mathrm{Cu}$ & 3.56727731198147 & 10.09282815715680 & 2.58772727605750 \\
\hline $\mathbf{C u}$ & 3.56727716341718 & 12.62374991119550 & 2.58448999374276 \\
\hline $\mathbf{C u}$ & 3.56728466740515 & 15.13998435431260 & 2.58480568695307 \\
\hline $\mathbf{C u}$ & 3.56728717608968 & 17.65748681194010 & 2.59309489027727 \\
\hline $\mathbf{C u}$ & 3.56728515305514 & 20.18130057302570 & 2.58244842471073 \\
\hline $\mathbf{C u}$ & 3.56727664437304 & 22.69614008390990 & 2.58440 \\
\hline $\mathbf{C u}$ & 3.56727996948448 & 25.21865703645480 & 2.5522938 \\
\hline $\mathbf{C u}$ & 3.56727870322896 & 27.74847958296740 & 2.54090146417452 \\
\hline $\mathbf{C u}$ & 3.56727729491232 & 30.26799272936590 & 2.54199763282390 \\
\hline $\mathbf{C u}$ & 3.56727915259403 & 32.79018180820180 & 2.54260727855069 \\
\hline $\mathbf{C u}$ & 7.13273168519125 & 35.31386794435650 & 2.53944213536443 \\
\hline $\mathbf{C u}$ & 7.13732186961729 & 2.52394552023219 & 2.54083708199505 \\
\hline $\mathbf{C u}$ & 7.13139196451164 & 5.04528190887823 & 2.54200221436148 \\
\hline $\mathbf{C u}$ & 7.13567254806134 & 7.56525118212059 & 2.54313703164884 \\
\hline $\mathbf{C u}$ & 7.13578642458622 & 10.09072540905610 & 2.54064655108525 \\
\hline $\mathbf{C u}$ & 7.13508904250063 & 12.61183554029570 & 2.54834663309252 \\
\hline $\mathbf{C u}$ & 7.14041934866724 & 15.13607657704400 & 2.53676299471613 \\
\hline $\mathbf{C u}$ & 7.13557667306691 & 17.65808496456500 & 2.54290511601464 \\
\hline $\mathbf{C u}$ & 7.13843924129020 & 20.17947812506920 & 2.53737983679731 \\
\hline $\mathbf{C u}$ & 7.13915519430812 & 22.70350182647860 & 2.54415626619578 \\
\hline $\mathbf{C u}$ & 7.13650227670389 & 25.22840892673540 & 2.53934397768522 \\
\hline $\mathbf{C u}$ & 7.13513070825928 & 27.74786017472100 & 2.54389851069548 \\
\hline $\mathbf{C u}$ & 7.13367618114837 & 30.26877062861680 & 2.54085121174139 \\
\hline $\mathbf{C u}$ & 7.13501886372478 & 32.79002919753550 & 2.54074157849288 \\
\hline $\mathbf{C u}$ & 1.78384268087342 & 1.25967086776422 & 3.85274887134961 \\
\hline $\mathbf{C u}$ & 1.77831658999961 & 3.77968191800588 & 3.85565724497765 \\
\hline $\mathbf{C u}$ & 1.79643659672817 & 6.30469024995472 & 3.85886433165585 \\
\hline $\mathbf{C u}$ & 1.78204413217945 & 8.82817810007127 & 3.83136437918035 \\
\hline $\mathbf{C u}$ & 1.80107647239469 & 11.35041338015460 & 3.85472675155561 \\
\hline $\mathbf{C u}$ & 1.77557629002251 & 13.87960230287430 & 3.84320883378015 \\
\hline $\mathbf{C u}$ & 1.77983033829995 & 16.39820973487110 & 3.84544823061340 \\
\hline $\mathbf{C u}$ & 1.78257854328862 & 18.92796292673270 & 3.85006300207215 \\
\hline $\mathbf{C u}$ & 1.77579638879024 & 21.44367401471020 & 3.84169335546318 \\
\hline $\mathbf{C u}$ & 1.78482637332968 & 23.96876807839020 & 3.84776987403773 \\
\hline $\mathbf{C u}$ & 1.78137587684135 & 26.48863214088750 & 3.85547617107385 \\
\hline $\mathbf{C u}$ & 1.78476392575017 & 29.00898377390000 & 3.85667433893344 \\
\hline $\mathbf{C u}$ & 1.78341443940194 & 31.52926120610530 & 3.85527857081851 \\
\hline $\mathbf{C u}$ & 1.78458138256069 & 34.04921865036570 & 3.85310450344979 \\
\hline $\mathbf{C u}$ & 5.35070663425366 & 1.25966902038292 & 3.85273057282823 \\
\hline
\end{tabular}




\begin{tabular}{|c|c|c|c|}
\hline $\mathrm{Cu}$ & 5.35622719497781 & 3.77968141312462 & 3.85565882107969 \\
\hline $\mathrm{Cu}$ & 5.33812363962476 & 6.30469286791418 & 3.85886511498396 \\
\hline $\mathrm{Cu}$ & 5.35251400838289 & 8.82818206539912 & 3.83136890821143 \\
\hline $\mathbf{C u}$ & 5.33348061932851 & 11.35040466405980 & 3.85470801075617 \\
\hline $\mathbf{C u}$ & 5.35898779720826 & 13.87959424266220 & 3.84320697307107 \\
\hline $\mathbf{C u}$ & 5.35472257103622 & 16.39821542626840 & 3.84547688157045 \\
\hline $\mathrm{Cu}$ & 5.35199216627548 & 18.92796603483830 & 3.85008328972945 \\
\hline $\mathrm{Cu}$ & 5.35875602940239 & 21.44367155603610 & 3.84170376521109 \\
\hline $\mathbf{C u}$ & 5.34971940204757 & 23.96877062668840 & 3.84777985964219 \\
\hline $\mathbf{C u}$ & 5.35318001038987 & 26.48863562071650 & 3.85547702122203 \\
\hline $\mathbf{C u}$ & 5.34979056087095 & 29.00898018653060 & 3.85666719419885 \\
\hline $\mathrm{Cu}$ & 5.35113377185504 & 31.52925907854990 & 3.85528404074109 \\
\hline $\mathrm{Cu}$ & 5.34997541444029 & 34.04920755694150 & 3.85309592693086 \\
\hline $\mathrm{Cu}$ & 8.91818713574203 & 1.26018600577807 & 3.85025077307574 \\
\hline $\mathbf{C u}$ & 8.91818448649152 & 3.78376653856127 & 3.84912896610749 \\
\hline $\mathbf{C u}$ & 8.91819111476339 & 6.30423211084686 & 3.85100229973878 \\
\hline $\mathrm{Cu}$ & 8.91818540098733 & 8.82739523089924 & 3.85708317871393 \\
\hline $\mathbf{C u}$ & 8.91819271243481 & 11.35568845543220 & 3.85632797687343 \\
\hline $\mathbf{C u}$ & 8.91818980565075 & 13.87279918307420 & 3.85476711272460 \\
\hline $\mathrm{Cu}$ & 8.91818734977388 & 16.39983947933090 & 3.85256324854607 \\
\hline $\mathbf{C u}$ & 8.91818953552742 & 18.91920401377980 & 3.85071405813166 \\
\hline $\mathbf{C u}$ & 8.91819187709484 & 21.43977223974210 & 3.85496808871653 \\
\hline $\mathrm{Cu}$ & 8.91818866351503 & 23.96336615297540 & 54941893 \\
\hline $\mathrm{Cu}$ & 48156203 & 26.49050280809530 & 25414 \\
\hline $\mathrm{Cu}$ & 8.91818909865938 & 29.00644944224720 & 23772 \\
\hline $\mathrm{Cu}$ & 8.91818649109830 & 31.53555743827300 & 024249 \\
\hline $\mathrm{Cu}$ & 8.91818334143221 & 34.05231468473760 & 3.85057157793141 \\
\hline $\mathrm{Cu}$ & 0.00198040914158 & 35.31283405637270 & 5.05522072637761 \\
\hline $\mathbf{C u}$ & 10.70158566859270 & 2.52190055892144 & 5.05844799879486 \\
\hline $\mathrm{Cu}$ & 0.00110861194809 & 5.04840484933065 & 5.05714767683543 \\
\hline $\mathrm{Cu}$ & 0.00652979421042 & 7.56641933546055 & 5.05156929720601 \\
\hline $\mathrm{Cu}$ & 0.00946405399214 & 10.09430647187790 & 5.04628957857672 \\
\hline $\mathrm{Cu}$ & 0.01114503609161 & 12.61298935264460 & 5.05970020218320 \\
\hline $\mathrm{Cu}$ & 0.00105809647235 & 15.13987278980610 & 5.05316498907626 \\
\hline $\mathrm{Cu}$ & 10.70100902199740 & 17.66114456740440 & 5.05424870489651 \\
\hline $\mathbf{C u}$ & 0.00397040304863 & 20.18085242149760 & 5.05746460158088 \\
\hline $\mathrm{Cu}$ & 0.00457489378982 & 22.70391048959290 & 5.05768225260417 \\
\hline $\mathbf{C u}$ & 0.00288965132281 & 25.22226479466850 & 5.05475709373208 \\
\hline $\mathrm{Cu}$ & 0.00064656829140 & 27.74969074397710 & 5.06510246207715 \\
\hline $\mathbf{C u}$ & 10.69951639448570 & 30.26824473158080 & 5.05467005585291 \\
\hline $\mathbf{C u}$ & 0.00500737816916 & 32.79023233156070 & 5.05875983663117 \\
\hline $\mathbf{C u}$ & 3.56728850130694 & 35.30992612292390 & 5.05685172135460 \\
\hline $\mathbf{C u}$ & 3.56727539227922 & 2.51671794919146 & 5.05746180930670 \\
\hline $\mathbf{C u}$ & 3.56727841359484 & 5.02214343499227 & 5.04967058790256 \\
\hline $\mathbf{C u}$ & 3.56728323901723 & 7.52035022265488 & 5.15757220134732 \\
\hline $\mathbf{C u}$ & 3.56727419371499 & 10.11119897249150 & 5.15612444719427 \\
\hline $\mathrm{Cu}$ & 3.56727559297321 & 12.62908544234430 & 5.14692845721466 \\
\hline $\mathrm{Cu}$ & 3.56727536160998 & 15.16207328639920 & 5.09449782228837 \\
\hline $\mathrm{Cu}$ & 3.56725698435000 & 17.67371578936610 & 5.14731786173780 \\
\hline $\mathrm{Cu}$ & 3.56726993226529 & 20.17979475266560 & 5.10029816055672 \\
\hline $\mathrm{Cu}$ & 3.56728554874204 & 22.71464468610190 & 5.13899184205764 \\
\hline $\mathbf{C u}$ & 3.56727587361060 & 25.24203486665880 & 5.06216264320426 \\
\hline $\mathbf{C u}$ & 3.56727115399045 & 27.75130725249840 & 5.06120569860382 \\
\hline $\mathbf{C u}$ & 3.56727898579994 & 30.27368858479630 & 5.06091571278076 \\
\hline
\end{tabular}




\begin{tabular}{|c|c|c|c|}
\hline $\mathrm{Cu}$ & 3.56728347796655 & 32.79177523338070 & 5.06008950723683 \\
\hline $\mathbf{C u}$ & 7.13255447487358 & 35.31283684562940 & 5.05521772091014 \\
\hline $\mathbf{C u}$ & 7.13477823210431 & 2.52189996563730 & 5.05844362974586 \\
\hline $\mathbf{C u}$ & 7.13343776650031 & 5.04840181318821 & 5.05715022470216 \\
\hline $\mathbf{C u}$ & 7.12802108200727 & 7.56641991788917 & 5.05157172604991 \\
\hline $\mathbf{C u}$ & 7.12509530618773 & 10.09430296557110 & 5.04628400349473 \\
\hline $\mathbf{C u}$ & 7.12341503999649 & 12.61297873579940 & 5.05968945557347 \\
\hline $\mathbf{C u}$ & 7.13349621470799 & 15.13987045480860 & 5.05316594226267 \\
\hline $\mathbf{C u}$ & 7.13539037514289 & 17.66114474016690 & 5.05426173385726 \\
\hline $\mathbf{C u}$ & 7.13059798010390 & 20.18086334013990 & 5.05747242362118 \\
\hline $\mathbf{C u}$ & 7.12998078118896 & 22.70391037373080 & 5.05768592857007 \\
\hline $\mathbf{C u}$ & 7.13166534936280 & 25.22226994556420 & 5.05475694281020 \\
\hline $\mathbf{C u}$ & 7.13390697521399 & 27.74969737829290 & 5.06510278506598 \\
\hline $\mathbf{C u}$ & 7.13685813362225 & 30.26824652112190 & 5.05467179532786 \\
\hline $\mathbf{C u}$ & 40437236 & 32.79023366528460 & 5.05876137413076 \\
\hline \multicolumn{4}{|c|}{ CI-NEB Image 16} \\
\hline Element & X-Coordinate $(\AA)$ & Y-Coordinate $(\AA))$ & $(\AA)$ \\
\hline $\mathbf{F}$ & 4.66325575388927 & 12.61774910783930 & 0095988 \\
\hline $\mathbf{F}$ & 2.47124799639836 & 12.61776531179240 & 7.765527 \\
\hline $\mathbf{F}$ & 3.56727012627171 & 8.81727684864815 & 6.62968446950770 \\
\hline $\mathbf{F}$ & 4.63311350775018 & 17.70993685872530 & 7.80173582992309 \\
\hline $\mathbf{F}$ & 2.50142828947513 & 17.70992698589560 & 7.80179668508025 \\
\hline $\mathbf{F}$ & 3.56727080191591 & 14.48646794040200 & 7.31827783317734 \\
\hline $\mathbf{F}$ & 4.66372886787895 & 22.79133147385210 & 7.77124766912405 \\
\hline $\mathbf{F}$ & 2.47081325375464 & 22.79135371493590 & 7.77122007380004 \\
\hline $\mathbf{F}$ & 3.56726267562032 & 20.91782247225200 & 7.35340645280722 \\
\hline C & 3.56726777883411 & 13.05594423183010 & 7.06571885485345 \\
\hline C & 3.56725313051869 & 17.69178739707250 & 7.01024560999980 \\
\hline C & 3.56727577191464 & 22.33448459010630 & 7.07626442437291 \\
\hline $\mathbf{C u}$ & 0.00000000000000 & 0.00000000000000 & 0.00000000000000 \\
\hline $\mathbf{C u}$ & 0.00000000000000 & 2.52244512012115 & 0.00000000000000 \\
\hline $\mathbf{C u}$ & 0.00000000000000 & 5.04488953395804 & 0.00000000000000 \\
\hline $\mathbf{C u}$ & 0.00000000000000 & 7.56733465407854 & 0.00000000000000 \\
\hline $\mathbf{C u}$ & 0.00000000000000 & 10.08977871477400 & 0.00000000000000 \\
\hline $\mathbf{C u}$ & 0.00000000000000 & 12.61222383489520 & 0.00000000000000 \\
\hline $\mathbf{C u}$ & 0.00000000000000 & 15.13466895501400 & 0.00000000000000 \\
\hline $\mathbf{C u}$ & 0.00000000000000 & 17.65711336885320 & 0.00000000000000 \\
\hline $\mathbf{C u}$ & 0.00000000000000 & 20.17955848897460 & 0.00000000000000 \\
\hline $\mathbf{C u}$ & 0.00000000000000 & 22.70200254966930 & 0.00000000000000 \\
\hline $\mathbf{C u}$ & 0.00000000000000 & 25.22444766979040 & 0.00000000000000 \\
\hline $\mathbf{C u}$ & 0.00000000000000 & 27.74689208362270 & 0.00000000000000 \\
\hline $\mathbf{C u}$ & 0.00000000000000 & 30.26933720374380 & 0.00000000000000 \\
\hline $\mathbf{C u}$ & 0.00000000000000 & 32.79178232386990 & 0.00000000000000 \\
\hline $\mathbf{C u}$ & 3.56727419238642 & 0.00000000000000 & 0.00000000000000 \\
\hline $\mathbf{C u}$ & 3.56727419238642 & 2.52244512012115 & 0.00000000000000 \\
\hline $\mathbf{C u}$ & 3.56727419238642 & 5.04488953395804 & 0.00000000000000 \\
\hline $\mathbf{C u}$ & 3.56727419238642 & 7.56733465407854 & 0.00000000000000 \\
\hline $\mathbf{C u}$ & 3.56727419238642 & 10.08977871477400 & 0.00000000000000 \\
\hline $\mathbf{C u}$ & 3.56727419238642 & 12.61222383489520 & 0.00000000000000 \\
\hline $\mathbf{C u}$ & 3.56727419238642 & 15.13466895501400 & 0.00000000000000 \\
\hline $\mathbf{C u}$ & 3.56727419238642 & 17.65711336885320 & 0.00000000000000 \\
\hline $\mathbf{C u}$ & 3.56727419238642 & 20.17955848897460 & 0.00000000000000 \\
\hline $\mathbf{C u}$ & 3.56727419238642 & 22.70200254966930 & 0.00000000000000 \\
\hline $\mathbf{C u}$ & 3.56727419238642 & 25.22444766979040 & 0.00000000000000 \\
\hline
\end{tabular}




\begin{tabular}{|c|c|c|c|}
\hline $\mathrm{Cu}$ & 3.56727419238642 & 27.74689208362270 & 0.00000000000000 \\
\hline $\mathbf{C u}$ & 3.56727419238642 & 30.26933720374380 & 0.00000000000000 \\
\hline $\mathbf{C u}$ & 3.56727419238642 & 32.79178232386990 & 0.00000000000000 \\
\hline $\mathbf{C u}$ & 7.13454838477291 & 0.00000000000000 & 0.00000000000000 \\
\hline $\mathbf{C u}$ & 7.13454838477291 & 2.52244512012115 & 0.00000000000000 \\
\hline $\mathbf{C u}$ & 7.13454838477291 & 5.04488953395804 & 0.00000000000000 \\
\hline $\mathbf{C u}$ & 7.13454838477291 & 7.56733465407854 & 0.00000000000000 \\
\hline $\mathbf{C u}$ & 7.13454838477291 & 10.08977871477400 & 0.00000000000000 \\
\hline $\mathbf{C u}$ & 7.13454838477291 & 12.61222383489520 & 0.00000000000000 \\
\hline $\mathbf{C u}$ & 7.13454838477291 & 15.13466895501400 & 0.00000000000000 \\
\hline $\mathbf{C u}$ & 7.13454838477291 & 17.65711336885320 & 0.00000000000000 \\
\hline $\mathbf{C u}$ & 7.13454838477291 & 20.17955848897460 & 0.00000000000000 \\
\hline $\mathrm{Cu}$ & 7.13454838477291 & 22.70200254966930 & 0.00000000000000 \\
\hline $\mathrm{Cu}$ & 7.13454838477291 & 25.22444766979040 & 0.00000000000000 \\
\hline $\mathbf{C u}$ & 7.13454838477291 & 27.74689208362270 & 0.00000000000000 \\
\hline $\mathbf{C u}$ & 7.13454838477291 & 30.26933720374380 & 0.00000000000000 \\
\hline $\mathbf{C u}$ & 7.13454838477291 & 32.79178232386990 & 0.00000000000000 \\
\hline $\mathbf{C u}$ & 1.78363955761358 & 1.26122414920059 & 1.26122399999993 \\
\hline $\mathbf{C u}$ & 1.78363955761358 & 3.78366926932167 & 1.26122399999993 \\
\hline $\mathbf{C u}$ & 1.78363955761358 & 6.30611333001662 & 1.26122399999993 \\
\hline $\mathbf{C u}$ & 1.78363955761358 & 8.82855845013778 & 1.26122399999993 \\
\hline $\mathbf{C u}$ & 1.78363955761358 & 11.35100286397460 & 1.26122399999993 \\
\hline $\mathbf{C u}$ & 1.78363955761358 & 13.87344798409320 & 1.26122399999993 \\
\hline $\mathrm{Cu}$ & 1.783639 & 16.39589310421720 & 1.26122399999993 \\
\hline $\mathrm{Cu}$ & 1.783 & 16491180 & 1.26122399999993 \\
\hline $\mathrm{Cu}$ & 1.78363955761358 & 21.44078263817520 & 1.26122399999993 \\
\hline $\mathbf{C u}$ & 1.78363955761358 & 23.96322669886980 & 1.26122399999993 \\
\hline $\mathbf{C u}$ & 1.78363955761358 & 26.48567181899090 & 1.26122399999993 \\
\hline $\mathbf{C u}$ & 1.78363955761358 & 29.00811693910780 & 1.26122399999993 \\
\hline $\mathrm{Cu}$ & 1.78363955761358 & 31.53056135294410 & 1.26122399999993 \\
\hline $\mathrm{Cu}$ & 1.78363955761358 & 34.05300647307050 & 1.26122399999993 \\
\hline $\mathrm{Cu}$ & 5.35091375000000 & 1.26122414920059 & 1.26122399999993 \\
\hline $\mathrm{Cu}$ & 5.35091375000000 & 3.78366926932167 & 1.26122399999993 \\
\hline $\mathbf{C u}$ & 5.35091375000000 & 6.30611333001662 & 1.26122399999993 \\
\hline $\mathbf{C u}$ & 5.35091375000000 & 8.82855845013778 & 1.26122399999993 \\
\hline $\mathbf{C u}$ & 5.35091375000000 & 11.35100286397460 & 1.26122399999993 \\
\hline $\mathbf{C u}$ & 5.35091375000000 & 13.87344798409320 & 1.26122399999993 \\
\hline $\mathbf{C u}$ & 5.35091375000000 & 16.39589310421720 & 1.26122399999993 \\
\hline $\mathbf{C u}$ & 5.35091375000000 & 18.91833716491180 & 1.26122399999993 \\
\hline $\mathbf{C u}$ & 5.35091375000000 & 21.44078263817520 & 1.26122399999993 \\
\hline $\mathbf{C u}$ & 5.35091375000000 & 23.96322669886980 & 1.26122399999993 \\
\hline $\mathbf{C u}$ & 5.35091375000000 & 26.48567181899090 & 1.26122399999993 \\
\hline $\mathbf{C u}$ & 5.35091375000000 & 29.00811693910780 & 1.26122399999993 \\
\hline $\mathbf{C u}$ & 5.35091375000000 & 31.53056135294410 & 1.26122399999993 \\
\hline $\mathbf{C u}$ & 5.35091375000000 & 34.05300647307050 & 1.26122399999993 \\
\hline $\mathrm{Cu}$ & 8.91818794238642 & 1.26122414920059 & 1.26122399999993 \\
\hline $\mathbf{C u}$ & 8.91818794238642 & 3.78366926932167 & 1.26122399999993 \\
\hline $\mathbf{C u}$ & 8.91818794238642 & 6.30611333001662 & 1.26122399999993 \\
\hline $\mathbf{C u}$ & 8.91818794238642 & 8.82855845013778 & 1.26122399999993 \\
\hline $\mathbf{C u}$ & 8.91818794238642 & 11.35100286397460 & 1.26122399999993 \\
\hline $\mathbf{C u}$ & 8.91818794238642 & 13.87344798409320 & 1.26122399999993 \\
\hline $\mathbf{C u}$ & 8.91818794238642 & 16.39589310421720 & 1.26122399999993 \\
\hline $\mathbf{C u}$ & 8.91818794238642 & 18.91833716491180 & 1.26122399999993 \\
\hline $\mathbf{C u}$ & 8.91818794238642 & 21.44078263817520 & 1.26122399999993 \\
\hline
\end{tabular}




\begin{tabular}{|c|c|c|c|}
\hline $\mathbf{C u}$ & 8.91818794238642 & 23.96322669886980 & 1.26122399999993 \\
\hline $\mathbf{C u}$ & 8.91818794238642 & 26.48567181899090 & 1.26122399999993 \\
\hline $\mathbf{C u}$ & 8.91818794238642 & 29.00811693910780 & 1.26122399999993 \\
\hline $\mathbf{C u}$ & 8.91818794238642 & 31.53056135294410 & 1.26122399999993 \\
\hline $\mathbf{C u}$ & 8.91818794238642 & 34.05300647307050 & 1.26122399999993 \\
\hline $\mathbf{C u}$ & 0.00188090310005 & 35.31384472917500 & 2.53943030713914 \\
\hline $\mathbf{C u}$ & 10.69903867016890 & 2.52385716038444 & 2.54084249203222 \\
\hline $\mathbf{C u}$ & 0.00315039483157 & 5.04531698986763 & 2.54194325410139 \\
\hline $\mathbf{C u}$ & 10.70062646909460 & 7.56524313748577 & 2.54320312511810 \\
\hline $\mathbf{C u}$ & 10.70004445645350 & 10.09066671774190 & 2.54043861847018 \\
\hline $\mathbf{C u}$ & 10.70084288649380 & 12.61264561859300 & 2.54838926510824 \\
\hline $\mathbf{C u}$ & 10.69593001721380 & 15.13665393610430 & 2.53680152968352 \\
\hline $\mathbf{C u}$ & 10.70110047122040 & 17.65792930433390 & 2.54292855547539 \\
\hline $\mathbf{C u}$ & 10.69815148379540 & 20.17957006909280 & 2.53755387763626 \\
\hline $\mathbf{C u}$ & 10.69769831136280 & 22.70313494932330 & 2.54442283135814 \\
\hline $\mathbf{C u}$ & 10.69986573730930 & 25.22820821751300 & 2.53913633661457 \\
\hline $\mathbf{C u}$ & 10.70130438793870 & 27.74782216397150 & 2.54378879275932 \\
\hline $\mathbf{C u}$ & 0.00089700418292 & 30.26871559882080 & 2.54074079362747 \\
\hline $\mathbf{C u}$ & 10.70137915341720 & 32.78995908634750 & 2.54073243837962 \\
\hline $\mathbf{C u}$ & 3.56726912199547 & 0.00079257849693 & 2.53 \\
\hline $\mathbf{C u}$ & 6647503 & 8786 & 2.54346529015393 \\
\hline $\mathbf{C u}$ & 3960603 & 857 & 2.54087477291102 \\
\hline $\mathbf{C u}$ & 3.56726739910402 & 7.57067023733479 & 2.57941375164151 \\
\hline $\mathbf{C u}$ & 3.56725683501654 & 10.09319745298380 & 2.58755249857361 \\
\hline $\mathbf{C u}$ & 3.56725926854540 & 12.62452537819430 & 2.58160932743608 \\
\hline $\mathbf{C u}$ & 3.56725673115365 & 15.13989446679240 & 2.58492495928350 \\
\hline $\mathbf{C u}$ & 3.56724281427637 & 17.65803528818270 & 2.59036653005221 \\
\hline $\mathbf{C u}$ & 3.56726034977992 & 20.18142780312130 & 2.58443459718164 \\
\hline $\mathbf{C u}$ & 6598183091 & 22.69593208126920 & 2.58446436822590 \\
\hline $\mathbf{C u}$ & 3.56728331954256 & 25.21845144787760 & 2.55211914818717 \\
\hline $\mathbf{C u}$ & 3.56728273111778 & 27.74838692952180 & 2.54079715015129 \\
\hline $\mathbf{C u}$ & 3.56728017247255 & 30.26801487703270 & 2.54193258768957 \\
\hline $\mathbf{C u}$ & 3.56727470356621 & 32.79001465351790 & 2.54251757084898 \\
\hline $\mathbf{C u}$ & 7.13267205423864 & 35.31384993692380 & 2.53941880499280 \\
\hline $\mathbf{C u}$ & 7.13733965410817 & 2.52386082232662 & 2.54084675330702 \\
\hline $\mathbf{C u}$ & 7.13141432575860 & 5.04528305173173 & 2.54197676117887 \\
\hline $\mathbf{C u}$ & 7.13576377751228 & 7.56524862436290 & 2.54318246392509 \\
\hline $\mathbf{C u}$ & 7.13634230140858 & 10.09065984839540 & 2.54040094154820 \\
\hline $\mathbf{C u}$ & 7.13557818763686 & 12.61264053960760 & 2.54838011511923 \\
\hline $\mathbf{C u}$ & 7.14043014120146 & 15.13665500918790 & 2.53680545318261 \\
\hline $\mathbf{C u}$ & 7.13528288147913 & 17.65793976487620 & 2.54291402095540 \\
\hline $\mathbf{C u}$ & 7.13824045592125 & 20.17959644146560 & 2.53756307484279 \\
\hline $\mathbf{C u}$ & 7.13871308813262 & 22.70315659287670 & 2.54440594846972 \\
\hline $\mathbf{C u}$ & 7.13652052948675 & 25.22821127471000 & 2.53916062010850 \\
\hline $\mathbf{C u}$ & 7.13507757205675 & 27.74781580797810 & 2.54379102296780 \\
\hline $\mathbf{C u}$ & 7.13366184468479 & 30.26870036312310 & 2.54073821996141 \\
\hline $\mathbf{C u}$ & 7.13499615537558 & 32.78998244671150 & 2.54071934485652 \\
\hline $\mathbf{C u}$ & 1.78417055432284 & 1.25971856535328 & 3.85240929525346 \\
\hline $\mathbf{C u}$ & 1.77831361593491 & 3.77971489947733 & 3.85555550913449 \\
\hline $\mathbf{C u}$ & 1.79651936649232 & 6.30487148150680 & 3.85848386162327 \\
\hline $\mathbf{C u}$ & 1.78200981044626 & 8.82843198349774 & 3.83120013214334 \\
\hline $\mathbf{C u}$ & 1.79960766484057 & 11.35028344052800 & 3.85255610861797 \\
\hline $\mathbf{C u}$ & 1.77490622087913 & 13.88066165309820 & 3.84256274848596 \\
\hline $\mathbf{C u}$ & 1.78179201597392 & 16.39725687987470 & 3.8466787149 \\
\hline
\end{tabular}




\begin{tabular}{|c|c|c|c|}
\hline $\mathbf{C u}$ & 1.78187502432960 & 18.92808877994730 & 3.84903530939261 \\
\hline $\mathbf{C u}$ & 1.77727331880397 & 21.44336230726050 & 3.84372787428668 \\
\hline $\mathbf{C u}$ & 1.78446131289157 & 23.96883957122890 & 3.84732043475873 \\
\hline $\mathbf{C u}$ & 1.78138824301116 & 26.48863258773260 & 3.85507070697030 \\
\hline $\mathbf{C u}$ & 1.78488387594156 & 29.00899370764530 & 3.85651406335756 \\
\hline $\mathbf{C u}$ & 1.78347790662511 & 31.52924356538030 & 3.85523145988589 \\
\hline $\mathbf{C u}$ & 1.78469579116092 & 34.04914691664230 & 3.85301033939485 \\
\hline $\mathbf{C u}$ & 5.35038374826892 & 1.25970800024303 & 3.85241168123093 \\
\hline $\mathbf{C u}$ & 5.35621984304633 & 3.77971753946024 & 3.85562240797957 \\
\hline $\mathbf{C u}$ & 5.33800295271880 & 6.30486921617008 & 3.85848092553288 \\
\hline $\mathbf{C u}$ & 5.35252863412352 & 8.82841571371667 & 3.83119253458960 \\
\hline $\mathbf{C u}$ & 5.33493008545924 & 11.35025910925590 & 3.85247879656754 \\
\hline $\mathbf{C u}$ & 5.35962898879876 & 13.88065743282370 & 3.84253263716030 \\
\hline $\mathrm{Cu}$ & 5.35273545232843 & 16.39728391931820 & 3.84659971433484 \\
\hline $\mathbf{C u}$ & 5.35265116031717 & 18.92809438692420 & 3.84905731485724 \\
\hline $\mathbf{C u}$ & 5.35723757205692 & 21.44335276421280 & 3.84374079728889 \\
\hline $\mathbf{C u}$ & 5.35008578502617 & 23.96878958361650 & 3.84736467903133 \\
\hline $\mathbf{C u}$ & 5.35316382570988 & 26.48859449589050 & 3.85509916242451 \\
\hline $\mathbf{C u}$ & 5.34968978647198 & 29.00895539998090 & 3.85651112534159 \\
\hline $\mathbf{C u}$ & 5.35109385114888 & 31.52924674818910 & 3.85521335768762 \\
\hline $\mathbf{C u}$ & 5.34984343976631 & 34.04913821326990 & 3.85300760288197 \\
\hline $\mathbf{C u}$ & 8.91816589313567 & 1.26011061316099 & 3.85034559535564 \\
\hline $\mathbf{C u}$ & 8.91819870184561 & 30683199142 & 5055276 \\
\hline $\mathbf{C u}$ & 66647 & 41868 & 178409914 \\
\hline $\mathbf{C u}$ & 060956505 & 15739 & 097987 \\
\hline $\mathrm{Cu}$ & 17118606 & 961980 & 3.85662702036933 \\
\hline $\mathbf{C u}$ & 8.91819860820812 & 13.87378107644500 & 3.85514489332102 \\
\hline $\mathbf{C u}$ & 8.91818606467760 & 16.40063172228480 & 3.85288529986643 \\
\hline $\mathrm{Cu}$ & 8.91818687093248 & 18.91972896157260 & 3.85084163348089 \\
\hline $\mathrm{Cu}$ & 8.91820316925795 & 21.44038926289730 & 3.85518884740415 \\
\hline $\mathrm{Cu}$ & 8.91820717901717 & 23.96345026486700 & 3.85024056016811 \\
\hline $\mathbf{C u}$ & 8.91819588710370 & 26.49056290744730 & 3.85625379180480 \\
\hline $\mathrm{Cu}$ & 8.91819126875127 & 29.00640563560830 & 3.84811847976040 \\
\hline $\mathbf{C u}$ & 8.91818382556734 & 31.53551283877010 & 3.85014023060249 \\
\hline $\mathbf{C u}$ & 8.91819248140553 & 34.05253097259420 & 3.85055748310916 \\
\hline $\mathbf{C u}$ & 0.00261963370886 & 35.31290791523260 & 5.05510336142610 \\
\hline $\mathbf{C u}$ & 0.00012709711447 & 2.52194438678492 & 5.05837009581108 \\
\hline $\mathbf{C u}$ & 0.00132212052282 & 5.04861193966963 & 5.05695414352333 \\
\hline $\mathbf{C u}$ & 0.00683512617588 & 7.56641072580561 & 5.05160358631797 \\
\hline $\mathbf{C u}$ & 0.00940082464389 & 10.09452087420970 & 5.04602691763320 \\
\hline $\mathbf{C u}$ & 0.01137628315163 & 12.61328939347360 & 5.05984903023537 \\
\hline $\mathbf{C u}$ & 0.00153987064563 & 15.14098369664730 & 5.05327101518153 \\
\hline $\mathbf{C u}$ & 10.70159030356950 & 17.66177446682750 & 5.05387606689138 \\
\hline $\mathbf{C u}$ & 0.00409593767034 & 20.18168717533200 & 5.05757215300907 \\
\hline $\mathbf{C u}$ & 0.00503888392482 & 22.70423212868620 & 5.05800894386059 \\
\hline $\mathbf{C u}$ & 0.00287555156519 & 25.22236541262950 & 5.05455314333393 \\
\hline $\mathbf{C u}$ & 0.00085875951094 & 27.74993462069480 & 5.06491305970895 \\
\hline $\mathbf{C u}$ & 10.69980930881420 & 30.26829644546350 & 5.05449264309162 \\
\hline $\mathbf{C u}$ & 0.00517003963820 & 32.79030671692230 & 5.05874111942616 \\
\hline $\mathbf{C u}$ & 3.56727110046165 & 35.30984469053860 & 5.05669839254593 \\
\hline $\mathbf{C u}$ & 3.56727050060381 & 2.51656091386084 & 5.05743471880178 \\
\hline $\mathbf{C u}$ & 3.56724915888151 & 5.02217702177562 & 5.04952208363363 \\
\hline $\mathbf{C u}$ & 3.56727309849805 & 7.52054521962827 & 5.15670894017159 \\
\hline $\mathbf{C u}$ & 3.56728406634531 & 10.11361615231980 & 5.15562950220339 \\
\hline
\end{tabular}




\begin{tabular}{|c|c|c|c|}
\hline $\mathbf{C u}$ & 3.56729411877744 & 12.63140663191720 & 5.13618723709722 \\
\hline $\mathbf{C u}$ & 3.56725003432999 & 15.15648470891390 & 5.10182001511042 \\
\hline $\mathbf{C u}$ & 3.56726344016630 & 17.66714124005030 & 5.14134800363021 \\
\hline $\mathbf{C u}$ & 3.56722646409844 & 20.17669420905440 & 5.10642700496760 \\
\hline $\mathbf{C u}$ & 3.56724120308468 & 22.71626798074330 & 5.13944611148777 \\
\hline $\mathbf{C u}$ & 3.56728425742685 & 25.24221497916410 & 5.06201563293013 \\
\hline $\mathbf{C u}$ & 3.56727957424737 & 27.75117853127650 & 5.06079719790055 \\
\hline $\mathbf{C u}$ & 3.56731110440418 & 30.27351440496430 & 5.06080846976697 \\
\hline $\mathbf{C u}$ & 3.56727186994331 & 32.79162863666930 & 5.05986850761983 \\
\hline $\mathbf{C u}$ & 7.13190644014097 & 35.31289293811960 & 5.05510474839463 \\
\hline $\mathbf{C u}$ & 7.13442451949245 & 2.52194569580024 & 5.05834548805002 \\
\hline $\mathbf{C u}$ & 7.13324855292562 & 5.04859305793243 & 5.05694883679635 \\
\hline $\mathbf{C u}$ & 7.12773827186285 & 7.56637901807874 & 5.05155692861561 \\
\hline $\mathbf{C u}$ & 7.12516281241854 & 10.09449187421350 & 5.04596219692858 \\
\hline $\mathbf{C u}$ & 7.12317772947776 & 12.61328057539610 & 981197898396 \\
\hline $\mathbf{C u}$ & 7.13294505013391 & 15.14099649260670 & 5.05326441058169 \\
\hline $\mathbf{C u}$ & 7.13478947942195 & 17.66178608159040 & 128916 \\
\hline $\mathrm{Cu}$ & 7.13044998688350 & 20.18167429937210 & 87796 \\
\hline $\mathbf{C u}$ & 7.12955484563826 & 22.70417205360510 & 5.05796 \\
\hline $\mathbf{C u}$ & 7.13170946812201 & 25.22233622778350 & 5.05455614309851 \\
\hline $\mathbf{C u}$ & 7.13370195625675 & 27.74990471902700 & 5.06491516413844 \\
\hline $\mathbf{C u}$ & 7.13656380981564 & 30.26831451907210 & 5.05450093574816 \\
\hline $\mathbf{C u}$ & 7.12938328353884 & 32.79030917176870 & 5.05874064639806 \\
\hline \multicolumn{4}{|c|}{ CI-NEB Image 17} \\
\hline Element & X-Coordinate (Å) & Y-Coordinate (Å) & Z-Coordinate (Å) \\
\hline $\mathbf{F}$ & 4.66320958463685 & 12.66180677365040 & 7.77276182945720 \\
\hline $\mathbf{F}$ & 2.47137339780001 & 12.66180328083430 & 7.77280662728702 \\
\hline $\mathbf{F}$ & 3.56728819308635 & 8.81718581081764 & 6.62956433104315 \\
\hline $\mathbf{F}$ & 4.63323815495645 & 17.85157859696610 & 7.79804568887239 \\
\hline $\mathbf{F}$ & 2.50096934647240 & 17.85148175453190 & 7.79784293959462 \\
\hline $\mathbf{F}$ & 3.56730186850640 & 14.52373931760400 & 7.29730165105185 \\
\hline $\mathbf{F}$ & 4.66444384617118 & 22.87680882163900 & 7.75652572887291 \\
\hline $\mathbf{F}$ & 2.47011404015061 & 22.87680735386270 & 7.75649675929504 \\
\hline $\mathbf{F}$ & 3.56728477271579 & 20.99161946877670 & 7.40742983756497 \\
\hline C & 3.56727746612735 & 13.08713330667850 & 7.06578300691644 \\
\hline $\mathrm{C}$ & 3.56718248296724 & 17.79048066951270 & 7.00815613497695 \\
\hline C & 3.56728702213846 & 22.38896655488820 & 7.08064621903423 \\
\hline $\mathbf{C u}$ & 0.00000000000000 & 0.00000000000000 & 0.00000000000000 \\
\hline $\mathbf{C u}$ & 0.00000000000000 & 2.52244512012115 & 0.00000000000000 \\
\hline $\mathbf{C u}$ & 0.00000000000000 & 5.04488953395804 & 0.00000000000000 \\
\hline $\mathbf{C u}$ & 0.00000000000000 & 7.56733465407864 & 0.00000000000000 \\
\hline $\mathbf{C u}$ & 0.00000000000000 & 10.08977871477400 & 0.00000000000000 \\
\hline $\mathbf{C u}$ & 0.00000000000000 & 12.61222383489520 & 0.00000000000000 \\
\hline $\mathbf{C u}$ & 0.00000000000000 & 15.13466895501450 & 0.00000000000000 \\
\hline $\mathbf{C u}$ & 0.00000000000000 & 17.65711336885320 & 0.00000000000000 \\
\hline $\mathbf{C u}$ & 0.00000000000000 & 20.17955848897460 & 0.00000000000000 \\
\hline $\mathbf{C u}$ & 0.00000000000000 & 22.70200254966930 & 0.00000000000000 \\
\hline $\mathbf{C u}$ & 0.00000000000000 & 25.22444766979040 & 0.00000000000000 \\
\hline $\mathbf{C u}$ & 0.00000000000000 & 27.74689208362360 & 0.00000000000000 \\
\hline $\mathbf{C u}$ & 0.00000000000000 & 30.26933720374470 & 0.00000000000000 \\
\hline $\mathbf{C u}$ & 0.00000000000000 & 32.79178232386990 & 0.00000000000000 \\
\hline $\mathbf{C u}$ & 3.56727419238642 & 0.00000000000000 & 0.00000000000000 \\
\hline $\mathbf{C u}$ & 3.56727419238642 & 2.52244512012115 & 0.00000000000000 \\
\hline $\mathbf{C u}$ & 3.56727419238642 & 5.04488953395804 & 0.00000000000000 \\
\hline
\end{tabular}




\begin{tabular}{|c|c|c|c|}
\hline $\mathrm{Cu}$ & 3.56727419238642 & 7.56733465407864 & 0.00000000000000 \\
\hline $\mathbf{C u}$ & 3.56727419238642 & 10.08977871477400 & 0.00000000000000 \\
\hline $\mathbf{C u}$ & 3.56727419238642 & 12.61222383489520 & 0.00000000000000 \\
\hline $\mathbf{C u}$ & 3.56727419238642 & 15.13466895501450 & 0.00000000000000 \\
\hline $\mathbf{C u}$ & 3.56727419238642 & 17.65711336885320 & 0.00000000000000 \\
\hline $\mathbf{C u}$ & 3.56727419238642 & 20.17955848897460 & 0.00000000000000 \\
\hline $\mathbf{C u}$ & 3.56727419238642 & 22.70200254966930 & 0.00000000000000 \\
\hline $\mathbf{C u}$ & 3.56727419238642 & 25.22444766979040 & 0.00000000000000 \\
\hline $\mathbf{C u}$ & 3.56727419238642 & 27.74689208362360 & 0.00000000000000 \\
\hline $\mathbf{C u}$ & 3.56727419238642 & 30.26933720374470 & 0.00000000000000 \\
\hline $\mathbf{C u}$ & 3.56727419238642 & 32.79178232386990 & 0.00000000000000 \\
\hline $\mathbf{C u}$ & 7.13454838477291 & 0.00000000000000 & 0.00000000000000 \\
\hline $\mathrm{Cu}$ & 7.13454838477291 & 2.52244512012115 & 0.00000000000000 \\
\hline $\mathrm{Cu}$ & 7.13454838477291 & 5.04488953395804 & 0.00000000000000 \\
\hline $\mathrm{Cu}$ & 7.13454838477291 & 7.56733465407864 & 0.00000000000000 \\
\hline $\mathbf{C u}$ & 7.13454838477291 & 10.08977871477400 & 0.00000000000000 \\
\hline $\mathrm{Cu}$ & 7.13454838477291 & 12.61222383489520 & 0.00000000000000 \\
\hline $\mathbf{C u}$ & 7.13454838477291 & 15.13466895501450 & 0.00000000000000 \\
\hline $\mathbf{C u}$ & 7.13454838477291 & 17.65711336885320 & 0.00000000000000 \\
\hline $\mathbf{C u}$ & 7.13454838477291 & 20.17955848897460 & 0.00000000000000 \\
\hline $\mathbf{C u}$ & 7.13454838477291 & 22.70200254966930 & 0.00000000000000 \\
\hline $\mathbf{C u}$ & 7.13454838477291 & 25.22444766979040 & 0.00000000000000 \\
\hline $\mathbf{C u}$ & 7.13454838477291 & 27.74689208362360 & 0.00000000000000 \\
\hline $\mathrm{Cu}$ & 7.13454838477291 & 30.26933720374470 & 0.00000000000000 \\
\hline $\mathbf{C u}$ & 7.13454838477291 & 32.79178232386990 & 0.00000000000000 \\
\hline $\mathbf{C u}$ & 1.78363955761358 & 1.26122414920059 & 1.26122399999993 \\
\hline $\mathbf{C u}$ & 1.78363955761358 & 3.78366926932172 & 1.26122399999993 \\
\hline $\mathbf{C u}$ & 1.78363955761358 & 6.30611333001662 & 1.26122399999993 \\
\hline $\mathbf{C u}$ & 1.78363955761358 & 8.82855845013778 & 1.26122399999993 \\
\hline $\mathbf{C u}$ & 1.78363955761358 & 11.35100286397460 & 1.26122399999993 \\
\hline $\mathbf{C u}$ & 1.78363955761358 & 13.87344798409370 & 1.26122399999993 \\
\hline $\mathbf{C u}$ & 1.78363955761358 & 16.39589310421720 & 1.26122399999993 \\
\hline $\mathbf{C u}$ & 1.78363955761358 & 18.91833716491180 & 1.26122399999993 \\
\hline $\mathbf{C u}$ & 1.78363955761358 & 21.44078263817520 & 1.26122399999993 \\
\hline $\mathbf{C u}$ & 1.78363955761358 & 23.96322669886980 & 1.26122399999993 \\
\hline $\mathbf{C u}$ & 1.78363955761358 & 26.48567181899090 & 1.26122399999993 \\
\hline $\mathbf{C u}$ & 1.78363955761358 & 29.00811693910870 & 1.26122399999993 \\
\hline $\mathbf{C u}$ & 1.78363955761358 & 31.53056135294510 & 1.26122399999993 \\
\hline $\mathbf{C u}$ & 1.78363955761358 & 34.05300647307050 & 1.26122399999993 \\
\hline $\mathbf{C u}$ & 5.35091375000000 & 1.26122414920059 & 1.26122399999993 \\
\hline $\mathbf{C u}$ & 5.35091375000000 & 3.78366926932172 & 1.26122399999993 \\
\hline $\mathbf{C u}$ & 5.35091375000000 & 6.30611333001662 & 1.26122399999993 \\
\hline $\mathbf{C u}$ & 5.35091375000000 & 8.82855845013778 & 1.26122399999993 \\
\hline $\mathbf{C u}$ & 5.35091375000000 & 11.35100286397460 & 1.26122399999993 \\
\hline $\mathbf{C u}$ & 5.35091375000000 & 13.87344798409370 & 1.26122399999993 \\
\hline $\mathbf{C u}$ & 5.35091375000000 & 16.39589310421720 & 1.26122399999993 \\
\hline $\mathbf{C u}$ & 5.35091375000000 & 18.91833716491180 & 1.26122399999993 \\
\hline $\mathbf{C u}$ & 5.35091375000000 & 21.44078263817520 & 1.26122399999993 \\
\hline $\mathrm{Cu}$ & 5.35091375000000 & 23.96322669886980 & 1.26122399999993 \\
\hline $\mathbf{C u}$ & 5.35091375000000 & 26.48567181899090 & 1.26122399999993 \\
\hline $\mathbf{C u}$ & 5.35091375000000 & 29.00811693910870 & 1.26122399999993 \\
\hline $\mathbf{C u}$ & 5.35091375000000 & 31.53056135294510 & 1.26122399999993 \\
\hline $\mathbf{C u}$ & 5.35091375000000 & 34.05300647307050 & 1.26122399999993 \\
\hline $\mathbf{C u}$ & 8.91818794238642 & 1.26122414920059 & 1.26122399999993 \\
\hline
\end{tabular}




\begin{tabular}{|c|c|c|c|}
\hline $\mathrm{Cu}$ & 8.91818794238642 & 3.78366926932172 & 1.26122399999993 \\
\hline $\mathbf{C u}$ & 8.91818794238642 & 6.30611333001662 & 1.26122399999993 \\
\hline $\mathbf{C u}$ & 8.91818794238642 & 8.82855845013778 & 1.26122399999993 \\
\hline $\mathbf{C u}$ & 8.91818794238642 & 11.35100286397460 & 1.26122399999993 \\
\hline $\mathbf{C u}$ & 8.91818794238642 & 13.87344798409370 & 1.26122399999993 \\
\hline $\mathbf{C u}$ & 8.91818794238642 & 16.39589310421720 & 1.26122399999993 \\
\hline $\mathbf{C u}$ & 8.91818794238642 & 18.91833716491180 & 1.26122399999993 \\
\hline $\mathbf{C u}$ & 8.91818794238642 & 21.44078263817520 & 1.26122399999993 \\
\hline $\mathbf{C u}$ & 8.91818794238642 & 23.96322669886980 & 1.26122399999993 \\
\hline $\mathbf{C u}$ & 8.91818794238642 & 26.48567181899090 & 1.26122399999993 \\
\hline $\mathbf{C u}$ & 8.91818794238642 & 29.00811693910870 & 1.26122399999993 \\
\hline $\mathbf{C u}$ & 8.91818794238642 & 31.53056135294510 & 1.26122399999993 \\
\hline $\mathbf{C u}$ & 8.91818794238642 & 34.05300647307050 & 1.26122399999993 \\
\hline $\mathrm{Cu}$ & 0.00206658588730 & 35.31371143333460 & 2.53963476925794 \\
\hline $\mathbf{C u}$ & 10.69917462679010 & 2.52377448409921 & 2.54074881054623 \\
\hline $\mathbf{C u}$ & 0.00307940723844 & 5.04530929997921 & 2.54187705083059 \\
\hline $\mathbf{C u}$ & 10.70076263212960 & 7.56526480843403 & 2.54314851644879 \\
\hline $\mathbf{C u}$ & 10.70057721616270 & 10.09084627246910 & 2.54073985964959 \\
\hline $\mathbf{C u}$ & 10.70099372726820 & 12.61206581857290 & 2.54842181111000 \\
\hline $\mathbf{C u}$ & 10.69596332478440 & 15.13598317799150 & 2.53675998849419 \\
\hline $\mathbf{C u}$ & 10.70076484698660 & 17.65737634937800 & 2.54292709951966 \\
\hline $\mathbf{C u}$ & 10.69796160119620 & 20.17896578782870 & 2.53760171919491 \\
\hline $\mathbf{C u}$ & 10.69775651994550 & 22.7022612 & 2.54500244907528 \\
\hline $\mathbf{C u}$ & 7249190 & 25.22736402280730 & 2.53879974391995 \\
\hline $\mathrm{Cu}$ & 10.70098 & 27.7468 & 2768493 \\
\hline $\mathrm{Cu}$ & 0.00072201947325 & 30.2683568 & 885561641 \\
\hline $\mathbf{C u}$ & 10.70149829702800 & 32.78981742389090 & 2.54071586903714 \\
\hline $\mathbf{C u}$ & 3.56727219344170 & 0.00044834919132 & 2.54005037122335 \\
\hline $\mathbf{C u}$ & 3.56727399437662 & 2.52300129170189 & 2.54341813839030 \\
\hline $\mathrm{Cu}$ & 3.56725689944628 & 5.04482461636141 & 2.54053024804349 \\
\hline $\mathrm{Cu}$ & 3.56722038234292 & 7.57073945616226 & 2.57936577886297 \\
\hline $\mathbf{C u}$ & 3.56728209403745 & 10.09345182540110 & 2.58760379615353 \\
\hline $\mathrm{Cu}$ & 3.56730225930168 & 12.62427642873070 & 2.58270072200743 \\
\hline $\mathbf{C u}$ & 3.56726152077887 & 15.13933327915730 & 2.58314087768149 \\
\hline $\mathbf{C u}$ & 3.56725178093735 & 17.65813729735100 & 2.59010370386710 \\
\hline $\mathbf{C u}$ & 3.56726946781492 & 20.17999512029220 & 2.58386100491515 \\
\hline $\mathbf{C u}$ & 3.56727786572994 & 22.69476020872210 & 2.58366058014717 \\
\hline $\mathbf{C u}$ & 3.56728677686532 & 25.21677109248380 & 2.55219591825067 \\
\hline $\mathbf{C u}$ & 3.56729580850503 & 27.74754110618780 & 2.54000995233093 \\
\hline $\mathbf{C u}$ & 3.56729245130004 & 30.26684910489020 & 2.54208517675113 \\
\hline $\mathbf{C u}$ & 3.56727783725000 & 32.78937505574430 & 2.54251312875541 \\
\hline $\mathbf{C u}$ & 7.13248586938729 & 35.31371871109800 & 2.53964364390858 \\
\hline $\mathbf{C u}$ & 7.13722889819528 & 2.52371074282832 & 2.54079461836107 \\
\hline $\mathbf{C u}$ & 7.13155033260134 & 5.04530843121903 & 2.54193453714464 \\
\hline $\mathbf{C u}$ & 7.13562826979597 & 7.56529615481844 & 2.54306005828043 \\
\hline $\mathbf{C u}$ & 7.13578630394391 & 10.09080781417020 & 2.54077905434776 \\
\hline $\mathbf{C u}$ & 7.13534246682914 & 12.61210660214710 & 2.54839447420003 \\
\hline $\mathbf{C u}$ & 7.14036313899845 & 15.13598132566440 & 2.53675741609160 \\
\hline $\mathbf{C u}$ & 7.13559781280368 & 17.65735477040000 & 2.54292547302492 \\
\hline $\mathbf{C u}$ & 7.13839457142924 & 20.17898711302250 & 2.53758230668137 \\
\hline $\mathbf{C u}$ & 7.13867058204008 & 22.70227822081230 & 2.54495606185650 \\
\hline $\mathbf{C u}$ & 7.13673952590835 & 25.22735344765670 & 2.53881757968619 \\
\hline $\mathbf{C u}$ & 7.13543624195218 & 27.74682888830570 & 2.54397616235762 \\
\hline $\mathbf{C u}$ & 7.13380171498139 & 30.26831621825700 & 2.54074179797966 \\
\hline
\end{tabular}




\begin{tabular}{|c|c|c|c|}
\hline $\mathbf{C u}$ & 7.13487703580960 & 32.78981311405890 & 2.54071293154517 \\
\hline $\mathbf{C u}$ & 1.78445121392338 & 1.25883608922411 & 3.85232235811835 \\
\hline $\mathbf{C u}$ & 1.77842120688011 & 3.77960618240770 & 3.85546630827996 \\
\hline $\mathbf{C u}$ & 1.79662043639309 & 6.30467747290366 & 3.85841740871822 \\
\hline $\mathbf{C u}$ & 1.78242867894328 & 8.82865449403888 & 3.83135161451698 \\
\hline $\mathbf{C u}$ & 1.80091211357017 & 11.35079677732160 & 3.85417474229028 \\
\hline $\mathbf{C u}$ & 1.77466028078503 & 13.88019332478970 & 3.84231520784989 \\
\hline $\mathbf{C u}$ & 1.78197197016830 & 16.39746358173530 & 3.84670676620108 \\
\hline $\mathbf{C u}$ & 1.78082264397962 & 18.92685548811900 & 3.84810225246519 \\
\hline $\mathbf{C u}$ & 1.77829297202074 & 21.44016404216230 & 3.84424357399202 \\
\hline $\mathbf{C u}$ & 1.78425868419209 & 23.96646740993030 & 3.84605064558742 \\
\hline $\mathbf{C u}$ & 1.78163420735270 & 26.48594195815350 & 3.85390326178416 \\
\hline $\mathbf{C u}$ & 1.78434665407688 & 29.00652448330820 & 3.85609892959824 \\
\hline $\mathbf{C u}$ & 1.78319860444357 & 31.52710997556200 & 3.85551508293505 \\
\hline $\mathbf{C u}$ & 1.78497173729630 & 34.04754173796740 & 3.85282935249555 \\
\hline $\mathbf{C u}$ & 5.35011338998027 & 1.25881810355383 & 3.85236008321640 \\
\hline $\mathbf{C u}$ & 5.35611389341817 & 3.77962870198661 & 3.85539171814738 \\
\hline $\mathbf{C u}$ & 5.33791279498733 & 6.30463292419600 & 3.85840335291679 \\
\hline $\mathbf{C u}$ & 5.35211043151661 & 8.82862801938766 & 3.83135868829993 \\
\hline $\mathbf{C u}$ & 5.33354221025846 & 11.35084525784000 & 3.85416340093572 \\
\hline $\mathbf{C u}$ & 5.35987458712534 & 13.88019399349320 & 3.84230813738754 \\
\hline $\mathbf{C u}$ & 5.35249969561269 & 16.39745004246600 & 3.84665807832527 \\
\hline $\mathbf{C u}$ & 5.35371241881159 & 18.92678402237100 & 1514630 \\
\hline $\mathrm{Cu}$ & 5.35623676400337 & 21.440119 & 657013 \\
\hline $\mathrm{Cu}$ & 5.35033435182103 & 23.9664238 & 09914887 \\
\hline $\mathrm{Cu}$ & 5.35297951642996 & 26.48591069213450 & 968500214 \\
\hline $\mathbf{C u}$ & 5.35024501617872 & 29.00643679018500 & 3.85612765824831 \\
\hline $\mathbf{C u}$ & 5.35135342303721 & 31.52707670444700 & 3.85553176873607 \\
\hline $\mathbf{C u}$ & 5.34957507659019 & 34.04749230207330 & 3.85282494635383 \\
\hline $\mathbf{C u}$ & 8.91819263754657 & 1.25896858474367 & 3.85010590273927 \\
\hline $\mathbf{C u}$ & 8.91819006038147 & 3.78294270817859 & 3.84914124036460 \\
\hline $\mathbf{C u}$ & 8.91821968209736 & 6.30421415355269 & 3.85104887872507 \\
\hline $\mathrm{Cu}$ & 8.91820597869973 & 8.82706119348494 & 3.85748176410369 \\
\hline $\mathrm{Cu}$ & 8.91816767819810 & 11.35593289646550 & 3.85669028657126 \\
\hline $\mathrm{Cu}$ & 8.91819874623263 & 13.87305540820020 & 3.85498545636574 \\
\hline $\mathbf{C u}$ & 8.91811459399334 & 16.39914451161560 & 3.85266445670830 \\
\hline $\mathbf{C u}$ & 8.91819364348617 & 18.91731979700360 & 3.85051160056210 \\
\hline $\mathbf{C u}$ & 8.91821591248501 & 21.43753504578640 & 3.85520505728166 \\
\hline $\mathbf{C u}$ & 8.91821082151613 & 23.96136108702470 & 3.85081837950709 \\
\hline $\mathbf{C u}$ & 8.91822654148987 & 26.48787732032380 & 3.85738913245284 \\
\hline $\mathbf{C u}$ & 8.91819390514225 & 29.00347459658230 & 3.84854896532824 \\
\hline $\mathbf{C u}$ & 8.91819589721714 & 31.53344085390360 & 3.84926912354532 \\
\hline $\mathbf{C u}$ & 8.91815920468008 & 34.05128634894480 & 3.85040063740246 \\
\hline $\mathbf{C u}$ & 0.00304046819765 & 35.31156323715500 & 5.05466587121203 \\
\hline $\mathbf{C u}$ & 0.00038666527627 & 2.52053841572105 & 5.05796971527756 \\
\hline $\mathbf{C u}$ & 0.00123297279633 & 5.04772678275516 & 5.05667742775081 \\
\hline $\mathbf{C u}$ & 0.00725952600788 & 7.56617327061435 & 5.05124696514118 \\
\hline $\mathbf{C u}$ & 0.00990020219862 & 10.09434203142180 & 5.04600023978191 \\
\hline $\mathrm{Cu}$ & 0.01145798283297 & 12.61294259239880 & 5.06007762369101 \\
\hline $\mathrm{Cu}$ & 0.00169753403008 & 15.13980880087100 & 5.05376857183492 \\
\hline $\mathbf{C u}$ & 10.70117894580650 & 17.65949051327860 & 5.05488604661922 \\
\hline $\mathbf{C u}$ & 0.00418893620949 & 20.17894119065010 & 5.05787991088453 \\
\hline $\mathbf{C u}$ & 0.00580325463900 & 22.70176197007670 & 5.05786247116615 \\
\hline $\mathbf{C u}$ & 0.00363609883007 & 25.21991161503140 & 5.05355532039631 \\
\hline
\end{tabular}




\begin{tabular}{|c|c|c|c|}
\hline $\mathbf{C u}$ & 0.00158495948841 & 27.74718770442740 & 5.06500942512828 \\
\hline $\mathbf{C u}$ & 10.69939729548310 & 30.26617418756920 & 5.05472480389231 \\
\hline $\mathbf{C u}$ & 0.00430986885630 & 32.78895406933700 & 5.05831117264570 \\
\hline $\mathbf{C u}$ & 3.56729339750554 & 35.30801332921560 & 5.05703528859458 \\
\hline $\mathbf{C u}$ & 3.56736500537107 & 2.51594958435308 & 5.05721537300277 \\
\hline $\mathbf{C u}$ & 3.56729667428985 & 5.02195706540047 & 5.04919075184742 \\
\hline $\mathbf{C u}$ & 3.56731732115290 & 7.52054315334907 & 5.15665920216627 \\
\hline $\mathbf{C u}$ & 3.56734369464991 & 10.11538161338530 & 5.15711425149101 \\
\hline $\mathbf{C u}$ & 3.56727794516267 & 12.63387021388690 & 5.14136014622739 \\
\hline $\mathbf{C u}$ & 3.56731753039047 & 15.16088358210570 & 5.09646371547790 \\
\hline $\mathbf{C u}$ & 3.56725911184794 & 17.66958256820200 & 5.14257805740365 \\
\hline $\mathbf{C u}$ & 3.56727475778253 & 20.17663498619600 & 5.11137522246433 \\
\hline $\mathbf{C u}$ & 3.56732696950020 & 22.71411135362720 & 5.13530443082830 \\
\hline $\mathbf{C u}$ & 3.56730109506303 & 25.24038181909620 & 5.06241924005204 \\
\hline $\mathbf{C u}$ & 3.56727564344225 & 27.74817648917200 & 5.05935065667964 \\
\hline $\mathbf{C u}$ & 3.56730428266163 & 30.26946985275130 & 5.06077003047994 \\
\hline $\mathbf{C u}$ & 3.56731057957439 & 32.78888945226230 & 2081602 \\
\hline $\mathrm{Cu}$ & 7.13144163907966 & 35.31157244940570 & 5460241 \\
\hline $\mathbf{C u}$ & 7.13411889618165 & 2.52052490680236 & 5.05796969570523 \\
\hline $\mathbf{C u}$ & 7.13331725876629 & 5.04773881492377 & 5.05669 \\
\hline $\mathbf{C u}$ & 7.12733568551908 & 7.56609226578344 & 5.051146 \\
\hline $\mathbf{C u}$ & 7.12462077700697 & 10.09435394698040 & 5.04593422206352 \\
\hline $\mathbf{C u}$ & 7.12310740447627 & 12.61299036665550 & 5.06006287723438 \\
\hline $\mathbf{C u}$ & 7.13284551401808 & 15.13976806634150 & 5.05370304542514 \\
\hline $\mathbf{C u}$ & 7.13514992382937 & 17.65938233739120 & 5.05489160888551 \\
\hline $\mathbf{C u}$ & 7.13029333148108 & 20.17887360802060 & 5.05789400313984 \\
\hline $\mathbf{C u}$ & 7.12877362162456 & 22.70162367268130 & 5.05771814178240 \\
\hline $\mathbf{C u}$ & 7.13097956302177 & 25.21978114185170 & 5.05352629585848 \\
\hline $\mathbf{C u}$ & 7.13301128454185 & 27.74709395521490 & 5.06501793210512 \\
\hline $\mathbf{C u}$ & 7.13697900824285 & 30.26610231060290 & 5.05475565310535 \\
\hline $\mathbf{C u}$ & 7.13026786080795 & 32.78890324241350 & 5.05831582971833 \\
\hline \multicolumn{4}{|c|}{ CI-NEB Image 18} \\
\hline Element & X-Coordin & Y-Coordinate (§̊) & Z-Coordin \\
\hline $\mathbf{F}$ & 4.66335836995430 & 12.64440651378800 & 7.76738070276761 \\
\hline $\mathbf{F}$ & 2.47124344329227 & 12.64435973486040 & 7.76736358964744 \\
\hline $\mathbf{F}$ & 3.56738568628991 & 8.81612867414812 & 6.63064608119547 \\
\hline $\mathbf{F}$ & 4.63519373413578 & 17.82665525735000 & 7.79557851219587 \\
\hline $\mathbf{F}$ & 2.50366387647437 & 17.82608749686380 & 7.79750438669476 \\
\hline $\mathbf{F}$ & 3.56726706286093 & 14.50926157331650 & 7.30390027741588 \\
\hline $\mathbf{F}$ & 4.66380394808486 & 22.74038026451390 & 7.78325129612739 \\
\hline $\mathbf{F}$ & 2.47117160484707 & 22.74072594914930 & 7.78328269801284 \\
\hline $\mathbf{F}$ & 3.56721338583549 & 20.87336128623520 & 7.34091271925722 \\
\hline C & 3.56729839987867 & 13.07501447665580 & 7.06349776312376 \\
\hline C & 3.56868854936949 & 17.76709126086500 & 7.00694127772371 \\
\hline C & 3.56741000603083 & 22.29531841086990 & 7.08132677348790 \\
\hline $\mathbf{C u}$ & 0.00000000000000 & 0.00000000000000 & 0.00000000000000 \\
\hline $\mathbf{C u}$ & 0.00000000000000 & 2.52244512012115 & 0.00000000000000 \\
\hline $\mathbf{C u}$ & 0.00000000000000 & 5.04488953395804 & 0.00000000000000 \\
\hline $\mathbf{C u}$ & 0.00000000000000 & 7.56733465407861 & 0.00000000000000 \\
\hline $\mathbf{C u}$ & 0.00000000000000 & 10.08977871477400 & 0.00000000000000 \\
\hline $\mathbf{C u}$ & 0.00000000000000 & 12.61222383489520 & 0.00000000000000 \\
\hline $\mathbf{C u}$ & 0.00000000000000 & 15.13466895501420 & 0.00000000000000 \\
\hline $\mathbf{C u}$ & 0.00000000000000 & 17.65711336885320 & 0.00000000000000 \\
\hline $\mathbf{C u}$ & 0.00000000000000 & 20.17955848897460 & 0.00000000000000 \\
\hline
\end{tabular}




\begin{tabular}{|c|c|c|c|}
\hline $\mathbf{C u}$ & 0.00000000000000 & 22.70200254966930 & 0.00000000000000 \\
\hline $\mathbf{C u}$ & 0.00000000000000 & 25.22444766979040 & 0.00000000000000 \\
\hline $\mathbf{C u}$ & 0.00000000000000 & 27.74689208362300 & 0.00000000000000 \\
\hline $\mathbf{C u}$ & 0.00000000000000 & 30.26933720374410 & 0.00000000000000 \\
\hline $\mathbf{C u}$ & 0.00000000000000 & 32.79178232386990 & 0.00000000000000 \\
\hline $\mathbf{C u}$ & 3.56727419238642 & 0.00000000000000 & 0.00000000000000 \\
\hline $\mathbf{C u}$ & 3.56727419238642 & 2.52244512012115 & 0.00000000000000 \\
\hline $\mathbf{C u}$ & 3.56727419238642 & 5.04488953395804 & 0.00000000000000 \\
\hline $\mathbf{C u}$ & 3.56727419238642 & 7.56733465407861 & 0.00000000000000 \\
\hline $\mathbf{C u}$ & 3.56727419238642 & 10.08977871477400 & 0.00000000000000 \\
\hline $\mathbf{C u}$ & 3.56727419238642 & 12.61222383489520 & 0.00000000000000 \\
\hline $\mathbf{C u}$ & 3.56727419238642 & 15.13466895501420 & 0.00000000000000 \\
\hline $\mathrm{Cu}$ & 3.56727419238642 & 17.65711336885320 & 0.00000000000000 \\
\hline $\mathrm{Cu}$ & 3.56727419238642 & 20.17955848897460 & 0.00000000000000 \\
\hline $\mathbf{C u}$ & 3.56727419238642 & 22.70200254966930 & 0.00000000000000 \\
\hline $\mathbf{C u}$ & 3.56727419238642 & 25.22444766979040 & 0.00000000000000 \\
\hline $\mathbf{C u}$ & 3.56727419238642 & 27.74689208362300 & 0.00000000000000 \\
\hline $\mathbf{C u}$ & 3.56727419238642 & 30.26933720374410 & 0.00000000000000 \\
\hline $\mathbf{C u}$ & 3.56727419238642 & 32.79178232386990 & 0.00000000000000 \\
\hline $\mathbf{C u}$ & 7.13454838477291 & 0.00000000000000 & 0.00000000000000 \\
\hline $\mathbf{C u}$ & 7.13454838477291 & 2.52244512012115 & 0.00000000000000 \\
\hline $\mathbf{C u}$ & 7.13454838477291 & 5.04488953395804 & 0.00000000000000 \\
\hline $\mathbf{C u}$ & 7.13454838477291 & 7.56733465407861 & 0.00000000000000 \\
\hline $\mathbf{C u}$ & 7.13454838477291 & 10.08977871477400 & 0.00000000000000 \\
\hline $\mathbf{C u}$ & 3477291 & 3489520 & 0.00000000000000 \\
\hline $\mathbf{C u}$ & 38477291 & 66895501420 & 0.00000000000000 \\
\hline $\mathbf{C u}$ & 7.13454838477291 & 17.65711336885320 & 0.00000000000000 \\
\hline $\mathbf{C u}$ & 7.13454838477291 & 20.17955848897460 & 0.00000000000000 \\
\hline $\mathbf{C u}$ & 7.13454838477291 & 22.70200254966930 & 0.00000000000000 \\
\hline $\mathrm{Cu}$ & 7.13454838477291 & 25.22444766979040 & 0.00000000000000 \\
\hline $\mathrm{Cu}$ & 7.13454838477291 & 27.74689208362300 & 0.00000000000000 \\
\hline $\mathrm{Cu}$ & 7.13454838477291 & 30.26933720374410 & 0.00000000000000 \\
\hline $\mathrm{Cu}$ & 7.13454838477291 & 32.79178232386990 & 0.00000000000000 \\
\hline $\mathbf{C u}$ & 1.78363955761358 & 1.26122414920059 & 1.26122399999993 \\
\hline $\mathbf{C u}$ & 1.78363955761358 & 3.78366926932170 & 1.26122399999993 \\
\hline $\mathbf{C u}$ & 1.78363955761358 & 6.30611333001662 & 1.26122399999993 \\
\hline $\mathbf{C u}$ & 1.78363955761358 & 8.82855845013778 & 1.26122399999993 \\
\hline $\mathbf{C u}$ & 1.78363955761358 & 11.35100286397460 & 1.26122399999993 \\
\hline $\mathbf{C u}$ & 1.78363955761358 & 13.87344798409340 & 1.26122399999993 \\
\hline $\mathbf{C u}$ & 1.78363955761358 & 16.39589310421720 & 1.26122399999993 \\
\hline $\mathbf{C u}$ & 1.78363955761358 & 18.91833716491180 & 1.26122399999993 \\
\hline $\mathbf{C u}$ & 1.78363955761358 & 21.44078263817520 & 1.26122399999993 \\
\hline $\mathbf{C u}$ & 1.78363955761358 & 23.96322669886980 & 1.26122399999993 \\
\hline $\mathbf{C u}$ & 1.78363955761358 & 26.48567181899090 & 1.26122399999993 \\
\hline $\mathbf{C u}$ & 1.78363955761358 & 29.00811693910810 & 1.26122399999993 \\
\hline $\mathbf{C u}$ & 1.78363955761358 & 31.53056135294440 & 1.26122399999993 \\
\hline $\mathbf{C u}$ & 1.78363955761358 & 34.05300647307050 & 1.26122399999993 \\
\hline $\mathbf{C u}$ & 5.35091375000000 & 1.26122414920059 & 1.26122399999993 \\
\hline $\mathbf{C u}$ & 5.35091375000000 & 3.78366926932170 & 1.26122399999993 \\
\hline $\mathbf{C u}$ & 5.35091375000000 & 6.30611333001662 & 1.26122399999993 \\
\hline $\mathbf{C u}$ & 5.35091375000000 & 8.82855845013778 & 1.26122399999993 \\
\hline $\mathbf{C u}$ & 5.35091375000000 & 11.35100286397460 & 1.26122399999993 \\
\hline $\mathbf{C u}$ & 5.35091375000000 & 13.87344798409340 & 1.26122399999993 \\
\hline $\mathbf{C u}$ & 5.35091375000000 & 16.39589310421720 & 1.26122399999993 \\
\hline
\end{tabular}




\begin{tabular}{|c|c|c|c|}
\hline $\mathrm{Cu}$ & 5.35091375000000 & 18.91833716491180 & 1.26122399999993 \\
\hline $\mathrm{Cu}$ & 5.35091375000000 & 21.44078263817520 & 1.26122399999993 \\
\hline $\mathrm{Cu}$ & 5.35091375000000 & 23.96322669886980 & 1.26122399999993 \\
\hline $\mathbf{C u}$ & 5.35091375000000 & 26.48567181899090 & 1.26122399999993 \\
\hline $\mathbf{C u}$ & 5.35091375000000 & 29.00811693910810 & 1.26122399999993 \\
\hline $\mathbf{C u}$ & 5.35091375000000 & 31.53056135294440 & 1.26122399999993 \\
\hline $\mathrm{Cu}$ & 5.35091375000000 & 34.05300647307050 & 1.26122399999993 \\
\hline $\mathrm{Cu}$ & 8.91818794238642 & 1.26122414920059 & 1.26122399999993 \\
\hline $\mathbf{C u}$ & 8.91818794238642 & 3.78366926932170 & 1.26122399999993 \\
\hline $\mathbf{C u}$ & 8.91818794238642 & 6.30611333001662 & 1.26122399999993 \\
\hline $\mathbf{C u}$ & 8.91818794238642 & 8.82855845013778 & 1.26122399999993 \\
\hline $\mathrm{Cu}$ & 8.91818794238642 & 11.35100286397460 & 1.26122399999993 \\
\hline $\mathrm{Cu}$ & 8.91818794238642 & 13.87344798409340 & 1.26122399999993 \\
\hline $\mathrm{Cu}$ & 8.91818794238642 & 16.39589310421720 & 1.26122399999993 \\
\hline $\mathbf{C u}$ & 8.91818794238642 & 18.91833716491180 & 1.26122399999993 \\
\hline $\mathrm{Cu}$ & 8.91818794238642 & 21.44078263817520 & 1.26122399999993 \\
\hline $\mathrm{Cu}$ & 8.91818794238642 & 23.96322669886980 & 1.26122399999993 \\
\hline $\mathbf{C u}$ & 8.91818794238642 & 26.48567181899090 & 1.26122399999993 \\
\hline $\mathbf{C u}$ & 8.91818794238642 & 29.00811693910810 & 1.26122399999993 \\
\hline $\mathrm{Cu}$ & 8.91818794238642 & 31.53056135294440 & 1.26122399999993 \\
\hline $\mathrm{Cu}$ & 8.91818794238642 & 34.05300647307050 & 9999993 \\
\hline $\mathbf{C u}$ & 0.00185535452955 & 35.31353916971730 & 2.53960987058759 \\
\hline $\mathrm{Cu}$ & 10.69907417005900 & 2.52358696127568 & 2.5407 \\
\hline $\mathrm{Cu}$ & 0.00306477511894 & 5.04515311823859 & 2.54190582028233 \\
\hline $\mathrm{Cu}$ & 10.70066995058140 & 7.56512543127593 & 2.54306017836360 \\
\hline $\mathrm{Cu}$ & 10.70027611012500 & 10.09039346397930 & 2.54038239185976 \\
\hline $\mathbf{C u}$ & 10.70081625290100 & 12.61168228084290 & 2.54799237487907 \\
\hline $\mathrm{Cu}$ & 10.69565685742430 & 15.13567047239540 & 2.53657203332402 \\
\hline $\mathbf{C u}$ & 10.70045955556820 & 17.65677742671200 & 2.54282491942859 \\
\hline $\mathrm{Cu}$ & 10.69765194930000 & 20.17902735161820 & 2.53723328033071 \\
\hline $\mathrm{Cu}$ & 10.69825624430730 & 22.70252580790090 & 2.54489740249074 \\
\hline $\mathrm{Cu}$ & 10.70051331437560 & 25.22697873877590 & 2.53956113676895 \\
\hline $\mathrm{Cu}$ & 10.70136344183480 & 27.74675900220740 & 2.54403297135419 \\
\hline $\mathbf{C u}$ & 0.00091163962397 & 30.26839829975380 & 2.54082258954511 \\
\hline $\mathbf{C u}$ & 10.70152669470470 & 32.78972836566270 & 2.54069903331998 \\
\hline $\mathbf{C u}$ & 3.56728211692026 & 0.00040013739670 & 2.54018578009047 \\
\hline $\mathrm{Cu}$ & 3.56727104240497 & 2.52258468193003 & 2.54371219783601 \\
\hline $\mathbf{C u}$ & 3.56727717830313 & 5.04477757056896 & 2.54074023781071 \\
\hline $\mathrm{Cu}$ & 3.56728224046559 & 7.57008576637868 & 2.57964166183743 \\
\hline $\mathbf{C u}$ & 3.56727785235976 & 10.09249320458130 & 2.58769007057882 \\
\hline $\mathrm{Cu}$ & 3.56726215234860 & 12.62345198804640 & 2.58230913855698 \\
\hline $\mathbf{C u}$ & 3.56728238305852 & 15.13761380841460 & 2.58391743120574 \\
\hline $\mathbf{C u}$ & 3.56724635479412 & 17.65539950084850 & 2.59028050331159 \\
\hline $\mathbf{C u}$ & 3.56728143788086 & 20.17853939781600 & 2.58296285131726 \\
\hline $\mathbf{C u}$ & 3.56727651499383 & 22.69386140920520 & 2.58683652050424 \\
\hline $\mathrm{Cu}$ & 3.56727326285814 & 25.21673282309440 & 2.55241460674930 \\
\hline $\mathrm{Cu}$ & 3.56729435190278 & 27.74749263898320 & 2.54051260536544 \\
\hline $\mathrm{Cu}$ & 3.56728064677267 & 30.26749763478760 & 2.54216731906658 \\
\hline $\mathbf{C u}$ & 3.56727980320756 & 32.78951687199420 & 2.54262619179398 \\
\hline $\mathrm{Cu}$ & 7.13271649943453 & 35.31355414285450 & 2.53959555931619 \\
\hline $\mathbf{C u}$ & 7.13729824367638 & 2.52359670394992 & 2.54082453230418 \\
\hline $\mathbf{C u}$ & 7.13149647685599 & 5.04524640734115 & 2.54196841132533 \\
\hline $\mathbf{C u}$ & 7.13570315712905 & 7.56519393604302 & 2.54306823178992 \\
\hline $\mathbf{C u}$ & 7.13611815033819 & 10.09037316228120 & 2.54040384478544 \\
\hline
\end{tabular}




\begin{tabular}{|c|c|c|c|}
\hline $\mathrm{Cu}$ & 7.13554624017804 & 12.61168594598300 & 2.54799439020734 \\
\hline $\mathrm{Cu}$ & 7.14070353015978 & 15.13571073870560 & 2.53665487031151 \\
\hline $\mathrm{Cu}$ & 7.13591881499724 & 17.65689585529230 & 2.54294925673031 \\
\hline $\mathbf{C u}$ & 7.13878246080732 & 20.17906034445030 & 2.53727372669383 \\
\hline $\mathbf{C u}$ & 7.13814514576408 & 22.70246320661540 & 2.54488257552778 \\
\hline $\mathbf{C u}$ & 7.13586401866927 & 25.22702226882660 & 2.53953906190908 \\
\hline $\mathrm{Cu}$ & 7.13505423595203 & 27.74674763263320 & 2.54407249736691 \\
\hline $\mathrm{Cu}$ & 7.13364366594284 & 30.26841786203970 & 2.54081998887516 \\
\hline $\mathbf{C u}$ & 7.13488197072021 & 32.78972931396700 & 2.54068993460994 \\
\hline $\mathbf{C u}$ & 1.78411963066287 & 1.25916671130605 & 3.85270868512652 \\
\hline $\mathbf{C u}$ & 1.77841718824548 & 3.77951890436091 & 3.85551242684558 \\
\hline $\mathrm{Cu}$ & 1.79661767989991 & 6.30447123105214 & 3.85859293836103 \\
\hline $\mathrm{Cu}$ & 1.78214405462857 & 8.82804576745543 & 3.83126028342379 \\
\hline $\mathrm{Cu}$ & 1.80007874423935 & 11.34946513545210 & 3.85330998269293 \\
\hline $\mathbf{C u}$ & 1.77358263879153 & 13.87816665721690 & 3.84207540694693 \\
\hline $\mathrm{Cu}$ & 1.78101496992703 & 16.39457796201240 & 3.84654261600247 \\
\hline $\mathrm{Cu}$ & 1.77989404289580 & 18.92423978160060 & 3.84630764010008 \\
\hline $\mathbf{C u}$ & 1.77776367483158 & 21.43978954408690 & 3.84384394438242 \\
\hline $\mathbf{C u}$ & 1.78693034478150 & 23.96516469808010 & 3.84967323328861 \\
\hline $\mathrm{Cu}$ & 1.78225519915167 & 26.48595609824170 & 3.85494581868841 \\
\hline $\mathrm{Cu}$ & 1.78496278285510 & 29.00765902463650 & 3.85636 \\
\hline $\mathbf{C u}$ & 1.78344237225254 & 31.52845733356830 & 3.85539090704161 \\
\hline $\mathrm{Cu}$ & 1.78479336500835 & 34.04846748741000 & 3.85311210560234 \\
\hline $\mathrm{Cu}$ & 5.35047388474347 & 1.25916630475152 & 3.85269015909493 \\
\hline $\mathrm{Cu}$ & 5.35634810249545 & 3.77947945803765 & 3.85547842586359 \\
\hline $\mathrm{Cu}$ & 5.33799299052436 & 6.30446264427911 & 3.85852085984558 \\
\hline $\mathbf{C u}$ & 5.35238973362007 & 8.82806972629858 & 3.83125703179991 \\
\hline $\mathrm{Cu}$ & 5.33448865021658 & 11.34950957702980 & 3.85325128684276 \\
\hline $\mathbf{C u}$ & 5.36094424474321 & 13.87822289080250 & 3.84208591771181 \\
\hline $\mathrm{Cu}$ & 5.35346412261827 & 16.39466901414940 & 3.84665826055057 \\
\hline $\mathrm{Cu}$ & 5.35456453581167 & 18.92422591773160 & 3.84630540868155 \\
\hline $\mathrm{Cu}$ & 5.35672385637510 & 21.43982613497170 & 3.84388922501604 \\
\hline $\mathrm{Cu}$ & 5.34768659441440 & 23.96518509058030 & 3.84967125439121 \\
\hline $\mathbf{C u}$ & 5.35231383153093 & 26.48598123270820 & 3.85495461548184 \\
\hline $\mathbf{C u}$ & 5.34962495134946 & 29.00762537215230 & 3.85636889888376 \\
\hline $\mathbf{C u}$ & 5.35115292230192 & 31.52847913286440 & 3.85539953619980 \\
\hline $\mathrm{Cu}$ & 5.34975775212657 & 34.04845272003480 & 3.85310802565672 \\
\hline $\mathbf{C u}$ & 8.91818970534728 & 1.25944024104117 & 3.85009499942828 \\
\hline $\mathrm{Cu}$ & 8.91819578918184 & 3.78316894299333 & 3.84937930236732 \\
\hline $\mathbf{C u}$ & 8.91820461181769 & 6.30416576952197 & 3.85107831902508 \\
\hline $\mathrm{Cu}$ & 8.91824723045042 & 8.82676050476732 & 3.85740894136059 \\
\hline $\mathbf{C u}$ & 8.91820519619166 & 11.35520891804990 & 3.85683294181192 \\
\hline $\mathrm{Cu}$ & 8.91818938431249 & 13.87228866826940 & 3.85468287967111 \\
\hline $\mathbf{C u}$ & 8.91821943979275 & 16.39844302480160 & 3.85233148991630 \\
\hline $\mathbf{C u}$ & 8.91823929772157 & 18.91744194862750 & 3.85060603766257 \\
\hline $\mathrm{Cu}$ & 8.91820776190851 & 21.43855129848400 & 3.85565806491377 \\
\hline $\mathrm{Cu}$ & 8.91816722471731 & 23.96153195491920 & 3.85075947555177 \\
\hline $\mathrm{Cu}$ & 8.91817868147719 & 26.48843725364430 & 3.85695528454839 \\
\hline $\mathbf{C u}$ & 8.91824060054299 & 29.00435222761780 & 3.84842607958658 \\
\hline $\mathbf{C u}$ & 8.91822252721911 & 31.53361124466060 & 3.84972049096313 \\
\hline $\mathbf{C u}$ & 8.91820102037428 & 34.05120552553470 & 3.85033476559566 \\
\hline $\mathbf{C u}$ & 0.00217850493626 & 35.31177307000560 & 5.05510695574620 \\
\hline $\mathbf{C u}$ & 0.00008782481164 & 2.52103731178656 & 5.05823137222140 \\
\hline $\mathbf{C u}$ & 0.00143817225412 & 5.04810365208911 & 5.05703867445299 \\
\hline
\end{tabular}




\begin{tabular}{|c|c|c|c|}
\hline $\mathbf{C u}$ & 0.00702493295282 & 7.56614503464654 & 5.05143478870541 \\
\hline $\mathbf{C u}$ & 0.00979729981265 & 10.09363636067010 & 5.04633588593436 \\
\hline $\mathrm{Cu}$ & 0.01010887364444 & 12.61183265576850 & 5.06014397112622 \\
\hline $\mathrm{Cu}$ & 0.00051605199323 & 15.13803596126350 & 5.05402135891080 \\
\hline $\mathbf{C u}$ & 10.70109116517230 & 17.65859852319570 & 5.05469233563623 \\
\hline $\mathbf{C u}$ & 0.00419334637027 & 20.17850179947230 & 5.05699325333931 \\
\hline $\mathbf{C u}$ & 0.00633061267372 & 22.70189155408190 & 5.05796901038968 \\
\hline $\mathbf{C u}$ & 0.00364332088741 & 25.21998780938450 & 5.05396545019168 \\
\hline $\mathrm{Cu}$ & 0.00150243029877 & 27.74758807256730 & 5.06442887947608 \\
\hline $\mathrm{Cu}$ & 10.69988602954100 & 30.26649325240490 & 5.05451339001190 \\
\hline $\mathbf{C u}$ & 0.00444173100793 & 32.78910023980400 & 5.05862234669043 \\
\hline $\mathrm{Cu}$ & 3.56729206021025 & 35.30867835245950 & 5.05688667552753 \\
\hline $\mathrm{Cu}$ & 3.56724478112861 & 2.51627260007876 & 5.05755449343136 \\
\hline $\mathrm{Cu}$ & 3.56725448344666 & 5.02171426698852 & 5.04928337449988 \\
\hline $\mathrm{Cu}$ & 3.56728281956208 & 7.52015263464816 & 5.15689364534711 \\
\hline $\mathbf{C u}$ & 3.56735200826889 & 10.11254208298020 & 5.15642454791901 \\
\hline $\mathrm{Cu}$ & 3.56730683051419 & 12.63045577452550 & 5.13780488067100 \\
\hline $\mathrm{Cu}$ & 3.56724868160773 & 15.15322212729410 & 5.09657266600934 \\
\hline $\mathrm{Cu}$ & 3.56692012132244 & 97959392580 & 5.14011916031686 \\
\hline $\mathrm{Cu}$ & 3.56727163415434 & 20.16613432409890 & 5.10112308857549 \\
\hline $\mathbf{C u}$ & 3.56734285081283 & 22.70621067036890 & 5.14844278559616 \\
\hline $\mathbf{C u}$ & 3.56729797753705 & 25.23892118783760 & 5.06390740889451 \\
\hline $\mathrm{Cu}$ & 3.56728791397996 & 27.74899058309500 & 5.06042348587737 \\
\hline $\mathrm{Cu}$ & 3.56730744629291 & 30.27101343117850 & 5.06094448042450 \\
\hline $\mathrm{Cu}$ & 3.56730375399803 & 32.78955840425870 & 5.05977535067768 \\
\hline $\mathrm{Cu}$ & 7.13238286786452 & 35.31176523376480 & 5.05508447091994 \\
\hline $\mathrm{Cu}$ & 7.13443781006363 & 2.52104401726684 & 5.05820579768008 \\
\hline $\mathrm{Cu}$ & 7.13316099642118 & 5.04809046070751 & 5.05697431093509 \\
\hline $\mathrm{Cu}$ & 7.12757604145248 & 7.56611678164374 & 5.05141119657703 \\
\hline $\mathrm{Cu}$ & 7.12481339189307 & 10.09361039554290 & 5.04630496138798 \\
\hline $\mathrm{Cu}$ & 7.12441240148752 & 12.61184545270260 & 5.06016293153359 \\
\hline $\mathrm{Cu}$ & 7.13398393536598 & 15.13807260620850 & 5.05402873410305 \\
\hline $\mathrm{Cu}$ & 7.13541254879325 & 17.65864962476950 & 5.05469291319166 \\
\hline $\mathrm{Cu}$ & 7.13040880736393 & 20.17849543554040 & 5.05700340001866 \\
\hline $\mathrm{Cu}$ & 7.12825548228795 & 22.70194421546450 & 5.05797471029978 \\
\hline $\mathbf{C u}$ & 7.13090104950172 & 25.22003265936430 & 5.05391305324336 \\
\hline $\mathbf{C u}$ & 7.13296748816692 & 27.74752089143060 & 5.06445750247591 \\
\hline $\mathbf{C u}$ & 7.13644705598701 & 30.26655240268740 & 5.05449307754571 \\
\hline $\mathbf{C u}$ & 7.13015612498426 & 32.78910296650320 & 5.05860531079152 \\
\hline \multicolumn{4}{|c|}{ CI-NEB Image 19} \\
\hline Element & X-Coordinate $(\AA))$ & Y-Coordinate (§̊) & Z-Coordinate (§̊) \\
\hline $\mathbf{F}$ & 4.66343112135952 & 12.65847122033730 & 7.76795828066308 \\
\hline $\mathbf{F}$ & 2.47115319001914 & 12.65842733330780 & 7.76794214286330 \\
\hline $\mathbf{F}$ & 3.56731908482632 & 8.81630228605649 & 6.63138634453887 \\
\hline $\mathbf{F}$ & 4.63789570481987 & 17.84028372518700 & 7.80748003020511 \\
\hline $\mathbf{F}$ & 2.50763244794679 & 17.83855756406430 & 7.81281702003470 \\
\hline $\mathbf{F}$ & 3.56726236042628 & 14.52119745834020 & 7.29742895565828 \\
\hline $\mathbf{F}$ & 4.66282453504114 & 22.60090704866530 & 7.81766298638445 \\
\hline $\mathbf{F}$ & 2.47317901127116 & 22.60104823890580 & 7.81887643839538 \\
\hline $\mathbf{F}$ & 3.56748467272604 & 20.76217934194130 & 7.26127403741235 \\
\hline $\mathbf{C}$ & 3.56728891836818 & 13.08510945755690 & 7.06204721288244 \\
\hline C & 3.57080249536107 & 17.77162736468380 & 7.02131936414280 \\
\hline $\mathbf{C}$ & 3.56756441401490 & 22.21257515486650 & 7.08621175656631 \\
\hline $\mathbf{C u}$ & 0.00000000000000 & 0.00000000000000 & 0.00000000000000 \\
\hline
\end{tabular}




\begin{tabular}{|c|c|c|c|}
\hline $\mathbf{C u}$ & 0.00000000000000 & 2.52244512012115 & 0.00000000000000 \\
\hline $\mathbf{C u}$ & 0.00000000000000 & 5.04488953395804 & 0.00000000000000 \\
\hline $\mathbf{C u}$ & 0.00000000000000 & 7.56733465407861 & 0.00000000000000 \\
\hline $\mathbf{C u}$ & 0.00000000000000 & 10.08977871477400 & 0.00000000000000 \\
\hline $\mathbf{C u}$ & 0.00000000000000 & 12.61222383489520 & 0.00000000000000 \\
\hline $\mathbf{C u}$ & 0.00000000000000 & 15.13466895501390 & 0.00000000000000 \\
\hline $\mathbf{C u}$ & 0.00000000000000 & 17.65711336885320 & 0.00000000000000 \\
\hline $\mathbf{C u}$ & 0.00000000000000 & 20.17955848897460 & 0.00000000000000 \\
\hline $\mathbf{C u}$ & 0.00000000000000 & 22.70200254966930 & 0.00000000000000 \\
\hline $\mathbf{C u}$ & 0.00000000000000 & 25.22444766979040 & 0.00000000000000 \\
\hline $\mathbf{C u}$ & 0.00000000000000 & 27.74689208362270 & 0.00000000000000 \\
\hline $\mathrm{Cu}$ & 0.00000000000000 & 30.26933720374380 & 0.00000000000000 \\
\hline $\mathrm{Cu}$ & 0.00000000000000 & 32.79178232386990 & 0.00000000000000 \\
\hline $\mathrm{Cu}$ & 3.56727419238642 & 0.00000000000000 & 0.00000000000000 \\
\hline $\mathbf{C u}$ & 3.56727419238642 & 2.52244512012115 & 0.00000000000000 \\
\hline $\mathbf{C u}$ & 3.56727419238642 & 5.04488953395804 & 0.00000000000000 \\
\hline $\mathbf{C u}$ & 3.56727419238642 & 7.56733465407861 & 0.00000000000000 \\
\hline $\mathbf{C u}$ & 3.56727419238642 & 10.08977871477400 & 0.00000000000000 \\
\hline $\mathbf{C u}$ & 3.56727419238642 & 12.61222383489520 & 0.00000000000000 \\
\hline $\mathbf{C u}$ & 3.56727419238642 & 15.13466895501390 & 0.00000000000000 \\
\hline $\mathbf{C u}$ & 3.56727419238642 & 17.65711336885320 & 0.00000000000000 \\
\hline $\mathbf{C u}$ & 3.56727419238642 & 20.17955848897460 & 0.00000000000000 \\
\hline $\mathbf{C u}$ & 3.56727419238642 & 22.70200254966930 & 0.00000000000000 \\
\hline $\mathbf{C u}$ & 3.56727419238642 & 25.22444766979040 & 0.00000000000000 \\
\hline $\mathrm{Cu}$ & 3.56727419238642 & 27.74689 & 0.00000000000000 \\
\hline $\mathrm{Cu}$ & 3.56727419238642 & 30.26933720374380 & 0.00000000000000 \\
\hline $\mathbf{C u}$ & 3.56727419238642 & 32.79178232386990 & 0.00000000000000 \\
\hline $\mathbf{C u}$ & 7.13454838477291 & 0.00000000000000 & 0.00000000000000 \\
\hline $\mathbf{C u}$ & 7.13454838477291 & 2.52244512012115 & 0.00000000000000 \\
\hline $\mathrm{Cu}$ & 7.13454838477291 & 5.04488953395804 & 0.00000000000000 \\
\hline $\mathrm{Cu}$ & 7.13454838477291 & 7.56733465407861 & 0.00000000000000 \\
\hline $\mathrm{Cu}$ & 7.13454838477291 & 10.08977871477400 & 0.00000000000000 \\
\hline $\mathrm{Cu}$ & 7.13454838477291 & 12.61222383489520 & 0.00000000000000 \\
\hline $\mathbf{C u}$ & 7.13454838477291 & 15.13466895501390 & 0.00000000000000 \\
\hline $\mathbf{C u}$ & 7.13454838477291 & 17.65711336885320 & 0.00000000000000 \\
\hline $\mathbf{C u}$ & 7.13454838477291 & 20.17955848897460 & 0.00000000000000 \\
\hline $\mathbf{C u}$ & 7.13454838477291 & 22.70200254966930 & 0.00000000000000 \\
\hline $\mathbf{C u}$ & 7.13454838477291 & 25.22444766979040 & 0.00000000000000 \\
\hline $\mathbf{C u}$ & 7.13454838477291 & 27.74689208362270 & 0.00000000000000 \\
\hline $\mathbf{C u}$ & 7.13454838477291 & 30.26933720374380 & 0.00000000000000 \\
\hline $\mathbf{C u}$ & 7.13454838477291 & 32.79178232386990 & 0.00000000000000 \\
\hline $\mathbf{C u}$ & 1.78363955761358 & 1.26122414920059 & 1.26122399999993 \\
\hline $\mathbf{C u}$ & 1.78363955761358 & 3.78366926932170 & 1.26122399999993 \\
\hline $\mathbf{C u}$ & 1.78363955761358 & 6.30611333001662 & 1.26122399999993 \\
\hline $\mathbf{C u}$ & 1.78363955761358 & 8.82855845013778 & 1.26122399999993 \\
\hline $\mathrm{Cu}$ & 1.78363955761358 & 11.35100286397460 & 1.26122399999993 \\
\hline $\mathbf{C u}$ & 1.78363955761358 & 13.87344798409310 & 1.26122399999993 \\
\hline $\mathbf{C u}$ & 1.78363955761358 & 16.39589310421720 & 1.26122399999993 \\
\hline $\mathbf{C u}$ & 1.78363955761358 & 18.91833716491180 & 1.26122399999993 \\
\hline $\mathbf{C u}$ & 1.78363955761358 & 21.44078263817520 & 1.26122399999993 \\
\hline $\mathbf{C u}$ & 1.78363955761358 & 23.96322669886980 & 1.26122399999993 \\
\hline $\mathbf{C u}$ & 1.78363955761358 & 26.48567181899090 & 1.26122399999993 \\
\hline $\mathbf{C u}$ & 1.78363955761358 & 29.00811693910780 & 1.26122399999993 \\
\hline $\mathbf{C u}$ & 1.78363955761358 & 31.53056135294420 & 1.26122399999993 \\
\hline
\end{tabular}




\begin{tabular}{|c|c|c|c|}
\hline $\mathbf{C u}$ & 1.78363955761358 & 34.05300647307050 & 1.26122399999993 \\
\hline $\mathbf{C u}$ & 5.35091375000000 & 1.26122414920059 & 1.26122399999993 \\
\hline $\mathbf{C u}$ & 5.35091375000000 & 3.78366926932170 & 1.26122399999993 \\
\hline $\mathbf{C u}$ & 5.35091375000000 & 6.30611333001662 & 1.26122399999993 \\
\hline $\mathbf{C u}$ & 5.35091375000000 & 8.82855845013778 & 1.26122399999993 \\
\hline $\mathbf{C u}$ & 5.35091375000000 & 11.35100286397460 & 1.26122399999993 \\
\hline $\mathbf{C u}$ & 5.35091375000000 & 13.87344798409310 & 1.26122399999993 \\
\hline $\mathbf{C u}$ & 5.35091375000000 & 16.39589310421720 & 1.26122399999993 \\
\hline $\mathbf{C u}$ & 5.35091375000000 & 18.91833716491180 & 1.26122399999993 \\
\hline $\mathbf{C u}$ & 5.35091375000000 & 21.44078263817520 & 1.26122399999993 \\
\hline $\mathbf{C u}$ & 5.35091375000000 & 23.96322669886980 & 1.26122399999993 \\
\hline $\mathbf{C u}$ & 5.35091375000000 & 26.48567181899090 & 1.26122399999993 \\
\hline $\mathbf{C u}$ & 5.35091375000000 & 29.00811693910780 & 1.26122399999993 \\
\hline $\mathbf{C u}$ & 5.35091375000000 & 31.53056135294420 & 1.26122399999993 \\
\hline $\mathbf{C u}$ & 5.35091375000000 & 34.05300647307050 & 1.26122399999993 \\
\hline $\mathbf{C u}$ & 8.91818794238642 & 1.26122414920059 & 1.26122399999993 \\
\hline $\mathbf{C u}$ & 8.91818794238642 & 3.78366926932170 & 1.26122399999993 \\
\hline $\mathbf{C u}$ & 8.91818794238642 & 6.30611333001662 & 1.26122399999993 \\
\hline $\mathbf{C u}$ & 8.91818794238642 & 8.82855845013778 & 1.26122399999993 \\
\hline $\mathbf{C u}$ & 8.91818794238642 & 11.35100286397460 & 1.26122399999993 \\
\hline $\mathbf{C u}$ & 8.91818794238642 & 13.87344798409310 & 1.26122399999993 \\
\hline $\mathbf{C u}$ & 8.91818794238642 & 16.39589310421720 & 1.26122399999993 \\
\hline $\mathbf{C u}$ & 8.91818794238642 & 716491180 & 399999993 \\
\hline $\mathrm{Cu}$ & 8.91818794238642 & 3817520 & 399999993 \\
\hline $\mathrm{Cu}$ & 4238642 & 23.963 & 9999993 \\
\hline $\mathrm{Cu}$ & 8.91818794238642 & 26.4856718 & 1.261223 \\
\hline $\mathrm{Cu}$ & 8.91818794238642 & 29.00811693910780 & 1.26122399999993 \\
\hline $\mathbf{C u}$ & 8.91818794238642 & 31.53056135294420 & 1.26122399999993 \\
\hline $\mathbf{C u}$ & 8.91818794238642 & 34.05300647307050 & 1.26122399999993 \\
\hline $\mathbf{C u}$ & 0.00152002736400 & 35.31377842094530 & 2.53943229236753 \\
\hline $\mathbf{C u}$ & 10.69902966292480 & 2.52377270942156 & 2.54083596146091 \\
\hline $\mathbf{C u}$ & 0.00316684609305 & 5.04527913254833 & 2.54201033624505 \\
\hline $\mathrm{Cu}$ & 10.70059380600130 & 7.56522522253610 & 2.54310854316299 \\
\hline $\mathrm{Cu}$ & 10.70030572774410 & 10.09041337080270 & 2.54042885130939 \\
\hline $\mathrm{Cu}$ & 10.70069703146670 & 12.61175461254010 & 2.54783627172083 \\
\hline $\mathbf{C u}$ & 10.69572293125530 & 15.13583389652110 & 2.53661421678580 \\
\hline $\mathbf{C u}$ & 10.70026312665870 & 17.65696858959520 & 2.54285785959835 \\
\hline $\mathbf{C u}$ & 10.69793603174430 & 20.17971408391290 & 2.53737205633974 \\
\hline $\mathbf{C u}$ & 10.69919624276560 & 22.70379553591600 & 2.54443344053773 \\
\hline $\mathbf{C u}$ & 10.70142259828490 & 25.22733143620050 & 2.54009869146967 \\
\hline $\mathbf{C u}$ & 10.70132810602190 & 27.74706520615330 & 2.54369877209937 \\
\hline $\mathbf{C u}$ & 0.00087170193036 & 30.26864869204700 & 2.54082540832388 \\
\hline $\mathbf{C u}$ & 10.70139865011510 & 32.78986451077360 & 2.54058395082200 \\
\hline $\mathbf{C u}$ & 3.56728565778651 & 0.00026100268454 & 2.54058379873520 \\
\hline $\mathbf{C u}$ & 3.56727743415279 & 2.52234163337845 & 2.54395965675718 \\
\hline $\mathbf{C u}$ & 3.56728317795833 & 5.04475739593275 & 2.54059905045845 \\
\hline $\mathbf{C u}$ & 3.56727745620687 & 7.56998799736733 & 2.57960481369286 \\
\hline $\mathbf{C u}$ & 3.56727807340823 & 10.09252209130420 & 2.58778870872244 \\
\hline $\mathrm{Cu}$ & 3.56728531735308 & 12.62361770211050 & 2.58218543489812 \\
\hline $\mathrm{Cu}$ & 3.56728393202288 & 15.13852494133420 & 2.58345614398954 \\
\hline $\mathbf{C u}$ & 3.56725471435754 & 17.65586867865150 & 2.59561214192813 \\
\hline $\mathbf{C u}$ & 3.56725507514166 & 20.17936450097740 & 2.58290316967344 \\
\hline $\mathbf{C u}$ & 3.56726773370423 & 22.69453899295100 & 2.59128352008920 \\
\hline $\mathbf{C u}$ & 3.56728466671623 & 25.21746567391930 & 2.55244262323497 \\
\hline
\end{tabular}




\begin{tabular}{|c|c|c|c|}
\hline $\mathbf{C u}$ & 3.56728276426788 & 27.74803224545240 & 2.54118633164104 \\
\hline $\mathbf{C u}$ & 3.56728591051736 & 30.26789333294670 & 2.54219457080668 \\
\hline $\mathbf{C u}$ & 3.56727900477308 & 32.79022544428510 & 2.54276340282419 \\
\hline $\mathbf{C u}$ & 7.13304029619759 & 35.31379316954260 & 2.53942843193130 \\
\hline $\mathbf{C u}$ & 7.13735292285477 & 2.52377513200958 & 2.54085069029128 \\
\hline $\mathbf{C u}$ & 7.13138934881489 & 5.04528376493905 & 2.54200073708791 \\
\hline $\mathbf{C u}$ & 7.13579221611234 & 7.56523298590121 & 2.54311141426730 \\
\hline $\mathbf{C u}$ & 7.13608562447714 & 10.09041274298770 & 2.54042620615402 \\
\hline $\mathbf{C u}$ & 7.13568876743150 & 12.61177688423490 & 2.54782489246220 \\
\hline $\mathbf{C u}$ & 7.14063783677691 & 15.13586362643820 & 2.53662063153153 \\
\hline $\mathbf{C u}$ & 7.13608185909109 & 17.65696515384240 & 2.54289345807085 \\
\hline $\mathbf{C u}$ & 7.13844538441593 & 20.17971417150170 & 2.53741021788036 \\
\hline $\mathbf{C u}$ & 7.13717786745893 & 22.70378232958620 & 2.54446707237673 \\
\hline $\mathrm{Cu}$ & 7.13495176761217 & 25.22729486285010 & 2.54010983713521 \\
\hline $\mathbf{C u}$ & 7.13506145656505 & 27.74704409662300 & 2.54371161490665 \\
\hline $\mathbf{C u}$ & 7.13368392444565 & 30.26865322484060 & 2.54083132676124 \\
\hline $\mathbf{C u}$ & 7.13498575942053 & 32.78986326845830 & 2.54057729639063 \\
\hline $\mathbf{C u}$ & 1.78379108600893 & 1.25939871090146 & 3.85290794911647 \\
\hline $\mathbf{C u}$ & 1.77847353809692 & 3.77945177230592 & 3.85545631379289 \\
\hline $\mathbf{C u}$ & 1.79670950230427 & 6.30456137542160 & 3.85861813891185 \\
\hline $\mathbf{C u}$ & 1.78210905227697 & 8.82796447110571 & 3.83143715999562 \\
\hline $\mathbf{C u}$ & 1.80020525139883 & 11.34962488126270 & 3.85355531182570 \\
\hline $\mathbf{C u}$ & 1.77281616618126 & 13.87821968814650 & 3.84096909415009 \\
\hline $\mathbf{C u}$ & 203015037 & 16.395 & 231217 \\
\hline $\mathrm{Cu}$ & 82130351 & 091520 & 111031 \\
\hline $\mathrm{Cu}$ & 1.77730507833174 & 21.44263761281430 & 3.84373165026585 \\
\hline $\mathbf{C u}$ & 1.78957452942955 & 23.96578179835040 & 3.85421550554155 \\
\hline $\mathbf{C u}$ & 1.78204762001432 & 26.48743117923660 & 3.85564155567761 \\
\hline $\mathrm{Cu}$ & 1.78450190905215 & 29.00893243209540 & 3.85666280593511 \\
\hline $\mathrm{Cu}$ & 1.78318785380411 & 31.52929189865310 & 3.85535086824419 \\
\hline $\mathrm{Cu}$ & 1.78397206530727 & 34.04917145412370 & 3.85346295839126 \\
\hline $\mathbf{C u}$ & 5.35077132298842 & 1.25940426526635 & 3.85289865328168 \\
\hline $\mathrm{Cu}$ & 5.35608343595304 & 3.77945072549312 & 3.85547347463440 \\
\hline $\mathbf{C u}$ & 5.33786035256455 & 6.30455612729173 & 3.85860287236856 \\
\hline $\mathbf{C u}$ & 5.35244831068410 & 8.82798318473444 & 3.83143391570272 \\
\hline $\mathbf{C u}$ & 5.33434539399700 & 11.34962476656710 & 3.85354820238523 \\
\hline $\mathbf{C u}$ & 5.36174597432028 & 13.87826167979170 & 3.84100223553387 \\
\hline $\mathbf{C u}$ & 5.35320891029112 & 16.39552086751090 & 3.84854448609629 \\
\hline $\mathbf{C u}$ & 5.35516985227858 & 18.92326637870550 & 3.84602350729944 \\
\hline $\mathbf{C u}$ & 5.35718098754201 & 21.44259816154570 & 3.84379433296201 \\
\hline $\mathbf{C u}$ & 5.34495461505136 & 23.96573626880190 & 3.85423396514590 \\
\hline $\mathbf{C u}$ & 5.35251591489472 & 26.48739976228650 & 3.85565667115018 \\
\hline $\mathbf{C u}$ & 5.35008606419696 & 29.00891650766300 & 3.85665683321431 \\
\hline $\mathbf{C u}$ & 5.35137426526049 & 31.52928749258460 & 3.85534414459932 \\
\hline $\mathbf{C u}$ & 5.35060196355469 & 34.04916799932300 & 3.85345733356046 \\
\hline $\mathbf{C u}$ & 8.91818692868679 & 1.26036791918737 & 3.85029450018227 \\
\hline $\mathbf{C u}$ & 8.91819840402967 & 3.78362449325403 & 3.84969844605523 \\
\hline $\mathbf{C u}$ & 8.91819679102922 & 6.30421266644359 & 3.85102677610876 \\
\hline $\mathbf{C u}$ & 8.91818842188602 & 8.82679902365626 & 3.85757407690420 \\
\hline $\mathbf{C u}$ & 8.91819172473797 & 11.35516269872710 & 3.85691149063243 \\
\hline $\mathbf{C u}$ & 8.91819935638412 & 13.87255335028960 & 3.85480362378889 \\
\hline $\mathbf{C u}$ & 8.91818645944203 & 16.39892210127550 & 3.85247776486957 \\
\hline $\mathbf{C u}$ & 8.91823108186894 & 18.91893235585900 & 3.85064334906108 \\
\hline $\mathbf{C u}$ & 8.91820178589210 & 21.44072260126460 & 3.85509155373230 \\
\hline
\end{tabular}




\begin{tabular}{|c|c|c|c|}
\hline $\mathbf{C u}$ & 8.91820381918568 & 23.96362191722790 & 3.84995077783558 \\
\hline $\mathbf{C u}$ & 8.91819326326257 & 26.48924352450030 & 3.85648178018745 \\
\hline $\mathbf{C u}$ & 8.91818972543377 & 29.00573134239070 & 3.84785657501117 \\
\hline $\mathbf{C u}$ & 8.91819751180234 & 31.53454578561390 & 3.84959375065391 \\
\hline $\mathrm{Cu}$ & 8.91816920034434 & 34.05169693114730 & 3.85023328185540 \\
\hline $\mathbf{C u}$ & 0.00112446821423 & 35.31241735639010 & 5.05541251759372 \\
\hline $\mathrm{Cu}$ & 10.70175422545570 & 2.52146041362834 & 5.05845956962707 \\
\hline $\mathbf{C u}$ & 0.00180370287457 & 5.04800659549964 & 5.05706305085223 \\
\hline $\mathbf{C u}$ & 0.00682015083432 & 7.56602187941770 & 5.05173510572292 \\
\hline $\mathbf{C u}$ & 0.00968077822472 & 10.0936188 & 5.046 \\
\hline $\mathbf{C u}$ & 0.00994233690854 & 12.61181349422300 & 5.05993428939781 \\
\hline $\mathbf{C u}$ & 0.00063651014574 & 15.13844496082450 & 5.05397265824218 \\
\hline $\mathbf{C u}$ & 10.70076204269590 & 17.65990933164840 & 5.05470738149139 \\
\hline $\mathbf{C u}$ & 0.00398302883474 & 20.18032127504940 & 5.05603808551098 \\
\hline $\mathbf{C u}$ & 0.00517684102171 & 22.70353172633450 & 5.05754261467600 \\
\hline $\mathbf{C u}$ & 0.00374894872902 & 25.22147232231200 & 5.05492035634877 \\
\hline $\mathrm{Cu}$ & 0.00050144504045 & 27.74911598453530 & 5.064 \\
\hline $\mathbf{C u}$ & 10.69899351371810 & 30.26780286432920 & 5.05506285693393 \\
\hline $\mathbf{C u}$ & 0.00363386692652 & 32.78954541566340 & 5.0589777 \\
\hline $\mathbf{C u}$ & 3.56727813760823 & 35.30939053901310 & 5.05676618317245 \\
\hline $\mathbf{C u}$ & 3.56727397291590 & 2.51652371698283 & 5.05753990687532 \\
\hline $\mathbf{C u}$ & 3.56727219699924 & 5.02163703375206 & 5.0493470 \\
\hline $\mathbf{C u}$ & 3.56730908388333 & 7.51988021988599 & 5.1578778 \\
\hline $\mathbf{C u}$ & 3.56727983972126 & 10.11199180532790 & 5.15632846457098 \\
\hline $\mathrm{Cu}$ & 3.56727747367831 & 12.63042382182940 & 5.13798328424930 \\
\hline $\mathbf{C u}$ & 3.56724562015068 & 15.14975787019300 & 5.09337683181406 \\
\hline $\mathbf{C u}$ & 3.56664094212062 & 17.65417402116970 & 5.15274467604630 \\
\hline $\mathbf{C u}$ & 3.56715394471146 & 20.16157939995570 & 5.08747065422025 \\
\hline $\mathbf{C u}$ & 3.56715826960040 & 22.70758956887340 & 5.16661980769475 \\
\hline $\mathbf{C u}$ & 3.56725521230069 & 25.24026056821500 & 5.06497830204587 \\
\hline $\mathbf{C u}$ & 3.56729075397588 & 27.75107708292730 & 5.06066377038400 \\
\hline $\mathbf{C u}$ & 3.56730190592978 & 30.27352083692750 & 5.06068930026045 \\
\hline $\mathbf{C u}$ & 3.56728280283617 & 32.79101858309680 & 5.05950078522929 \\
\hline $\mathbf{C u}$ & 7.13341426094272 & 35.31241625551190 & 5.05539243670603 \\
\hline $\mathbf{C u}$ & 7.13465076770110 & 2.52146733175720 & 5.05847282523435 \\
\hline $\mathbf{C u}$ & 7.13277184449459 & 5.04800932930976 & 5.05706408340821 \\
\hline $\mathbf{C u}$ & 7.12774032750216 & 7.56601495421640 & 5.05173276944682 \\
\hline $\mathbf{C u}$ & 7.12486566824622 & 10.09362944624150 & 5.04645397970455 \\
\hline $\mathbf{C u}$ & 7.12461704456819 & 12.61186425222380 & 5.05991215164787 \\
\hline $\mathbf{C u}$ & 7.13396614400825 & 15.13850842979770 & 5.05394004901390 \\
\hline $\mathbf{C u}$ & 7.13580051161425 & 17.65997997956600 & 5.05462269660154 \\
\hline $\mathbf{C u}$ & 7.13058595679227 & 20.18033384827170 & 5.05603666897178 \\
\hline $\mathbf{C u}$ & 7.12938547004474 & 22.70352957174760 & 5.05755711802743 \\
\hline $\mathbf{C u}$ & 7.13081519886193 & 25.22145313324480 & 5.05488488979703 \\
\hline $\mathbf{C u}$ & 7.13407464991301 & 27.74909398988680 & 5.06409459997844 \\
\hline $\mathbf{C u}$ & 7.13739916064868 & 30.26779415535860 & 5.05508011553914 \\
\hline $\mathbf{C u}$ & 7.13094265343769 & 32.78953031726470 & 5.05897054453171 \\
\hline \multicolumn{4}{|c|}{ CI-NEB Image 20} \\
\hline Element & X-Coordinate ( $(\AA)$ & Y-Coordinate (Å) & Z-Coordinate (Å) \\
\hline $\mathbf{F}$ & 4.66332796089174 & 12.66914885420040 & 7.76653989051713 \\
\hline $\mathbf{F}$ & 2.47127843530116 & 12.66906381847480 & 7.76650070580406 \\
\hline $\mathbf{F}$ & 3.56750820669095 & 8.81679559078813 & 6.63492947141456 \\
\hline $\mathbf{F}$ & 4.63761023055590 & 17.84934497568590 & 7.83795312035207 \\
\hline $\mathbf{F}$ & 2.51003280648604 & 17.84701725592160 & 7.84441903186359 \\
\hline
\end{tabular}




\begin{tabular}{|c|c|c|c|}
\hline $\mathbf{F}$ & 3.56724756934304 & 14.53012489391190 & 7.28926077613556 \\
\hline $\mathbf{F}$ & 4.64936706228936 & 22.50907396446980 & 7.85611872121234 \\
\hline $\mathbf{F}$ & 2.48717957658347 & 22.50918976640730 & 7.85744323676271 \\
\hline $\mathbf{F}$ & 3.56758255815958 & 20.61615863878940 & 7.12443838968299 \\
\hline C & 3.56730037457716 & 13.09297209380540 & 7.05878324316465 \\
\hline C & 3.57139283291195 & 17.80912676429790 & 7.05236444986879 \\
\hline C & 3.56777354022383 & 22.23727131880730 & 7.08670550264662 \\
\hline $\mathbf{C u}$ & 0.00000000000000 & 0.00000000000000 & 0.00000000000000 \\
\hline $\mathbf{C u}$ & 0.00000000000000 & 2.52244512012115 & 0.00000000000000 \\
\hline $\mathbf{C u}$ & 0.00000000000000 & 5.04488953395804 & 0.00000000000000 \\
\hline $\mathbf{C u}$ & 0.00000000000000 & 7.56733465407861 & 0.00000000000000 \\
\hline $\mathbf{C u}$ & 0.00000000000000 & 10.08977871477400 & 0.00000000000000 \\
\hline $\mathbf{C u}$ & 0.00000000000000 & 12.61222383489520 & 0.00000000000000 \\
\hline $\mathbf{C u}$ & 0.00000000000000 & 15.13466895501370 & 0.00000000000000 \\
\hline $\mathbf{C u}$ & 0.00000000000000 & 17.65711336885320 & 0.00000000000000 \\
\hline $\mathbf{C u}$ & 0.00000000000000 & 20.17955848897460 & 0.00000000000000 \\
\hline $\mathbf{C u}$ & 0.00000000000000 & 22.70200254966930 & 0.00000000000000 \\
\hline $\mathbf{C u}$ & 0.00000000000000 & 25.22444766979040 & 0.00000000000000 \\
\hline $\mathbf{C u}$ & 0.00000000000000 & 27.74689208362250 & 0.00000000000000 \\
\hline $\mathbf{C u}$ & 0.00000000000000 & 30.26933720374360 & 0.00000000000000 \\
\hline $\mathbf{C u}$ & 0.00000000000000 & 32.79178232386990 & 0.00000000000000 \\
\hline $\mathbf{C u}$ & 3.56727419238642 & 0.00000000000000 & 0.00000000000000 \\
\hline $\mathbf{C u}$ & 3.56727419238642 & 2.52244512012115 & 0.00000000000000 \\
\hline $\mathbf{C u}$ & 3.56727419238642 & 5.04488953395804 & 0.00000000000000 \\
\hline $\mathbf{C u}$ & 3.56727419238642 & 7.56733465407861 & 0.00000000000000 \\
\hline $\mathbf{C u}$ & 3.56727419238642 & 10.08977871477400 & 0.00000000000000 \\
\hline $\mathbf{C u}$ & 3.56727419238642 & 12.61222383489520 & 0.00000000000000 \\
\hline $\mathbf{C u}$ & 3.56727419238642 & 15.13466895501370 & 0.00000000000000 \\
\hline $\mathbf{C u}$ & 3.56727419238642 & 17.65711336885320 & 0.00000000000000 \\
\hline $\mathbf{C u}$ & 3.56727419238642 & 20.17955848897460 & 0.00000000000000 \\
\hline $\mathbf{C u}$ & 3.56727419238642 & 22.70200254966930 & 0.00000000000000 \\
\hline $\mathbf{C u}$ & 3.56727419238642 & 25.22444766979040 & 0.00000000000000 \\
\hline $\mathbf{C u}$ & 3.56727419238642 & 27.74689208362250 & 0.00000000000000 \\
\hline $\mathbf{C u}$ & 3.56727419238642 & 30.26933720374360 & 0.00000000000000 \\
\hline $\mathbf{C u}$ & 3.56727419238642 & 32.79178232386990 & 0.00000000000000 \\
\hline $\mathbf{C u}$ & 7.13454838477291 & 0.00000000000000 & 0.00000000000000 \\
\hline $\mathbf{C u}$ & 7.13454838477291 & 2.52244512012115 & 0.00000000000000 \\
\hline $\mathbf{C u}$ & 7.13454838477291 & 5.04488953395804 & 0.00000000000000 \\
\hline $\mathbf{C u}$ & 7.13454838477291 & 7.56733465407861 & 0.00000000000000 \\
\hline $\mathbf{C u}$ & 7.13454838477291 & 10.08977871477400 & 0.00000000000000 \\
\hline $\mathbf{C u}$ & 7.13454838477291 & 12.61222383489520 & 0.00000000000000 \\
\hline $\mathbf{C u}$ & 7.13454838477291 & 15.13466895501370 & 0.00000000000000 \\
\hline $\mathbf{C u}$ & 7.13454838477291 & 17.65711336885320 & 0.00000000000000 \\
\hline $\mathbf{C u}$ & 7.13454838477291 & 20.17955848897460 & 0.00000000000000 \\
\hline $\mathbf{C u}$ & 7.13454838477291 & 22.70200254966930 & 0.00000000000000 \\
\hline $\mathbf{C u}$ & 7.13454838477291 & 25.22444766979040 & 0.00000000000000 \\
\hline $\mathbf{C u}$ & 7.13454838477291 & 27.74689208362250 & 0.00000000000000 \\
\hline $\mathbf{C u}$ & 7.13454838477291 & 30.26933720374360 & 0.00000000000000 \\
\hline $\mathbf{C u}$ & 7.13454838477291 & 32.79178232386990 & 0.00000000000000 \\
\hline $\mathbf{C u}$ & 1.78363955761358 & 1.26122414920059 & 1.26122399999993 \\
\hline $\mathbf{C u}$ & 1.78363955761358 & 3.78366926932170 & 1.26122399999993 \\
\hline $\mathbf{C u}$ & 1.78363955761358 & 6.30611333001662 & 1.26122399999993 \\
\hline $\mathbf{C u}$ & 1.78363955761358 & 8.82855845013778 & 1.26122399999993 \\
\hline $\mathbf{C u}$ & 1.78363955761358 & 11.35100286397460 & 1.26122399999993 \\
\hline
\end{tabular}




\begin{tabular}{|c|c|c|c|}
\hline $\mathrm{Cu}$ & 1.78363955761358 & 13.87344798409290 & 1.26122399999993 \\
\hline $\mathbf{C u}$ & 1.78363955761358 & 16.39589310421720 & 1.26122399999993 \\
\hline $\mathrm{Cu}$ & 1.78363955761358 & 18.91833716491180 & 1.26122399999993 \\
\hline $\mathbf{C u}$ & 1.78363955761358 & 21.44078263817520 & 1.26122399999993 \\
\hline $\mathbf{C u}$ & 1.78363955761358 & 23.96322669886980 & 1.26122399999993 \\
\hline $\mathbf{C u}$ & 1.78363955761358 & 26.48567181899090 & 1.26122399999993 \\
\hline $\mathrm{Cu}$ & 1.78363955761358 & 29.00811693910760 & 1.26122399999993 \\
\hline $\mathrm{Cu}$ & 1.78363955761358 & 31.53056135294390 & 1.26122399999993 \\
\hline $\mathbf{C u}$ & 1.78363955761358 & 34.05300647307050 & 1.26122399999993 \\
\hline $\mathbf{C u}$ & 5.35091375000000 & 1.26122414920059 & 1.26122399999993 \\
\hline $\mathbf{C u}$ & 5.35091375000000 & 3.78366926932170 & 1.26122399999993 \\
\hline $\mathrm{Cu}$ & 5.35091375000000 & 6.30611333001662 & 1.26122399999993 \\
\hline $\mathrm{Cu}$ & 5.35091375000000 & 8.82855845013778 & 1.26122399999993 \\
\hline $\mathrm{Cu}$ & 5.35091375000000 & 11.35100286397460 & 1.26122399999993 \\
\hline $\mathbf{C u}$ & 5.35091375000000 & 13.87344798409290 & 1.26122399999993 \\
\hline $\mathbf{C u}$ & 5.35091375000000 & 16.39589310421720 & 1.26122399999993 \\
\hline $\mathrm{Cu}$ & 5.35091375000000 & 18.91833716491180 & 1.26122399999993 \\
\hline $\mathbf{C u}$ & 5.35091375000000 & 21.44078263817520 & 1.26122399999993 \\
\hline $\mathbf{C u}$ & 5.35091375000000 & 23.96322669886980 & 1.26122399999993 \\
\hline $\mathrm{Cu}$ & 5.35091375000000 & 26.48567181899090 & 1.26122399999993 \\
\hline $\mathbf{C u}$ & 5.35091375000000 & 29.00811693910760 & 1.26122399999993 \\
\hline $\mathbf{C u}$ & 5.35091375000000 & 31.53056135294390 & 1.26122399999993 \\
\hline $\mathrm{Cu}$ & 5.35091375000000 & 307050 & 399999993 \\
\hline $\mathrm{Cu}$ & 8.91818794238642 & 2414920059 & 2399999993 \\
\hline $\mathrm{Cu}$ & 8.91818794238642 & 32170 & 399999993 \\
\hline $\mathrm{Cu}$ & 8.91818794238642 & 3001662 & 1.26122399999993 \\
\hline $\mathrm{Cu}$ & 8.91818794238642 & 8.82855845013778 & 1.26122399999993 \\
\hline $\mathrm{Cu}$ & 8.91818794238642 & 11.35100286397460 & 1.26122399999993 \\
\hline $\mathbf{C u}$ & 8.91818794238642 & 13.87344798409290 & 1.26122399999993 \\
\hline $\mathrm{Cu}$ & 8.91818794238642 & 16.39589310421720 & 1.26122399999993 \\
\hline $\mathrm{Cu}$ & 8.91818794238642 & 18.91833716491180 & 1.26122399999993 \\
\hline $\mathrm{Cu}$ & 8.91818794238642 & 21.44078263817520 & 1.26122399999993 \\
\hline $\mathrm{Cu}$ & 8.91818794238642 & 23.96322669886980 & 1.26122399999993 \\
\hline $\mathrm{Cu}$ & 8.91818794238642 & 26.48567181899090 & 1.26122399999993 \\
\hline $\mathrm{Cu}$ & 8.91818794238642 & 29.00811693910760 & 1.26122399999993 \\
\hline $\mathbf{C u}$ & 8.91818794238642 & 31.53056135294390 & 1.26122399999993 \\
\hline $\mathrm{Cu}$ & 8.91818794238642 & 34.05300647307050 & 1.26122399999993 \\
\hline $\mathbf{C u}$ & 0.00113195356816 & 35.31392826837780 & 2.53903839951184 \\
\hline $\mathrm{Cu}$ & 10.69912680308660 & 2.52357100705222 & 2.54072950669165 \\
\hline $\mathbf{C u}$ & 0.00356539220235 & 5.04536259440698 & 2.54213247347176 \\
\hline $\mathrm{Cu}$ & 10.70063892251630 & 7.56519289624602 & 2.54298359906981 \\
\hline $\mathbf{C u}$ & 10.70046336246740 & 10.09053431158400 & 2.54066547545279 \\
\hline $\mathbf{C u}$ & 10.70036621922290 & 12.61226460115180 & 2.54768748188271 \\
\hline $\mathbf{C u}$ & 10.69630114600790 & 15.13694147141060 & 2.53729603518403 \\
\hline $\mathbf{C u}$ & 10.70069703152920 & 17.65739870247600 & 2.54279852719813 \\
\hline $\mathbf{C u}$ & 10.69844243478980 & 20.18016477540080 & 2.53767195205953 \\
\hline $\mathbf{C u}$ & 10.69933213922690 & 22.70444842936710 & 2.54206026210621 \\
\hline $\mathbf{C u}$ & 10.70153551127170 & 25.22727467106410 & 2.53967038282332 \\
\hline $\mathbf{C u}$ & 10.70084540063810 & 27.74714964753360 & 2.54257940079057 \\
\hline $\mathrm{Cu}$ & 0.00035427983521 & 30.26889359753620 & 2.54090517363296 \\
\hline $\mathbf{C u}$ & 10.70129213358000 & 32.78977347823850 & 2.54027549949222 \\
\hline $\mathbf{C u}$ & 3.56727884407420 & 0.00002097716090 & 2.54154171464902 \\
\hline $\mathbf{C u}$ & 3.56727733133813 & 2.52178625634952 & 2.54452025510599 \\
\hline $\mathbf{C u}$ & 3.56727646263558 & 5.04451085707474 & 2.54061331389284 \\
\hline
\end{tabular}




\begin{tabular}{|c|c|c|c|}
\hline $\mathbf{C u}$ & 3.56727921019007 & 7.56996884756443 & 2.58011612511625 \\
\hline $\mathbf{C u}$ & 3.56727798613776 & 10.09256969019700 & 2.58839548462057 \\
\hline $\mathbf{C u}$ & 3.56728867633423 & 12.62328270473770 & 2.58140175946431 \\
\hline $\mathbf{C u}$ & 3.56731389329191 & 15.14024998767090 & 2.57929697841571 \\
\hline $\mathbf{C u}$ & 3.56726957165078 & 17.65910197998150 & 2.60342901864236 \\
\hline $\mathbf{C u}$ & 3.56727918055104 & 20.18075270929310 & 2.59609366382962 \\
\hline $\mathbf{C u}$ & 3.56727024808140 & 22.69649969202570 & 2.59797637143690 \\
\hline $\mathbf{C u}$ & 3.56729140428665 & 25.21940360431620 & 2.54867116142605 \\
\hline $\mathbf{C u}$ & 3.56727833140453 & 27.74865026169960 & 2.54104301158947 \\
\hline $\mathbf{C u}$ & 3.56728266334086 & 30.26829084361640 & 2.54245966492652 \\
\hline $\mathbf{C u}$ & 3.56727449590905 & 32.79068685434070 & 2.54300792717683 \\
\hline $\mathbf{C u}$ & 7.13341912787852 & 35.31393421435160 & 2.53903955460745 \\
\hline $\mathbf{C u}$ & 7.13724968343027 & 2.52356715981505 & 2.54074116538335 \\
\hline $\mathrm{Cu}$ & 7.13099072288576 & 5.04536043411806 & 2.54213432109078 \\
\hline $\mathbf{C u}$ & 7.13574308841471 & 7.56519450770490 & 2.54298168370019 \\
\hline $\mathrm{Cu}$ & 7.13592258886269 & 10.09052349114390 & 2.54065719434439 \\
\hline $\mathbf{C u}$ & 7.13600923743307 & 12.61226741682990 & 2.54770934313792 \\
\hline $\mathbf{C u}$ & 7.14007720980337 & 15.13694021972370 & 2.53729583287890 \\
\hline $\mathbf{C u}$ & 7.13561896747908 & 17.65739266441080 & 2.54288809022975 \\
\hline $\mathbf{C u}$ & 7.13789060783944 & 20.18013309263650 & 2.53771984784786 \\
\hline $\mathbf{C u}$ & 7.13702685129996 & 22.70440109851630 & 2.54205676514307 \\
\hline $\mathbf{C u}$ & 7.13483823353634 & 25.22725381077340 & 2.53967070313805 \\
\hline $\mathbf{C u}$ & 7.13553757642357 & 27.74713636366050 & 2.54257681909992 \\
\hline $\mathrm{Cu}$ & 7.13418989078404 & 30.26889276102220 & 2.54090 \\
\hline $\mathrm{Cu}$ & 7.13508437454764 & 32.78977454 & 2.5402 \\
\hline $\mathrm{Cu}$ & 1.78323514119392 & 1.25883424689601 & 3.85326254577920 \\
\hline $\mathbf{C u}$ & 1.77858031302100 & 3.77875525042945 & 3.85523080734815 \\
\hline $\mathbf{C u}$ & 1.79718293667283 & 6.30487589826154 & 3.85888891260939 \\
\hline $\mathrm{Cu}$ & 1.78168604914815 & 8.82747273093280 & 3.83241085006513 \\
\hline $\mathrm{Cu}$ & 1.80020808947543 & 11.34945264859810 & 3.85361162019985 \\
\hline $\mathrm{Cu}$ & 1.77243013329422 & 13.87845778101860 & 3.83869220121720 \\
\hline $\mathbf{C u}$ & 1.78408653529961 & 16.39877989719460 & 3.85121294600910 \\
\hline $\mathrm{Cu}$ & 1.78047159294899 & 18.92401302236870 & 3.84822546940017 \\
\hline $\mathbf{C u}$ & 1.77780343942132 & 21.44631460156440 & 3.84325079607961 \\
\hline $\mathbf{C u}$ & 1.78864200559549 & 23.96919459045910 & 3.85346168654465 \\
\hline $\mathbf{C u}$ & 1.78067165066777 & 26.49007409368230 & 3.85430446606842 \\
\hline $\mathbf{C u}$ & 1.78246218809695 & 29.01040709504600 & 3.85685412022123 \\
\hline $\mathbf{C u}$ & 1.78207950977411 & 31.52957865315550 & 3.85592799823348 \\
\hline $\mathbf{C u}$ & 1.78286176864961 & 34.04898182055730 & 3.85357824366153 \\
\hline $\mathbf{C u}$ & 5.35132589606074 & 1.25883910719192 & 3.85326684713213 \\
\hline $\mathbf{C u}$ & 5.35598017956162 & 3.77875843063130 & 3.85523882807403 \\
\hline $\mathbf{C u}$ & 5.33738519797896 & 6.30487355703020 & 3.85885442347418 \\
\hline $\mathbf{C u}$ & 5.35286831432720 & 8.82748708390391 & 3.83240389530744 \\
\hline $\mathbf{C u}$ & 5.33436098847034 & 11.34945186536250 & 3.85361536392925 \\
\hline $\mathbf{C u}$ & 5.36210520614799 & 13.87853410778690 & 3.83875919971920 \\
\hline $\mathbf{C u}$ & 5.35044781528753 & 16.39887294981160 & 3.85143730909209 \\
\hline $\mathbf{C u}$ & 5.35398328287020 & 18.92397009747420 & 3.84838556946920 \\
\hline $\mathbf{C u}$ & 5.35665962076544 & 21.44626205047630 & 3.84336890988044 \\
\hline $\mathbf{C u}$ & 5.34587720434115 & 23.96913305186240 & 3.85349418965453 \\
\hline $\mathbf{C u}$ & 5.35388727570151 & 26.49004174396950 & 3.85432878110326 \\
\hline $\mathbf{C u}$ & 5.35210724079704 & 29.01039686118550 & 3.85685940586356 \\
\hline $\mathbf{C u}$ & 5.35247666718354 & 31.52957337722640 & 3.85593504648838 \\
\hline $\mathbf{C u}$ & 5.35169889165343 & 34.04897650985210 & 3.85357632470300 \\
\hline $\mathbf{C u}$ & 8.91818676202816 & 1.26105990113044 & 3.84987648262187 \\
\hline
\end{tabular}




\begin{tabular}{|c|c|c|c|}
\hline $\mathbf{C u}$ & 8.91819394693058 & 3.78396436009604 & 3.84989345413907 \\
\hline $\mathbf{C u}$ & 8.91819314636115 & 6.30380184716846 & 3.85115640592347 \\
\hline $\mathbf{C u}$ & 8.91818840294182 & 8.82628966196202 & 3.85729860283954 \\
\hline $\mathbf{C u}$ & 8.91819314921995 & 11.35516974126710 & 3.85690824316646 \\
\hline $\mathbf{C u}$ & 8.91820096270553 & 13.87344875304340 & 3.85512109256645 \\
\hline $\mathbf{C u}$ & 8.91821340485495 & 16.39920453400470 & 3.85289005359818 \\
\hline $\mathbf{C u}$ & 8.91822610667903 & 18.92015304058490 & 3.85095459025526 \\
\hline $\mathbf{C u}$ & 8.91821304004622 & 21.44220302182670 & 3.85448064145818 \\
\hline $\mathbf{C u}$ & 8.91819530446737 & 23.96662197465720 & 3.84838054081195 \\
\hline $\mathbf{C u}$ & 8.91819158584367 & 26.48855340251260 & 3.85477818962031 \\
\hline $\mathbf{C u}$ & 8.91818600428090 & 29.00616900428560 & 3.84788110858771 \\
\hline $\mathbf{C u}$ & 8.91819221734051 & 31.53327467229900 & 3.84940795364956 \\
\hline $\mathbf{C u}$ & 8.91819075787526 & 34.05094949403620 & 3.84910894135364 \\
\hline $\mathbf{C u}$ & -0.00066263932336 & 35.31181905714590 & 5.05544482230987 \\
\hline $\mathbf{C u}$ & 10.70148832735720 & 2.52105078682033 & 5.05868227222837 \\
\hline $\mathbf{C u}$ & 0.00267218789506 & 5.04764215327370 & 5.05725729380155 \\
\hline $\mathbf{C u}$ & 0.00586635657086 & 7.56538414461010 & 5.05203691147966 \\
\hline $\mathbf{C u}$ & 0.00908172737647 & 10.09312138878050 & 5.04693382005449 \\
\hline $\mathbf{C u}$ & 0.00980823065970 & 12.61225142894780 & 5.05940458437184 \\
\hline $\mathbf{C u}$ & 0.00228944580100 & 15.13970656659370 & 5.05379759674000 \\
\hline $\mathbf{C u}$ & 10.70151173651840 & 17.66164784028470 & 5.05430053767126 \\
\hline $\mathbf{C u}$ & 0.00326121341515 & 20.18267047587390 & 5.05528613900632 \\
\hline $\mathbf{C u}$ & 1616078 & 96098267990 & 354146 \\
\hline $\mathbf{C u}$ & 20879383 & 25.22349060540300 & 79171 \\
\hline $\mathbf{C u}$ & 10.70115525054050 & 27.74896584001870 & 5.06 \\
\hline $\mathbf{C u}$ & 10.69768012741230 & 30.26776648177250 & 7634003 \\
\hline $\mathbf{C u}$ & 0.00121001777571 & 32.78900851405090 & 5.05960460200320 \\
\hline $\mathbf{C u}$ & 3.56727738582173 & 35.30894788221810 & 5.05682242255078 \\
\hline $\mathbf{C u}$ & 3.56727642475000 & 2.51640389655001 & 5.05812466934841 \\
\hline $\mathbf{C u}$ & 3.56728038604143 & 5.02164798269641 & 5.04943888912201 \\
\hline $\mathbf{C u}$ & 3.56728542452884 & 7.51937451801535 & 5.16070804709786 \\
\hline $\mathbf{C u}$ & 3.56727472050932 & 10.10790922638170 & 5.15565821662608 \\
\hline $\mathbf{C u}$ & 3.56729335955030 & 12.62804188070720 & 5.13724123111373 \\
\hline $\mathbf{C u}$ & 3.56716645563105 & 15.14441570790040 & 5.08515483309579 \\
\hline $\mathbf{C u}$ & 3.56642931071202 & 17.65045854521620 & 5.17753056972212 \\
\hline $\mathbf{C u}$ & 3.56716351971495 & 20.17165450592970 & 5.09375042164439 \\
\hline $\mathbf{C u}$ & 3.56708091855960 & 22.73058863886670 & 5.17529075417144 \\
\hline $\mathbf{C u}$ & 3.56725183363930 & 25.25151822432090 & 5.06006724667701 \\
\hline $\mathbf{C u}$ & 3.56727708577307 & 27.75569740415710 & 5.05902950684155 \\
\hline $\mathbf{C u}$ & 3.56727617836059 & 30.27551032783970 & 5.05972389075034 \\
\hline $\mathbf{C u}$ & 3.56726989300564 & 32.79096601090740 & 5.05895337215869 \\
\hline $\mathbf{C u}$ & 7.13521466112693 & 35.31182142921240 & 5.05544555354542 \\
\hline $\mathbf{C u}$ & 7.13490805639264 & 2.52106187292858 & 5.05869953402622 \\
\hline $\mathbf{C u}$ & 7.13189201025995 & 5.04764414258787 & 5.05725336930658 \\
\hline $\mathbf{C u}$ & 7.12868655783514 & 7.56537964857353 & 5.05203085833145 \\
\hline $\mathbf{C u}$ & 7.12550180731372 & 10.09314597437190 & 5.04692735387088 \\
\hline $\mathbf{C u}$ & 7.12472420728581 & 12.61226490303560 & 5.05941445812197 \\
\hline $\mathbf{C u}$ & 7.13231843964520 & 15.13975367624050 & 5.05377924549140 \\
\hline $\mathbf{C u}$ & 7.13509448147650 & 17.66172640598350 & 5.05444202310185 \\
\hline $\mathbf{C u}$ & 7.13134881538091 & 20.18268218238250 & 5.05521670965909 \\
\hline $\mathbf{C u}$ & 7.13046120871331 & 22.70595591896680 & 5.05278127709383 \\
\hline $\mathbf{C u}$ & 7.13096880334899 & 25.22346503426530 & 5.05628167877520 \\
\hline $\mathbf{C u}$ & 7.13523863685984 & 27.74894528615770 & 5.06347287028905 \\
\hline $\mathbf{C u}$ & 7.13871451185779 & 30.26775859062240 & 5.05716955941271 \\
\hline
\end{tabular}




\begin{tabular}{|c|c|c|c|}
\hline $\mathbf{C u}$ & 7.13335530665680 & 32.78900650540350 & 5.05960692645839 \\
\hline \multicolumn{4}{|c|}{ CI-NEB Image 21} \\
\hline Element & X-Coordinate $(\AA))$ & Y-Coordinate $(\AA))$ & Z-Coordinate (Å) \\
\hline $\mathbf{F}$ & 4.66317553645919 & 12.67082520800160 & 7.76643635748138 \\
\hline $\mathbf{F}$ & 2.47145035772862 & 12.67072191269500 & 7.76639666428726 \\
\hline $\mathbf{F}$ & 3.56760471598747 & 8.81692009461194 & 6.63632329359409 \\
\hline $\mathbf{F}$ & 4.63679026953962 & 17.84698146479580 & 7.84504734511478 \\
\hline $\mathbf{F}$ & 2.51124562519093 & 17.84399020378810 & 7.85212513555539 \\
\hline $\mathbf{F}$ & 3.56724474303206 & 14.53237508239160 & 7.28786576829567 \\
\hline $\mathbf{F}$ & 4.63811718946367 & 22.52469055922850 & 7.86434211764490 \\
\hline $\mathbf{F}$ & 2.49854054366788 & 22.52467337788160 & 7.86570788241658 \\
\hline $\mathbf{F}$ & 3.56753281527696 & 20.40703364713650 & 7.06894826210047 \\
\hline C & 3.56730709651339 & 13.09398455858570 & 7.05812448493626 \\
\hline C & 3.57135022871762 & 17.84269955242030 & 7.06413831498786 \\
\hline C & 3.56783968631788 & 22.36946330999150 & 7.08663509695302 \\
\hline $\mathbf{C u}$ & 0.00000000000000 & 0.00000000000000 & 0.00000000000000 \\
\hline $\mathbf{C u}$ & 0.00000000000000 & 2.52244512012115 & 0.00000000000000 \\
\hline $\mathrm{Cu}$ & 0.00000000000000 & 5.04488953395804 & 0.00000000000000 \\
\hline $\mathbf{C u}$ & 0.00000000000000 & 7.56733465407861 & 0.00000000000000 \\
\hline $\mathbf{C u}$ & 0.00000000000000 & 10.08977871477400 & 0.00000000000000 \\
\hline $\mathbf{C u}$ & 0.00000000000000 & 12.61222383489520 & 0.00000000000000 \\
\hline $\mathbf{C u}$ & 0.00000000000000 & 15.13466895501340 & 0.00000000000000 \\
\hline $\mathbf{C u}$ & 0.00000000000000 & 17.65711336885320 & 0.00000000000000 \\
\hline $\mathbf{C u}$ & 0.00000000000000 & 20.17955848897460 & 0.00000000000000 \\
\hline $\mathbf{C u}$ & 0.00000000000000 & 22.70200254966930 & 0.00000000000000 \\
\hline $\mathbf{C u}$ & 0.00000000000000 & 25.22444766979040 & 0.00000000000000 \\
\hline $\mathbf{C u}$ & 0.00000000000000 & 27.74689208362220 & 0.00000000000000 \\
\hline $\mathbf{C u}$ & 0.00000000000000 & 30.26933720374340 & 0.00000000000000 \\
\hline $\mathbf{C u}$ & 0.00000000000000 & 32.79178232386990 & 0.00000000000000 \\
\hline $\mathbf{C u}$ & 3.56727419238642 & 0.00000000000000 & 0.00000000000000 \\
\hline $\mathbf{C u}$ & 3.56727419238642 & 2.52244512012115 & 0.00000000000000 \\
\hline $\mathbf{C u}$ & 3.56727419238642 & 5.04488953395804 & 0.00000000000000 \\
\hline $\mathbf{C u}$ & 3.56727419238642 & 7.56733465407861 & 0.00000000000000 \\
\hline $\mathbf{C u}$ & 3.56727419238642 & 10.08977871477400 & 0.00000000000000 \\
\hline $\mathbf{C u}$ & 3.56727419238642 & 12.61222383489520 & 0.00000000000000 \\
\hline $\mathbf{C u}$ & 3.56727419238642 & 15.13466895501340 & 0.00000000000000 \\
\hline $\mathbf{C u}$ & 3.56727419238642 & 17.65711336885320 & 0.00000000000000 \\
\hline $\mathbf{C u}$ & 3.56727419238642 & 20.17955848897460 & 0.00000000000000 \\
\hline $\mathbf{C u}$ & 3.56727419238642 & 22.70200254966930 & 0.00000000000000 \\
\hline $\mathbf{C u}$ & 3.56727419238642 & 25.22444766979040 & 0.00000000000000 \\
\hline $\mathbf{C u}$ & 3.56727419238642 & 27.74689208362220 & 0.00000000000000 \\
\hline $\mathbf{C u}$ & 3.56727419238642 & 30.26933720374340 & 0.00000000000000 \\
\hline $\mathbf{C u}$ & 3.56727419238642 & 32.79178232386990 & 0.00000000000000 \\
\hline $\mathbf{C u}$ & 7.13454838477291 & 0.00000000000000 & 0.00000000000000 \\
\hline $\mathbf{C u}$ & 7.13454838477291 & 2.52244512012115 & 0.00000000000000 \\
\hline $\mathbf{C u}$ & 7.13454838477291 & 5.04488953395804 & 0.00000000000000 \\
\hline $\mathbf{C u}$ & 7.13454838477291 & 7.56733465407861 & 0.00000000000000 \\
\hline $\mathbf{C u}$ & 7.13454838477291 & 10.08977871477400 & 0.00000000000000 \\
\hline $\mathbf{C u}$ & 7.13454838477291 & 12.61222383489520 & 0.00000000000000 \\
\hline $\mathbf{C u}$ & 7.13454838477291 & 15.13466895501340 & 0.00000000000000 \\
\hline $\mathbf{C u}$ & 7.13454838477291 & 17.65711336885320 & 0.00000000000000 \\
\hline $\mathbf{C u}$ & 7.13454838477291 & 20.17955848897460 & 0.00000000000000 \\
\hline $\mathbf{C u}$ & 7.13454838477291 & 22.70200254966930 & 0.00000000000000 \\
\hline $\mathbf{C u}$ & 7.13454838477291 & 25.22444766979040 & 0.00000000000000 \\
\hline
\end{tabular}




\begin{tabular}{|c|c|c|c|}
\hline $\mathrm{Cu}$ & 7.13454838477291 & 27.74689208362220 & 0.00000000000000 \\
\hline $\mathbf{C u}$ & 7.13454838477291 & 30.26933720374340 & 0.00000000000000 \\
\hline $\mathrm{Cu}$ & 7.13454838477291 & 32.79178232386990 & 0.00000000000000 \\
\hline $\mathbf{C u}$ & 1.78363955761358 & 1.26122414920059 & 1.26122399999993 \\
\hline $\mathbf{C u}$ & 1.78363955761358 & 3.78366926932170 & 1.26122399999993 \\
\hline $\mathbf{C u}$ & 1.78363955761358 & 6.30611333001662 & 1.26122399999993 \\
\hline $\mathrm{Cu}$ & 1.78363955761358 & 8.82855845013778 & 1.26122399999993 \\
\hline $\mathrm{Cu}$ & 1.78363955761358 & 11.35100286397460 & 1.26122399999993 \\
\hline $\mathbf{C u}$ & 1.78363955761358 & 13.87344798409260 & 1.26122399999993 \\
\hline $\mathbf{C u}$ & 1.78363955761358 & 16.39589310421720 & 1.26122399999993 \\
\hline $\mathbf{C u}$ & 1.78363955761358 & 18.91833716491180 & 1.26122399999993 \\
\hline $\mathrm{Cu}$ & 1.78363955761358 & 21.44078263817520 & 1.26122399999993 \\
\hline $\mathrm{Cu}$ & 1.78363955761358 & 23.96322669886980 & 1.26122399999993 \\
\hline $\mathrm{Cu}$ & 1.78363955761358 & 26.48567181899090 & 1.26122399999993 \\
\hline $\mathrm{Cu}$ & 1.78363955761358 & 29.00811693910730 & 1.26122399999993 \\
\hline $\mathbf{C u}$ & 1.78363955761358 & 31.53056135294370 & 1.26122399999993 \\
\hline $\mathrm{Cu}$ & 1.78363955761358 & 34.05300647307050 & 1.26122399999993 \\
\hline $\mathbf{C u}$ & 5.35091375000000 & 1.26122414920059 & 1.26122399999993 \\
\hline $\mathbf{C u}$ & 5.35091375000000 & 3.78366926932170 & 1.26122399999993 \\
\hline $\mathrm{Cu}$ & 5.35091375000000 & 6.30611333001662 & 1.26122399999993 \\
\hline $\mathbf{C u}$ & 5.35091375000000 & 8.82855845013778 & 1.26122399999993 \\
\hline $\mathbf{C u}$ & 5.35091375000000 & 11.35100286397460 & 1.26122399999993 \\
\hline $\mathrm{Cu}$ & 5.35091375000000 & 13.87344798409260 & 1.26122399999993 \\
\hline $\mathrm{Cu}$ & 00000 & 0421720 & 1.26122399999993 \\
\hline $\mathrm{Cu}$ & 000000 & 18.91 & 2399999993 \\
\hline $\mathrm{Cu}$ & 5.35091 & 3263817520 & 1.26122399999993 \\
\hline $\mathbf{C u}$ & 5.35091375000000 & 23.96322669886980 & 1.26122399999993 \\
\hline $\mathrm{Cu}$ & 5.35091375000000 & 26.48567181899090 & 1.26122399999993 \\
\hline $\mathbf{C u}$ & 5.35091375000000 & 29.00811693910730 & 1.26122399999993 \\
\hline $\mathrm{Cu}$ & 5.35091375000000 & 31.53056135294370 & 1.26122399999993 \\
\hline $\mathbf{C u}$ & 5.35091375000000 & 34.05300647307050 & 1.26122399999993 \\
\hline $\mathrm{Cu}$ & 8.91818794238642 & 1.26122414920059 & 1.26122399999993 \\
\hline $\mathrm{Cu}$ & 8.91818794238642 & 3.78366926932170 & 1.26122399999993 \\
\hline $\mathbf{C u}$ & 8.91818794238642 & 6.30611333001662 & 1.26122399999993 \\
\hline $\mathbf{C u}$ & 8.91818794238642 & 8.82855845013778 & 1.26122399999993 \\
\hline $\mathbf{C u}$ & 8.91818794238642 & 11.35100286397460 & 1.26122399999993 \\
\hline $\mathrm{Cu}$ & 8.91818794238642 & 13.87344798409260 & 1.26122399999993 \\
\hline $\mathbf{C u}$ & 8.91818794238642 & 16.39589310421720 & 1.26122399999993 \\
\hline $\mathrm{Cu}$ & 8.91818794238642 & 18.91833716491180 & 1.26122399999993 \\
\hline $\mathbf{C u}$ & 8.91818794238642 & 21.44078263817520 & 1.26122399999993 \\
\hline $\mathrm{Cu}$ & 8.91818794238642 & 23.96322669886980 & 1.26122399999993 \\
\hline $\mathbf{C u}$ & 8.91818794238642 & 26.48567181899090 & 1.26122399999993 \\
\hline $\mathbf{C u}$ & 8.91818794238642 & 29.00811693910730 & 1.26122399999993 \\
\hline $\mathbf{C u}$ & 8.91818794238642 & 31.53056135294370 & 1.26122399999993 \\
\hline $\mathbf{C u}$ & 8.91818794238642 & 34.05300647307050 & 1.26122399999993 \\
\hline $\mathbf{C u}$ & 0.00098072260541 & 35.31388695573130 & 2.53873760934810 \\
\hline $\mathrm{Cu}$ & 10.69924020015760 & 2.52328716722534 & 2.54066356309312 \\
\hline $\mathrm{Cu}$ & 0.00367993707495 & 5.04534505639474 & 2.54223607070594 \\
\hline $\mathrm{Cu}$ & 10.70065284612780 & 7.56523728550808 & 2.54290713060676 \\
\hline $\mathrm{Cu}$ & 10.70057143147930 & 10.09069094034900 & 2.54079320848439 \\
\hline $\mathbf{C u}$ & 10.70032208567230 & 12.61236545203880 & 2.54780638227438 \\
\hline $\mathbf{C u}$ & 10.69636171096820 & 15.13686586902190 & 2.53741884687815 \\
\hline $\mathbf{C u}$ & 10.70059220051240 & 17.65739036129540 & 2.54255120169070 \\
\hline $\mathbf{C u}$ & 10.69813043154860 & 20.18037327918470 & 2.53767427211449 \\
\hline
\end{tabular}




\begin{tabular}{|c|c|c|c|}
\hline $\mathbf{C u}$ & 10.69965709669130 & 22.70441091893770 & 2.54145139720662 \\
\hline $\mathbf{C u}$ & 10.70162435001350 & 25.22670594075260 & 2.53974677808211 \\
\hline $\mathbf{C u}$ & 10.70081064472510 & 27.74689578314950 & 2.54222183150571 \\
\hline $\mathbf{C u}$ & 10.70181233493890 & 30.26920216711180 & 2.54105443267612 \\
\hline $\mathbf{C u}$ & 10.70120793142520 & 32.78982332162630 & 2.54019791266950 \\
\hline $\mathbf{C u}$ & 3.56727274554463 & 35.31408888624010 & 2.54188496116636 \\
\hline $\mathbf{C u}$ & 3.56727543600175 & 2.52184895002897 & 2.54465979920861 \\
\hline $\mathbf{C u}$ & 3.56727461597263 & 5.04427989862400 & 2.54055474591456 \\
\hline $\mathbf{C u}$ & 3.56728099309911 & 7.56998166055348 & 2.58045611409404 \\
\hline $\mathbf{C u}$ & 3.56727312661598 & 10.09273755328930 & 2.58896850505213 \\
\hline $\mathbf{C u}$ & 3.56728629634024 & 12.62283774823890 & 2.58097592619615 \\
\hline $\mathbf{C u}$ & 3.56731114456034 & 15.14034643933910 & 2.57809504615812 \\
\hline $\mathbf{C u}$ & 3.56726445101898 & 17.66028757676540 & 2.60459849866840 \\
\hline $\mathbf{C u}$ & 3.56729019272217 & 20.18071862728720 & 2.60183777640832 \\
\hline $\mathrm{Cu}$ & 3.56727120082874 & 22.69676026792660 & 2.60012369192089 \\
\hline $\mathrm{Cu}$ & 3.56729061603354 & 25.22037825019510 & 2.54739345474062 \\
\hline $\mathrm{Cu}$ & 3.56728381639436 & 27.74855282538630 & 2.54139196307857 \\
\hline $\mathbf{C u}$ & 3.56728204865687 & 30.26843260898700 & 2.54251115292602 \\
\hline $\mathbf{C u}$ & 3.56727876222401 & 32.79101281302780 & 2.54285908556322 \\
\hline $\mathbf{C u}$ & 7.13357069214404 & 35.31389352653000 & 2.53873962408243 \\
\hline $\mathbf{C u}$ & 7.13713818229122 & 2.52328338561684 & 2.54067495412282 \\
\hline $\mathbf{C u}$ & 7.13087493398438 & 5.04534016397707 & 2.54222940666926 \\
\hline $\mathbf{C u}$ & 7.13573022178986 & 7.56524491335956 & 2.54290166237100 \\
\hline $\mathbf{C u}$ & 7.13580961069142 & 10.09068926028300 & 2.54076913139754 \\
\hline $\mathbf{C u}$ & 7.13604426387551 & 12.61237756416660 & 2.54782752071821 \\
\hline $\mathbf{C u}$ & 7.14000464719892 & 15.13684692113370 & 2.53745476591881 \\
\hline $\mathbf{C u}$ & 7.13570974366310 & 17.65737347925040 & 2.54258844855433 \\
\hline $\mathbf{C u}$ & 7.13820512048128 & 20.18032659363450 & 2.53773841221113 \\
\hline $\mathbf{C u}$ & 7.13671233145382 & 22.70436722660280 & 2.54146929222405 \\
\hline $\mathbf{C u}$ & 7.13475556230908 & 25.22669004366980 & 2.53974915775567 \\
\hline $\mathbf{C u}$ & 7.13556852675160 & 27.74688283661970 & 2.54223663320054 \\
\hline $\mathbf{C u}$ & 7.13456066701667 & 30.26919818228770 & 2.54105576169497 \\
\hline $\mathbf{C u}$ & 7.13517035270895 & 32.78982193393030 & 2.54020132901543 \\
\hline $\mathbf{C u}$ & 1.78298287487447 & 1.25879776235249 & 3.85348419024834 \\
\hline $\mathbf{C u}$ & 1.77867291667203 & 3.77844829859884 & 3.85519672915927 \\
\hline $\mathbf{C u}$ & 1.79743937420272 & 6.30493530317351 & 3.85893195028324 \\
\hline $\mathbf{C u}$ & 1.78147868970135 & 8.82741131139596 & 3.83273630351511 \\
\hline $\mathbf{C u}$ & 1.80033785851331 & 11.34977864978440 & 3.85373792732782 \\
\hline $\mathbf{C u}$ & 1.77260292692203 & 13.87876621012100 & 3.83849019527371 \\
\hline $\mathbf{C u}$ & 1.78483183949848 & 16.39952926165380 & 3.85215270869719 \\
\hline $\mathbf{C u}$ & 1.78012070139851 & 18.92412384555850 & 3.84715979815244 \\
\hline $\mathbf{C u}$ & 1.77890826125487 & 21.44565876906310 & 3.84283184938913 \\
\hline $\mathbf{C u}$ & 1.78811932105301 & 23.96896819598420 & 3.85346854952072 \\
\hline $\mathbf{C u}$ & 1.78034548335096 & 26.49019657089430 & 3.85430479498196 \\
\hline $\mathbf{C u}$ & 1.78182342262875 & 29.01077774126770 & 3.85708204362498 \\
\hline $\mathbf{C u}$ & 1.78208215985587 & 31.52958715735140 & 3.85599685353796 \\
\hline $\mathbf{C u}$ & 1.78271650333196 & 34.04893036232460 & 3.85358365824217 \\
\hline $\mathbf{C u}$ & 5.35157197886320 & 1.25880750562936 & 3.85348941935007 \\
\hline $\mathbf{C u}$ & 5.35588378243155 & 3.77845000016667 & 3.85520750618951 \\
\hline $\mathbf{C u}$ & 5.33712913039728 & 6.30493055114729 & 3.85889847850791 \\
\hline $\mathbf{C u}$ & 5.35306575097498 & 8.82742271660820 & 3.83273040243418 \\
\hline $\mathbf{C u}$ & 5.33422406633846 & 11.34976939559550 & 3.85371449117035 \\
\hline $\mathbf{C u}$ & 5.36192690230592 & 13.87885055500860 & 3.83856589457396 \\
\hline $\mathbf{C u}$ & 5.34961179414572 & 16.39969446423260 & 3.85239180006198 \\
\hline
\end{tabular}




\begin{tabular}{|c|c|c|c|}
\hline $\mathrm{Cu}$ & 5.35430154930491 & 18.92406582692880 & 3.84745275759248 \\
\hline $\mathrm{Cu}$ & 5.35556657756117 & 21.44559852717980 & 3.84292769336249 \\
\hline $\mathrm{Cu}$ & 5.34642080690807 & 23.96892453934460 & 3.85348571863236 \\
\hline $\mathbf{C u}$ & 5.35422100694876 & 26.49016687795540 & 3.85433639712943 \\
\hline $\mathbf{C u}$ & 5.35274441730905 & 29.01077754945840 & 3.85708743444721 \\
\hline $\mathbf{C u}$ & 5.35247208307847 & 31.52958324129270 & 3.85600104254064 \\
\hline $\mathrm{Cu}$ & 5.35184482018435 & 34.04892515438670 & 3.85359547797479 \\
\hline $\mathrm{Cu}$ & 8.91818748591555 & 1.26158551446529 & 3.84971979235556 \\
\hline $\mathbf{C u}$ & 8.91819595012808 & 3.78429684724669 & 3.85003922747319 \\
\hline $\mathbf{C u}$ & 8.91819078490994 & 6.30380968220928 & 3.85130006360064 \\
\hline $\mathbf{C u}$ & 8.91819549283833 & 8.82605996419695 & 3.85717510389239 \\
\hline $\mathrm{Cu}$ & 8.91819458099162 & 11.35518295677980 & 3.85682269166893 \\
\hline $\mathrm{Cu}$ & 8.91818856565823 & 13.87330244748980 & 3.85507143231740 \\
\hline $\mathrm{Cu}$ & 8.91822303803496 & 16.39844834088630 & 3.85293595532611 \\
\hline $\mathbf{C u}$ & 8.91827449450663 & 18.92052327878220 & 3.85131618792405 \\
\hline $\mathbf{C u}$ & 8.91821169316655 & 21.44257089036330 & 3.85451067301796 \\
\hline $\mathrm{Cu}$ & 8.91820082432493 & 23.96731294922270 & 3.84798208467855 \\
\hline $\mathbf{C u}$ & 8.91819459280918 & 26.48761771913500 & 3.85440771738405 \\
\hline $\mathbf{C u}$ & 8.91818794765327 & 29.00647001988460 & 3.84810264706531 \\
\hline $\mathrm{Cu}$ & 8.91819144934551 & 31.53273987160400 & 3.84951634259214 \\
\hline $\mathbf{C u}$ & 8.91818532791890 & 34.05050431311380 & 3.84886636054331 \\
\hline $\mathbf{C u}$ & 10.70065114794690 & 35.31174386898960 & 5.05532540958871 \\
\hline $\mathrm{Cu}$ & 10.70114257168010 & 2.52089792750203 & 5078829272 \\
\hline $\mathrm{Cu}$ & 0.00296510034314 & 5.04768866063758 & 7475 \\
\hline $\mathrm{Cu}$ & 0.00567908082971 & 7.5653113 & 3134 \\
\hline $\mathrm{Cu}$ & 0.00878026849653 & 10.09293048565570 & 07998 \\
\hline $\mathrm{Cu}$ & 0.00973525861428 & 12.61220066003210 & 5.05935269084815 \\
\hline $\mathrm{Cu}$ & 0.00269701545440 & 15.13945447342180 & 5.05349470222859 \\
\hline $\mathbf{C u}$ & 0.00007956878394 & 17.66179202216780 & 5.05416154877149 \\
\hline $\mathrm{Cu}$ & 0.00304366299521 & 20.18299037151640 & 5.05511251790121 \\
\hline $\mathrm{Cu}$ & 0.00394095269457 & 22.70596434351870 & 5.05061165134190 \\
\hline $\mathrm{Cu}$ & 0.00348470713027 & 25.22391189258990 & 5.05696843442836 \\
\hline $\mathrm{Cu}$ & 10.70071136734820 & 27.74838777438880 & 5.06307547764606 \\
\hline $\mathrm{Cu}$ & 10.69764033450750 & 30.26787818843730 & 5.05768746557053 \\
\hline $\mathrm{Cu}$ & 0.00073694415282 & 32.78885644255030 & 5.05984543013739 \\
\hline $\mathbf{C u}$ & 3.56727708120098 & 35.30889437703230 & 5.05685431570348 \\
\hline $\mathrm{Cu}$ & 3.56727189116004 & 2.51647414127392 & 5.05873948790706 \\
\hline $\mathbf{C u}$ & 3.56727651936533 & 5.02175031537331 & 5.04969112369458 \\
\hline $\mathrm{Cu}$ & 3.56727887878739 & 7.51969041817644 & 5.16101818766901 \\
\hline $\mathbf{C u}$ & 3.56727344931274 & 10.10649670943700 & 5.15517622532226 \\
\hline $\mathrm{Cu}$ & 3.56729291138878 & 12.62676722072220 & 5.13743524040672 \\
\hline $\mathbf{C u}$ & 3.56713023357494 & 15.14190974227490 & 5.08290720212984 \\
\hline $\mathbf{C u}$ & 3.56625090097483 & 17.64986338834820 & 5.17986706198769 \\
\hline $\mathbf{C u}$ & 3.56700738164767 & 20.18033769319440 & 5.10797711219686 \\
\hline $\mathbf{C u}$ & 3.56713106762506 & 22.73296595437400 & 5.17660377713242 \\
\hline $\mathbf{C u}$ & 3.56725664469494 & 25.25282876295640 & 5.05852452766909 \\
\hline $\mathbf{C u}$ & 3.56727966193778 & 27.75593321422600 & 5.05860155963246 \\
\hline $\mathrm{Cu}$ & 3.56726912937116 & 30.27573108790460 & 5.05981601188257 \\
\hline $\mathrm{Cu}$ & 3.56727969643114 & 32.79056879976320 & 5.05903800561432 \\
\hline $\mathrm{Cu}$ & 7.13573039635900 & 35.31176244999920 & 5.05533238659205 \\
\hline $\mathbf{C u}$ & 7.13525513354384 & 2.52090829226217 & 5.05896720891131 \\
\hline $\mathbf{C u}$ & 7.13160143842661 & 5.04769072327066 & 5.05721954738280 \\
\hline $\mathbf{C u}$ & 7.12889443038357 & 7.56531892310107 & 5.05186110508865 \\
\hline $\mathbf{C u}$ & 7.12580223311766 & 10.09296121893540 & 5.04710230304732 \\
\hline
\end{tabular}




\begin{tabular}{|c|c|c|c|}
\hline $\mathbf{C u}$ & 7.12481064377467 & 12.61223694600240 & 5.05935431966142 \\
\hline $\mathbf{C u}$ & 7.13190691159700 & 15.13952226498240 & 5.05347143862514 \\
\hline $\mathbf{C u}$ & 7.13480723865396 & 17.66185351043410 & 5.05421596410957 \\
\hline $\mathbf{C u}$ & 7.13158204679020 & 20.18300343187440 & 5.05503636860049 \\
\hline $\mathbf{C u}$ & 7.13060917077472 & 22.70596972162070 & 5.05058853287629 \\
\hline $\mathbf{C u}$ & 7.13110224594390 & 25.22387306338230 & 5.05695690134062 \\
\hline $\mathbf{C u}$ & 7.13568769944275 & 27.74836185649720 & 5.06310091939254 \\
\hline $\mathbf{C u}$ & 7.13874197569605 & 30.26786510906900 & 5.05768700156798 \\
\hline $\mathbf{C u}$ & 7.13381865645285 & 32.78886253444970 & 5.05983338373174 \\
\hline \multicolumn{4}{|c|}{ CI-NEB Image 22} \\
\hline Element & X-Coordinate (Å) & Y-Coordinate (Å) & Z-Coordinate (§̊) \\
\hline $\mathbf{F}$ & 4.66310332192774 & 12.67205851018880 & 7.76601894828813 \\
\hline $\mathbf{F}$ & 2.47155729856229 & 12.67192410548570 & 7.76597787909055 \\
\hline $\mathbf{F}$ & 3.56761030753242 & 8.81718626942891 & 6.63772615205724 \\
\hline $\mathbf{F}$ & 4.63820701448224 & 17.84371541194070 & 7.85400671825082 \\
\hline $\mathbf{F}$ & 2.51024130369541 & 17.84018607044420 & 7.86211863376886 \\
\hline $\mathbf{F}$ & 3.56723886112362 & 14.53362313518530 & 7.28615481400663 \\
\hline $\mathbf{F}$ & 4.63278513222799 & 22.53933759331420 & 7.86081033238943 \\
\hline $\mathbf{F}$ & 2.50358823523311 & 22.53940426888550 & 7.86158426063768 \\
\hline $\mathbf{F}$ & 3.56749711771237 & 20.18574958399550 & 7.04884433458178 \\
\hline $\mathbf{C}$ & 3.56731851118175 & 13.09521924667310 & 7.05749178626192 \\
\hline $\mathbf{C}$ & 3.57107056912063 & 17.90219645799130 & 7.07862045849379 \\
\hline C & 3.56789349624351 & 22.48145903950150 & 7.07728656102181 \\
\hline $\mathbf{C u}$ & 0.00000000000000 & 0.00000000000000 & 0.00000000000000 \\
\hline $\mathbf{C u}$ & 0.00000000000000 & 2.52244512012115 & 0.00000000000000 \\
\hline $\mathbf{C u}$ & 0.00000000000000 & 5.04488953395804 & 0.00000000000000 \\
\hline $\mathbf{C u}$ & 0.00000000000000 & 7.56733465407835 & 0.00000000000000 \\
\hline $\mathbf{C u}$ & 0.00000000000000 & 10.08977871477400 & 0.00000000000000 \\
\hline $\mathbf{C u}$ & 0.00000000000000 & 12.61222383489520 & 0.00000000000000 \\
\hline $\mathbf{C u}$ & 0.00000000000000 & 15.13466895501340 & 0.00000000000000 \\
\hline $\mathbf{C u}$ & 0.00000000000000 & 17.65711336885320 & 0.00000000000000 \\
\hline $\mathbf{C u}$ & 0.00000000000000 & 20.17955848897460 & 0.00000000000000 \\
\hline $\mathbf{C u}$ & 0.00000000000000 & 22.70200254966930 & 0.00000000000000 \\
\hline $\mathbf{C u}$ & 0.00000000000000 & 25.22444766979040 & 0.00000000000000 \\
\hline $\mathbf{C u}$ & 0.00000000000000 & 27.74689208362170 & 0.00000000000000 \\
\hline $\mathbf{C u}$ & 0.00000000000000 & 30.26933720374290 & 0.00000000000000 \\
\hline $\mathbf{C u}$ & 0.00000000000000 & 32.79178232386990 & 0.00000000000000 \\
\hline $\mathbf{C u}$ & 3.56727419238642 & 0.00000000000000 & 0.00000000000000 \\
\hline $\mathbf{C u}$ & 3.56727419238642 & 2.52244512012115 & 0.00000000000000 \\
\hline $\mathbf{C u}$ & 3.56727419238642 & 5.04488953395804 & 0.00000000000000 \\
\hline $\mathbf{C u}$ & 3.56727419238642 & 7.56733465407835 & 0.00000000000000 \\
\hline $\mathbf{C u}$ & 3.56727419238642 & 10.08977871477400 & 0.00000000000000 \\
\hline $\mathbf{C u}$ & 3.56727419238642 & 12.61222383489520 & 0.00000000000000 \\
\hline $\mathbf{C u}$ & 3.56727419238642 & 15.13466895501340 & 0.00000000000000 \\
\hline $\mathbf{C u}$ & 3.56727419238642 & 17.65711336885320 & 0.00000000000000 \\
\hline $\mathbf{C u}$ & 3.56727419238642 & 20.17955848897460 & 0.00000000000000 \\
\hline $\mathbf{C u}$ & 3.56727419238642 & 22.70200254966930 & 0.00000000000000 \\
\hline $\mathbf{C u}$ & 3.56727419238642 & 25.22444766979040 & 0.00000000000000 \\
\hline $\mathbf{C u}$ & 3.56727419238642 & 27.74689208362170 & 0.00000000000000 \\
\hline $\mathbf{C u}$ & 3.56727419238642 & 30.26933720374290 & 0.00000000000000 \\
\hline $\mathbf{C u}$ & 3.56727419238642 & 32.79178232386990 & 0.00000000000000 \\
\hline $\mathbf{C u}$ & 7.13454838477291 & 0.00000000000000 & 0.00000000000000 \\
\hline $\mathbf{C u}$ & 7.13454838477291 & 2.52244512012115 & 0.00000000000000 \\
\hline $\mathbf{C u}$ & 7.13454838477291 & 5.04488953395804 & 0.00000000000000 \\
\hline
\end{tabular}




\begin{tabular}{|c|c|c|c|}
\hline $\mathbf{C u}$ & 7.13454838477291 & 7.56733465407835 & 0.00000000000000 \\
\hline $\mathbf{C u}$ & 7.13454838477291 & 10.08977871477400 & 0.00000000000000 \\
\hline $\mathrm{Cu}$ & 7.13454838477291 & 12.61222383489520 & 0.00000000000000 \\
\hline $\mathbf{C u}$ & 7.13454838477291 & 15.13466895501340 & 0.00000000000000 \\
\hline $\mathbf{C u}$ & 7.13454838477291 & 17.65711336885320 & 0.00000000000000 \\
\hline $\mathbf{C u}$ & 7.13454838477291 & 20.17955848897460 & 0.00000000000000 \\
\hline $\mathbf{C u}$ & 7.13454838477291 & 22.70200254966930 & 0.00000000000000 \\
\hline $\mathbf{C u}$ & 7.13454838477291 & 25.22444766979040 & 0.00000000000000 \\
\hline $\mathbf{C u}$ & 7.13454838477291 & 27.74689208362170 & 0.00000000000000 \\
\hline $\mathbf{C u}$ & 7.13454838477291 & 30.26933720374290 & 0.00000000000000 \\
\hline $\mathbf{C u}$ & 7.13454838477291 & 32.79178232386990 & 0.00000000000000 \\
\hline $\mathbf{C u}$ & 1.78363955761358 & 1.26122414920059 & 1.26122399999993 \\
\hline $\mathbf{C u}$ & 1.78363955761358 & 3.78366926932145 & 1.26122399999993 \\
\hline $\mathrm{Cu}$ & 1.78363955761358 & 6.30611333001662 & 1.26122399999993 \\
\hline $\mathbf{C u}$ & 1.78363955761358 & 8.82855845013778 & 1.26122399999993 \\
\hline $\mathbf{C u}$ & 1.78363955761358 & 11.35100286397460 & 1.26122399999993 \\
\hline $\mathbf{C u}$ & 1.78363955761358 & 13.87344798409260 & 1.26122399999993 \\
\hline $\mathbf{C u}$ & 1.78363955761358 & 16.39589310421720 & 1.26122399999993 \\
\hline $\mathbf{C u}$ & 1.78363955761358 & 18.91833716491180 & 1.26122399999993 \\
\hline $\mathbf{C u}$ & 1.78363955761358 & 21.44078263817520 & 1.26122399999993 \\
\hline $\mathbf{C u}$ & 1.78363955761358 & 23.96322669886980 & 1.26122399999993 \\
\hline $\mathbf{C u}$ & 1.78363955761358 & 26.48567181899090 & 1.26122399999993 \\
\hline $\mathbf{C u}$ & 1.783639 & 29.00811693910680 & 1.26122399999993 \\
\hline $\mathbf{C u}$ & 1.783639 & 31.53056135294320 & 1.26122399999993 \\
\hline $\mathbf{C u}$ & 761358 & 7050 & 1.26122399999993 \\
\hline $\mathbf{C u}$ & 5.35091375000000 & 1.26122414920059 & 1.26122399999993 \\
\hline $\mathbf{C u}$ & 5.35091375000000 & 3.78366926932145 & 1.26122399999993 \\
\hline $\mathbf{C u}$ & 5.35091375000000 & 6.30611333001662 & 1.26122399999993 \\
\hline $\mathbf{C u}$ & 5.35091375000000 & 8.82855845013778 & 1.26122399999993 \\
\hline $\mathbf{C u}$ & 5.35091375000000 & 11.35100286397460 & 1.26122399999993 \\
\hline $\mathbf{C u}$ & 5.35091375000000 & 13.87344798409260 & 1.26122399999993 \\
\hline $\mathbf{C u}$ & 5.35091375000000 & 16.39589310421720 & 1.26122399999993 \\
\hline $\mathbf{C u}$ & 5.35091375000000 & 18.91833716491180 & 1.26122399999993 \\
\hline $\mathbf{C u}$ & 5.35091375000000 & 21.44078263817520 & 1.26122399999993 \\
\hline $\mathbf{C u}$ & 5.35091375000000 & 23.96322669886980 & 1.26122399999993 \\
\hline $\mathbf{C u}$ & 5.35091375000000 & 26.48567181899090 & 1.26122399999993 \\
\hline $\mathbf{C u}$ & 5.35091375000000 & 29.00811693910680 & 1.26122399999993 \\
\hline $\mathbf{C u}$ & 5.35091375000000 & 31.53056135294320 & 1.26122399999993 \\
\hline $\mathbf{C u}$ & 5.35091375000000 & 34.05300647307050 & 1.26122399999993 \\
\hline $\mathbf{C u}$ & 8.91818794238642 & 1.26122414920059 & 1.26122399999993 \\
\hline $\mathbf{C u}$ & 8.91818794238642 & 3.78366926932145 & 1.26122399999993 \\
\hline $\mathbf{C u}$ & 8.91818794238642 & 6.30611333001662 & 1.26122399999993 \\
\hline $\mathbf{C u}$ & 8.91818794238642 & 8.82855845013778 & 1.26122399999993 \\
\hline $\mathbf{C u}$ & 8.91818794238642 & 11.35100286397460 & 1.26122399999993 \\
\hline $\mathbf{C u}$ & 8.91818794238642 & 13.87344798409260 & 1.26122399999993 \\
\hline $\mathbf{C u}$ & 8.91818794238642 & 16.39589310421720 & 1.26122399999993 \\
\hline $\mathbf{C u}$ & 8.91818794238642 & 18.91833716491180 & 1.26122399999993 \\
\hline $\mathbf{C u}$ & 8.91818794238642 & 21.44078263817520 & 1.26122399999993 \\
\hline $\mathbf{C u}$ & 8.91818794238642 & 23.96322669886980 & 1.26122399999993 \\
\hline $\mathbf{C u}$ & 8.91818794238642 & 26.48567181899090 & 1.26122399999993 \\
\hline $\mathbf{C u}$ & 8.91818794238642 & 29.00811693910680 & 1.26122399999993 \\
\hline $\mathbf{C u}$ & 8.91818794238642 & 31.53056135294320 & 1.26122399999993 \\
\hline $\mathbf{C u}$ & 8.91818794238642 & 34.05300647307050 & 1.26122399999993 \\
\hline $\mathbf{C u}$ & 0.00084220447389 & 35.31376242955450 & 2.53849027241278 \\
\hline
\end{tabular}




\begin{tabular}{|c|c|c|c|}
\hline $\mathbf{C u}$ & 10.69932057769210 & 2.52293892153281 & 2.54046148596066 \\
\hline $\mathbf{C u}$ & 0.00383146074802 & 5.04521700059274 & 2.54243882730258 \\
\hline $\mathbf{C u}$ & 10.70066425568640 & 7.56528414091093 & 2.54290943001634 \\
\hline $\mathbf{C u}$ & 10.70071511572240 & 10.09084867454700 & 2.54094457562218 \\
\hline $\mathbf{C u}$ & 10.70042071784020 & 12.61251047850090 & 2.54794708038677 \\
\hline $\mathbf{C u}$ & 10.69647934815110 & 15.13673006526880 & 2.53760764548996 \\
\hline $\mathbf{C u}$ & 10.70047589013230 & 17.65735234818400 & 2.54217220453593 \\
\hline $\mathbf{C u}$ & 10.69787122656900 & 20.18054033214390 & 2.53760539238744 \\
\hline $\mathbf{C u}$ & 10.70002100076100 & 22.70425286756510 & 2.54143767503055 \\
\hline $\mathbf{C u}$ & 10.70165708129120 & 25.22628211631060 & 2.53982028453268 \\
\hline $\mathbf{C u}$ & 10.70065008340690 & 27.74684048542440 & 2.54169341537662 \\
\hline $\mathbf{C u}$ & 10.70150889143300 & 30.26954748352220 & 2.54108437716922 \\
\hline $\mathbf{C u}$ & 10.70109616110670 & 32.78994883021530 & 2.54016365215519 \\
\hline $\mathrm{Cu}$ & 3.56728212599261 & 35.31403346154040 & 2.54201855067626 \\
\hline $\mathbf{C u}$ & 3.56727860784616 & 2.52199761655047 & 2.54454355637175 \\
\hline $\mathbf{C u}$ & 3.56727538183292 & 5.04433791156476 & 2.54052385604460 \\
\hline $\mathbf{C u}$ & 3.56727384818757 & 7.57000867145265 & 2.58110336356235 \\
\hline $\mathbf{C u}$ & 3.56727294573525 & 10.09265793186680 & 2.58980653736523 \\
\hline $\mathbf{C u}$ & 3.56728130631310 & 12.62256801260420 & 2.58046319584962 \\
\hline $\mathbf{C u}$ & 2092990336 & 15.14006814392200 & 2.57716904436943 \\
\hline $\mathbf{C u}$ & 3.56727730145673 & 17.66088976758100 & 2.60513474519822 \\
\hline $\mathbf{C u}$ & 3.56732979719399 & 20.18140370358310 & 2.60272668929434 \\
\hline $\mathbf{C u}$ & 3.56726621702201 & 22.69755878209930 & 2.60104821362219 \\
\hline $\mathrm{Cu}$ & 3.56729365336331 & 25.22096844583660 & 037383250 \\
\hline $\mathrm{Cu}$ & 3.56727778271059 & 27.748463 & 5008018634 \\
\hline $\mathrm{Cu}$ & 3.56728605024690 & 30.268144 & 2.54247274497657 \\
\hline $\mathbf{C u}$ & 3.56727438424029 & 32.79153278294280 & 2.54253593979972 \\
\hline $\mathbf{C u}$ & 7.13370136313892 & 35.31376397956650 & 2.53849584586314 \\
\hline $\mathbf{C u}$ & 7.13706134994081 & 2.52294337118363 & 2.54047114791632 \\
\hline $\mathrm{Cu}$ & 7.13072056143372 & 5.04521310249903 & 2.54243350673599 \\
\hline $\mathrm{Cu}$ & 7.13571947575947 & 7.56529444856923 & 2.54290611186049 \\
\hline $\mathrm{Cu}$ & 7.13568078250491 & 10.09084054953520 & 2.54093256656186 \\
\hline $\mathrm{Cu}$ & 7.13595077927820 & 12.61252564715140 & 2.54797165112100 \\
\hline $\mathbf{C u}$ & 7.13983937533083 & 15.13675364212200 & 2.53768154888062 \\
\hline $\mathbf{C u}$ & 7.13581434327282 & 17.65734110805010 & 2.54234342099127 \\
\hline $\mathbf{C u}$ & 7.13847423812336 & 20.18051425742840 & 2.53764986498925 \\
\hline $\mathbf{C u}$ & 7.13637128462132 & 22.70422847273150 & 2.54142232157443 \\
\hline $\mathbf{C u}$ & 7.13473184099846 & 25.22628277339040 & 2.53981901574164 \\
\hline $\mathbf{C u}$ & 7.13573345255829 & 27.74683156800470 & 2.54170849555142 \\
\hline $\mathbf{C u}$ & 7.13485993278349 & 30.26953784773340 & 2.54109037411870 \\
\hline $\mathbf{C u}$ & 7.13528433757104 & 32.78994809687190 & 2.54016057236586 \\
\hline $\mathbf{C u}$ & 1.78279059505408 & 1.25867975109464 & 3.85354078499188 \\
\hline $\mathbf{C u}$ & 1.77887414288094 & 3.77815060808007 & 3.85523367720750 \\
\hline $\mathbf{C u}$ & 1.79779930015437 & 6.30486742903460 & 3.85874177521784 \\
\hline $\mathbf{C u}$ & 1.78141425215751 & 8.82731735697021 & 3.83290976158392 \\
\hline $\mathbf{C u}$ & 1.80055003378045 & 11.35002212231830 & 3.85392581307770 \\
\hline $\mathbf{C u}$ & 1.77298270607696 & 13.87935392672660 & 3.83844747078694 \\
\hline $\mathbf{C u}$ & 1.78573561764220 & 16.40020641290030 & 3.85289591185883 \\
\hline $\mathbf{C u}$ & 1.77870445018873 & 18.92383559940080 & 3.84605678925231 \\
\hline $\mathbf{C u}$ & 1.78048193734912 & 21.44556089923190 & 3.84275930252668 \\
\hline $\mathbf{C u}$ & 1.78769859585335 & 23.96936216093940 & 3.85329247079341 \\
\hline $\mathbf{C u}$ & 1.78014690221367 & 26.49065328010770 & 3.85439680105573 \\
\hline $\mathbf{C u}$ & 1.78148046773978 & 29.01109692377030 & 3.85731380944768 \\
\hline $\mathbf{C u}$ & 1.78216970593100 & 31.52962647626380 & 3.85603383653661 \\
\hline
\end{tabular}




\begin{tabular}{|c|c|c|c|}
\hline $\mathrm{Cu}$ & 1.78259722202973 & 34.04886143008740 & 3.85359080092654 \\
\hline $\mathbf{C u}$ & 5.35176434803581 & 1.25869006710619 & 3.85354062996563 \\
\hline $\mathbf{C u}$ & 5.35568102565861 & 3.77814870854875 & 3.85525247181986 \\
\hline $\mathbf{C u}$ & 5.33676865338939 & 6.30485620512263 & 3.85870795788460 \\
\hline $\mathbf{C u}$ & 5.35311736667001 & 8.82732130353905 & 3.83290630403322 \\
\hline $\mathbf{C u}$ & 5.33401198649493 & 11.35001001923030 & 3.85390193467116 \\
\hline $\mathbf{C u}$ & 5.36154543336859 & 13.87940851763590 & 3.83851111499938 \\
\hline $\mathbf{C u}$ & 5.34856842232736 & 16.40041713444630 & 3.85336568935013 \\
\hline $\mathbf{C u}$ & 5.35581064290762 & 18.92374317508460 & 3.84641983443458 \\
\hline $\mathbf{C u}$ & 5.35408543381533 & 21.44555682722970 & 3.84275727529446 \\
\hline $\mathbf{C u}$ & 5.34688238295753 & 23.96934812233780 & 3.85328070429172 \\
\hline $\mathbf{C u}$ & 5.35443048413283 & 26.49062372493590 & 3.85443507032654 \\
\hline $\mathbf{C u}$ & 5.35309393648027 & 29.01107302607550 & 3.85731546743637 \\
\hline $\mathbf{C u}$ & 5.35238258029375 & 31.52961711852200 & 3.85603624885041 \\
\hline $\mathrm{Cu}$ & 5.35195467607210 & 34.04885526630130 & 3.85358974913420 \\
\hline $\mathbf{C u}$ & 8.91818852605295 & 1.26211168855553 & 3.84946318053063 \\
\hline $\mathrm{Cu}$ & 8.91819342933665 & 3.78476253935392 & 3.85023533960756 \\
\hline $\mathrm{Cu}$ & 8.91819897652693 & 6.30358937135825 & 3.85158803223709 \\
\hline $\mathrm{Cu}$ & 8.91819269707793 & 8.82567990783767 & 3.85696323773543 \\
\hline $\mathrm{Cu}$ & 8.91820351712521 & 11.35520361875060 & 3.85640242826190 \\
\hline $\mathbf{C u}$ & 8.91820813842554 & 13.87307113463290 & 3.85497511127325 \\
\hline $\mathbf{C u}$ & 8.91823265145943 & 16.39783124873090 & 3.85331602336595 \\
\hline $\mathbf{C u}$ & 8.91828908538807 & 18.92088863343830 & 3.85165489684120 \\
\hline $\mathrm{Cu}$ & 8.91823669285104 & 21.44318351731460 & 3.85438945280924 \\
\hline $\mathbf{C u}$ & 8.91819713145908 & 23.96747690684290 & 3.84767334139015 \\
\hline $\mathbf{C u}$ & 8.91819883980159 & 26.48681257036760 & 3.85416895369197 \\
\hline $\mathrm{Cu}$ & 8.91818670977104 & 29.00670060694690 & 3.84835139038703 \\
\hline $\mathbf{C u}$ & 8.91819051216436 & 31.53206617490730 & 3.84990219387113 \\
\hline $\mathbf{C u}$ & 8.91818692622439 & 34.04989627502870 & 3.84846854117464 \\
\hline $\mathbf{C u}$ & 10.70039280309530 & 35.31181911554290 & 5.05545454426172 \\
\hline $\mathbf{C u}$ & 10.70085264807110 & 2.52061641966376 & 5.05910608241057 \\
\hline $\mathbf{C u}$ & 0.00304011635177 & 5.04771749890400 & 5.05716860713479 \\
\hline $\mathbf{C u}$ & 0.00556831039006 & 7.56528440701968 & 5.05159433583373 \\
\hline $\mathbf{C u}$ & 0.00848708156447 & 10.09275868198000 & 5.04697568684360 \\
\hline $\mathbf{C u}$ & 0.00976084883981 & 12.61204708551380 & 5.05917567014523 \\
\hline $\mathbf{C u}$ & 0.00323945620380 & 15.13931365018670 & 5.05309033502245 \\
\hline $\mathbf{C u}$ & 0.00038367875086 & 17.66170078705310 & 5.05455856050161 \\
\hline $\mathbf{C u}$ & 0.00296713561338 & 20.18358893930160 & 5.05483279130163 \\
\hline $\mathbf{C u}$ & 0.00368303607632 & 22.70607612303150 & 5.04949691774092 \\
\hline $\mathbf{C u}$ & 0.00309659573794 & 25.22427948094550 & 5.05749742433107 \\
\hline $\mathbf{C u}$ & 10.70019164194620 & 27.74790046706860 & 5.06267267970053 \\
\hline $\mathbf{C u}$ & 10.69781176513660 & 30.26779509618750 & 5.05819516173110 \\
\hline $\mathbf{C u}$ & 0.00066165159926 & 32.78881208382500 & 5.05986755303147 \\
\hline $\mathbf{C u}$ & 3.56727372424684 & 35.30890991015220 & 5.05706499842923 \\
\hline $\mathbf{C u}$ & 3.56726497784927 & 2.51644631045175 & 5.05901665822751 \\
\hline $\mathbf{C u}$ & 3.56727141353381 & 5.02169850551674 & 5.04986583999565 \\
\hline $\mathbf{C u}$ & 3.56727724973695 & 7.51993950861896 & 5.16125361687434 \\
\hline $\mathbf{C u}$ & 3.56727245784354 & 10.10509302711560 & 5.15459742735439 \\
\hline $\mathbf{C u}$ & 3.56728243327537 & 12.62541266398120 & 5.13767280035503 \\
\hline $\mathbf{C u}$ & 3.56706562260830 & 15.14111434630900 & 5.08148052493492 \\
\hline $\mathbf{C u}$ & 3.56607134100680 & 17.65418073599620 & 5.17925053383157 \\
\hline $\mathbf{C u}$ & 3.56703397098596 & 20.19334452986710 & 5.10984813645628 \\
\hline $\mathbf{C u}$ & 3.56726620195704 & 22.73733855529700 & 5.18281775197837 \\
\hline $\mathbf{C u}$ & 3.56726889802256 & 25.25310334104630 & 5.05982167903370 \\
\hline
\end{tabular}




\begin{tabular}{|c|c|c|c|}
\hline $\mathbf{C u}$ & 3.56728037052578 & 27.75614882460950 & 5.05816092994136 \\
\hline $\mathbf{C u}$ & 3.56727708677788 & 30.27604000063170 & 5.06009308378617 \\
\hline $\mathbf{C u}$ & 3.56726111779681 & 32.79013457157460 & 5.05920763607595 \\
\hline $\mathbf{C u}$ & 7.13598721483653 & 35.31182944867340 & 5.05547149022159 \\
\hline $\mathbf{C u}$ & 7.13554506181874 & 2.52062260340704 & 5.05912775087974 \\
\hline $\mathbf{C u}$ & 7.13153267110221 & 5.04770765725990 & 5.05716815846639 \\
\hline $\mathbf{C u}$ & 7.12899914837982 & 7.56528848270253 & 5.05156071993481 \\
\hline $\mathbf{C u}$ & 7.12612237224922 & 10.09275368575320 & 5.04694029134080 \\
\hline $\mathbf{C u}$ & 7.12480496608199 & 12.61207785139280 & 5.05916853667049 \\
\hline $\mathbf{C u}$ & 7.13136275911271 & 15.13938831782990 & 5.05308851397348 \\
\hline $\mathbf{C u}$ & 7.13461773152973 & 17.66175159733090 & 5.05460545761815 \\
\hline $\mathbf{C u}$ & 7.13163310638084 & 20.18364804060000 & 5.05474407015364 \\
\hline $\mathbf{C u}$ & 7.13088605023638 & 22.70603367252080 & 5.04948436559867 \\
\hline $\mathbf{C u}$ & 7.13151157807946 & 25.22423540211300 & 5.05749190454707 \\
\hline $\mathbf{C u}$ & 7.13620661891921 & 27.74785840562020 & 70308805183 \\
\hline $\mathbf{C u}$ & 7.13856243888133 & 30.26779187639700 & 0865 \\
\hline $\mathbf{C u}$ & 7.13388774837591 & 32.78881604058840 & 5.05986046277402 \\
\hline \multicolumn{4}{|c|}{ CI-NEB Image 23} \\
\hline Element & X-Coordinate (Å) & Y-Coordinate (Å) & Z-Coordinate (Å) \\
\hline $\mathbf{F}$ & 4.66333583932689 & 12.67303808764150 & 7.75516541541425 \\
\hline $\mathbf{F}$ & 2.47132676773793 & 12.67294841550590 & 7.75515350681981 \\
\hline $\mathbf{F}$ & 3.56742715183085 & 8.81397290950060 & 6.63782330141647 \\
\hline $\mathbf{F}$ & 4.66348176519558 & 17.82767886838450 & 7.81584452725843 \\
\hline $\mathbf{F}$ & 2.47950059801314 & 17.82461658740840 & 7.82120470600576 \\
\hline $\mathbf{F}$ & 3.56727119659804 & 14.53246518295400 & 7.27210276808432 \\
\hline $\mathbf{F}$ & 4.63495070837511 & 22.56107789176940 & 7.82321407233886 \\
\hline $\mathbf{F}$ & 2.50197312271671 & 22.56127674587690 & 7.82435925650992 \\
\hline $\mathbf{F}$ & 3.56744731287227 & 19.67221324270730 & 7.22569379527360 \\
\hline C & 3.56732160511211 & 13.10026179009750 & 7.04953995040360 \\
\hline C & 3.56914519141610 & 18.18962764009430 & 7.08098642513895 \\
\hline $\mathrm{C}$ & 3.56802879843329 & 22.61366412012610 & 7.02954099416741 \\
\hline $\mathbf{C u}$ & 0.00000000000000 & 0.00000000000000 & 0.00000000000000 \\
\hline $\mathbf{C u}$ & 0.00000000000000 & 2.52244512012115 & 0.00000000000000 \\
\hline $\mathbf{C u}$ & 0.00000000000000 & 5.04488953395804 & 0.00000000000000 \\
\hline $\mathbf{C u}$ & 0.00000000000000 & 7.56733465407835 & 0.00000000000000 \\
\hline $\mathbf{C u}$ & 0.00000000000000 & 10.08977871477400 & 0.00000000000000 \\
\hline $\mathbf{C u}$ & 0.00000000000000 & 12.61222383489520 & 0.00000000000000 \\
\hline $\mathbf{C u}$ & 0.00000000000000 & 15.13466895501370 & 0.00000000000000 \\
\hline $\mathbf{C u}$ & 0.00000000000000 & 17.65711336885320 & 0.00000000000000 \\
\hline $\mathbf{C u}$ & 0.00000000000000 & 20.17955848897460 & 0.00000000000000 \\
\hline $\mathbf{C u}$ & 0.00000000000000 & 22.70200254966930 & 0.00000000000000 \\
\hline $\mathbf{C u}$ & 0.00000000000000 & 25.22444766979040 & 0.00000000000000 \\
\hline $\mathbf{C u}$ & 0.00000000000000 & 27.74689208362220 & 0.00000000000000 \\
\hline $\mathbf{C u}$ & 0.00000000000000 & 30.26933720374340 & 0.00000000000000 \\
\hline $\mathbf{C u}$ & 0.00000000000000 & 32.79178232386990 & 0.00000000000000 \\
\hline $\mathbf{C u}$ & 3.56727419238642 & 0.00000000000000 & 0.00000000000000 \\
\hline $\mathbf{C u}$ & 3.56727419238642 & 2.52244512012115 & 0.00000000000000 \\
\hline $\mathbf{C u}$ & 3.56727419238642 & 5.04488953395804 & 0.00000000000000 \\
\hline $\mathbf{C u}$ & 3.56727419238642 & 7.56733465407835 & 0.00000000000000 \\
\hline $\mathbf{C u}$ & 3.56727419238642 & 10.08977871477400 & 0.00000000000000 \\
\hline $\mathbf{C u}$ & 3.56727419238642 & 12.61222383489520 & 0.00000000000000 \\
\hline $\mathbf{C u}$ & 3.56727419238642 & 15.13466895501370 & 0.00000000000000 \\
\hline $\mathbf{C u}$ & 3.56727419238642 & 17.65711336885320 & 0.00000000000000 \\
\hline $\mathbf{C u}$ & 3.56727419238642 & 20.17955848897460 & 0.00000000000000 \\
\hline
\end{tabular}




\begin{tabular}{|c|c|c|c|}
\hline $\mathbf{C u}$ & 3.56727419238642 & 22.70200254966930 & 0.00000000000000 \\
\hline $\mathbf{C u}$ & 3.56727419238642 & 25.22444766979040 & 0.00000000000000 \\
\hline $\mathbf{C u}$ & 3.56727419238642 & 27.74689208362220 & 0.00000000000000 \\
\hline $\mathbf{C u}$ & 3.56727419238642 & 30.26933720374340 & 0.00000000000000 \\
\hline $\mathbf{C u}$ & 3.56727419238642 & 32.79178232386990 & 0.00000000000000 \\
\hline $\mathbf{C u}$ & 7.13454838477291 & 0.00000000000000 & 0.00000000000000 \\
\hline $\mathbf{C u}$ & 7.13454838477291 & 2.52244512012115 & 0.00000000000000 \\
\hline $\mathbf{C u}$ & 7.13454838477291 & 5.04488953395804 & 0.00000000000000 \\
\hline $\mathbf{C u}$ & 7.13454838477291 & 7.56733465407835 & 0.00000000000000 \\
\hline $\mathbf{C u}$ & 7.13454838477291 & 10.08977871477400 & 0.00000000000000 \\
\hline $\mathbf{C u}$ & 7.13454838477291 & 12.61222383489520 & 0.00000000000000 \\
\hline $\mathrm{Cu}$ & 7.13454838477291 & 15.13466895501370 & 0.00000000000000 \\
\hline $\mathrm{Cu}$ & 7.13454838477291 & 17.65711336885320 & 0.00000000000000 \\
\hline $\mathrm{Cu}$ & 7.13454838477291 & 20.17955848897460 & 0.00000000000000 \\
\hline $\mathbf{C u}$ & 7.13454838477291 & 22.70200254966930 & 0.00000000000000 \\
\hline $\mathbf{C u}$ & 7.13454838477291 & 25.22444766979040 & 0.00000000000000 \\
\hline $\mathbf{C u}$ & 7.13454838477291 & 27.74689208362220 & 0.00000000000000 \\
\hline $\mathbf{C u}$ & 7.13454838477291 & 30.26933720374340 & 0.00000000000000 \\
\hline $\mathbf{C u}$ & 7.13454838477291 & 32.79178232386990 & 0.00000000000000 \\
\hline $\mathbf{C u}$ & 1.78363955761358 & 1.26122414920059 & 1.26122399999993 \\
\hline $\mathbf{C u}$ & 1.78363955761358 & 3.78366926932145 & 1.26122399999993 \\
\hline $\mathbf{C u}$ & 1.78363955761358 & 6.30611333001662 & 1.26122399999993 \\
\hline $\mathbf{C u}$ & 1.78363955761358 & 5013778 & 1.26122399999993 \\
\hline $\mathrm{Cu}$ & 1.78363955761358 & 11.35100286397460 & 1.26122399999993 \\
\hline $\mathrm{Cu}$ & 1.783 & 8409290 & 1.26122399999993 \\
\hline $\mathrm{Cu}$ & 1.78363955761358 & 310421720 & 1.26122399999993 \\
\hline $\mathbf{C u}$ & 1.78363955761358 & 18.91833716491180 & 1.26122399999993 \\
\hline $\mathbf{C u}$ & 1.78363955761358 & 21.44078263817520 & 1.26122399999993 \\
\hline $\mathbf{C u}$ & 1.78363955761358 & 23.96322669886980 & 1.26122399999993 \\
\hline $\mathbf{C u}$ & 1.78363955761358 & 26.48567181899090 & 1.26122399999993 \\
\hline $\mathbf{C u}$ & 1.78363955761358 & 29.00811693910730 & 1.26122399999993 \\
\hline $\mathrm{Cu}$ & 1.78363955761358 & 31.53056135294370 & 1.26122399999993 \\
\hline $\mathrm{Cu}$ & 1.78363955761358 & 34.05300647307050 & 1.26122399999993 \\
\hline $\mathbf{C u}$ & 5.35091375000000 & 1.26122414920059 & 1.26122399999993 \\
\hline $\mathbf{C u}$ & 5.35091375000000 & 3.78366926932145 & 1.26122399999993 \\
\hline $\mathbf{C u}$ & 5.35091375000000 & 6.30611333001662 & 1.26122399999993 \\
\hline $\mathbf{C u}$ & 5.35091375000000 & 8.82855845013778 & 1.26122399999993 \\
\hline $\mathbf{C u}$ & 5.35091375000000 & 11.35100286397460 & 1.26122399999993 \\
\hline $\mathbf{C u}$ & 5.35091375000000 & 13.87344798409290 & 1.26122399999993 \\
\hline $\mathbf{C u}$ & 5.35091375000000 & 16.39589310421720 & 1.26122399999993 \\
\hline $\mathbf{C u}$ & 5.35091375000000 & 18.91833716491180 & 1.26122399999993 \\
\hline $\mathbf{C u}$ & 5.35091375000000 & 21.44078263817520 & 1.26122399999993 \\
\hline $\mathbf{C u}$ & 5.35091375000000 & 23.96322669886980 & 1.26122399999993 \\
\hline $\mathbf{C u}$ & 5.35091375000000 & 26.48567181899090 & 1.26122399999993 \\
\hline $\mathbf{C u}$ & 5.35091375000000 & 29.00811693910730 & 1.26122399999993 \\
\hline $\mathbf{C u}$ & 5.35091375000000 & 31.53056135294370 & 1.26122399999993 \\
\hline $\mathbf{C u}$ & 5.35091375000000 & 34.05300647307050 & 1.26122399999993 \\
\hline $\mathbf{C u}$ & 8.91818794238642 & 1.26122414920059 & 1.26122399999993 \\
\hline $\mathbf{C u}$ & 8.91818794238642 & 3.78366926932145 & 1.26122399999993 \\
\hline $\mathrm{Cu}$ & 8.91818794238642 & 6.30611333001662 & 1.26122399999993 \\
\hline $\mathbf{C u}$ & 8.91818794238642 & 8.82855845013778 & 1.26122399999993 \\
\hline $\mathbf{C u}$ & 8.91818794238642 & 11.35100286397460 & 1.26122399999993 \\
\hline $\mathbf{C u}$ & 8.91818794238642 & 13.87344798409290 & 1.26122399999993 \\
\hline $\mathbf{C u}$ & 8.91818794238642 & 16.39589310421720 & 1.26122399999993 \\
\hline
\end{tabular}




\begin{tabular}{|c|c|c|c|}
\hline $\mathbf{C u}$ & 8.91818794238642 & 18.91833716491180 & 1.26122399999993 \\
\hline $\mathrm{Cu}$ & 8.91818794238642 & 21.44078263817520 & 1.26122399999993 \\
\hline $\mathbf{C u}$ & 8.91818794238642 & 23.96322669886980 & 1.26122399999993 \\
\hline $\mathbf{C u}$ & 8.91818794238642 & 26.48567181899090 & 1.26122399999993 \\
\hline $\mathbf{C u}$ & 8.91818794238642 & 29.00811693910730 & 1.26122399999993 \\
\hline $\mathbf{C u}$ & 8.91818794238642 & 31.53056135294370 & 1.26122399999993 \\
\hline $\mathbf{C u}$ & 8.91818794238642 & 34.05300647307050 & 1.26122399999993 \\
\hline $\mathbf{C u}$ & 0.00100617526351 & 35.31362899414290 & 2.53779364543469 \\
\hline $\mathbf{C u}$ & 10.69979002833450 & 2.52199629123690 & 2.54054190380552 \\
\hline $\mathbf{C u}$ & 0.00407326463677 & 5.04481185621732 & 2.54312008095075 \\
\hline $\mathbf{C u}$ & 10.70004912134380 & 7.56541710208684 & 2.54325538462739 \\
\hline $\mathrm{Cu}$ & 10.70108528054390 & 10.09106169952110 & 2.54067684041273 \\
\hline $\mathbf{C u}$ & 10.70149337893280 & 12.61292571395590 & 2.54990483952336 \\
\hline $\mathrm{Cu}$ & 10.69623485670990 & 15.13707007806420 & 2.53811314661661 \\
\hline $\mathbf{C u}$ & 10.70044412976630 & 17.65750734436310 & 2.54461391858779 \\
\hline $\mathbf{C u}$ & 10.69844231867040 & 20.18164511208900 & 2.53799618480687 \\
\hline $\mathbf{C u}$ & 10.70015016641760 & 22.70287758682000 & 2.54158842038688 \\
\hline $\mathbf{C u}$ & 10.70070342516010 & 25.22722729985930 & 2.53883112324874 \\
\hline $\mathbf{C u}$ & 10.70096762577960 & 27.74762115643270 & 2.54115087967843 \\
\hline $\mathbf{C u}$ & 10.70171644132700 & 30.27004909363840 & 2.54097447192989 \\
\hline $\mathbf{C u}$ & 10.70059014486330 & 32.79074600621210 & 2.54011257771376 \\
\hline $\mathbf{C u}$ & 3.56730158988175 & 35.31279053638790 & 2.54076886920347 \\
\hline $\mathbf{C u}$ & 3.56729530359661 & 2.52199120029481 & 2.54337023033665 \\
\hline $\mathrm{Cu}$ & 3.56728924873518 & 5.0451 & 520205 \\
\hline $\mathrm{Cu}$ & 3.56727331911424 & 7.5701690 & 2.581 \\
\hline $\mathrm{Cu}$ & 3.56730302317827 & 10.09240592093390 & 3671743 \\
\hline $\mathbf{C u}$ & 3.56729390550132 & 12.62283389543790 & 2.57734831162383 \\
\hline $\mathbf{C u}$ & 3.56729145745541 & 15.14137068527180 & 2.58140653426996 \\
\hline $\mathrm{Cu}$ & 3.56727492071371 & 17.66251180015520 & 2.59680655623316 \\
\hline $\mathrm{Cu}$ & 3.56731199022970 & 20.17748531485740 & 2.58515587915490 \\
\hline $\mathrm{Cu}$ & 3.56728245455613 & 22.70061333410930 & 2.59307156918754 \\
\hline $\mathbf{C u}$ & 3.56728616891799 & 25.22294400589700 & 2.55231221920350 \\
\hline $\mathrm{Cu}$ & 3.56729027054050 & 27.74717586346110 & 2.54397078758779 \\
\hline $\mathbf{C u}$ & 3.56731330584084 & 30.26752642130780 & 2.54289784373234 \\
\hline $\mathbf{C u}$ & 3.56728465411250 & 32.79161373291270 & 2.54182744089390 \\
\hline $\mathbf{C u}$ & 7.13355692671113 & 35.31361023381130 & 2.53779356919895 \\
\hline $\mathbf{C u}$ & 7.13660394570982 & 2.52200313629731 & 2.54053827385336 \\
\hline $\mathbf{C u}$ & 7.13049243412920 & 5.04480444416656 & 2.54310335016097 \\
\hline $\mathbf{C u}$ & 7.13634139369371 & 7.56541895106844 & 2.54326885060082 \\
\hline $\mathbf{C u}$ & 7.13532011887516 & 10.09107661691190 & 2.54066048015913 \\
\hline $\mathbf{C u}$ & 7.13489572582235 & 12.61296153122720 & 2.54991195397039 \\
\hline $\mathbf{C u}$ & 7.14008869846266 & 15.13709211642990 & 2.53813626306824 \\
\hline $\mathbf{C u}$ & 7.13591834330865 & 17.65749150018940 & 2.54471700872366 \\
\hline $\mathbf{C u}$ & 7.13792378319948 & 20.18164017719870 & 2.53804455184436 \\
\hline $\mathrm{Cu}$ & 7.13624996454712 & 22.70287354654640 & 2.54159182047013 \\
\hline $\mathbf{C u}$ & 7.13568896947350 & 25.22722451385620 & 2.53882998010918 \\
\hline $\mathbf{C u}$ & 7.13543917369853 & 27.74760873228890 & 2.54117081201437 \\
\hline $\mathbf{C u}$ & 7.13469049029068 & 30.27005522505720 & 2.54098017974495 \\
\hline $\mathbf{C u}$ & 7.13579954935508 & 32.79075215563530 & 2.54013053829397 \\
\hline $\mathbf{C u}$ & 1.78340692035504 & 1.25777972605077 & 3.85312564229722 \\
\hline $\mathbf{C u}$ & 1.77952599775537 & 3.77757657819668 & 3.85454225552996 \\
\hline $\mathbf{C u}$ & 1.79814674727453 & 6.30543992226194 & 3.85774816231136 \\
\hline $\mathbf{C u}$ & 1.78079544820912 & 8.82746013968978 & 3.83374319033574 \\
\hline $\mathbf{C u}$ & 1.80185906160692 & 11.34920492491190 & 3.85298570252484 \\
\hline
\end{tabular}




\begin{tabular}{|c|c|c|c|}
\hline $\mathbf{C u}$ & 1.77534089477951 & 13.88212767885340 & 3.83840980942317 \\
\hline $\mathbf{C u}$ & 1.78881739476036 & 16.40290393155410 & 3.85436364460475 \\
\hline $\mathbf{C u}$ & 1.77540510894598 & 18.92432562649380 & 3.84723110008198 \\
\hline $\mathbf{C u}$ & 1.78390064425060 & 21.44521492245820 & 3.84201544466832 \\
\hline $\mathbf{C u}$ & 1.78295078724882 & 23.97304860186340 & 3.84739293914228 \\
\hline $\mathbf{C u}$ & 1.78119248430801 & 26.49057180536690 & 3.85565766163866 \\
\hline $\mathbf{C u}$ & 1.78142470517117 & 29.01151439172610 & 3.85774899881577 \\
\hline $\mathbf{C u}$ & 1.78219928671589 & 31.53012151537160 & 3.85572519993469 \\
\hline $\mathbf{C u}$ & 1.78299511253240 & 34.04861645988980 & 3.85327319342943 \\
\hline $\mathbf{C u}$ & 5.35119083488197 & 1.25777477831987 & 3.85314211795979 \\
\hline $\mathbf{C u}$ & 5.35506581585738 & 3.77757191706063 & 3.85453923853381 \\
\hline $\mathbf{C u}$ & 5.33640711398142 & 6.30542563776052 & 3.85774039329501 \\
\hline $\mathbf{C u}$ & 5.35380205423343 & 8.82745476855756 & 3.83373148155629 \\
\hline $\mathbf{C u}$ & 5.33275306672832 & 11.34921734390530 & 3.85296990801572 \\
\hline $\mathbf{C u}$ & 5.35919236942441 & 13.88220037078610 & 3.83838136886573 \\
\hline $\mathbf{C u}$ & 5.34561866999992 & 16.40306488136850 & 3.85449211879240 \\
\hline $\mathbf{C u}$ & 5.35912783150425 & 18.92430197513910 & 3.84737187769582 \\
\hline $\mathbf{C u}$ & 5.35063032550074 & 21.44518315608930 & 3.84212458073076 \\
\hline $\mathbf{C u}$ & 5.35158669087403 & 23.97303530140020 & 3.84739924164138 \\
\hline $\mathbf{C u}$ & 5.35340220056096 & 26.49055104524640 & 3.85 \\
\hline $\mathbf{C u}$ & 5.35320277656118 & 29.01 & 3.85775699509387 \\
\hline $\mathbf{C u}$ & 5.35 & 31.53 & 3.85578338994994 \\
\hline $\mathbf{C u}$ & 5.35162632363192 & 34.04859859909570 & 3.85328808139664 \\
\hline $\mathbf{C u}$ & 8.91818257392579 & 1.26242519386114 & 3.84824921657880 \\
\hline $\mathbf{C u}$ & 8.91819812286657 & 3.78574270852817 & 3.85082184902732 \\
\hline $\mathbf{C u}$ & 8.91816888284068 & 6.30193982745210 & 3.85243733187269 \\
\hline $\mathbf{C u}$ & 8.91819944529489 & 8.82612137248673 & 3.85549934985365 \\
\hline $\mathbf{C u}$ & 8.91818685892488 & 11.35574273992030 & 3.85581998377152 \\
\hline $\mathbf{C u}$ & 8.91819554564828 & 13.87366883809440 & 3.85297589673708 \\
\hline $\mathbf{C u}$ & 8.91822719558057 & 16.40174560463410 & 3.85693427097131 \\
\hline $\mathbf{C u}$ & 8.91823491610985 & 18.92347827780600 & 3.85208028143455 \\
\hline $\mathbf{C u}$ & 8.91821622063770 & 21.44413215842690 & 3.85396541556872 \\
\hline $\mathbf{C u}$ & 8.91820188098139 & 23.96590105005380 & 3.84654287468349 \\
\hline $\mathbf{C u}$ & 8.91821669888891 & 26.48665516762430 & 3.85229797139020 \\
\hline $\mathbf{C u}$ & 8.91820126698223 & 29.00681368559940 & 3.84806962850114 \\
\hline $\mathbf{C u}$ & 8.91822116675685 & 31.53248244916500 & 3.85112756365410 \\
\hline $\mathbf{C u}$ & 8.91820893917952 & 34.04966215426010 & 3.84772273571939 \\
\hline $\mathbf{C u}$ & -0.00081227842138 & 35.31179288554740 & 5.05449170665857 \\
\hline $\mathbf{C u}$ & 0.00050071137173 & 2.51963385524948 & 5.05806391048906 \\
\hline $\mathbf{C u}$ & 0.00505238538361 & 5.04795030572959 & 5.05704295903378 \\
\hline $\mathbf{C u}$ & 0.00526683976345 & 7.56590351783425 & 5.05047557225597 \\
\hline $\mathbf{C u}$ & 0.00773080628529 & 10.09352022903170 & 5.04649024459083 \\
\hline $\mathbf{C u}$ & 0.01227532394632 & 12.61228673012420 & 5.05949861445726 \\
\hline $\mathbf{C u}$ & 0.00516595729707 & 15.14181955299540 & 5.05193828076006 \\
\hline $\mathbf{C u}$ & 0.00109465941593 & 17.66428567979420 & 5.06162763460998 \\
\hline $\mathbf{C u}$ & 0.00468554294514 & 20.18710801877950 & 5.05593143633825 \\
\hline $\mathbf{C u}$ & 0.00415149493848 & 22.70744652229650 & 5.04904515525056 \\
\hline $\mathbf{C u}$ & 0.00115702992867 & 25.22474794580100 & 5.05727096577623 \\
\hline $\mathbf{C u}$ & 10.70064834583260 & 27.74759528009430 & 5.06170868455961 \\
\hline $\mathbf{C u}$ & 10.69788511523080 & 30.26720888822710 & 5.05817769511749 \\
\hline $\mathbf{C u}$ & 0.00155279402967 & 32.78870435278370 & 5.06034923614506 \\
\hline $\mathbf{C u}$ & 3.56730811347400 & 35.30925266161670 & 5.05678542781749 \\
\hline $\mathbf{C u}$ & 3.56729024799477 & 2.51684243308802 & 5.05834703130127 \\
\hline $\mathbf{C u}$ & 3.56729284082754 & 5.02028928843586 & 5.04850865739090 \\
\hline
\end{tabular}




\begin{tabular}{|c|c|c|c|}
\hline $\mathbf{C u}$ & 3.56730095238468 & 7.51958425242346 & 5.15956341024929 \\
\hline $\mathbf{C u}$ & 3.56727991305832 & 10.10379079086130 & 5.15520386673964 \\
\hline $\mathbf{C u}$ & 3.56731479372141 & 12.62409589191480 & 5.13271307307653 \\
\hline $\mathbf{C u}$ & 3.56717693129682 & 15.15271491961630 & 5.09142047091848 \\
\hline $\mathbf{C u}$ & 3.56646702547924 & 17.67801977719450 & 5.16655367357806 \\
\hline $\mathbf{C u}$ & 3.56721865361437 & 20.21574122361280 & 5.09493583421343 \\
\hline $\mathbf{C u}$ & 3.56711914547565 & 22.73260563049450 & 5.16179491937728 \\
\hline $\mathbf{C u}$ & 3.56726543373921 & 25.24170907047110 & 5.07003671283540 \\
\hline $\mathbf{C u}$ & 3.56732275143541 & 27.75426879783070 & 5.05653084101981 \\
\hline $\mathbf{C u}$ & 3.56730663067650 & 30.27551498051910 & 5.06031600178997 \\
\hline $\mathbf{C u}$ & 3.56729212005637 & 32.78890636822010 & 5.05947387665741 \\
\hline $\mathbf{C u}$ & 7.13541224746700 & 35.31177209660610 & 5.05451324177540 \\
\hline $\mathbf{C u}$ & 7.13408799426380 & 2.51962880172934 & 5.05807139559195 \\
\hline $\mathbf{C u}$ & 7.12951317966757 & 5.04795341949839 & 5.05702479064066 \\
\hline $\mathbf{C u}$ & 7.12932695820825 & 7.56590771687292 & 5.050473 \\
\hline $\mathbf{C u}$ & 7.12683984373225 & 10.09351864930420 & 5.04647522525835 \\
\hline $\mathbf{C u}$ & 7.12227016951951 & 12.61228733513070 & 163671 \\
\hline $\mathrm{Cu}$ & 7.12941944509331 & 15.14190788843690 & 521477 \\
\hline $\mathbf{C u}$ & 7.13367499446099 & 17.66430463272210 & 5.06165 \\
\hline $\mathbf{C u}$ & 7.12997090972342 & 3734319690 & 5.055926 \\
\hline $\mathbf{C u}$ & 7.13044641332578 & 22.70744264668510 & 5.048975 \\
\hline $\mathbf{C u}$ & 7.13344679854676 & 25.22474117496390 & 5.05722170045718 \\
\hline $\mathbf{C u}$ & 7.13578758971885 & 27.74760358949890 & 5.06175954579146 \\
\hline $\mathbf{C u}$ & 7.13859997198744 & 30.26720903225040 & 5.05821322587639 \\
\hline $\mathbf{C u}$ & 7.13308388199558 & 32.78867166640030 & 5.06034599664520 \\
\hline \multicolumn{4}{|c|}{ CI-NEB Image 24} \\
\hline Element & X-Coordinate (Å) & Y-Coordinate (Å) & Z-Coordinate (Å) \\
\hline $\mathbf{F}$ & 4.66337365812031 & 12.66698091514450 & 7.74737024870332 \\
\hline $\mathbf{F}$ & 2.47146072210086 & 12.66674817845080 & 7.74733259443681 \\
\hline $\mathbf{F}$ & 3.56730079216030 & 8.81342963681047 & 6.63776298646369 \\
\hline $\mathbf{F}$ & 4.66490885717191 & 17.74754915207700 & 7.77677631482186 \\
\hline $\mathbf{F}$ & 2.47261639435945 & 17.74677479752300 & 7.77885130450297 \\
\hline $\mathbf{F}$ & 3.56724571167714 & 14.52753712679850 & 7.26805926887758 \\
\hline $\mathbf{F}$ & 4.63664854277558 & 22.94729528427680 & 7.78770483811281 \\
\hline $\mathbf{F}$ & 2.49994209754765 & 22.94819012871200 & 7.78874280732632 \\
\hline $\mathbf{F}$ & 3.56755359588932 & 19.60722725892230 & 7.29871377912787 \\
\hline C & 3.56738182410505 & 13.09684160087200 & 7.04333626642421 \\
\hline $\mathrm{C}$ & 3.56790397604319 & 18.16983702140050 & 7.06973451035384 \\
\hline $\mathbf{C}$ & 3.56787940213959 & 22.90253102284070 & 6.99191156644389 \\
\hline $\mathbf{C u}$ & 0.00000000000000 & 0.00000000000000 & 0.00000000000000 \\
\hline $\mathbf{C u}$ & 0.00000000000000 & 2.52244512012115 & 0.00000000000000 \\
\hline $\mathbf{C u}$ & 0.00000000000000 & 5.04488953395804 & 0.00000000000000 \\
\hline $\mathbf{C u}$ & 0.00000000000000 & 7.56733465407835 & 0.00000000000000 \\
\hline $\mathbf{C u}$ & 0.00000000000000 & 10.08977871477400 & 0.00000000000000 \\
\hline $\mathbf{C u}$ & 0.00000000000000 & 12.61222383489520 & 0.00000000000000 \\
\hline $\mathbf{C u}$ & 0.00000000000000 & 15.13466895501390 & 0.00000000000000 \\
\hline $\mathbf{C u}$ & 0.00000000000000 & 17.65711336885320 & 0.00000000000000 \\
\hline $\mathbf{C u}$ & 0.00000000000000 & 20.17955848897460 & 0.00000000000000 \\
\hline $\mathbf{C u}$ & 0.00000000000000 & 22.70200254966930 & 0.00000000000000 \\
\hline $\mathbf{C u}$ & 0.00000000000000 & 25.22444766979040 & 0.00000000000000 \\
\hline $\mathbf{C u}$ & 0.00000000000000 & 27.74689208362270 & 0.00000000000000 \\
\hline $\mathbf{C u}$ & 0.00000000000000 & 30.26933720374380 & 0.00000000000000 \\
\hline $\mathbf{C u}$ & 0.00000000000000 & 32.79178232386990 & 0.00000000000000 \\
\hline $\mathbf{C u}$ & 3.56727419238642 & 0.00000000000000 & 0.00000000000000 \\
\hline
\end{tabular}




\begin{tabular}{|c|c|c|c|}
\hline $\mathbf{C u}$ & 3.56727419238642 & 2.52244512012115 & 0.00000000000000 \\
\hline $\mathbf{C u}$ & 3.56727419238642 & 5.04488953395804 & 0.00000000000000 \\
\hline $\mathbf{C u}$ & 3.56727419238642 & 7.56733465407835 & 0.00000000000000 \\
\hline $\mathbf{C u}$ & 3.56727419238642 & 10.08977871477400 & 0.00000000000000 \\
\hline $\mathbf{C u}$ & 3.56727419238642 & 12.61222383489520 & 0.00000000000000 \\
\hline $\mathbf{C u}$ & 3.56727419238642 & 15.13466895501390 & 0.00000000000000 \\
\hline $\mathbf{C u}$ & 3.56727419238642 & 17.65711336885320 & 0.00000000000000 \\
\hline $\mathbf{C u}$ & 3.56727419238642 & 20.17955848897460 & 0.00000000000000 \\
\hline $\mathbf{C u}$ & 3.56727419238642 & 22.70200254966930 & 0.00000000000000 \\
\hline $\mathbf{C u}$ & 3.56727419238642 & 25.22444766979040 & 0.00000000000000 \\
\hline $\mathbf{C u}$ & 3.56727419238642 & 27.74689208362270 & 0.00000000000000 \\
\hline $\mathrm{Cu}$ & 3.56727419238642 & 30.26933720374380 & 0.00000000000000 \\
\hline $\mathrm{Cu}$ & 3.56727419238642 & 32.79178232386990 & 0.00000000000000 \\
\hline $\mathrm{Cu}$ & 7.13454838477291 & 0.00000000000000 & 0.00000000000000 \\
\hline $\mathbf{C u}$ & 7.13454838477291 & 2.52244512012115 & 0.00000000000000 \\
\hline $\mathbf{C u}$ & 7.13454838477291 & 5.04488953395804 & 0.00000000000000 \\
\hline $\mathbf{C u}$ & 7.13454838477291 & 7.56733465407835 & 0.00000000000000 \\
\hline $\mathbf{C u}$ & 7.13454838477291 & 10.08977871477400 & 0.00000000000000 \\
\hline $\mathbf{C u}$ & 7.13454838477291 & 12.61222383489520 & 0.00000000000000 \\
\hline $\mathbf{C u}$ & 7.13454838477291 & 15.13466895501390 & 0.00000000000000 \\
\hline $\mathbf{C u}$ & 7.13454838477291 & 17.65711336885320 & 0.00000000000000 \\
\hline $\mathbf{C u}$ & 7.13454838477291 & 20.17955848897460 & 0.00000000000000 \\
\hline $\mathbf{C u}$ & 7.13454838477291 & 22.70200254966930 & 0.00000000000000 \\
\hline $\mathbf{C u}$ & 7.13454838477291 & 25.22444766979040 & 0.00000000000000 \\
\hline $\mathbf{C u}$ & 8477291 & 27.74689 & 0000000000 \\
\hline $\mathbf{C u}$ & 38477291 & 30.26933720 & 0.00000000000000 \\
\hline $\mathbf{C u}$ & 7.13454838477291 & 32.79178232386990 & 0.00000000000000 \\
\hline $\mathbf{C u}$ & 1.78363955761358 & 1.26122414920059 & 1.26122399999993 \\
\hline $\mathbf{C u}$ & 1.78363955761358 & 3.78366926932145 & 1.26122399999993 \\
\hline $\mathrm{Cu}$ & 1.78363955761358 & 6.30611333001662 & 1.26122399999993 \\
\hline $\mathrm{Cu}$ & 1.78363955761358 & 8.82855845013778 & 1.26122399999993 \\
\hline $\mathrm{Cu}$ & 1.78363955761358 & 11.35100286397460 & 1.26122399999993 \\
\hline $\mathrm{Cu}$ & 1.78363955761358 & 13.87344798409310 & 1.26122399999993 \\
\hline $\mathbf{C u}$ & 1.78363955761358 & 16.39589310421720 & 1.26122399999993 \\
\hline $\mathbf{C u}$ & 1.78363955761358 & 18.91833716491180 & 1.26122399999993 \\
\hline $\mathbf{C u}$ & 1.78363955761358 & 21.44078263817520 & 1.26122399999993 \\
\hline $\mathbf{C u}$ & 1.78363955761358 & 23.96322669886980 & 1.26122399999993 \\
\hline $\mathbf{C u}$ & 1.78363955761358 & 26.48567181899090 & 1.26122399999993 \\
\hline $\mathbf{C u}$ & 1.78363955761358 & 29.00811693910780 & 1.26122399999993 \\
\hline $\mathbf{C u}$ & 1.78363955761358 & 31.53056135294420 & 1.26122399999993 \\
\hline $\mathbf{C u}$ & 1.78363955761358 & 34.05300647307050 & 1.26122399999993 \\
\hline $\mathbf{C u}$ & 5.35091375000000 & 1.26122414920059 & 1.26122399999993 \\
\hline $\mathbf{C u}$ & 5.35091375000000 & 3.78366926932145 & 1.26122399999993 \\
\hline $\mathbf{C u}$ & 5.35091375000000 & 6.30611333001662 & 1.26122399999993 \\
\hline $\mathbf{C u}$ & 5.35091375000000 & 8.82855845013778 & 1.26122399999993 \\
\hline $\mathrm{Cu}$ & 5.35091375000000 & 11.35100286397460 & 1.26122399999993 \\
\hline $\mathbf{C u}$ & 5.35091375000000 & 13.87344798409310 & 1.26122399999993 \\
\hline $\mathbf{C u}$ & 5.35091375000000 & 16.39589310421720 & 1.26122399999993 \\
\hline $\mathbf{C u}$ & 5.35091375000000 & 18.91833716491180 & 1.26122399999993 \\
\hline $\mathbf{C u}$ & 5.35091375000000 & 21.44078263817520 & 1.26122399999993 \\
\hline $\mathbf{C u}$ & 5.35091375000000 & 23.96322669886980 & 1.26122399999993 \\
\hline $\mathbf{C u}$ & 5.35091375000000 & 26.48567181899090 & 1.26122399999993 \\
\hline $\mathbf{C u}$ & 5.35091375000000 & 29.00811693910780 & 1.26122399999993 \\
\hline $\mathbf{C u}$ & 5.35091375000000 & 31.53056135294420 & 1.26122399999993 \\
\hline
\end{tabular}




\begin{tabular}{|c|c|c|c|}
\hline $\mathrm{Cu}$ & 5.35091375000000 & 34.05300647307050 & 1.26122399999993 \\
\hline $\mathrm{Cu}$ & 8.91818794238642 & 1.26122414920059 & 1.26122399999993 \\
\hline $\mathbf{C u}$ & 8.91818794238642 & 3.78366926932145 & 1.26122399999993 \\
\hline $\mathrm{Cu}$ & 8.91818794238642 & 6.30611333001662 & 1.26122399999993 \\
\hline $\mathbf{C u}$ & 8.91818794238642 & 8.82855845013778 & 1.26122399999993 \\
\hline $\mathbf{C u}$ & 8.91818794238642 & 11.35100286397460 & 1.26122399999993 \\
\hline $\mathbf{C u}$ & 8.91818794238642 & 13.87344798409310 & 1.26122399999993 \\
\hline $\mathrm{Cu}$ & 8.91818794238642 & 16.39589310421720 & 1.26122399999993 \\
\hline $\mathbf{C u}$ & 8.91818794238642 & 18.91833716491180 & 1.26122399999993 \\
\hline $\mathbf{C u}$ & 8.91818794238642 & 21.44078263817520 & 1.26122399999993 \\
\hline $\mathbf{C u}$ & 8.91818794238642 & 23.96322669886980 & 1.26122399999993 \\
\hline $\mathbf{C u}$ & 8.91818794238642 & 26.48567181899090 & 1.26122399999993 \\
\hline $\mathbf{C u}$ & 8.91818794238642 & 29.00811693910780 & 1.26122399999993 \\
\hline $\mathbf{C u}$ & 8.91818794238642 & 31.53056135294420 & 1.26122399999993 \\
\hline $\mathrm{Cu}$ & 8.91818794238642 & 34.05300647307050 & 1.26122399999993 \\
\hline $\mathrm{Cu}$ & 0.00107989266812 & 35.31310666239730 & 2.53769482233905 \\
\hline $\mathrm{Cu}$ & 10.69972303940250 & 2.52167425297430 & 2.54058791622698 \\
\hline $\mathbf{C u}$ & 0.00387275966498 & 5.04466169864810 & 2.54325061349604 \\
\hline $\mathrm{Cu}$ & 10.70013001738360 & 7.56533648930741 & 2.54317780057675 \\
\hline $\mathbf{C u}$ & 10.70085710709790 & 10.09047109165050 & 2.540368 \\
\hline $\mathbf{C u}$ & 10.70136661996530 & $12.6124 t$ & 7865 \\
\hline $\mathbf{C u}$ & 0436169620 & 15.1 & $2.53774 \mathrm{C}$ \\
\hline $\mathbf{C u}$ & 5014003390 & 17.65735196223750 & 2.54477494581463 \\
\hline $\mathrm{Cu}$ & 10.69779725123540 & 20.18158228707680 & 2.53754481051888 \\
\hline $\mathrm{Cu}$ & 10.69873996701340 & 22.70121798424680 & 2.541446 \\
\hline $\mathrm{Cu}$ & 10.69967274241210 & 25.22800425994280 & 2.53807271636197 \\
\hline $\mathrm{Cu}$ & 10.70178000721450 & 27.74738048814550 & 2.54213891829797 \\
\hline $\mathrm{Cu}$ & 10.70207142052600 & 30.26955811343710 & 2.54107070072599 \\
\hline $\mathrm{Cu}$ & 10.70079424848280 & 32.79042189641370 & 2.540304 \\
\hline $\mathrm{Cu}$ & 3.56727297379086 & 35.31224610501730 & 2.54049222785835 \\
\hline $\mathrm{Cu}$ & 3.56727752617758 & 2.52150533641162 & 2.54276067660033 \\
\hline $\mathrm{Cu}$ & 3.56727282646495 & 5.04491822193291 & 2.53902508922115 \\
\hline $\mathbf{C u}$ & 3.56727130920971 & 7.57012789130701 & 2.58123830020869 \\
\hline $\mathbf{C u}$ & 3.56726930802351 & 10.09246344890330 & 2.59145889894774 \\
\hline $\mathbf{C u}$ & 3.56727467104394 & 12.62235480922850 & 2.57549058840933 \\
\hline $\mathbf{C u}$ & 3.56728247076181 & 15.14138048329960 & 2.58188953882659 \\
\hline $\mathrm{Cu}$ & 3.56726404057015 & 17.66228385002820 & 2.58869427596770 \\
\hline $\mathbf{C u}$ & 3.56728322908524 & 20.17356824611400 & 2.58040106862504 \\
\hline $\mathrm{Cu}$ & 3.56728966549144 & 22.70007617724300 & 2.58443626662544 \\
\hline $\mathbf{C u}$ & 3.56728926501850 & 25.22168207021340 & 2.55712085122354 \\
\hline $\mathrm{Cu}$ & 3.56727381614136 & 27.74520665052510 & 2.54448011842630 \\
\hline $\mathbf{C u}$ & 3.56726660523149 & 30.26629209317860 & 2.54326474839900 \\
\hline $\mathbf{C u}$ & 3.56727052468031 & 32.79031442959840 & 2.54124901604603 \\
\hline $\mathbf{C u}$ & 7.13349703099952 & 35.31310891937430 & 2.53770604424430 \\
\hline $\mathbf{C u}$ & 7.13668699458563 & 2.52167352829363 & 2.54059076288442 \\
\hline $\mathrm{Cu}$ & 7.13070856710194 & 5.04465229294300 & 2.54323190108312 \\
\hline $\mathrm{Cu}$ & 7.13626045932802 & 7.56533647562216 & 2.54319349416477 \\
\hline $\mathbf{C u}$ & 7.13557539752102 & 10.09047764723360 & 2.54035880140658 \\
\hline $\mathrm{Cu}$ & 7.13505615420270 & 12.61246798914980 & 2.55001232841870 \\
\hline $\mathrm{Cu}$ & 7.14057145621132 & 15.13702105243510 & 2.53777192062219 \\
\hline $\mathrm{Cu}$ & 7.13678652583739 & 17.65735696907500 & 2.54485472908855 \\
\hline $\mathbf{C u}$ & 7.13856321043020 & 20.18154416509420 & 2.53758056357971 \\
\hline $\mathrm{Cu}$ & 7.13769558602122 & 22.70122456156230 & 2.54147489924051 \\
\hline $\mathrm{Cu}$ & 7.13671465251353 & 25.22799642401340 & 2.53809957014662 \\
\hline
\end{tabular}




\begin{tabular}{|c|c|c|c|}
\hline $\mathbf{C u}$ & 7.13458242005678 & 27.74737230215000 & 2.54213300528024 \\
\hline $\mathbf{C u}$ & 7.13433046734000 & 30.26957295024280 & 2.54106087352132 \\
\hline $\mathbf{C u}$ & 7.13559141694368 & 32.79042632995900 & 2.54030763168308 \\
\hline $\mathbf{C u}$ & 1.78366176319267 & 1.25716245021798 & 3.85304270807734 \\
\hline $\mathbf{C u}$ & 1.77974809412962 & 3.77757408521896 & 3.85375591518664 \\
\hline $\mathbf{C u}$ & 1.79801641521895 & 6.30530311724556 & 3.85758334491803 \\
\hline $\mathbf{C u}$ & 1.78061595354347 & 8.82717486409488 & 3.83410974898320 \\
\hline $\mathbf{C u}$ & 1.80142499232474 & 11.34883636211590 & 3.85185093499155 \\
\hline $\mathbf{C u}$ & 1.77511612998364 & 13.88292701050150 & 3.83743400056897 \\
\hline $\mathbf{C u}$ & 1.78845770758546 & 16.40324010264010 & 3.85238845940613 \\
\hline $\mathbf{C u}$ & 1.77320466300985 & 18.92453946489780 & 3.84464065793177 \\
\hline $\mathbf{C u}$ & 1.78557739218702 & 21.44171544103230 & 3.84252819871073 \\
\hline $\mathbf{C u}$ & 1.77693952937653 & 23.97127438626920 & 3.83725276687841 \\
\hline $\mathrm{Cu}$ & 1.78425154246951 & 26.48560833878130 & 3.85890538427319 \\
\hline $\mathbf{C u}$ & 1.78210871320932 & 29.00878971053630 & 3.85875454608078 \\
\hline $\mathbf{C u}$ & 1.78335234259152 & 31.52861802854400 & 3.85575579132633 \\
\hline $\mathbf{C u}$ & 1.78383553174441 & 34.04752317322420 & 3.85230386575711 \\
\hline $\mathbf{C u}$ & 5.35090403628376 & 1.25713856355977 & 3.85305193948016 \\
\hline $\mathbf{C u}$ & 5.35483099674260 & 3.77754242937385 & 3.85376441118310 \\
\hline $\mathbf{C u}$ & 5.33654938572998 & 6.30527151532587 & 3.85757155988432 \\
\hline $\mathbf{C u}$ & 5.35393162730818 & 8.82718119193443 & 3.83411125150196 \\
\hline $\mathbf{C u}$ & 5.33314815190740 & 11.34882833854950 & 3.85181728813740 \\
\hline $\mathbf{C u}$ & 5.35944840566390 & 13.88294260916580 & 969829323 \\
\hline $\mathbf{C u}$ & 5.34598968171007 & 372322490 & 93560 \\
\hline $\mathbf{C u}$ & 49455195 & 18.92460695694880 & 30605 \\
\hline $\mathrm{Cu}$ & 5.34899323463846 & 21.44168089243750 & 3.84259326126941 \\
\hline $\mathbf{C u}$ & 5.35763281618289 & 23.97122317998490 & 3.83731642572289 \\
\hline $\mathbf{C u}$ & 5.35030987806308 & 26.48561307187810 & 3.85890839551003 \\
\hline $\mathrm{Cu}$ & 5.35246835693811 & 29.00880793938160 & 3.85874512902816 \\
\hline $\mathrm{Cu}$ & 5.35119169252155 & 31.52863084622030 & 3.85576644120446 \\
\hline $\mathbf{C u}$ & 5.35073603485578 & 34.04752685096980 & 3.85231600558206 \\
\hline $\mathbf{C u}$ & 8.91822155052970 & 1.26147878867097 & 3.84879888152151 \\
\hline $\mathrm{Cu}$ & 8.91820444117336 & 3.78534399340447 & 3.85118702280323 \\
\hline $\mathbf{C u}$ & 8.91820142162425 & 6.30173137798296 & 3.85274680038420 \\
\hline $\mathbf{C u}$ & 8.91822651756686 & 8.82595333928992 & 3.85557362007825 \\
\hline $\mathbf{C u}$ & 8.91822044443911 & 11.35558038241390 & 3.85540361979069 \\
\hline $\mathbf{C u}$ & 8.91821584371777 & 13.87336307334940 & 3.85264114877810 \\
\hline $\mathbf{C u}$ & 8.91819525943933 & 16.40216483397560 & 3.85832436627374 \\
\hline $\mathbf{C u}$ & 8.91819146778741 & 18.92361059183880 & 3.85209994732537 \\
\hline $\mathbf{C u}$ & 8.91821500905127 & 21.44279783847230 & 3.85415500628049 \\
\hline $\mathbf{C u}$ & 8.91820648254475 & 23.96483856112680 & 3.84579887151487 \\
\hline $\mathbf{C u}$ & 8.91819898559669 & 26.48699108929310 & 3.85251570812197 \\
\hline $\mathbf{C u}$ & 8.91819147090318 & 29.00584830299800 & 3.84825775509714 \\
\hline $\mathbf{C u}$ & 8.91820704957814 & 31.53230215513390 & 3.85168440860189 \\
\hline $\mathbf{C u}$ & 8.91819846598207 & 34.04872774518540 & 3.84881360267909 \\
\hline $\mathbf{C u}$ & 0.00050755707236 & 35.31089675460360 & 5.05392333620354 \\
\hline $\mathbf{C u}$ & 0.00178466110462 & 2.51881176273387 & 5.05728679414590 \\
\hline $\mathbf{C u}$ & 0.00518293224648 & 5.04749734659634 & 5.05700083870584 \\
\hline $\mathbf{C u}$ & 0.00527078151254 & 7.56564612226892 & 5.05023557806988 \\
\hline $\mathbf{C u}$ & 0.00751340606669 & 10.09342208739650 & 5.04628455758250 \\
\hline $\mathbf{C u}$ & 0.01267674838955 & 12.61232073568860 & 5.05958609932807 \\
\hline $\mathbf{C u}$ & 0.00592036915301 & 15.14203362129110 & 5.05146063765921 \\
\hline $\mathbf{C u}$ & 0.00091659033883 & 17.66406649470130 & 5.06219737840576 \\
\hline $\mathbf{C u}$ & 0.00469393649854 & 20.18692350607280 & 5.05684582295170 \\
\hline
\end{tabular}




\begin{tabular}{|c|c|c|c|}
\hline $\mathbf{C u}$ & 0.00435121035917 & 22.70585520986330 & 5.04821420848373 \\
\hline $\mathbf{C u}$ & 0.00030995531620 & 25.22322687375590 & 5.05616911898751 \\
\hline $\mathbf{C u}$ & 10.70182335746550 & 27.74572211024590 & 5.06268811514774 \\
\hline $\mathbf{C u}$ & 10.69865659572100 & 30.26601905854270 & 5.05723203386523 \\
\hline $\mathbf{C u}$ & 0.00325834096999 & 32.78804881738020 & 5.06011731881780 \\
\hline $\mathbf{C u}$ & 3.56724376649630 & 35.30824328141100 & 5.05701629467961 \\
\hline $\mathbf{C u}$ & 3.56727459667670 & 2.51660367414228 & 5.05782171175310 \\
\hline $\mathbf{C u}$ & 3.56727497424472 & 5.01964894808107 & 5.04807550337635 \\
\hline $\mathbf{C u}$ & 3.56730870201564 & 7.51955738635485 & 5.15891650777426 \\
\hline $\mathbf{C u}$ & 3.56726595931094 & 10.10379962841010 & 5.15516152259449 \\
\hline $\mathbf{C u}$ & 3.56729745254828 & 12.62426913882180 & 5.12698256487468 \\
\hline $\mathbf{C u}$ & 3.56732518013297 & 15.15597593744610 & 5.09146718676805 \\
\hline $\mathbf{C u}$ & 3.56695430760083 & 17.68915308656180 & 5.14844634149104 \\
\hline $\mathbf{C u}$ & 3.56722863695916 & 20.21388744464210 & 5.09960917432268 \\
\hline $\mathrm{Cu}$ & 3.56713676650015 & 22.71847728898690 & 5.13480290608727 \\
\hline $\mathrm{Cu}$ & 3.56724538122156 & 25.22426825988570 & 5.08578847361915 \\
\hline $\mathrm{Cu}$ & 3.56728970769208 & 27.74436984970370 & 5.0584914 \\
\hline $\mathbf{C u}$ & 3.56729018880149 & 30.26866540774900 & 5.06134363901107 \\
\hline $\mathbf{C u}$ & 3.56727873654712 & 32.78773981658280 & 5.05944959041135 \\
\hline $\mathbf{C u}$ & 7.13408330777442 & 35.31092177568610 & 5.05396108729280 \\
\hline $\mathbf{C u}$ & 7.13282211788761 & 2.51880740003795 & 5.05730796000646 \\
\hline $\mathbf{C u}$ & 7.12940651103598 & 5.04750617863029 & 5.05697395298608 \\
\hline $\mathbf{C u}$ & 7.12930804897073 & 7.56566480261123 & 5.05022370132222 \\
\hline $\mathbf{C u}$ & 7.12704517077797 & 10.09341387935490 & 5.04621713726909 \\
\hline $\mathbf{C u}$ & 7.12191612593000 & 12.61230865578310 & 5.05954303790821 \\
\hline $\mathbf{C u}$ & 7.12866312577860 & 15.14203199743970 & 5.05145003323499 \\
\hline $\mathbf{C u}$ & 7.13365212817964 & 17.66403950386320 & 5.06223895840719 \\
\hline $\mathbf{C u}$ & 7.12990095545033 & 20.18687987944000 & 5.05689303045026 \\
\hline $\mathbf{C u}$ & 7.13030515942128 & 22.70581793486550 & 5.04824786982041 \\
\hline $\mathbf{C u}$ & 7.13425939719757 & 25.22322373808780 & 5.05620642361624 \\
\hline $\mathbf{C u}$ & 7.13458485811218 & 27.74570005815420 & 5.06267534784490 \\
\hline $\mathbf{C u}$ & 7.13773015123849 & 30.26603240679020 & 5.05725007818603 \\
\hline $\mathbf{C u}$ & 7.13132454427337 & 32.78803336604930 & 5.06011334391659 \\
\hline \multicolumn{4}{|c|}{ CI-NEB Image 25} \\
\hline Element & X-Coordinate (§̊) & Y-Coordin & Z-Coo \\
\hline $\mathbf{F}$ & 4.66329525995733 & 12.66376201753860 & 7.74420157470181 \\
\hline $\mathbf{F}$ & 2.47132277105405 & 12.66369726952110 & 7.74419613788030 \\
\hline $\mathbf{F}$ & 3.56727007498844 & 8.81250488390232 & 6.63786026594228 \\
\hline $\mathbf{F}$ & 4.66539078017047 & 17.74334441925000 & 7.76687049984402 \\
\hline $\mathbf{F}$ & 2.47228910106993 & 17.74165188947820 & 7.76838880132193 \\
\hline $\mathbf{F}$ & 3.56726410799073 & 14.52488692013750 & 7.26743376250696 \\
\hline $\mathbf{F}$ & 4.64006854562766 & 23.30228654252590 & 7.69573359780942 \\
\hline $\mathbf{F}$ & 2.49618773861310 & 23.30223038453720 & 7.69663168809944 \\
\hline $\mathbf{F}$ & 3.56707435368134 & 19.60466849965690 & 7.30149889736393 \\
\hline C & 3.56729910810301 & 13.09518012925470 & 7.04129087704931 \\
\hline C & 3.56800631663617 & 18.17293548548980 & 7.06410316326051 \\
\hline C & 3.56779090440210 & 23.25130373071460 & 6.89324216984107 \\
\hline $\mathrm{Cu}$ & 0.00000000000000 & 0.00000000000000 & 0.00000000000000 \\
\hline $\mathbf{C u}$ & 0.00000000000000 & 2.52244512012115 & 0.00000000000000 \\
\hline $\mathbf{C u}$ & 0.00000000000000 & 5.04488953395804 & 0.00000000000000 \\
\hline $\mathrm{Cu}$ & 0.00000000000000 & 7.56733465407835 & 0.00000000000000 \\
\hline $\mathbf{C u}$ & 0.00000000000000 & 10.08977871477400 & 0.00000000000000 \\
\hline $\mathbf{C u}$ & 0.00000000000000 & 12.61222383489520 & 0.00000000000000 \\
\hline $\mathbf{C u}$ & 0.00000000000000 & 15.13466895501420 & 0.00000000000000 \\
\hline
\end{tabular}




\begin{tabular}{|c|c|c|c|}
\hline $\mathrm{Cu}$ & 0.00000000000000 & 17.65711336885320 & 0.00000000000000 \\
\hline $\mathrm{Cu}$ & 0.00000000000000 & 20.17955848897460 & 0.00000000000000 \\
\hline $\mathrm{Cu}$ & 0.00000000000000 & 22.70200254966930 & 0.00000000000000 \\
\hline $\mathrm{Cu}$ & 0.00000000000000 & 25.22444766979040 & 0.00000000000000 \\
\hline $\mathbf{C u}$ & 0.00000000000000 & 27.74689208362320 & 0.00000000000000 \\
\hline $\mathbf{C u}$ & 0.00000000000000 & 30.26933720374440 & 0.00000000000000 \\
\hline $\mathrm{Cu}$ & 0.00000000000000 & 32.79178232386990 & 0.00000000000000 \\
\hline $\mathrm{Cu}$ & 3.56727419238642 & 0.00000000000000 & 0.00000000000000 \\
\hline $\mathbf{C u}$ & 3.56727419238642 & 2.52244512012115 & 0.00000000000000 \\
\hline $\mathbf{C u}$ & 3.56727419238642 & 5.04488953395804 & 0.00000000000000 \\
\hline $\mathbf{C u}$ & 3.56727419238642 & 7.56733465407835 & 0.00000000000000 \\
\hline $\mathrm{Cu}$ & 3.56727419238642 & 10.08977871477400 & 0.00000000000000 \\
\hline $\mathrm{Cu}$ & 3.56727419238642 & 12.61222383489520 & 0.00000000000000 \\
\hline $\mathrm{Cu}$ & 3.56727419238642 & 15.13466895501420 & 0.00000000000000 \\
\hline $\mathbf{C u}$ & 3.56727419238642 & 17.65711336885320 & 0.00000000000000 \\
\hline $\mathbf{C u}$ & 3.56727419238642 & 20.17955848897460 & 0.00000000000000 \\
\hline $\mathrm{Cu}$ & 3.56727419238642 & 22.70200254966930 & 0.00000000000000 \\
\hline $\mathbf{C u}$ & 3.56727419238642 & 25.22444766979040 & 0.00000000000000 \\
\hline $\mathbf{C u}$ & 3.56727419238642 & 27.74689208362320 & 0.00000000000000 \\
\hline $\mathrm{Cu}$ & 3.56727419238642 & 30.26933720374440 & 0.00000000000000 \\
\hline $\mathbf{C u}$ & 3.56727419238642 & 32.79178232386990 & 0.00000000000000 \\
\hline $\mathbf{C u}$ & 7.13454838477291 & 0.00000000000000 & 0.00000000000000 \\
\hline $\mathrm{Cu}$ & 7.13454838477291 & 2.52244512012115 & 000000000 \\
\hline $\mathrm{Cu}$ & 7.13454838477291 & 53395804 & 0000000 \\
\hline $\mathrm{Cu}$ & 7.13454838477291 & 7.56733465407835 & 0000000 \\
\hline $\mathrm{Cu}$ & 7.13454838477291 & 10.08977871477400 & 0.00000000000000 \\
\hline $\mathbf{C u}$ & 7.13454838477291 & 12.61222383489520 & 0.00000000000000 \\
\hline $\mathrm{Cu}$ & 7.13454838477291 & 15.13466895501420 & 0.00000000000000 \\
\hline $\mathbf{C u}$ & 7.13454838477291 & 17.65711336885320 & 0.00000000000000 \\
\hline $\mathbf{C u}$ & 7.13454838477291 & 20.17955848897460 & 0.00000000000000 \\
\hline $\mathbf{C u}$ & 7.13454838477291 & 22.70200254966930 & 0.00000000000000 \\
\hline $\mathrm{Cu}$ & 7.13454838477291 & 25.22444766979040 & 0.00000000000000 \\
\hline $\mathrm{Cu}$ & 7.13454838477291 & 27.74689208362320 & 0.00000000000000 \\
\hline $\mathbf{C u}$ & 7.13454838477291 & 30.26933720374440 & 0.00000000000000 \\
\hline $\mathbf{C u}$ & 7.13454838477291 & 32.79178232386990 & 0.00000000000000 \\
\hline $\mathbf{C u}$ & 1.78363955761358 & 1.26122414920059 & 1.26122399999993 \\
\hline $\mathrm{Cu}$ & 1.78363955761358 & 3.78366926932145 & 1.26122399999993 \\
\hline $\mathbf{C u}$ & 1.78363955761358 & 6.30611333001662 & 1.26122399999993 \\
\hline $\mathrm{Cu}$ & 1.78363955761358 & 8.82855845013778 & 1.26122399999993 \\
\hline $\mathbf{C u}$ & 1.78363955761358 & 11.35100286397460 & 1.26122399999993 \\
\hline $\mathrm{Cu}$ & 1.78363955761358 & 13.87344798409340 & 1.26122399999993 \\
\hline $\mathbf{C u}$ & 1.78363955761358 & 16.39589310421720 & 1.26122399999993 \\
\hline $\mathbf{C u}$ & 1.78363955761358 & 18.91833716491180 & 1.26122399999993 \\
\hline $\mathbf{C u}$ & 1.78363955761358 & 21.44078263817520 & 1.26122399999993 \\
\hline $\mathbf{C u}$ & 1.78363955761358 & 23.96322669886980 & 1.26122399999993 \\
\hline $\mathrm{Cu}$ & 1.78363955761358 & 26.48567181899090 & 1.26122399999993 \\
\hline $\mathbf{C u}$ & 1.78363955761358 & 29.00811693910830 & 1.26122399999993 \\
\hline $\mathrm{Cu}$ & 1.78363955761358 & 31.53056135294470 & 1.26122399999993 \\
\hline $\mathrm{Cu}$ & 1.78363955761358 & 34.05300647307050 & 1.26122399999993 \\
\hline $\mathrm{Cu}$ & 5.35091375000000 & 1.26122414920059 & 1.26122399999993 \\
\hline $\mathrm{Cu}$ & 5.35091375000000 & 3.78366926932145 & 1.26122399999993 \\
\hline $\mathbf{C u}$ & 5.35091375000000 & 6.30611333001662 & 1.26122399999993 \\
\hline $\mathbf{C u}$ & 5.35091375000000 & 8.82855845013778 & 1.26122399999993 \\
\hline $\mathbf{C u}$ & 5.35091375000000 & 11.35100286397460 & 1.26122399999993 \\
\hline
\end{tabular}




\begin{tabular}{|c|c|c|c|}
\hline $\mathrm{Cu}$ & 5.35091375000000 & 13.87344798409340 & 1.26122399999993 \\
\hline $\mathrm{Cu}$ & 5.35091375000000 & 16.39589310421720 & 1.26122399999993 \\
\hline $\mathrm{Cu}$ & 5.35091375000000 & 18.91833716491180 & 1.26122399999993 \\
\hline $\mathbf{C u}$ & 5.35091375000000 & 21.44078263817520 & 1.26122399999993 \\
\hline $\mathbf{C u}$ & 5.35091375000000 & 23.96322669886980 & 1.26122399999993 \\
\hline $\mathbf{C u}$ & 5.35091375000000 & 26.48567181899090 & 1.26122399999993 \\
\hline $\mathrm{Cu}$ & 5.35091375000000 & 29.00811693910830 & 1.26122399999993 \\
\hline $\mathrm{Cu}$ & 5.35091375000000 & 31.53056135294470 & 1.26122399999993 \\
\hline $\mathbf{C u}$ & 5.35091375000000 & 34.05300647307050 & 1.26122399999993 \\
\hline $\mathbf{C u}$ & 8.91818794238642 & 1.26122414920059 & 1.26122399999993 \\
\hline $\mathbf{C u}$ & 8.91818794238642 & 3.78366926932145 & 1.26122399999993 \\
\hline $\mathrm{Cu}$ & 8.91818794238642 & 6.30611333001662 & 1.26122399999993 \\
\hline $\mathrm{Cu}$ & 8.91818794238642 & 8.82855845013778 & 1.26122399999993 \\
\hline $\mathrm{Cu}$ & 8.91818794238642 & 11.35100286397460 & 1.26122399999993 \\
\hline $\mathbf{C u}$ & 8.91818794238642 & 13.87344798409340 & 1.26122399999993 \\
\hline $\mathrm{Cu}$ & 8.91818794238642 & 16.39589310421720 & 1.26122399999993 \\
\hline $\mathrm{Cu}$ & 8.91818794238642 & 18.91833716491180 & 1.26122399999993 \\
\hline $\mathbf{C u}$ & 8.91818794238642 & 21.44078263817520 & 1.26122399999993 \\
\hline $\mathbf{C u}$ & 8.91818794238642 & 23.96322669886980 & 1.26122399999993 \\
\hline $\mathrm{Cu}$ & 8.91818794238642 & 26.48567181899090 & 1.26122399999993 \\
\hline $\mathbf{C u}$ & 8.91818794238642 & 29.0081169 & 9999993 \\
\hline $\mathbf{C u}$ & 8.91818794238642 & 31.53056135294470 & 22399999993 \\
\hline $\mathrm{Cu}$ & 8.91818794238642 & 34.05300647307050 & 1.261223 \\
\hline $\mathrm{Cu}$ & 0.00160954146495 & 35.31219328409580 & 2.53765450095347 \\
\hline $\mathrm{Cu}$ & 10.69973193036370 & 2.52143997736990 & 2.54072384496659 \\
\hline $\mathrm{Cu}$ & 0.00336462310242 & 5.04443569750111 & 2.54313616293444 \\
\hline $\mathbf{C u}$ & 10.70040723701890 & 7.56490670921400 & 2.54298378229947 \\
\hline $\mathrm{Cu}$ & 10.70015638689580 & 10.08963358324560 & 2.53992915748926 \\
\hline $\mathbf{C u}$ & 10.70128036979110 & 12.61108327446490 & 2.54934603472701 \\
\hline $\mathrm{Cu}$ & 10.69543347520280 & 15.13639343125600 & 2.53740895582302 \\
\hline $\mathrm{Cu}$ & 10.69907975459670 & 17.65639697829570 & 2.54412471049980 \\
\hline $\mathrm{Cu}$ & 10.69727408462180 & 20.17977997618940 & 2.53808885022784 \\
\hline $\mathrm{Cu}$ & 10.69519758635940 & 22.69775130888330 & 2.53946063581951 \\
\hline $\mathbf{C u}$ & 10.69893607615480 & 25.22888549664250 & 2.53761407384749 \\
\hline $\mathbf{C u}$ & 10.70461611264170 & 27.74435945652320 & 2.54442997647437 \\
\hline $\mathbf{C u}$ & 10.70252354336440 & 30.26710996847360 & 2.54065583128983 \\
\hline $\mathrm{Cu}$ & 10.70056530940690 & 32.78885318887530 & 2.54055009498879 \\
\hline $\mathbf{C u}$ & 3.56727579386471 & 35.31046879462160 & 2.54122313347023 \\
\hline $\mathrm{Cu}$ & 3.56728076802013 & 2.52075701594384 & 2.54259056636239 \\
\hline $\mathbf{C u}$ & 3.56727920384424 & 5.04397090627801 & 2.53812913508738 \\
\hline $\mathrm{Cu}$ & 3.56727245821076 & 7.56951957971336 & 2.58080081425727 \\
\hline $\mathbf{C u}$ & 3.56727850318044 & 10.09209890921950 & 2.59112872242803 \\
\hline $\mathbf{C u}$ & 3.56727375346763 & 12.62009068738130 & 2.57491419589711 \\
\hline $\mathbf{C u}$ & 3.56725325337601 & 15.14029078929870 & 2.58201389855041 \\
\hline $\mathbf{C u}$ & 3.56725496057139 & 17.65997844494810 & 2.58722722425434 \\
\hline $\mathrm{Cu}$ & 3.56727861866343 & 20.16939647322840 & 2.57716692818913 \\
\hline $\mathrm{Cu}$ & 3.56727556166615 & 22.70110774035060 & 2.57195178555822 \\
\hline $\mathrm{Cu}$ & 3.56727393026507 & 25.21768537184560 & 2.57549320951345 \\
\hline $\mathbf{C u}$ & 3.56727843161770 & 27.74026955369260 & 2.54617999139754 \\
\hline $\mathrm{Cu}$ & 3.56728860210125 & 30.26392030631260 & 2.54364110074838 \\
\hline $\mathbf{C u}$ & 3.56727435819195 & 32.78762163355260 & 2.54209802107914 \\
\hline $\mathbf{C u}$ & 7.13295975145689 & 35.31219987193730 & 2.53767021765848 \\
\hline $\mathbf{C u}$ & 7.13665875389526 & 2.52144143948236 & 2.54073674997071 \\
\hline $\mathbf{C u}$ & 7.13119720264085 & 5.04443272557936 & 2.54313300990124 \\
\hline
\end{tabular}




\begin{tabular}{|c|c|c|c|}
\hline $\mathrm{Cu}$ & 7.13597827482644 & 7.56490954499318 & 2.54300306171228 \\
\hline $\mathbf{C u}$ & 7.13625600908856 & 10.08963070598940 & 2.53991408900987 \\
\hline $\mathrm{Cu}$ & 7.13512646497673 & 12.61108045018720 & 2.54937727050341 \\
\hline $\mathbf{C u}$ & 7.14093747220274 & 15.13639509941160 & 2.53740947755111 \\
\hline $\mathbf{C u}$ & 7.13727584473015 & 17.65640959900530 & 2.54416466099759 \\
\hline $\mathbf{C u}$ & 7.13908185949971 & 20.17978859713150 & 2.53811840876623 \\
\hline $\mathbf{C u}$ & 7.14122059775870 & 22.69778319593820 & 2.53948622390634 \\
\hline $\mathbf{C u}$ & 7.13747117640477 & 25.22887072046770 & 2.53761593318747 \\
\hline $\mathbf{C u}$ & 7.13176351931728 & 27.74437822899540 & 2.54444360176374 \\
\hline $\mathbf{C u}$ & 7.13386741522585 & 30.26710073565380 & 2.54066164748872 \\
\hline $\mathbf{C u}$ & 7.13576286042078 & 32.78884901972250 & 2.54059747616993 \\
\hline $\mathbf{C u}$ & 1.78450140691536 & 1.25526956596257 & 3.85282587453455 \\
\hline $\mathbf{C u}$ & 1.78038822605878 & 3.77721140009629 & 3.85266323127089 \\
\hline $\mathrm{Cu}$ & 1.79731183318485 & 6.30455468620261 & 3.85767930465318 \\
\hline $\mathbf{C u}$ & 1.78034185301974 & 8.82580312440983 & 3.83429399105693 \\
\hline $\mathbf{C u}$ & 1.80132281017083 & 11.34711973418510 & 3.85029432481352 \\
\hline $\mathbf{C u}$ & 1.77415797760106 & 13.88173580206240 & 3.83637690481502 \\
\hline $\mathbf{C u}$ & 1.78787893756965 & 16.40120715533120 & 3.85153519689594 \\
\hline $\mathbf{C u}$ & 1.77186961661833 & 18.92100541686920 & 3.84402921052836 \\
\hline $\mathbf{C u}$ & 1.78449011186406 & 21.43662141908390 & 3.84031463889290 \\
\hline $\mathbf{C u}$ & 1.76735014971013 & 23.96583996023080 & 3.82333663283781 \\
\hline $\mathbf{C u}$ & 1.79494405154490 & 26.47169363137340 & 3.86944220426525 \\
\hline $\mathbf{C u}$ & 1.78455207241055 & 28.99939236584340 & 3.86021506576008 \\
\hline $\mathbf{C u}$ & 160977107 & 31.52205819022400 & 059876380 \\
\hline $\mathbf{C u}$ & 1.78469332487100 & 34.04423330014020 & 73599 \\
\hline $\mathbf{C u}$ & 5.34999469935666 & 1.25530391043191 & 3.85289935237290 \\
\hline $\mathbf{C u}$ & 5.35417925444589 & 3.77717692690162 & 3.85266544351038 \\
\hline $\mathbf{C u}$ & 5.33721171730604 & 6.30451014624169 & 3.85768268318106 \\
\hline $\mathbf{C u}$ & 5.35423686547273 & 8.82580368986347 & 3.83427420125493 \\
\hline $\mathbf{C u}$ & 5.33325527607010 & 11.34713332386850 & 3.85025393402613 \\
\hline $\mathbf{C u}$ & 5.36039216302888 & 13.88182371851620 & 3.83635551421126 \\
\hline $\mathbf{C u}$ & 5.34661077536566 & 16.40123194257340 & 3.85153479602955 \\
\hline $\mathbf{C u}$ & 5.36256867001392 & 18.92101957808170 & 3.84405585475831 \\
\hline $\mathbf{C u}$ & 5.35002711444491 & 21.43656962486790 & 3.84036596853058 \\
\hline $\mathbf{C u}$ & 5.36725645959167 & 23.96583007291010 & 3.82342342864585 \\
\hline $\mathbf{C u}$ & 5.33965242457441 & 26.47168817156960 & 3.86941055392498 \\
\hline $\mathbf{C u}$ & 5.35004340619429 & 28.99939556156290 & 3.86019702945276 \\
\hline $\mathbf{C u}$ & 5.35087496171755 & 31.52206337550090 & 3.85633734070077 \\
\hline $\mathbf{C u}$ & 5.34987107768328 & 34.04425654599540 & 3.85235721080888 \\
\hline $\mathbf{C u}$ & 8.91819810050234 & 1.25913324281191 & 3.84993493509355 \\
\hline $\mathbf{C u}$ & 8.91819918398457 & 3.78305108425932 & 3.85173937720805 \\
\hline $\mathbf{C u}$ & 8.91819502538582 & 6.30140189773557 & 3.85328197682571 \\
\hline $\mathbf{C u}$ & 8.91818682760511 & 8.82504459503093 & 3.85653532358613 \\
\hline $\mathbf{C u}$ & 8.91819342570022 & 11.35483704722590 & 3.85396656150484 \\
\hline $\mathbf{C u}$ & 8.91817608215192 & 13.87132089623290 & 3.85202125476689 \\
\hline $\mathbf{C u}$ & 8.91817646439359 & 16.39935799245660 & 3.85790894708084 \\
\hline $\mathbf{C u}$ & 8.91818367318877 & 18.92140934482280 & 3.85220590509430 \\
\hline $\mathbf{C u}$ & 8.91819799919130 & 21.43888636749160 & 3.85367595975432 \\
\hline $\mathbf{C u}$ & 8.91821438079065 & 23.96243402066890 & 3.84414046826326 \\
\hline $\mathbf{C u}$ & 8.91820020238783 & 26.48550366283810 & 3.85366038228255 \\
\hline $\mathbf{C u}$ & 8.91820846914573 & 29.00094252349180 & 3.84849876108845 \\
\hline $\mathbf{C u}$ & 8.91819120889662 & 31.52854137484930 & 3.85188946735767 \\
\hline $\mathbf{C u}$ & 8.91820909753146 & 34.04669693069500 & 3.84939840757251 \\
\hline $\mathbf{C u}$ & 0.00299980082885 & 35.30859402342270 & 5.05297745557459 \\
\hline
\end{tabular}




\begin{tabular}{|c|c|c|c|}
\hline $\mathbf{C u}$ & 0.00405435895858 & 2.51734459119823 & 5.05626862135388 \\
\hline $\mathbf{C u}$ & 0.00469633600403 & 5.04666871386797 & 5.05665907836491 \\
\hline $\mathrm{Cu}$ & 0.00428473142102 & 7.56466906856073 & 5.05112413689930 \\
\hline $\mathbf{C u}$ & 0.00776376194968 & 10.09232708156170 & 5.04532218620303 \\
\hline $\mathbf{C u}$ & 0.01319122858757 & 12.61012378624610 & 5.05952986443293 \\
\hline $\mathbf{C u}$ & 0.00577475753889 & 15.13873148626680 & 5.05174798081866 \\
\hline $\mathbf{C u}$ & -0.00039292668135 & 17.66006406706960 & 5.06245901714779 \\
\hline $\mathbf{C u}$ & 0.00437930532575 & 20.18343463290750 & 5.05856583553964 \\
\hline $\mathbf{C u}$ & 0.00328462194800 & 22.70153395896000 & 5.04737686144734 \\
\hline $\mathbf{C u}$ & 10.70190025976290 & 25.21913656017510 & 5.05495592098052 \\
\hline $\mathbf{C u}$ & 10.70393548818930 & 27.74063596190680 & 5.06288795941088 \\
\hline $\mathbf{C u}$ & 10.69941211882200 & 30.26114801208830 & 5.05551890594084 \\
\hline $\mathbf{C u}$ & 0.00372697095121 & 32.78563967139910 & 5.05906389422547 \\
\hline $\mathbf{C u}$ & 3.56727892797668 & 35.30346713858370 & 5.05842768024649 \\
\hline $\mathbf{C u}$ & 3.56723929291936 & 2.51351533326111 & 5.05691800914771 \\
\hline $\mathbf{C u}$ & 3.56727038586522 & 5.01861498080597 & 5.04776760010473 \\
\hline $\mathrm{Cu}$ & 3.56724697426612 & 7.51904951327920 & 5.15790175154819 \\
\hline $\mathbf{C u}$ & 3.56732095394638 & 10.10232591590170 & 5.15459377884594 \\
\hline $\mathbf{C u}$ & 3.56729706375706 & 12.62366738339310 & 5.12509111586578 \\
\hline $\mathbf{C u}$ & 3.56727865167557 & 15.15532971107300 & 5.09016249124077 \\
\hline $\mathbf{C u}$ & 3.56724543589022 & 17.68830177162030 & 5.14436533459337 \\
\hline $\mathbf{C u}$ & 3.56716876880081 & 20.21191519500710 & 5.10064250104324 \\
\hline $\mathbf{C u}$ & 091625 & 7188030 & 51036 \\
\hline $\mathbf{C u}$ & 3.56729961519919 & 25.17524094 & 5.1597 \\
\hline $\mathbf{C u}$ & 3.5672991 & 27.721442 & 5.066 \\
\hline $\mathrm{Cu}$ & 08229 & 30.2 & 65628 \\
\hline $\mathbf{C u}$ & 3.567 & 32.780 & 494900 \\
\hline $\mathbf{C u}$ & 7.13159 & 35.308 & 5.053011 \\
\hline $\mathrm{Cu}$ & 7.13051024450892 & 2.5173 & 5.056294 \\
\hline $\mathrm{Cu}$ & 7.12980930540514 & 5.04661993893295 & 5.05667743325640 \\
\hline $\mathrm{Cu}$ & 7.13023561730174 & 7.56470644337622 & 5.05112039564752 \\
\hline $\mathrm{Cu}$ & 7.12677959733953 & 10.09235739273750 & 5.04531324764437 \\
\hline $\mathrm{Cu}$ & 7.12133267302203 & 12.61014071980240 & 5.05960798500126 \\
\hline $\mathbf{C u}$ & 7.12875279447041 & 15.13877159834500 & 5.05172237865353 \\
\hline $\mathbf{C u}$ & 7.13489607498858 & 17.66008252285590 & 5.06248252035495 \\
\hline $\mathbf{C u}$ & 7.13017993730418 & 20.18345578095680 & 5.05857519555696 \\
\hline $\mathbf{C u}$ & 7.13132973292799 & 22.70152582916800 & 5.04738976076138 \\
\hline $\mathbf{C u}$ & 7.13447498082905 & 25.21909055250280 & 5.05496971728637 \\
\hline $\mathbf{C u}$ & 7.13248235257882 & 27.74062991083450 & 5.06290110531086 \\
\hline $\mathbf{C u}$ & 7.13701356242503 & 30.26113705965530 & 5.05553398130307 \\
\hline $\mathbf{C u}$ & 7.13086631818637 & 32.78564560524320 & 5.05908294233115 \\
\hline \multicolumn{4}{|c|}{ CI-NEB Image 26} \\
\hline Element & X-Coordinate (§̊) & Y-Coordinate (§̊) & Z-Coordinate (§̊) \\
\hline $\mathbf{F}$ & 4.66312253880296 & 12.65406165807620 & 7.74385997335362 \\
\hline $\mathbf{F}$ & 2.47151373374714 & 12.65398470999980 & 7.74388161147357 \\
\hline $\mathbf{F}$ & 3.56729617283447 & 8.81260937585887 & 6.63660292546956 \\
\hline $\mathbf{F}$ & 4.66505699178080 & 17.72846981027050 & 7.75925268119587 \\
\hline $\mathbf{F}$ & 2.47197969722983 & 17.72720634647080 & 7.76058749658683 \\
\hline $\mathbf{F}$ & 3.56725500764999 & 14.51609038241330 & 7.26762557123569 \\
\hline $\mathbf{F}$ & 4.64738961288091 & 23.58209149009170 & 7.55950681670074 \\
\hline $\mathbf{F}$ & 2.48773203928179 & 23.58212637415890 & 7.55989381156700 \\
\hline $\mathbf{F}$ & 3.56716871256980 & 19.59191124095110 & 7.29954831606035 \\
\hline C & 3.56729679998914 & 13.08663503793750 & 7.04154415814452 \\
\hline C & 3.56782056842785 & 18.16223101003570 & 7.05881070512521 \\
\hline
\end{tabular}




\begin{tabular}{|c|c|c|c|}
\hline $\mathbf{C}$ & 3.56741776229510 & 23.68260925079640 & 6.74605830099697 \\
\hline $\mathbf{C u}$ & 0.00000000000000 & 0.00000000000000 & 0.00000000000000 \\
\hline $\mathbf{C u}$ & 0.00000000000000 & 2.52244512012115 & 0.00000000000000 \\
\hline $\mathbf{C u}$ & 0.00000000000000 & 5.04488953395804 & 0.00000000000000 \\
\hline $\mathbf{C u}$ & 0.00000000000000 & 7.56733465407835 & 0.00000000000000 \\
\hline $\mathbf{C u}$ & 0.00000000000000 & 10.08977871477400 & 0.00000000000000 \\
\hline $\mathbf{C u}$ & 0.00000000000000 & 12.61222383489520 & 0.00000000000000 \\
\hline $\mathbf{C u}$ & 0.00000000000000 & 15.13466895501440 & 0.00000000000000 \\
\hline $\mathbf{C u}$ & 0.00000000000000 & 17.65711336885320 & 0.00000000000000 \\
\hline $\mathbf{C u}$ & 0.00000000000000 & 20.17955848897460 & 0.00000000000000 \\
\hline $\mathbf{C u}$ & 0.00000000000000 & 22.70200254966930 & 0.00000000000000 \\
\hline $\mathrm{Cu}$ & 0.00000000000000 & 25.22444766979040 & 0.00000000000000 \\
\hline $\mathbf{C u}$ & 0.00000000000000 & 27.74689208362370 & 0.00000000000000 \\
\hline $\mathbf{C u}$ & 0.00000000000000 & 30.26933720374490 & 0.00000000000000 \\
\hline $\mathbf{C u}$ & 0.00000000000000 & 32.79178232386990 & 0.00000000000000 \\
\hline $\mathbf{C u}$ & 3.56727419238642 & 0.00000000000000 & 0.00000000000000 \\
\hline $\mathrm{Cu}$ & 3.56727419238642 & 2.52244512012115 & 0.00000000000000 \\
\hline $\mathbf{C u}$ & 3.56727419238642 & 5.04488953395804 & 0.00000000000000 \\
\hline $\mathbf{C u}$ & 3.56727419238642 & 7.56733465407835 & 0.00000000000000 \\
\hline $\mathbf{C u}$ & 3.56727419238642 & 10.08977871477400 & 0.00000000000000 \\
\hline $\mathbf{C u}$ & 3.56727419238642 & 12.61222383489520 & 0.00000000000000 \\
\hline $\mathbf{C u}$ & 3.56727419238642 & 15.13466895501440 & 0.00000000000000 \\
\hline $\mathbf{C u}$ & 3.56727419238642 & 17.65711336885320 & 0.00000000000000 \\
\hline $\mathbf{C u}$ & 3.56727419238642 & 20.17955848897460 & 0.00000000000000 \\
\hline $\mathbf{C u}$ & 3.56727419238642 & 22.70200254966930 & 0.00000000000000 \\
\hline $\mathbf{C u}$ & 3.56727419238642 & 25.22444766979040 & 0.00000000000000 \\
\hline $\mathbf{C u}$ & 3.56727419238642 & 27.74689208362370 & 0.00000000000000 \\
\hline $\mathbf{C u}$ & 3.56727419238642 & 30.26933720374490 & 0.00000000000000 \\
\hline $\mathbf{C u}$ & 3.56727419238642 & 32.79178232386990 & 0.00000000000000 \\
\hline $\mathbf{C u}$ & 7.13454838477291 & 0.00000000000000 & 0.00000000000000 \\
\hline $\mathbf{C u}$ & 7.13454838477291 & 2.52244512012115 & 0.00000000000000 \\
\hline $\mathbf{C u}$ & 7.13454838477291 & 5.04488953395804 & 0.00000000000000 \\
\hline $\mathbf{C u}$ & 7.13454838477291 & 7.56733465407835 & 0.00000000000000 \\
\hline $\mathbf{C u}$ & 7.13454838477291 & 10.08977871477400 & 0.00000000000000 \\
\hline $\mathbf{C u}$ & 7.13454838477291 & 12.61222383489520 & 0.00000000000000 \\
\hline $\mathbf{C u}$ & 7.13454838477291 & 15.13466895501440 & 0.00000000000000 \\
\hline $\mathbf{C u}$ & 7.13454838477291 & 17.65711336885320 & 0.00000000000000 \\
\hline $\mathbf{C u}$ & 7.13454838477291 & 20.17955848897460 & 0.00000000000000 \\
\hline $\mathbf{C u}$ & 7.13454838477291 & 22.70200254966930 & 0.00000000000000 \\
\hline $\mathbf{C u}$ & 7.13454838477291 & 25.22444766979040 & 0.00000000000000 \\
\hline $\mathbf{C u}$ & 7.13454838477291 & 27.74689208362370 & 0.00000000000000 \\
\hline $\mathbf{C u}$ & 7.13454838477291 & 30.26933720374490 & 0.00000000000000 \\
\hline $\mathbf{C u}$ & 7.13454838477291 & 32.79178232386990 & 0.00000000000000 \\
\hline $\mathbf{C u}$ & 1.78363955761358 & 1.26122414920059 & 1.26122399999993 \\
\hline $\mathbf{C u}$ & 1.78363955761358 & 3.78366926932145 & 1.26122399999993 \\
\hline $\mathbf{C u}$ & 1.78363955761358 & 6.30611333001662 & 1.26122399999993 \\
\hline $\mathbf{C u}$ & 1.78363955761358 & 8.82855845013778 & 1.26122399999993 \\
\hline $\mathbf{C u}$ & 1.78363955761358 & 11.35100286397460 & 1.26122399999993 \\
\hline $\mathbf{C u}$ & 1.78363955761358 & 13.87344798409360 & 1.26122399999993 \\
\hline $\mathbf{C u}$ & 1.78363955761358 & 16.39589310421720 & 1.26122399999993 \\
\hline $\mathbf{C u}$ & 1.78363955761358 & 18.91833716491180 & 1.26122399999993 \\
\hline $\mathbf{C u}$ & 1.78363955761358 & 21.44078263817520 & 1.26122399999993 \\
\hline $\mathbf{C u}$ & 1.78363955761358 & 23.96322669886980 & 1.26122399999993 \\
\hline $\mathbf{C u}$ & 1.78363955761358 & 26.48567181899090 & 1.26122399999993 \\
\hline
\end{tabular}




\begin{tabular}{|c|c|c|c|}
\hline $\mathbf{C u}$ & 1.78363955761358 & 29.00811693910880 & 1.26122399999993 \\
\hline $\mathbf{C u}$ & 1.78363955761358 & 31.53056135294520 & 1.26122399999993 \\
\hline $\mathbf{C u}$ & 1.78363955761358 & 34.05300647307050 & 1.26122399999993 \\
\hline $\mathbf{C u}$ & 5.35091375000000 & 1.26122414920059 & 1.26122399999993 \\
\hline $\mathbf{C u}$ & 5.35091375000000 & 3.78366926932145 & 1.26122399999993 \\
\hline $\mathbf{C u}$ & 5.35091375000000 & 6.30611333001662 & 1.26122399999993 \\
\hline $\mathbf{C u}$ & 5.35091375000000 & 8.82855845013778 & 1.26122399999993 \\
\hline $\mathbf{C u}$ & 5.35091375000000 & 11.35100286397460 & 1.26122399999993 \\
\hline $\mathbf{C u}$ & 5.35091375000000 & 13.87344798409360 & 1.26122399999993 \\
\hline $\mathbf{C u}$ & 5.35091375000000 & 16.39589310421720 & 1.26122399999993 \\
\hline $\mathbf{C u}$ & 5.35091375000000 & 18.91833716491180 & 1.26122399999993 \\
\hline $\mathbf{C u}$ & 5.35091375000000 & 21.44078263817520 & 1.26122399999993 \\
\hline $\mathbf{C u}$ & 5.35091375000000 & 23.96322669886980 & 1.26122399999993 \\
\hline $\mathbf{C u}$ & 5.35091375000000 & 26.48567181899090 & 1.26122399999993 \\
\hline $\mathbf{C u}$ & 5.35091375000000 & 29.00811693910880 & 1.26122399999993 \\
\hline $\mathbf{C u}$ & 5.35091375000000 & 31.53056135294520 & 1.26122399999993 \\
\hline $\mathbf{C u}$ & 5.35091375000000 & 34.05300647307050 & 1.26122399999993 \\
\hline $\mathbf{C u}$ & 8.91818794238642 & 1.26122414920059 & 1.26122399999993 \\
\hline $\mathbf{C u}$ & 8.91818794238642 & 3.78366926932145 & 1.26122399999993 \\
\hline $\mathbf{C u}$ & 8.91818794238642 & 33001662 & 99999993 \\
\hline $\mathbf{C u}$ & 8.91818794238642 & 78 & 2399 \\
\hline $\mathbf{C u}$ & 8.91818794238642 & 11. & 1.26122399999993 \\
\hline $\mathbf{C u}$ & 8.9181 & 13.87344798409360 & 1.26122399999993 \\
\hline $\mathbf{C u}$ & 8.91818794238642 & 16.39589310421720 & 1.26122399999993 \\
\hline $\mathbf{C u}$ & 8.91818794238642 & 18.91833716491180 & 1.26122399999993 \\
\hline $\mathbf{C u}$ & 8.91818794238642 & 21.44078263817520 & 1.26122399999993 \\
\hline $\mathbf{C u}$ & 8.91818794238642 & 23.96322669886980 & 1.26122399999993 \\
\hline $\mathbf{C u}$ & 8.91818794238642 & 26.48567181899090 & 1.26122399999993 \\
\hline $\mathbf{C u}$ & 8.91818794238642 & 29.00811693910880 & 1.26122399999993 \\
\hline $\mathbf{C u}$ & 8.91818794238642 & 31.53056135294520 & 1.26122399999993 \\
\hline $\mathbf{C u}$ & 8.91818794238642 & 34.05300647307050 & 1.26122399999993 \\
\hline $\mathbf{C u}$ & 0.00122692531848 & 35.31192205506660 & 2.53791510383672 \\
\hline $\mathbf{C u}$ & 10.69999194383190 & 2.52176946160558 & 2.54042780594990 \\
\hline $\mathbf{C u}$ & 0.00246082192890 & 5.04472385149220 & 2.54207805829523 \\
\hline $\mathbf{C u}$ & 10.70047781572090 & 7.56370921596306 & 2.54334428709806 \\
\hline $\mathbf{C u}$ & 10.69912065740100 & 10.08878450373550 & 2.53979430293683 \\
\hline $\mathbf{C u}$ & 10.70027764331520 & 12.61015913883540 & 2.54836683579246 \\
\hline $\mathbf{C u}$ & 10.69602061069170 & 15.13555835091810 & 2.53729082683258 \\
\hline $\mathbf{C u}$ & 10.69812240546500 & 17.65489883449680 & 2.54335775454655 \\
\hline $\mathbf{C u}$ & 10.69718293951770 & 20.17763993064640 & 2.53826484578581 \\
\hline $\mathbf{C u}$ & 10.69099799689600 & 22.69368783578260 & 2.53723297811589 \\
\hline $\mathbf{C u}$ & 10.69830960518850 & 25.22844612282740 & 2.53715828880688 \\
\hline $\mathbf{C u}$ & 0.00477070548668 & 27.74301037499750 & 2.54701114503326 \\
\hline $\mathbf{C u}$ & 10.70258798758860 & 30.26416972983670 & 2.54063022618833 \\
\hline $\mathbf{C u}$ & 10.70071616773540 & 32.78783851261470 & 2.54082855746536 \\
\hline $\mathbf{C u}$ & 3.56727819439765 & 35.30949463724950 & 2.54185597609360 \\
\hline $\mathbf{C u}$ & 3.56727784836611 & 2.51978921025118 & 2.54201945043445 \\
\hline $\mathbf{C u}$ & 3.56728058608039 & 5.04185022419409 & 2.53844681566700 \\
\hline $\mathbf{C u}$ & 3.56727643384742 & 7.56964273643120 & 2.58012500273551 \\
\hline $\mathbf{C u}$ & 3.56728175471246 & 10.09215384617970 & 2.59037176226017 \\
\hline $\mathbf{C u}$ & 3.56728196769955 & 12.61789075636770 & 2.57551671489988 \\
\hline $\mathbf{C u}$ & 3.56728262935551 & 15.13915655385210 & 2.58342638355055 \\
\hline $\mathbf{C u}$ & 3.56727600767502 & 17.65727929065550 & 2.58399842868279 \\
\hline $\mathbf{C u}$ & 3.56727090214300 & 20.16795084821300 & 2.57553245840022 \\
\hline
\end{tabular}




\begin{tabular}{|c|c|c|c|}
\hline $\mathbf{C u}$ & 3.56728992228534 & 22.70180841270030 & 2.55363152456260 \\
\hline $\mathbf{C u}$ & 3.56728397392510 & 25.21422869891440 & 2.59317630363123 \\
\hline $\mathbf{C u}$ & 3.56728003413668 & 27.73868468079880 & 2.54742192624054 \\
\hline $\mathbf{C u}$ & 3.56728380552381 & 30.26279748644480 & 2.54594147623772 \\
\hline $\mathbf{C u}$ & 3.56727762094241 & 32.78601695416070 & 2.54293935807875 \\
\hline $\mathbf{C u}$ & 7.13333030218527 & 35.31192496636930 & 2.53791200456312 \\
\hline $\mathbf{C u}$ & 7.13639753302613 & 2.52177170729033 & 2.54043403856036 \\
\hline $\mathbf{C u}$ & 7.13210360155906 & 5.04472413995198 & 2.54207222938218 \\
\hline $\mathbf{C u}$ & 7.13591163836153 & 7.56372183801994 & 2.54334249372790 \\
\hline $\mathbf{C u}$ & 7.13727288909902 & 10.08878471321470 & 2.53977794918064 \\
\hline $\mathbf{C u}$ & 7.13611217350222 & 12.61017567585000 & 2.54837736139794 \\
\hline $\mathbf{C u}$ & 7.14036071476171 & 15.13556641875100 & 2.53729398338357 \\
\hline $\mathbf{C u}$ & 7.13825437872781 & 17.65490454091260 & 2.54335665187576 \\
\hline $\mathrm{Cu}$ & 7.13919370751443 & 20.17765327405200 & 2.53827678989595 \\
\hline $\mathbf{C u}$ & 7.14538390974987 & 22.69369974164680 & 2.53726607910840 \\
\hline $\mathbf{C u}$ & 7.13807591683761 & 25.22844414136230 & 2.53716355914398 \\
\hline $\mathbf{C u}$ & 7.12977841962636 & 27.74300876693450 & 2.54701006246298 \\
\hline $\mathbf{C u}$ & 7.13379600547345 & 30.26419053836240 & 2.54062874092752 \\
\hline $\mathbf{C u}$ & 7.13566789819574 & 32.78784307024250 & 2.54082926389585 \\
\hline $\mathbf{C u}$ & 1.78553514285945 & 1.25403627076842 & 3.85136108162617 \\
\hline $\mathbf{C u}$ & 1.78048493405787 & 3.77764450477367 & 3.85208952649987 \\
\hline $\mathbf{C u}$ & 1.79539576866694 & 6.30379303863558 & 3.85804946061922 \\
\hline $\mathbf{C u}$ & 1.77882038134447 & 0377490146 & 14983183 \\
\hline $\mathbf{C u}$ & 1.79995639099775 & 11.34523779343690 & 549619 \\
\hline $\mathbf{C u}$ & 77098 & 13.878 & 26648 \\
\hline $\mathrm{Cu}$ & 1.78780994399488 & 16.39940671451930 & 78533410 \\
\hline $\mathbf{C u}$ & 1.77211403830371 & 18.91873492817290 & 3.84291773746128 \\
\hline $\mathbf{C u}$ & 1.78496019323200 & 21.43311570305810 & 3.84069771465467 \\
\hline $\mathrm{Cu}$ & 1.75769177567101 & 23.95613386499010 & 3.80988583724733 \\
\hline $\mathrm{Cu}$ & 1.80306740209202 & 26.46057165702840 & 3.87539242173752 \\
\hline $\mathrm{Cu}$ & 1.78602759704436 & 28.99209242276380 & 3.86035078203499 \\
\hline $\mathbf{C u}$ & 1.78355030948649 & 31.51713335426450 & 3.85687035509309 \\
\hline $\mathrm{Cu}$ & 1.78539241465668 & 34.04206631591320 & 3.85255981034938 \\
\hline $\mathbf{C u}$ & 5.34902494851491 & 1.25404880264234 & 3.85136706404849 \\
\hline $\mathbf{C u}$ & 5.35407117426179 & 3.77764879772875 & 3.85209270919759 \\
\hline $\mathbf{C u}$ & 5.33918360817432 & 6.30378286378921 & 3.85804199317051 \\
\hline $\mathbf{C u}$ & 5.35576985972883 & 8.82469140349880 & 3.83417894162149 \\
\hline $\mathbf{C u}$ & 5.33463406995930 & 11.34523846671910 & 3.84839622488149 \\
\hline $\mathbf{C u}$ & 5.36089039615093 & 13.87838159075830 & 3.83570437725377 \\
\hline $\mathbf{C u}$ & 5.34674423180256 & 16.39940836877490 & 3.85001970316148 \\
\hline $\mathbf{C u}$ & 5.36241687944688 & 18.91874411274160 & 3.84291861050706 \\
\hline $\mathbf{C u}$ & 5.34957799765629 & 21.43312482125130 & 3.84075576405714 \\
\hline $\mathbf{C u}$ & 5.37688625575730 & 23.95613204682830 & 3.80991976833827 \\
\hline $\mathbf{C u}$ & 5.33150404800023 & 26.46057051069620 & 3.87536908741034 \\
\hline $\mathrm{Cu}$ & 5.34855443128140 & 28.99209831499050 & 3.86034881206491 \\
\hline $\mathbf{C u}$ & 5.35103421652422 & 31.51713201039030 & 3.85691765055506 \\
\hline $\mathbf{C u}$ & 5.34917440054940 & 34.04205345780990 & 3.85256161596471 \\
\hline $\mathbf{C u}$ & 8.91819591111907 & 1.25681289073265 & 3.85188149074497 \\
\hline $\mathbf{C u}$ & 8.91819281329893 & 3.78024656106393 & 3.85174758692399 \\
\hline $\mathbf{C u}$ & 8.91818890644629 & 6.30118692933976 & 3.85405067704708 \\
\hline $\mathbf{C u}$ & 8.91820541716730 & 8.82545028713089 & 3.85749740378667 \\
\hline $\mathbf{C u}$ & 8.91819166399379 & 11.35457306409480 & 3.85384706456356 \\
\hline $\mathbf{C u}$ & 8.91819180437064 & 13.87062830935510 & 3.85205815214943 \\
\hline $\mathbf{C u}$ & 8.91819783429832 & 16.39567333986490 & 3.85682422004232 \\
\hline
\end{tabular}




\begin{tabular}{|c|c|c|c|}
\hline $\mathbf{C u}$ & 8.91818688419136 & 18.91950172330400 & 3.85251763417547 \\
\hline $\mathbf{C u}$ & 8.91819989428896 & 21.43372606003720 & 3.85284451722434 \\
\hline $\mathrm{Cu}$ & 8.91820428596771 & 23.95828279201630 & 3.84290834951122 \\
\hline $\mathrm{Cu}$ & 8.91820155035390 & 26.48414138470680 & 3.85289568518075 \\
\hline $\mathbf{C u}$ & 8.91816310187095 & 28.99807161050940 & 3.84818708806237 \\
\hline $\mathbf{C u}$ & 8.91819758384467 & 31.52617358641460 & 3.85360935586804 \\
\hline $\mathbf{C u}$ & 8.91819503493497 & 34.04680439136960 & 3.85195778268534 \\
\hline $\mathrm{Cu}$ & 0.00626953037958 & 35.30674079129790 & 5.05301382337359 \\
\hline $\mathbf{C u}$ & 0.00668759848063 & 2.51640316256886 & 5.05428414877924 \\
\hline $\mathbf{C u}$ & 0.00280995593096 & 5.04574736639967 & 5.05620199276348 \\
\hline $\mathbf{C u}$ & 0.00123544896740 & 7.56367262472824 & 5.05478588774108 \\
\hline $\mathbf{C u}$ & 0.00733285030090 & 10.09217482495020 & 5.04538617386777 \\
\hline $\mathbf{C u}$ & 0.01415368142779 & 12.60846628834280 & 5.05926293318087 \\
\hline $\mathrm{Cu}$ & 0.00760516180906 & 15.13548768902410 & 5.05210661899214 \\
\hline $\mathrm{Cu}$ & -0.00072524843086 & 17.65719384792560 & 5.05937488450234 \\
\hline $\mathbf{C u}$ & 0.00494303389672 & 20.17988458606820 & 5.05895096756733 \\
\hline $\mathrm{Cu}$ & 0.00274087134493 & 22.69587834750930 & 5.049 \\
\hline $\mathrm{Cu}$ & 10.70064974673850 & 25.21485486022680 & 5.0496760 \\
\hline $\mathrm{Cu}$ & 148869480 & 27.73505761548450 & 5.0638 \\
\hline $\mathrm{Cu}$ & 10.69860722375770 & 30.25595465709250 & $5.0556251 \xi$ \\
\hline $\mathrm{Cu}$ & 0.0037930 & 32.782760 & 5.05787978161088 \\
\hline $\mathbf{C u}$ & 3.56728797130384 & 35.30034858524940 & 5.0594913 \\
\hline $\mathrm{Cu}$ & 3.56728155112945 & 2.51005687850277 & 5.05504644206362 \\
\hline $\mathrm{Cu}$ & 3.56729209381635 & 5.01962609434971 & 5.04740553458445 \\
\hline $\mathrm{Cu}$ & 3.56727652971788 & 7.51945565394388 & 5.15578383499462 \\
\hline $\mathrm{Cu}$ & 3.56734087369484 & 10.10015893785110 & 5.15230260952665 \\
\hline $\mathrm{Cu}$ & 3.56728839421628 & 12.62236845327730 & 5.12413808276142 \\
\hline $\mathrm{Cu}$ & 3.56727505643676 & 15.15344242184930 & 5.08850690368659 \\
\hline $\mathrm{Cu}$ & 3.56722633741645 & 17.68288697129980 & 5.13875280958041 \\
\hline $\mathrm{Cu}$ & 3.56723974713555 & 20.20462280107190 & 5.10119228479032 \\
\hline $\mathrm{Cu}$ & 3.56723035993418 & 22.67412130778120 & 5.07609390027116 \\
\hline $\mathrm{Cu}$ & 3.56728259582493 & 25.13463818854000 & 5.24701269888563 \\
\hline $\mathrm{Cu}$ & 3.56728054719391 & 27.69752659699340 & 5.07047273338084 \\
\hline $\mathrm{Cu}$ & 3.56729526123428 & 30.24491869584420 & 5.06144638758207 \\
\hline $\mathbf{C u}$ & 3.56727602366371 & 32.77844407106590 & 5.05900419478805 \\
\hline $\mathbf{C u}$ & 7.12830055102266 & 35.30673115921970 & 5.05300713627508 \\
\hline $\mathbf{C u}$ & 7.12788622103179 & 2.51640354740454 & 5.05428541524022 \\
\hline $\mathbf{C u}$ & 7.13176620990429 & 5.04574876834164 & 5.05619972425856 \\
\hline $\mathrm{Cu}$ & 7.13335448751707 & 7.56367443436015 & 5.05479621987964 \\
\hline $\mathrm{Cu}$ & 7.12724586034605 & 10.09216265682500 & 5.04537460197615 \\
\hline $\mathbf{C u}$ & 7.12040456857677 & 12.60844011576590 & 5.05928400634833 \\
\hline $\mathrm{Cu}$ & 7.12697469548029 & 15.13547291745210 & 5.05209691141562 \\
\hline $\mathrm{Cu}$ & 7.13529290944689 & 17.65719375520140 & 5.05939444925717 \\
\hline $\mathrm{Cu}$ & 7.12960899481377 & 20.17987945802500 & 5.05893838918054 \\
\hline $\mathbf{C u}$ & 7.13184634159495 & 22.69589046685720 & 5.04920983313488 \\
\hline $\mathrm{Cu}$ & 7.13575950660189 & 25.21486769096350 & 5.04967782440697 \\
\hline $\mathbf{C u}$ & 7.13185119250274 & 27.73506537866450 & 5.06381182261929 \\
\hline $\mathbf{C u}$ & 7.13778405067095 & 30.25596361723290 & 5.05565327489722 \\
\hline $\mathrm{Cu}$ & 7.13079148938648 & 32.78276003839050 & 5.05788855173896 \\
\hline \multicolumn{4}{|c|}{ CI-NEB Image 27} \\
\hline Element & X-Coordinate (Å) & Y-Coordinate (Å) & Z-Coordinate (§̊) \\
\hline $\mathbf{F}$ & 4.66312385223830 & 12.57924742465230 & 7.73367634854224 \\
\hline $\mathbf{F}$ & 2.47144143117409 & 12.57924548665900 & 7.73365996008043 \\
\hline $\mathbf{F}$ & 3.56729485216573 & 8.80941357754255 & 6.63627296133745 \\
\hline
\end{tabular}




\begin{tabular}{|c|c|c|c|}
\hline $\mathbf{F}$ & 4.66367523889115 & 17.64774372741720 & 7.75038279351476 \\
\hline $\mathbf{F}$ & 2.47088087025930 & 17.64775439582320 & 7.75039385073328 \\
\hline $\mathbf{F}$ & 3.56727970755624 & 14.44924058036750 & 7.29090159070650 \\
\hline $\mathbf{F}$ & 4.64904939821109 & 23.94308119589500 & 7.52718241984622 \\
\hline $\mathbf{F}$ & 2.48552421089570 & 23.94306834460380 & 7.52719916637128 \\
\hline $\mathbf{F}$ & 3.56727675584131 & 19.51975592482610 & 7.32200024607926 \\
\hline C & 3.56728658622126 & 13.02873819485240 & 7.04071477572698 \\
\hline C & 3.56727661296699 & 18.09796407432040 & 7.05775026149829 \\
\hline C & 3.56728150158070 & 23.95942220674950 & 6.70742766192012 \\
\hline $\mathbf{C u}$ & 0.00000000000000 & 0.00000000000000 & 0.00000000000000 \\
\hline $\mathrm{Cu}$ & 0.00000000000000 & 2.52244512012115 & 0.00000000000000 \\
\hline $\mathbf{C u}$ & 0.00000000000000 & 5.04488953395804 & 0.00000000000000 \\
\hline $\mathbf{C u}$ & 0.00000000000000 & 7.56733465407875 & 0.00000000000000 \\
\hline $\mathbf{C u}$ & 0.00000000000000 & 10.08977871477400 & 0.00000000000000 \\
\hline $\mathbf{C u}$ & 0.00000000000000 & 12.61222383489520 & 0.00000000000000 \\
\hline $\mathbf{C u}$ & 0.00000000000000 & 15.13466895501500 & 0.00000000000000 \\
\hline $\mathbf{C u}$ & 0.00000000000000 & 17.65711336885320 & 0.00000000000000 \\
\hline $\mathbf{C u}$ & 0.00000000000000 & 20.17955848897460 & 0.00000000000000 \\
\hline $\mathbf{C u}$ & 0.00000000000000 & 22.70200254966930 & 0.00000000000000 \\
\hline $\mathrm{Cu}$ & 0.00000000000000 & 25.22444766979040 & 0.00000000000000 \\
\hline $\mathrm{Cu}$ & 0.00000000000000 & 27.74689208362480 & 0.00000000000000 \\
\hline $\mathbf{C u}$ & 0.00000000000000 & 30.26933720374600 & 0.00000000000000 \\
\hline $\mathbf{C u}$ & 0.00000000000000 & 232386990 & 0.00000000000000 \\
\hline $\mathbf{C u}$ & 3.56727419238642 & 000000000 & 000 \\
\hline $\mathrm{Cu}$ & 19238642 & 2115 & 0.00000000000000 \\
\hline $\mathbf{C u}$ & 3642 & 5.0448 & 0.00000000000000 \\
\hline $\mathbf{C u}$ & 3.56727419238642 & 7.56733465407875 & 0.00000000000000 \\
\hline $\mathrm{Cu}$ & 3.56727419238642 & 10.08977871477400 & 0.00000000000000 \\
\hline $\mathbf{C u}$ & 3.56727419238642 & 12.61222383489520 & 0.00000000000000 \\
\hline $\mathbf{C u}$ & 3.56727419238642 & 15.13466895501500 & 0.00000000000000 \\
\hline $\mathbf{C u}$ & 3.56727419238642 & 17.65711336885320 & 0.00000000000000 \\
\hline $\mathbf{C u}$ & 3.56727419238642 & 20.17955848897460 & 0.00000000000000 \\
\hline $\mathbf{C u}$ & 3.56727419238642 & 22.70200254966930 & 0.00000000000000 \\
\hline $\mathrm{Cu}$ & 3.56727419238642 & 25.22444766979040 & 0.00000000000000 \\
\hline $\mathrm{Cu}$ & 3.56727419238642 & 27.74689208362480 & 0.00000000000000 \\
\hline $\mathbf{C u}$ & 3.56727419238642 & 30.26933720374600 & 0.00000000000000 \\
\hline $\mathbf{C u}$ & 3.56727419238642 & 32.79178232386990 & 0.00000000000000 \\
\hline $\mathbf{C u}$ & 7.13454838477291 & 0.00000000000000 & 0.00000000000000 \\
\hline $\mathbf{C u}$ & 7.13454838477291 & 2.52244512012115 & 0.00000000000000 \\
\hline $\mathbf{C u}$ & 7.13454838477291 & 5.04488953395804 & 0.00000000000000 \\
\hline $\mathbf{C u}$ & 7.13454838477291 & 7.56733465407875 & 0.00000000000000 \\
\hline $\mathbf{C u}$ & 7.13454838477291 & 10.08977871477400 & 0.00000000000000 \\
\hline $\mathbf{C u}$ & 7.13454838477291 & 12.61222383489520 & 0.00000000000000 \\
\hline $\mathbf{C u}$ & 7.13454838477291 & 15.13466895501500 & 0.00000000000000 \\
\hline $\mathrm{Cu}$ & 7.13454838477291 & 17.65711336885320 & 0.00000000000000 \\
\hline $\mathbf{C u}$ & 7.13454838477291 & 20.17955848897460 & 0.00000000000000 \\
\hline $\mathbf{C u}$ & 7.13454838477291 & 22.70200254966930 & 0.00000000000000 \\
\hline $\mathbf{C u}$ & 7.13454838477291 & 25.22444766979040 & 0.00000000000000 \\
\hline $\mathbf{C u}$ & 7.13454838477291 & 27.74689208362480 & 0.00000000000000 \\
\hline $\mathbf{C u}$ & 7.13454838477291 & 30.26933720374600 & 0.00000000000000 \\
\hline $\mathrm{Cu}$ & 7.13454838477291 & 32.79178232386990 & 0.00000000000000 \\
\hline $\mathrm{Cu}$ & 1.78363955761358 & 1.26122414920059 & 1.26122399999993 \\
\hline $\mathrm{Cu}$ & 1.78363955761358 & 3.78366926932190 & 1.26122399999993 \\
\hline $\mathbf{C u}$ & 1.78363955761358 & 6.30611333001662 & 1.2612239 \\
\hline
\end{tabular}




\begin{tabular}{|c|c|c|c|}
\hline $\mathbf{C u}$ & 1.78363955761358 & 8.82855845013778 & 1.26122399999993 \\
\hline $\mathbf{C u}$ & 1.78363955761358 & 11.35100286397460 & 1.26122399999993 \\
\hline $\mathbf{C u}$ & 1.78363955761358 & 13.87344798409420 & 1.26122399999993 \\
\hline $\mathbf{C u}$ & 1.78363955761358 & 16.39589310421720 & 1.26122399999993 \\
\hline $\mathbf{C u}$ & 1.78363955761358 & 18.91833716491180 & 1.26122399999993 \\
\hline $\mathbf{C u}$ & 1.78363955761358 & 21.44078263817520 & 1.26122399999993 \\
\hline $\mathbf{C u}$ & 1.78363955761358 & 23.96322669886980 & 1.26122399999993 \\
\hline $\mathbf{C u}$ & 1.78363955761358 & 26.48567181899090 & 1.26122399999993 \\
\hline $\mathbf{C u}$ & 1.78363955761358 & 29.00811693910990 & 1.26122399999993 \\
\hline $\mathbf{C u}$ & 1.78363955761358 & 31.53056135294630 & 1.26122399999993 \\
\hline $\mathbf{C u}$ & 1.78363955761358 & 34.05300647307050 & 1.26122399999993 \\
\hline $\mathbf{C u}$ & 5.35091375000000 & 1.26122414920059 & 1.26122399999993 \\
\hline $\mathbf{C u}$ & 5.35091375000000 & 3.78366926932190 & 1.26122399999993 \\
\hline $\mathbf{C u}$ & 5.35091375000000 & 6.30611333001662 & 1.26122399999993 \\
\hline $\mathbf{C u}$ & 5.35091375000000 & 8.82855845013778 & 1.26122399999993 \\
\hline $\mathbf{C u}$ & 5.35091375000000 & 11.35100286397460 & 1.26122399999993 \\
\hline $\mathbf{C u}$ & 5.35091375000000 & 13.87344798409420 & 1.26122399999993 \\
\hline $\mathbf{C u}$ & 5.35091375000000 & 16.39589310421720 & 1.26122399999993 \\
\hline $\mathbf{C u}$ & 5.35091375000000 & 18.91833716491180 & 1.26122399999993 \\
\hline $\mathbf{C u}$ & 5.35091375000000 & 21.44078263817520 & 1.26122399999993 \\
\hline $\mathbf{C u}$ & 5.35091375000000 & 23.96322669886980 & 1.26122399999993 \\
\hline $\mathbf{C u}$ & 5.35091375000000 & 26.48567181899090 & 1.26122399999993 \\
\hline $\mathbf{C u}$ & 5.350913 & 910990 & 399999993 \\
\hline $\mathbf{C u}$ & 000000 & 5294630 & 399999993 \\
\hline $\mathbf{C u}$ & 000000 & 34.0 & 9993 \\
\hline $\mathrm{Cu}$ & 8.91818794238642 & 20059 & 1.26122399999993 \\
\hline $\mathrm{Cu}$ & 8.91818794238642 & 3.78366926932190 & 1.26122399999993 \\
\hline $\mathbf{C u}$ & 8.91818794238642 & 6.30611333001662 & 1.26122399999993 \\
\hline $\mathbf{C u}$ & 8.91818794238642 & 8.82855845013778 & 1.26122399999993 \\
\hline $\mathbf{C u}$ & 8.91818794238642 & 11.35100286397460 & 1.26122399999993 \\
\hline $\mathbf{C u}$ & 8.91818794238642 & 13.87344798409420 & 1.26122399999993 \\
\hline $\mathbf{C u}$ & 8.91818794238642 & 16.39589310421720 & 1.26122399999993 \\
\hline $\mathrm{Cu}$ & 8.91818794238642 & 18.91833716491180 & 1.26122399999993 \\
\hline $\mathrm{Cu}$ & 8.91818794238642 & 21.44078263817520 & 1.26122399999993 \\
\hline $\mathrm{Cu}$ & 8.91818794238642 & 23.96322669886980 & 1.26122399999993 \\
\hline $\mathbf{C u}$ & 8.91818794238642 & 26.48567181899090 & 1.26122399999993 \\
\hline $\mathbf{C u}$ & 8.91818794238642 & 29.00811693910990 & 1.26122399999993 \\
\hline $\mathbf{C u}$ & 8.91818794238642 & 31.53056135294630 & 1.26122399999993 \\
\hline $\mathbf{C u}$ & 8.91818794238642 & 34.05300647307050 & 1.26122399999993 \\
\hline $\mathbf{C u}$ & 0.00082804613130 & 35.31267490828740 & 2.53762633549802 \\
\hline $\mathbf{C u}$ & 10.70009990612060 & 2.52184910179464 & 2.54023345727522 \\
\hline $\mathbf{C u}$ & 0.00246838304992 & 5.04444393925914 & 2.54192423129280 \\
\hline $\mathbf{C u}$ & 10.70049174664560 & 7.56305239138387 & 2.54328841295274 \\
\hline $\mathbf{C u}$ & 10.69829982363110 & 10.08813480428020 & 2.53929159327613 \\
\hline $\mathbf{C u}$ & 10.69974255770940 & 12.61000620807290 & 2.54822484390196 \\
\hline $\mathbf{C u}$ & 10.69571925505330 & 15.13577457725970 & 2.53717716163390 \\
\hline $\mathbf{C u}$ & 10.69902774577170 & 17.65637980647240 & 2.54330612218482 \\
\hline $\mathbf{C u}$ & 10.70129193985120 & 20.18010106215370 & 2.54018757672846 \\
\hline $\mathbf{C u}$ & 10.69120847615160 & 22.69223181055700 & 2.53794115692221 \\
\hline $\mathbf{C u}$ & 10.69540163577360 & 25.22851765883260 & 2.53474782011756 \\
\hline $\mathbf{C u}$ & 0.00057738892418 & 27.74752244574830 & 2.54395174361312 \\
\hline $\mathbf{C u}$ & 10.70164738855780 & 30.26705376861300 & 2.54030389644730 \\
\hline $\mathbf{C u}$ & 10.70134636772670 & 32.78943510908360 & 2.54032182618485 \\
\hline $\mathbf{C u}$ & 3.56730125714698 & 35.31017525203570 & 2.54128835804988 \\
\hline
\end{tabular}




\begin{tabular}{|c|c|c|c|}
\hline $\mathbf{C u}$ & 3.56723272243581 & 2.51994764256773 & 2.54149445799978 \\
\hline $\mathbf{C u}$ & 3.56725889266721 & 5.04140019195885 & 2.53833586096574 \\
\hline $\mathbf{C u}$ & 3.56727581552539 & 7.56895582669007 & 2.57977824321227 \\
\hline $\mathbf{C u}$ & 3.56729386025491 & 10.09055544556690 & 2.58871055028891 \\
\hline $\mathbf{C u}$ & 3.56724908719136 & 12.61710989872930 & 2.57372297664363 \\
\hline $\mathbf{C u}$ & 3.56727379638469 & 15.13846338420060 & 2.58356841864827 \\
\hline $\mathbf{C u}$ & 3.56729658126545 & 17.65755710373220 & 2.58115204080390 \\
\hline $\mathbf{C u}$ & 3.56734094310404 & 20.17014617359870 & 2.57685689408414 \\
\hline $\mathbf{C u}$ & 3.56725144425170 & 22.70391171765570 & 2.56789512796288 \\
\hline $\mathbf{C u}$ & 3.56727645114081 & 25.21878576477900 & 2.57477577385115 \\
\hline $\mathbf{C u}$ & 3.56727360185956 & 27.74453203699770 & 2.54450787783427 \\
\hline $\mathbf{C u}$ & 3.56727905997126 & 30.26604713288990 & 2.54635719083491 \\
\hline $\mathbf{C u}$ & 3.56728412339377 & 32.78735826148270 & 2.54313737095030 \\
\hline $\mathrm{Cu}$ & 7.13368296380231 & 35.31267797538140 & 2.53767385050908 \\
\hline $\mathbf{C u}$ & 7.13625297145697 & 2.52186493718786 & 2.54020314167447 \\
\hline $\mathbf{C u}$ & 7.13209212495740 & 5.04445393368237 & 2.54189499850391 \\
\hline $\mathbf{C u}$ & 7.13591792258717 & 7.56302809026176 & 2.54333136809895 \\
\hline $\mathbf{C u}$ & 7.13814979250130 & 10.08816362180110 & 2.53927596442696 \\
\hline $\mathbf{C u}$ & 7.13662361659860 & 12.61006140975200 & 2.54820111513594 \\
\hline $\mathbf{C u}$ & 7.14064317897257 & 15.13578450509230 & 2.53714787220112 \\
\hline $\mathbf{C u}$ & 7.13737642895272 & 17.65641041974330 & 2.54329995786151 \\
\hline $\mathbf{C u}$ & 7.13508733665626 & 20.18009236206970 & 2.54017992844331 \\
\hline $\mathbf{C u}$ & 0897932080 & 22.69223691096990 & 2.53793525265967 \\
\hline $\mathrm{Cu}$ & 3344964051 & 25.22850515011700 & 583914 \\
\hline $\mathrm{Cu}$ & 8777795 & 18960 & 4269008527 \\
\hline $\mathrm{Cu}$ & 7.13471438893139 & 30.26701918267940 & 2.54032450370893 \\
\hline $\mathbf{C u}$ & 7.13501044122393 & 32.78942599048570 & 2.54033969386546 \\
\hline $\mathbf{C u}$ & 1.78603058002529 & 1.25423612526072 & 3.85034055795956 \\
\hline $\mathbf{C u}$ & 1.78031696104668 & 3.77774899348318 & 3.85175356361330 \\
\hline $\mathrm{Cu}$ & 1.79460618718088 & 6.30305706823522 & 3.85786558798789 \\
\hline $\mathrm{Cu}$ & 1.77814900052735 & 8.82259472763543 & 3.83340977151035 \\
\hline $\mathbf{C u}$ & 1.79794172709766 & 11.34104256108660 & 3.84495687479688 \\
\hline $\mathrm{Cu}$ & 1.77408087990677 & 13.87580703607900 & 3.83467248763380 \\
\hline $\mathbf{C u}$ & 1.78803997073173 & 16.39732825391400 & 3.84841779548257 \\
\hline $\mathbf{C u}$ & 1.77628851842798 & 18.92278260509470 & 3.84405850464564 \\
\hline $\mathbf{C u}$ & 1.79676353247950 & 21.44340172721810 & 3.85507426447547 \\
\hline $\mathbf{C u}$ & 1.75401873720148 & 23.95894817965100 & 3.80421537062826 \\
\hline $\mathbf{C u}$ & 1.79087446531967 & 26.47626003271190 & 3.85817419443773 \\
\hline $\mathbf{C u}$ & 1.78197566377727 & 29.00086744666240 & 3.86014148305940 \\
\hline $\mathbf{C u}$ & 1.78235358513004 & 31.52038453007550 & 3.85658717075920 \\
\hline $\mathbf{C u}$ & 1.78558058628562 & 34.04361574079990 & 3.85226621412377 \\
\hline $\mathbf{C u}$ & 5.34853908267326 & 1.25427280067637 & 3.85032416380239 \\
\hline $\mathbf{C u}$ & 5.35422091086113 & 3.77776463253432 & 3.85167835355106 \\
\hline $\mathbf{C u}$ & 5.33998028112077 & 6.30301863094740 & 3.85779829688869 \\
\hline $\mathrm{Cu}$ & 5.35651873929964 & 8.82259789512809 & 3.83329363164193 \\
\hline $\mathbf{C u}$ & 5.33670001715666 & 11.34103990271730 & 3.84489707977367 \\
\hline $\mathbf{C u}$ & 5.36042429256436 & 13.87584742478080 & 3.83467921766131 \\
\hline $\mathbf{C u}$ & 5.34648806977462 & 16.39735240156480 & 3.84841646784194 \\
\hline $\mathbf{C u}$ & 5.35827388628920 & 18.92279045886280 & 3.84404445674172 \\
\hline $\mathbf{C u}$ & 5.33777962637738 & 21.44334375204280 & 3.85504628326572 \\
\hline $\mathbf{C u}$ & 5.38053677917580 & 23.95888863863890 & 3.80411162696732 \\
\hline $\mathbf{C u}$ & 5.34369634732029 & 26.47627247394570 & 3.85813087799349 \\
\hline $\mathbf{C u}$ & 5.35259904667179 & 29.00089505702730 & 3.86012030221221 \\
\hline $\mathbf{C u}$ & 5.35224167681994 & 31.52037159031560 & 3.85674845026633 \\
\hline
\end{tabular}




\begin{tabular}{|c|c|c|c|}
\hline $\mathrm{Cu}$ & 5.34895922444336 & 34.04362692258550 & 3.85227913501461 \\
\hline $\mathrm{Cu}$ & 8.91813174374388 & 1.25626821523414 & 3.85263213053190 \\
\hline $\mathbf{C u}$ & 8.91816216122949 & 3.77856786867498 & 3.85111615999193 \\
\hline $\mathbf{C u}$ & 8.91821041967704 & 6.29950123506609 & 3.85333217521270 \\
\hline $\mathbf{C u}$ & 8.91821601742917 & 8.82424020772482 & 3.85745507057599 \\
\hline $\mathbf{C u}$ & 8.91815225446297 & 11.35364670010570 & 3.85442100623214 \\
\hline $\mathbf{C u}$ & 8.91815649294470 & 13.87034774889020 & 3.85206689353112 \\
\hline $\mathbf{C u}$ & 8.91818572372205 & 16.39647796090350 & 3.85698596442315 \\
\hline $\mathbf{C u}$ & 8.91819234343654 & 18.92141806130320 & 3.85259052102860 \\
\hline $\mathbf{C u}$ & 8.91818943001978 & 21.43345113003110 & 3.85302895153834 \\
\hline $\mathbf{C u}$ & 8.91820183101858 & 23.95822511248070 & 3.84275335639771 \\
\hline $\mathbf{C u}$ & 8.91818031870280 & 26.48798988820650 & 3.85073958397808 \\
\hline $\mathbf{C u}$ & 8.91817972321530 & 29.00411531267190 & 3.84745982540827 \\
\hline $\mathrm{Cu}$ & 8.91819498015397 & 31.52932073466260 & 3.85446736850017 \\
\hline $\mathbf{C u}$ & 8.91816430758765 & 34.04835309356450 & 3.85224228372686 \\
\hline $\mathbf{C u}$ & 0.00761676830383 & 35.30720672839280 & 5.05323246834629 \\
\hline $\mathbf{C u}$ & 0.00801266810863 & 2.51556050827186 & 5.05399630808931 \\
\hline $\mathbf{C u}$ & 0.00195816012989 & 5.04428531036728 & 5.05564362524519 \\
\hline $\mathbf{C u}$ & 10.70168065470370 & 7.56214367886219 & 5.05512367638085 \\
\hline $\mathbf{C u}$ & 0.00671910174035 & 10.08997149673520 & 5.04539822540831 \\
\hline $\mathbf{C u}$ & 0.01556441378214 & 12.60682826947590 & 5.05849677799051 \\
\hline $\mathbf{C u}$ & 0.00919933599368 & 15.13417702215180 & 5.05124665271159 \\
\hline $\mathbf{C u}$ & 0.00175881343727 & 17.65860497761430 & 2367620 \\
\hline $\mathrm{Cu}$ & 0.00799790060724 & 20.18166897476400 & 60969 \\
\hline $\mathrm{Cu}$ & 0.0036660 & 17950 & 5725107 \\
\hline $\mathrm{Cu}$ & 10.69875921339270 & 25.21869275079780 & 1595868 \\
\hline $\mathbf{C u}$ & 10.70126433579270 & 27.74117891735360 & 5.06313735238462 \\
\hline $\mathbf{C u}$ & 10.69664724149740 & 30.26147203563100 & 5.05811898902784 \\
\hline $\mathbf{C u}$ & 0.00398723462356 & 32.78486139349660 & 5.05798652937023 \\
\hline $\mathrm{Cu}$ & 3.56730388654979 & 35.30044301273670 & 5.05957092482772 \\
\hline $\mathrm{Cu}$ & 3.56732007030201 & 2.50940137415441 & 5.05433793999211 \\
\hline $\mathrm{Cu}$ & 3.56727951855705 & 5.01843233186805 & 5.04672837967523 \\
\hline $\mathrm{Cu}$ & 3.56733112441074 & 7.51664416240829 & 5.15535119986570 \\
\hline $\mathbf{C u}$ & 3.56741158326632 & 10.09296090028820 & 5.14919811900813 \\
\hline $\mathbf{C u}$ & 3.56735421875018 & 12.61050399160020 & 5.11481361030231 \\
\hline $\mathbf{C u}$ & 3.56725141428748 & 15.14257653203440 & 5.09161581713676 \\
\hline $\mathbf{C u}$ & 3.56733122956108 & 17.67529789547550 & 5.12920150284844 \\
\hline $\mathbf{C u}$ & 3.56717691349342 & 20.21723264552690 & 5.11095282716502 \\
\hline $\mathbf{C u}$ & 3.56734615170102 & 22.72531526533710 & 5.13815781302472 \\
\hline $\mathbf{C u}$ & 3.56729623924477 & 25.20920480900460 & 5.15259606295060 \\
\hline $\mathbf{C u}$ & 3.56728168692169 & 27.72273374605670 & 5.06243213089757 \\
\hline $\mathbf{C u}$ & 3.56723562554009 & 30.25359661715420 & 5.06185142655863 \\
\hline $\mathbf{C u}$ & 3.56725718028855 & 32.78016275103910 & 5.05886260047965 \\
\hline $\mathbf{C u}$ & 7.12688023016262 & 35.30733880650000 & 5.05329474106287 \\
\hline $\mathbf{C u}$ & 7.12647452371181 & 2.51577892242352 & 5.05401411369970 \\
\hline $\mathbf{C u}$ & 7.13256941611184 & 5.04437891162803 & 5.05563290642621 \\
\hline $\mathbf{C u}$ & 7.13473322488761 & 7.56217384410670 & 5.05511324765522 \\
\hline $\mathbf{C u}$ & 7.12785201190486 & 10.09000365063460 & 5.04536781409515 \\
\hline $\mathbf{C u}$ & 7.11891665892824 & 12.60695327104390 & 5.05855405662841 \\
\hline $\mathrm{Cu}$ & 7.12532324537050 & 15.13425375511900 & 5.05126771709686 \\
\hline $\mathbf{C u}$ & 7.13280182592191 & 17.65865357422410 & 5.05636505559505 \\
\hline $\mathbf{C u}$ & 7.12659170694584 & 20.18168942623190 & 5.05804720685060 \\
\hline $\mathbf{C u}$ & 7.13083349538984 & 22.69784718056870 & 5.05075912553709 \\
\hline $\mathbf{C u}$ & 7.13759065164080 & 25.21863092477490 & 5.04516200861023 \\
\hline
\end{tabular}




$\begin{array}{llll}\mathbf{C u} & 7.13509923717727 & 27.74110631984350 & 5.06314796163822 \\ \mathbf{C u} & 7.13975046474616 & 30.26142413984810 & 5.05822311493348 \\ \mathbf{C u} & 7.13057197508415 & 32.78490550099390 & 5.05801266098298\end{array}$

